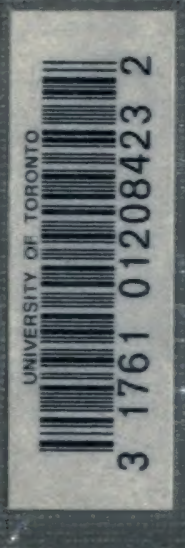




Digitized by the Internet Archive in 2008 with funding from Microsoft Corporation 


(92)

$I$

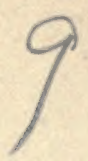



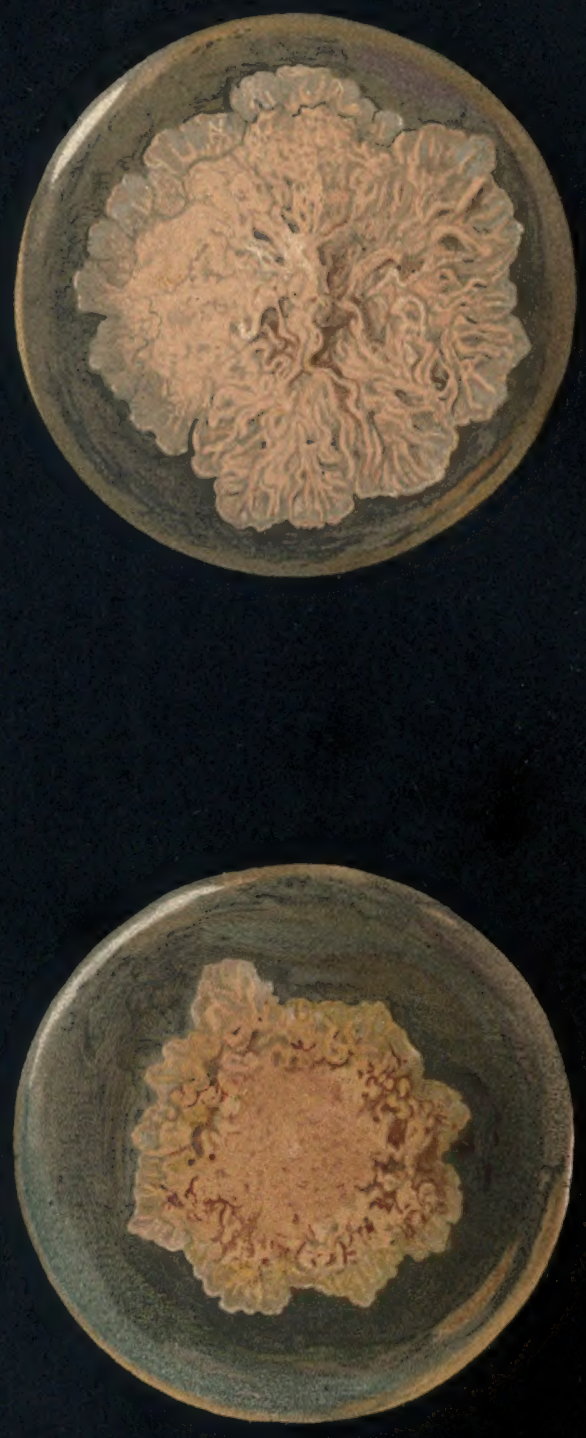


\section{Bacteriology of Milk}

BY HAROLD SWITHINBAYK

we tas macrian Acid-fast bacillus from butter of Berlin

(Petri-Rabinowitsch).

Flask culture on glycerine-agar-3 months at $22^{\circ} \mathrm{C}$.

GBORGE NPWMA Single colony-actual sizeD.P.

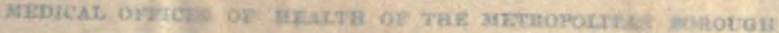

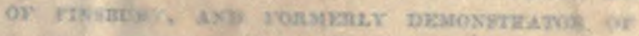

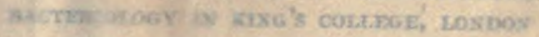

WITH SPBCIAL CHAPTERS ALSQ 8 Y DR NEWMAN ON THE SPREAD WY DISEASB BY. MILK AND THE CONY OF TMB MILK SUPPLY

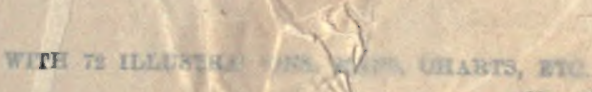

Acid-1 bacillus from milk of Belzig (A. Moeller).

Flask culturn glycerine-agar-3 months at $22^{\circ} \mathrm{C}$.

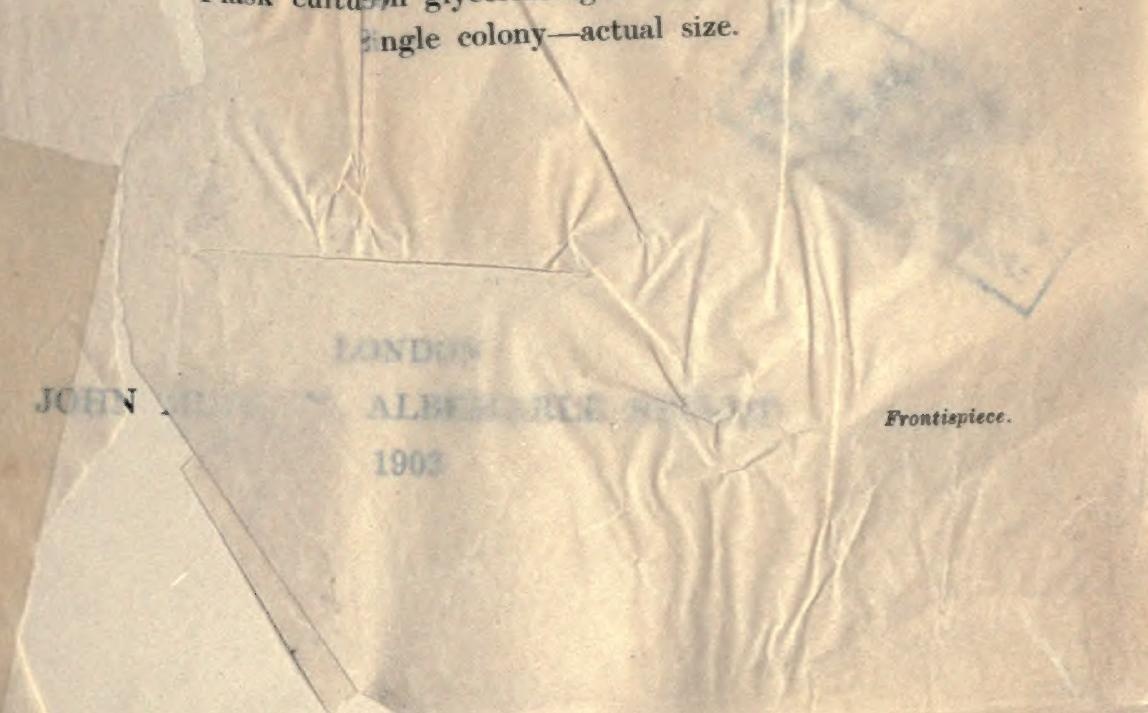




\section{Bacteriology of Milk}

\section{BY HAROLD SWITHINBANK}

OF THE BACTERIOLOGICAI RESARCR LABORATORY, DEVTAM

AND

GEORGE NEWMAN, M.D., F.R.S.E., D.P.H.

MEDICAL OFFICER OF HEALTH OF THE METROPOLTTAN BOROLGH

OF FISSERT, AND FORMERLY DEMONSTRATOR OF BACTERIOLOGY IN KTKG'S COLIJGE, LONDON

WITH SPECIAL CHAPTERS ALSO BY DR NEWMAN ON THE SPREAD OF DISEASE BY MILK AND THE CONTROL OF THE MILK SUPPLY

WITH 72 ILLUSTRATION8, MAPS, CHART8, ETC.

\section{LONDON}

JOHN MURRAY, ALBEMARLE STREET 


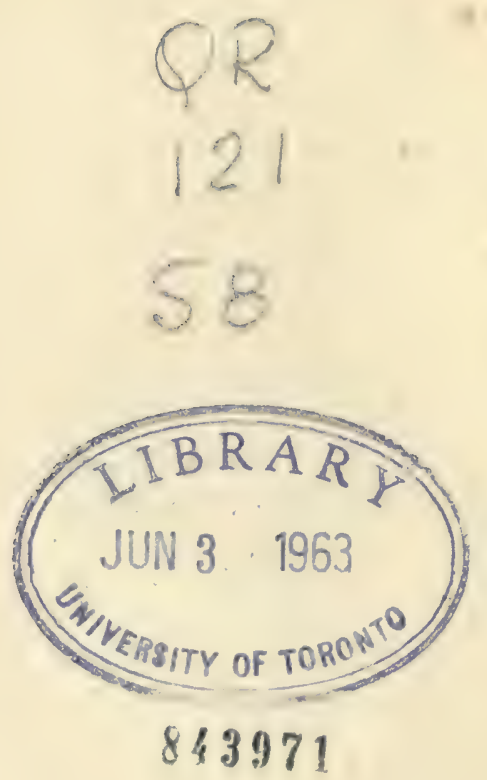

wiollest 


\section{DEDICATED}

TO

LORD LISTER 



\section{PREFACE}

THIS is, we believe, the first occasion on which an attempt has been made to deal in a systematic manner with the bacteriology of milk, a subject which is year by year engrossing more of the attention of the public and of the medical profession, on account of the advances which have been made in preventive medicine in general and bacteriology in particular.

We commenced the serious work involved in writing the present volume in 1899, and have thus spent, in available time at our disposal, a period of some five years. The length of this period is due in part to the ever-widening field which had to be covered, and in part to unavoidable delays. Whilst this is to be regretted, there is perhaps the advantage that no part of the work has been hastily done. Yet for obvious reasons, the chief of which is the extremely rapid growth of bacteriology, much of the work must be looked upon as provisional only. The conditions affecting bacteria in nature, the potentiality of bacteria in butter and cheese making, and the species of bacteria belonging to milk are subjects still requiring much more investigation than they have hitherto received. The practical and applied aspect of the bacteriology of milk also claims much fuller inquiry. For instance, comparatively little is known of the relationship between disease in the cow and its transmission to man by means of milk. Questions of preventive medicine and of the control of the milk supply are also still open to revision. We are under no illusion as to finality or completeness in this volume. Few, probably, know better than ourselves of the 
immensity of the field which he must study who would learn even the elementary principles controlling the life and action of microorganisms in milk. Our aim has been to systematise the bacteriological study of milk rather than incorporate every known or tested fact concerning the subject. For those who desire to follow the matter more minutely we commend the references in the footnotes and the Bibliographia Lactaria.

The chapters on technique have been written in more detail than is perhaps usual in books of this nature. But, anyone who has worked at practical bacteriology is aware how necessary it still is to describe, with minuteness, the different steps taken. We have also added a chapter on the examination of air and water as they affect the milk supply.

The relationship existing between milk-borne disease and the bacteriology of milk is, of course, intimate and essential, and, therefore, several chapters have been devoted to pathogenic bacteria in milk and milk-borne epidemics. This has naturally led on to the questions of prevention and control. Whilst we recognise that these latter questions have no direct relation to the bacteriology of milk, the indirect relation is so strong, and a wise control depends so entirely upon a knowledge of the bacteriology of milk, that we have decided on the whole that it is most convenient to all concerned to bind up the chapters on the academical and practical sides of the subject in one volume.

Whilst the authors share jointly the views expressed throughout the book, it should be understood that to Dr Newman has fallen the writing of the chapters on the pathogenic organisms in milk and the control of the milk supply. The whole of the laboratory work necessary in the production of the book has been done in $\mathrm{Mr}$ Swithinbank's laboratories at Denham, and he is also responsible for the photographs, with the exception of the micro-photographs, which were taken from our own preparations by Dr Harold Spitta of the Bacteriological Department of St George's Hospital.

We owe our thanks to Dr Mervyn H. Gordon of the Bacteriological Laboratories of St Bartholomew's Hospital for the four 
hitherto unpublished photographs of the Streptococcus scarlatine; to Dr D. S. Davies of Bristol for the two graphic-method charts illustrating milk-borne outbreaks ; to Mrs George Newman for the water-colour drawings of cultures and the text illustrations drawn from apparatus used by us; and to various workers too numerous to name, in England, France, Germany, and America, who have responded to our requests for information. We are also under obligation to the American Government and various State Boards for many official reports. In conclusion, we desire to express our appreciation of the unfailing courtesy and assistance of the publisher.

LoNDON, October 1903. 



\title{
CONTENTS
}

\author{
CHAPTER I
}

SOME GENERAL CONDITIONS AFFECTING BACTERIA IN MILK

General Properties and Composition of Milk:-The Carbohydrates of Milk; the Proteids or Albuminoids of Milk; the Fats and Salts of Milk. Chemical Composition of Milk Products. The Physiology of Milk:-The Secretion of Milk and Influences bearing upon it; Variation in the Composition of Milk-(1) The Race of Cow ; (2) the Period of Lactation; (3) Kind of Fodder ; (4) Seasonal Variations ; (5) Intervals between Times of Milking. Milk as a Medium 1 for Bacteria:-The Sources of Bacteria in Milk; Elements of Contamination. The Pathology of Milk

\section{H A P T E R I I}

THE BACTERIOLOGICAL EXAMINATION OF MILK: METHODS AND TECHNIQUE

Culture Media. Quantitative Examination of Milk and Method of Milk Dilution. The Making and Staining of Preparations for the Microscope. Qualitative Examination of Milk :-Aërobic Examinations. Method of Examination of Special Organisms. Special Methods. Routine Procedure in Examination of Milk . - 30,

\section{CHAPTER I I}

\section{ANAËROBIC ORGANISMS: THEIR ISOLATION AND CULTURE}

General Note. Cultivation in Hydrogen and in vacuo. Hydrogen Production. Methods of Isolation and Culture . . . . 


\section{CHAPTER I V \\ EXAMINATION OF AIR AND WATER IN RELATION TO THE MILK SUPPLY}

Examination of Air-General Note. Air-borne Organisms. Seasonal Variations. Air of byres, dairies, milk-shops, etc. Methods of Examination. Examination of Water: Quantitative and Qualitative. Pathogenic Organisms in Water

\section{CHAPTER V}

\section{THE BACTERIAL CONTENT OF MILK}

Numbers of Bacteria present in Milk. The Milk of Towns. Natural Conditions affecting Bacteria in Milk: ( $\mathrm{I}$ ) The Influence of Temperature. (2) The Influence of Time. The Toxicity of Milk. (3) The Inter-relationship of Bacteria in Milk and its Germicidal * Power. Bacteria in Separated Milk. Species of Bacteria present in Milk .

\section{CHAPTER VI}

\section{FERMENTATION IN MILK}

General Introductory Notes. Kinds of Fermentation:-1. Lactic Acid Fermentation; 2. Butyric Acid Fermentation; 3. Alcoholic Fermentation; 4. The Coagulative Fermentations; 5. Diseases of Milk: (1) Blue Milk, (2) Red Milk, (3) Yellow Milk, (4) Bitter Milk, (5) Soapy Milk, (6) Ropy Milk. The Prevention of Milk Anomalies

\section{CHAPTER VII}

\section{ECONOMIC BACTERIA IN MILK AND MILK PRODUCTS}

Cream Ripening. Bacteria in Butter-making. Control of the Ripening Process. Bacteria in Cheese-making. Number of Bacteria in Cheese. Kinds of Bacteria present in Cheese. The Use of Artificial Cultures in Cheese-making. Abnormal Cheese Ripening and Poisonous Cheese. 


\section{CHAPTER VIII}

\section{PATHOGENIC BACTERIA IN MILK. TUBERCULOSIS AS A TYPE}

Infective Diseases of the Cow communicable to Man by Milk. Patho-

PAGR genic Bacteria in Milk. Clinical Evidence; Bacteriological Evidence; The Tubercle Bacillus in Milk and Butter. Streptococcus in Milk. Tuberculosis as a Type. The Specificity of Tuberculosis; The Biology of the Bacillus Tuberculosis. Tubercle Bacilli of Bovine and Human Origin compared. Polymorphism; Pseudo-Tuberculosis. Bovine Tuberculosis; The Entrance of Tubercle Bacilli into Milk. Virulence of Milk containing Tubercle Bacilli. Powers of Resistance of Tubercle Bacillus. The Acid-Fast Bacilli allied to the Tubercle Bacillus .

\section{CHAPTER IX}

\section{PATHOGENIC BACTERIA IN MILK (continued)}

Introductory Note. Signs and Characteristics of Milk-borne Epidemics:-(I) Special Incidence, $(a)$ Local, $(b)$ as regards Social Position of those attacked, $(c)$ Incidence on Milk-drinkers, $(d)$ Age and Sex Incidence; (2) Incubation; (3) Sudden Onset and Rapid Decline ; (4) Clinical Characters ; (5) Mortality-rate in Milk-borne Disease .

\section{CHAPTER X}

\section{PATHOGENIC BACTERIA IN MILK (continued)}

Scarlet Fever. The Hendon Outbreak. Channels of Infection. Abstracts of Typical Epidemics. Typhoid Fever: the Vehicle of Infection, Bacteriology, and Channels of Infection. Abstracts of Typical Epidemics. Diphtheria: the Question of Bovine Diphtheria. The Bacillus of Diphtheria and Milk: Channels of Infection. Abstracts of Typical Epidemics. Throat Illnesses spread by Milk. Epidemic Diarrhœea and Milk. The Bacteriology and Conditions of Diarrhœea. The Relationship of Milk to Epidemic Diarrhœe. Preventive Measures. Cholera

\section{CHA PTER XI}

\section{THE INVESTIGATION AND PREVENTION OF MILK-BORNE EPIDEMICS}

The Investigation of Milk-borne Epidemics. Methods of Prevention. Modern Bacteriological Methods applied to Milk-borne Disease: Diagnosis and Preventive Inoculation . 


\section{CHAPTER X I I}

DESCRIPTION OF SOME SPECIES OF MILK BACTERIA

0. Description, Differentiation, Cultural Characters, etc., of Bacteria found in Milk.

\section{HAPTER XIII}

THE CONTROL OF THE MILK SUPPLY:(i.) BY THE STATE

General Note. Milk Legislation in England and Wales. Model Milk Clauses. The Local Authority and the Milk Supply. Milk Legislation in Scotland: In Ireland: On the Continent of Europe: In the United States of America : In the British Colonies

\section{CHAPTER XIV}

THE CONTROL OF THE MILK SUPPLY : (ii.) BY PRIVATE ENTERPRISE

The Aylesbury Dairy Company, London. The Dairy Supply Company of Copenhagen. The Danish Milk Company. The Milk Supply Pasteur, Copenhagen. The Philadelphia Milk Commission. The Rotch System.

\section{CHAPTER X V}

THE CONTROL OF THE MILK SUPPLY: (iii.) BY THE TRADE

General Note; (I) Milk Herds:-Freedom from Disease, Inspection, Bacteriological Examination, Tuberculin ; (2) The Housing of Milk Herds; (3) Milkers and Milking; (4) Treatment of Milk after Milking :-Clean Milking, Straining, Cooling, Transport; (5) The Final Treatment of Milk:-Filtration, Preservation, Pasteurisation, Sterilisation; the Retail Sale. Summary . . . . 504 


\section{A P PEN DICES}

A.-Elementary Glass Blowing with Bunsen Flame . . . 547

B. - The Contagious Diseases (Animals) Act, 1878 . . . 550

C.-Public Health (London) Act, 1891 . . . . . . . 551

D. -The Dairies, Cowsheds and Milk-shops Order of 1885 . 555

E.-The Dairies, Cowsheds and Milk-shops Amending Order, I886 • 559

F.-The Dairies, Cowsheds and Milk-shops Order of 1899 . . 56I

G.-Model Regulations : Dairies, Cowsheds and Milk-shops Order . 562

H.-Infectious Diseases (Prevention) Act, 1890 . . . . 567

I.-Public Health Act, 1875 . $\quad$. . . . 568

J.-City of Liverpool-Tuberculosis and Milk . . . . . 569

K.-City of Manchester-Farmers' Circular . . . . 582

L.-Borough of Sunderland-Milk Certification . . . 576

M.-Public Health (Scotland) Act, 1897 . . . . 578

N.-The Aylesbury Dairy Company, Limited-Form $A$. . $\quad 581$

O.-The Aylesbury Dairy Company, Limited-Form $B \quad$. 582

P.-The Aylesbury Dairy Company, Limited-Form C . . 584

Q.-Copenhagen Dairy Company's Regulations ․ . 589

R.-Tuberculosis in London Cowsheds; Manchester, Liverpool, and Melbourne • • • • . . . . 592

S.-The Danish Method of Milk Supply in England . . . 594

T.-Infant Milk Depôts . . . . . . . 596

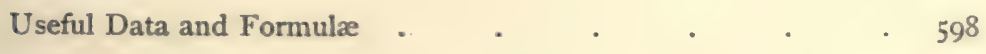

INDEX $\quad . \quad . \quad . \quad . \quad . \quad . \quad . \quad 599$ 



\section{LIST OF ILLUSTRATIONS}

\section{PLATE ILLUSTRATIONS}

No.

I. Colonies of Acid-Fast Bacilli from Butter (Petri-Rabinowitsch), and Milk (Moeller)

- Chromo. frontispiece

2. Constituent Elements of Milk - - . To face page 8

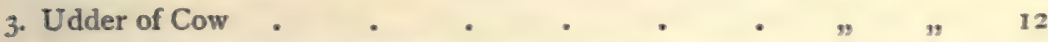

4. Air Pollution in Towns, and Tyndall's "Raft Theory" " " 22

5. Sources of Contamination in Milk-Air . . " " 24

6. Sources of Contamination in Milk-Water . . " " 26

7. Method of Drawing and Storing Sterile Milk . - " " " 42

8. Pakes' Counting Disc, etc. . . . . . " " 60

9. Flasks for Anaërobic Cultivation - . - " " " 78

10. Comparative Air Plates-(a) Country Air, (b) London

Air . . . . . . Between pp. 100 and 101

II. Comparative Air Plates-(c) Labourer's Cottage in

Bucks, (d) Tenement House in London - . " " "

12. Comparative Air Plates-(e) London Milk-Shop, $(f)$

Country Milk-Cooling Room

13. Apparatus for Bacteriological Examination of Air - To face page IO4

I4. Typical Milk Plates on Nutrient Agar (a) and (b)

I5. Typical Milk Plates on Nutrient Agar $(c)$ and $(d)$ Between pp. 130 and 131

16. Typical Milk Plates on Nutrient Agar (e) and $(f)$

17. Typical Milk Plates on Nutrient Agar $(g)$ and $(k)$ )

132 and 133

18. Relative Distribution of Organisms in (i) Set Milk

and Cream; (ii) Separated Milk and Cream . To face page I 44 xrii 
No.

19. Relative Distribution of Organisms in (iii) Milk before

Separation, and Separator Bowl Sediment . . To face page 146

20. Hyphomycetes (moulds) . - . . . " " 202

21. Microphotographs; B. tuberculosis; Perlsucht; Staphy-

lococcus ; Diplococcus • • • • " " 222

22. Colonies of Acid-Fast Bacilli. Woodbury-type-Between pp. 234 and 235

23. Power of Resistance of the B. tuberculosis . . To face page 252

24. Colonies of Acid-Fast Bacilli from Butter (Korn I. and II.) - . . . C Chromo. " " 254

25. Colonies of Acid-Fast Bacilli from Butter (Binot and Grassberger) . . $\quad$ - $\quad$ C Chromo. 256

26. Microphotographs - Aroma Bacterium (Weigmann);

B. butyricus (Hueppe); B. cyanogenes (Flügge);

$B$. erythrogenes (Grotenfeld) .

27. Microphotographs - Acid-Fast Bacillus from Butter

(Petri-Rabinowitsch) and B. lactis niger (Gorini) .

400

28. Microphotographs-B. Friburgensis (Korn I. and II.).

418

29. Acid-Fast Organisms from Milk and Butter; Tube

Cultures

30. Varieties of $B$. tuberculosis; Tube Cultures

426

3I. Microphotographs - Staphylococcus pyogenes aureus;

Streptococcus pyogenes; $B$. actinobacter; Micrococcus Freudenreichii

32. Microphotographs-Streptococcus scarlatince (Gordon)

, $\quad$,

33. Microphotographs-Streptococcus scarlatina (Gordon) 


\section{TEXT ILLUSTRATIONS}

No.

1. Potato Borer

PAGR

2. Roux Potato Tube

3. Milking Tube and Accessories .

4. Wire Cage Stand for Petri Dishes

5. Xylol Washing .

6. Conical Glass for Staining

7. Simple Holder for Stain Filtration

8. Heating Stage .

9. Slide prepared for Flagella Staining

ro. Cornet's Forceps for Glass Slides

11. Isolation by Stroke Culture

12. Sterile Platinum Needle in Protecting Glass Tube . . . 67

13. Metzger's Centrifuge . . . . . . . . 76

14. Gärtner's Centrifuge - . $\quad$ - . $\quad$ - 76

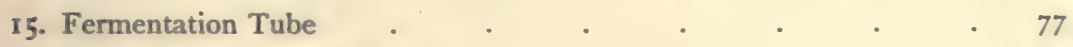

16. Best form of Screw Pinchcock . $\quad$. $\quad$. $\quad$. 80

17. Combined Hydrogen Apparatus and Vacuum Pump . . . 8 I

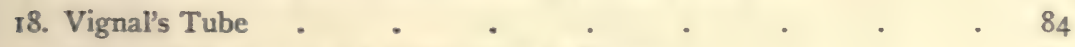

19. Roux's Tube for Liquid Cultivation in Vacuo . $\quad . \quad$. 85

20. Buchner's Tube . . . . . . . . 87

21. Platinum Spatula for Klein's Method . . . . 88

22. Vacuum Desiccator for Petri Dish Cultivations $\quad$. $\quad$ - 89

23. Vacuum Desiccator for Test-Tube Cultures . . . $\quad$. 89

24. Esmarck Roll Culture . . . . . . . . 90

25. Fraenkel's Tube . $\quad$. $\quad$. . . . . . 9 . 9

26. Yeast Flask Culture in Hydrogen . . . . . 92

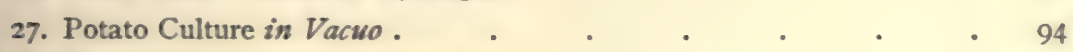

28. Agar Culture in Vacuo . . . . . . . . . . 94

29. Pasteur's Tubes for Cultivation of Anaërobes . . . . 95

30. Air-Filtering Tube . . . . . . . . 103

31. Laveran's Tube for Air Analysis . . . . . . 105

32. Apparatus of Strauss and Wurtz . . . . . 106

33. Sedgwick's Tube. . . . . . . . . . 109

34. Frankland's Air Tube . . . . . . . . Iro

35. Pasteur Pipettes . $\quad$ - . . . . 547 


\section{MAPS AND CHARTS}

No.

I. Chart illustrating the Spread of Enteric Fever by Infected

$$
\text { Milk . . . . . To face page } 380
$$

II. Chart illustrating the Spread of Scarlet Fever by Infected

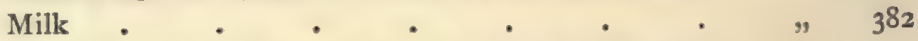

III. Map illustrating Sources of Milk Supply of a London District . . . . . . " , 462

IV. Chart illustrating Relationship of Contractor to Milk Supply , " 466 


\title{
BACTERIOLOGY OF MILK
}

\author{
CHAPTER I
}

\section{SOME GENERAL CONDITIONS AFFECTING BACTERIA IN MILK}

General Properties and Composition of Milk:-The Carbohydrates of Milk; the Proteids or Albuminoids of Milk; the Fats and Salts of Milk. Chemical Composition of Milk Products. The Physiology of Milk:-The Secretion of Milk and Influences bearing upon it; Variation in the Composition of Milk-(I) The Race of Cow ; (2) the Period of Lactation; (3) Kind of Fodder; (4) Seasonal Variations; (5) Interval between Times of Milking. Milk as a Medium for Bacteria:-The Sources of Bacteria in Milk; Elements of Contamination. The Pathology of Milk.

BEFORE entering upon the study of milk from a bacteriological point of view it is necessary to consider briefly some of its physical and physiological characters. The further our research has led us the more convinced have we become of the influence, direct and indirect, of the external and internal conditions of milk upon its bacterial flora. The physiological and biological characters of milk and the varied conditions of dairying, and not simply the species of bacteria which happen to gain access to the milk, demand the careful and systematic study of the bacteriologist. It may be that in the future we shall find that much more is dependent upon these external conditions than upon particular species of bacteria. It has been said that upwards of two hundred distinct species of ordinary milk bacteria have been found and described. Without reflecting in the least upon the excellent work which such results prove, we would venture to suggest in the first place that many of these so-called "milk bacteria" are in reality purely saprophyticexisting naturally in soil and dust, in manure, in hay and straw, and in the general environment of cattle-rather than specifically related to milk; and in the second that a more careful study of the exact biological characters, conditions, and life history of known species would prove more fruitful and of greater permanent service 
to the bacteriology of the dairy than further extensive additions to the already formidable number of species. Hence our investigations have been specially devoted to such a general study rather than to the isolation of supposed new organisms, or of such as do not exactly answer to any of the already published descriptions of bacteria. We would indeed go so far as to suggest that the more the conditions of growth, culture, and environment are studied the more is it likely that there will be a reduction rather than an increase in the number of clearly differentiated species of milk organisms.

However that may be, and however much or little such a result will in the future be due to clearer nomenclature, we are convinced that a careful study of those conditions which in one way or another affect all bacteria, and especially bacteria in milk, is of the first importance to the bacteriologist in a clear understanding of their rôle.

\section{General Properties and Composition of Milk}

It is desirable then to state briefly the chief physical and chemical properties of milk. ${ }^{1}$ Milk consists, like the blood, of two main elements, a fluid or milk plasma, and suspended therein innumerable minute globules of fat. It is therefore when fresh a perfect emulsion, and its white colour is produced, as in other emulsions, by reflection of light from the surface of the globules. In addition to the fat globules there are also in suspension smaller particles of proteid matter. ${ }^{2}$

The globules of fat vary in size, but probably the average diameter of the globules in cow's milk is about $\frac{1}{500}$ of an inch. The size varies considerably with different breeds, and even with different cows. As a rule the fat globules of Jersey and Guernsey milk are large and uniform, and of the Ayrshire small and variable. Lloyd has, however, pointed out that the presence of exceptionally large fat globules in milk is rare, and uniformity in size is the rule (varying from or to $.0016 \mathrm{~mm}$. in diameter). He states that in Jersey milk the globules appear to have a narrow range of variation in size whilst in Shorthorn the range is considerable. ${ }^{3}$ The number of globules in a given volume of milk varies greatly according to their size and in the percentage of fat.

${ }^{1}$ It is only necessary in a book of this character to refer to those points which have a relationship direct or indirect to bacteria in milk. The general chemistry of milk will be found recorded in Foods: Their Composition and Analysis (Wynter Blyth), 1903, pp. 189-310.

${ }^{2}$ Text-book of Physiology, edited by E. A. Schäfer, F.R.S. (W. D. Halliburion, F.R.S., p. 125.)

${ }^{3}$ Journal of Bath and West of England Society, vol. xii. (1902), p. 128. 
Babcock pointed out a number of years ago that the chief differences in the composition of normal milks are due to variations in the amount of fat, the milk plasma being fairly uniform in all milks. The variation in the amount of serum solids in milk from the same cow is rarely more than $\frac{1}{2}$ per cent., in milk from different cows of the same breed usually less than I per cent., and in milk from cows of different breeds not more than $2 \frac{1}{2}$ per cent. ${ }^{1}$

Statements have been somewhat frequently made to the effect that each globule of milk fat possesses a capsule or membrane composed of caseinogen, the rupture of which by churning, it was supposed, enabled the fat to assume the form of butter. There is now, however, considerable evidence in support of the view that each fat globule by molecular attraction is surrounded by a more closely adherent layer of caseinogen solution (milk plasma). Béchamp holds that each globule is surrounded by a true membrane. Storch, on the other hand, maintains that the membrane, the existence of which he admits, is not a true capsule but a gelatinous mucoid ground substance. The general consensus of opinion among chemists is probably opposed to the view of Béchamp, though further evidence is necessary before concluding as to the exact nature of the layer around the fat globules. From observation with the microscope it would appear that the fat globules in milk are nearly always distributed unevenly in, and not uniformly throughout, the plasma. It is, however, almost impossible to examine milk quite naturally and this grouping may arise from mechanical causes.

The reaction of milk must be described on the whole as amphoteric. The milk of most animals is normally alkaline, but in the carnivora fresh milk has an acid reaction. Normal acidity or alkalinity in milk is due to the presence of acid or alkaline phosphates respectively, the amphoteric reaction to the presence of both. The herds of cows which feed on certain grasses in various parts of the world, such, for example, as the blue-grass region of Kentucky, are found to give a slightly alkaline milk, and by experiment it has been found that a ration of 10 lbs. a day of sugar beets given to a cow will render her milk slightly alkaline. As is well known, in a comparatively short time, owing to lactic acid or other fermentations, milk assumes a definitely acid reaction. The specific gravity varies from 1028 to 1034 (average 1032). It is approximately the same in cow's and human milk. ${ }^{2}$

1 Wisconsin Agricultural Experiments, Bulletin 18 (1899), p. 34 .

2 See also footnote on p. I54. 
The percentage constituents of milk may be approximately stated as follows :-
I. Water
2. Carbohydrates (lactose, etc.)
$84-87$
3. Proteids or Albuminoids (casein, albumin, etc.) .
4-5
$4-4 \cdot 5$
$3 \cdot 9$
4. Fats
5. Salts

There are in addition traces of extractives (urea, creatinin, lecithin, cholesterin, etc.), and a small quantity of oxygen, nitrogen, and carbon dioxide.

Picton and Linder have shown that there is no sharp dividing line between colloidal substances in milk and substances in complete solution. But broadly speaking we may represent the various conditions as follows:- The fat is in suspension, the casein in pseudo-solution, the albumin in solution as a colloid, and the lactose in solution as a crystalloid. These four states are probably due to the size of the conglomerates of molecules or particles (Richmond).

The carbohydrates of milk. - The most important carbohydrate present in milk is milk-sugar or lactose $\left(\mathrm{C}_{12} \mathrm{H}_{22} \mathrm{O}_{11}+\mathrm{H}_{2} \mathrm{O}\right)$. This is a member of the cane-sugar group and is found in varying quantities in the milk of most, if not all, mammals except the Egyptian buffalo (Richmond and Pappel). It is the most constant constituent of milk, about 5 per cent. being present in human milk and 4 per cent. in that of the cow. It possesses only a faint sweet taste, and is much less soluble than cane sugar, being soluble in six parts of cold, and two or three parts of hot, water. It crystallises in the form of rhombic prisms. Lactose is very resistant to the inverting ferment of yeast and therefore undergoes alcoholic fermentation very slowly. It is unacted upon by diastase, rennet, pepsin, and trypsin, but is rapidly inverted by the kephir fungus (lactase). Of all the sugars it is most readily affected by the micro-organisms which are the causal agents in lactic acid fermentation. This fermentation occurs in two stages :

$$
\begin{aligned}
& \text { I. } \mathrm{C}_{12} \underset{\text { (lactose) }}{\mathrm{H}_{22} \mathrm{O}_{11}}+\mathrm{H}_{2} \mathrm{O}={ }_{4} \mathrm{C}_{3} \mathrm{H}_{6} \mathrm{O}_{3} \\
& \text { 2. }{ }_{\text {(lactic acid) }} \mathrm{C}_{3} \mathrm{H}_{6} \mathrm{O}_{3}=\mathrm{C}_{4} \mathrm{H}_{8} \mathrm{O}_{2}+2 \mathrm{CO}_{2}+2 \mathrm{H}_{2}
\end{aligned}
$$

To this matter we shall have occasion to refer at a later stage.

A point of some interest and importance is the non-assimilability of lactose. Like cane-sugar and maltose, the other two chief members of the cane-sugar group, lactose is incapable of assimilation, for when injected into the blood-vessels it appears unaltered 
in the urine. Presumably when lactose is ingested it is changed in the alimentary canal into some form of sugar, possibly dextrose or galactose, which is assimilable. ${ }^{1}$ Very large amounts of dextrose can be absorbed into the blood without producing glycosuria in a normal animal, unless, indeed, the assimilative powers have been reduced, as, for example, by starvation. The change from unassimilable lactose to some assimilable form may take place rather during than before the passage of the sugar through the intestinal walls. This non-assimilability of lactose is certainly remarkable when it is remembered that it in this form that young animals receive their carbohydrate food.

The proteids or albuminoids of milk are now generally considered to be four, namely, casein, lactalbumin, lacto-globulin, and Storch's mucoid. The first and by far the most abundant is casein, or as it occurs in milk, according to Professor Halliburton, caseinogen. ${ }^{2}$ It is this proteid which is acted upon by rennet and converted into casein. The lactalbumin and lacto-globulin only exist in small quantities (about one sixth to one seventh of all the proteid) and of Storch's mucoid there is only a trace. For all practical purposes, indeed, we may say that the albuminoids of milk are two, casein and lactalbumin. During lactic acid fermentation-due to lactic acid bacteria-the caseinogen of milk is precipitated, and certain nther fermentative bacteria are able to produce the same effect. But the agency by which coagulation is most readily formed is that of rennet. Rennet is an enzyme produced in the stomachs of mammals, and generally prepared from the fourth stomach of the calf. Caseinogen is not, as we have already pointed out, in perfect solution in the milk plasma, but is present in all probability along with the fat in both the milk globules and the proteid particles, in a state of minute division, and in partnership with phosphate of lime. This opalescent solution has been credited with causing, in part, the whiteness of the milk. The globules contain a maximum of fat and a minimum of caseinogen. The particles contain a minimum of fat and a maximum of caseinogen. In nature caseinogen is probably never seen apart from fat, and in milk the fat never apart from caseinogen. The two substances appear to be produced side by side by the metabolic activity of the protoplasm of the cells of the acini of the mammary gland, so that caseinogen

1 The Chemical Basis of the Animal Body, by Sheridan Lea, F.R.S., 1892 edition, P. II5.

${ }^{2}$ Caseinogen may be precipitated from milk by the addition of such acids as acetic, or by saturation with salts such as sodium chlorideand magnesium sulphate. 
is not a chemical entity sui generis, it and the fat together constituting a highly complex organic substance. ${ }^{1}$

Details concerning the exact chemical constitution of casein, lactalbumin, lacto-globulin, and mucoid are not necessary to our purpose here, which is merely a brief survey of the general constitution of milk. Such details may be obtained from any standard work on organic chemistry.

The fats of milk.-Milk fat consists of palmitin (about 25 per cent.), myristin (about 20 per cent.), and olein (about 35 per cent.), with small quantities of the tri-glycerides (ethereal salts of glycerol), of butyric, caproic, caprylic, capric, stearic, and other fatty acids. The amount of fat in cream varies from 15 to 50 per cent. In butter, besides fat, there are small quantities of proteid and lactose, and it is important to remember that the fat itself is characterised by the presence in considerable quantity of glycerides of the fatty acids, the most important being butyric acid. The fat of cow's butter averages 68 per cent. of palmitin and stearin, 30 per cent. of olein, and 2 per cent. of the specific butter fats. Their melting point is $3 \mathrm{I}-34^{\circ} \mathrm{C}$. The composition of good butter is, however, variable. It should not contain more than about I2-I4 per cent. of water. The amount of butter fat in butter prepared from cow's milk is about 85 per cent. and there are, of course, small quantities of casein and salts.

Fat, as is stated above, occurs in milk in the form of small globules, varying in size from $.01 \mu$ to $.0016 \mu$ in diameter. According to Rothschild a drop of milk not larger than a pin's head contains $1,500,000$ separate fat globules. Milk fat is not soluble in water, but owing to its fine division is easy of digestion. Its decomposition is the first step in the rancidity of butter, which is set up by fat being split up into fatty acids and glycerol.

As, of course, is perfectly well known, the fat of milk, being lighter than the water, rises to the surface as cream whenever milk is allowed to stand. Such cream may be skimmed off, leaving behind skim milk which, although being milk devoid of its cream, still retains a small percentage of fat. By centrifugal separation the fat of milk may be almost wholly removed. The result is known as separated milk. Forty-five to fifty per cent. of fat in cream has been found to be the normal (Vieth, Richmond, etc.).

By a simple experiment Lloyd ${ }^{2}$ has shown that it is the large globules of fat which form the cream. He took a vessel 15 inches

1 Brit. Med. Jour., 1898, vol ii., p. 779.

2 Jour. of Bath and West of England Society, vol. xii. (1902), p. 129. 
high, and allowed some Shorthorn milk to stand for twelve hours, after which the fat globules at different stages were examined by the microscope and so measured. The result was as follows :-

\begin{tabular}{|l|c|c|c|c|}
\hline & \multicolumn{3}{|c|}{ Diameter in Micro-millimetres of Fat Globules. } \\
\cline { 2 - 4 } & At bottom. & 5 inches up. & 10 inches up. & 10 to 16 inches up. \\
\hline Largest & 4 & $\begin{array}{c}7-8 \\
2\end{array}$ & $\begin{array}{c}8-10 \\
2\end{array}$ & $\begin{array}{r}12 \\
2\end{array}$ \\
\hline
\end{tabular}

Lloyd has also made observations, as we have seen, on the size of fat globules in the milk of various species of cows. He finds a marked uniformity in the size of the globules in each sample, the milk from Jerseys having a narrow range of size and that from Shorthorns a wider range.

The Salts present in cow's milk have been represented by Soldner thus :-

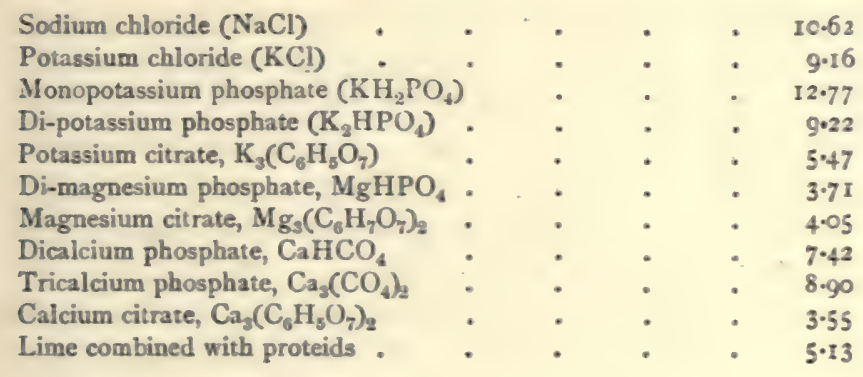

Of the total phosphoric acid from 36 to 56 per cent, and of the lime from 53 to 72 per-cent. is not simply dissolved in the fluid, but is united more or less firmly in the caseinogen. It will be noted that 13 per cent. of the mineral ingredient is constituted of compounds of citric acid. Besides the bodies mentioned above, milk also contains certain organic compounds of the nature of urea, hypoxanthine, and other nitrogenous basic substances. Béchamp has described a starch-liquefying enzyme, and Babcock and Russell a proteolytic enzyme, in milk.

Chemical composition of milk products.-We may briefly summarise the chemical characters of the various products of milk, of which what has been said already applies as a general 
standard. The following is König's table setting forth the differences succinctly:-

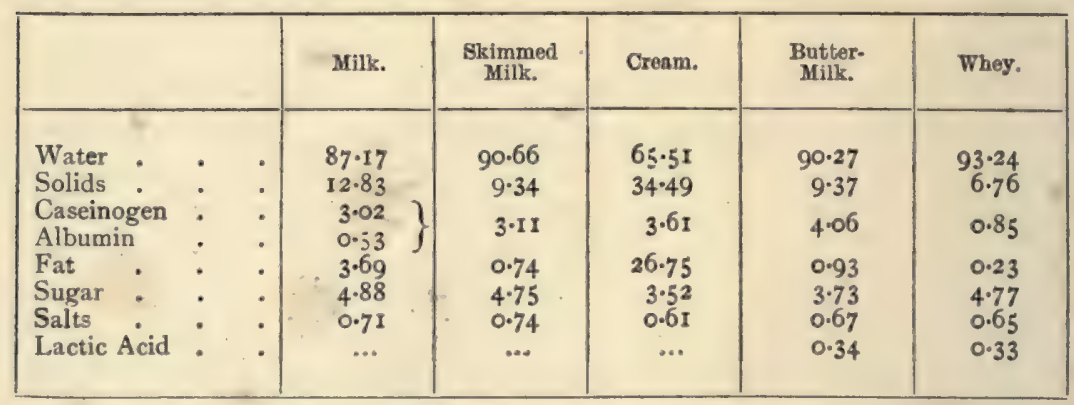

These have been briefly referred to already with the exception of the butter-milk and whey. Of the former it may be said that it is the fluid remaining after the fat has been removed from cream by churning: its sourness is due to lactic acid; its proteids are present in finely flocculent form. Whey is the watery fluid which exudes from clotted milk and possesses a small degree of nutriment.

The physiology of milk.-Under this heading we propose, for convenience, to deal with two matters, leaving for treatment elsewhere the question of the secretion of milk, and the circumstances affecting it. The two points to which reference will be made here are the curdling and coagulation of milk and the effects of heat upon it, apart from the question of sterilisation, to which reference will be made at a later stage.

The natural curdling of milk is brought about by the precipitation of caseinogen, owing to the action of lactic acid fermentation. The caseinogen is broken from its union with lime salts, and not being soluble is thrown down in bulk. At first the precipitation is partial and gives an appearance of flakiness and flocculence. But eventually it is complete, the separation being even more definite than in the clotting of blood. The accompanying Plate illustrates the natural curdling of milk almost to perfection. This particular case occurred in the laboratory, where incidentally a bottle of whole milk had been left for some months at rest. At the top is seen the cream (or fat): in the middle is the clear serum (milk plasma): and at the bottom is the curdled precipitate (caseinogen).

Such curdling which invariably occurs in standing milk, though milk is rarely allowed to remain for a sufficiently long period to provide such a clear demonstration, must not be confused with the 
coagulation of milk, which is an altogether different change. Coagulation occurs when milk is brought, either in the stomach or outside the body, into contact with rennin, the active ferment of rennet. Rennet is produced in the stomachs of mammals, and is generally prepared from the fourth stomach of the calf. It acts on casein only in neutral or acid solution. The larger the amount of acidity which the milk possesses, the more will be the action. It requires for its efficient action the presence of calcium salts, generally calcium phosphate (Hammarsten), from which latter body it is with difficulty separated. "The action of rennet is to split up the casein into a dyscaseose, the calcium compound of which is insoluble and which forms curd and a soluble caseose" (Richmond). If calcium salts be completely removed, no insoluble dyscaseose is produced. The curd carries down with it the fat entangled with the casein : the whey contains the other proteids, sugar and salts of milk. Probably the entire fermentation consists of two stages, the first, by which the ferment changes caseinogen into casein; the second, by which the calcium salt precipitates the casein as curd. The clotting of caseinogen is due then to a specific action of the enzyme and not to the formation of lactic acid from milk-sugar. When milk is clotted the separation is so complete that no casein is found in the whey. ${ }^{1}$

Effects of Heat.-When milk is heated various changes occur. Coagulation is facilitated, changes occur in the fat globules, the flavour and colour are altered and a skin forms on the surface at about $60^{\circ} \mathrm{C}$. This "scum" consists in part of coagulated lactalbumin and in part of caseinogen and salts. These latter bodies would appear to become separated by heat, and the caseinogen becomes entangled in the fat and carried to the surface where, by evaporation, it forms part of the dried wrinkled scum or skin. The brown colour which occurs is probably due to the burning of the sugar (caramel).

So many and varied seemed to be the views accepted on this question that we made a series of simple investigations which we may briefly mention. Our only justification for differing from various authorities is that we have experimented again and again and arrived at certain results. We found that milk taken off the fire as "boiling" by the ordinary cook or nurse rarely exceeds $90^{\circ} \mathrm{C}$, and often falls short of this, the custom being to judge by the

${ }^{1}$ The Chemical Basis of the Animal Body, by Sheridan Lea, F.R.S., Pp. 2 I-22. See also Jour. of Physiology, 1890, pp. 307, 369, 378, 448; and Jour. of Anatomy and Physiology, 1894-95, p. 188. 
naked eye appearance of surface bubbling. In the experiments in question, the temperature was taken by independent individuals at the moment of the supposed boiling milk being taken off the fire by the cook. In only one case did the temperature exceed $90^{\circ} \mathrm{C}$.

Milk scalded as above has a pronounced odour and flavour, and the temperatures at which milk heated with ordinary precautions against burning, etc., changes in odour and flavour we found to be as follows :-

Up to $80^{\circ} \mathrm{C}$. No perceptible change.

Between $80^{\circ}$ and $85^{\circ}$. A slight change in odour and flavour can be perceived.

Between $85^{\circ}$ and $90^{\circ}$. A decided scalded flavour and odour becomes perceptible.

At $90^{\circ}$. . . The flavour and odour are pronounced, and become accentuated as the temperature rises to $100^{\circ} \mathrm{C}$.

A noteworthy point with regard to the above is that the resultant cream from the heated milk undergoes no change in flavour unless the milk temperature has exceeded $90^{\circ} \mathrm{C}$.

It should also be noted that milk heated in an ordinary pan begins to change flavour at a lower temperature than if heated in a water-lined pan such as is now in frequent use for milk purposes.

The objections on the part of the public to boiled milk as an article of food are chiefly the following :-

I. That milk so treated has distinctly a "cooked" taste.

2. The formation of surface scum on the milk.

The former it is difficult to avoid altogether when milk is heated to a temperature above $80^{\circ} \mathrm{C}$., but the latter, being an oxidation product, can be almost wholly avoided by the simple expedient of not exposing the boiled milk to the air until it has cooled down to below $60^{\circ} \mathrm{C}$.

If the following simple rules are observed in heating milk, the change in flavour will be but slight, and the formation of surface scum practically nil :-

I. Use an ordinary double milk-pan, or a smaller covered saucepan containing the milk placed inside a larger one containing the water.

2. Let the water in the outer pan be cold when placed on the fire.

3. Bring the water up to boiling point, and maintain it at this for three or four minutes without removing the lid of the inner milk-pan.

4. Cool the milk down quickly by placing the inner pan in one or two changes of cold water without removing the lid.

5. When cooled down aerate the milk by stirring well with a spoon.

Boiling milk does not materially affect its power of clotting in the stomach, nor does it materially affect its digestibility (Cautley). 
Certain recent experiences, however, tend to show that it may not be wise wholly to ignore the theory put forward some years ago by Barlow that children fed exclusively on boiled milk are inclined to develop a scorbutic tendency, and it is possible that further examination of the physiological changes which milk undergoes in process of heating might tend to throw some light upon this. These may be briefly summarised as follows :-

$\mathrm{Up}$ to $70^{\circ} \mathrm{C}$. there is no apparent change, but about this temperature a change takes place in the albumin : it is not precipitated, but it is converted into a form which is easy of precipitation by acids, magnesium sulphate, and other precipitants of casein. The view most generally held that casein and albumin exist in milk in combination with bases explains the behaviour of albumin in heated milk. The mucoid proteid of Storch loses water at, and probably below, $70^{\circ} \mathrm{C}$.

At or a little below $80^{\circ} \mathrm{C}$. the milk begins, as we have seen, to alter in flavour, and certain organised principles (enzymes), the nature of which is not fully known, undergo a change. The presence of these principles in normal milk, and in milk which has been heated up to a point not exceeding $80^{\circ} \mathrm{C}$, and the fact that they undergo a change when this temperature has been passed may be shown by the following simple experiment:- If a small quantity of a I per cent. solution of Hydroquinone is added to milk at varying temperature up to $80^{\circ}$, and hydrogen peroxide is then dropped into the solution, a distinct rose coloration, turning later to a crushed strawberry tint, will take place on the milk being allowed to stand a few moments, a reaction which is absent if the milk has been raised to a temperature above that point.

At about $100^{\circ} \mathrm{C}$. calcium citrate may be deposited in a slight degree, and if the milk be kept for some time at this temperature slight oxidation sets in, with production of traces of formic acid, and a deposition of salts takes place on the fat globules, causing them to rise but slowly to the surface when the milk is subsequently cooled. The alteration in taste is more marked, and fusion of the fat globules occurs.

It should be mentioned also that milk which has been raised to the boiling point is resistant to the action of rennet.

The secretion of milk and influences bearing upon it.-The mammary gland forms milk from the materials derived through the lymph from the blood, and cannot be regarded simply as filtering apparatus. It is well known that the gland cells are functionally active only for a certain period after parturition. The whole 
gland becomes enlarged by an increase in the size of existing, and possibly, by the formation of new, alveoli. Within the alveolar cell granules of a proteid and fatty nature become collected, and the alveoli become filled with a clear fluid which contains a few fatty globules and composite corpuscles. These latter cells are amoeboid and probably derived from migrated leucocytes. Their occurrence produces a thickened slimy secretion known as colostrum, which is yellower and more alkaline than fully-formed milk, and sometimes contains a certain amount of blood.

Schäfer ${ }^{1}$ gives the exact composition of colostrum by various authorities as follows:-

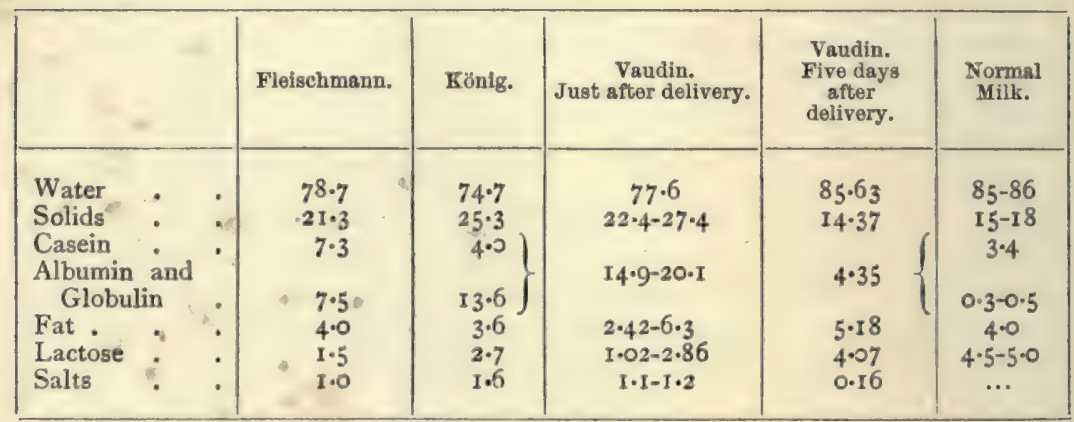

Colostrum therefore differs from milk in that it contains less sugar, a fat which is very poor in volatile acids, and a high percentage of nitrogenous compounds. Milk containing colostrum is not used for dairy purposes; from three to five days should elapse after parturition before the milk is employed for human consumption. It may be useful for reference to append the following table, for the data of which Houdet is responsible :-

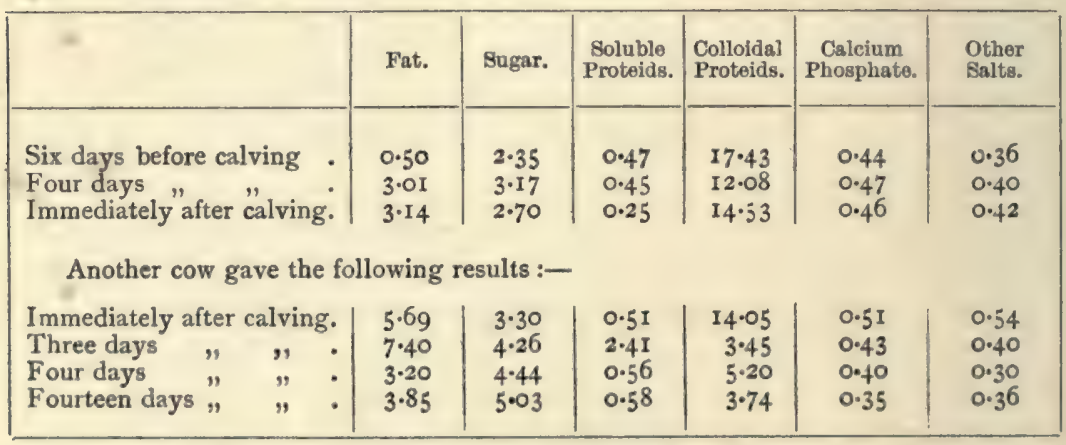

${ }^{1}$ Text-book of Physiology. E. A. Schäfer, F.R.S., p. 129. 
PI.ATE 3.

COW'S UDDER.

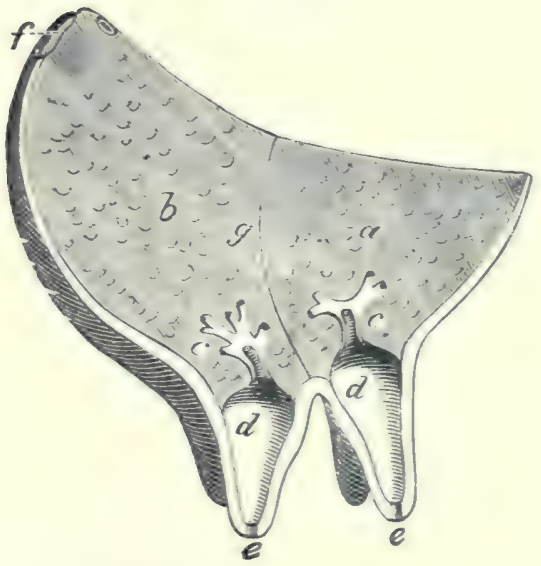

Section of Udder of Cow.

The seation shows the anterior quartor ( $($ ) separatel from the posterior quarter $(b)$ by a septum (g). At the top of the pjsterior quarter is situate 1 a large lymph gland ( $D$. Near the centre of each gland an'l at the base of the teat there is a single large lactiferous sinus or unilk cistern $(d)$, which is the general conflnent of all the lactiferuus ducts $(c, c)$, and opens externally throngh the teat by a single excretory canal (e). This canal is widest at its commeneement and nsrrow at its termination at the end of the teat. The walls of the latter are very thick, elastic, and retractile.
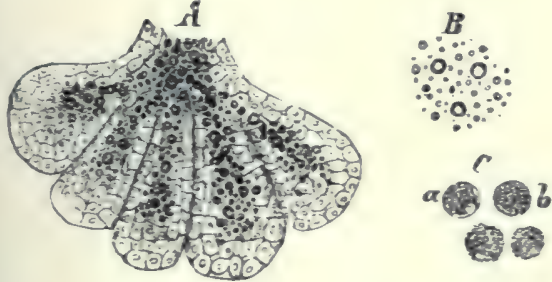

Section of Gland Lobule.

$A$, Lobule of mamma filled with milk. $B$, Milk globules. $C$, Colostrum- $(a)$ cell with a visible nucleus, (b) cells from which nucleus has disappeared.

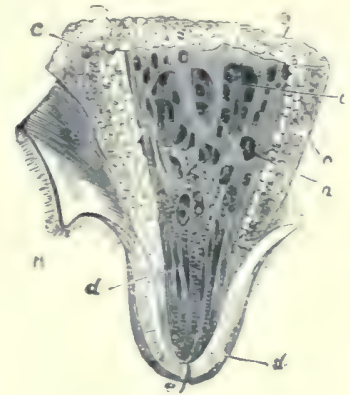

Section of Teat.

$a, a$, Principal lactiferous ducts; $b$, lactiferous sinus; $c, c$, acini; $d$, elastic or dartoid tissue; $e$, oritice of teat.

The dartoid tissue acts as a sphincter, preventing the passive escape of the mill from the oritices of the excretory ducts. If a small camnula be inserted beyond the orifice, the secretion imuediately flows. 

During lactation the alveoli secrete milk in the intervals of suckling so that the milk accumulates both in the alveoli and in the ducts, which, in some animals, are provided with sacs and dilatations which serve as reservoirs. The later drawn portions of milk contain more solids, in proportion, than that which is first drawn.

The exact source of the various constituents comprising fullyformed milk is not known. The probabilities are that the casein is produced by a molecular change in the composition of the serum albumin or globulin, which is supplied to the cells from the blood or lymph. The fat may be formed from proteid, or even carbohydrates, present in the blood, or it may be taken up directly from fat which has been formed elsewhere. There is evidence to show that the sugar is derived from dextrose.

Variation in the composition of milk.-It is obvious that whilst we know generally speaking the standard quality of milk, such standard is not strictly applicable to all kinds of milks. We must bear in mind that in nature, and quite apart from adulteration, we have constantly to deal with considerable variations from the normal standard. These variations affect the constitution of the milk rather as regards its percentage composition than as regards its constituents. The chief causes for natural variation of milk are :-

(I) The race of cow - The milk of different herds varies within comparatively wide limits. Probably the best all-round good milk is obtained from dairy Shorthorns or their cross. Jersey and Guernsey cattle yield a milk having large fat globules which readily rise to the surface. The characteristics of their milk are the high percentage of fat and the richness of colour combined with a liberal quantity. Kerrys and Dexter Kerrys yield a milk approaching in standard that of the Shorthorn, the latter of the two however being as a rule richer in fat. The milk of Ayrshire cattle contains a high percentage of casein, but is low in fat. The globules are of small average size and the milk rises slowly and has comparatively little colour. In Devons the quality is good but the quantity small, while the Holstein, on the other hand, gives a large quantity of milk of comparatively poor quality. In the emulsion of milk from Jersey cows the corpuscles are much larger than in the Holstein, while the Holstein emulsion, when broken down, is much more easily restored; that is, it emulsifies better and resists a destruction of its emulsion, such as occurs when cream is produced, more than do the milks of Jerseys and Guernseys. The emulsion 
of the latter is easily broken up, more apt to produce oil drops on the top of the vessel, and is not so easily restored. These facts are of importance in relation to the transportation of milk and the examination of milks and cream from transported cows. ${ }^{1}$

The following table may be added as an illustration of chemical differences in the milk composition of various herds of cattle:-

\begin{tabular}{|c|c|c|c|c|c|c|c|}
\hline \multicolumn{3}{|c|}{ Breed. } & Total Solids. & Fat. & Milk Sugar. & Proteids. & Ash. \\
\hline \multicolumn{3}{|c|}{ American Grade Shorthorn } & $13 \cdot 17$ & $4 \cdot 0 \mathrm{r}$ & $4 \cdot 36$ & 4.06 & 0.74 \\
\hline Ayrshire. & . &.$\quad 0$. & $12 \cdot 70$ & 3.68 & 4.84 & $3 \cdot 48$ & 0.69 \\
\hline Devon & . & . & 13.21 & 4.09 & $4 \cdot 32$ & 4.04 & 0.76 \\
\hline Guernsey & & . & 14.48 & 5.02 & 4.80 & 3.92 & 0.75 \\
\hline Holstein & . & 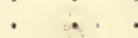 & 12.12 & $3.5 I$ & $4 \cdot 69$ & $3 \cdot 28$ & 0.64 \\
\hline Jersey & & . & $14 \cdot 34$ & $4 \cdot 78$ & 4.85 & 3.96 & 0.75 \\
\hline Shorthorn & & . & $12 \cdot 45$ & 3.65 & 4.80 & $3 \cdot 27$ & 0.73 \\
\hline Brown Swi & & . & 13.06 & 4.00 & $4 \cdot 30$ & 4.00 & 0.76 \\
\hline
\end{tabular}

A somewhat similar table has been constructed by Droop Richmond, and is as follows:-

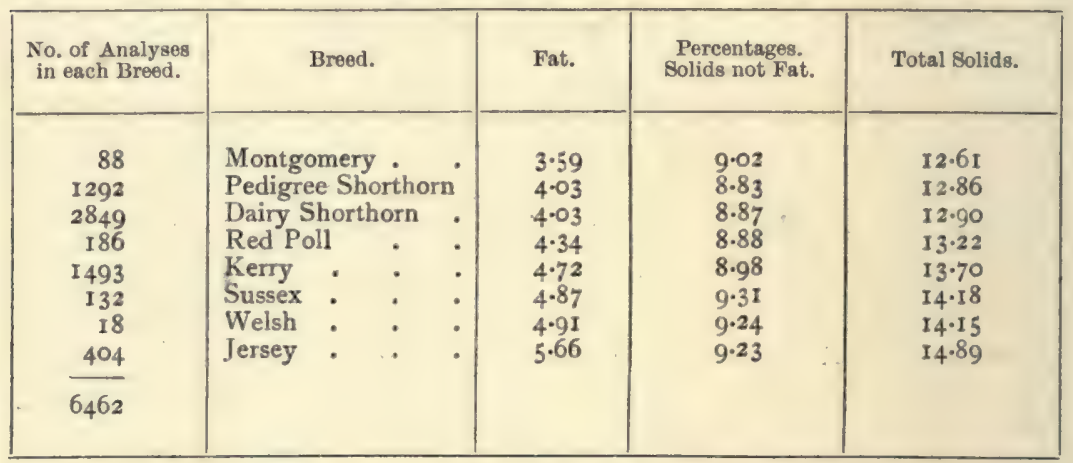

(2) The period of lactation.--Age per se exerts a certain influence upon the quality of the milk. A cow not only arrives at a time of maximum yield as regards quantity of milk, but also as regards quality. Yet a finer distinction may be drawn, for the quality undergoes considerable change according to the period of time which has elapsed since calving. There is, as referred to above,

1 For American breeds of dairy cattle and their chief distinguishing characteristics, see U.S. Dep. of Agriculture, Fifteenth Ann. Rep. Bureau of Animal Industry, 1898, pp. I36-200. 
the colostrum, which is the first milk secreted in lactation, and the constitution of which has been fully described. Some of its characters are carried on into the first weeks of lactation. During the first two months after delivery the casein and fat are increased in amount. The sugar is diminished during the first month, but increased from the eighth to the tenth. The salts increase up to the third month, after which they steadily diminish. ${ }^{1}$ The changes may be expressed in a general way by saying that every newlycalved cow gives the richest milk the first week after calving. About the second or third or fourth week the milk falls fairly rapidly to the normal. From this time forward the cow gradually moves upwards in milk standard until near the drying period (Spier).

(3) Kind of fodder.-The nature of the diet exerts considerable influence upon the quantity and quality of the secretion. ${ }^{2}$ The amount of casein is increased by a nitrogenous diet, owing to the assimilation of proteid. It is possible that fat may be increased in the same way but in a less degree. Food rich in carbohydrates, e.g. beetroot, carrot, etc., causes an increase of the amount of sugar in the milk. The value of forage is determined by its yield, composition, palatability, season of the year in which it may be grown, time required for it to mature, and its effect upon the flavour of milk. ${ }^{3}$

(4) Seasonal variations. - Distinct variations according to season may also occur. Richmond divides the year into four periods having the following characteristics :-

I. November, December, and January-the milk is rich, both in fat and in solids not fat.

2. February, March, and April-the solids not fat do not show appreciable diminution, but the fat becomes less in quality.

3. May, June, July, and August - the fat is low, though there is a tendency to rise at the end of the period. In July and August the solids not fat are below the average.

4. September and October-an improvement in quality both in fat and solids not fat is noticed.

A period of drought tends, while diminishing the yield, to raise

1 Text-book of Physiology. Landois \& Stirling. Fourth Edition, p. 428.

2 Departmental Committee (Board of Agriculture) on Milk and Cream Regulations (1901), p. 7. Sections 24 and 30 , also footnote.

${ }^{3}$ Special Report after six years' investigation. New Jersey Agricultural Experiment Station (1902). See also Sixteenth Annual Report of the Bureau of Animal Industry (U.S.A.), $1899, \mathrm{pp} .450-463$, on Feed and Care of the Dairy Cow. 
the percentage of fat in milk, and the solids not fat frequently decrease. The average quality of milk is usually lower during the spring and early summer, that is, especially during the months of April, May, and June, than at other times of the year. Analyses of the milk supplied to the Aylesbury Dairy Company show that, on the average of ten years, the percentage of fat is lowest in May and June, the months coming next being April and July. The period of lowest quality may, however, vary slightly in particular years. The relative poverty of milk in spring is mainly due to two causes, viz., the number of freshly-calved cows coming in the ordinary course into milk at that time, and the stimulating effect of the first flush of grass exciting an increased flow and a consequent lowering of quality.

(5) Interval between times of milking.-Perhaps the greatest variation in the quality of milk as regards fat is that which arises from the difference in the interval of time which elapses between one milking and another. That the milk yielded by the cow in the morning is almost invariably less rich in milk fat than that yielded in the evening is a well-known fact. It may be accepted as a general axiom that the longer the interval the greater is the quantity of milk and the lower is its quality, and vice versa. If the day were divided into two equal periods, this difference between the evening and the morning milk would be greatly reduced. But such an equal division is, for various reasons, impracticable. ${ }^{1}$

(6) Miscellaneous circumstances.-There are a number of minor circumstances which are held to influence the composition of milk, such as change of milkers, methods and rapidity of milking, exposure to rain, unusual excitement, or temporary or trivial illnesses. Thus individual animals of the same herd have a continually varying milk capacity and quality. This point in regard to a cow's individual variation in quality of milk supply is one of some importance. It may be well illustrated by two features to which attention has recently been called by Professor Rotch of Harvard. ${ }^{2}$ In the first place, not only do the conditions we have discussed affect the individual cow, but the quality of the milk varies chemically as well as bacteriologically in the course of a single milking. We shall have occasion to refer to the bacterial variations

${ }^{1}$ See also Droop Richmond's Annual Returns for the Aylesbury Dairy Company-Jour. of State Medicine, vol. viii. (1900), p. 830, and Jour. of Brit. Dairy Farmers Assoc., vol. xv.

${ }^{2}$ Brit. Med. Jour, 1902, vol. ii., p. 657. 
elsewhere. The chemical variation is shown in the following table :-

\begin{tabular}{|c|c|c|c|c|c|}
\hline $\begin{array}{l}\text { Stages in Milking } \\
\text { Period. }\end{array}$ & & Fat. & Total Solids. & Water. & Mineral Matter. \\
\hline $\begin{array}{l}\text { Fore Milk } \\
\text { Middle Milk . } \\
\text { Strippings . }\end{array}$ & : & $\begin{array}{l}3 \cdot 88 \\
6 \cdot 74 \\
8 \cdot 12\end{array}$ & $\begin{array}{l}13 \cdot 34 \\
15 \cdot 40 \\
17 \cdot 13\end{array}$ & $\begin{array}{l}86.66 \\
84 \cdot 60 \\
82 \cdot 87\end{array}$ & $\begin{array}{l}0.85 \\
0.31 \\
0.82\end{array}$ \\
\hline
\end{tabular}

Boussingault, Heaton, Dyer, and Richmond have given other illustrations of this point. ${ }^{1}$

In the second place, though it is well known that cream from different herds yields a varying percentage of fat, it is probably not so well known that the cream of the same herds and same cows varies from day to day in such percentage. For example, it has been found that a cow will yield a cream containing 6 per cent. of fat on one day and 8 per cent. on the next day.

\section{Milik as a Medium for Bacteria}

The most elementary definition of bacteria describes them as living vegetable cells of microscopic size, and composed of two chief parts, a body or cell substance, and a capsule or cell wall. It is generally held that they contain no nucleus. But in some species internal differentiation of protoplasm has been demonstrated. Bacteria certainly contain no chlorophyll. The cell substance consists of protoplasm, 84 per cent., which is composed of a nitrogenous body called myco-protein, which in its turn is half composed of carbon, and contains about 15 per cent. of nitrogen and half as much hydrogen. It differs from ordinary proteid, according to Nencki, in not being precipitated by alcohol, and in not containing sulphur.

The protoplasm of some species of bacteria, however, contains in addition to myco-protein other substances, such as starch, sulphur, or pigment. The cell membrane is in some species composed of cellulose $\left(\mathrm{C}_{6} \mathrm{H}_{10} \mathrm{O}_{5}\right)$, but in others it is believed to be formed by a concentration of the body protoplasm.

Hence it is clear that to maintain their vitality and fulfil their functions like other vegetable cells, bacteria require carbon, hydrogen, nitrogen, oxygen, certain salts, and water. Parasitic and saprophytic bacteria will, of course, differ from each other in many of the conditions of their nutrition. The source from which all plants containing chlorophyll derive their carbon is the carbonic

1 Dairy Chemistry, H. Droop Richmond (1899), pp. 128-135. 
acid $\left(\mathrm{CO}_{2}\right)$ of the atmosphere (or in case of submerged plants that which is held in solution by the water), which is decomposed under the influence of sunlight by the chlorophyll-containing cells. Vegetable cells not furnished with chlorophyll are unable thus to decompose carbonic acid. Hence they are compelled to derive their carbon, hydrogen, and nitrogen directly, and at first hand, from more complex organic compounds. These elements are chiefly derived by bacteria from proteids and carbohydrates, which form therefore their staple diet. We might roughly express the favourable diet of bacteria in three words, a neutral (or alkaline) proteid solution. But, as a matter of fact, their nutrition cannot be arranged quite so readily as this. For, in regard to their diet, bacteria reveal very great differences indeed-differences not only between species but between individuals of a species, and even these individuals differ under various extrinsic circumstances. What suits one species will not suit another species; what suited one species some hours or days ago will not suit it now. These differences are due, not only to the specific myco-protein of various species, but often to the effect which the organism has had upon its special medium-e.g. the production of lactic acid and proteolytic enzymes. Again, while some bacteria are capable of growing and multiplying upon simple nitrogenous compounds, such as ammonia or urea, many other show no growth whatever under such conditions. Common saprophytic bacteria are examples of the first class, and pathogenic bacteria (parasitic) are examples of the second, these latter thriving only in the presence of albumen or albuminoid nitrogenous material. As regards the amount of albuminous material, some, like the common water bacteria, flourish on little; whereas others, accustomed to live on more copious nitrogenous soil, if transferred to ordinary water would diminish and eventually die. The greater the amount of albuminoid material present, the greater will be found, cateris paribus, the capability of multiplication. ${ }^{1}$

As regards the lowest limit of proteid matter possible to sustain bacterial life no exact figures can be quoted. Most bacteria flourish in media containing $\frac{1}{2}$ per cent., yet some will not grow if the proteid matter is much below 2 per cent. $B$. tuberculosis and $B$. diphtherice require a comparatively large amount of albuminoid material; and some organisms can grow well on no higher organic material than starch (Prazmowski).

1 Pathology and Etiology of Infectious Diseases (Stevenson \& Murphy), vol. ii., p.17, E. Klein, M.D., F.R.S. 
In addition to carbon and nitrogen, salts are requisite in a favourable food for bacteria. Sulphates, phosphates, sodium chloride, potassium, and calcium salts all seem necessary for full development. These substances are presented to bacteria in solution in water.

Briefly, then, we may summarise the full diet of bacteria as nitrogenous matter (proteids); non-nitrogenous matter containing carbon and hydrogen (carbohydrates); calcium, potassium, phosphates, etc. (salts); and, for some species, oxygen.

When we turn our attention to milk as a medium for bacteria, what do we find? We find a complete bacterial diet-proteids represented by casein and lactalbumin, 4 per cent. in total-carbohydrates represented by lactose, the most readily affected of all the sugars by bacteria; fat as palmitin and olein; salts, potassium and calcium largely as phosphates, the calcium phosphate being united with casein. Even the normal reaction of milk, neutral or amphoteric, is favourable to the growth of bacteria, most of which find a definitely acid or a definitely alkaline reaction inimical to their growth. It is true that changes, mostly of a fermentative nature, rapidly set in, which affect milk as a medium for bacteria. But in its fresh, normal, untreated condition we have theoretically an almost ideal medium for both saprophytic and parasitic bacteria. Notwithstanding the truth of this general statement, we must not pass over the experiments of Fokker, Freudenreich, Cunningham, and others, which appear to demonstrate that freshly drawn milk possesses for certain species of bacteria a germicidal power. To this, however, we shall have occasion to refer again.

In the healthy condition of animals we have, generally speaking, no micro-organisms whatever in their secretions, whatever may be the condition of their excretions. Hence though milk affords, from its constitution, such an ideal nidus for the growth and multiplication of bacteria, it is, as secreted, a perfectly sterile fluid. This was demonstrated more than twenty years ago by Lister, who states that "unboiled milk as coming from a healthy cow, really contains no material capable of giving rise to any fermentative change, or to the development of any kind of organism which we have the means of discovering." 1 Subsequent experiment has only confirmed the general truth of this statement. ${ }^{2}$ With efficient precautions (which will be set forth in detail at a later stage)

1 Transactions of Pathological Society of London, 1878, p. 440.

${ }^{2}$ See also Rotch, Pediatrics, the Hygiene and Medical Treatment of Children, London, 1896. 
it is possible to draw from the udder of a healthy cow perfectly sterile milk, which retains its sterility unchanged for long periods of time in a sterilised and sealed flask. Yet we know by practical experience as well as by ultimate changes in the milk that, generally speaking, the presence therein of bacteria is very marked. We must therefore next consider whence comes this pollution.

\section{The Sources of Bacteria in Milk}

These are various and depend upon many minor circumstances and conditions.

For all practical purposes there are four chief opportunities between the cow and the consumer when milk may become contaminated with bacteria :-

I. At the time of milking.

2. During treatment and preparation.

3. During transit to the town or dairy or consumer.

4. After arrival at its destination.

The following table, compiled from the experiments of Backhaus of Königsberg, will show the possible sources of contamination at a glance :-

Elements of Contamination.

I. Infection.

2. Bodily cleauliness.

3. Litter.
Fresh milk - + . + 6,660 per c.c. After passage through six vessels 97,600 ,

Milk from clean cow . . . 20,600 " Milk from dirty cow . . . 170,000 "

Peat . . . . . 2,000,000 per gramme

Good straw . . . . 7,500,000

Bad straw . . . . 10,000,000 "

4. Influence of the litter on the number of bacteria
in milk.

With peat litter

With straw litter

5. Food (dust of, in the byre). Oil-cake .

- 3,500 per c.c.

- 7,330 "

6. Milking.

Bran

457,500 per gramme

Milked dry

Milked wet

First milk

Last milk

Washed udder

Unwashed udder

7. Vessels.

Enamelled vessel

Tin vessel

$1,361,900$

- 5,600 per c.c.

- 9,000 ,

. 10,400

- sterile

- 2,200

- 3,800

. 1,105

- 1,690

Wooden vessel . . . . 279,000

8. Cleansing of vessels.

Sterilised pail . . . : . 1,300

Simply rinsed . . . . . 28,600 
Somewhat similar results to the above were obtained by $\mathrm{H} . \mathrm{H}$. Deane in Canada. We would suggest, however, that such figures as the above be accepted with reserve and only as a general illustration on the point under consideration.

Pollution at the time of milking arises from the animal, the byre, the milker, or the utensils used for holding the milk. It is now well known that in certain diseases of cows, particularly those affecting the udder, we may find the milk impregnated with pathogenic, putrefactive, and other bacteria. Tuberculosis of the cow affecting the udder is the best known example. According to Klein, ${ }^{1}$ scarlet fever and diphtheria are diseases which may, under certain circumstances, affect cows and render the milk infective. In ordinary bovine mastitis a well-known organism makes its appearance in the milk. We do not, however, in this place propose to enter into the question of the pathogenic organisms which may occur in milk. We only draw attention to these points in order to establish the fact that any departure from health, any temporary or permanent disease of the cow affecting the milk-secreting apparatus and especially all forms of udder-disease (even slight diseases and eruptions) must inevitably add their quota of bacteria to milk.

But it is not the diseased cow which alone adds bacteria to the milk. A far more extensive pollution due to the cow arises, not from disease, but from uncleanliness. The unclean coat of a cow will more materially influence the number of micro-organisms in milk than either disease or the popularly supposed fermenting food which the animal may eat. It is from this external source rather than from the diet that organisms occur in the milk. The dry hairy coat offers many facilities for harbouring dust and dirt. The impurities upon the body of a cow may contain pathogenic as well as saprophytic bacteria, as, for example, when a cow standing in water polluted by sewage is splashed (Axe). The mud and filth of every kind that may habitually be seen on the hinder quarters of cattle all contribute largely to polluted milk. Nor is this surprising. Such filth contains large quantities of those organic compounds upon which bacteria thrive, and plastered upon cattle at or near the temperature of the blood, nothing is wanting to make it a perfect environment for bacteria. It is impossible for the milker to prevent some of the dust and dirt from the cow finding

1 Annual Report of Medical Officer to Local Government Board, vol. xix. (1890), p. 171. 
its way into the milk. It may not appear to be extensive contamination, but every hair and dust particle which finds its way into the milk-pail carries with it a large number of bacteria, and whilst it may be possible, by muslin straining, to remove the hairs and microscopic particles, the conveyed bacteria will remain. No one having in his mind Tyndall's "raft-theory" can watch an ordinary milking process without being convinced that it is by such rafts that countless germs, most of which are fortunately innocent and harmless, are introduced into the milk. Further reference is made subsequently to this question of dust in the air as a vehicle of organisms. The accompanying Plate is made from a photograph of a Petri dish of nutrient medium exposed at a spot in Central London where milk is delivered daily. A close examination of the colonies will show the particle of dust stranded on the summit of the colony, itself consisting of the progeny of the bacteria carried on to the plate by the particle of dust acting as a raft or vehicle, as suggested by Tyndall.

But even yet, it is to be feared, we have not exhausted the cowelement in the pollution of milk. Its diseases, its uncleanliness, and its diet, do not complete its contribution. There still remain the open milk-ducts of the udder. The mammary gland of the cow, as is well known, is divided into four quarters, to each of which a teat is attached. The teat is composed of spongy elastic tissue surrounding the main milk-duct, which differs from the secretory ducts in function and in size, and is indeed the final channel through which the milk passes from the udder. Now this milk-duct is practically open to the air, and from its valvular shape, it will contain at the close of milking, however thorough the manipulation, some small amount of milk adherent to its walls. The temperature of such retained milk is at or near blood-heat. Bacteria in the air or from the soil have no difficulty whatever in gaining admittance to such residual milk, and under such favouring circumstances can and do multiply with enormous rapidity. It is, in fact, an excellent incubator. At the next milking the first or fore-milk contains this residual, highly-contaminated, milk, which for some hours at least has been lying in the milk-duct. The result is that the fore-milk always contains an excess of bacteria, which, in the case of an experiment by Schultz, were found to amount to 80,000 micro-organisms per c.c., while the last drawn milk was practically sterile ; and in a similar experiment by Russell under ordinary circumstances, 2800 bacteria per c.c. were found in the fore-milk, whilst the average of the total yield was only 330 
PLATE 4.

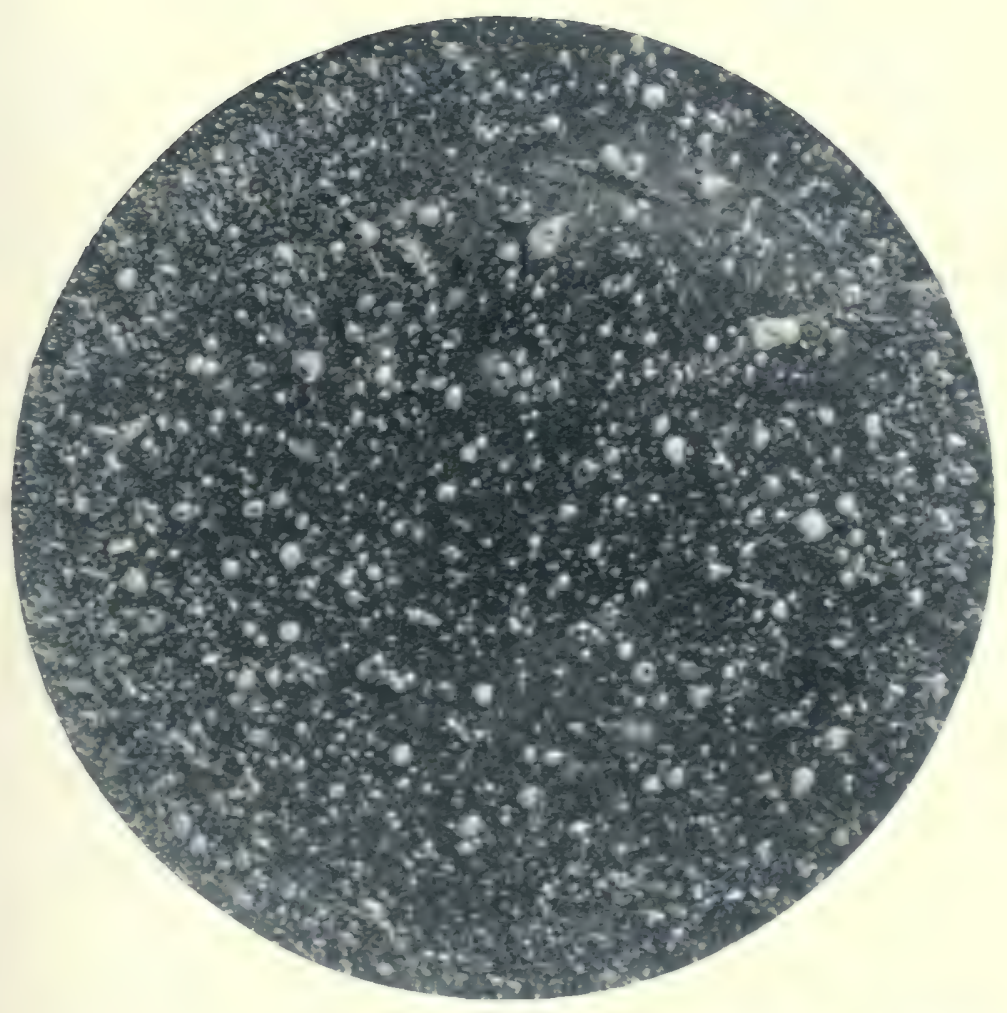

j

Agar plate exposed for 30 minutes in open air at entrance to "moxtel i weitin ss" in Finstury, London, E.C., where milk is delivered daily. Incubation, 4 days at 1 (s (C. This phate illustrates (a) pollution of air in towns; and (b) the "raft theory" of Tyndall. 

per c.c. It is clear that if such quantities of organisms pass into the general milk, they will rapidly pollute the entire quantity which might otherwise, at the outset, have been comparatively free from germs. Ward and others have pointed out that the species of bacteria normally inhabiting the ducts are limited to a few. It appears that only certain kinds of bacteria, including some species of streptococcus, are able to live at this site. These species, as a rule, are more or less persistent. The lactic acid group of organisms do not appear to inhabit the milk duct, but probably gain entrance to the milk from sources external to the cow.

Two other agencies contributing to contamination at the milking time are the milker and the byre. The milker's hands and sometimes his clothes may convey bacteria to the milk. Clothed in dust-laden garments, and frequently characterised by dirty hands, the milker may readily act as an excellent purveyor of germs. Not a few cases are also on record where it is certain that milkers have conveyed germs of disease from some infectious case in their homes, such as scarlet fever. Under the more efficient registration of such diseases, which has recently been a feature in the policy of the more public spirited dairy companies, the danger of infection from this source has, however, now been considerably reduced.

The influence of the barn air, and the cleanliness or otherwise of the barn itself, is obviously great in this matter. We do not suggest that the air of byres is a medium in which bacteria live or multiply. The air-organisms to which we refer are in point of fact dust and dirt organisms present in the air only at times of molecular disturbance, and having their origin in dry fodder, hay, straw, etc., as well as the ungroomed surface of the cow's body. But such disturbance at milking time is actually a normal state of affairs. We know that moist surfaces retain any bacteria lodged upon them, and that a wet perimeter results in a germ-free or nearly germ-free atmosphere. But in a dry byre the air is often heavily laden with microbic life. The evidence obtainable, if evidence be necessary, on this point is two-fold. There is the mass of evidence laid before the Royal Commission on Tuberculosis respecting the evil infection and predisposing influences of the byre air on cattle in the direction of conveying tuberculosis. Sir George Brown, Sir Richard Thorne Thorne, and many other authorities, have again and again drawn attention to the bad state of the air in byres. The second and still stronger evidence is derived from experimental examination of the air of byres. Russell, Conn, and other American bacteriologists, as well as many workers in 
this country, have furnished results of such investigation. We have ourselves made a very large number of bacterial examinations of the air of byres, both during molecular disturbance and otherwise, and have isolated a large number of micro-organisms. A statement concerning these will be found elsewhere.

During treatment or preparation the main source of pollution arises from the air of the dairy or the apparatus used for separation. We have examined dairy air and dairy utensils and have found them far from bacteriologically pure. On the ordinary farm it is difficult to cleanse utensils thoroughly, and it is the custom to leave the milk exposed to the air of the dairy. Through these two sources it is remarkable how many bacteria gain access to the milk. But in efficiently and well-conducted dairies both these agencies should be reduced to a minimum. ${ }^{1}$

The risks in transit differ according to many circumstances. Probably the commonest source of contamination is in the use of unclean utensils and milk-cans. Any unnecessary delay in transit affords opportunity for multiplication; particularly is this the case in the summer months, for at such times all the conditions are favourable for an enormous increase of any extraneous germs which may have gained admittance at the time of milking. The milk churns are left standing on railway platforms unprotected from the sun or dust. The churns may be insufficiently closed, and as a rule are neither locked or sealed. Thus we have $(a)$ the milk itselfa perfect pabulum for bacteria ; $(b)$ a more or less lengthened railway journey or period of transit, giving ample time for multiplication; (c) the favourable temperature of summer heat.

The period occupied by transit depends, of course, upon the size of the community to be supplied. The great growth of cities has widened the area from which milk is obtained, so that at the present time it may be said that most of the milk consumed in large cities has undergone a more or less lengthened railway journey, and must have been kept, say from twelve to twenty-four hours, before it reaches the consumer. The milk supply of London and $P$ aris is in each case derived from a very wide area. ${ }^{2}$ In London the milk is delivered by rail at the stations about 2 A.M.

1 As a result of experiment on an extended scale (published since the above was written), Delépine holds that infection of the milk at the farm or through vessels infected at the farm and used by the farmers for the storage and carriage of milk are of paramount importance, and play a much greater part in the bacterial pollution of milk than infection in the house of the consumer.-Jour. of Hygiene, I903, vol. iii., No. I, pp. 79-87.

2 See also Jour. Roy. Stat. Soc., 1892, pp. 244-273. 


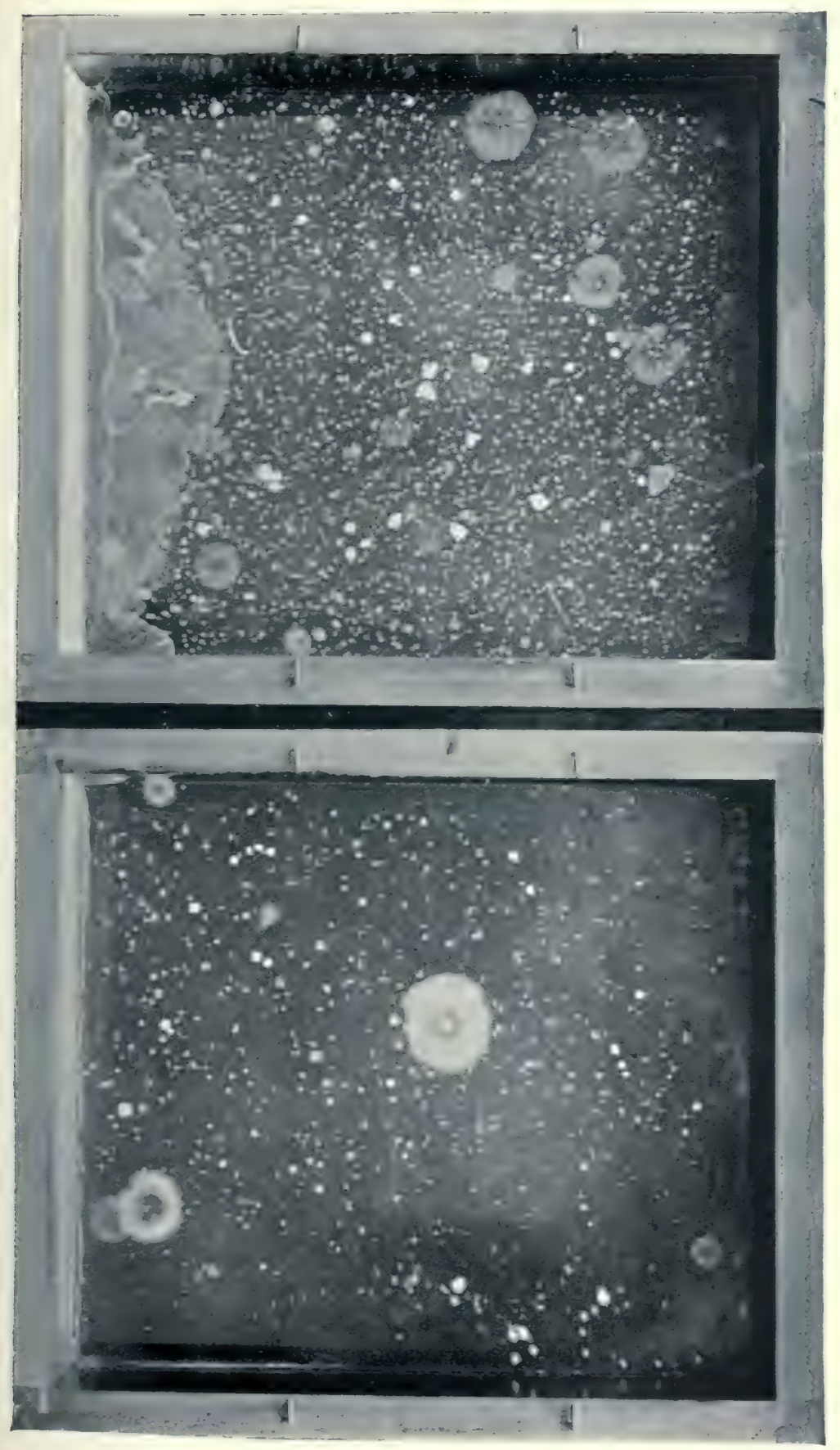

I. Air of byre. -30 minutre exposure during milking time Agar plate. Incubation, 24 hours at $37^{\circ} \mathrm{C}$; 48 hours at $18^{\circ} \mathrm{C}$. Size of plate, $13 \times 11$ inches.

II. Air of dairy. - 6 hour's exposure, incluling 2 working hours. Agar plate. Incubation, $2 ;$ hours at $37^{\circ} \mathrm{C}$; 48 hours at $18^{\circ} \mathrm{C}$. Size of plate, $13 \times 11$ inches. 

or $3 \mathrm{A.M}$. It is then distributed by the milk contractors to the wholesale and retail shops in the metropolis, and reaches the customer at the earliest twelve or fourteen hours after milking. A certain amount of milk, of course, that obtained from the nearer home counties, will reach the consumer a little sooner. ${ }^{1}$ The milk supply of New York is transported by railroad, some of it travelling a distance of 350 miles. It is delivered in the early morning in 40-quart cans or in quart jars, and the dealers distribute. They have no control over the bacterial content of the milk as it arrives,

' From evidence brought before the Committee on Food Preservatives (I gor), it appears that the average distance from which seven of the great railway companies bring the milk supply into London is more than 200 miles. The chief facts may be given in the following form :-

\begin{tabular}{|c|c|c|c|c|}
\hline RAILWAY. & $\begin{array}{l}\text { Maximum } \\
\text { Distance in } \\
\text { miles from } \\
\text { which Milk is } \\
\text { Carried. }\end{array}$ & $\begin{array}{c}\text { Time } \\
\text { occupied by } \\
\text { the Railway } \\
\text { Journey in } \\
\text { hours. }\end{array}$ & $\begin{array}{l}\text { Whether } \\
\text { Special Ysins } \\
\text { and Carriages } \\
\text { are used for } \\
\text { Conveysnce of } \\
\text { Milk. }\end{array}$ & $\begin{array}{l}\text { Whether } \\
\text { Refrigerator } \\
\text { Vans are } \\
\text { Used for the } \\
\text { Yilk. }\end{array}$ \\
\hline $\begin{array}{l}\text { Great Eastern Railway } \\
\text { Great Northern Railway } \\
\text { Great Western Railway } \\
\text { Lond. Brighton, and S.C. } \\
\text { Lond. and N.W. Railway . } \\
\text { Lond. and S.W. Railway : } \\
\text { Midland Railway }\end{array}$ & $\begin{array}{r}130 \\
150 \\
300 \\
86 \\
359 \\
150 \\
423\end{array}$ & $\begin{array}{c}4 \\
5 \frac{1}{8} \\
\mathrm{II} \\
3 \frac{1}{3} \\
8 \frac{8}{4} \\
5 \\
\mathrm{I} 2\end{array}$ & $\begin{array}{l}\text { Yes } \\
\text { Yes } \\
\text { Yes } \\
\text { No } \\
\text { Yes } \\
\text { Yes } \\
\text { Yes }\end{array}$ & $\begin{array}{l}\text { No } \\
\text { No } \\
\text { No } \\
\text { No } \\
\text { No } \\
\text { No } \\
\text { No }\end{array}$ \\
\hline
\end{tabular}

It appears that the Great Western Railway Company are the largest carriers of milk in the United Kingdom, the quantity conveyed by them during I 899 being $23,495,925$ gallons in $1,642,380$ cans. Ninety-nine per cent. of the milk traffic received in London by this company is from stations within 150 miles, and the average distance is probably about 60 miles. The chief centre is the Wiltshire Dairy District. The London and North-Western Railway brings large quantities of milk from the Midlands and Staffordshire district. Its most distant supply comes from Tarff in Kirkcudbright ( 359 miles). The Midland Company conveys milk from Glasgow (max. distance). As a rule, most of the milk (80 to go per cent.) conveyed on the English railways is carried in special milk-trains. Some of it, in small quantities, is conveyed in vans attached to passenger trains, and a small amount by the ordinary goods trains. No special precautions are taken to keep milk churns or railway trucks carrying churns in the shade in summer time. Special sheds are exceptional. Generally speaking, the heavy milk traffic passes at night time. Cows are milked in the morning or afternoon, and the milk is frequently mixed and carted by the farmer to the nearest station, whence it is worked to London as most convenient to the company. Railway companies do not collect or deliver milk. They transport from platform to platform only. At the London terminus the milk contractor arranges for delivery. The companies do not insist upon the milk churns being sealed or locked, and there is no difference in the rates according to whether or not the churns are locked or only fastened. 
though they may prevent further contamination or multiplication by clean manipulation and refrigeration. ${ }^{1}$ The milk problem, therefore, in all great cities, is to furnish milk in a condition suitable for food after it has been kept twenty-four or thirty-six hours.

Lastly, many are the advantages given to bacteria after arrival of milk at its destination. In milk-shops, and in the house, there are not a few risks to be added to the already imposing category. Water is sometimes added to milk to increase its volume. Such water, unless sterilised, will add its own contribution to the flora of the milk. Again, in small houses-or homes perhaps of only one room-where the milk stands in unsterilised vessels for some hours, it is impossible to suppose that pollution is avoidable. In such dwellings, milk runs the risk of being contaminated with bacteria, and every kind of dust and dirt, from a hundred different sources. Particularly is this so in the one-room dwellings of London and other large cities, where the cooking, eating, sleeping, cleansing, and sometimes even trade employment, are all conducted, for the entire

1 The quantity of milk and cream daily conveyed into New York City to supply the wants of its $3 \frac{1}{2}$ million inhabitants is, according to a recent estimate of the local board of health, about $1,230,000$ quarts. From 85 to 90 per cent. of the total is carried by the railroads, the remainder being taken in by waggons from the suburban districts, or supplied by herds within the city itself, the latter embracing altogether some 23,200 cows. The milk transported by rail has its origin in the States of New York, New Jersey, Pennsylvania, Connecticut, and Massachusetts, and some of it is conveyed as far as 350 miles. Much is forwarded from the northern and north-eastern sections of New York State, and it is noteworthy that in some cases consignments destined respectively for New York City in the east and Buffalo in the west originate only a dozen miles apart. Steamers calling at places on the Hudson River carry about 40,000 quarts of milk and cream into New York every day. There are, however, some districts from which the shipments, formerly very large, have almost or entirely ceased. From Long Island, for example, the quantities sent by rail have steadily declined from $6 \frac{1}{2}$ million quarts in 1885 to practically nothing in 1901 . This is largely due to the intensive cultivation of market garden crops, which has developed into an industry far more profitable than dairying. A less important cause of the practical disappearance of this supply is the steady absorption of farms adjacent to New York City into suburban villages, a movement which has taken place to a remarkable degree within the last five years. Baggage cars, refrigerator cars, and cars specially constructed for the purpose, are employed in the transportation of milk, each railway adopting the style of car best suited to its own requirements. A large milk car will hold 325 cans of 40 quarts each. Most stations where there is a large milk traffic have covered receiving stations, or cooling vats containing ice. By this means milk is reduced to $40^{\circ} \mathrm{F}$. in about 40 minutes, and ready for immediate transference to the refrigerator cars of the train. The freight vats are not uniform, not all the lines having adopted "the zone system" recommended by the Interstate Commerce Commission. 
SOURCES OF CONTAMINATION IN MILK.

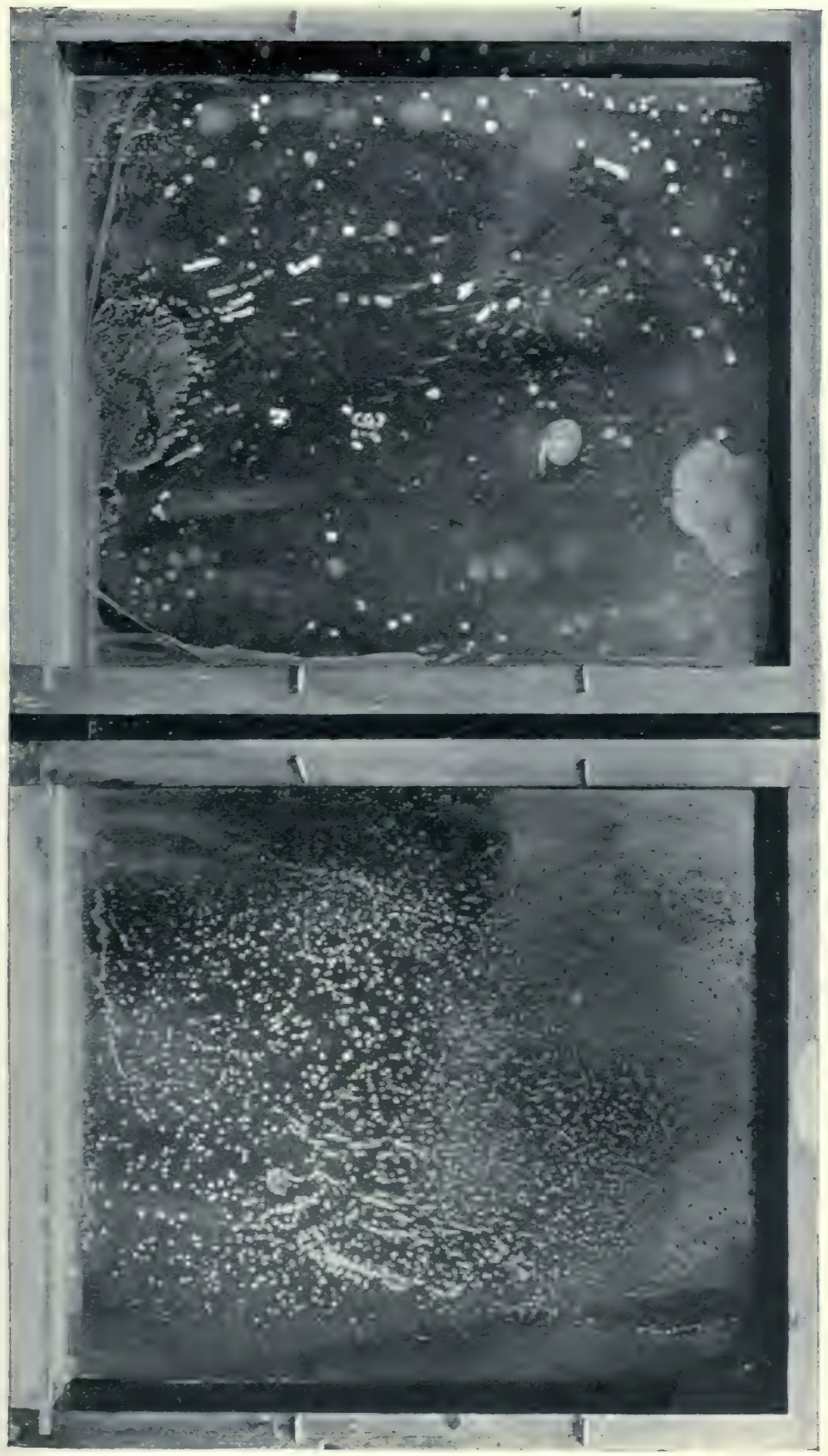

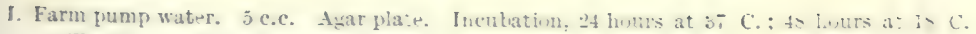
Size of plate, $13 \times 11$ inches.

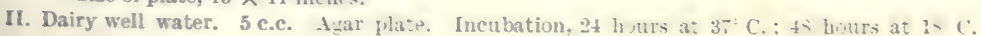
Size of plate $13 \times 11$ inches. 

family, in one and the same room, in which there is no pantry, larder, or separate accommodation provided. ${ }^{1}$

The pathology of milk.- It will have been seen that there is no hard and fast standard of healthy milk, chemically, physically, and physiologically. Vital secretions do not admit of such standardisation. As a consequence, the exact demarcation between the physiology and pathology of milk is an arbitrary one, and only determinable by a careful recognition of all the facts of the case. But in what has been said up to the present, no reference has been made to various departures in milk from the normal and healthy standard. The chief of these elementary facts concerning the pathology of milk must now be mentioned.

First in importance stand those conditions of milk by which specific disease is conveyed. Enteric fever, scarlet fever, diphtheria, epidemic diarrhoea, sore throats, and other diseases have been conveyed by means of milk, acting as the vehicle either of the infective agent (bacteria), or of some product (toxins), ${ }^{2}$ or other materia morbi. This subject will be considered at a later stage, and need not be more than named here.

Secondly, there are the fermentations of milk, which may have direct or indirect effects upon the consumer, either mechanically or physiologically. Our knowledge of these effects is very limited, and it is only in recent years that the elementary facts concerning fermentation have been elucidated. The subject is dealt with subsequently.

Thirdly, it must not be forgotten that milk may contain various substances or bodies intermediate between the healthy and normal fat globules on the one hand, and pathogenic bacteria on the other. It is convenient that such contaminations should be mentioned briefly here, for they constitute an important item in the pathology of milk.

At the beginning of the first week of lactation, cow's milk contains two kinds of cells in addition to fat globules. These are colostrum corpuscles and leucocytes. The former are known under the microscope by their size, granulation, and contents, which consist of a conglomeration of small fat particles, or one large fat globule resulting from a coalescence of many small ones. The latter, leucocytes, correspond to the poly-morpho-nuclear neutro-

${ }^{1}$ According to the census of 1901, there are in London 149,524 one-room tenement dwellings, in which 304,874 persons reside. The total number of tenement dwellings with less than five rooms is 672,030 .

${ }^{2}$ Lyon Medical, vol. xcvi. (1900), p. 56r (Leblanc). 
philes of human blood, and are present in small numbers in healthy milk. In the early stage of lactation, however, they are present in considerable number, and may occur so generally as to constitute leucocytosis.

A third cell not infrequently present in milk is the pus cell pure and simple. It is always present in the milk of cows suffering from infectious mammitis. Stokes and Wegefarth carried out a series of experiments to show the percentage of milk which under various conditions contains pus cells. They examined the milks of three grades or groups of cows as follows :-

(a) Ioo cows in the country, kept in a well-ventilated stable with roomy stalls and upon good pasturage. They were fed while in stall on bran, ground corn, and hay. They were groomed and cleaned daily, and inspected by competent veterinary inspectors. All sick animals were isolated from the herd, and the milking operations were carried out in a cleanly manner.

On microscopical examination of the milk of this group it was found that it gave a general average of $\mathbf{I} \cdot \mathrm{I}$ pus cell to the field of the microscope. ${ }^{1}$ There were practically no pus organisms, such as streptococci or staphylococci ; 75 cows gave an average of less than I pus cell to the field; 93 cows out of 100 gave less than 5 cells; whilst 98 cows out of IOO gave less than IO pus cells; and 45 cows gave a percentage of 0 . I pus cell to the field. In each case fair samples and fields were chosen.

(b) The second group consisted of 50 cows in the country, stalled in badly ventilated narrow stalls, and running upon bad pasturage. They were fed on distillery grains, cut hay, and bran. The sanitary precautions carried out in the first group were not adopted.

On microscopical examination of the milk this group gave an average of II. 3 pus cells in the field of the microscope, as compared with $\mathbf{I} \cdot \mathbf{I}$ in the first group.

(c) The third group consisted of 100 cows kept in the city always confined to stables, with narrow stalls, no ventilation, little or no light, and no fresh pasturage. They were fed on brewery grains, distillery slops, bran, and hay. The cows remained in the stables for months together until dry. The milk was collected in such a way as to obtain a fair average from the whole herd, and was drawn under veterinary supervision.

On microscopical examination, the milk of this group gave a

1 These averages are all based upon counts of ten microscopic fields, with $1_{1}^{\frac{1}{2}}$ oil immersion lens. 
general average of 19.2 pus cells in the field of the microscope. Streptococci and other bacteria were present in large numbers. The highest number of pus cells to any one cow was 158.5 in the field of the microscope. Five cows only gave a percentage of $\mathrm{O}$. I pus cell to the field (as compared with 45 in the first group); only I 2 cows out of this IOO gave an average of less than I pus cell to the field; only 38 gave an average of less than 5 pus cells; and only 54 (as compared with 98 in the first group) gave less than 10 pus cells to the field. ${ }^{1}$

From these results Stokes and Wegefarth were led to the obvious conclusion that cows kept in the country under sanitary and hygienic conditions are less capable of causing disease in persons consuming their milk than cows kept in the city under the conditions of group 3. They consider any standard for exclusion of milk containing pus cells must of necessity be arbitrary, but recommend that an average of more than 5 pus cells to the field of the oil immersion lens should suffice to exclude the milk of such animal. Taking I pus cell in the field as a standard, it would appear that Stokes and Wegefarth found 25 per cent. of the milks of country cows, kept under good conditions, and 88 per cent. of town cows, kept under bad conditions, contained pus cells. Eastes, who made an examination of 186 London milks, found pus cells present in 30 per cent. ${ }^{2}$ Too much emphasis must not be laid upon the difference between these results, as somewhat different methods were adopted. The effect of drinking milk containing pus is referred to subsequently.

Mucous threads are commonly found in milk containing pus. Such threads probably consist of nucleo-albumin, and when occur. ring with pus cells, the condition of "muco-pus" is present. This is held to indicate inflammatory lesion of the ducts of the udder, and not abscess formation in the substance of the gland. Blood corpuscles are not rare in milk, particularly soon after lactation.

The last and least important kind of cell is that of the epithelium. Such scales may be derived either from the hand of the milker or from the teats of the udder. Epithelial cells are large and nucleated.

Milk containing many blood cells, mucous threads, or leucocytes, and milk containing any pus cells should be looked upon as unfit for human consumption.

1 Jour. of State Med., I897, vol. v., pp. 439-443.

1 Brit. Med. Jour., 1899, vol. ii., p. 1342. 


\section{CHAPTER II}

THE BACTERIOLOGICAL EXAMINATION OF MILK: METHODS AND

TECHNIQUE

Culture Media. Quantitative Examination of Milk and Method of Milk Dilution. The Making and Staining of Preparations for the Microscope. Qualitative Examination of Milk :-Aërobic Examinations. Method of Examination of Special Organisms. Special Methods. Routine Procedure in Examination of Milk.

\section{Culture Media}

ALTHOUGH for the culture of micro-organisms finding their natural habitat therein milk itself may be looked upon as the most favourable medium, yet for differentiation, comparative, and even cultural purposes, the ordinary media of the laboratory are essential, and too much stress cannot be laid upon the necessity for care and uniformity in their preparation. In the course of a long investigation extending over some years, which has been complicated by a series of irreconcilable discrepancies in the description by various workers of evidently identical organisms, the conviction has been gradually forced upon us that until some general standard of media has been adopted by bacteriologists, results obtained will never be really comparable. It is in point of reaction especially that the greatest divergence would appear to occur, and it must be remembered that Alkalinity, Neutrality, and Acidity, are terms that have no practical meaning as applied to culture media unless it is stated at the same time in reference to what special indicator the terms are used. A medium may be alkaline to litmus, yet at the same time acid to phenolphthalein, or acid to both of these and yet alkaline to fluorescine. To a medium which is just neutral to sensitive litmus paper a quantity of caustic soda solution corresponding to 0.068 per cent. of $\mathrm{NaOH}$ must be added before the same medium becomes neutral to phenolphthalein. Media neutralised with the latter, therefore, are more alkaline by 0.068 per cent. of 
$\mathrm{NaOH}$ than litmus neutral media, and if for the neutralisation not caustic soda but sodium carbonate is used the discrepancy becomes still greater. For some years past we have ourselves adopted in our investigations a standard reaction midway between the neutral point of litmus and phenolphthalein, but a series of experiments, lately undertaken, has convinced us that an equally favourable reaction for the growth of all micro-organisms likely to be found in milk is a point at which the first appearance of a pinkish tinge can be observed in a dilution of the medium when at the boiling point, on the addition to it of a few drops of a 50 per cent alcoholic solution of phenolphthalein. This reaction has the advantage of representing a very exact point, and it is, with an ordinary amount of care, so easy of attainment that we strongly recommend its adoption for all media, except those in which a certain degree of acidity is desired.

In regard to this question of standard media we propose to make reference to two chief points-(a) neutralisation of media, (b) peptones.

\section{Neutralisation of Media}

This may be looked upon as the most important stage in the preparation of media, and too much care cannot be exercised if accurate results and a constant standard of reaction is to be obtained. One of two methods may be adopted.

I. The fractional method, as originally recommended in the report of the Bacteriological Committee appointed by the American Public Health Association issued in the year 1897 ; and

2. The method of neutralisation in bulk. - Of the two, the authors, after experience of both, prefer the latter as simpler, less liable to error, and equally accurate in results, but as the former has been generally adopted in America, and, with various modifications, in certain of the schools of this and other European countries, both are given.

\section{Fractional method ${ }^{1}-$}

The following solutions are required:-

I. A 0.5 per cent. solution of commercial phenolphthalein in 50 per cent. alcohol.

2. A $\frac{1}{20}$ normal solution of sodium hydrate.

3. A $\frac{1}{20}$ normal solution of $\mathrm{HCl}$.

1 Reports and Papers of the American Public Health Association, vol. xxiil., p. 73 et seg. 
Solutions Nos. 2 and 3 must be accurately made up, and must correspond with the normal solutions referred to below. Solutions of sodium hydrate are prone to deterioration from the absorption of carbon dioxide and the consequent formation of sodium carbonate. To prevent as much as possible this change it is well to place in the bottle containing the stock solution a small amount of calcium hydroxid, while the air entering the burettes or the supply bottles should be made to pass through a " $U$ " tube containing caustic soda to extract from it the carbon dioxide.

The medium to be tested is brought to the prescribed volume by the addition of distilled water to replace that lost by boiling, and after being thoroughly stirred, 5 c.c. are transferred to a sixinch porcelain evaporating dish; to this 45 c.c. of distilled water are added, and the 50 c.c. of fluid are boiled for three minutes over a flame. One c.c. of the solution of phenolphthalein (No. I) is then added and by titration with the required reagent (No. 2 or 3) the reaction is determined. In the majority of cases the reaction will be found to be acid, so that the $\frac{1}{20}$ normal sodium hydrate is the reagent most frequently required. This determination should be made not less than three times and the average of the results obtained taken as the degree of reaction.

One of the most difficult things to determine in this process is exactly when the neutral point is reached as shown by the colour developed, and to be able in every instance to obtain the same shade of colour. In regard to this, it may be remarked that in bright daylight the first change that can be seen on the addition of alkali is a very faint darkening of the fluid, which on the addition of more alkali develops into what may be described as an Italian pink. A still further addition of alkali suddenly develops a clear and bright pink colour, and this is the reaction always to be obtained. All titrations should be made quickly and in the hot solutions, to avoid complications arising from the presence of carbon dioxide.

The next step in the process is to add to the bulk of the medium the calculated amount of reagent, either alkali or acid as may be determined. For the purpose of neutralisation a normal solution of sodium hydrate or of $\mathrm{HCl}$ is used, and after being thoroughly stirred the fluid thus neutralised is again tested in the same manner as at first to ensure the proper reaction of the medium being attained. When neutralisation is to be effected by the addition of alkali, it not infrequently happens that after the calculated amount of normal solution of sodium hydrate has been added, the 
test by titration will show that the medium is still acid to phenolphthalein, to the extent sometimes of from 0.5 to I per cent. This discrepancy is perhaps due to minor reactions which are not understood; the reaction of the medium, however, must be brought to the desired point by the further addition of sodium hydrate, and the titrations and additions of alkali must be repeated until the medium has the desired reaction. ${ }^{1}$

\section{Neutralisation in bulk (Procedure recommended) -}

Place the medium in a wide-mouthed enamelled saucepan, and neutralise to litmus with caustic soda solution. Accurate standardisation is not necessary at this stage, the only object being to save time in the subsequent neutralisation to phenolphthalein. Bring slowly to the boiling-point over the Bunsen flame. All risk of burning can be avoided by the employment of a water-jacketed pan, or the interposition of an asbestos mat between the pan and the flame. Boil for five minutes. Remove and place on one side in a beaker about IOO c.c. of the medium, to be subsequently returned as explained below. Hold ready to hand a beaker of boiling water, half a dozen small white porcelain evaporating dishes, and two ordinary dessert spoons marked on their inner surface with a line showing the level of 5 c.c. of liquid. By means of one of these, place quickly 5 c.c. of the boiling medium in one of the evaporating dishes, and add with the other an equal quantity of the boiling water. Drop in two drops of a I per cent. solution of phenolphthalein in 50 per cent. alcohol. ${ }^{2}$ If the mixture remains colourless as seen against the white porcelain after stirring with a glass rod, add a sufficient quantity of normal soda solution to the bulk, and repeat until a pinkish tinge is seen. A slight excess of alkali does not matter at this stage as the addition of the 100 c.c. withdrawn

1 The method of expressing the degree of reaction is as follows:-Acid media are designated by the plus sign, and alkaline media by the minus sign, and the degree of acidity or alkalinity is noted in parts per hundred; thus, a medium marked +1.5 would indicate that the medium was acid, and that I. 5 per cent. of normal sodium hydrate solution is required to make it neutral to phenolphthalein, while -1.5 would indicate that the medium was alkaline, and that $\mathrm{I} \cdot 5$ per cent. of normal acid must be added to make it neutral to the same indicator.

${ }^{2}$ In view of the slightly acid properties of this indicator this solution should, in course of preparation, be rendered neutral by first cautiously adding a few drops of centi-normal alkali until the appearance of a very faint redness, and then adding one drop of centi-normal acid. 
as above, which is now returned to the bulk, will counteract this. Proceed now more cautiously, and repeat the test, adding the soda solution to the bulk drop by drop from a pipette, until the first tinge of pink is seen against the white porcelain after stirring the mixture with a glass rod. The medium is now neutral to phenolphthalein. Keep it at the boiling-point for another five minutes, and then proceed as laid down under preparation of media.

Care must be taken to thoroughly cleanse the spoon employed for the medium by dipping in hot water between each of the above operations, and the medium itself must be kept constantly and well stirred by an assistant.

\section{Peptones}

Very little reliance can be placed in the uniformity of the dry peptones of commerce. Even those of the best makers vary at times in a high degree, and all quickly lose their nutritive properties with age. The authors have found the liquid peptone (bouillon d'estomac de porc) originally suggested by Dr Louis Martin for the production of diphtheria toxin so easy in manipulation, economical in use, and uniform in result, that they have for some years past, in the preparation of media, used it to the exclusion of all others. Its preparation is as follows, and as it improves somewhat with age a sufficient stock can be made at one time and kept in hand for use.

Preparation.-Take two or three stomachs of freshly killed pigs, and mince together I kilogramme of the mucous and muscular coats. Place in a strong earthenware jar containing 5 litres of water acidulated with 50 c.c. (I per cent.) pure hydrochloric acid, and heat up to $50^{\circ}$. Allow to digest for twenty-four hours, maintaining the temperature at $50^{\circ}$, at which the pepsin of the mucous lining digests the tissues more actively and transforms them into peptone. When digestion has taken place the bouillon is brought slowly to the boiling point, by heating the jar over the Bunsen flame, in order to destroy the excess of pepsin. The liquid obtained by this self-digestion of the stomach is a true solution of peptone. It is then filtered through a fine sieve, or a layer of loosely packed cotton wool, and set aside to cool. In this condition it can be stored for apparently an unlimited time without deterioration. A slight layer of mould will form on the surface of the jar, but this can be disregarded, and the strong acidity prevents the 
multiplication of other organisms. When required for use, a given quantity is filtered through filter paper moistened with water (the filtration takes some hours), heated up to the boiling point, and made neutral to phenolphthalein with normal caustic soda solution. In the preparation of nutrient bouilion or other media, equal quantities of this liquid and beef bouillon prepared as below are mixed together.

\section{PREPARATION OF MEdia}

The method of preparation will now be given, according to recognised formulæ, of the ordinary laboratory media likely to be of service to the bacteriologist engaged in work upon milk, with the addition of those peculiarly applicable to this special branch of research.

\section{Nutrient beef bouillon-}

Take I pound of beef free from fat and connective tissue, mince, add I litre of water, and allow to digest in a cool place for eighteen hours, stirring occasionally. Strain through a fine sieve and muslin, place the liquid in a clean enamelled saucepan, heat up slowly to boiling point and allow to boil for five minutes. Filter through damp paper into a clean flask.

To make 2 litres of media, add to the litre of liquid thus obtained I litre of the pig's stomach broth prepared as above, and 10 grammes of common salt, and bring the liquid to the required reaction in the manner described under the head of neutralisation of media. Make up to 2 litres with distilled water, boil for ten minutes, ${ }^{1}$ and filter through damp filter paper into flasks or tubes. Sterilise in the autoclave for fifteen minutes at $115^{\circ}$, or in the Koch steriliser at $100^{\circ}$ for thirty minutes on three successive days.

The bouillon can be cleared with white of egg, but if the above instructions are carefully carried out this will be found unnecessary, and a perfectly limpid fluid will be the result.

\section{Glycerine bouillon-}

Add to bouillon prepared as above 6 per cent. of pure glycerine immediately after the final filtration, and thoroughly incorporate by stirring or shaking.

1 If final sterilisation is to take place in the autoclave the bouillon should, in order to get rid of the excess of earthy phosphates which may subsequently trouble it, be autoclaved at this stage for fifteen minutes at $115^{\circ}$. 


\section{Glucose or lactose bouillon-}

To 500 c.c. of nutrient bouillon add 10 grammes (2 per cent.) of anhydrous glucose or lactose before final filtration.

\section{Glucose formate bouillon-}

To 500 c.c. of nutrient bouillon add Io grammes ( 2 per cent.) of anhydrous glucose, and 2 grammes ( 4 per cent.) of sodium formate before final filtration.

\section{Phenol bouillon-}

Dissolve in nutrient bouillon, before final filtration, carbolic acid crystals in the proportion of 5 grammes ( 5 per cent.), I gramme (. I per cent.), or 5 decigrammes (.05 per cent.) per litre, according to strength desired.

\section{Dextrose-free bouillon-}

Inoculate alkaline bouillon with a pure culture of $B$. coli or other organism known to ferment dextrose, and incubate for forty-eight hours at $37^{\circ} \mathrm{C}$. Place in the Koch steriliser at $100^{\circ} \mathrm{C}$. for twenty minutes, allow to stand for twelve hours in a cool place, decant supernatant fluid, filter, tube, and sterilise in the autoclave at II $5^{\circ} \mathrm{C}$. for fifteen minutes. To test freedom from dextrose fill a fermentation tube (see p. 77) with the medium at the same time, and sterilise with the tubes; re-inoculate this when cool with a culture of $B$. coli. If no fresh gas formation is observed after incubation at $37^{\circ} \mathrm{C}$. for forty-eight hours, the bouillon may be considered dextrose-free.

\section{Parietti's bouillon-}

To tubes containing Io c.c. of sterile nutrient bouillon add, by means of a sterile calibrated pipette, $\cdot I, \cdot 2$, and $\cdot 3$ c.c. of Parietti's solution prepared as follows:-Dissolve 5 grammes carbolic acid crystals in 100 c.c. of distilled water, and add 4 c.c. of pure hydrochloric acid.

\section{MacConkey's liquid medium-}

An emulsion of 20 grammes of dry peptone (Chapoteaut or Witte) is made in 200 c.c. of distilled water at $60^{\circ}$ C., and 5 grammes of commercial sodium taurocholate and 5 grammes of glucose are then added and dissolved by stirring. The emulsion is placed in a flask with 800 c.c. of distilled water, and heated in the Koch at $100^{\circ} \mathrm{C}$. for twenty minutes. It is then filtered through Swedish filter paper into a sterile flask, and sufficient litmus solu- 
tion is added to produce a deep purple coloration. Fermentation tubes are filled with a measured quantity of the liquid, and sterilised in the Koch at $100^{\circ} \mathrm{C}$. on three consecutive days.

\section{Drigalski and Conradi's medium ${ }^{1}$ -}

Prepare as follows:-(a) Agar solution; (b) Litmus solution (a) Put $3 \mathrm{lb}$. finely-minced beef into 2 litres of water, and allow to stand till following day. Press out the "Fleischwasser," boil for one hour, filter, add 20 gr. dry Witte's peptone (pep. siccum), $20 \mathrm{gr}$. neutrose, and Io gr. $\mathrm{NaCl}$; boil again for one hour, and add $60 \mathrm{gr}$. finest bar agar; boil for three hours, make faintly alkaline to litmus, filter, and boil for half an hour. (b) Take 260 c.cm. of Kubel and Tiemann's litmus solution, boil for ten minutes, add 30 gr. chemically pure milk-sugar, and boil for fifteen minutes. Well $\operatorname{mix} a$ and $b$, and re-establish the faintly alkaline reaction; add $4 \mathrm{c.cm}$. of a hot sterile solution of Io per cent. anhydrous soda and $20 \mathrm{c.cm}$. of freshly-prepared solution of I gr. crystal violet B. (Hochst) in $100 \mathrm{c.cm}$. of warm distilled water. The authors give many details of manipulation and technique, for which the original memoir should be consulted.

\section{ro. Nutrient gelatine (to make I litre)-}

Take half a litre of liquid peptone prepared as above and filter cold. Place in clean enamelled saucepan, bring slowly up to boiling point, and boil for five minutes. Filter, and make alkaline to litmus with caustic soda solution. Very exact neutralisation is not necessary at this point, as the whole medium will subsequently be made neutral to phenolphthalein. Add half a litre of beef bouillon prepared as above up to and including the first filtration, and 5 grammes of common salt. Bring up to boiling point, boil for ten minutes, filter hot, and make up to I litre with distilled water. Remove the flame, and add slowly I Io grammes (winter) or I 20 grammes (summer) of best gold label gelatine, stirring constantly until dissolved. Replace flame, and bring up again to boiling point. Neutralise to phenolphthalein in the manner described under the head of neutralisation of media. Boil for five minutes, cool down to $60^{\circ}$, add the white of one egg mixed with 60 c.c. of distilled water, and thoroughly incorporate by stirring. Steam for one hour in Koch's steriliser. Filter hot through double filter paper, run into tubes, and sterilise by intermittent heating in the Koch steriliser at $100^{\circ}$ for thirty minutes on three successive days. 


\section{I1. Carbolised gelatine-}

Dissolve in the liquefied gelatine prior to final filtration carbolic acid crystals in the proportion of 5 grammes $(\cdot 5$ per cent.), I gramme (. I per cent.), or 5 decigrammes (.05 per cent.) per litre, according to strength desired.

\section{Glucose formate gelatine (Kitasato)-}

To 500 c.c. of hot nutrient gelatine just prior to final filtration, add 2 grammes ( 4 per cent.) of sodium formate, and Io grammes ( 2 per cent.) of anhydrous glucose. Dissolve and thoroughly mix before filtering.

\section{Glucose (or lactose) gelatine-}

As above, with omission of the sodium formate.

\section{Litmus gelatine-}

Liquefy a tube or tubes of nutrient gelatine by placing in hot water, and by means of a finely-drawn Pasteur pipette introduced between the cotton wool plug and the tube wall, add sufficient sterile litmus solution to tint the medium a deep lavender colour. Roll well in the hand to ensure an even distribution of the colour and allow to cool either upright or on the slope as required.

Preparation of neutral litmus solution.-Extract all the colouring-matter from solid litmus by repeated digestion with hot water. Evaporate this solution to a moderate volume, and convert all carbonates present into acetates by adding a slight excess of acetic acid. Again evaporate the solution over a waterbath until it becomes pasty, and then add an excess of alcohol. The spirit will precipitate the blue colouring-matter, while a red colouring-matter, together with the alkaline acetates, will remain in solution. The precipitate is transferred to a filter, and is washed with spirit. It is then dissolved in warm water, and the solution is rendered purple by the cautious addition of dilute nitric acid.

\section{Nutrient agar (to make I litre)-}

Proceed as in the case of nutrient gelatine up to and including the addition of the salt. Bring up to the boiling point and neutralise to phenolphthalein in the manner described under neutralisation of media. Boil for five minutes. Filter hot and make up to I litre with distilled water. Add, after compressing in a cloth to get rid of excess of moisture, 20 grammes of agar cut into small pieces and previously soaked in distilled water for eighteen hours. Steam in water bath until agar is thoroughly dissolved, stirring constantly. Cool down to $60^{\circ}$. Add the white of one egg in 60 c.c. of distilled water, and thoroughly incor- 
porate with the medium by stirring. Steam in the Koch steriliser for one hour at $100^{\circ}$. Filter hot through "papier Chardin." Run into tubes or flasks, and autoclave for fifteen minutes at I $5^{\circ}$, or sterilise by intermittent heating in the Koch steriliser for thirty minutes on three successive days.

Nutrient agar (Ravenel's method).--If dry peptone is employed, or when it is necessary to prepare a stock of agar at short notice, the method devised by Dr Mazyck P. Ravenel of the University of Pennsylvania is recommended. ${ }^{1}$ The whole process, with the exception of the time the meat is digesting need not exceed two hours, and the result is a remarkably clear jelly.

The method of preparation is as follows:-To make I litre, take

$$
\begin{array}{lllll}
\text { A. Chopped meat } & * & * & * & 500 \text { grammes. } \\
\text { Water } \quad & \cdot & * & \text {. } 500 \text { c.c. }
\end{array}
$$

Mix and place in a cool place over-night, then strain through a towel or muslin.

$$
\begin{aligned}
& \text { B. Agar-agar - . } 12 \text { grammes ( } 1 \cdot 2 \text { per cent.). } \\
& \text { Water . . } 500 \text { c.c. }
\end{aligned}
$$

Put into autoclave, run up to two atmospheres ( $134^{\circ} \mathrm{C}$.) put out flame, allow to cool until below $100^{\circ} \mathrm{C}$. before opening. Let the solution of agar cool still further to about $75^{\circ} \mathrm{C}$. : then add $\mathrm{B}$ to A. Add Io grammes (I per cent) of dried peptone and 5 grammes ( 5 per cent.) of common salt : bring to boiling point and boil for five minutes, neutralise and filter. The filtration is rapid, no hot water funnel being necessary if the filter paper is wetted with boiling water immediately before using. The clarification of the medium is effected by the coagulation of the albumen in the meat water on the raising of the temperature after the addition of the salt and peptone. Hence solution A must not be added to B until cool enough to avoid premature coagulation.

\section{I6. Glycerine agar-}

To nutrient agar prepared as above add 6 per cent. of glycerine after filtration, and before final sterilisation in tubes.

17. Glucose (or lactose) agar-

To nutrient agar prepared in the manner above described, add 2 per cent. of glucose or lactose at the same time as the salt.

18. Agar gelatine-

To nutrient bouillon prepared as above, add 5 per cent. of gelatine, and .8 per cent. of agar. The temperature of sterilisation 1 Journal of Applied Microscopy, vol. i., No. 6. 
should on no account exceed $105^{\circ}$. This medium is specially useful for showing the peptonising properties of certain organisms at blood heat. If carefully made the medium remains solid up to $48^{\circ}$, liquefaction, however, by peptonisation taking place as in the case of gelatine alone, although in a lesser degree. A very valuable medium for use in the tropics.

\section{Glucose formate agar-}

Add to hot melted agar during preparation, glucose and sodium formate as under the head of "glucose formate gelatine."

\section{Nutrient milk agar-}

Thoroughly melt a tube of nutrient agar in a beaker of boiling water. Place a tube containing 5 c.c. of sterile milk in the beaker and heat up to between $70^{\circ}$ and $80^{\circ}$, but do not allow it to approach the boiling point. Remove the cotton-wool plugs and quickly pour an equal quantity ( 5 c.c.) of the melted agar into the tube containing the milk. Replace the cotton-wool plug and allow to cool either vertically or on the slope, as required. A nutrient milk gelatine can be made in the same manner provided the proportion of gelatine in the medium is not less than 15 per cent.

\section{Potato (in tubes) -}

Choose long, sound, and healthy potatoes of the same crop if possible. ${ }^{1}$ Thoroughly scrub them with a hard brush in a current of water to remove all trace of soil. Cut off both ends of the potato with a clean knife, and bore through the longer axis with a cork borer ${ }^{2}$ of $\frac{3}{4}$ in. dia-

FIG. I.-Potato borer : Emporte-piéce. meter (for tubes of $\frac{7}{8}$ in. internal diameter). Push out the cylinder, and allow it to drop on to a clean filter paper. Without touching it with the hands, and using a clean knife previously boiled, cut it through on its longer axis into two

1 The different varieties of potato vary greatly in composition and reaction, and the difference in cultural properties of some of the varieties is very striking.

${ }^{2}$ If a special "emporte-piéce" is procurable, it should be used, as with this the cylinders are, in one and the same operation, bored and divided into equal halves, and can be dropped directly into the soda solution without handling. This can now be procured from Messrs Baird \& Tatlock of 14 Cross Street, Hatton Garden. A nickled borer should in any case be employed, as the slight traces of iron salts left on the potato when bare steel is used will often modify considerably the colouring properties of chromogenic organisms. 
equal halves, and place them in a weak solution of carbonate of soda ( 1 gramme to a litre of water). Allow the cut pieces to remain in the solution for an hour, stirring occasionally with a glass rod. Renew the solution at least twice, or until all milkiness of the water on stirring has disappeared. Pour off the water, and, removing the superfluous moisture with a piece of clean filter paper, place the sections in tubes as figured, without touching them with the bare fingers. The bulbs of the tubes should have been previously filled to two-thirds of their height with either alkaline bouillon, sterile milk, distilled water, or a Io per cent. solution of glycerine in water, in which, by inclining the tube, the lower portion of the potato should be allowed to bathe when cultural growth is taking place. Sterilise in the autoclave at $115^{\circ}$ for fifteen minutes, or in the steam steriliser at $100^{\circ}$ for thirty minutes on three successive days. After sterilisation, it is well to lay the tubes, while cooling, with the plain surface of the potato downwards in order to prevent the curling up which takes place when cooled in an upright position, but to prevent undue soften-

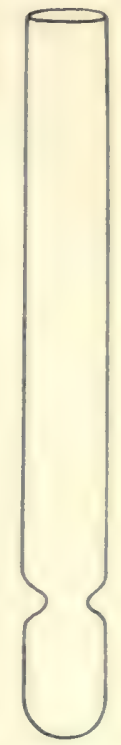

FiG. 2.-Roux potato tube. ing, care must be taken that at this stage the tubes are not sloped at such an angle as will allow the fluid in the bulb to reach the potato. When cool, a slight tap with the finger will cause the potato to resume its proper position in the tube.

\section{Potato gelatine (Elsner's medium)-}

Choose a sufficient number of sound well-grown potatoes. Peel, remove eyes, and reduce to paste by means of a coarse grater. Take 500 grammes of this paste and macerate in a litre of water for twelve hours in a cool place. Strain through muslin, make up to one litre, and bring slowly up to boiling point, adding gradually, before this point is reached, 150 grammes of best gelatine. Boil for five minutes, and neutralise to litmus with caustic soda solution. Add a sufficient quantity of normal $\mathrm{HCl}$ to leave the medium feebly and yet distinctly acid. Autoclave for twenty-five minutes at $115^{\circ}$, and filter hot through "Chardin" paper. Run into tubes or flasks and sterilise in the autoclave at $115^{\circ}$ for fifteen minutes, or steam in the Koch steriliser at $100^{\circ}$ for twenty minutes on three consecutive days. 
Add, immediately before use, one per cent. of potassium iodide to the melted gelatine.

23. Milk : (a) sterile milk, (b) sterilised milk-

(a) Sterile milk.-No great difficulty need be experienced in obtaining sterile milk direct from the udder of the normally healthy cow. Such milk is a more favourable medium for the growth of bacterial flora than that in which the process of sterilisation by heat has produced chemical or physical change. With the simple apparatus figured on Plate 7 , the authors have for some years past found no difficulty in holding ready to hand a constant supply of sterile milk in its natural condition. The apparatus, which is shown both in the position of drawing milk, and with the milk stored ready for drawing off into tubes or flasks as required, consists of a Kitasato filtering flask of 2 litres capacity, fitted with a two-hole india-rubber stopper, and with the lateral orifice plugged lightly with cotton-wool. Through one of the holes in the cork is led a curved glass tube as shown, reaching in the interior to within a quarter of an inch of the bottom of the flask, and a short length of india-rubber tubing, fitted with a screw pinchcock, connects the terminal curve with a further length of straight glass tube reaching on the outside to within about 2 inches from the bottom level of the flask. (After the milk is drawn, this tube can be fitted, in order to minimise the risk of air contamination when drawing off the milk into test tubes, with a one-holed india-rubber stopper, and a small lamp glass of sufficiently large capacity to allow of the insertion into it from below of an ordinary test tube.)

Through the second hole of the neck stopper is led a short and right-angled length of glass tubing, projecting into the flask for about I inch, and connected up, at the other end, with about 2 inches of "vacuum tubing" fitted in the centre with a screw pinchcock. The end of this tubing is covered with loose fitting coverings of white wrapping paper tied on with string, and the whole apparatus is then ready for sterilisation.

The milking tube and accessories consist of a 3-foot length of indiarubber tubing fitted at one end with an ordinary silver plated " milking tube" such as is supplied by all veterinary instrument makers, ${ }^{1}$

${ }^{1} \mathrm{~A}$ better form still is the tube supplied with the apparatus for injecting a solution of potassium iodide into the udder in the case of milk fever in cows, and which can be procured from Messrs Arnold \& Son, veterinary instrument makers, Smithfield. 
a.

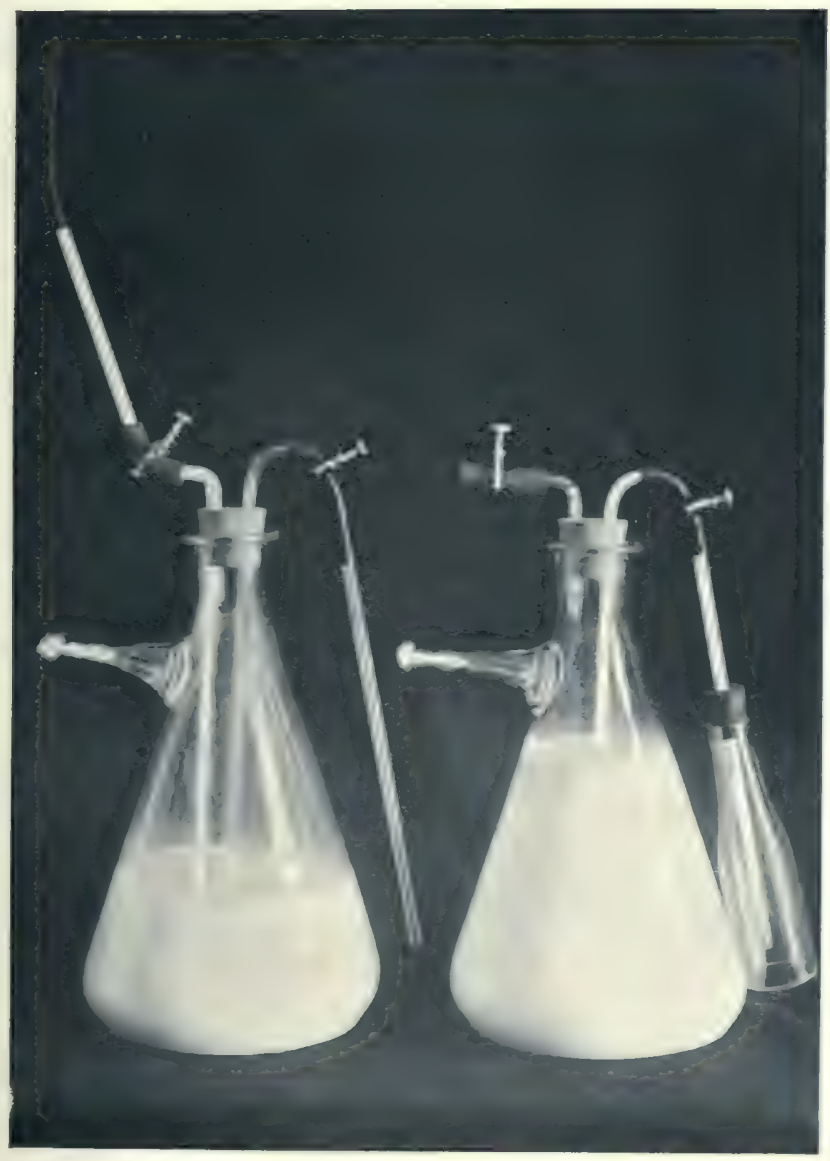

Method of drawng and storing sterile milk.

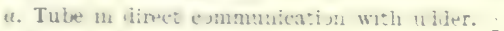



and at the other with a length of about 5 inches of glass tubing drawn slightly to a point in order to allow it more readily to enter the tube orifice of the flask in subsequent manipulations. A Mohr pinchcock is placed on the indiarubber tubing midway between the two terminal tubes, and these latter are placed in test tubes plugged with cotton-wool, and in which a few drops of water have been placed in order to ensure perfect sterilisation. The whole apparatus, including the flask, is then sterilised in the autoclave and held in reserve.

\section{Method of drawing the milk.-} Select a healthy cow in full milk, and which a careful inspection shows to be free from any signs of indurated udder. Place in a stall or box from which all litter has previously been cleared away, and

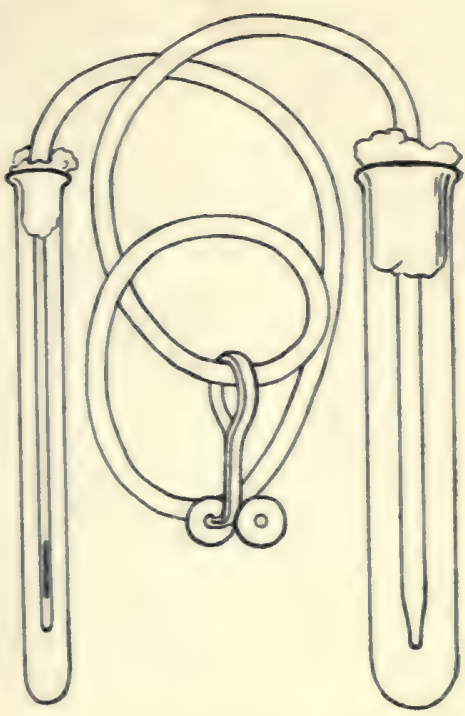

FIG. 3.-Milking tube and accessories. the floor of which has been well washed down with water. Wash carefully the flanks, belly, end of tail, udder, and teats, with clean water followed by bichloride solution, or other effective germicide. Let the attendant, after rinsing his hands well with the antiseptic solution, draw about a dozen draughts of milk on to the ground in order thoroughly to clear the milk duct of any organisms which may have worked their way up it. After this is done, take from an assistant the test tube containing the milking tube, remove this latter from the tube, and, holding the teat in the left hand, pass the milking tube into the orifice of the teat and up until the constrictor valve is felt to be passed. The milk will at once begin to flow into the india-rubber tubing. Open the pinchcock and the milk will flow freely into the test tube containing the glass outlet tube. It is better to let this fill almost to overflowing in order that any stray organisms which may possibly have got on to the milking tube in the previous manipulations may be washed away. Compress the tube with the pinchcock, remove the paper covering from the vacuum tubing, and taking the glass tube out of the test tube, pass it quickly into the open end of the tubing. The milk will then flow freely into the flask on opening 
the pinchcock. When one quarter of the udder is exhausted, the operation, if a sufficient quantity has not been drawn, can be repeated with a second quarter.

The screw pinchcock on the vacuum tubing is then screwed down permanently, the milking tubes are withdrawn, and the milk stored ready for use. The cream will rise to the top, and the delivery tube will draw from the lower layer of milk free from fat. When the cream has fully risen, charge the syphon by blowing through the lateral orifice of the flask, and the milk can then, by releasing the pinchcock, be drawn off at any time and in any quantity required. The authors have kept milk in this manner without contamination for more than two years, drawing off the quantity required at intervals.

(b) Sterilised milk.-Milk sterilised by heating cannot, owing to certain organic or chemical changes which take place in it at or near the boiling point, be looked upon as so favourable a medium for the growth of micro-organisms as that which is drawn sterile from the cow in the manner referred to above. Under ordinary circumstances, however, it may be necessary to employ it, and in this case it is well, if possible, to procure freshly separated milk and allow it to stand in a cold place for twelve hours in order that any fat left by imperfect separation may rise to the surface. The fatfree milk is then syphoned off from the bottom, run into sterile tubes or flasks, and steamed in the Koch steriliser for one hour on three successive days. As, however, this may be insufficient in some cases to destroy the spores of the bacillus subtilis if they exist in quantity in the milk, the containing tubes should be tested after sterilisation by being placed in the incubator at $37^{\circ}$ for twentyfour hours.

\section{Litmus milk-}

Sterilise neutral litmus solution by boiling in a test tube, and when litmus milk is required, introduce into a tube of sterile milk by means of a finely drawn flamed pipette, and without withdrawing the cotton-wool plug, a sufficient quantity of the solution to give the milk a distinct lavender tint.

\section{Gelatinised milk-}

Prepare a 15 per cent. solution of gelatine in distilled water. Neutralise or make feebly alkaline to litmus ${ }^{1}$ with caustic soda solution. Run into test tubes in the proportion of 5 c.c. per

${ }^{1}$ Neutralisation to phenolphthalein would, in this case, make the resulting medium of much greater alkalinity than the normal milk itself. 
tube, and hold ready to hand. When gelatinised milk is required as a medium, place one of these tubes, and a tube containing 5 c.c. of sterile milk, in a beaker of water. Heat up to $45^{\circ}$, and then, removing the cotton-wool plugs, quickly pour the milk into the gelatine. Replace the cotton-wool plug, roll the tube in the hands for a few seconds and place on one side to cool either upright or on the slope as required. Exactness of measurement is not necessary in the case of the milk, provided the quantity added does not greatly exceed that of the gelatine solution. If the manipulations are carefully carried out, and the tube orifices flamed, there should be no fear of contamination.

\section{Sterilised whey-}

Heat a sufficient quantity (say I litre) of separated milk to $4 \mathrm{I}^{\circ} \mathrm{C}$. in a water bath. ${ }^{1}$ Make a solution of .5 of a gramme of a reliable rennet powder in 100 c.c. of distilled water. Thoroughly stir or agitate the mixture, and place on one side for precipitation of the casein, which ought to take place within a few minutes. Allow to stand for one hour. Filter off the clear whey by means of coarse muslin, add the white of one egg well mixed with 50 c.c. of distilled water, and thoroughly incorporate by vigorous shaking. Place in the Koch steriliser and steam for one hour at $100^{\circ} \mathrm{C}$. to allow precipitation of the albumen. Filter, run into test tubes and sterilise by intermittent heating in the Koch for twenty minutes on three successive days. It should be noted that rennet acts on casein only in neutral or acid solution. If, therefore, alkaline whey is desired it will be necessary to alkalinise as laid down under the reaction of media.

\section{Gelatinised litmus whey-}

Take whey prepared as above up to and including the first filtering off of the clear whey from the precipitated casein. Allow to stand in a cool place for twenty-four hours and decant off clear supernatant liquid. Heat up to $65^{\circ} \mathrm{C}$. in a water bath, and add $i 5$ per cent. of gelatine cut into small pieces. When thoroughly dissolved neutralise the medium to litmus with caustic soda solution. Cool down to $45^{\circ} \mathrm{C}$., add the white of one egg well beaten up, and thoroughly incorporate with the medium. Steam in the Koch at $100^{\circ} \mathrm{C}$. for one hour. Filter through Chardin paper (this final filtration is a somewhat tedious process), and add sufficient

1 The optimum temperature for the action of rennet was found by Fleischmann in his experiments to be at $4 \mathrm{r}^{\circ} \mathrm{C}$,, but free precipitation will take place at any temperature between $35^{\circ}$ and $45^{\circ} \mathrm{C}$. 
neutral litmus solution to produce a deep lavender colour. Run into flasks or test tubes in measured quantities, and sterilise at $100^{\circ} \mathrm{C}$. in the Koch for half an hour on three successive days. Sloped tubes will be found preferable, as there is a tendency to unequal coloration of the medium when the tubes are allowed to cool in a vertical position.

\section{Petruschky's litmus whey ${ }^{1}$}

Fresh milk is warmed up to $40^{\circ}$, and sufficient very dilute $\mathrm{HCl}$ is added to cause precipitation of the casein. This latter is then filtered off, and the clear whey neutralised to litmus with dilute caustic soda solution. The fluid is then steamed in the Koch for two hours, by which procedure any casein converted into acid albumen by the hydrochloric acid is precipitated. This is filtered off, and the filtrate should now be clear, colourless, and neutral in reaction, its chief constituent being lactose. To this 5 per cent. of a saturated solution of litmus is added, and the medium run in equal quantities into test tubes and then sterilised. When growth has taken place in an inoculated tube the amount of acid can be estimated by dropping in from a burette standardised soda solution until the tint of an uninoculated tube is reached.

\section{Litmus lactose solution-}

Dissolve 2 grammes of pure lactose in 40 c.c. of nutrient bouillon, and make up to IOO c.c. with distilled water. Neutralise to litmus, steam in Koch steriliser for half an hour and filter. Add a sufficient quantity of neutral litmus to produce a deep lavender colour. Run into test tubes and sterilise in the Koch steriliser for half an hour on three successive days.

30. Artificial lacto-serum (Bordas and Joulin) ${ }^{2}$ -

This medium, which it is claimed has the same cultural properties as milk, is prepared as follows :-

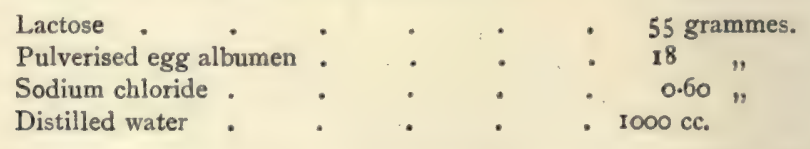

1 The recognised formula of this medium, which was extensively used by Petruschky in his researches upon the Bacillus coli, is given, although the authors have found that the precipitation of the casein by $\mathrm{HCl}$ is not so reliable or satisfactory in its final results as that by rennet in the method described above.

${ }^{2}$ Societé de Biologie, 9 th Jan. 1897. 
Neutralise with caustic soda until the reaction is distinctly alkaline, filter into test tubes, and sterilise in the autoclave at $110^{\circ}$ for ten minutes, allowing the tubes, during sterilisation, to lie on a bed of cotton-wool in order to prevent the alkaline solution of lactose turning brown through too sudden heating.

\section{I. Extract of malt-}

Thoroughly crush 100 grammes of freshly malted barley, and place in a litre of water. Shake well and allow to macerate for one hour. Place in a water bath and raise the temperature very slowly to from $50^{\circ}$ to $55^{\circ}$. Maintain it at this for one hour, care being taken not to exceed a temperature of $58^{\circ}$ or the diastase transforming the starch into maltose will be destroyed. At the expiration of the hour bring up quickly to boiling point, filter through Chardin paper, run into tubes, and sterilise in the Koch at $100^{\circ}$ for half an hour on three successive days.

\section{Gelatinised beer-wort-}

Take I litre of fresh beer-wort (preferably un-hopped). Sterilise in the autoclave at $115^{\circ}$ for twenty minutes, and allow to stand for a fortnight. Decant, filter, heat up to $80^{\circ}$, add 100 grammes of best gelatine, stirring well. Clarify and filter as in the case of ordinary nutrient gelatine, run into test tubes, and steam in the Koch steriliser for half an hour at $100^{\circ}$ on three successive days. If a liquid medium is desired omit the gelatine and heat up to $60^{\circ}$ only before the addition of the white of egg. Steam in the Koch to ensure good coagulation and proceed as above.

\section{Peptone solution (Dunham)-}

Make into a paste of the consistency of thick cream by the addition of a little distilled water 10 grammes of dry peptone (Chapoteaut or Witte), and 5 grammes of sodium chloride. Add slowly I litre of boiling water, stirring well. Boil for five minutes, stirring constantly. Filter, and run into test tubes. Sterilise in the autoclave for fifteen minutes. Owing to the natural alkalinity of the dry peptone this medium rarely requires neutralisation. Used for indol reaction.

\section{Hay infusion-}

Cut into small pieces 15 grammes of hay, and allow to macerate in I litre of water for three hours. Bring slowly to boiling point, and allow to boil for five minutes. Filter, test reaction, and neutralise, acidify, or make alkaline, as desired. Steam in Koch's 
steriliser for half an hour, filter again, and run into test tubes. Sterilise in the autoclave at two atmospheres in order to ensure destruction of the spores of bacillus subtilis.

\section{Choice of medium-}

This must be left very largely to individual experience and the objects of the investigation. In a general way the constituents of the various media described indicate the purposes to be obtained. The general standard liquid media are bouillon and milk, the solid media are gelatine (for room temperature cultivation) and agar (for blood heat). In tropical countries a combination of the two may be used. Further, just as gelatine is a solid bouillon, so gelatinised milk may be used when a solid milk medium is required. For anaërobes glucose and formate media are commonly used. There are, of course, various media used for different species of organisms. For the streptothrix group, including B. tuberculosis, glycerine media and potato are used. To isolate the B. typhosus carbolised media and Elsner are taken. Chromogenic bacteria nearly always grow well on potato. The use of litmus milk, beer wort, wort gelatine, milk agar, etc., is sufficiently designated in the names of the media.

Preservation of media.-Media may be kept in good condition for months if a few simple precautions are borne in mind. The tubes or flasks containing the medium must be effectually sealed, either with caps, corks, or paraffin. The store of media must then be kept in a closed metal box, and in a cool dark place.

\section{Quantitative Examination of Milk and Method of Milk Dilution}

In the many experiments we have carried out with fresh milk we have not found it necessary as a rule to carry dilution further than 500 times. But in some cases it will be found necessary to dilute up to 1000 or even 5000 times if a satisfactory result is to be obtained. The procedure we have adopted for this dilution, though simple, is likely to become involved unless undertaken with care and proceeded with a step at a time. The method is as follows :-

Apparatus required (for six samples) -

I. Six ordinary I c.c. pipettes.

2. ${ }^{1}$ Six dropping pipettes specially calibrated to drop exactly twenty drops to every cubic centimetre.

1 Very accurate calibration is required in the pipettes, and those used by us have been supplied by Ruelle of to Rue Chouin, Paris. 
3. A liberal supply of test tubes marked on the outside, with a diamond or fine file dipped in turpentine, at the level of 9 c.c. of water.

4. A liberal supply of small conical flasks marked at the level of 49 c.c. of water.

5. A sufficient number of sterile Petri dishes.

The pipettes should be carefully cleaned and dried, lightly plugged with cotton-wool at the upper end, placed either in a cylindrical box made of copper or thin sheet iron (or a glass cylinder of suitable length cut from stout tubing of about $I_{\frac{1}{8}}$ in. diameter and plugged at each end with cotton-wool), and sterilised in the hot air steriliser for one hour at $150^{\circ} \mathrm{C}$. To avoid contamination of the exterior they should not be removed from the box or cylinder until required for use.

Into the test tubes and conical flasks, previously plugged with cotton-wool and sterilised in the hot air steriliser for one hour at $150^{\circ} \mathrm{C}$, are run 9 c.c. and 49 c.c. of water respectively. It is better to allow a slight margin over the exact quantity in order to avoid possible loss during sterilisation. Any excess is easily withdrawn subsequently by means of a sterile Pasteur pipette $^{1}$ passed between the stopper and the neck. The tubes and flasks are then either sterilised in the autoclave at $115^{\circ}$ C. for thirty minutes, or in the Koch for half an hour on three successive days. After sterilisation they should be provided with an india-rubber cap to prevent evaporation, and placed on one side until required for use.

Procedure. Dilution to 500 times.-I. By means of a sterilised I c.c. pipette remove from the original sample I cubic centimetre of the milk under examination, and place it in a test tube of 9 c.c. of sterile water. Aspirate the mixed milk and water into the pipette at least 3 times in order to well wash down the interior walls, and, replacing the cotton-wool plug of the tube, but keeping the pipette within it, roll the tube well in the hands to ensure an even distribution of the organisms in the original milk. The tube now contains Io c.c. of liquid containing I c.c. of the original milk. This is termed the primary dilution- $\mathrm{I}$ in $\mathrm{IO}$.

2. Transfer, by means of the same pipette, I c.c. of this primary dilution to a flask containing 49 c.c. of sterile water. Aspirate 3

${ }^{1}$ For the method of making these pipettes, which will be found of the greatest possible service in almost every branch of bacteriological work, see Appendix A, p. 547. 
times as in the former case, withdraw the pipette, replace the cotton-wool plug and shake for some minutes in order to obtain an even distribution of the organisms. The flask now contains 50 c.c. of liquid containing $\frac{1}{10}$ of the original milk, or a dilution of $\mathrm{I}$ in 500. This is termed the final dilution.

It will easily be seen that by means of these test-tubes and flasks of water almost any desired dilution can be made. Thus if a dilution of $\frac{1}{1000}$ is desired, it is only necessary to transfer I c.c. of the primary dilution to a second tube containing 9 c.c. of water, and again I c.c. of this admixture to a third tube under the same conditions as above.

Or if a dilution of $\frac{1}{5000}$ is thought desirable, then I c.c. of the primary dilution is transferred to a second tube of 9 c.c. of water, and I c.c. of this admixture to a flask containing 49 c.c. of water. The flask will contain the final dilution of $I$ in 5000 . A dilution of I in 10,000 will require four tubes of 9 c.c. of water, etc., etc., etc.

Whatever the dilution may be, the subsequent procedure is the same up to the enumeration of the colonies. For each milk three or four tubes of liquefied gelatine and an equal number of Petri dishes will be required. The tubes of gelatine should be clearly marked I, 2,3 , etc., to correspond with numbers on the covers of the Petris, and each of these latter should be clearly labelled both with the amount of the final dilution in each case, and the exact fraction of this dilution which has been used for plating purposes. Otherwise confusion is likely to occur in the subsequent estimation of the number of organisms.

The most convenient fractions will be found to be .05 c.c. (one drop from the calibrated pipette), $\cdot$ I c.c. (two drops), $\cdot 2$ c.c. (four drops), 5 c.c. (ten drops).

The procedure is as follows:-

I. Remove the plug of the flask or tube containing the final dilution and aspire into the calibrated dropping pipette a sufficient quantity of the liquid.

2. Take the tube of gelatine marked $\mathrm{I}$, from which the attendant will have withdrawn the plug, and drop carefully, without allowing it to touch the tube walls, one drop from the pipette into the liquefied medium.

3. Replace the plug and place the tube in a beaker of warm water. Proceed in the same manner with the tube marked 2, but add two drops of the medium. In the third tube four drops will be required, and in the fourth ten drops. 
4. Take the first tube from the beaker and well roll it in the hand (inclining it occasionally) in order to obtain an even distribution of the organisms. Raise the lid of the Petri dish marked to correspond with it, remove the cotton-wool plug, pass the tube orifice through the flame, and pour out the gelatine into the dish with an even flow. Place aside on a level surface until solidification has taken place.

5. Proceed as rapidly as possible with the other tubes in a similar fashion, and when solidification has taken place on the four plates, place them at a suitable temperature for the development of the colonies. We have ourselves found that the temperature of an ordinary room, $15^{\circ}-18^{\circ} \mathrm{C}$. gives the best result.

In practice it will be found that under ordinary circumstances the plate containing .5 c.c. of the final dilution will be superfluous. But, especially in the examination of town milk, it should on no account be omitted, for in the event of the addition of antiseptics to the milk, it will often be found that the colonies on the plates containing a less quantity of the dilution will be so few in number that error will be likely to occur if they are relied upon alone.

A very convenient form of wire stand or cage, fitted for Petri dish cultivations when made is shown in Fig. 4. The arrangement of the stages is such that the lids are held close down and cannot be removed without withdrawing the dish itself from cage. ${ }^{1}$

Counting the colonies. - The colonies

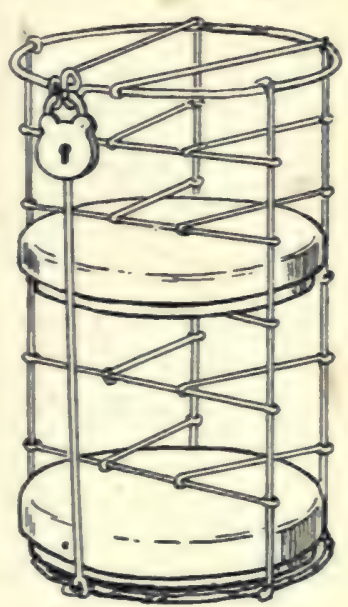

FIG. 4.- Wire cage stand for Petri dishes. should be counted upon the second, third, and fourth days, and an estimate made, not only of the total number of organisms per c.c. of milk, but also of those producing liquefaction of the gelatine. For the enumeration we recommend the circular card form of counter such as that of Pakes, illustrated on Plate 8 . The plates should be carefully adjusted in the centre of the black disc, the covers removed, and the organisms counted with the aid of a magnifying glass. Unless this latter precaution is taken it is almost impossible to distinguish between

1 These and other apparatus of a like nature, referred to in this work, can be obtained from E. Cogit \& Cie., 49 Boulevard St Michel, Paris. 
minute sub-surface colonies and small air bubbles contained in the medium. If the colonies on the plates are comparatively few, it is more satisfactory to count the whole of them, and with the assistance of the divisions on the black disc this can be done without difficulty. If numerous, however, it will suffice to count say half the number of sectors, to estimate from them the average number per sector, and multiply by the total number of sectors on the counting card. This will give approximately the total number of organisms on the plate, and the subsequent calculations will be as follows :-

$x \times y \times z=$ number of organisms per c.c. of the original milk, $x$ being number of organisms on the plate, $y$ the multiple of fraction of the final dilution taken, and $z$ the amount of such dilution.

Example.-Given 05 of a dilution of $\mathrm{I}$ in 500 , and 98 as the total number of organisms on the plate. Then

$98 \times 20 \times 500=980,000$ - total number of organisms per c.c.

or, Given $\cdot 2$ of a I in 5000 dilution, and 476 as the total number of organisms. Then

$476 \times 5 \times 5000=I 1,900,000$ - total number of organisms per c.c.

At least two and if possible three plates should be counted for each sample of milk, and the average of these taken as the approximate number of organisms per c.c. of the milk.

Petri dishes.-Those of 9 centimetres internal diameter will be found the most convenient. They should be carefully selected, and any showing unevenness on the interior bottom surface should be rejected. A regular distribution of organisms over the plate is impossible when the medium is of varying depth.

A supply of these dishes should always be kept sterile ready to hand. The best way of ensuring this is to wrap up each dish in the form of a small paper packet before sterilisation, ${ }^{1}$ and only remove the paper covering when the dishes are required for use. In making the packet the dish should be placed lid downwards, so that when finished the folds come at the bottom side when the plate is in proper position. After sterilisation for one hour at $150^{\circ} \mathrm{C}$. they are taken out of the hot air steriliser, and stored in a drawer or other convenient place free from dust. If stored with the folded side of the paper downwards and the paper unbroken, there is practically no chance of contamination under ordinary circumstances. To avoid carbonisation of the paper care should be taken that the dishes during sterilisation do not come in

${ }^{1}$ A very suitable paper for this purpose is that known to the trade as " $22 \frac{1}{2}$ white Demy." 
contact with the hot iron of the bottom or sides of the steriliser. Funnels, conical glasses, and other glass apparatus of small size can be kept sterile by treating in a similar manner.

\section{The Making and Staining of Preparations for the MICROSCOPE}

\section{General note-}

It will be found convenient to stain on the glass slide rather than on the cover glass, which should be kept for its legitimate purpose. The slides are easier to clean and handle, are less liable to breakage, and the result is the same. If it is desired to keep the preparation as a permanent cabinet specimen a cover glass may be applied with Canada balsam in exactly the same manner as in the case of sections, but under ordinary circumstances this will be found unnecessary. Microscopic examination of the preparation can be made as soon as the film has dried after staining, by placing a drop of cedar-wood oil directly upon the stained surface and bringing the lens of the microscope down upon it without the intervention of a cover glass. If it is desired to preserve the specimen, either for further examination, or for mounting, the oil is easily removed with

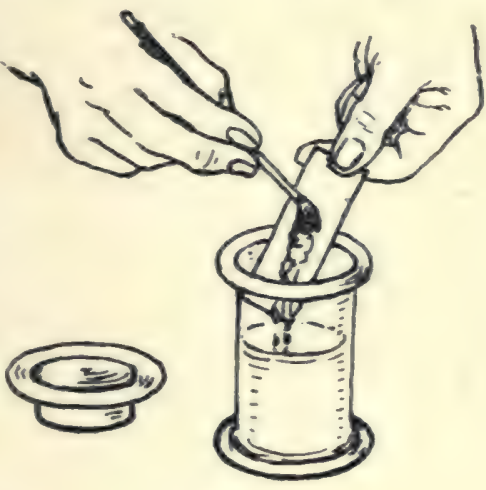

FIG. the aid of a little xylol or benzole applied with a camel's hair brush. With a due amount of care there is but little chance of removing any of the stained film in so doing. It will be found convenient to have upon the working bench a small wide mouth specimen jar accurately stoppered, and containing sufficient waste xylol or benzole for the purpose. The method of removing the oil is shown on Fig. 5.

\section{Cleaning slides and cover glasses-}

Slides.-Slides which have been already used should be allowed to soak for some days in a strong solution of Hudson's or other extract of soap in hot water, care being taken that they do not cling together surface to surface. All traces of the film or stain should then be carefully removed with a pad of cotton-wool dipped 
in a 10 per cent. solution of $\mathrm{HCl}$, and the slides should be thoroughly washed in running water. They should then be placed in a beaker containing a mixture of concentrated sulphuric acid 30 parts, bichromate of potassium 30 parts, and water 500 parts, and allowed to remain for some hours, care being taken that they do not cling together by capilliary attraction and so prevent the acid coming in contact with the whole surface of the slide. On removal from the acid they should be thoroughly washed in running water, dried with a soft cloth free from any trace of grease and stored in alcohol until required for use.

New slides should be carefully dusted, placed in the acid solution, and allowed to soak as above. They should then be thoroughly washed in a stream of running water, dried with a soft cloth free from any trace of grease, and placed in a jar of alcohol until required for use. Care must be taken that from the moment they leave the acid bath they are not handled with the bare fingers except by the edges, and if they are to be subsequently used for flagella staining, even this should be carefully avoided.

It will be found in practice a good plan, when taking new slides into use to mark, at the extreme end of each slide, one surface with a writing diamond or sharp flint, and to make a rule of always using the marked side for the preparation. Unless some such method is adopted it is at times difficult, especially when staining by Gram's method or when the film is very thin, to ascertain on which side the preparation has been made.

Cover glasses. - If cover glasses are to be used for making preparations upon they should be treated in similar fashion to slides as described above, but if, as recommended, staining is to be carried out on the slide, and the cover glass used only as a cover, it will be found sufficient to clean them between the thumb and index finger with a clean soft rag moistened with a mixture of equal parts of alcohol and ether, and drop them one by one into a jar of alcohol. When required for use they are removed from the alcohol and carefully dried and polished with a clean soft cambric rag.

\section{Preparation of the film-}

The staining of the film when milk is under examination is described below, and in examining cultural growth in bouillon or other liquid media of a like nature it will be found advisable after fixing to clear the film with a 5 per cent. solution of acetic acid 
as there described. If, however, the growth is upon solid media, the following method should be adopted :-

Place a small droplet of distilled water in the centre of a thoroughly clean slide. With the point of a fine platinum needle take up a trace of the culture under examination and inoculate with it the water on the slide. Spread the droplet, which now ought to have a cloudy appearance, in a thin even film by gently moving the needle in a circular direction. Dry by waving the slide to and fro in the heated air above a Bunsen burner or spirit lamp. A well-spread film should have the appearance of a thin opaque cloud of even density, just visible on the surface of the slide.

Fixation.-The object of fixing is to coagulate the albuminous material and cause perfect adhesion of the prepared film to the slide. The following alternative methods are recommended:-

(a) Heat.-Holding the glass slide by one extremity between the thumb and index finger of the right hand, pass it, film side upwards, gently through the flame three times, allowing the under surface to rest on the back of the left hand between each passage. The judging of the exact amount of heat required is a matter of practice, but it should on no account be greater than the back of the hand can comfortably bear when the under surface of the slide is placed upon it.

(b) Alcohol-ether.-Place one or two drops of a mixture of equal parts of absolute alcohol and ether upon the dried film, and allow it to evaporate.

(c) Formol-alcohol--Formalin I part, absolute alcohol 9 parts. Leave in contact for from three to four minutes, wash well in water, blot off excess of moisture, and stain.

(d) Perchloride of mercury. - Saturated aqueous solution. Leave in contact with the film for from four to five minutes. Wash off with a stream of water and apply Gram's iodine solution in order to dissolve out any formed crystals of the salt. Wash again in water, blot off excess of moisture, and apply stain. This fixing agent should be used on all occasions when dealing with morbid material or cultures of a specially virulent nature.

\section{Simple Staining of Milk Specimens}

The ordinary methods of staining bacteria in use in the laboratory may be readily applied in the case of milk. In certain 
samples it is best however to dilute the milk, or secure a deposit by the gravity flask method or centrifuge. The only difficulty which presents itself in the preparation of milk for the microscope is the simultaneous staining of the casein and fat as well as the organisms, which may seriously confuse the issue. Hence the removal of the two former substances is recommended.

In the course of our investigations we have tried a large number of staining methods, and we have obtained the best results from one or other of the following :-

(a) Staining after clearing with 5 per cent. acetic acid.-The slides are thoroughly cleaned in the ordinary way, and immediately before use are again washed with equal parts of alcohol and ether. Several loopfuls of the milk to be examined are now placed on the slide and allowed to dry at the temperature of the room, being protected from the air by means of a small glass cover. When the film is dry it is fixed, preferably with alcohol and ether, in the manner above described. It is then washed alternately with a 5 per cent. solution of acetic acid and distilled water until there is but little apparent film left upon the slide, which is then dried between layers of "papier de soie" or fine filter paper. The specimen may now be stained by means of any of the ordinary aniline dyes, washed in distilled water, again dried, and examined under the microscope. We have tried ether, chloroform, various strengths of alcohol, and many other clearing agents, but we have obtained the best results from acetic acid.

(b) Saponification.-If it be desired to retain the background of casein and fat, it will be found best to saponify the milk in the following manner:-

Prepare the film of milk as before, but before drying it add an equal number of loopfuls of a sodium carbonate or sodium hydrate solution ( 5 per cent. to 50 per cent. dilution). The loopfuls of milk and soda solution should be placed in immediate proximity to each other on the slide, and thoroughly mixed by means of the platinum loop. By this means an even distribution of the bacteria is obtained. The film is then dried by gentle heating, stained, washed, and cleared with xylol. The result will be that the organisms will be stained more deeply in colour than the background of saponified matter.

(c) Clearing with acetic acid after saponification.-We have found as a general rule that the best preparations are obtained by a combination of the above two simple methods. For this purpose the films are prepared exactly as in the ordinary saponification 
method above described, but as soon as the films have become saponified, instead of at once proceeding to stain with the desired dye, the film is thoroughly cleared by several alternate washings with the 5 per cent. solution of acetic acid, and distilled water. The subsequent procedure is as in (a). In our hands this simple plan has yielded excellent results. The organisms are both clearly defined and well stained.

\section{StAining REAGENTS}

There is no need for a multiplicity of staining reagents in ordinary work upon milk or its bacteria. ${ }^{1}$ We have found in practice that, except when engaged in special work, the following concentrated solutions are quite sufficient as stock:-

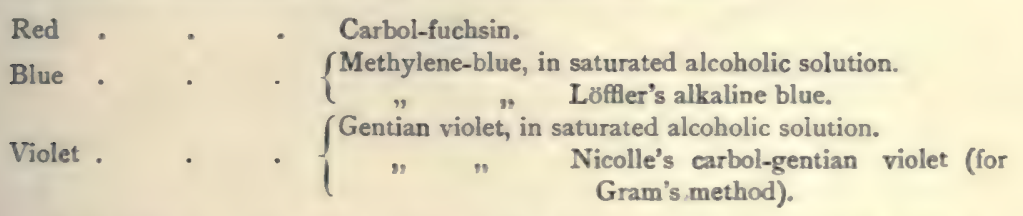

The above, when made up, will keep for a lengthened period, and from them the watery solutions employed in staining ordinary bacteria can be made up in a few moments when required for use. It is advisable, however, as they will probably be exposed to the full light of the working bench, that they should be stored in drop bottles of a deep amber colour. Convenient forms of these bottles are illustrated on Plate 8 . The stains are made up as follows :-

\section{Carbol-fuchsin (Ziehl-Neelsen).}

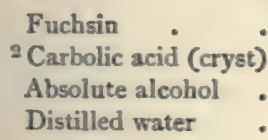

Triturate the fuchsin and the alcohol in a small glass mortar; add the carbolic acid and well mix. Add by degrees to the above two-thirds of the distilled water and pour into a small conical flask or other convenient receptacle. Wash out the mortar with the remainder of the water, and add it to the bulk. Shake well and

${ }^{1}$ Care should be taken, however, that whatever stains are used are fresh. We have found that the best stains are those which bear the name of Grüber of Leipsig, or Marck of Darmstadt.

2 Solution of carbolic acid ( $\mathrm{I}$ in 20 ) may be employed in the proportion of 90 parts, and the carbolic crystals and distilled water omitted. The authors however, prefer the formula as above. 
allow to stand for twenty-four hours. Filter three times and place in a drop bottle, of a deep amber colour preferably.

Loffler's Alkaline Blue.

Methylene-blue, saturated alcoholic solution .

Caustic potash, or per cent. aqueous solution $\begin{array}{r}-\quad 30 \text { c.c. } \\ \hline\end{array}$

Shake well and filter. Should be used fresh, as staining power weakens with age.

Saturatea Alcohol Solutions of Methylene-blue or Gentian Violet.

Colouring matter

Absolute alcohol

Shake the above well together, and allow to stand for twentyfour hours, shake again, allow to settle, and filter twice, and again

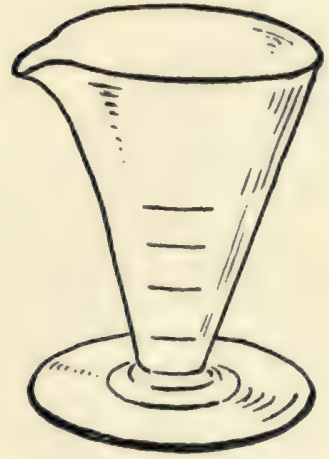

FIG. 6.-Conical glass.

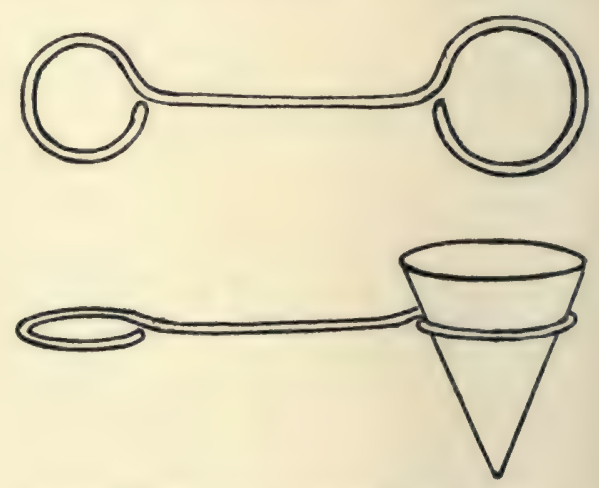

FIG. 7.-Simple holder for stain filtration.

finally into a drop bottle of colour as above. These solutions will keep for a long time if not exposed to strong light.

Dilute aqueous solutions of the above are used in the staining of all ordinary bacteria. The solutions are made by adding to every Io cubic centimetres of distilled water from two to five drops of the concentrated solution. In the case of Ziehl-Neelsen or other carbol stain the appearance on the surface of the liquid of a bronze iridescence will indicate that a proper strength for the dilute solution has been reached. The dilute stains should be prepared only when required for use, and a convenient form of conical glass for this purpose, as well as for many others in the general work of the laboratory, is shown on Fig. 6. The glass used should be marked on the outside, with a diamond or a fine file dipped in turpentine, at the level of 5 , IO, 15 , and 20 c.c. of water. In use it is filled to the required level with distilled water and the 
stain is then dropped in from a drop bottle. The dilute solution should be fltered on to the preparation and allowed to remain in contact for from two to five minutes according to the potency of the stain. For this filtration a convenient little apparatus is shown on Fig. 7. It consists of a piece of stout copper wire turned to a circle at each end and with an over-all length when finished of about 5 inches. When required for use a circular filter paper of the smallest size is folded and inserted in one of the loops as shown. The ring will be found to grasp the paper better if the inner surface of the loop has been slightly roughened by means of a blunt knife or a file.

\section{Compound Staining and Special Stains}

(a) Gram's method of staining (Nicolle's modification)-

The method of Gram enables us to classify bacteria into two great groups. Certain organisms when coloured with a basic stain in aniline or carbolic acid solution and afterwards treated with a special mordant of which iodine is the base, resist decolorisation by means of absolute alcohol or other like reagent. Others on the contrary, when treated in the same fashion, readily give up their stain and decolorise when treated with such reagents. The bacillus anthracis may be taken as a type of the former, the bacillus typhosus of the latter.

All bacteria then can be grouped in two great classes, viz. :-

I. Those which retain the stain when treated by the method of Gram.

2. Those which do not.

In the original method of Gram, aniline-gentian-violet was the stain invariably employed, but this is gradually being discarded in favour of Nicolle's carbol-gentian-violet as more stable and of equal efficacy in result.

The staining fluid consists of 10 c.c. of saturated alcoholic solution of gentian-violet added to 100 c.c. of I per cent. carbolic acid solution.

The mordant is composed as follows :-

\section{Gram's Todine Solution.}

\begin{tabular}{|c|c|c|c|c|c|}
\hline Iodine & & - & & 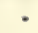 & I gramme. \\
\hline Potassium iodide & ${ }^{\circ}$ & - & & * & 2 gramme \\
\hline Distilled water & & . & . & . & 200 c.c. \\
\hline
\end{tabular}

The film is prepared and fixed in the usual manner. The procedure then is as follows:- 
I. Allow two or three drops of the stain to fall upon the slide and remain in contact with the film for five seconds.

2. Wash off the stain with the iodine solution applied from a drop bottle for five or six seconds. The film should then be black or dark brown.

3. Wash off the iodine solution with a mixture of $I$ part acetone and 2 parts alcohol absolute, but allow to remain in contact for two or three seconds only.

4. Wash off with absolute alcohol, applied until no more stain comes away.

5. Wash in water, blot off superfluous water, and set aside to dry. If thought desirable the preparation may be counterstained by the application of a very weak solution of Ziehl-Neelsen.

As the above manipulation follow one another with some rapidity, it is well to have the bottles containing the reagents clearly labelled and arranged in their proper order, so as to come readily to hand. A very easily made and convenient stand for this purpose is illustrated on Plate 8.

\section{(b) Ziehl-Neelsen method of staining-}

If milk is under examination it should first be centrifugalised. ${ }^{1}$ The centrifuge tubes must be thoroughly cleaned with water, followed by soaking for some minutes in strong sulphuric acid or nitric acid, then rinsed in tap water, distilled water, and finally rectified spirit. It is better not to place more than about an inch depth of milk in the tube. The centrifuge should be whirled for at least two minutes.

The film is prepared in the usual manner, either from the particulate matter of the milk after centrifugalisation or from the lesions of an experimental animal, and fixed with alcohol and ether. The procedure then is as follows :-

I. Allow two or three drops of Ziehl's carbol-fuchsin to fall upon the film, and heat either over the flame or upon a heating stage as shown on Fig. 8 until the steam rises.

2. Allow to act for three minutes, replacing with fresh stain any loss by evaporation.

1 If no centrifuge is available the milk must be placed in a sterilised conical flask and treated in the following manner : To 50 c.c. of milk set for sedimentation, Io c.c. of liquefied carbolic acid crystals are added. The mixture is thoroughly shaken and poured into a tall conical glass. After standing for twenty-four hours a little of the sediment is taken by means of a sterilised pipette and the film made. 
PLATE 8 .

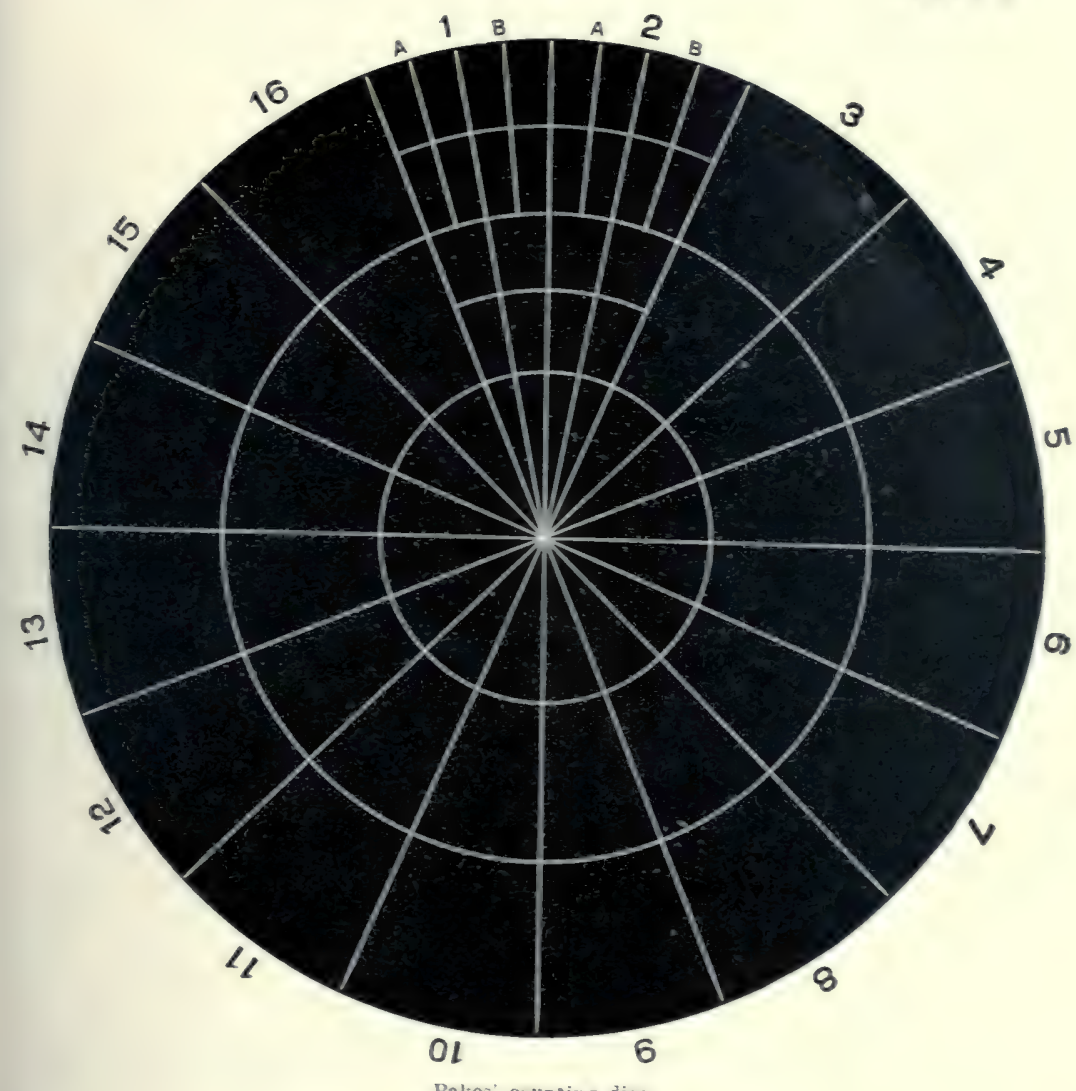

Pakes counting dise.

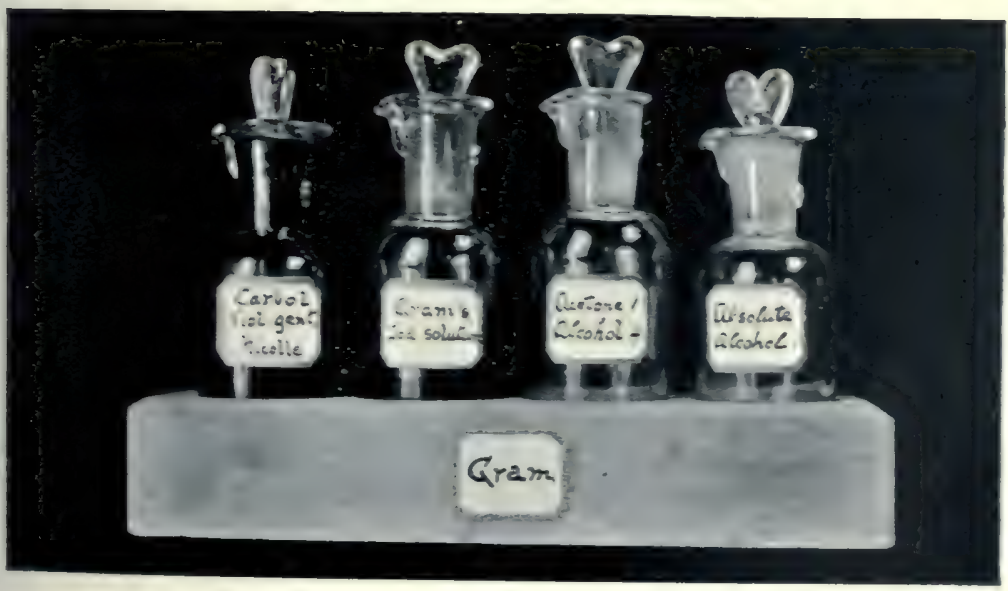

Dropuing bottles and stand. 

3. Wash well in water, and treat with 25 per cent. sulphuric acid, or 33 per cent. nitric acid, until the film remains decolorised when washed with water.

4. Dry between layers of fine filter paper, and counterstain with Löffler's alkaline blue.

5. Wash thoroughly, dry, and examine.

In the case of milk, the bacilli of tubercle or other acid-

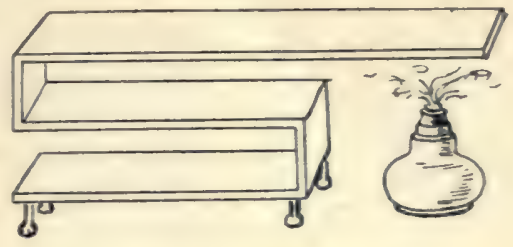

FIG. 8.- Heating stage. fast organisms will be stained red, and the milk or casein cells blue.

Fraenkel's modification of Ziehl-Neelsen method.-In this process the contrast stain is combined with the decolorising agent. The films are stained as in the ordinary method, and then placed in a solution composed as follows :-

\begin{tabular}{|c|c|c|c|c|c|c|}
\hline Nitric acid . & . & • & . & . & t & 20 parts. \\
\hline Absolute alcohol & - & - & . & - & - & $30 "$ \\
\hline Distilled water & - & . & . & * & . & $50 "$ \\
\hline
\end{tabular}

The stained films are treated with the above until the whole of the red colour has been discharged and replaced by blue.

\section{Flagella Staining}

Successful staining of flagella is a question of practice, and of careful and exact technique. Whateyer the method of staining adopted, the preparation of the film is the same, and too much care cannot be exercised at this stage. The slides should in no case have been previously used, and they should be most carefully cleaned in the manner described on p. 54. When taken out of the alcohol, care should be taken not to touch the edges with the fingers, and it is well to mark clearly one extreme corner of the slide, by which it can be held between the forefinger and thumb during the succeeding manipulations. The slide should be carefully dried and polished with a clean piece of old cambric, without handling with the bare fingers. It should then be passed several times through the flame, and set aside to cool.

Preparation of the film.-I. The cultures for examination should be upon agar, and should not be less than six, or more than eighteen, hours old, if incubation has taken place at $37^{\circ}$. If incubated at $22^{\circ}$, slightly older cultures may be employed.

2. Inoculate with a trace of the youngest growth of the culture 
taken up on the point of the platinum needle, a test tube containing from 30 to 40 c.c. of tap water at room temperature. Repeat this until the water shows a slight turbidity, and set aside for half an hour or longer; do not shake or handle the tube roughly.

3. With a finely drawn pipette, take up a small quantity of the surface water from the inoculated tube, and distribute it in small

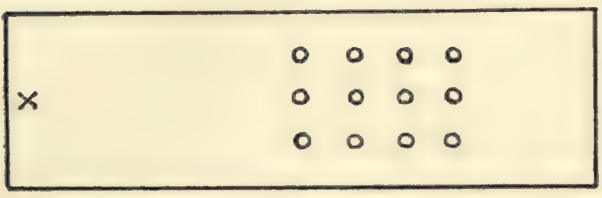

FIG. 9.-Slide prepared for flagella staining. droplets upon the slide, as shown on Fig. 9.

4. Place aside to dry, carefully covered from chance of dust. When dry, the staining can be proceeded with according to the method adopted. Do not fix the films in the flame; the flagella are apt to be injured thereby, and it will be found that the subsequent manipulations will cause the organisms to adhere sufficiently to the slide.

\section{Staining the Film}

The three ordinary methods practised in this country are :-

(1) Pitfield's method (Muir's modification).

The following solutions are required :-

A. The Mordant.

Tannic acid, 10 per cent. aqueous solution

Corrosive sublimate, saturated aqueous solution

Alum, saturated aqueous solution

Carbol-fuchsin (Ziehl) .

The above must be thoroughly mixed, and the precipitate which forms must be allowed to deposit. The clear supernatant fluid is then drawn off with a pipette and placed in a clean dropping bottle. The mordant will remain good for one or two weeks, but not longer.

B. The Stain.

Alum, saturated aqueous solution - . . . 10 c.c.

Gentian violet, saturated alcoholic solution . . 2 c.c.

Filter twice. The stain must be freshly prepared and will not keep.

The film is prepared as described above. The mordant is then dropped on to the slide and heated gently over the flame until the steam begins to rise. Allow to steam for from one to two minutes, wash well in running water and dry carefully. When thoroughly dry apply a sufficient quantity of the stain, 
and heat as before, allowing to steam for from one to two minutes. Wash well in distilled water, dry and examine. A pair of Cornet's special forceps for slides will be found convenient in the above manipulations.

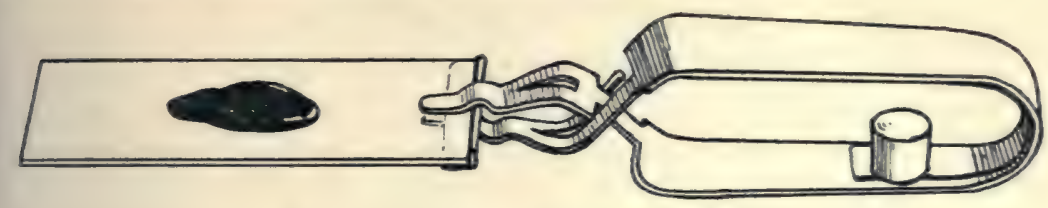

FIG. 10.-Cornet's forceps for glass slides.

(2) Van Ermengem's method.

Three solutions are required in this method :-

A. Fixing solution.

Osmic acid, 2 per cent. solution . . . . . I part.

Tannin, 20 per cent, solution . . : . 2 parts.

To each 100 c.c. of this mixture add four to five drops of glacial acetic acid.

B. Sensitising Solution.

Nitrate of silver . . . . 0.5 aqueous solution.

This solution should be kept in the dark and filtered before use.

C. Reducing Solution.

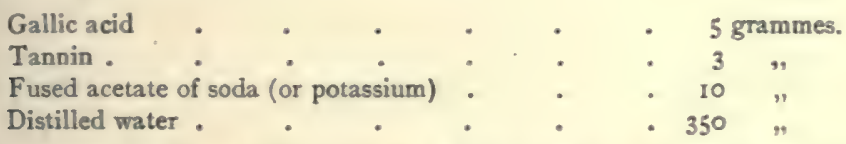

(a) Cover the film with solution "A" and allow to act for five minutes at $37^{\circ} \mathrm{C}$, or one hour at room temperature. Or heat gently until steam rises, and allow the staining fluid to act for fifteen minutes.

(b) Wash well with distilled water, then in absolute alcohol, and then again in distilled water.

(c) Treat with solution " $\mathrm{B}$ " and allow it to act for thirty seconds, keeping the fluid in movement on the slide.

(d) Allow the fluid to run off the slide, and without washing treat with "C" for thirty seconds in the same manner.

(e) Allow fluid to run off and again treat with sensitising solution till the preparation begins to turn black.

(f) Wash in distilled water, mount in water, and examine under the microscope.

\section{(3) M'Crorie's method.}

A combined mordant stain is made by dissolving I gramme of tannic acid, and I gramme of potash alum in 40 c.c. of distilled water, and adding to this, 0.5 gramme of "night-blue" dissolved in 20 c.c. of absolute alcohol. Filter the stain at least twice before use. Stain with the solution for about ten minutes, changing the stain two or three times during the operation. Then wash gently 
in running-water. Dilute carbol-gentian-violet may be used as a counterstain if desired. In such a specimen the bacilli will be stained violet and the flagella blue.

\section{The Staining of Spores}

The following are the methods commonly adopted:Möller's method.

(a) Prepare the film as usual, fix and dry, observing the precautions taken in preparing milk specimens.

(b) Treat with alcohol for two minutes, and then with chloroform for two minutes; wash in water.

(c) Treat with chromic acid, 5 per cent. aqueous solution, for from one to two minutes ; wash and dry.

(d) Pour on freshly filtered carbol-fuchsin and warm gently till it steams; allow it to act for ten minutes and wash off with water.

(e) Decolorise with sulphuric acid (5 per cent.) and water alternately, to remove the carbol-fuchsin from the bacilli but not the spores.

(f) Dry and counter stain with Loffler's blue until the film is of a faint bluish tint. Wash off stain, dry and examine. The spores will be stained red and the bacilli blue.

\section{Ziehl-Neelsen method.}

(a) Stain the film as for tubercle bacilli.

(b) Decolorise with I per cent. aqueous solution of sulphuric acid, or alcohol 2 parts, acetic acid I per cent., I part.

(c) Counter stain with Löfller's blue.

(d) Wash, dry, and examine.

\section{Capsule Staining}

MacConkey's method.-I.5 gramme methyl-green crystals and 5 gramme dahlia are rubbed up in 100 c.c. distilled water: add Io c.c. of saturated alcoholic solution of fuchsin, and make up to 200 c.c. with distilled water. Treat with stain for five minutes or longer, and wash thoroughly in a stream of water. The stain should be allowed to stand for a fortnight before use, and must be kept in a dark place.

\section{Qualitative Examination of Milk}

\section{Isolation of Aërobic Organisms}

\section{Plate cultivation on nutrient gelatine-}

Apparatus required.-Three Petri dishes with their paper coverings removed, and clearly marked 1, 2, and 3. Three tubes of nutrient gelatine previously melted in warm water at a temperature not exceeding $37^{\circ} \mathrm{C}$. and with the tubes marked I, 2, and 3 , as in the case of the dishes. Three Pasteur pipettes, or a platinum needle with looped end. 


\section{Procedure-}

I. Introduce into the tube marked I, by means of one of the pipettes or the platinum needle, a single drop of the fluid under examination. Replace the cotton-wool plug and roll the tube in the hand for at least two minutes in order to ensure an even distribution of organisms throughout the medium.

2. With a second pipette, lightly passed through the flame, or with the platinum needle freshly flamed, remove three drops of the liquid from tube $\mathrm{I}$, into the second tube. Roll in the hand for one minute as above.

3. Transfer three drops from tube 2 to tube 3 in the same fashion. Roll the tube as above and set aside for a few moments with the others.

4. Take the tube marked I, and the first Petri dish, and, after removing the cotton-wool plug from the former and flaming the tube orifice, raise the cover of the dish sufficiently to allow the liquefied medium to be poured into it with an even flow. Replace the cover as soon as the last drop of gelatine has fallen, and, keeping it as level as possible, oscillate the dish gently in order to obtain an even surface of the medium. Set aside on a level surface, and when complete solidification has taken place incubate at $20^{\circ} \mathrm{C}$. or lower temperature. Treat tubes 2 and 3 in a similar manner.

Examine the dishes carefully each day. Note the characters of the developing colonies, and inoculate a tube of broth from each distinctive colony ; from this tube subsequent cultures can be made on any media desired. The tubes should be clearly marked to correspond with the colonies from which the growth was taken and the colonies themselves may be marked $(a, b, c$, etc. $)$ in ink on the under side of the bottom dish.

\section{On nutrient agar-}

Proceed as in the case of gelatine. It is necessary to act quickly, as the medium, of which the melting point is $100^{\circ} \mathrm{C}$., resolidifies on the fall of the temperature to $40^{\circ} \mathrm{C}$. The tubes of agar are first melted by boiling in a beaker of water, then allowed to cool until th. reach a temperature just above the solidifying point. They should then be placed, and kept during the succeeding manipulations, in a beaker of water maintained at from $40^{\circ}$ to $45^{\circ} \mathrm{C}$. The manipulations should be carried out as quickly as possible, and to prevent an uneven surface due to the too rapid solidification of the medium, the Petri dishes should be previously 
warmed by being placed in the incubator at $37^{\circ} \mathrm{C}$. for from twenty minutes to half an hour.

\section{Stroke cultivation on agar-}

I. Prepare an agar plate by pouring the contents of a tube of liquefied nutrient agar into a sterile Petri dish, and allow it to solidify by placing the dish on a cold stone slab or other level surface.

2. When solidification has taken place and the medium has thoroughly hardened, remove on the extreme point of a platinum needle a minute quantity of the material from which it is desired to isolate the organisms, or, in the case of milk, dip the needle in the liquid.

3. Raise carefully the cover of the Petri dish and, beginning at one side of it, draw with the

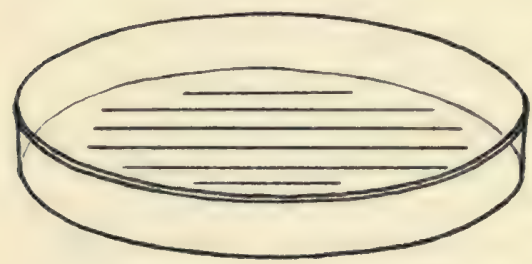

FIG. II.-Isolation by stroke culture. point of the needle a series of parallel lines across the medium at a distance of $\frac{1}{2}$ inch apart, without recharging the needle. At each stroke the needle leaves upon the medium a certain number of organisms, the number becoming fewer and fewer, and the individual organisms more

and more scattered, as the lines proceed.

4. Incubate at $37^{\circ} \mathrm{C}$. or any desired temperature. The colonies will be found to develop abundantly on the early tracks of the needle and to become more and more scattered and discrete on the later ones.

5. Remove from the more isolated colonies a small portion of the growth for microscopic examination and the inoculation of media.

\section{Upon nutrient agar by Klein's method-}

See p. 88 under Isolation of Anaërobes.

\section{Upon blood serum-}

Apparatus required.-A thick platinum needle (No. I8 or 19, Imperial gauge, will be found a convenient size) flattened at the end in spatula form, and three tubes of solidified blood serum.

\section{Procedure-}

I. Remove upon the flattened portion of the platinum needle a small quantity of the material or liquid under examination and, 
withdrawing the plug of one of the tubes of serum with the usual precautions, carry the end of the needle down to the bottom of the tube. Placing it upon the lowest portion of the medium withdraw the needle gently, making at the same time with it a series of transverse zig-zag lines across from side to side of the medium.

2. Replace the plug in the first tube, and, opening the second, proceed in the same manner without recharging the needle.

3. Carry out the same operation with the third tube, again without charging the needle.

4 Incubate at $37^{\circ} \mathrm{C}$.

In the course of the above operations it will be found that the needle gradually becomes freed from the organisms with which it was charged. In the first tube a large number of colonies, most of them confluent, will be found to develop, but the colonies will become rarer and more isolated upon the serum of the second and third tube. It is upon either one or other of these two tubes that the various colonies will be found sufficiently isolated for examination.

METHODS OF EXAMINATION FOR SPECIAL Micro-ORGanisMS IN MILK

Bacillus pseudo-tuberculosis of Pfeiffer (found in London milk by Klein)-

By the centrifuge or by sedimentation in an ice chest for twenty-four hours obtain the particulate matter of the milk to be examined. Inoculate 2 c.c. into a guinea-pig subcutaneously or intraperitoneally. In the course of three to four weeks caseo-purulent nodules will occur in the inguinal glands (if subcutaneously inoculated), or in the omentum and pancreas and other organs (if intraperitoneally). Cultures may be obtained best from glands, spleen, pancreas, or liver. Examine the nodules by staining and culture. They will have the following characters if the disease be pseudo-tuberculosis: (a) Absence of 
giant cells; $(b)$ absence of the true tubercle bacillus; $(c)$ presence of large numbers of $B$.pseudo-tuberculosis; and $(d)$ signs of a rapid rather than a slow development.

Method of staining.-Make films in the ordinary way and stain with Löffler's methylene-blue, heating the stain till it steams (Klein). Wash in distilled water. Nodules may be hardened in Müller's fluid and spirit and sections cut, and stained by placing in Löffler's blue for twenty-four hours and counterstaining in a mixture of eosin and methylene-blue. Löffler's blue may also be used for staining the bacillus in milk-films made from sediment. Gram's method is also applicable, but the bacillus is not acid-fast and will not hold the Ziehl-Neelsen stain.

\section{Bacillus diphtheriæ-}

By centrifuge or sedimentation obtain the particulate matter of the milk under examination and inoculate it into a guinea-pig. Subculturing from the tissues of the guinea-pig, or, having obtained sediment as above, inoculate six tubes or plates of Löffler's medium (ox serum 3 parts, veal broth I part-the broth to contain glucose I per cent., peptone I per cent., and sodium chloride 0.5 per cent.). Upon this medium the Klebs-Löffler bacillus grows rapidly in twelve to twenty hours, producing scattered, nucleated, round, white colonies which later become yellow.

Method of staining.-Gram's method as modified by Nicolle (see p. 59) will be found the most satisfactory, but the methylene-blue solution of Löffler is often used. This consists of 30 c.c. of a saturated alcoholic solution of methylene-blue added to 100 c.c. of a $\cdot 0$ I per cent. solution of caustic potash. By this stain the striped appearance of the bacilli in older cultures on blood serum is obtained. For differential purposes it is best to use Neisser's method.

\section{Streptococcus in milk-}

By centrifuge or sedimentation obtain the particulate matter of the milk. Take a sterilised platinum loop, dip in the sediment, and remove a drop of it. Distribute this in a test tube containing I to 2 c.c. of sterile salt solution. Inoculate agar plates with a drop of this dilution, and incubate at $37^{\circ} \mathrm{C}$. When the colonies appear, subculture those resembling streptococcus colonies in bouillon, and on blood serum. Subculture from the bouillon in milk, gelatine, and agar, carefully noting the characters of growth, etc. Or guinea-pigs may be inoculated in the subcutaneous tissue of the groin or intraperitoneally. An acute purulent inflammation will 
be set up, in the exudation of which streptococci will occur in large numbers.

Method of staining.-Gram's method is the most satisfactory. Next to Gram's method the most useful is Löffler's blue. It may be noted that most of the putrefactive organisms do not hold Gram's stain.

\section{Bacillus coli communis-}

(a) Dilute the milk to be examined 500 or 1000 times. Take a sterilised brush, dip it in the dilution, and brush over the surface of six agar plates without recharging the brush. Incubate at $42^{\circ} \mathrm{C}$, and subculture the coliform colonies (bouillon, milk, litmus milk, gelatine "shake" cultures, etc.).

(b) Take six tubes of phenol bouillon ( 0.05 per cent. of carbolic acid), and inoculate them with crude or diluted milk. Those which show abundant turbidity after twenty-four to forty-eight hours at $37^{\circ} \mathrm{C}$. may be plated out on phenol-gelatine, incubated at $20^{\circ} \mathrm{C}$., and the coli colonies subcultured; or diluted milk may be at once plated out on phenol-gelatine, and colonies subcultured on such media as will show the characteristics of the organisms.

The main characters of the $B$. coli group of organisms may be briefly stated here, though particulars will be foundelsewhere in the present volume:-(I) They are non-sporing and non-liquefying; (2) they rarely stain by Gram's method; (3) they are motile; (4) they produce acid and gas in glucose and lactose media; (5) they produce acid in milk, and usually coagulate it ; $(6)$ they grow well at a temperature of $42^{\circ} \mathrm{C}$. Referring to the isolation of $B$. coli Houston writes: "No test based on observation of a change or changes produced in the nutrient medium, and supposed to be characteristic of $\mathrm{B}$. coli, can compare with isolation from plate cultivations of the microbes suspected to be B. coli, and the subsequent attentive study of the biological characters of pure cultures of these bacteria grown in various media." 1

\section{Bacillus enteritidis sporogenes of Klein-}

Take six tubes containing 15 c.c. of fresh milk and sterilise them by boiling for half an hour. Rapidly cool them by placing them in a beaker of cold water, add to each tube I c.c. of a I in 500 dilution of the milk to be examined, or if it be preferred O.I c.c. of the crude milk. Heat the inoculated tubes at $80^{\circ} \mathrm{C}$. for fifteen minutes. Then remove and cool, and place in Buchner

1 Second Report of Royal Commission on Servage Disposal, 1902, p. $41 \mathrm{I}$. 
tubes or cylinder containing freshly prepared mixture of pyrogallic acid and potassium hydrate solution. Seal the Buchner tubes or cylinder with great care, making it absolute. Place the Buchner apparatus, including the milk tubes, in the incubator at $37^{\circ} \mathrm{C}$. After forty-eight hours take out the tubes, and examine them for the $B$. enteritidis sporogenes. If necessary inoculate guinea-pigs subcutaneously with I c.c. of the whey, which in a few hours causes swelling at the point of inoculation, and extensive gangrene of the subcutaneous and muscular tissues with sanguineous exudation; the animal dies in twenty-four or thirty hours. The $B$. butyricus of Botkin may produce similar changes in milk tubes, but it has no pathogenic action. Milk may be examined directly by placing 20 c.c. in tubes and treating as above. The "enteritidis change" in the milk thus treated consists in copious gas formation, clear or slightly turbid whey in the middle of the tube, with faintly pinkish flocculi of casein floating on top, and casein masses at sides and bottom of tube.

\section{Bacillus tuberculosis-}

Obtain the sediment of the milk under examination and inoculate 2 c.c. of it into the subcutaneous tissue of a guinea-pig. In about four weeks time, local, if not general, tuberculosis will have been set up (see p. 74). Take some of the discharge and stain it after the Ziehl-Neelsen method. The sediment of tuberculous milk may be stained forthwith, without inoculation, by the same method, and in some cases the tubercle bacillus may be thus detected, but generally speaking the only sure test is inoculation of animals. The pathological process is slower than in pseudotuberculosis, and on examination the diseased tissues show giant cells and numerous tubercle bacilli arranged within the giant cell (see Plate 2I).

Differentiation between the tubercle bacillus and other acid-fast bacilli.-For the differentiation of the bacillus tuberculosis from other acid-fast organisms in butter the only reliable method is that of inoculation. The following is the procedure employed in the examination of butter as originally devised by Obermuller, ${ }^{1}$ and subsequently modified by Rabinowitsch, Coggi, and others. The butter under examination, received into a sterile conical glass, with all aseptic precautions possible, is placed in the incubator at $37^{\circ} \mathrm{C}$., where on melting it will be found to arrange itself in ${ }_{1}$ Hyg., Rundschau, 1895, No. 19, p. 877, and 1900, No. 17, p. 845. 
two layers. Three c.c. of the supernatant fatty liquid are injected into the peritoneal cavity of a guinea-pig. A similar quantity of the deposit is treated in a like manner, and finally, two or three other animals are inoculated intraperitoneally with the semi-liquid substance obtained on mixing together the two layers into which the butter has formed. At the end of expiration of seventy days the animals which have not already succumbed are killed, and a careful post-mortem examination is made. Microscopic preparations and cultures are made from any organs affected. The latter, taken together with the general aspect of the lesions, will, in the majority of cases, be sufficient to enable a diagnosis to be made between the true bacilli of tuberculosis, and other acid-fast organisms resembling it. Bacilli which resist in a moderate or somewhat feeble manner decolorisation by acids, which develop rapidly at a temperature of $37^{\circ} \mathrm{C}$., and grow feebly at ordinary room temperature, which exhibit chromogenic properties in culture, and give rise in the guinea-pig to lesions which are not characteristically those of tuberculosis, must be regarded as organisms of the acid-fast group, non-pathogenic for man, though possibly related in some degree to the true bacillus of tuberculosis (see also p. 253).

Another method is that indicated by Roth. Five grammes of butter are vigorously shaken up in sterile water, and the whole is then centrifugalised. A fat-free deposit is thus obtained, and given quantities of this are injected into animals in the ordinary manner.

\section{SPECIAL METHODS}

\section{Examination of colostrum-}

Colostrum is the term applied to the first milk yielded by the cow after parturition. It differs considerably from ordinary milk and generally appears as a thick, turbid, yellowish, viscid fluid. When examined under the microscope it is found to contain, in addition to the ordinary milk corpuscles, peculiar conglomerations of very minute fat granules which are known as "colostrum corpuscles." The chief chemical differences between colostrum (or beastings) and milk are mainly three. First, colostrum is deficient in casein. Secondly, it is proportionately rich in albumen. Thirdly, it contains nearly three times more salts than milk. Probably it is this excess of salts that usually causes it to exert a purgative effect upon the new-born calf, and thus to remove the meconium which has accumulated in the foetal intestine. 
The difficulties of bacteriological examination of such an article as colostrum are considerable. At the outset, a fair sample is only obtainable by adopting the following precautions: $(a)$ the teat's and udder to be cleansed; $(b)$ milking to be carried out as soon after calving as possible, when the calf has sucked; (c) the first part of the "milking" to be discarded, and the last part only to be examined. When the colostrum reaches the laboratory it must be diluted in precisely the same manner as thick cream. After abundant dilution treat the solution in the ordinary way, by staining preparations for the microscope, plating out on various media, and subculturing.

\section{Bacteriological examination of butter-}

Take a quarter of a pound of butter and place it in a sterilised flask with 150 c.c. of sterile salt solution. Place the flask in the water bath at about $35^{\circ} \mathrm{C}$, and shake gently until the butter has melted. The contents of the flask now appear as a milk-like emulsion. A small quantity of this mixture may be used for plate cultivation on gelatine and agar, as in milk. The remainder should be placed in a sedimentation flask in the refrigerator for twenty-four hours. By this means the particulate matter of the butter, including the contained organisms, are deposited. After removing the superficial solidified fat by means of a sterile spatula, the turbid fluid may be decanted, and the sediment collected for microscopical examination or the injection of guineapigs. (See p. 70, also Inoculation of Animals, p. 74.)

\section{Examination of cheese-}

With a knife previously sterilised by passing through the flame, cut off from the piece of cheese under examination a thin slice parallel to the surface. Remove this, and with a second sterile knife cut perpendicularly downward from the bared surface. Pass down into the latter cut a coarse sterile platinum needle of which a small portion near the extremity has been slightly roughened with a file.

Inoculate with this needle a sufficient number of tubes of bouillon, from which plate cultivations can subsequently be made for isolation purposes, and placed under both aërobic and anaërobic conditions.

\section{Examination of moulds-}

The examination of hyphomycetes or mould fungi is, for differentiation purposes, best carried out on the Petri dish itself, where 
the construction of the microscope will admit of this being placed on the stage.

By the following method there is but little difficulty in at once recognising the various species, and a beautiful demonstration is given of the hyphæ with interstitial cells, and germinating conidia of the Oidium lactis, the conidiophore and sclerotium of the Pencillium glaucum, or the ramified mycelium, sporangia, and germinating zygospores of the various species of Mucor, without disturbance of the growth.

By means of a finely drawn pipette allow to fall upon the centre of the mould colony a small drop of aqueous solution (I per cent.) of eosin. It is necessary to exercise a little care in this, or the liquid will at once run off the colony on to the surrounding medium. Place carefully upon the centre of the drop a thin coverglass, and press in order to obtain close contact. Remove the Petri dish to the stage of the microscope and examine the margins of the growth with a sixth objective.

If the construction of the microscope will not allow examination on the Petri dish, or if a permanent specimen is desired, the following method can be recommended:-Detach by means of a pair of fine pointed forceps a portion of the young growth, holding it by the base, and place it carefully on a slide. Place near it one drop of ammoniated alcohol, and bring this in contact with the specimen by means of a finely pointed needle. The absorption of the alcohol will allow the subsequent penetration of the tissues by the liquids employed. Drop on to the preparation a small quantity of Flemming's solution, and allow it to remain for four or five minutes. Wash carefully with water, cover with a cover-glass, and examine.

To make a permanent preparation, replace the water with glycerine by placing a drop of the latter at one side of the coverglass, and absorb the water from the other by means of filter paper. Dry carefully with filter paper damped with alcohol, and ring with paraffin.

\section{Flemming's Solution.}

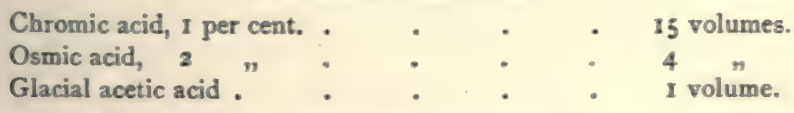

\section{Examination of milk for pus cells-}

Place ro c.c. of the milk to be examined in each tube of the centrifuge (Fig. 13) and centrifugalise for two minutes. Pour off 
the superfluous fluid, and with a sterilised needle or pipette take up a small quantity of the sediment remaining in the tube. Spread the sediment evenly over the surface of an ordinary glass slide and dry over the flame of a Bunsen burner or on the drying stage. Wash the fixed film with ether (or alternately with absolute alcohol and ether) until all the superfluous fat is removed, and stain. The preparation may be stained $(a)$ by one of the ordinary solutions, such as Löffler's blue, etc.; or (b) by Gram's method. Examine under the microscope with a $\frac{1}{12}$ th oil immersion lens.

\section{Inoculation of animals-}

For details respecting the inoculation of animals, books dealing more especially with general technique may be consulted. Here it will be sufficient to remark that the simplest forms of inoculation are all that are usually required in milk investigation, namely, the intraperitoneal and the subcutaneous. In some cases it may be sufficient to inoculate a few c.c. of the original milk; but, as a rule, it is advisable to centrifugalise, or use the sedimentation flask containing about 250 c.c. From the deposit or sediment two guinea-pigs may be inoculated, the one subcutaneously in the groin, the other intraperitoneally. Particularly is this necessary in making a reliable and exhaustive search for the $B$.tuberculosis. Microscopic examination alone for this organism is not reliable (see p. 70). The details of the process as carried out in practice are as follows :-

After centrifugalisation the deposit is mixed with the 2 c.c. of milk remaining in the tube after aspiration of that which is superfluous. Two guinea-pigs (of say 250 grammes weight each) are taken and inoculated with the deposit from about 40 c.c. of milk. The fluid is inoculated subcutaneously on the inner side of the leg under strict aseptic precautions (the skin having been washed with I-IOOO corrosive sublimate, and shaved). In less than a fortnight's time, if the inoculated milk contained a considerable number of tubercle bacilli, typical infection of the popliteal and inguinal glands can be detected. If the milk contained very few bacilli the infection is much slower (fifth week). After the animal has been killed the presence of the tubercle bacilli can be detected in the inguinal glands and the spleen. Some workers make it a rule to inoculate two guinea-pigs from the sediment of the milk, one receiving half of the sediment subcutaneously in the groin, the other receiving the remaining half intraperitoneally. 
Rottine Procedure in the Systematic Examination OF MILK

Physical examination (temperature, reaction, colour, cream, deposit, specific gravity, etc.) of the milk should be made if necessary. The microscopical examination of the milk before and after centrifugalisation or sedimentation will likewise often yield useful results.

I. Plate cultivation.-Dilute as required (see p. 49) and make plate cultivations in Petri dishes or flat-bottomed flasks. Six or more gelatine plates should be made and incubated at room temperature. Plates should also be made with nutrient agar for incubation at $37^{\circ} \mathrm{C}$. Other media may also be selected.

The plates should be counted on the second, third, and fourth days, and the necessary subcultures made. Agar plates incubated wholly at $18^{\circ}$ or $22^{\circ} \mathrm{C}$., will in the long run show more colonies than when incubated at $37^{\circ} \mathrm{C}$. and then at $22^{\circ} \mathrm{C}$, or at $37^{\circ} \mathrm{C}$. throughout.

2. Anaërobic cultivation.-At the same time that the primary aërobic plate cultivations are made, similar plates should be made on lactose-gelatine and lactose-agar for anaërobic culture (see p. 83 et seq.).

3. Primary tube cultivation-Take ten tubes of 10 c.c. of the milk under examination and place three of them in the incubator at room temperature and three of them at $37^{\circ} \mathrm{C}$. Place four of them in a water bath heated to $80^{\circ} \mathrm{C}$. for fifteen minutes, and then enclose each of the four tubes in a Buchner's tube or vacuum desiccator (p. 89). These primary cultures may be tested in forty-eight hours for B. coli, the presence of indol, and B. enteritidis sporogenes.

4. Secondary or subcultures.-From the primary cultivations make subcultures on selected media for the isolation of organisms making their appearance on the plates, or what is often preferable make a separate set of plates for qualitative examination only.

5. Examination for special micro-organisms. - The milk must be centrifugalised or the particulate matter allowed to gravitate by sedimentation. It is, as a rule, useless to attempt examination microscopically or otherwise without first using the centrifuge or sedimentation flask. The deposit is then to be stained for the particular organism for which search is being made.

For centrifugalisation take two or three samples of the milk under examination to the amount of about 40 c.c. each and place 
it in the sterilised tubes of the centrifuge. In these tubes the milk may be centrifugalised for ten or fifteen minutes at 3000 revolutions a minute. At the end of such a period the milk in each tube has separated into three layers-at the top there is a dense layer of cream, at the bottom there is the sediment or "slime" containing all the particulate matter, and between these two is the separated milk. Aspirate off the cream by means of a sterile glass tube connected with an aspirator or vacuum pump, and examine separately; aspirate all the separated milk except 2 c.c. The remaining sediment is so compact and dense

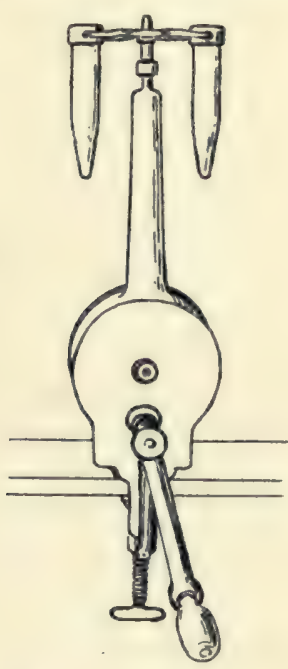

FiG. 13.-Metzger's centrifuge.

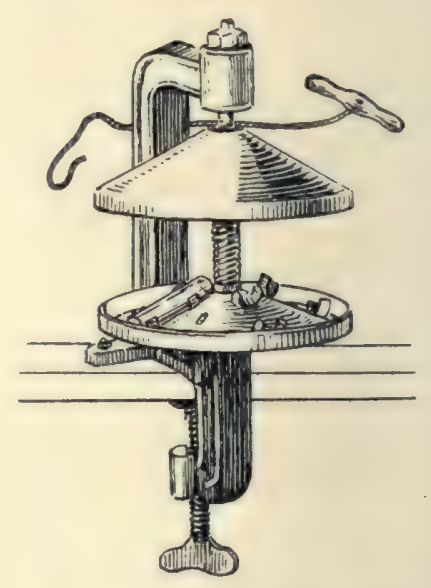

FIG. I4,-Gärtner's centrifuge.

that the tube may now be inclined and the sediment fully exposed without displacement. By means of a sterilised platinum loop a small portion may be taken up and spread on the surface of half a dozen slides and stained. The remainder of the sediment is well mixed with the 2 c.c. of milk and used for inoculation of guineapigs.

For sedimentation take two conical sedimentation glasses and fill them with the milk under examination, allowing them to stand in the refrigerator for twelve to fourteen hours. It is customary to add a few small carbolic crystals to each flask. On the completion of sedimentation the milk has separated into three main strata, the cream at the top, the sediment at the apex of the flask, and the 
separated milk in the middle. The cream and milk may then be carefully decanted, and the sediment will be available for examination.

For methods of examination for special organisms see page 67.

\section{Products FORMED IN CULTURES}

\section{(a) Gas-}

To test an organism for gas production, inoculate a fermentation tube (see Fig. I 5) containing sterile glucose or lactose bouillon (or dextrose-free bouillon to which a definite amount of sugar has been added), and incubate at $37^{\circ} \mathrm{C}$. Care must be taken that the culture liquid, prior to inoculation, completely fills the upright branch. The gas generated gathers at the top of this branch, and when the evolution has ceased the length of the column of gas can, if desired, be measured with a scale and expressed as a percentage of the total length of the column of liquid prior to inoculation.

\section{(b) Indol-}

To test for the presence of indol, inoculate test-tubes of peptone water with the organism to be tested and incubate at $37^{\circ} \mathrm{C}$.

When growth has taken place remove the cultures from the incubator and, after

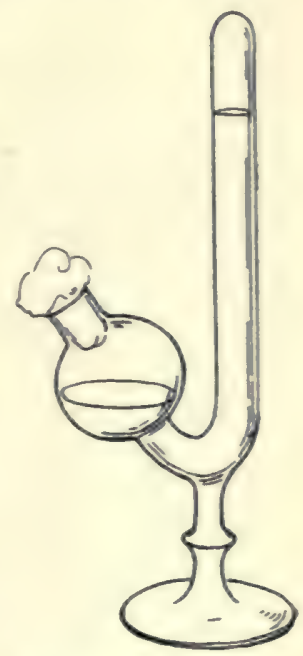

FIG. 15.-Fermentation tube. allowing them to cool down to room temperature, run in by means of a sterile pipette I c.c. of pure sulphuric acid.

Allow the tubes to stand upright in a rack for ten minutes. If a red or rose-pink colour is developed within this time it will indicate the presence of both indol and a nitrite.

If no colour change takes place, add I c.c. of 02 per cent. solution of potassium nitrite and place aside for a further period of ten minutes. The production of a red coloration will indicate the presence of indol. Should the coloration prove feeble, a small quantity of amylic alcohol may be shaken up with the culture, when the rose colour will show very distinctly after standing. 


\section{CHAPTER III}

\section{ANAËROBIC ORGANISMS: THEIR ISOLATION AND CULTURE}

General Note. Cultivation in Hydrogen and in vacuo. Hydrogen Production. Methods of Isolation and Culture.

\section{General note-}

The fermentative and other changes produced in milk by organisms of the anaërobic type are of so important a nature that a thorough knowledge of the technique employed in this special branch of research is essential to those who would study the bacteriology of milk, and it is proposed in this chapter to explain in some detail the principal methods employed in the various English, German, and French schools, as well as those which the writers have themselves found most useful in research work.

For the study of the phenomena attending anaërobic fermentation the special flasks illustrated on Plate 9 will be found convenient, and are easily set up. Flask " A" is specially useful for the study of the progressive phenomena of anaërobic fermentation, as it permits, up to a certain stage, of the examination of the milk at intervals without admission of outward air, or the disturbance of the existing anaërobic conditions, the pressure set up in the interior of the flask by the disengagement of gas being sufficient to cause the liquid under examination to fall drop by drop into a receptacle placed underneath the curved outlet tube. The apparatus consists of a three-necked Wolff flask of 400 to 500 c.c. capacity, arranged as in figure. The flask is closed with indiarubber one-holed corks, through the two side ones of which are led lengths of glass tubing reaching in each case to the bottom of the flask, the one being bent at the top at a right angle with a return of about $I$ inch, constricted at the point of entry into the cork, lightly plugged with cotton-wool, and terminating with a short length of vacuum tubing fitted with a screw pinchcock as 
PLATE 9.

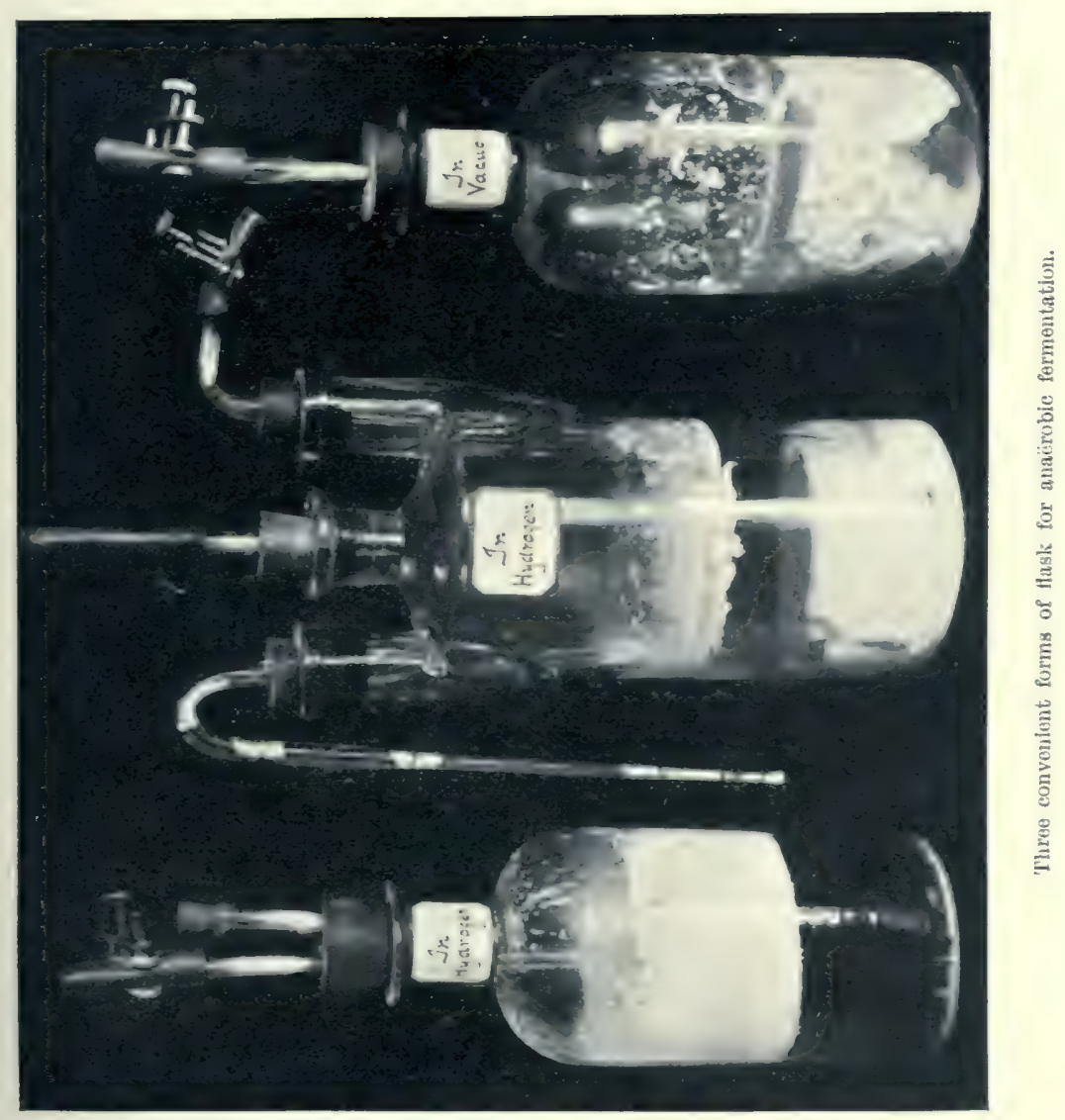



shown; the other curved in a downward direction on the exterior, drawn in the form of a pipette, and prolonged downwards to within about $\mathbf{I}$ inch of the bottom level, the end being sealed off in the flame. Through the centre stopper a short length of similar tubing is inserted, the lower end (the orifice of which should be slightly constricted in the flame) projecting not more than half an inch beyond the lower level of the cork, and the upper to about I inch above it, this latter being constricted at the point of entry into the cork, lightly plugged with cotton-wool, and fitted with a tightly fitting india-rubber cap of the form shown, which must be removed when sterilising liquid in the flask or when passing a current of hydrogen through it.

The flask, after sterilisation in the autoclave, is filled to onethird its capacity with fresh milk, or (if it is desired to study the action of some particular organism) is sterilised with the milk or bouillon in it, ${ }^{1}$ and then inoculated in the usual manner by slightly withdrawing the centre stopper. In this latter case the central stopper with its fitting should be covered with a loosely fitting paper cap lightly tied on during process of sterilisation and until the inoculation is made. The short length of india-rubber tubing is then connected up with the hydrogen apparatus (as explained on p. 82) and a current of hydrogen (escaping by the central orifice) is passed through the medium for ten or fifteen minutes. The cap is then placed on the outlet tube, and the inlet closed with the pinchcock. If thought desirable successive currents of hydrogen can be passed through the medium in a similar manner. The flask is then incubated at $37^{\circ}$ or other temperature.

As soon as pressure of gas induced by fermentation is set up in the flask - the first indication of which will be increased tension of the india-rubber cap, and the rising of the liquid in the inner portion of the outlet tube-the extreme point of the bent tube is snipped off, and the contents allowed to fall drop by drop into a receptacle placed below. If it is desired to obtain a freer flow of the culture it is only necessary to connect up the inlet with the hydrogen apparatus, and allow a current of gas under pressure to enter the flask.

Flasks " $\mathrm{B}$ " and " $\mathrm{C}$ " are convenient when it is desired only to study the final results of the fermentative processes. They consist of flasks of bottle shape, of sufficient temper to withstand exposure to a high temperature during course of sterilisation, and yet of

1 Care must be taken in this case to remove the india-rubber cap and close down tightly the pinchcock of the inlet tube before placing in the autoclave. 
sufficient strength to allow (in the case of " $\mathrm{C}$ ") of a vacuum being set up in the interior. The former is useful when hydrogen or other inert gas is alone employed, the latter when it is desired to study the phenomena of fermentation "in vacuo."

Flask "B" is fitted with a two-holed india-rubber stopper, through one hole of which a long length of glass tubing is led to the bottom of the flask, and through the other a shorter length extending into the flask to a point just below the under surface of the stopper. Both tubes are constricted at the point at which they enter the cork, are plugged lightly with cotton-wool, and fitted at their exterior extremities as shown-the one with a short length of pressure tubing fitted with a screw pinchcock-the other with a small india-rubber stopper fitting tightly into the tube ending, which should have been previously "coned" in the flame. The flask is filled to about one-third its capacity with the medium

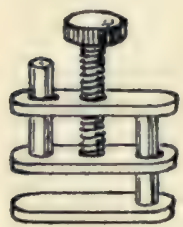

FIG. I6.-Best form of screw pinchcock. required, a piece of wrapping paper is tied loosely over the tube terminals and neck of the flask, and the whole is then sterilised in the autoclave, care being taken that the screw pinchcock is tightly closed and the small stopper removed during the process. Inoculation is made in the usual manner by withdrawing the central stopper sufficiently to allow the insertion of a finely drawn pipette, but without the removal of the loose paper covering mentioned above. The stopper is then replaced, the pinchcock opened, and a current of hydrogen passed through the liquid for ten or fifteen minutes. The small stopper is then fitted tightly into place, the screw pinchcock closed, and the flask incubated at any desired temperature. As soon as interior pressure is set up by the evolution of gas, the small stopper is slightly loosened in its place in order to allow the escape of any great excess.

Flask " $\mathrm{C}$ " is treated in a similar manner, the only difference being that instead of a current of hydrogen being passed through the liquid, a vacuum is formed in the flask.

\section{Cultivation in hydrogen-}

Although a supply of hydrogen is not absolutely essential in anaërobic work, yet a convenient apparatus for generating and holding in reserve a sufficient supply of this gas will be found of the greatest possible value, and will considerably extend the range of methods at the disposal of the worker. 
with a solution of pyrogallate of potash (4 grammes pyrogallic acid in 200 c.c. of 50 per cent. caustic potash solution; place the pyrogallic acid in the flask, pour in the potash solution by means of a thistle funnel, and stopper immediately), through which the current of hydrogen is passed to remove any existing traces of oxygen. To avoid accident the three flasks are enclosed in a box as figured, which can be put together by any handy carpenter, and to the corner of this a two-way tap " $e$ " connected up with the exit tube of flask " $c$ " is attached.

To start the apparatus, in the first instance, it is only necessary to fill the flask " $a$ " up to a little more than two-thirds its capacity with dilute acid (a 10 or 15 per cent. solution of $\mathrm{H}_{2} \mathrm{SO}_{4}$ is recommended in order to obtain pressure by rapid evolution of gas), and then to blow through the inlet tube " $d$ " until the acid rises well above the layers of zinc in " $b$. " The gas generated by the contact of the acid with the zinc will then issue with a sufficient force by the two-way tap " $e$ " turned vertically, after bubbling through the pyrogallate of potash solution in flask " $c$." By placing a pinchcock on the tube " $d$," and keeping open the tap " $e$ " the generation of gas can be kept up as long as desired, and at once stopped by reversing the process-i.e. shutting off the tap " $e$ " and at once opening the inlet tube " $d$. " 1 The pressure of gas will then force the liquid back into the flask " $a$," and well below the level of the zinc in flask " $b$," leaving in the latter a constant reserve of hydrogen under the pressure of the column of liquid in " $a$ ". The process then becomes automatic-the abstraction of gas from " $b$ " causes descent of the liquid in " $a$ " until the level of the zinc is reached in " $b$," when fresh evolution of gas takes place, the liquid being again forced back into the flask " $a$ " as soon as the exit tube or tap is closed.

To renew the acid solution without loss of gas it is only necessary to close the pinchcock on the connecting tube " $f$ " and syphon out from the flask " $a$ " the solution of sulphate of zinc, replacing it by a fresh acid solution.

For use with the air or other vacuum pump one way of the tap " $e$ " is joined up with the pump itself by turning the tap horizontally, when the pump can be connected up with any desired apparatus by means of a length of pressure tubing as shown. The

1 To prevent a sudden regurgitation of the liquid from the wash-bottle " $c$ " it is advisable when opening the inlet tube to pinch with the fingers the connecting tube between it and flask " $b$ " for a few moments until the pressure is equalised. 
working of the combined apparatus presents no difficulty, and is very effective. By turning the tap " $e$ " horizontally, connection is made between the pump and any receptacle or apparatus in which it is desired to create a vacuum. By turning it vertically, connection is at once made between the reserve of hydrogen and the vacuum so caused, and this latter is at once filled by an inrush of gas. By repeating the process as many successive "washings" in hydrogen as desired can be made, and the receptacle can then be sealed off in vacuo or in hydrogen as desired. The addition of a vacuum gauge to the air pump, although not actually necessary, will be found of great advantage generally, and of special assistance in these successive washings in hydrogen.

\section{Methods of Isolation and Culture of Anaërobic Organisms ${ }^{1}$}

\section{Vignal's tubes-}

For gelatine cultures this method, given a certain amount of dexterity in the manipulation of the blow-pipe flame or Bunsen burner, is one of the most simple and at the same time most efficient means of isolating or culturing anaërobic organisms, inasmuch as during the whole period of their growth there is ocular evidence of the presence or absence of free oxygen in the culture medium.

To prepare a Vignal tube.-Take a length of thin glass tubing about $\frac{1}{8}$ inch internal diameter and some 15 inches long. With the aid of the blow-pipe flame or Bunsen burner make a constriction at about 2 or 3 inches from one end, and insert well above it a light cotton-wool plug. Partially close the external orifice of the tube by turning the end in the flame. Place the tube again in

${ }^{1}$ A method by which fairly accurate information may be obtained as to whether an organism under examination is $(a)$ aërobic, or $(b)$ anaërobic, has been suggested by the Committee of Bacteriologists appointed by the American Public Health Association (Reports and Papers, American Public Health Association, vol. xxiii., p. 78). It consists in the inoculation of a fermentation tube, of the pattern shown on Fig. 15 (p. 77), filled with glucose bouillon from which the free oxygen has been expelled in process of sterilisation, with the organism in question. "Growth in the bulb, to which access to oxygen is allowed, is aërobic, while growth in the closed branch, from which oxygen is excluded, is anaërobic." Growth in both, on the other hand, will be evidence in favour of the organism being a facultative anaërobe. The bubbles which collect in the closed branch during sterilisation should be removed by tilting the tube while the medium is still very hot; otherwise they will again be absorbed by the fluid. 
the flame at such a distance from the other extremity as will allow it to be conveniently held by the fingers of the left hand, and, turning it during the process of heating, draw it out to a fine point. Pass the whole of the tube through the flame a sufficient number of times to ensure thorough sterilisation both of the interior of the tube and of the cottonwool plug, and place it on one side to cool.

Prepare a tube of glucose gelatine by boiling it in a beaker of water for some minutes in order to get rid of the free oxygen, and by means of a finely drawn pipette introduce into the medium a sufficient quantity (three or four drops will suffice) of a strong solution of sulphindigotate of soda, previously sterilised by boiling in a plugged test-tube, to tint it a distinct blue colour. Cool down the tube of gelatine by placing it under the tap until it reaches a temperature at which the hand can without discomfort bear the heat of the tube when it is held in it (about $40^{\circ} \mathrm{C}$.), and inoculate the medium with a trace of the material to be studied. ${ }^{1}$ Thoroughly incorporate this with the medium in the same manner as in the case of ordinary plate cultures. Break off the finely-drawn-out point of the Vignal tube and, after passing the extremity through the flame, insert it into the tube of gelatine. Aspirate the air slowly through the cotton-wool plug until the gelatine reaches the lower part of the constriction of the upper extremity. Withdraw it from the test-tube and, holding it in the right hand with the forefinger pressed tightly on the upper aperture, slightly elevate the other extremity and seal it off in a small Bunsen flame. Seal the upper end in a similar

1 Successful culture in a Vignal tube depends upon the complete isolation of the colonies. In order to ensure this, it is well to make a second or even third test-tube dilution before aspiration into the tube.

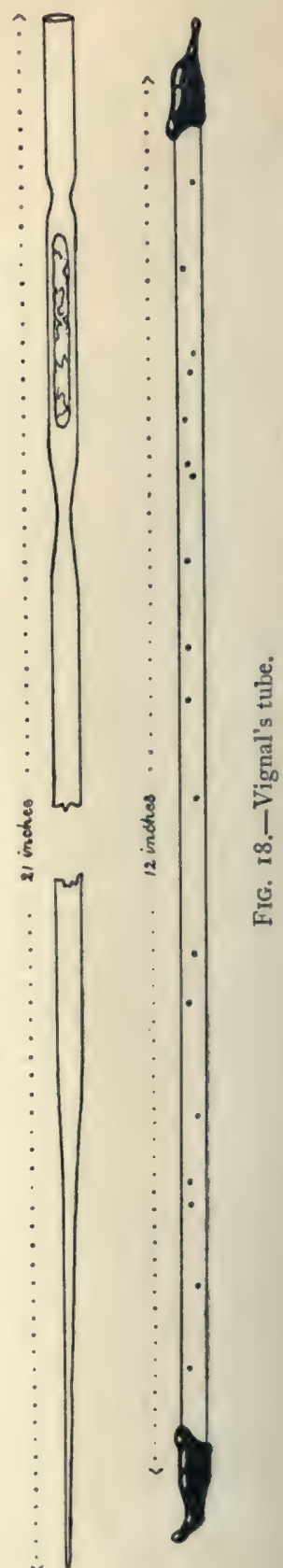


manner at the point of constriction, and strengthen the points of closure by the application of a little Golatz wax while the glass is still hot. Allow solidification of the medium to take place, and place in the incubator at $22^{\circ} \mathrm{C}$.

The colonies quickly develop, scattered in the gelatine, the first indication of growth being the decoloration of the medium due to the reduction of any remaining trace of oxygen by the organisms introduced.

When it is desired to culture, reculture, or to examine any particular colony, it is only necessary to make a file mark at the point desired, pass the tube lightly two or three times through the flame in order to sterilise the exterior and slightly to liquefy the gelatine adhering to the walls of the tube, break off first one end of the tube and then the tube itself at the file mark, and, by blowing gently through the tube orifice, expel the contents into a sterile Petri dish. Any desired colony can then without difficulty be picked out on the point of a platinum needle and either examined under the microscope, or recultured as desired.

\section{Roux tube for cultures in milk, bouillon, or other liquid media-}

For cultures in liquid media under strict anaërobic conditions, the method of Roux will be found most efficacious. The tube consists of a piece of $\frac{5}{8}$ inch glass tubing, drawn out at an extremity to a gradually reducing pipette of from 8 to 9 inches in length, leaving above it some 4 or 5 inches of the full-sized tube, to the other extremity of which is fused on a length of smaller tubing of about 7 inches. In this latter two constrictions are made, a cotton-wool plug being placed in position between the two. The tubes when made are sterilised at $150^{\circ} \mathrm{C}$.

A tube of sterile milk or bouillon is inoculated in the ordinary manner. After breaking off the pipette point, sterilising the exterior

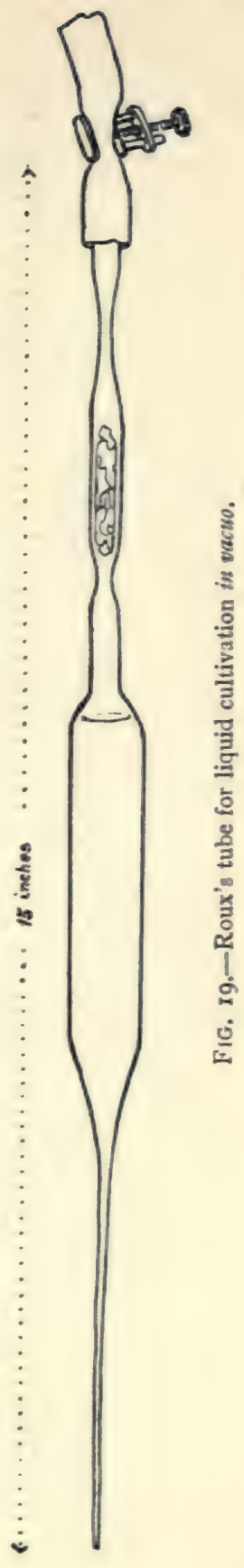


by passing it lightly two or three times through the flame, and loosening the cotton-wool plug of the test-tube so as to admit of its passage, the pipette is inserted into the inoculated medium and its contents gently aspirated into the tube, which, however, it is well not to fill to more than within from $\mathrm{I} \frac{1}{2}$ to 2 inches from the top. Holding the tube in the right hand, with the upper aperture closed by pressure of the forefinger, elevate the point slightly, and seal it off in a small flame.

Connect the tube with the tubing of the combined vacuum pump and hydrogen apparatus, and after exhausting, wash some four or five times in hydrogen (see p. 83). Exhaust again, and in order to ensure the expulsion of any oxygen held in suspension in the liquid, it is well at this stage to provoke ebullition in vacuo by raising the temperature to $30^{\circ}$ or $35^{\circ} \mathrm{C}$. The simplest method of doing this is by the gentle application to the exterior of the tube of the small flame of a spirit or other lamp. Great care, however, must be exercised to avoid such application of the flame as will cause violent ebullition, as in this case the liquid will have a tendency to pass into the exhaust tube. To prevent this, commence always by applying the heat first to the higher part of the tube, just above the level of the liquid. After ebullition has ceased, wash again with hydrogen, re-exhaust, and either seal off at the lower constriction, strengthening the point of closure by the application of a little Golatz wax, or screw down tightly the screw pinchcock upon a short length of pressure tubing fitted as shown on Fig. 19. This latter, which must of course be fitted previous to exhaustion of the air within the tube, has the advantage of permitting the examination of the culture at intervals. It is only necessary, if such examination is desired, to attach the tube to the hydrogen apparatus, fill the vacuum with the gas, snip off the extreme point, allow a drop of the culture to fall on a slide, and seal up afresh in the flame.

\section{Cultures in nitrogen (pyrogallate of potash method)-}

Although not so rigorous as many of the methods to be hereafter described, this form of culture is a most valuable accessory, owing to its extreme simplicity and the ready way in which it lends itself to anaërobic cultivations both in liquid and upon solid media. It depends upon the avidity with which oxygen is taken up by a solution of pyrogallate of potash, and is applicable both to test-tube and plate cultivations.

Test-tube cultures.-In the lower portion of a Buchner's tube, 
which consists of a strong glass tube of about $\frac{3}{4}$ inch diameter with a constriction about $\mathrm{I} \frac{1}{2}$ inch from the sealed end, is placed I gramme of pyrogallic acid. Within this tube is then placed the test-tube containing the cultivation, the top of which should come sufficiently below the mouth of the larger tube to enable the latter to be effectually stoppered with an india-rubber cork. By means of a thistle funnel io c.c. of a ro per cent. solution of caustic potash is then delivered into the bulb containing the pyrogallic acid, and the tube is immediately stoppered with the india-rubber cork, which should be previously moistened with benzole to make an air-tight joint. By gently tilting the tube a complete solution of the acid in the potash solution is then made in the bulb. The absorption of the oxygen takes some time, but if the operation has been performed with care it becomes finally so complete that growths even of the stricter forms of anaërobes will take place in the lesser tube.

This method of culture is both suitable for cultures in milk, bouillon, or other liquid media, and those upon potato, gelatinised whey, wort gelatine, or ordinary nutrient gelatine or agar. It is well, however, in the case of such media as will not be injured thereby, previously to boil, and cool quickly prior to inoculation, in order to, as far as possible, get rid of the oxygen held in suspension.

Test-tube cultures (Wright's method). - The tube cultivations are prepared in the ordinary manner, and that portion of the cotton-wool plug which projects above the mouth of the tube is cut off with scissors. The plug is then pushed down into the tube for a distance of about I inch, and about I c.c. of a Io per cent. aqueous solution

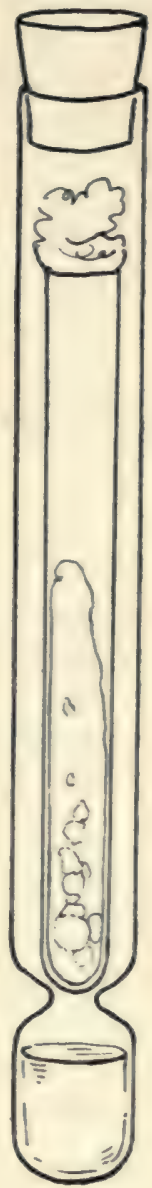

FIG. 20.-Buchner's
tube. of pyrogallic acid is dropped upon the plug, by which it will be at once absorbed. An equal quantity of a ro per cent. solution of caustic potash is then run in, and the mouth of the tube at once closed with a tightly fitting india-rubber stopper, sterilised by boiling. Incubation can then take place at any desired temperature according to the medium employed. 
Petri dish isolation on agar (Klein's method).-Melt a tube of glucose formate agar, pour out into a Petri dish and set aside to cool. After the medium has thoroughly set, a trace of the material under examination, or a drop of the whey of a typical milk culture, or a drop of fluid from a serum culture, is diluted in a sterile watch glass with from $\frac{1}{2}$ to I c.c. of sterile salt solution. A platinum spatula of the form shown on Fig. 21 is sterilised in the flame, dipped into the dilution, and is then drawn over the surface of the set agar, so as to rub the fluid adhering to the spatula uniformly over the whole surface of the agar. The Petri dish is closed with its cover and placed on a small wire triangle above a smaller and deeper glass dish, set upon a ground-glass plate con-

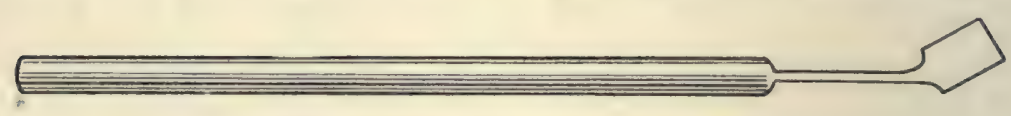

FIG. 21.-Platinum spatula for Klein's method.

taining a sufficient quantity of pyrogallic acid. A sufficient quantity of liquor potassæ is added to the pyrogallic acid in the lower glass dish, and the whole apparatus is then covered with a small bell glass, the free rim of which has been previously well smeared with resin ointment so as to ensure the bell glass, on being well pressed down on the ground-glass plate, an air-tight seal.

\section{Petri dish eultivation in vacuo or in hydrogen ${ }^{1}$}

When a supply of hydrogen and the vacuum pump are available, a modification of the ordinary method of isolation by means of Petri dish cultivation can be employed. Either gelatine or agar media can be used, but in each case glucose should be added in the proportion of 2 to 3 per cent.

\section{Cultures in glucose gelatine-}

The medium should be boiled for ten minutes in a water bath in order to expel the free oxygen, and then quickly cooled down under the tap to below $40^{\circ}$ (a temperature approaching that of the solidifying point). The tube is then rapidly inoculated in the usual manner, and poured out without delay into the Petri dish,

1 If the most stringent anaërobic conditions are desired, solidification should take place either in vacuo or in a current of hydrogen, but under ordinary circumstances the above will be found sufficient for the purpose, the glucose acting as a reducer of any free oxygen present in the medium. 
which must be placed on a cold slab to ensure rapid solidification. It is then transferred to a vacuum desiccator of the form shown. The desiccator is connected up with the hydrogen apparatus, a vacuum formed, and after repeated washings in hydrogen the cultivation is either left in an atmosphere of that gas, or in vacuo as thought desirable.

\section{Cultures in glucose agar-}

For cultures on agar similar technique is employed, care being taken, however, that the medium, before inocu-

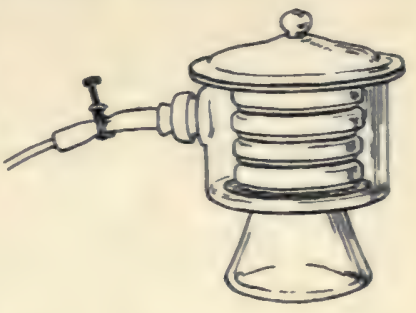
lation, is not allowed to cool down to a

FIG. 22.-Vacuum desiccator for Petri dish culkivations. temperature approaching too nearly the solidifying point. The operations must be performed quickly, or solidification, which occurs at about $40^{\circ}$, will take place before the medium is evenly distributed in the Petri dish.

Another method in the case of agar media is that of stroke cultivation. The media after being allowed to boil for some minutes

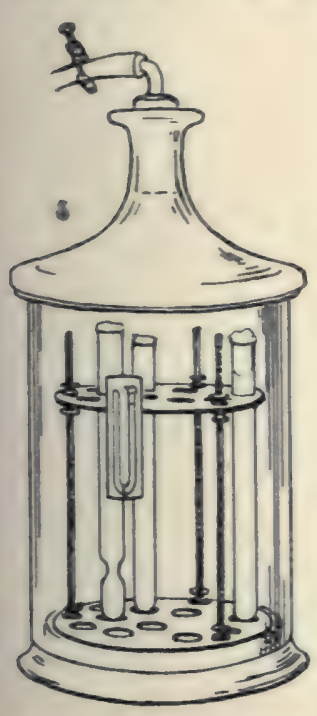

FIG. 23.- Vacuum desiccator for test-tube cultures.

is quickly poured out into a Petri dish placed on a cold slab in the usual way. When solid, a platinum needle charged with a trace of the culture or material under examination is drawn lightly across the medium in parallel lines extending across the dish. The dishes are then placed in the desiccator and treated as above. Growth can be allowed to take place either at room temperature or at $37^{\circ}$ as desired. (See also Klein's method of isolation described above.)

\section{hydrogen- \\ 5. Test-tube cultures in vacuo or}

When it is desired to place ordinary test-tube cultures under anaërobic conditions, a vacuum desiccator of the form shown on Fig. 23 will be found of great service for daily use. The tubes are placed upright in the nickel support as shown, the lid replaced, and an air-tight joint made with resin ointment. The air is then exhausted, replaced by hydrogen, 
exhausted again, and the cultures left in vacuo or hydrogen as desired, by screwing down tightly the screw pinchcock on the short length of vacuum tubing. The ease with which cultures can be examined and again replaced under anaërobic conditions renders this method at times of great service.

\section{Esmarck roll culture (modified) for isolation of organisms-}

Take tube as shown in Fig. 24, plug with cotton-wool, and sterilise in the hot-air steriliser. Take two tubes of liquefied gelatine and inoculate the one with a trace of the culture to be studied, or from which it is desired to isolate any particular organism. Tint the other with sodium sulphindigotate solution, and boil in a beaker of water to get rid of the oxygen in suspension. Cool down rapidly to $36^{\circ} \mathrm{C}$., and inoculate with one or two loopfuls of the first dilution. Thoroughly incorporate, and by means of a fine drawn sterile pipette transfer to the Esmarck tube, after removal of the cotton-wool plug, sufficient of this second dilution to give a thin coating to the walls of the tube when making the roll culture (from $\frac{1}{4}$ to $\frac{1}{2}$ an inch from the bottom of the tube will be found sufficient). Replace the cotton-wool plug, and slightly constrict the tube-opening by turning the extremity in the Bunsen flame. Allow the tube to cool, and connect up with the hydrogen apparatus. Exhaust, and wash four or five times with hydrogen in the manner already explained. Exhaust again, and seal off in vacuo at the point of constriction. Liquefy the medium by placing in a beaker of warm water not exceeding $36^{\circ} \mathrm{C}$, and, holding the tube horizontally, turn and tilt it until the medium is seen to cover the whole of the interior walls of the tube.

Solidify by turning under the tap or in a basin

FIG. 24.-Esmarck roll culture. of cold water, care being taken to get a perfectly even layer distributed over the tube walls. Incubate at $22^{\circ} \mathrm{C}$. When growth has taken place, snip off the sealed point in the heated air above the Bunsen flame (to prevent accidental contamina- 
tion by the inrush of air to fill the vacuum), make a file mark in the centre of the tube, and carry the crack round with a red-hot wire. The tube can then without difficulty be divided in two, and any desired colonies picked out with a sterile platinum needle for examination or culture.

\section{media- \\ 7. Fraenkel's tubes for anaërobic cultivation in liquid}

Take an ordinary large sized and sufficiently strong test-tube and plug with an india-rubber stopper pierced with two holes. Through one of these introduce a glass tube, bent at right angles and of such a length that one extremity reaches almost to the bottom of the test-tube, whilst the other projects about I inch above the stopper, with a right-angled projection of some $2 \frac{1}{2}$ to 3 inches. A similar tube, but of such a length that it will just project beyond the bottom edge of the stopper, is inserted through the second hole. Before inserting the tubes make a constriction in the right-angled return of each, plug with cotton-wool and, turning the ends in the Bunsen flame, partially close the openings in order to prevent the possible exit of the cotton-wool plug.

Introduce a sufficient quantity of medium into the tube, ${ }^{1}$ replace the indiarubber stopper with its tubes, and sterilise in the ordinary manner. When required for use, liquefy the medium, in-

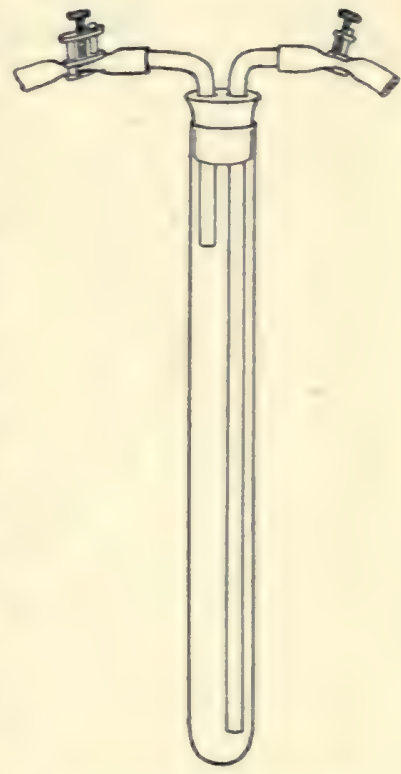

FiG. 25.-Fraenkel's tube. oculate with a trace of the culture to be studied, thoroughly incorporate, make an air-tight joint with benzole or paraffin, and connect the longer tube with the hydrogen apparatus.

Pass a current of hydrogen under pressure (see p. 82) through the medium for a sufficient length of time to expel all the contained oxygen, and seal off both tubes (the exit tube first) in the flame, or screw down the pinchcocks on india-rubber fittings as shown on Fig. 25. Incubate at $22^{\circ}$, or lower temperature.

${ }^{1}$ To avoid excessive frothing when subsequently passing a current of hydrogen through the medium, it is well to place one drop of sterile oil on the surface of the gelatine. 


\section{Yeast flask method-}

This method is specially suitable for the study of anaërobic organisms producing a large quantity of gas, all danger of rupture of the flask being avoided by means of a simple automatic mercury valve. The apparatus consists of a yeast flask as figured, which is filled with milk, glucose bouillon, or other liquid media to about two-thirds of its volume. The mouth is closed with a perforated india-rubber cork, through which is inserted a tightly-

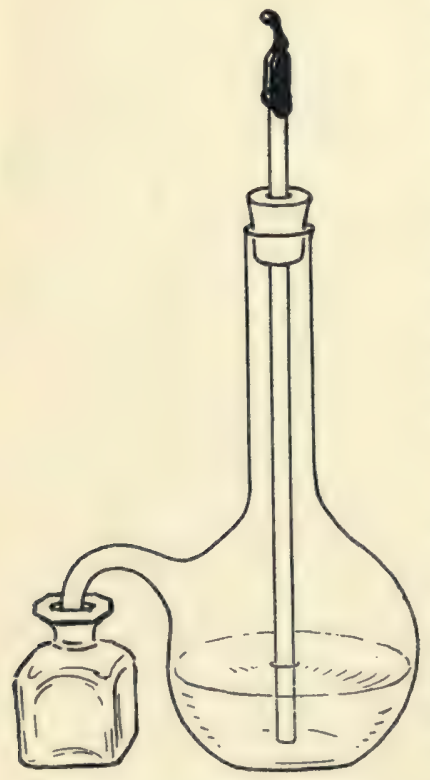

Fig. 26.-Yeast flask culture in hydrogen. fitting glass tube, reaching almost to the bottom of the flask, and extending to 2 or 3 inches above the level of the cork. A constriction is made in the upper portion of this, and above it is inserted a loose cotton-wool plug kept in position by slightly closing the tube orifice in the flame. The whole is then sterilised (except in the case of milk, which should be introduced sterile into an already sterilised flask).

After inoculation, the tube passing down into the medium is connected up with the hydrogen apparatus, and a current of the gas is passed through the liquid for some twenty minutes or half an hour. The extremity of the bent tube is then inserted into a small phial containing a sufficient quantity of mercury to well cover the exit, the longer tube is sealed off at the point of constriction, and the whole placed in the incubator at the temperature desired. The phial of mercury forms a valve which, whilst allowing the escape of all gas generated in excess within the flask, effectually prevents the admission of air.

\section{Veillon's method-}

(Glucose agar or glucose gelatine).-Veillon takes advantage of the property of glucose as a reducer of oxygen. Ordinary testtubes are filled with the medium to two-thirds of their height, and by means of a long needle or very finely drawn glass rod the inoculation is made to the bottom of the tube. To avoid subsequent 
absorption of oxygen along the line of puncture the tube should be stoppered by a layer of liquid agar, or a mixture of vaseline and paraffin as recommended by Miquel, run in over the top of the medium after the inoculation has been made. A simpler, and equally efficacious way, if a due amount of care in manipulation is exercised, is to heat gently the test-tube by turning it in a small Bunsen flame immediately above the upper level of the medium, until slight liquefaction of the medium at this point occurs. The subsequent solidification of this thin liquid layer will effectually seal the point of entry of the needle.

To render evident the absorption of the oxygen in the medium, it is well to tint it with the sodium sulphindigotate solution, as described under the head of Vignal's tubes.

\section{0xygen absorption by superflcial cultivation of a highly aërobic organism-}

Melt a tube of glucose agar or glucose gelatine (preferably tinted with sodium sulphindigotate solution) in a beaker of boiling water. Boil for some minutes in order to get rid of free oxygen. Cool rapidly down to $40^{\circ}$ and inoculate in the usual manner. Solidification should be accelerated by placing the tube in cold water in order to avoid further absorption of oxygen. A layer of liquid agar charged with a cultivation of the Bacillus subtilis or other strong aërobe is then poured on the top of the solidified medium. The avidity with which the oxygen is taken up by the bacteria in the upper layer will effectually prevent any further absorption of it in the lower one. To examine, or to reculture, it is only necessary to make a file mark on the glass at the point required and carry the crack round the tube with a red-hot wire. It is well, however, first to break the bottom of the tube in order to avoid the possible sudden expulsion of the contents by gas generated by the organisms. In the case of gelatine, another method is to plunge the test tube for a moment into boiling water so as slightly to liquefy the medium at the point of adherence to the tube walls, and then, after withdrawing the cotton-wool plug and flaming the tube orifice, to allow the solid medium to fall into a sterile Petri dish, when cultivations can be made from any desired point.

\section{Potato cultures in vacuo or in hydrogen-}

Take a Roux potato-tube containing the medium and liquid (as described on p. 4I), and substitute for the cotton-wool plug a 
sterile india-rubber stopper pierced by one hole, through which is

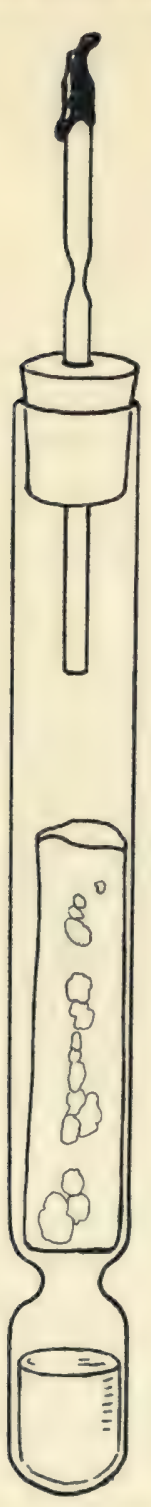

FIG. 27.-Potato culture in vacuo. inserted a piece of narrow glass tubing constricted as shown, and plugged lightly with cottonwool between the two constrictions. Thoroughly sterilise this latter in the flame before passing it through the stopper, and moisten with benzole to secure an air-tight joint. Inoculate the potato in the ordinary manner with a spatula-shaped needle, replace the stopper, and apply with a camel's hair brush, a few drops of benzole, or a mixture of paraffin and vaseline previously melted, in order to secure an air-tight joint. Connect the inlet-tube with the india-rubber tubing of the hydrogen apparatus, exhaust by means of the air pump, wash some four or five times with hydrogen as described above (p. 83), and seal off (either in vacuo or in hydrogen) at the upper constriction. ${ }^{1}$ When it is required to open the tube for examination or reculture, it is only necessary to break off the extreme point of the inlet-tube and allow the air to filter in through the cotton-wool plug. The stopper can then be removed and a platinum needle inserted in the ordinary manner, after which, if desired, the stopper can be replaced, the air

1 The ordinary method is to seal off at the lower constriction, in which case the opening of the tube causes almost certain contamination by the inrush of unfiltered air. By sealing off the upper constriction first this is avoided, and the tube can be re-exhausted if it is desired to proceed further with the culture. 
exhausted as before, and the inlet-tube sealed off at the lower constriction.

The above method is equally applicable to cultures upon agar, gelatine, and other media, provided that the usual precautions are taken to first get rid of the oxygen in suspension or solution.

\section{Pasteur's tubes-}

These tubes, three forms of which are shown on figure, are at times useful for cultivations in liquid media. Form " $\mathrm{A}$ " is of special service in studying the phenomena attendant upon the "association" of organisms. A constriction is made in the upright

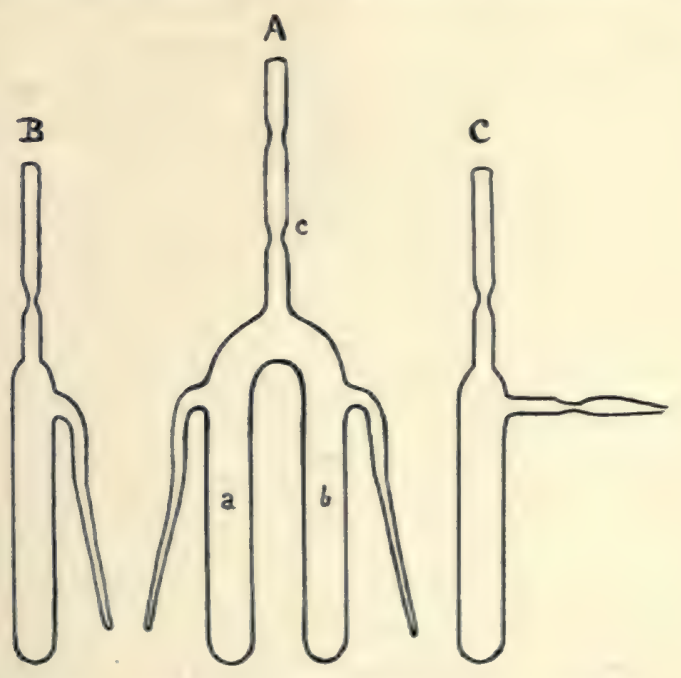

FIG. 29.-Pasteur's tubes for cultivation of anaërobes.

tube at the point " $c$," a light cotton-wool plug inserted above it, and the orifice of the tube is then slightly closed by turning the end in the Bunsen flame (or making a second constriction above the cotton-wool plug) in order to prevent the escape of the cottonwool on a vacuum being formed. The extreme points of the lateral tubes are sealed in the flame, and the whole is then sterilised at $150^{\circ}$ in the hot-air steriliser.

When required for use, snip off one of the lateral points, pass the pipette portion of the tube through the flame, plunge it into a tube of liquid medium inoculated with a trace of one of the organisms it is desired to study, and aspirate the contents into the tube " $a$." Seal off the point in the flame, snip off the point of 
the second lateral tube, and repeat with a culture of the second organism. Seal off the point, and connect up with the vacuum pump and hydrogen apparatus. Exhaust, wash well in hydrogen, and seal off in vacuo or hydrogen as desired, by screwing down tightly the screw pinchcock on a short fitting of pressure tubing fitted as shown on Fig. I9 (p. 85). Incubate at $37^{\circ}$, or room temperature as desired. When growth has taken place in one or both of the tubes, incline the apparatus and allow a few drops of the one culture to gain entry to the tube containing the other, without disturbing the vacuum. In order to obtain at any time a sufficient quantity of culture for examination, it is only necessary to connect up with the hydrogen apparatus, allow the tube to fill with the gas, snip off the end of one or other of the pipette tubes, and force out by means of the pressure of hydrogen, a few drops of the culture. The point can then be sealed and the tube closed without in any way disturbing the anaërobic conditions.

Form " B" is simply a modification of form " $A$," and is useful in the study of one organism only.

Form "C" can be used either for liquid or solid media, and has been specially used for the latter by Dr Roux at the Pasteur Institute. ${ }^{1}$ A sufficient quantity of nutrient gelatine or agar is introduced, the two tubes of entry are lightly plugged with cottonwool, and the whole is then sterilised. Inoculation is made, by means of a finely-drawn Pasteur pipette, through the vertical tube, which is then sealed off in the flame. The lateral tube, which should have in it a double constriction in order to maintain the cotton-wool in place, is connected up with the vacuum pump and hydrogen apparatus, the air exhausted, and the culture well washed in hydrogen while the medium is still liquid. The lateral aperture is then sealed off in the flame. The tube is then inclined and rolled in the hands in order to obtain an even distribution of the organisms introduced, and the medium is then allowed to solidify on the slope. A modification of Petri dish culture is thus obtained under strictly anaërobic conditions. To pick out the colonies after growth it is only necessary to admit air or hydrogen through the cotton-wool filter of the lateral orifice, to make a file mark on any portion of the tube, and to carry the crack round by means of a red-hot wire.

The methods described above may be said to comprise all those

1 Roux, "Sur la Culture des Microbes anaërobies" (Ann. de l'Inst. Pasteur, 1887 , p. 49). This article is specially worthy of study by those who wish to obtain a mastery of anaërobic technique. 
which the writers have found most useful in anaërobic research, but various modifications of these will naturally occur to the individual worker. The catalogues of the various English and Continental furnishers of bacteriological apparatus contain illustrations of various forms of flasks for the cultivation of anaërobic organisms, the use of which will at once be apparent to those who make a study of the subject, and are conversant with the general principles underlying the methods described above. 


\section{CHAPTER IV}

\section{EXAMINATION OF AIR AND WATER IN RELATION TO THE MILK SUPPLY}

Examination of Air-General Note. Air-borne Organisms. Seasonal Variations. Air of byres, dairies, milk-shops, etc. Methods of Examination. Examination of Water: Quantitative and Qualitative. Pathogenic Organisms in Water.

\section{General note on bacteria in air-}

We have already pointed out in a preceding chapter the important rôle played by air-borne organisms in the contamination of milk. In the byre, in the cooling-room, in transport to point of distribution, and in storage after reaching the hand of the consumer, this contamination continues in more or less degree, and although many of the organisms are harmless from a pathogenic point of view, yet others undoubtedly may at times play an important part in the spread of disease.

It must be borne in mind that fresh country air away from centres of population may be regarded in still weather as practically free from bacteria, although, according to the season of the year, it will contain a more or less considerable number of the hyphomycetes or mould fungi. At high altitudes, the air may be looked upon as practically germ free, although here again the lighter spores of the mould fungi may cause them to be carried by air currents to a very great height. The experiments of Pasteur are well known. In the recent researches of Dr Jean Binot of the Pasteur Institute, ${ }^{1}$ IOO litres of air taken at the summit of Mont Blanc did not contain a single microbe, and the total number of organisms varied between 4 and II per metre cube (I000 litres). An examination of the air of the interior of M. Janssen's Observatory, situated on the highest point of Mont Blanc, and taken in two different rooms, gave, on the other hand, 540 and 260 organisms per metre cube.

1 Communication al IAcadémie des Sciences de Paris, 17 Mars 1902. 
The gradual increase of the number of organisms as descent to lower level takes place is of interest. Thus 6 per metre cube were found on the Grand Plateau, 8 at the Grands Mulets and I4 at the Plan de l'Aiguille. Upon the Mer de Glace 23 organisms were found, and 49 at Montanvert. It is interesting to compare these figures with the records of M. Miquel at the Mont Souris Observatory, where a careful analysis of the air both at the Observatory itself, and at different quarters of Paris is systematically made at weekly intervals throughout the year. The Park of Mont Souris, in which the Observatory is situated, lies at the southern extremity of the city, and adjoins the line of fortifications, so that all winds blowing from N.W., N., or N.E., blow from and over the city towards the Observatory, whilst all those blowing from the S.W., S., and S.E., blow towards the Observatory and city from the open country. In the month of August, with the wind from the south, i.e. blowing from the country citywards, the number of organisms was found to be 40 in the Park around the Observatory, while at the same moment a record of 14,800 was obtained in the $4^{\text {th }}$ Arrondissement, which may be taken as the centre of Paris, and comprises the surroundings of Notre Dame and of the Hôtel de Ville. In the month of June, on the other hand, with the wind blowing from the N.E., i.e. across the city towards Mont Souris, the numbers were, in the $4^{\text {th }}$ Arrondissement 10,000 per metre cube, and in the Park of Mont Souris itself, II80 per metre cube.

The seasonal variations of the organisms present in the air are also worthy of note; the following table shows the mean over a period of ten years in the air taken at Mont Souris :-

Avarage per motre eube.

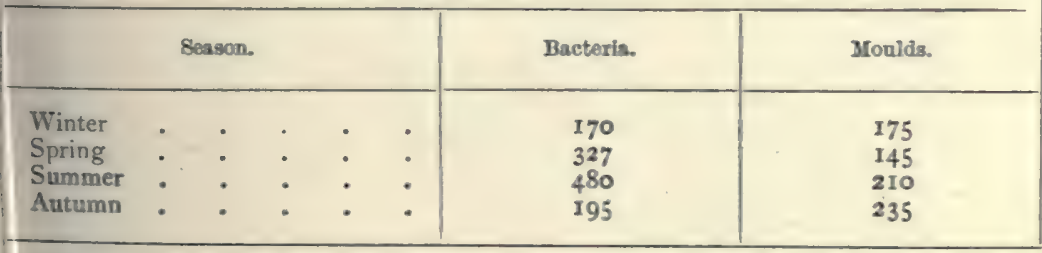

Similar experiments have been carried out by Frankland, lügge, Delalivesse, Neisser, Chick, Andrewes, and others. The est named conducted some experiments in London in 1902, and tported his results to the Pathological Society. $\mathrm{He}$ found the imber of organisms varied greatly, but no pathogenic species 
were isolated by him. Staphylococci, sarcinæ, streptothriceæ, and moulds were the most common species present. He confirmed the view of Chick as to the rarity of $B$. coli in air.

We have ourselves carried out a number of investigations into the bacteriology of air of byres, dairies, milk-shops, bakehouses, workshops, and the open air in town and country. We found no exact relationship to exist between percentage of $\mathrm{CO}_{2}$ in the air and number of organisms, although in a general way it may be said that where $\mathrm{CO}_{2}$ was most largely present organisms were most numerous. In addition to general investigations of this character, we have made several series of examinations of air in relation to the milk supply, both in town and country. The accompanying Plates are reproduced from photographs of the colonies on nutrient agar in Petri dishes. These were incubated for four days at ordinary room temperature, and the technique adopted was that described below. Plates 5, 6, and 4 also illustrate similar examinations of byre and dairy air, farm water, and the air of a certain spot in a London street, where milk is delivered daily.

These Plates illustrate as words cannot do $(a)$ the differences in the bacterial pollution of air in town and country, and $(b)$ the degree of contamination likely to occur in milk exposed in such atmospheres. It should be added that the plates have been selected from a large number as representative; they are not the result of accidental or haphazard examinations. As to the kind of organisms present, we have found them chiefly to be the following :- staphylococci, diplococci, a few chromogenic organisms, sarcinæ, saccharomyces, moulds, and a few representatives of the streptothrix group of organisms. Occasionally, but by no means uniformly, we have found lactic acid organisms. We have met with no $B$. coli, although a constant outlook has been made for it.

\section{Bacteriological Methods}

For the bacteriological examination of air many methods have been devised, and we propose to summarise below the various systems employed in this country and abroad, giving in greater detail those we ourselves have found most useful for the purpose in our investigations in relation to milk pollution.

\section{Koch's plate method-}

The simplest means of obtaining a purely qualitative bacteriological analysis of the air in any given locality or place is that 
PLATE 10.

COMPARATIVE AIR PLATES.

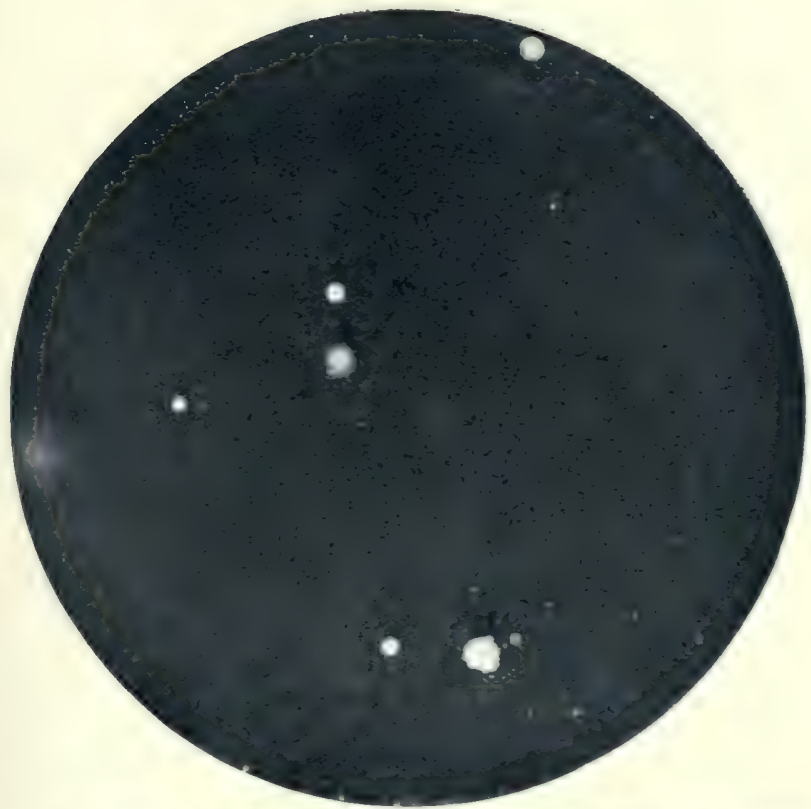

. Conntry air. Nearest habitation, 'uarter-mile. Half-hour's exqosure.

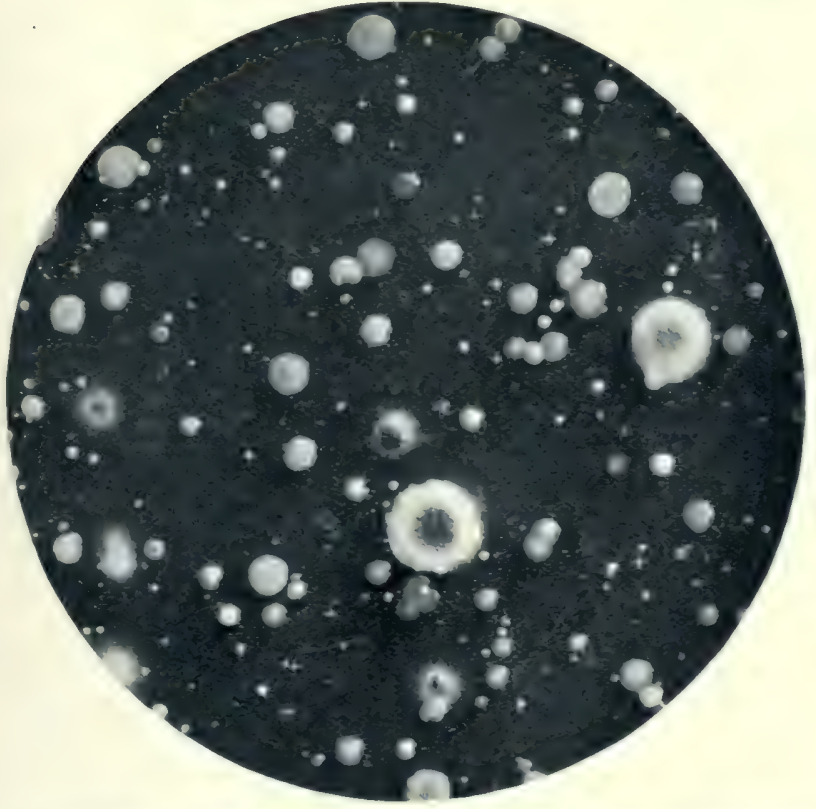

1. Iondon air. Bond Street, 50 feet above rsalway. Half-hour's exprosure. 

PLATE 11.

COMPARATIVE AIR PLATES.

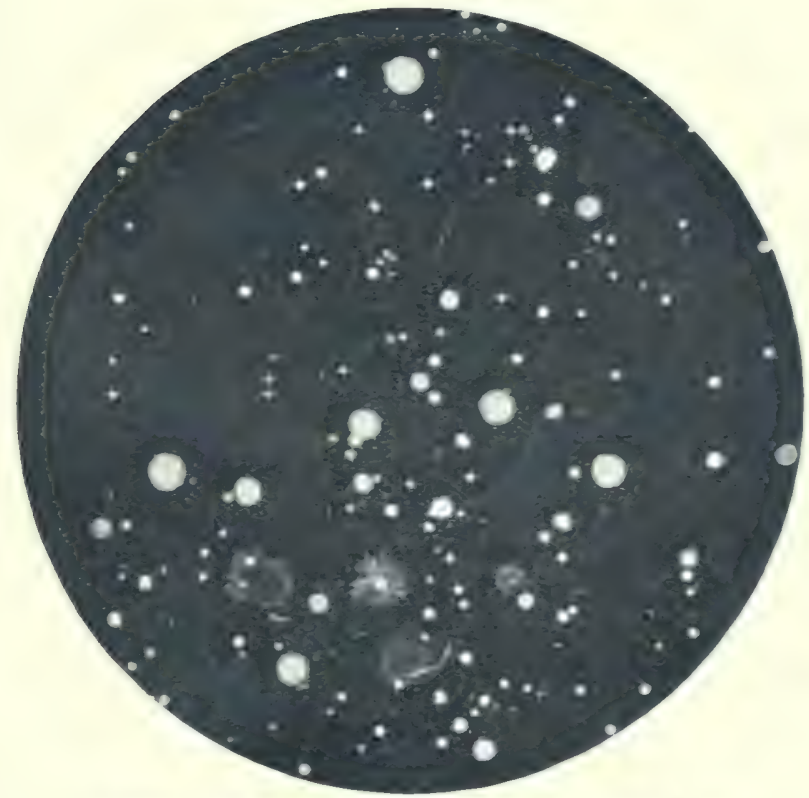

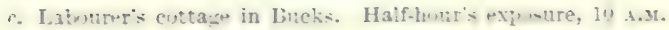

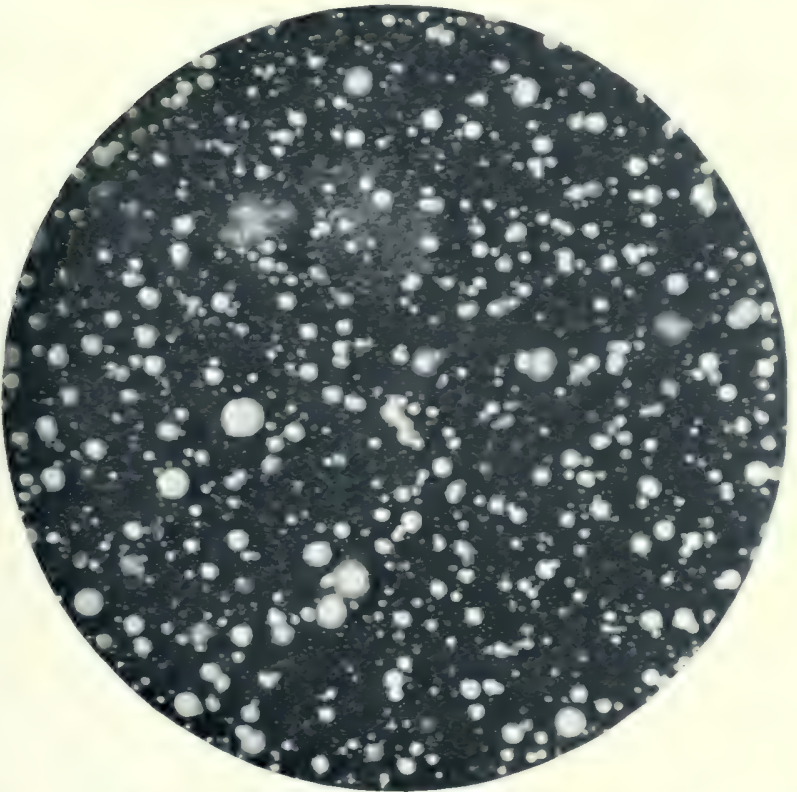

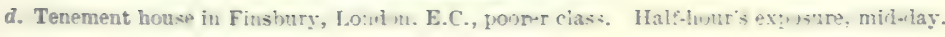



originally devised by Koch. This consists in the exposure of a plate of nutrient agar or gelatine for a given time to the air of which examination is desired, in such a manner that the organisms floating, or borne upon dust particles therein, will by gravity fall upon the plate, and, finding themselves in a suitable environment, start growth thereon. It will be found in practice that nutrient agar is better for the purpose than nutrient gelatine. Greater latitude is obtained both in point of temperature and length of incubation, and the result is uncomplicated by the, at times, very rapid liquefaction of the gelatine by liquefying organisms. Care should be taken in preparing the plates to allow them to cool on a perfectly level surface, and at least I 5 c.c. of the medium should be employed for each Petri dish in order to ensure a perfectly even surface and sufficient depth all over the plate. After exposure the plates are, under ordinary circumstances, best left at room temperature during the development of the colonies, but if it is desired to examine the bacteria alone it will be found well to favour the growth of these at the expense of the moulds by first incubating the dish at a temperature of $37^{\circ} \mathrm{C}$. for, say, eighteen hours. In any case the plate should be shielded from light, as otherwise many of the chromogenic organisms will not assume their typical coloration. Should it be desired to photograph the plates in order to obtain a permanent record, the growth should be arrested and the organisms killed about the third or fourth day of incubation. The best method of doing this is to reverse the dish, and to pour upon a piece of blotting-paper placed on the inner surface of the lid, which will now be undermost, a sufficient quantity of Formalin to saturate it.

We have ourselves in this method employed special dishes of large size (see Plates 5 and 6), but in ordinary practice it will be found that the $9 \mathrm{~cm}$. Petri dish is sufficient, and by means of these an accurate idea may be obtained of the relative richness of organisms of the air in different localities or situations. A series showing such comparative results will be found on Plates IO, II, and I2.

As will be seen, however, the above method is of no use when any approach to exactness as to the number of bacteria in a given volume of air is desired, although by a simple calculation an idea can be gained thereby of the number of organisms falling on a given area in a given space of time. ${ }^{1}$

1 To calculate the area of a circular dish, square the diameter, and multiply by .785 . According to Petri, the number of bacteria present in 1o litres of air are deposited upon 100 square centimetres in five minutes. 


\section{Miquel's method (modified)-}

The method of Miquel is the one which, for quantitative analysis, has given the best results in our hands, and this method will be found most reliable except when the air is very heavily charged with moisture. Advantage is taken of the filtering properties of an inert salt in a finely granulated condition, possessing no antiseptic power, and unalterable and nonfusible at a temperature of $170^{\circ}-180^{\circ} \mathrm{C}$. Such salts were found by Miquel, after a long series of experiments, to include the sulphates and phosphates of sodium, and the sulphate of magnesia, all of which were found to favour rather than to retard the growth of organisms. Of these three salts, sulphate of sodium was found to be the best, as being more soluble than the phosphate, and less deliquescent than the sulphate of magnesia. To prepare the filtering medium, a sufficient quantity of the salt should be thoroughly dried, and then reduced to coarse powder in a mortar. The powder thus obtained is then thrown upon a sieve containing between 15 and 18 meshes per $\mathrm{cm}$. That which passes this sieve is then thrown upon a second containing between 22 and 25 meshes per $\mathrm{cm}$. The finer particles of the powder pass through this latter mesh, leaving upon the sieve an evenly granulated powder of which the individual grains will be found to be of about 5 of a $\mathrm{mm}$. in diameter. A sufficient quantity of the powder should be prepared, and if stored in a dry place, and in a carefully stoppered bottle, will be found to remain unchanged for a lengthened period. ${ }^{1}$

The filtering apparatus itself is of the simplest description, and is represented by Fig. 30. It consists of a piece of stout glass tubing about $12 \mathrm{~cm}$. in length and $.75 \mathrm{~cm}$. internal diameter, constricted in the middle as shown. At one side of the constriction a light plug of glass wool $(a)$ is inserted in order to act as a support to the granulated salt used for the filtration. The filtering substance is shown at $b$, and consists of the granulated sulphate of soda, prepared as explained above. The extremities of the tube are plugged with asbestos plugs ( $c$ and $d$ ) to prevent contamination of the tube after sterilisation. It will be found convenient to prepare a number of these tubes at a time. The central plug of glass wool should be inserted and the tube itself heated in a Bunsen flame until all trace of moisture has disappeared. About I gramme of the pulverised salt in a thoroughly dry condition is then introduced into the tube

1 Messrs Baird \& Tatlock of I4 Cross Street, Hatton Garden, E.C., will supply on order this salt in a properly granulated condition. 
by the orifice $c$, the two asbestos plugs are fitted in position, each tube wrapped in a piece of wrapping paper and sterilised in the hot air steriliser for one hour at $150^{\circ} \mathrm{C}$. The paper will act as a further protection against possible contamination of the tube before it is required for use. Thus furnished, the tubes can be carried without risk in the pocket or elsewhere.

When required for use the paper covering is removed, the tube placed in a position approaching the vertical; the plug $d$ is removed, and the extremity of the tube joined up to the india-rubber tubing of an aspirating apparatus. One or two smart taps of the finger are then given to the tube in order to bring the salt particles into close contact, the plug $c$ is removed, and the aspirating apparatus set in action. After a given quantity of air has passed through the tube, the plug $c$, which should in the meantime have been carefully protected from contamination, is replaced, the tube itself detached from the aspirating apparatus, and conveyed to the laboratory for examination. As many tubes as thought desirable can be employed, either as controls or for the examination of the air in different localities. As the organisms are deposited upon the particles of an inert salt in a dry condition, the necessity of immediate examination is not so great as in the case of other methods.

On arriving at the laboratory, the end of the tube containing the sulphate of soda is lightly passed through the Bunsen flame, the plug $c$ withdrawn, and the contents allowed to fall into a tube containing Io c.c. of bouillon. The tube should be well rolled in the hand to ensure dissolution of the soda and a general distribution of the organisms in the medium, and given quantities withdrawn by means of a sterile calibrated pipette, and distributed into tubes of gelatine, from which plate cultivations are made in the ordinary manner. The plates are left for three days or longer at

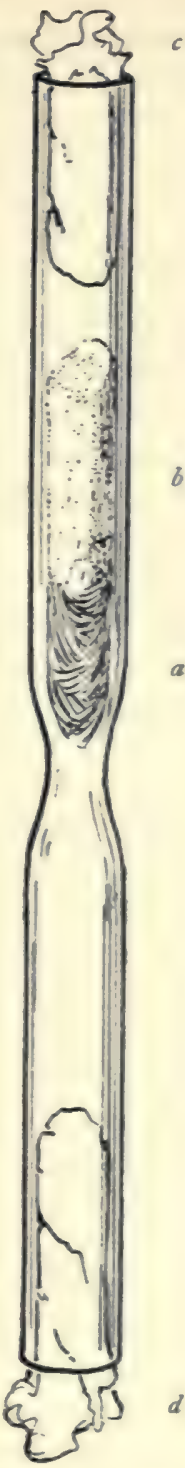

FIG. 30.-Air-filtering tube; actual size. room temperature, and an enumeration is then made of the colonies 
appearing. A simple calculation will give the number of organisms per metre cube, the standard usually employed. Thus, if 25 litres of air are passed through the apparatus and I c.c. of the bouillon gives, say, 5 colonies, then

and

25 litres of air contain $5 \times$ Io aërobic organisms

I metre cube of air (1000 litres) contains $\frac{5 \times 10 \times 1000}{25}=2000$ aërobic organisms per metre cube.

As a control the glass wool plug should, in all cases, be carefully withdrawn from the tube by means of a sterile needle or forceps, and dropped into a tube of melted gelatine. From this an ordinary plate cultivation should be made. If the filtration has been effective, no growth whatever should appear upon the plate. The withdrawal of the plug should be made through the end of the tube which was attached to the aspirator, in order to avoid the picking up of any chance organism which may have been left on the tube walls on the removal of the salt, and the orifice and end portion of the tube should be well heated in the flame and allowed to cool before the withdrawal is proceeded with.

Aspirating apparatus. - A convenient form is shown with the filtering tube in position, on Plate I3. It consists of a portable tripod stand with head, from the under side of which are suspended 2 chains of about 8 inches in length, and furnished at the ends with 2 large rings of $1 \frac{1}{4}$ inches in diameter. From these latter are suspended, the one below the other, 2 vessels of exactly 5 litres capacity, and furnished with chains terminating with large $\mathrm{S}$ hooks as shown. The vessels are furnished at the top with an opening of $\frac{3}{4}$ of an inch in diameter, to which is fitted a I-hole india-rubber stopper, and at the bottom with straight-way taps of about $\frac{1}{4}$ of an inch bore. Through this stopper a short length of tightly fitting glass tubing is run, and to the upper extremity of this a piece of india-rubber tubing of some 2 or 3 feet in length is attached. This tubing should lead through a hole in the supporting plate of the tripod, upon the upper surface of which is fixed a large spring pinchcock for the purpose of holding in position the tube containing the filtering medium. When required for use, the upper vessel is filled with water to its utmost capacity, the india-rubber stopper pressed tightly home so as to ensure an air-tight joint, and the free end of the tubing slipped on to the lower extremity of the filtering tube, which is then fixed by the spring pinchcock in a position approaching the vertical. The top of the 
PLATE 13.

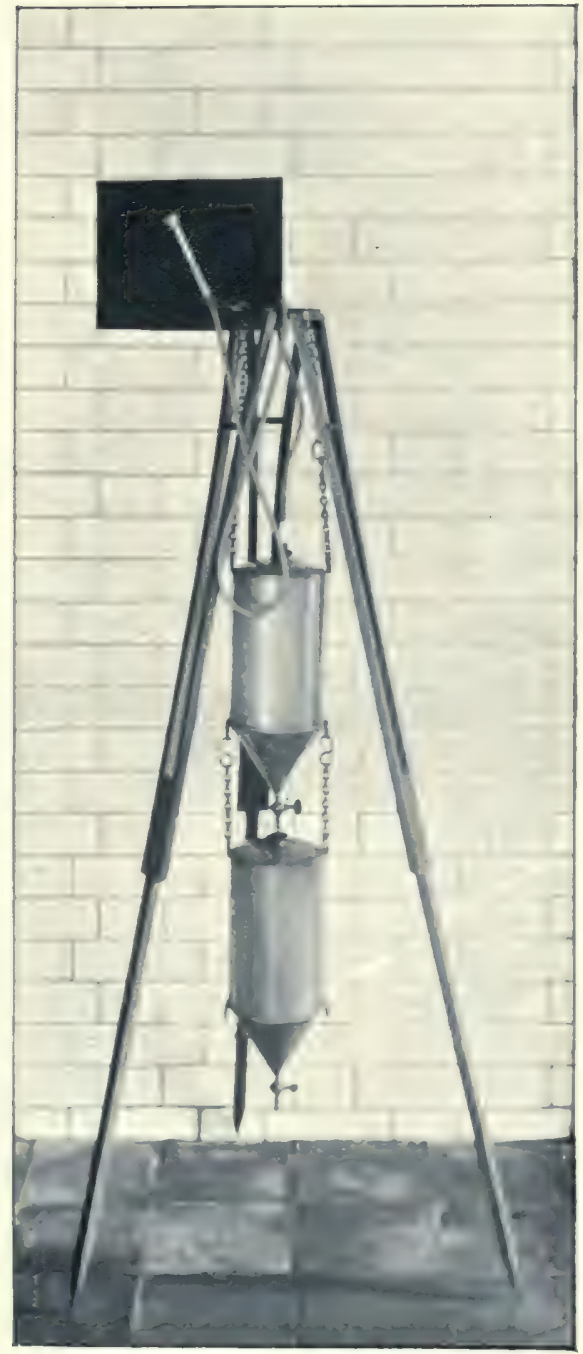

Apparatus for bacteriological examination of air. 

upper vessel is then opened, and the water allowed to run slowly into the lower one. As soon as the whole of the water has run off, the position of the vessels is reversed and the procedure repeated. By this means any number of litres, being a multiple of 5 , can be run through the aspirator, an equivalent number of litres of air being drawn through the filtering tube, depositing upon the filtering medium all organisms floating or suspended therein. ${ }^{1}$

\section{Method of Laveran-}

This method will be found of service when the atmosphere is so heavily charged with moisture as not to admit of the employment of soluble filtering medium as above. The apparatus employed is shown in Fig. 31, and consists of 2 tubes of stout glass of $2 \mathrm{~cm}$. internal diameter closed at the bottom, and joined together, as shown by a horizontal tubulure. The orifices of both tubes are fitted with india-rubber stoppers, through which are passed pipettes, the inferior extremities of which reach almost to the bottom of the tubes, and of which one is graduated in divisions of a c.c. One of the tubes is clearly marked with a line showing the level of 10 c.c. of water, and the superior orifices of the pipettes are lightly plugged with cotton-wool. Into the marked tube, Io c.c. of sterile water (the addition of I c.c. of pure cane sugar can be made if thought desirable) is then placed, and the whole is sterilised in the autoclave at $115^{\circ} \mathrm{C}$. for thirty minutes. When required for use, the graduated pipette in the empty tube is joined up to the aspirating apparatus, the cotton-wool plug of the other pipette removed, and the aspirator set in action. The aspired air bubbles through the sterile water, passing into the first tube, and thence by the hori-

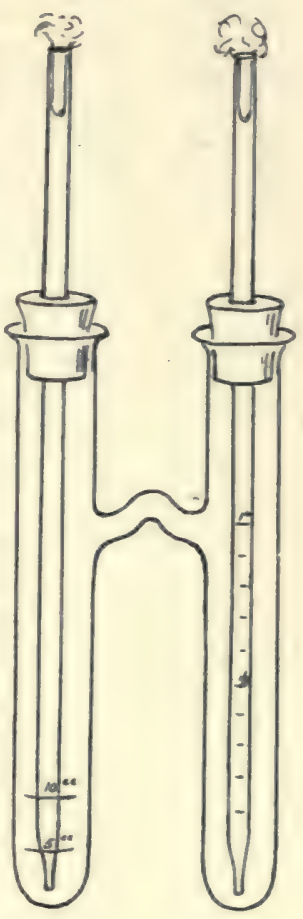

FiG. 31.-Laveran's tube. zontal tubulure into the second, finally escaping by the pipette in connection with the aspirator. When a sufficient quantity of air has been passed through, the cotton-wool plug is replaced, the apparatus detached from the aspirator, and the water gently drawn

${ }^{1}$ Various aspirating pumps are in common use, the particular form used is a matter of personal selection. 
up into the pipette two or three times by aspiration, so as to wash down into the tube any chance organisms adhering to its inner walls. By careful tilting, the water is then caused to flow by the horizontal tubulure into the second tube, washing the tube walls as it passes. The water is then aspired gently two or three times into the gauged pipette, after which any given quantity can be removed for the purpose of plate cultivation. The number of organisms per metre cube of

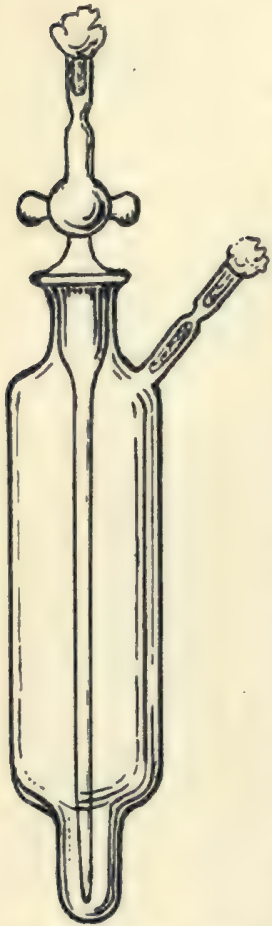

FIG. 32.-Apparatus of Strauss and Wurtz. air is calculated according to the formula given above. If the air taken is at any great distance from where the plate cultivations are to be made, the tubes must be kept in ice during transit in order to avoid "the multiplication of organisms.

The above two methods are those which have given the most satisfactory results in our hands.

\section{Method of Strauss and Wurtz-}

In this method, which has been largely used by Duclaux, the air is drawn through melted nutrient gelatine in the special form of tube shown in Fig. 32.

This consists of a glass cylinder drawn to a neck at the upper extremity, and terminating at the lower with a closed contraction in the tube of about 15 millimetres in diameter. Ground into the neck so as to close it hermetically, is a hollow glass stopper of the form shown. From its inferior surface this stopper is continued down almost to the bottom of the lower constriction in the tube, in the form of a pipette calibrated in divisions of I c.c., while the superior surface is fitted as shown, and furnished with a cottonwool plug. The lateral branch is constricted in the centre, and a plug of glass wool is placed in position on each side of the constriction. The apparatus is then sterilised in the hot air steriliser at I $50^{\circ} \mathrm{C}$. for one hour.

Ten c.c. of liquefied gelatine are placed in the tube with the usual precautions, one drop of sterile oil is dropped on to the surface of this, and the whole is then sterilised, and subsequently allowed to cool. When required for use, the gelatine is melted and 
kept liquid throughout the operation, either by being placed in a beaker of warm water, or more simply by being held in the hand. The lateral tube is connected up with the aspirator, the cottonwool plug removed from the orifice of entry, and the aspirator put in action. The aspired air passes through the central tube and bubbles through the gelatine, leaving behind it the greater proportion of the organisms contained therein. Excessive frothing is prevented by the thin coat of oil on the surface of the gelatine. Such organisms as may pass through the gelatine are either deposited in the tube itself, or caught by the lower glass wool plug in the lateral branch.

As soon as the required quantity of air has been passed through, the apparatus is detached, and by careful aspiration the liquid gelatine is drawn several times up into the pipette tube, in order to wash from its walls any organisms which may have remained in it. The glass wool plug above the constriction in the lateral branch is removed, and by means of a sterile needle or fine glass rod, the one below it is pushed forward and allowed to fall into the melted gelatine. The upper plug is then replaced, and the tube is well rolled in the hand and agitated in order to secure an even distribution of the organisms in the gelatine. Petri dish cultivations are then made in the ordinary manner, by means of the pipette tube, or, if preferred, this latter is withdrawn, and an Esmarck roll culture made upon the inner walls of the larger tube itself.

\section{Miquel's flask method-}

This method has been discarded by its originator in favour of the system of soluble filters. It consists in the aspiration of a given volume of air through 30 c.c. of sterile water or bouillon contained in a special flask. After aspiration the water or bouillon is distributed into a large number of ordinary flasks of sterile bouillon, from 30 to 50 at least. Calculation of the organisms present is made by noting proportion of flasks remaining non-turbid after incubation, it being presumed that each flask in which growth takes place contains but a single germ. The large number of flasks in use for a single operation, and the fact that, however great the fractioning, the possibility always arose that more than one organism would find its way into a certain proportion of the flasks, led to the discontinuance of this method.

\section{Hesse's method-}

In this method a given volume of air is caused to circulate in and pass through a glass tube, the interior walls of which are 
covered with a thin surface of solidified nutrient gelatine. The organisms in suspension in the air fall upon, and adhere to, the surface of the gelatine during the passage of the air through the tube, the subsequent colony growth being clearly visible through its walls. The apparatus consists of a large tube of glass from $50-70 \mathrm{~cm}$. in length, and from $3-5 \mathrm{~cm}$. internal diameter, one end of which is closed by a tightly drawn covering of india-rubber sheeting pierced in the centre by a circular hole of $\mathrm{I} \mathrm{cm}$. in diameter, this again being covered, until the moment of employment, with a second covering of india-rubber securely tied on. The other end of the tube is closed by an india-rubber stopper bored for a length of glass tubing of small calibre, which in use is connected up with the aspirating apparatus. This length of glass tubing should project about $\mathrm{I} \mathrm{cm}$. into the interior of the larger tube and be furnished at its outer orifice with a plug of cotton-wool. The apparatus is then sterilised at $115^{\circ} \mathrm{C}$. for half an hour. Before use the outer india-rubber covering is removed and from $40-50$ c.c. of sterile nutrient gelatine is introduced into the tube by means of a suitable delivery flask. The outer india-rubber covering is then quickly replaced, and the tube itself rolled in the hand upon ice, or under a cold water tap, until the gelatine becomes fixed in a thin layer on its interior walls. When in use the tube is fixed horizontally on a suitable stand, the length of glass tubing connected up with an aspirating apparatus, the outer india-rubber covering at the further end of the tube removed, and a current of air drawn through the tube. The air should pass through slowly, and at a rate not exceeding I litre per three minutes, in order to allow the organisms in suspension to deposit themselves upon the surface of the gelatine. After a sufficient quantity of air has been passed through, the outer india-rubber covering is replaced, the tube detached from the aspirator and the orifice of the exit tube again plugged with cotton-wool. The tube is then placed under suitable conditions for the development of the colonies, which should begin to appear about the second or third day. The method is somewhat uncertain in its results, owing to the fact that a certain proportion of the organisms are apt to pass through the tube without being deposited, or are left adhering to the surface of the india-rubber stopper. The gelatine too is apt to be deposited unevenly upon the tube walls, and when too thin desiccates quickly during incubation and becomes unsuitable for colony growth. 


\section{Sedgwick and Tucker's method-}

Sedgwick employs as a filtering medium finely granulated sugar in the special form of tube illustrated. This consists of a glass tube of about $30 \mathrm{~cm}$. in length, half of which has a bore of $2.5 \mathrm{~cm}$. and the other half a bore of $.5 \mathrm{~cm}$. A plug of glass wool is inserted at $a$ to act as a support to the filtering material, and the tube is then sterilised at $160^{\circ} \mathrm{C}$. for one hour in the hot air steriliser. When cool the narrow portion of the tube is filled, as far down as the glass wool plug and up to its opening into the lumen of the larger tube, with finely granulated cane sugar. Cotton-wool plugs are fitted into the orifices of both tubes and the whole is then again sterilised for two hours at $130^{\circ} \mathrm{C}$. If a higher temperature than this is reached there will be danger of melting the sugar, and forming caramel. Great care should be taken that the sugar is in a thoroughly dry condition before it is inserted into the tube. When the apparatus is required for use, the cotton-wool plug of the smaller tube is removed

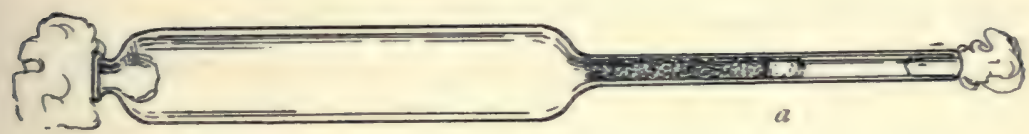

FIG. 33.--Sedgwick's tube.

and the tube itself connected up with the aspirator. The second cotton-wool plug is withdrawn and a measured quantity of air drawn through the tube. The wool plugs are then replaced. On arrival at the laboratory the sugar is shaken down to the wider portion of the tube, or pushed down by means of the central plug of glass wool, and 15 c.c. of melted nutrient gelatine are poured into the larger end, after which the two wool plugs are replaced. The sugar readily dissolves in the melted gelatine, and the tube is then rolled on ice or under a cold water tap in order to fix the gelatine in an even surface round its inner walls. The colonies will appear in two to three days according to the temperature of incubation. Unless very numerous their subsequent enumeration is comparatively easy, but some difficulty is experienced when it is required to pick out the different colonies for examination. Another objection to this method is that the rapid liquefaction of the gelatine under the influence of liquefying bacteria will, at times, render the counting and the isolation of particular organisms a matter of extreme difficulty. 


\section{Petri's method-}

In this method the air is aspirated through a glass tube of about $9 \mathrm{~cm}$. in length and from $\mathbf{I} \cdot 5-\mathbf{I} \cdot 8 \mathrm{~cm}$. internal bore containing sterilised sand, which is kept in position by wads of iron wire gauze. The sand is afterwards distributed into tubes of gelatine, from which plate cultivations are made in the ordinary manner. The presence of the opaque particles of sand in the gelatine renders the accurate enumeration of the minute colonies a matter of extreme difficulty, and is the principal objection to this method.

\section{Frankland's method-}

Frankland uses finely powdered cane sugar and glass wool as a filtering medium. The form of tube employed is shown in Fig. 34. A glass wool plug is inserted at $c$, and a second one at $b$, consisting of glass wool and cane sugar in a finely powdered condition; this again is supported on each side by plugs of glass wool. A cotton-wool plug is inserted at $a$ and the tube is

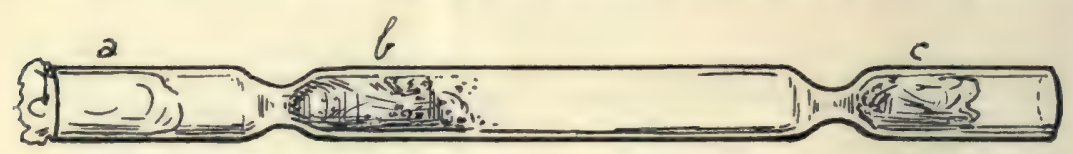

FIG. 34.-Frankland's air tube.

sterilised in the hot air steriliser. When required for use the plug $a$ is removed and the tube connected up by a length of indiarubber tubing to an aspirating apparatus. The plug $c$ is then withdrawn, and the aspirator set in action. After a sufficient quantity of air has been passed through, the tube or tubes are removed to the laboratory, a file mark is made across the centre of each, the tube itself is then broken in half and the plugs of glass wool and powdered sugar are pushed, by means of a sterile needle, into a flask containing Io or 15 c.c. of liquefied gelatine. The sugar dissolves, and the organisms contained in the glass wool are distributed through the gelatine. A roll culture is made on the walls of the flask, or Petri plates are made from the gelatine. The difficulty in distinguishing the young colonies, owing to the opalescence of the medium due to the presence of the glass wool, is a great objection to this method.

\section{BACTERIOLOGICAL EXAMINATION OF WATER}

Collection of samples.-Water from streams or wells should be collected in glass bottles or flasks, closed with glass stoppers, 
previously sterilised or washed out with pure sulphuric acid. When the latter method is adopted, the bottle should be well rinsed with the water which is to be examined before the sample is taken. In taking the sample the bottle should be held well below the surface before the stopper is removed, in order to obtain a sample of the main body of water and not the surface water only. If it is an ordinary water supply through pipes or from a cistern, the tap should be turned on and the water allowed to run for a few minutes before taking the sample : and the same principle applies to a well not in regular use. Such a well should be pumped for a considerable time before taking the sample. For obtaining samples from a considerable depth Miquel's special apparatus may be used, or, if that is not available, a weighted bottle.

After collection the bottle should be at once stoppered, labelled, and packed in ice and sawdust for transport to the laboratory, or placed in one of the various ice cases now in use (Delépine's or Pakes'). Below $5^{\circ} \mathrm{C}$. organisms do not multiply in water, and therefore it is important to keep samples previous to examination at a low temperature. In all cases where it is possible the water should be examined at once after collection.

Physical examination.-The temperature and reaction of the water should first be tested, and an examination made of any deposit or suspended matter. Bubbles of gas, if present, should be noted. The colour, character, and amount of particulate matter in suspension or sediment should be observed and noted. A record of the quantity of the sample, its source, and the date and time of its collection is also important.

Bacteriological examination.-This divides itself naturally into two divisions-( $a$ a quantitative examination, and $(b)$ a qualitative examination.

\section{(a) Quantitative Examination}

The sample should be gently mixed and plate cultivations made. Take five tubes of 1O-15 C.C. of gelatine and five Petri dishes, and melt the medium of the former in a water bath. The gelatine should be well liquefied but not overheated. The Petri dishes should be of even surface, equal size, and properly sterilised. Take a I c.c. sterilised pipette accurately calibrated, and pass it into the bottle, removing the necessary quantities of water. As a rule 0.5 c.c., 0.2 c.c., 0.2 c.c., 0.1 c.c., and 0.1 c.c. are suitable quantities for each of the five plates. Add these quantities to the five tubes of liquefied gelatine, and well mix and pour into the Petri dishes. 
Allow the gelatine to set; and incubate at $22^{\circ} \mathrm{C}$. for as long as possible before complete liquefaction occurs. Count the colonies which appear after forty-eight hours' incubation, take the average at the period of maximum growth, multiply up according to the raction of a c.c. which has been used, and return as so many organisms per cubic centimetre. ${ }^{1}$ It is necessary that each quantity of water from which the fractional part is added to the gelatine should be taken up separately, and not that I c.c. of water should

1 The number of bacteria per c.c. of course varies within wide limits. As an illustration the official returns may be given of the Metropolitan Water Supply for the first six months of 1903 (as reported by Sir W. Crookes and Professor James Dewar) :-

\begin{tabular}{|c|c|c|c|c|c|c|}
\hline \multirow{2}{*}{ SOUROE OF WATER. } & \multicolumn{6}{|c|}{ Micro-organisms per cubic centimetrø. } \\
\hline & $\begin{array}{l}\text { Jsn. } \\
\text { 1908. }\end{array}$ & $\begin{array}{l}\text { Feb. } \\
1903 .\end{array}$ & $\begin{array}{l}\text { March } \\
1903 .\end{array}$ & $\begin{array}{l}\text { April } \\
1908 .\end{array}$ & $\begin{array}{l}\text { May } \\
1908 .\end{array}$ & $\begin{array}{l}\text { June } \\
\text { 1908. }\end{array}$ \\
\hline 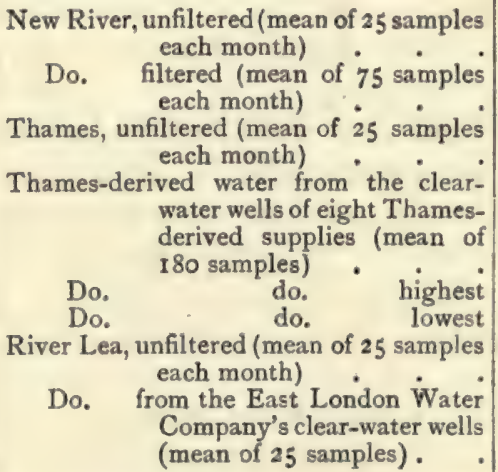 & $\begin{array}{r}69 \\
562 \\
0\end{array}$ & $\begin{array}{r}455 \\
8 \\
15,653\end{array}$ & $\begin{array}{r}27 \\
212 \\
0 \\
582\end{array}$ & $\begin{array}{r}149 \\
6 \\
2,820\end{array}$ & $\begin{array}{r}231 \\
I 1 \\
4,625\end{array}$ & $\begin{array}{r}42 \\
588 \\
0\end{array}$ \\
\hline
\end{tabular}

For January the Water Examiners' report :-

"Of the 298 daily samples taken from the filter wells of the Metropolitan Water Companies and examined bacteriologically by us, 13 samples, or $4 \cdot 3$ per cent., were sterile; 45 samples, or 15.1 per cent., contained more than 100 microbes per c.c. ; and 21 of these samples contained more than 150 microbes per c.c. The mean number of microbes in the 45 samples containing more than 100 microbes was 198 , against a corresponding mean of 177 in 23 samples in December.

"Owing to the great increase in the rainfall during the last month, the microbes in the unfiltered Thames water have risen from about 6,000 to 13,000 , that is, the bacteriological impurity has about doubled, whereas the unfiltered New River water has undergone little or no alteration. The result of this increase has been that the filters of the Thames-derived Companies, which were not working at their best, furnished a larger number of samples from the 
be taken up and the fractional amounts say of $0.5,0.2$, and 0.1 c.c. be added to the gelatine.

\section{(b) Qualitative Examination}

At the time of making the gelatine plates for quantitative examination several agar plates may be made for qualitative purposes. The plates must be poured immediately after inoculation of liquefied agar with small quantities of the water, as below $40^{\circ} \mathrm{C}$. the agar will re-solidify. When poured the agar plates should be placed on cold stone or metal until the medium has hardened and then incubated at blood heat. On the second or third day colonies will have appeared, and these should be studied and subcultured (as pure cultures) on suitable media.

Valuable facts as to the quality of the water may also be obtained from an examination of the five gelatine plates above referred to, particularly in respect of the liquefying organisms, which should be counted as carefully as any other colonies, and noted separately as well as in the total number of colonies present. But in addition to the facts obtained from gelatine and agar plates, other methods must be adopted in order to obtain information respecting the quality of the water.

Take a sterilised Berkefeld filter, and pump or aspirate through it a litre ( 1000 c.c.) of the water under examination, and with a sterilised brush transfer the particulate matter which has

filter wells showing an increase in the number of microbes. In our last Report we pointed out that this would be the inevitable result in the event of an increased rainfall."

A similar report is made monthly and it was found, as the above table shows, that the conditions improved in February, March, and April, but owing to the heavy rains and flood pollution the numbers of bacteria were greatly increased in June. These figures throw an interesting light on the bacteriology of water.-See Monthly Report under the Metropolis Water Act, $187 \mathrm{I}$.

Another illustration may be quoted. Out of thirty-eight streams entering Lake Vyrnwy which were bacteriologically examined only one yielded any $B$. coli; many land drains were practically free from organisms except when receiving pollution from neighbouring houses; the River Severn contained 5000 organisms per c.c. two miles above Shrewsbury, but 23,000 per c.c. three miles below.-Second Report of Royal Commission on Sewage Disposal, 1902, p. 99.

There is no fixed standard as to how many organisms potable water may contain. It is customary to say that waters containing under 100 bacteria per c.c. are potable; but the figure is, in fact, altogether arbitrary, and dependent also upon the kinds of bacteria present. In relation to the milk supply the chief importance of water examination is to detect sewage and surface pollution ( $B$. coli) and not to estimate merely the number of bacteria. 
collected on the candle to 5 or IO c.c. of sterile water. This is now a concentration or emulsion of the organismal content of the litre of water, and may be used for examination for special organisms.

(a) Place 0.5 or I c.c. of the concentrated emulsion in each of three tubes of IO-I 5 C.c. of fresh sterilised milk. Put the three tubes into the water bath for fifteen minutes at $80^{\circ} \mathrm{C}$, and after allowing them to cool place them in a Buchner's tube or cylinder containing pyrogallic solution (Pyrogallic acid, I20 grains, strong liquor potassæ, IO c.c.). Accurately seal up the Buchner, and place it, containing the tubes, in the incubator at $37^{\circ} \mathrm{C}$. The next day, or in thirty-six hours, examine for $B$. enteritidis sporogenes. If that organism is present the following characteristic appearances will be apparent (Klein). The cream of the milk will be torn and altogether dissociated by the development of gas, so that the surface of the medium becomes covered with stringy white masses of coagulated casein enclosing a number of gas bubbles. The main portion of the tube formerly occupied by the milk will contain a colourless, thin, watery whey, with a few lumps of casein adhering here and there to the sides of the tube. If the tube be opened there will be found to be a smell of butyric acid and an acid reaction. If some of the contents of the tube are stained, as slide preparations, the bacilli will be seen.

(b) Take from 0.1 to 0.5 of the concentrated emulsion and add to three tubes of phenolated gelatine and make plates as in the quantitative examination. Colonies developing in these plates should be suspected of being B. coli communis and tested accordingly; or, inoculate from the concentrated water three tubes of Parietti's broth, and incubate at $37^{\circ} \mathrm{C}$. Those tubes which show growth in one to three days should be plated out on ordinary or phenolated gelatine and colonies of $B$. coli examined for. Some authorities recommend incubating Parietti's tubes at $42^{\circ} \mathrm{C}$, a temperature favourable to $B$. coli but unfavourable to ordinary water organisms. Tubes of glucose formate bouillon may also be inoculated with 0.1 to 0.5 c.c. of the concentrated emulsion and incubated in a Buchner's tube at $42^{\circ} \mathrm{C}$. and all the tubes which show turbidity in twenty-four hours may be plated out on gelatine or alkaline glucose litmus agar and the $B$. coli, if present, thus isolated.

Subcultures should be made in gelatine or glucose gelatine (for gas production), milk (for acidity and coagulation), and peptone water (for indol). 
(c) B.typhosus may be examined for by adopting exactly the same methods as for $B$. coli. Its detection in, and isolation from, water supplies is so difficult as to be well-nigh impossible. The condition of a water is, however, ascertainable short of an absolute test for $B$. typhosus, valuable though that would be.

(d) Sewage organisms and the organisms indicative of surface pollution should also be examined for. If they be present in the water, it may be taken as proved that such water has been recently polluted, and should be condemned. Crude sewage generally zontains in I c.c. : (a) I-IO million bacteria; (b) I00,000 B. coli (or closely allied forms); (c) IOO spores of B.enteritidis sporogenes; and (d) 1000 streptococci (Houston). Further, so minute a quantity as $\frac{1}{1000}$ of a c.c. of crude sewage is usually sufficient to produce "gas" in a gelatine "shake" culture in twenty-four hours at $20^{\circ} \mathrm{C}$, and the inoculation of animals with crude sewage always leads to a local reaction and not uncommonly results in death. These three organisms, $B$. coli, $B$. enteritidis sporogenes, and streptococci have been termed the "microbes of indication." These bacteria are wholly, or relatively, absent from pure water, and their presence, at all events in considerable numbers, must be taken as indicating recent animal pollution. ${ }^{1}$. coli is a most accurate measure of intestinal pollution, and far greater information as regards the sewage pollution of water can be gathered by its estimation, than by simply counting the total number of organisms present in water. It is an intestinal parasite and tends to perish in other media. ${ }^{2}$ When it is present in a small stream, contamination from houses can generally be traced. ${ }^{3}$ 27.

1 Second Report of Royal Commission on Sewage Disposal, 1902, pp. 26 and 2 Ibid., p. 99. 3 lbid., p. Iog. 


\section{CHAPTER V}

\section{THE BACTERIAL CONTENT OF MILK}

Numbers of Bacteria present in Milk The Milk of Towns. Natural Conditions affecting Bacteria in Milk: (1) The Influence of Temperature. (2) The Influence of Time. The Toxicity of Milk. (3) The Inter-relationship of Bacteria in Milk and its Germicidal Power. Bacteria in Separated Milk. Species of Bacteria present in Milk.

IT cannot be a surprise to anyone who considers the elementary facts set forth in the first chapter to learn that the numbers of bacteria present in milk reach, even under normal circumstances, a very high figure. There is at present no fixed standard of microbes in milk with which it is possible to compare results. A host of observers have made quantitative investigations in which milk has been obtained, collected for examination, removed, stored, and examined, under widely varying circumstances. And as a result it has been found as a matter of fact that many apparently excellent milks contain more than 50,000 bacteria per c.c. Many town milks contain hundreds of thousands and frequently millions of bacteria per c.c. ${ }^{1}$ Owing, however, to differences of nomenclature, classification, and mode of examination at present existing in various countries, neither qualitative nor quantitative estimations can as yet be of much permanent value. When a common international standard is established, mathematical computations will be of worth. The presence of large numbers of

1 Gernhardt obtained 7,000,000 per c.c.; Watson and Loveland estimated $50,000,000$ to $1,000,000,000$ per c.c. "at the time when the curdling of milk begins"; Schmelck found 2,800,000 per c.c. in Christiania milk; Moore found 7,200 and 8,400 per c.c. in the first milk, and 546 and 504 in the last milk; Schweinitz found 200 organisms per c.c. in pasteurised milk, as many as 50,000 in "sanitary" milk, and Ir5,000 in ordinary city milk; Schultz found 97,000 bacteria per c.c. in fore-milk, 9000 in middle milk, 500 in milk at close of milking; Sedgwick and Batchelder 2,300,000 (in Boston); Russell 35,000 to 275,000 in April milk in Madison, U.S.A., and 380,000 to 2,000,000 during 116 
bacteria indicates a favourable nidus for micro-organisms-the larger the number the more favourable the nidus. And it must always be borne in mind that a fluid-especially a nutritious fluid like milk-which can support life in this way, even though such life be represented only by saprophytic, innocent, or even beneficial and economic bacteria, is a fluid which can at any moment, as we shall see at a later stage, harbour and favour the growth of bacteria possessing a high degree of pathogenic virulence.

As we have pointed out elsewhere, with strict precautions naturally sterile milk may be obtained, and has been repeatedly so obtained by us, in which no bacteria occur. Such naturally sterile milk if carefully sealed remains germ-free for an indefinite period, or at all events, as we found in practice, undergoes no bacterial or fermentative change whatever for two years and is sterile at the end of that period. Such sterile milk is obviously the highest standard. But it is obtained only under exceptional conditions, and the question arises, What is the best practicable standard for everyday dairying ?

An answer to this question may be obtained by a study of the results of examining milk drawn with every reasonable means for insuring cleanliness. On account of the exactness of the record we select from many such experiments one conducted by W. $\mathrm{H}$. Park of New York. ${ }^{1}$ To insure cleanliness Park collected milk under the following conditions:- The long hairs on the udder were clipped; the cows were roughly cleansed and placed in clean barns before milking; the udders were wiped just previous to milking; the hands of the men were washed and dried; the pails used had small (six-inch) openings and were thoroughly cleansed and sterilised by steam before use. The milk was cooled to $45^{\circ} \mathrm{F}$. within one hour after milking and subsequently kept at that temperature. The first six specimens were obtained from individual cows, the last six from mixed milk as it flowed at different times from the cooler.

May and June; Buchanan Young 24,000 in winter, 44,000 in spring, 173,000 in autumn (in Edinburgh.) Most London milks would exceed 500,000 per c.c. (over $4,000,000$ per c.c. has frequently been found), and we ourselves have examined a large number of town and country milks having over 50,000 bacteria (Bitter's Standard). A large number of similar investigations, with similar results, have been made by Clauss and Hohenkamp in Wurzburg market milk; Binwid in Warsaw; Genns in Amsterdam; Renk in Halle milk; Uhl in Giessen; Knochenstiern in Dorpat ; Baumann in Könisburg; Cunningham in India ; and by several investigators in London.

${ }^{1}$ Jour. of Hygiene, 1901 (July), p. 393. 
The temperature of the barns was $55^{\circ} \mathrm{F}$. The results were as follows :-

\begin{tabular}{|c|c|c|c|c|}
\hline & \multicolumn{4}{|c|}{ Number of Bacteria in 1 c.c. of Milk." } \\
\hline & \multicolumn{4}{|c|}{ From six individual Cows. } \\
\hline & $\begin{array}{l}5 \text { hours after } \\
\text { milking. }\end{array}$ & After 24 hours. & After 48 hours. & After 72 hours. \\
\hline \multirow{4}{*}{ Average. } & $\begin{array}{r}500 \\
700 \\
19,900 \\
400 \\
900 \\
13,600\end{array}$ & $\begin{array}{r}700 \\
700 \\
5,200 \\
200 \\
1,600 \\
3,200\end{array}$ & $\begin{array}{r}12,500 \\
29,400 \\
24,200 \\
8,600 \\
12,700 \\
19,500\end{array}$ & $\begin{array}{c}\text { Not counted. } \\
\text { " } \\
n \\
n \\
\text { " } \\
n\end{array}$ \\
\hline & 6,000 & 1,933 & 17,816 & $n$ \\
\hline & \multicolumn{4}{|c|}{ From mixed Milk of entire herd. } \\
\hline & $\begin{array}{l}6,900 \\
6,100 \\
4,100 \\
1,200 \\
6,000 \\
1,700\end{array}$ & $\begin{array}{r}12,000 \\
2,200 \\
700 \\
400 \\
900 \\
400\end{array}$ & $\begin{array}{r}19,800 \\
10,200 \\
7,900 \\
7,100 \\
9,800 \\
8,700\end{array}$ & $\begin{array}{l}494,000 \\
550,000 \\
361,000 \\
355,000 \\
445,000 \\
389,000\end{array}$ \\
\hline Average. & 4,333 & 2,766 & 10,583 & 329,000 \\
\hline
\end{tabular}

* The number of bacteria was obtained from development on agar in Petri plates. The nutrient medium contained 2 per cent. peptone and $I \cdot 2$ per cent. agar, and was faintly alkaline to litmus. One set of plates was usually left four days at $20^{\circ} \mathrm{C}$. and one set forty hours at $37^{\circ} \mathrm{C}$, and then twenty-four hours at $20^{\circ} \mathrm{C}$. From 5 to 30 per cent. more colonies developed as a rule in the plates kept at room temperature than in those kept for twenty-four hours at $37^{\circ} \mathrm{C}$. The milk was diluted as required with 100 or 10,000 parts of sterile water and I c.c. of the diluted milk was added to 8 c.c. of melted nutrient agar.

Park also examined milk taken during winter in well-ventilated, fairly clean, but dusty barns. Visible dirt was cleaned off the hair about the udder ; milkers' hands were wiped but not washed; milk pails and cans were clean, but the straining cloths were dusty. The milk was cooled within two hours after milking to $45^{\circ} \mathrm{F}$. Under these conditions Professor Park found an average of 15,500 bacteria per c.c. at the time of milking, 2I,666 after twenty-four hours, and 76,000 after forty-eight hours. In a third series the conditions were 
these:-The milk was taken in ordinary barns; the ground was covered with manure, and the cows more or less visibly dirty; the teats of the udder were cleansed slightly by running the unwashed hands over them once before milking; and the pails and cans were cleaned but not sterilised. The milk was cooled to $45^{\circ} \mathrm{F}$. within two hours of milking, and the results were as follows :-Average shortly after milking in warm weather 30,366 bacteria per c.c.; after twentyfour hours, 48,000 ; after forty-eight hours, 680,000 . Average shortly after milking in winter weather 16,650 bacteria per c.c.; after twenty-four hours, 3I,000; after forty-eight hours, 2 I0,000.

But even these high figures are at once dwarfed when we come to consider the bacterial content of milk not in any prearranged or academical sense but actually as it is placed on the market.

The researches of Zakherbekoff ${ }^{1}$ into the bacterial content of St Petersburg milk are of interest. An examination of (I) Farm milk, (2) Creamery milk, and (3) Milk at time of delivery from house to house, produced the following results :-

\begin{tabular}{|c|c|c|c|}
\hline & & Minimum per c.c. & Maximum per c.c. \\
\hline $\begin{array}{l}\text { Farm milk } \\
\text { Creamery Milk } \\
\text { Milk delivered at house }\end{array}$ & $: \quad:$ & $\begin{array}{r}450,000 \\
4,100,000 \\
10,200,000\end{array}$ & $\begin{array}{r}9,800,000 \\
1,153,600,000 \\
82,300,000\end{array}$ \\
\hline
\end{tabular}

Comparing average maximum figures of St Petersburg milk with that of other centres he found the milk of

\begin{tabular}{|c|c|c|c|c|c|c|}
\hline Munich & . & 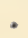 & & to contain & \multicolumn{2}{|c|}{$4,000,000$ per $c_{2} c_{2}$} \\
\hline Wurzburg & . & 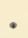 & . & , & $7,335,000$ & $n$ \\
\hline Odessa & . & ${ }^{*}$ & . & , & $29,850,000$ & , \\
\hline Halle. & . & . & . & $n$ & $30,700,000$ & $"$ \\
\hline St Petersburg & . & . & - & $"$ & I I $5,300,000$ & $\eta$ \\
\hline Yourieff-Dorpat & . & . & • & $"$ & II $6,817,200$ & $"$ \\
\hline Giessen & . & . & . & , & $169,032,000$ & " \\
\hline
\end{tabular}

The examination of Naples milk by Dr A. Montefusco ${ }^{2}$ shows interesting results, and is worthy of note owing to the well-known system in vogue of taking round the cows, asses, and goats, and milking them into receptacles provided by the householders at

1 Annales de Micrographie, 1895.

${ }^{2}$ Anali dell Instituto d'igiene sperimentale della $R$. Universita de Rcma, III., P. 539. 
their doors. The result of a quantitative bacteriological analysis was as follows :-

In milk from cows milked at domicile $\quad 5,847$ to 9,524 m.o. per c.c. $" \quad "$ laiterie . 17,716 "3,000,000 " "

The average "time" increase of micro-organisms noted by Montefusco was as follows :-

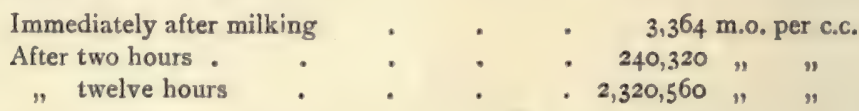

Freudenreich has found on an average in freshly drawn milk in Berne 10,000 to 20,000 bacteria per c.c., while Knopf has found 60,000 to 100,000 in Munich, i.e. 60 to 100 millions to the litre.

Park has investigated the milk supply of New York as delivered and as distributed. ${ }^{1}$ (I) Twenty samples of milk were taken late in March by the Inspectors of the Department of Health of New York City from cans of milk immediately upon their arrival in the city. The temperature of the atmosphere averaged $50^{\circ} \mathrm{F}$. during the previous twenty-four hours. The temperature of the milk when taken from the cans averaged $45^{\circ} \mathrm{F}$. Much of the milk had been carried over two hundred miles. From the time of the removal from the cans, which was at about 2 A.M., until the plating on nutrient agar at IO A.M., the milk was kept at about $45^{\circ} \mathrm{F}$. The results were as follows :-

\begin{tabular}{|c|c|c|c|}
\hline \multicolumn{2}{|c|}{ From New York and Hudson River Railroad. } & \multicolumn{2}{|c|}{ From Harlem Railroad. } \\
\hline No. of Sample. & No. of Bacteria per 1 c.c. & No. of Sample. & No. of Bacteria per I c.c. \\
\hline 50 & $35,200,000$ & 48 & $6,200,000$ \\
\hline 51 & $\mathrm{I} 3,000,000$ & 49 & $2,200,000$ \\
\hline 52 & $2,500,000$ & 50 & $15,000,000$ \\
\hline 53 & $I, 400,000$ & $5 \mathrm{I}$ & 70,000 \\
\hline 54 & 200,000 & 52 & 80,000 \\
\hline 55 & 600,000 & 53 & 320,000 \\
\hline 56 & $2,500,000$ & 54 & $5,000,000$ \\
\hline 57 & 100,000 & 55 & 140,000 \\
\hline 58 & $3,700,000$ & 56 & $25,000,000$ \\
\hline 59 & I 35,000 & 57 & 52,000 \\
\hline \multicolumn{2}{|c|}{ Average' per c.c. . $5,933,500$} & \multicolumn{2}{|c|}{ Average per c.c. . 5,406,200 } \\
\hline
\end{tabular}

(2) Milk as sold in the shops and dairy stores was examined by the same method. A poor district and a "well-to-do" district 1 Jour. of Hygiene roor (July), p. 395. 
were both tested in mid-winter and in September. The results were as follows :-

Number of Bacteria in 1 c.e. of the Milk.

\begin{tabular}{|c|c|c|c|}
\hline $\begin{array}{l}\text { Poor District. } \\
\text { Midwinter. } \\
\text { I3 Samples. }\end{array}$ & $\begin{array}{l}\text { Well-to-do District. } \\
\text { Mid winter. } \\
10 \text { Samples. }\end{array}$ & $\begin{array}{l}\text { Poor District. } \\
\text { September. } \\
5 \text { Samples. }\end{array}$ & $\begin{array}{l}\text { Well-to-do District. } \\
\text { Septem ber. } \\
\text { S Samples. }\end{array}$ \\
\hline $\begin{array}{r}I 10,000 \\
I 40,000 \\
I 40,000 \\
145,000 \\
I 75,000 \\
280,000 \\
320,000 \\
560,000 \\
640,000 \\
1,200,000 \\
6,400,000 \\
5,100,000 \\
10,500,000\end{array}$ & $\begin{array}{r}30,000 \\
30,000 \\
60,000 \\
60,000 \\
70,000 \\
155,000 \\
240,000 \\
560,000 \\
550,000 \\
1,420,000\end{array}$ & $\begin{array}{r}5,600,000 \\
6,100,000 \\
15,910,000 \\
16,320,000 \\
31,888,000\end{array}$ & $\begin{array}{r}80,000 \\
192,000 \\
355,000 \\
480,000 \\
4,200,000\end{array}$ \\
\hline Average . $\quad 1,977,692$ & 327,500 & I 5, I 63,600 & $I, 06 I, 400$ \\
\hline
\end{tabular}

These two tables of figures indicate, in the first place, that much of the milk sold in New York is not of exceptionally good quality when judged by the number of bacteria contained. Some of the returns are very high, and reveal improper handling of the milk. Secondly, the contrast between milk sold in well-to-do districts and that sold in poorer tenement districts is very striking. It may, we think, be safely assumed that the difference is due not to a difference of source so much as to difference of storage immediately preceding the sale. Uncleanly shops, dirty utensils, and unclean handling in the city is the cause of the high numbers of bacteria in such milks, rather than unclean milking at the farm from which the supply was obtained. Obviously, this remark does not apply to the results obtained by examination of the milk on arrival.

We have carried out a number of examinations of London milks, and have frequently found several millions of organisms per cubic centimetre to be present. In a general way it may be said that the features demonstrated by Park as characteristic of milk in New York are true of milk in London. Such milk has been brought from more or less remote parts of England, and has suffered in consequence. In London milk-shops also it must be said that careful protection of the milk from dust and similar contamination is the exception and not the rule. It is unnecessary 
to burden these pages with many tables of the numbers of bacteria in London milks, but we may insert here the results of an examination carried out by us in May 1903. Purchases were made in nine shops, five situated in the borough of Finsbury, which is as near the centre of the metropolis as possible, and four others from shops in the four other central districts. In three cases small poor shops were selected, but in the other six good class shops were sampled. The sample was asked for in the ordinary way, and it was not known that it was being taken for purposes of examination. It was collected in sterilised bottles, and examined within a few minutes of being purchased. Ordinary Petri dishes were used, the medium was gelatine, and the dilution was $\frac{1}{500}$. The technique described on p. 49 was rigidly adopted. The following table gives the results:-

\begin{tabular}{|c|c|c|}
\hline Kind of Milkshop. & $\begin{array}{l}\text { Total No. of } \\
\text { Bacteria } \\
\text { per c.c. }\end{array}$ & $\begin{array}{l}\text { No. of Lique- } \\
\text { fying Bacteria } \\
\text { per c.c. in- } \\
\text { cluded in total. }\end{array}$ \\
\hline Poor class : Finsbury & 340,000 & o \\
\hline Poor class : Finsbury & $3,200,000$ & 45,000 \\
\hline Good class : Finsbury & $\mathbf{I}, 280,000$ & 30,000 \\
\hline Good class: Finsbury & $2,300,000$ & 46,000 \\
\hline Poor class: Finsbury. & $2,700,000$ & \\
\hline Good class : City of London & $4,800,000$ & 120,000 \\
\hline Good class: Islington & $1,600,000$ & 10,000 \\
\hline Good class : Holborn . & $4,800,000$ & $145, \infty 00$ \\
\hline Good class : City of Westminster & $1,600,000$ & 125,000 \\
\hline
\end{tabular}

* These plates liquefied too rapidly for correct enumeration.

It should be stated that the samples above recorded were obtained in a haphazard manner, and do not necessarily represent the usual quality of the milk in these several districts. Obviously, a very large number of examinations would be necessary to form any opinion upon the comparative condition of the milk of different districts, and other conditions would also have to be considered. The table must be taken for what it represents, namely, the bacterial content of nine milks purchased by chance in nine London shops. The figures were checked by three counters.

It is, however, necessary to comment upon the first sample recorded in the table. This was obtained in a poor class shop in Finsbury, in which no precautions are taken to protect the milk from dust, and yet the returns yielded are the lowest of the series, namely, 340,000 bacteria per c.c., and none of which were liquefying organisms. We therefore suspected the addition of antiseptics. By accident, antiseptics had not been estimated at the 
time of the bacterial examination. A chemical examination (including detection of antiseptics) should always be adopted in examining town milks. To prove the point in this particular case, we therefore purchased twelve samples on twelve different occasions during the next few days, to discover if this vendor used preservatives. The results obtained by Mr Kear Colwell, F.I.C., showed that in eight samples out of twelve formalin was present, approximately to the amount of $I$ in I0,000 parts. In only three cases was the milk normal and genuine. The returns are interesting as showing the result of sampling a poor shop, consecutively, and we append them as a note. 1

Many similar investigations with very similar results might be quoted, but the above will suffice to convey an impression of the bacterial content of many milks. It is, of course, needless to add that quantitative records, whether represented by high or low figures, are in no sense an exact index as to the injurious nature or otherwise of the milk in question, or as to its value for human consumption. A knowledge of the exact quality of the milkof the kind of organisms and their rôle-is necessary before any valid conclusions can be drawn. These matters we treat of elsewhere. But it is desirable here to discuss the conditions influencing both the quantity and quality of milk bacteria. Before doing so, however, we may briefly summarise the answer to the question concerning the practical standard of the number of bacteria in milk. Bitter has suggested 50,000 organisms per c.c. as a suitable standard. The Philadelphia and New York Milk Commissions lay down 30,000 organisms as the standard for

\begin{tabular}{|c|c|c|c|c|c|c|c|}
\hline & $\begin{array}{l}\text { Date of } \\
\text { Purchase of } \\
\text { Sample. }\end{array}$ & $\begin{array}{l}\text { Specifle } \\
\text { gravity. }\end{array}$ & $\begin{array}{l}\text { Total } \\
\text { Solids. }\end{array}$ & Fat. & $\begin{array}{l}\text { Non- } \\
\text { fatty } \\
\text { Solids. }\end{array}$ & Preservative. & Remarks. \\
\hline i & 26th May 'o3 & $1029 \cdot 5$ & $12 \cdot 46$ & $3 \cdot 32$ & $9 \cdot 14$ & Not found & Genuine. \\
\hline 2 & 27 th $"$ & 1029 & 10.46 & $2 \cdot 79$ & $7 \cdot 67$ & Not found & Added water $9.8 \%$ \\
\hline 3 & 29 th & IO $28 \cdot 5$ & 10.75 & $2 \cdot 70$ & 8.05 & Not found & $\left\{\begin{array}{l}\text { Added water } 5 \cdot 3 \% \text {. } \\
\text { Fat remored } 5 \%\end{array}\right.$ \\
\hline 4 & 3rd June 'o 3 & 1032 & I $1 \cdot 28$ & 2.60 & 8.68 & Formalin & Fat removed $13.3 \%$. \\
\hline j & 4 ih & $1024 \cdot 5$ & $14 \cdot 65$ & 7.00 & $7 \cdot 65$ & Formalin & Abnormally high fat. \\
\hline 6 & 4th & 1028 & 10.75 & $3-\infty$ & $7 \cdot 75$ & Not found & Added water $8.9 \%$ \\
\hline 7 & 5 th & 1027 & I $3 \cdot 38$ & $5 \cdot 40$ & $7 \cdot 98$ & Formalin & Abnormal fat. \\
\hline 8 & gth & 1029 & $11 \cdot 60$ & $3 \cdot 50$ & 8.10 & Formalin & Added water $4.6 \%$. \\
\hline 9 & gth & $1029 \cdot 5$ & I I -6 I & $3 \cdot 40$ & $8 \cdot 21$ & Formalin & Added water $3.5 \%$ \\
\hline Io & roth & I03I & 12.00 & $3 \cdot 20$ & 8.80 & Formalin & Genuine. \\
\hline II & Ioth & 1029 & 11.98 & $3 \cdot 60$ & $8 \cdot 38$ & Formalin & Genuine; poor quality. \\
\hline 12 & I Ith & 1030.5 & II $\cdot 25$ & 2.90 & $8 \cdot 35$ & Formalin & $\left\{\begin{array}{l}\text { probably fat removed } \\
\text { and water added. }\end{array}\right.$ \\
\hline
\end{tabular}


certified milk. We admit the reasonableness, on the whole, of these figures, although we should not necessarily condemn milk which contained a larger number. The fact is that numerical estimation of organisms is not, by itself, a sufficient criterion. All the circumstances must be taken into consideration, including the condition of the farms, the presence of preservatives, and the species of the bacteria.

There are three chief special natural conditions affecting bacteria present in milk, which call for careful consideration :-

I. The influence of temperature.

2. The influence of time.

3. The inter-relationship of different species of bacteria, and the germicidal effect of the milk.

\section{The Influence of Temperature}

It is now well known that the vitality of bacteria depends upon the fulfilment of a number of external physical conditionsmedium, moisture, oxygen, temperature, etc. One of the chief among these is temperature, and in few media, if any, does it exert a more powerful or more permanent influence than in the case of milk.

Every species of micro-organism has, generally speaking, its three degrees of temperature: (a) The minimum temperature, varying as a rule from $10^{\circ}$ to $14^{\circ} \mathrm{C}$., though under exceptional circumstances growth may take place in some species at a very much lower temperature. (b) The optimum temperature, usually about the temperature of the natural habitat of the micro-organism in question. Ordinary saprophytes-those able to live upon dead organic matter-engaged in putrefactive functions flourish between $18^{\circ}$ and $24^{\circ} \mathrm{C}$. ; whilst the parasitic, living in the blood or body of

${ }^{1}$ Within recent years various workers have shown that although multiplication practically ceases at zero, the vitality of micro-organisms is maintained at much lower temperatures. For example, MacFadyen and Rowland have made experiments with organisms possessing varying degrees of resistance, ten organisms altogether being used and cooled down to $-190^{\circ} \mathrm{C}$., in the first instance for twenty hours, and eventually for seven days. These exposures did not produce any appreciable impairment in the vitality of the organisms, either as regards their growth or their characteristic physiological properties, such as pigment and gas production, pathogenicity, etc. Amongst the organisms tested were photogenic bacteria, and these likewise preserved their normal luminous properties. An exposure to the temperature of liquid hydrogen (about $-252^{\circ} \mathrm{C}$.), a temperature which was as far removed from that of liquid air as was that of liquid air from the average summer temperature, for ten hours had 
a living host, thrive best at or about blood heat, namely $36^{\circ}$ to $39^{\circ} \mathrm{C}$. (c) The temperature of the thermal death point varies considerably according to species, but lies not uncommonly between $52^{\circ}$ and $58^{\circ} \mathrm{C}$. Yet some species will flourish at $70^{\circ} \mathrm{C}$, and an exception to these figures is of course found in the thermophylic bacteria, which have been isolated from the intestinal tract, excreta, or sewage, and which do not flourish below $60^{\circ} \mathrm{C}$. It should be understood that while growth and multiplication do not take place as a general rule below or above the standards mentioned, it by no means always follows that death will take place immediately outside those limits. Organisms can withstand cold and heat beyond and below the minimum, or beyond the maximum, without being actually killed, although their virulence and biological characters may be modified. As is well known, many of the non-thermophilic spore-bearing bacilli occurring more or less frequently in milk can withstand a temperature of $80^{\circ} \mathrm{C}$. for as long as fifteen minutes (which would kill all non-spore-bearing bacilli) without being killed, e.g. Bacillus enteritidis sporogenes (Klein). Now whilst it is true that this resistance is a resistance of the spore-which by its concentrated protoplasm and more resistant capsule is of tougher substance than the adult bacillus-it must not be forgotten that it has a practical issue of the utmost importance. For, as Koch has stated, the standard of sterilisation should always be the highest resistant power possible of either bacillus or spore. Hence milk is only sterilised by heat when the heat is of such a degree that the most resistant spores succumb.

Following these general remarks, we may briefly consider the effect of temperature on bacteria in milk as shown by a large number of researches. The majority of bacteria met with in milk grow best at about $25^{\circ} \mathrm{C}$, but they will multiply at a temperature as low as $4^{\circ} \mathrm{C}$.

no appreciable effect on the vitality of the micro-organisms tested. At such temperatures it must be assumed that the chemical metabolism of the cell ceases, in the absence of heat and moisture. Bacteria were suspended in small loops of platinum wire or on cotton-wool swabs, and directly immersed in liquid air. The yeast, washed and pressed, was wrapped in rice-paper, and likewise directly immersed in the liquid air. Samples were taken and tested at intervals for a total period of six months. In no instance could any impairment of the vitality of the organisms be detected. Judging by the results, the experiments might have been prolonged for a much longer period than six months without appreciable influence on the vitality of the organisms in question. A further note on our own work in relation to this subject will be found in a subsequent chapter dealing with the tubercle bacillus. All such experiment is, of course, of a tentative character only. 
Freudenreich found that bacteria in a sample of milk increased in the following ratios when kept at the following temperatures:-

\begin{tabular}{|c|c|c|c|c|}
\hline & 3 hours. & 6 hours. & 9 hours. & 24 hours. \\
\hline & & & & \\
\hline $15^{\circ} \mathrm{C}$. & 2 & $18 \cdot 5$ & 5 & 163 \\
$25^{\circ} \mathrm{C}$. & 4 & 1290 & 107 & 62,100 \\
$35^{\circ} \mathrm{C}$. & & 3800 & 5,370 \\
\hline
\end{tabular}

If this table is read from above downwards, we obtain results which reveal the effect of temperature. If it be read across, from left to right, we obtain the effect of time upon bacterial multiplication. In the case of the temperature influence we see clearly the very small multiplication at $15^{\circ} \mathrm{C}$, and the excessive increase at blood heat. This is the simple but fundamental point to be borne in mind by the dairy bacteriologist. To refrigerate milk as soon as it is drawn from the cow is at once to check the increase of bacteria. Obviously, such treatment is but one step short of radi$\mathrm{cal}$, and in cases where the dairying from beginning to end is conducted with strict cleanliness, and the milk is derived from healthy cows, such refrigeration is ample protection. But where these conditions are not fulfilled, the only safe course is in sterilisation or pasteurisation of the milk.

Another series of results from the same worker will illustrate much the same point:-

Samples of milk which shortly after milking contained 23,000 m.o. per c.c. were kept at $25^{\circ} \mathrm{C}$. and $35^{\circ} \mathrm{C}$. respectively, with the following results :-

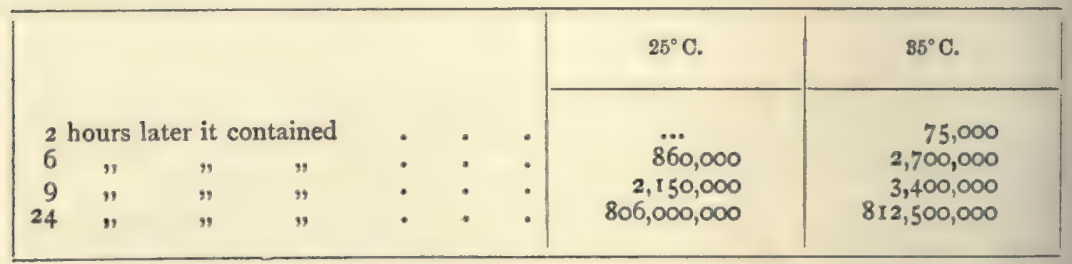

We ourselves have carried out a number of experiments which have invariably borne out the same principle as that enunciated, namely, that the warmer the milk up to blood heat the more marked is the increase of bacteria up to a certain point. As our results have relation to the effect of time as well as temperature, they will be considered at a later stage. 


\section{The Influence of Time}

Having considered the effect of temperature upon microorganisms in milk, we may now direct our attention to what we have, for the sake of convenience, termed the "influence of time." It is, of course, obvious that under this title it is intended briefly to summarise the changes which occur in milk during the period which passes between its withdrawal from the cow, and its domestic consumption.

As we have pointed out already, milk is a favourable medium for organisms, and it will therefore be understood that finding themselves in this suitable nidus, they commence rapid multiplication. Hence, in the course of a few hours after milking, the milk remaining at an even temperature (namely, the temperature of the surrounding air), there are vastly more bacteria in it, bulk for bulk, than was the case at the actual time of milking; and when such milk, though naturally sterile, reaches the consumer, it contains many thousands of bacteria in every cubic centimetre. The following facts fully illustrate this. A sample of Berne milk which shortly after milking contained 9000 bacteria per c.c. was kept by Freudenreich at an even temperature of $15^{\circ} \mathrm{C}$. It was regularly examined during the next twenty-four hours with the following result:-

I hour after milking it contained .

- 31,750 bacteria per c.c.

2

4

7

9

25

Freudenreich gives another example which resulted in the following figures:-

Milk drawn at $15.5^{\circ} \mathrm{C}$.

After 4 hours at the same temperature

$\begin{array}{crcc}\text { contained } & 27,000 \\ n & 34,000 & \text { bacteria per c.c. } \\ " & 100,000 & n & n \\ " & 4,000,000 & & n\end{array}$

Conn furnishes an example of milk yielding the following multiplication results :-

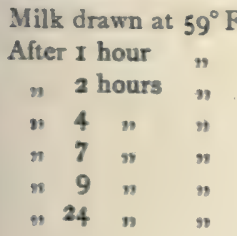

\begin{tabular}{|c|c|c|c|c|}
\hline & - & 153,000 & teri & ub. inch. \\
\hline ? & 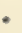 & 616,000 & " & $n$ \\
\hline & * & 539,000 & $"$ & $n$ \\
\hline & - & 680,000 & $"$ & $n$ \\
\hline & - & - $1,020,000$ & $n$ & $n$ \\
\hline & 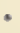 & - $2,040,000$ & $n$ & " \\
\hline & * & . $85, \infty \infty 0, \infty \infty$ & " & " \\
\hline
\end{tabular}


Quite recently further investigations have been made in milk maintained at a standard temperature by various workers. For the sake of comparison with other statistics which we have selected, we may take two series recorded by Park. In the first the temperature was $90^{\circ} \mathrm{F}$, a temperature common in New York in hot summer weather, and the samples of milk were of three degrees of quality, namely, fresh and good, fair, and bad. The result was as follows :-

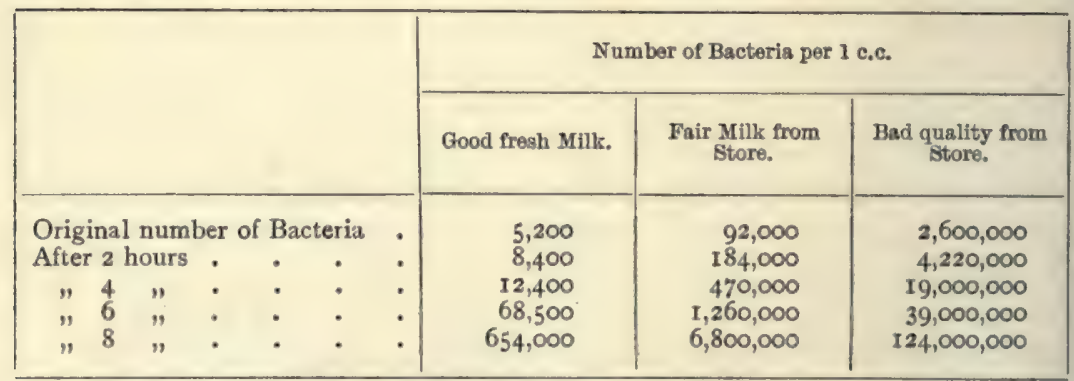

The second series of Park was milk taken from cows in common dirty stalls, twenty-four, thirty-six, and forty-eight hours after milking. The milk was cooled to $52^{\circ} \mathrm{F}$, three hours after milking, and maintained at that temperature for the forty-eight hours of the experiment. The result, therefore, shows the effect of time even more exactly than the first series :-

\begin{tabular}{|l|c|c|c|}
\hline \multicolumn{3}{|c|}{ Average Number of Bacteria per 1 c.c. of Milk at $52^{\circ}$ F. (six samples). } \\
\hline After 3 hours. & After 24 hours. & After 36 hours. * & After 48 hours. \\
30,366 & 69,433 & 348,833 & $\mathbf{1}, 668,333$ \\
\hline
\end{tabular}

* The figures at thirty-six hours were estimated from the test of one sample only.

Even a cursory examination of the figures already given will have shown how intimately the two influences of time and temperature act and interact in relation to the multiplication of microorganisms in milk. They are scarcely separable, and no hard-andfast line can be drawn by way of comparison of these two influences.

We must now, therefore, discuss the numerical changes occurring in the bacterial content of milk in as broad a way as possible. For this purpose reference will be made to two investigations carried out, one by Park of New York, and the other by ourselves 
in 1900. Park's record was published in 1901.1 Our investigation has not hitherto been published.

The following figures obtained by Park show the development of bacteria in two samples of milk maintained at different temperatures for twenty-four, forty-eight, and ninety-six hours respectively. The first sample was obtained under the best conditions possible, the second in the usual way (the figures of this sample are underlined). When received, Specimen No. I contained 3000 bacteria per c.c., and Specimen No. 2, 30,000 per c.c.

\begin{tabular}{|c|c|c|c|c|}
\hline \multirow{2}{*}{ Temperature. } & \multicolumn{4}{|c|}{ Time which elapeed before making the test. } \\
\hline & 24 hours. & 48 hours. & 96 houns. & 168 bours. \\
\hline \multirow[t]{2}{*}{$32^{\circ} \mathrm{F} .\left(0^{\circ} \mathrm{C}.\right)$} & 2,400 & 2,100 & 1,850 & 1,400 \\
\hline & 30,000 & 27,000 & 24.000 & 19.000 \\
\hline \multirow[t]{2}{*}{$39^{\circ} \mathrm{F} .\left(4^{\circ} \mathrm{C}.\right)$} & 2.500 & 3.600 & $2 \overline{18.000}$ & $4 \longdiv { 2 0 0 . 0 0 0 }$ \\
\hline & $\frac{38,000}{2600}$ & $\frac{56,000}{2600}$ & $\frac{4.300,000}{500000}$ & $38.000,000$ \\
\hline $42^{\circ} \mathrm{F} \cdot\left(5 \cdot 5^{\circ} \mathrm{C}.\right)$ & $\begin{array}{r}2,600 \\
43.000\end{array}$ & $\begin{array}{r}3,600 \\
210.000\end{array}$ & $\begin{array}{r}500,000 \\
5.760,000\end{array}$ & \\
\hline \multirow[t]{2}{*}{$46^{\circ} \mathrm{F} .\left(6^{\circ} \mathrm{C}.\right)$} & 3,100 & $\overline{12,000}$ & $I, 480,000$ & \\
\hline & 42,000 & 360,000 & $12,200,000$ & \\
\hline $50^{\circ} \mathrm{F} .\left(10^{\circ} \mathrm{C}.\right)$ & $\begin{array}{l}11,600 \\
89,000\end{array}$ & $\begin{array}{r}540,000 \\
1,940,000\end{array}$ & & \\
\hline $55^{\circ} \mathrm{F} .\left(13^{\circ} \mathrm{C}.\right)$ & $\overline{18,800}$ & $\overline{3,400,000}$ & & \\
\hline \multirow{2}{*}{$60^{\circ} \mathrm{F} .\left(16^{\circ} \mathrm{C}.\right)$} & $\frac{187,000}{180,000}$ & $\frac{38,000,000}{28,000,000}$ & & \\
\hline & 900.000 & $168,000,000$ & & \\
\hline $68^{\circ} \mathrm{F} .\left(20^{\circ} \mathrm{C}.\right)$ & $\begin{array}{r}450,000 \\
4.000,000\end{array}$ & $\begin{array}{l}25, \overline{000,000,000} \\
25,000,000,000\end{array}$ & & \\
\hline $86^{\circ} \mathrm{F} \cdot\left(30^{\circ} \mathrm{C}.\right)$ & $1,400,0000,000$ & & & \\
\hline \multirow[t]{2}{*}{$94^{\circ} \mathrm{F} \cdot\left(35^{\circ} \mathrm{C}.\right)$} & $\frac{14,000,000,000}{25,000,000,000}$ & & & \\
\hline & $\underline{25.000,000,000}$ & & & \\
\hline
\end{tabular}

We have selected this experiment of Park for comparative purposes on account of its fulness of detail, although it differs in important particulars from our own results which were arrived at two years previously. We have, for convenience, inserted the temperatures in degrees centigrade. The table should, of course, be read downwards to observe the effect of temperature, and across for the effect of time. The cultures were made on agar in Petri dishes. ${ }^{2}$

There are two points upon which we may remark. First, it may be noted that at $32^{\circ} \mathrm{F}$. $\left(0^{\circ} \mathrm{C}\right.$.) there is a decline in the number

1 Jour. of Hygiene, Igor (July), p. 398.

2 The agar used by Park contained 2 per cent. peptone, and I. 2 per cent. agar, and was faintly alkaline to litmus. 
of organisms both in good and bad milk during the first I 68 hours. At all the other temperatures, to which there is no exception, there is a rise in the number of organisms. Secondly, the numbers of bacteria at $20^{\circ} \mathrm{C}$. in forty-eight hours are equal to the numbers at $35^{\circ} \mathrm{C}$. in twenty-four hours, and in both instances the number is phenomenally high.

Our own examinations were made many times over, and involved a considerable amount of labour. The results seemed of such exceptional interest that the work was repeated on several occasions. Briefly, it appears that the differences between our work and Professor Park's is that we used I milk (instead of 2); that its initial number of bacteria was 812,000 per c.c. (instead of 3000 and 30,000 per c.c.) ; that we worked at 3 temperatures only (instead of I0); that we carried out an examination at 18 periods (instead of 4); and that in our experiment the organisms were counted up to thirty days in regular sequence, and then at the end of two years. The cultures were made in Petri dishes, but on gelatine instead of agar. Other particulars will be found in the Notes following the table.

The results were as follows:-

Number of Bacteria AT Commencement of EXPERIMent, 812,000.

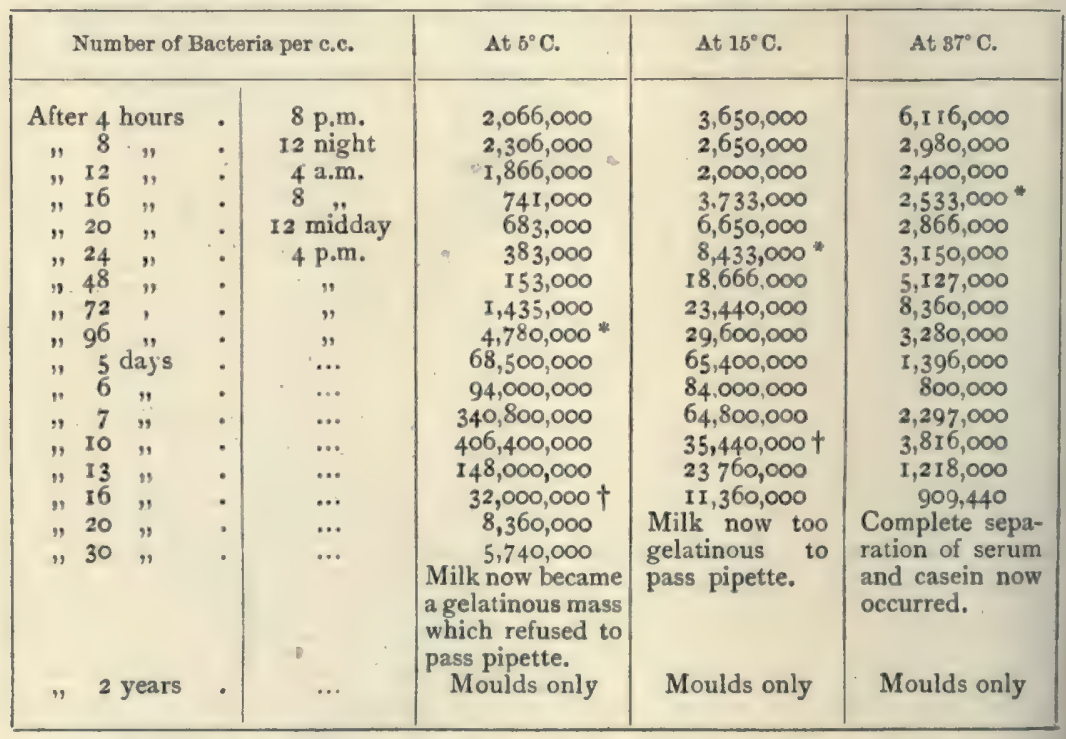

* Milk turned distinctly sour.

+ At this stage the Oidium lactis, which had made its appearance on the plates only rarely before, began to grow in profusion at the expense of other organisms, which became slower in development. 
TIPICAL MII.K PLATES.

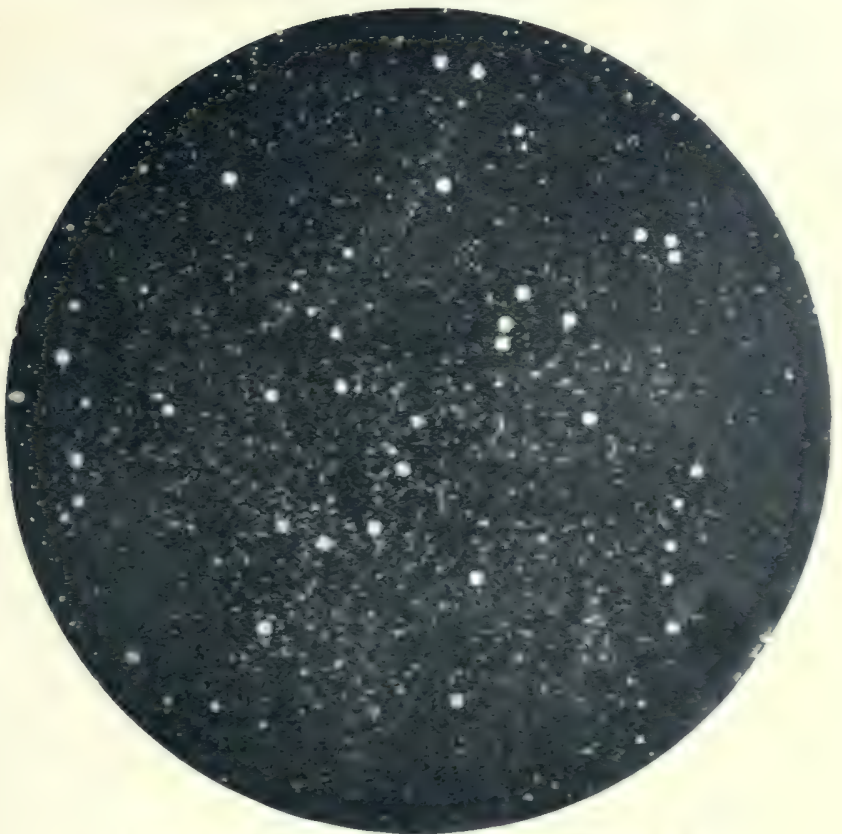

u. os c.e. uf a I in 50 dilutum ufter 24 hours at $15 \mathrm{C}$.

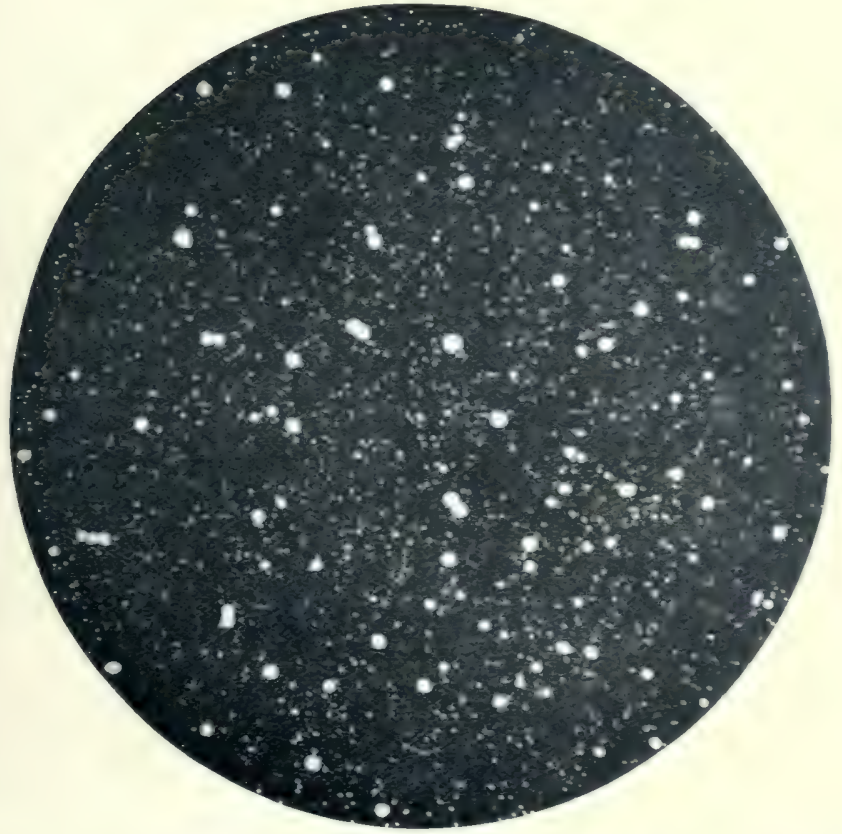

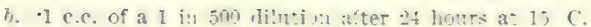



TYPICAL MILK PLATES.

PLATE 15.

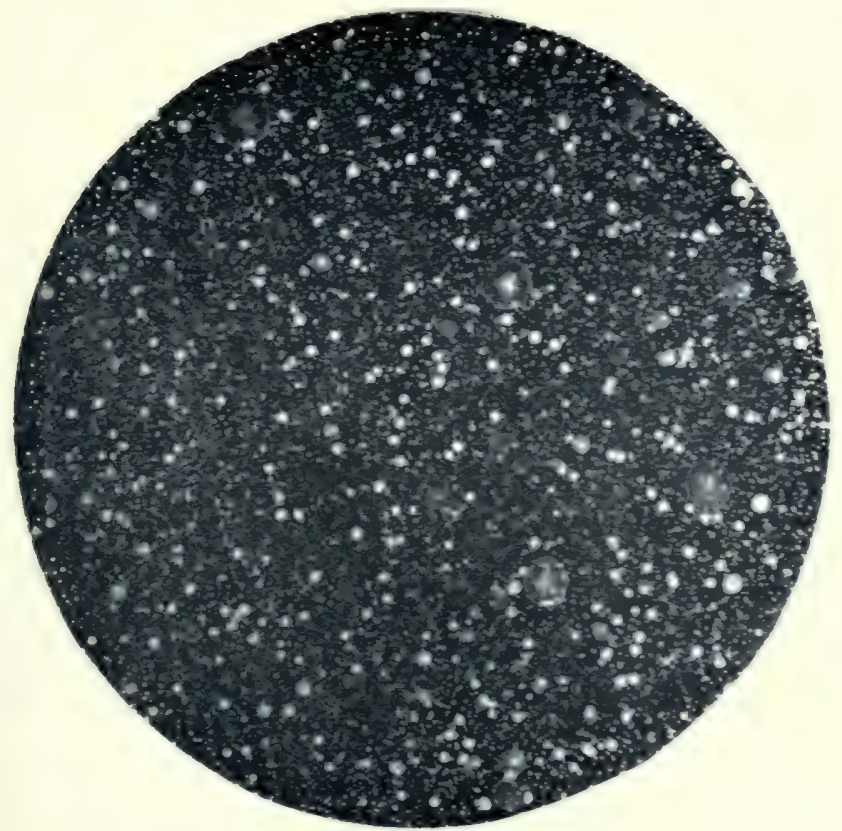

e. $\cdot 05$ c.c. of a I in 1000 dilution after 120 bours at $15 \mathrm{C}$.

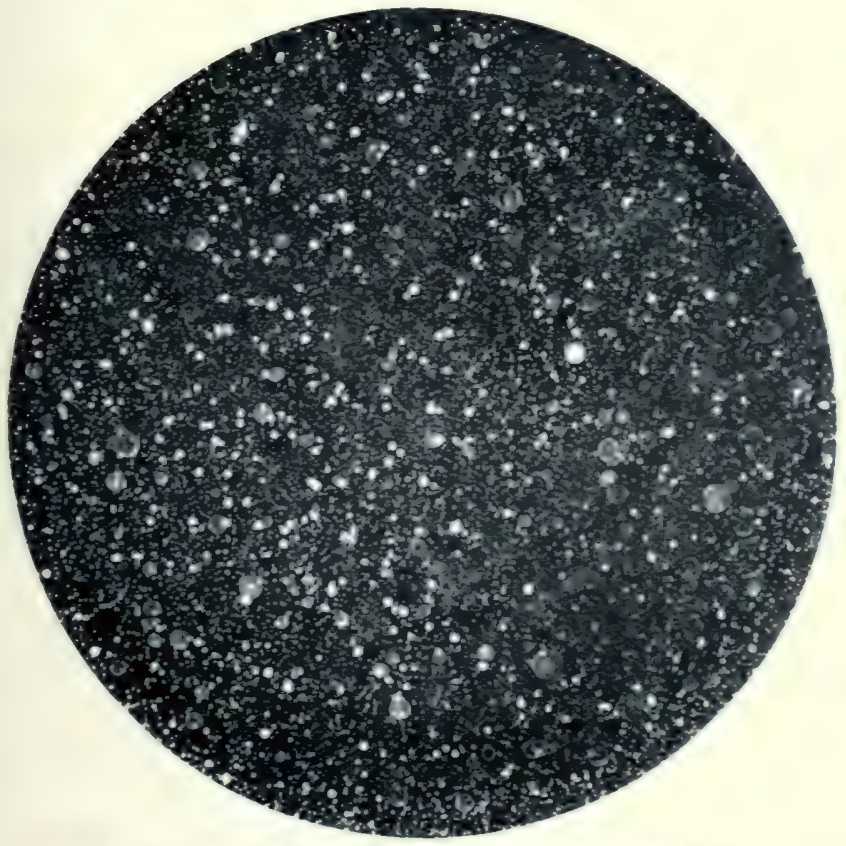

(1. - 1 c.c. of a 1 in 5000 dilution after 168 hours at 5 (c 

Notes.-(1) The experiments began in April rgoo at Denham.

(2) The milking was carried out in the usual way, without notice or warning to the milkmen. The cowshed was an ordinary country byre, and no precautions of any kind were taken, nor was the milk when drawn refrigerated or treated in any way. The temperature of the milk at the time of milking was $34^{\circ} \mathrm{C}$. Samples of milk were taken from the pail containing the mixed milk of three cows during the process of milking. The samples were received into sterilised flasks, and conveyed to the laboratory and incubated at $5^{\circ} \mathrm{C}_{\text {, , at }} 15^{\circ} \mathrm{C}$, and at $37^{\circ} \mathrm{C}$. These temperatures were chosen as representing in a general way a cold storage cellar, an ordinary dairy room temperature, and blood heat. The three temperatures were uniformly maintained throughout the experiment.

(3) Four Petri gelatine plates were made at once from the common stock of milk in the milk pail from which the samples had been taken. The average number of organisms found, which furnished the initial bacterial standard of the milk under observation, was 812,000 per c.c.

(4) The succeeding plates were made from a dilution in sterile water from each of the three samples $\left(\right.$ at $5^{\circ}, 15^{\circ}$, and $\left.37^{\circ}\right)$ of one in five hundred $\left(3{ }^{\circ} \sigma\right)$. These dilutions were maje with great care and absolute accuracy (see p. 48). Of the dilution, we took .05 of a c.c. ( $=\mathbf{I}$ drop from the specially calibrated pipette), -1 c.c. $(=2$ drops), and $\cdot 2$ c.c. (=4 drops), and added these amounts to tubes of melted gelatine, which were thoroughly mixed and poured into Petri dishes. Thus, for example, at eight o'clock one of the samples of milk which had been incubated for exactly four hours at $5^{\circ} \mathrm{C}$. was taken, a portion was diluted to $I$ in 500 , and three gelatine plates were made $(.05$ c.c.n. - I c.c., $\cdot 2$ c.c.) and incubated at $22^{\circ} \mathrm{C}$. The same was done with samples of milk at each of the three temperatures every four hours, as the table indicates. Upwards of 200 Petri plates were made during this experiment.

(5) The gelatine plates were in all cases incubated at $22^{\circ} \mathrm{C}$, and counted on the third anj fourth days. All countings were checked several times.

(6) On the fifth and sixth day of the experiment it was found necessary to increase the dilutions from $\mathrm{I}$ in 500 to $\mathrm{I}$ in $\mathrm{r} 000$ for the samples at $15^{\circ} \mathrm{C}$. and $5^{\circ} \mathrm{C}$. respectively. On the seventh day the dilution was increased for both samples to $\mathbf{I}$ in 5000 . In all respects the technique remained precisely the same throughout the experiment.

(7) On the thirteenth day the fall in the number of organisms in the sample maintained at $5^{\circ} \mathrm{C}$. was so marked that an interim analysis was made twenty-four hours afterwards (i.s. after fourteen days) as a check, and this analysis yielded $84,800,000$ bacteria per c.c., which showed at once that the fall was correctly estimated, and was but one of the steps in a uniformly declining gradation.

(8) The nutrient gelatine used throughout the experiment was made as follows: one pound and a quarter of lean beef was minced and macerated in one litre of water for twelve hours, and strained through sieve and muslin. The resultant fluid was made up to one litre with distilled water, put into a saucepan, and slowly brought up to boiling point. It was then filtered through damp filter paper. Five grammes of common salt $(\mathrm{NaCl})$ and 10 grammes of dry peptone were added. The peptone was rubbed down in a mortar with a small quantity of bouillon before being added to the bulk. The broth was then placed on the stove and stirred until the peptone had completely dissolved. The gelatine (120 grammes) was then added slowly and stirred constantly until fully dissolved. The medium was alkalinised with normal caustic soda, and made faintly alkaline to litmus, and cooled down to $60^{\circ} \mathrm{C}$. The white of one egg was well mixed with 60 c.c. of distilled water, and added. The gelatine was then made up to one litre with distilled water, and steamed at $110^{\circ} \mathrm{C}$. in Koch's steam steriliser for one hour. It was then filtered hot and run into sterilised test tubes. The tubes as finished were sterilised on three successive days at $100^{\circ} \mathrm{C}$. for thirty minutes each day. ${ }^{1}$

1 We bave discarded the above method of preparing nutrient gelatine since 1900 see P. 37). 
We submit this table with all the more confidence in that we had occasion to repeat several portions of it subsequently, and although the figures, of course, differed materially, the same kind of changes were invariably exhibited. It will be understood that the milk was ordinary country milk, and offered no complications or any special sources of contamination. We do not suggest that great emphasis can always be laid upon experimental records of this nature, and it would be undesirable to draw, from such data, conclusions of a far-reaching nature. There are, however, one or two points to which attention may be drawn, and which, we venture to think, have a somewhat wide application in the study of milk.

First, it will be noted that there was an extremely rapid increase in organisms in the first four hours, particularly at $37^{\circ} \mathrm{C}$. A slight fallacy occurs (as regards $5^{\circ}$ and $15^{\circ}$ ) respecting this point, in that the milk would not fall immediately from $34^{\circ} \mathrm{C}$, which temperature it was at the time of milking, to the two standards of $5^{\circ}$ and $15^{\circ}$; and hence in all probability it should be assumed that the increase from 812,000 to $2,066,000$ in four hours at $5^{\circ}$ is exceptional.

Secondly, speaking in a general way, the following great principle appears to emerge, namely, that there is at each temperature $(a)$ a sudden rise, (b) a sudden fall, (c) a steady rise to maximum, and $(d)$ a steady fall ultimately to sterility. In other words, there are tides of organisms, and we have found this to occur invariably in our study of "natural" milks. We do not suggest that it is an invariable phenomenon in all milks, but rather that it is the rule in respect to "natural" milk examined immediately after milking. It is obvious that if we had commenced our examination, as is frequently the case in the study of town milks, twelve or twenty hours after milking, we should, even if we had obtained the same figures, have drawn very different deductions, because the initial rise and initial fall would have been lost sight of.

Thirdly, it will be seen that the maximum number of bacteria occur in seventy-two hours at $37^{\circ} \mathrm{C}$., six days at $15^{\wedge} \mathrm{C}$., and ten days at $5^{\circ} \mathrm{C}$, and that the maximum is lowest at blood heat and highest at $5^{\circ} \mathrm{C}$. It is evident, therefore, that what occurs in a short time at a high temperature occurs in a longer period at a low temperature, but at a low temperature the bacteria eventually become most numerous. These facts are of great importance in relation to the time which milk is kept before use, and to the injurious properties which it may acquire during such a period in the direction of increased bacterial toxin production.

Fourthly, marked acidity commenced between the twelfth and 
PLATE 16.

TYPICAL MILK PI.ATES.

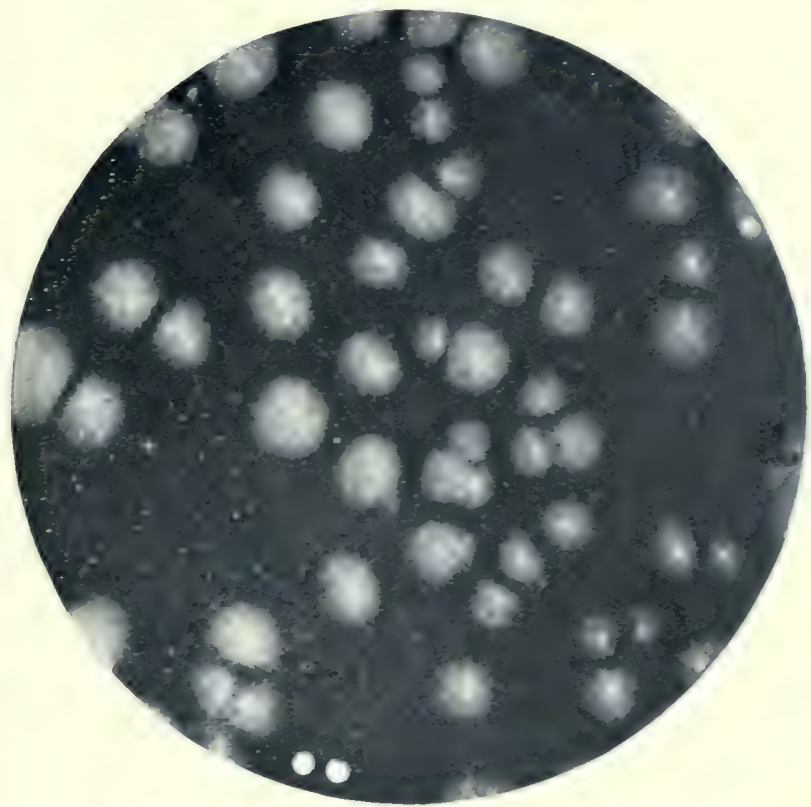

$\therefore 0.5 \mathrm{coc}$ of a 1 in loke duntiun after lis days at $15 \mathrm{C}$.

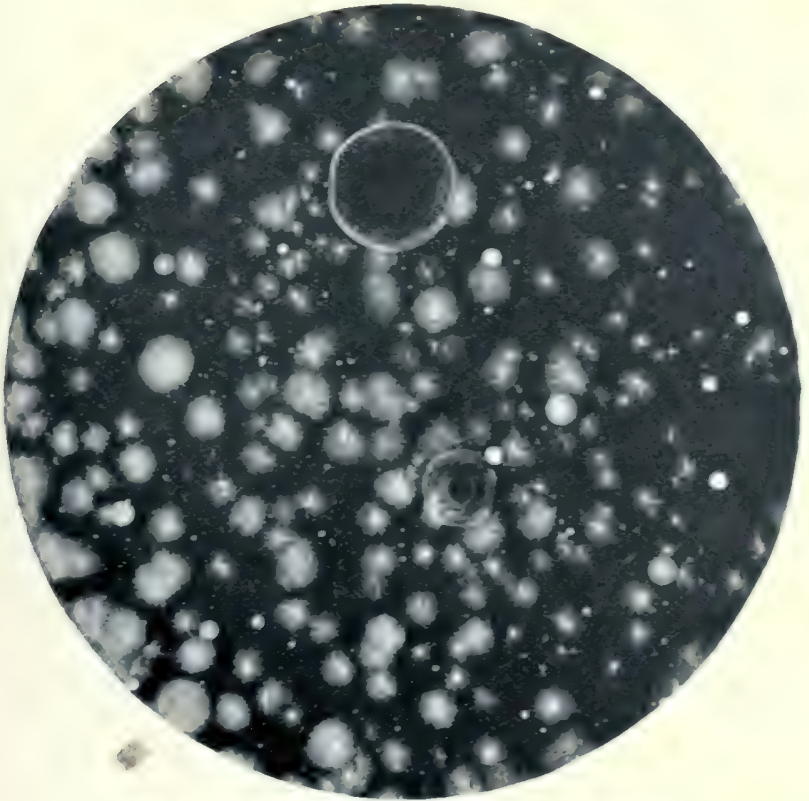

f. 05 c.e. of a 1 in 1000 dilution after lo ways at $5 . \mathrm{C}$. 

PLATE 17.

TYPICAL MIIK PLATES.

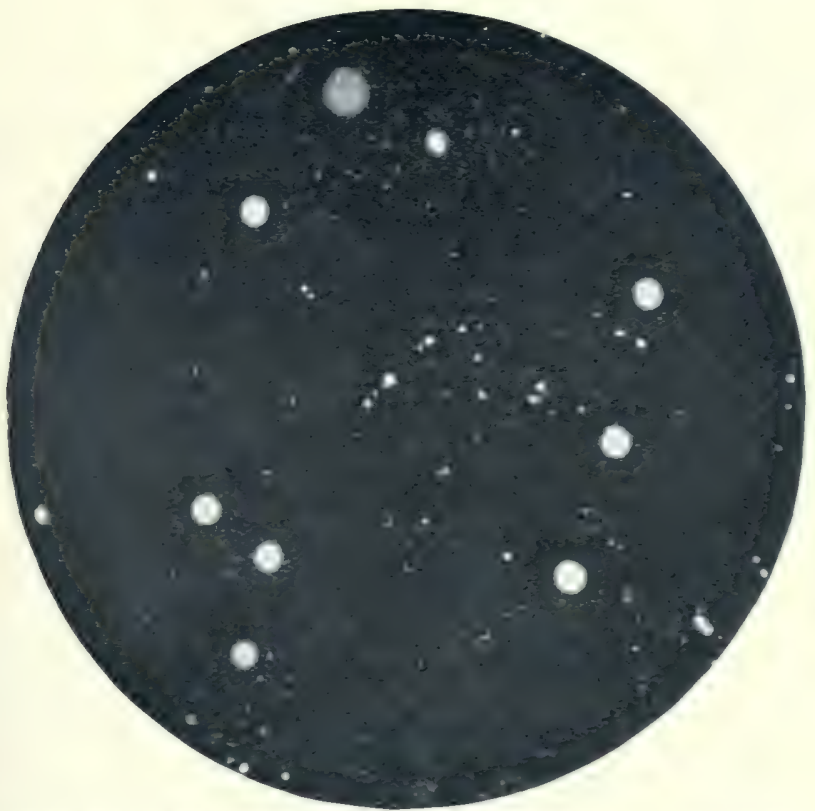

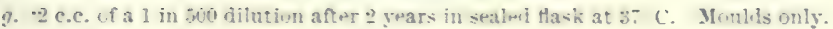

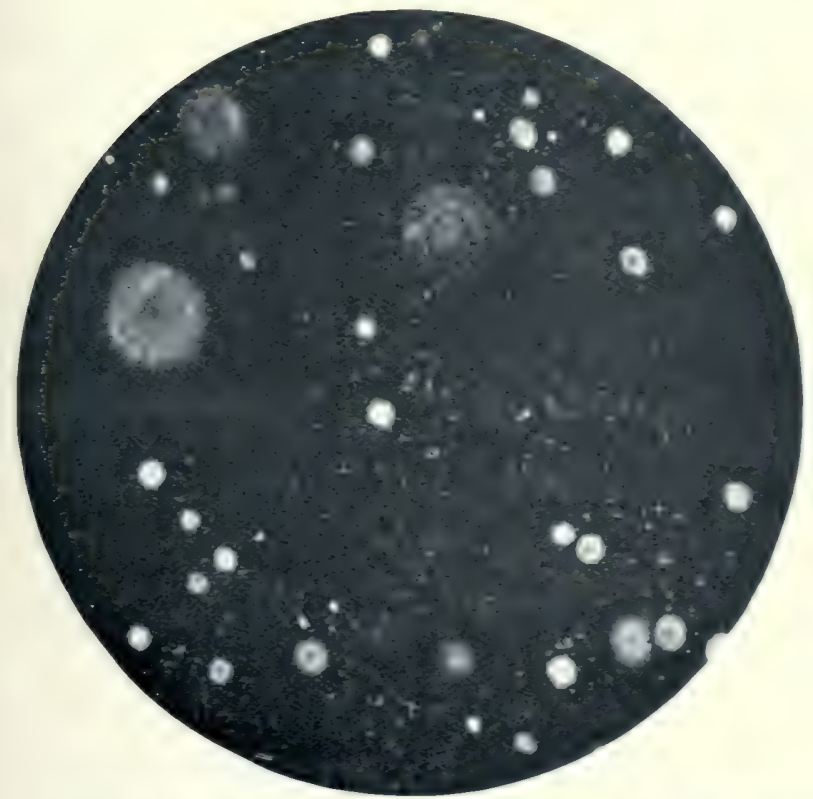

h. -2 c.c. of a 1 in 500 dilutiun after 2 years in se 1 lect flask at 5 C. Mundus only. 

sixteenth hours in the sample at $37^{\circ}$; between the twentieth and twenty-fourth hours at $15^{\circ}$; and between the seventy-second and ninety-sixth hours at $5^{\circ} \mathrm{C}$. At the end of these particular stages it will be noticed that there is a rising tide following the "low water mark " of organisms at each temperature.

The relation which the degree of acidity bears to the bacterial content is an intimate one. In this particular series of experiments we estimated the commencement of acidity by sourness of taste. But in order to check the results, we carried out some acid-testing experiments on similar milk, as follows:-Three flasks of milk, each containing I pint, were taken at the byre. The milk was milked direct from the cow into sterile flasks, and drawn from one quarter of the udder only.

One flask of the milk was kept at $37^{\circ} \mathrm{C}$.

$" \quad " \quad " \quad n \quad " \quad 15^{\circ} \mathrm{C}$.

For the purpose of acid-testing 50 c.c. of milk was placed in a porcelain vessel, and 2.5 c.c. of $a \cdot 1$ per cent. solution of phenolphthalein was added to and well mixed with the milk. The milk was then titrated with a solution of deci-normal caustic soda from a burette until a faint pink colour was obtained. Generally speaking, about 15 to 20 c.c. of $\frac{N}{\mathrm{IO}}$ alkali is required. Each c.c. of $\frac{\mathrm{N}}{\mathrm{IO}}$ alkali is termed "one degree of acidity" (Richmond). Hence a milk requiring 20 c.c. will have $20^{\circ}$ of acidity. Each c.c. of $\frac{N}{10}$ soda $=.009$ grammes of lactic acid. The results were as follows :

Dhorets of Acidiry.

Amount of deci-normal Caustic Soda necessary to render milk "faintly pink" to Phenolphthalein when kept at the temperatures as below.

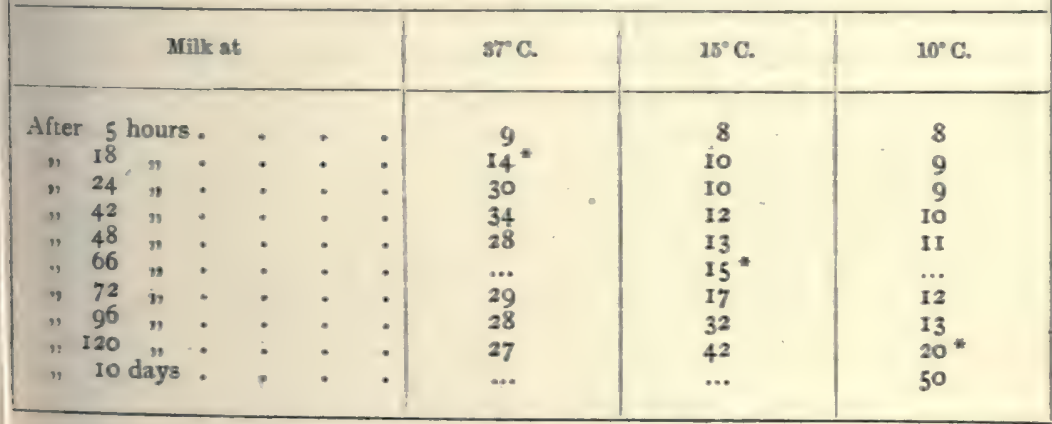

* Stage at which the milk became "sour" to the taste. 
The results may be briefly summarised thus :-

(1) Milk at $37^{\circ}$ after 18 hours was sour to taste.

" " 24 " curdled in flask.

(2) Milk at $15^{\circ}$ after 66 hours was sour to taste. " on 7 th day was curdled in flask.

(3) Milk at $10^{\circ}$ after 120 hours was sour to taste. $" \quad$ on 7 th day was curdled in flask.

These results are of interest, and compare closely with the results obtained during the bacteriological experiments above recorded. Thorner has investigated the acidity requisite to coagulate milk, and fixed it at 22 degrees or 0.207 per cent. of lactic acid. We have found the coagulating point somewhat higher than this, and would suggest that when the acidity is above 0.25 per cent. lactic acid the milk should be considered unfit for dietetic use.

Fifthly, as the flasks of milk were kept intact, we were able to repeat the experiment in every particular after the lapse of exactly two years (April 1902) from the commencement. The milk was the same milk, and the experiment was repeated as at 30 days. During the intervening period the flasks had been kept, hermetically sealed, at the three temperatures. The result was that the flasks were found to be germ free with the exception of an abundant growth of Oidium lactis and other moulds. (See Plates I4-17.)

As far back as 1878 Lister pointed out the marked inhibitory effect which the presence of a high degree of lactic acid had upon common moulds and ordinary saprophytic bacteria. ${ }^{1}$ When the lactic acid declines, these other forms commence growth, and eventually enormously preponderate.

.. The question naturally arises, What is the explanation of these facts? We believe the answer to this question will be found in a glance at the life-history of such a milk as that under consideration. At the time of milking there is, as we have seen, an introduction into the warm milk of vast numbers of common saprophytic and parasitic bacteria. Finding themselves in an ideal nidus, they multiply with almost incredible rapidity. Hence the first rise in numbers of bacteria. Competition and exhaustion of pabulum soon produce inevitable effects, and we obtain the first decline. At this stage it may be said that the common extraneous bacteria, whether putrefactive or simple saprophytes, practically die out, and that for a very simple reason, namely, that they cannot live in the presence of the new tide of acid-forming bacteria. Although

1 Path. Soc. Trans., 1878, p. 440. 
the lactic acid group of organisms do not multiply as rapidly as ordinary saprophytes, they reach a much higher maximum in the end. It is to this family of bacteria that the second and maximum rise is due. In time, also, the same inimical conditions begin to act, and the lactic acid bacteria decline owing to the acidity and to the lack of pabulum. Eventually, the medium, which twenty days before was an ideal one for any organism, and mostly so for those which came first, and which ten days before was favourable to lactic acid organisms, is now favourable to no bacteria at all. Accordingly, bacteria of all descriptions gradually die out, and the medium is eventually left in possession of Oidium lactis and the common moulds. That the destruction of large quantities of solid albuminous substances may occur simply through bacterial agencies has been conclusively shown in the so-called septic tank method of sewage disposal. The death of bacteria under these circumstances always follows shortly after their enormous multiplication, and how much is due to starvation or how much to poisoning by the products of their own activity it is impossible to say. It is, however, clear that the decomposition of large quantities of albuminous substance is first accompanied by great bacterial reproduction, and this is invariably followed by a season of speedy and extreme mortality of bacteria. ${ }^{1}$ In a general way that represents, we believe, the changes taking place as represented in the record we have considered. That there are two rises and two falls in the number of bacteria, the first rise being due to extraneous organisms, and the second rise to lactic acid organisms, we believe to be the almost universal rule in untreated " natural" milk.

Whilst we do not suggest that the record of, and deductions drawn from, this investigation are exceptional, we may say that in our opinion mere estimations of numbers of bacteria in milk appear to us to be scarcely worth the labour involved. What is required is a careful, prolonged, and systematic study of the behaviour of bacteria in milk. In relation to this matter of numbers of bacteria there are, however, two points which require to be briefly mentioned, and it may be convenient to do so here.

First, the enormously high figures reached in the above records may seem at first sight to be almost impossible. In considering this subject, it is important to remember the extremely rapid multiplication which occurs amongst bacteria, particularly in a favourable medium. This differs somewhat widely in different species. The

1 See also Journal of Experimental Medicine, vol. v., Igor, p. 3I3. (E. O. Jordan.) 
Staphylococcus family multiply much more rapidly than do the Streptococcus family; the B. subtilis, which is almost ubiquitous in its distribution and constantly occurring in milk, multiplies more rapidly than the $B$. butyricus. Experiments for determining the exact time of division of the Schizomycetes have been often made, and though the results obtained differ according to conditions and medium, we may take it that division of many of the common bacilli will, as a rule, take place within thirty minutes. At this rate in twenty-four hours one individual would give rise to many millions of similar individuals. Many organisms, however, do not multiply at this rate, and even this rate declines, owing to various circumstances, as time passes. From a number of experiments Klein calculated that for certain organisms the multiplication during the first twenty-four hours is eighty thousand-fold, during the second twenty-four hours four hundred-fold, and during the third twenty-four hours five-fold. ${ }^{1}$

In the second place, we must revert to a remark made earlier in the present chapter to the effect that there is no standard or uniformity in the numerical estimation in milk. A large number of observers have recorded widely-varying returns due in not a little measure to the widely-varying conditions under which the milk has been collected, stored, and examined. Nor is it possible to establish any standard which may be accepted as a normal or healthy number of bacteria, as in the case of water. Many London milks. would exceed 500,000 micro-organisms per c.c. ${ }^{2}$ Until some common international standard is established, mathematical computations are in reality practically worthless. They are for one thing needlessly alarming and sensational. Moreover, it should be remembered that, for comparative purposes, reliance cannot always be placed upon these numerical estimations. They vary from season to season, from day to day, and even from hour to hour, depending upon a hundred varying conditions. Furthermore, as we shall have occasion to point out at a later stage, large numbers of bacteria are economic in the best sense of the term, and the bacteria in milk are no exception to the rule. Large numbers are not therefore necessarily an evil.

Nor is it the number of organisms alone which is affected by the temperature of the milk. There is abundant evidence, obtain-

1 Stevenson and Murphy, Hygiene and Public Health, vol. ii., p. 21.

${ }^{2}$ British Medical Journal, 1895, vol. ii., p. 322. Bitter has suggested 50,000 micro-organisms per c.c. as a maximum limit for milk intended for human consumption. 
able from various sources, to show that the pathogenicity or virulence of milk is increased by rise of temperature. Many workers have made reference to this matter in its relation to the toxicity of milk, cream, butter, and ice-cream in hot weather. Recently, Delépine of Manchester prepared three tables which well illustrate the point. ${ }^{1}$ He found that mixed milk coming from a distance of more than 40 miles, and generally kept for from twenty-four to sixty hours or longer, showed an increase of virulence on inoculation into guinea-pigs in proportion to the mean temperature in the shade in Manchester during the time the specimens were kept (tuberculous samples excluded):-

\begin{tabular}{|c|c|c|c|c|}
\hline $\begin{array}{l}\text { Mean Temperatare in the } \\
\text { Shade (Manchester) } \\
\text { during Time the Specimens } \\
\text { were kept. }\end{array}$ & $\begin{array}{c}\text { Specimens } \\
\text { producing } \\
\text { no Noxious } \\
\text { Effects. }\end{array}$ & $\begin{array}{l}\text { Noxious } \\
\text { Specimens. }\end{array}$ & Totals. & $\begin{array}{l}\text { Percentage } \\
\text { of Good } \\
\text { Specimens. }\end{array}$ \\
\hline Deg. Fahr. & & & & \\
\hline 30 to 35 & 7 & 5 & 12 & 58.0 \\
\hline $35 " 40$ & 7 & II & I 8 & $38 \cdot 5$ \\
\hline 40 n 45 & 2 & 3 & 5 & 40.0 \\
\hline 45 n 50 & I & 4 & 5 & 20.0 \\
\hline 50 " 55 & 一 & 一 & - & - \\
\hline \multirow[t]{2}{*}{$55 n \quad 60$} & o & 2 & 2 & 0.0 \\
\hline & 17 & 25 & 42 & $39-0$ \\
\hline
\end{tabular}

Mixed milks coming from a short distance (generally under 20 miles), most of them kept for less than ten hours (with the exception of five out of the seven bad specimens, and four out of the twenty-two good specimens, which had been kept somewhat longer), gave a similar result (tuberculous samples excluded) :-

\begin{tabular}{|c|c|c|c|c|}
\hline $\begin{array}{l}\text { Mean Temperature in the } \\
\text { Shade (Manchester) } \\
\text { during Time the Specimens } \\
\text { were kept. }\end{array}$ & $\begin{array}{l}\text { Specimens } \\
\text { producing } \\
\text { no Noxious } \\
\text { Effects. }\end{array}$ & $\begin{array}{c}\text { Noxions } \\
\text { specimens. }\end{array}$ & Totals. & $\begin{array}{l}\text { Percentsge } \\
\text { of Bood } \\
\text { Specimens. }\end{array}$ \\
\hline \multirow[t]{2}{*}{\begin{tabular}{lll}
\multicolumn{3}{c}{ Deg. Fahr. } \\
50 & to & 55 \\
55 & $\prime$ & 60 \\
60 & $" 1$ & 65 \\
65 & $"$ & 70 \\
70 & $" 1$ & 75
\end{tabular}} & $\begin{array}{r}\mathrm{I} \\
8 \\
\mathrm{II} \\
2\end{array}$ & $\begin{array}{l}0 \\
1 \\
4 \\
2\end{array}$ & $\begin{array}{r}1 \\
9 \\
15 \\
4\end{array}$ & $\begin{array}{l}100 \cdot 0 \\
88 \cdot 3 \\
73 \cdot 2 \\
\frac{-10.0}{50.0}\end{array}$ \\
\hline & 22 & 7 & 29 & $75 \cdot 63$ \\
\hline
\end{tabular}

1 Jour. of Hygiene, 1903, vol. iii., No. 1, p. 83. 
Unmixed milks kept for various lengths of time, but collected from the udder in sterilised vessels (tuberculous sample excluded), also yielded a like result :-

\begin{tabular}{|c|c|c|c|c|}
\hline $\begin{array}{l}\text { Mean Temperature in the } \\
\text { Shade (Manchester) } \\
\text { during Time the Specimens } \\
\text { were kept. }\end{array}$ & $\begin{array}{l}\text { Specimens } \\
\text { producing } \\
\text { no Noxious } \\
\text { Effects. }\end{array}$ & $\begin{array}{c}\text { Noxious } \\
\text { Specimens. }\end{array}$ & Totals. & $\begin{array}{l}\text { Percentage } \\
\text { of Good } \\
\text { Specimens. }\end{array}$ \\
\hline \multirow[t]{2}{*}{\begin{tabular}{ccc}
\multicolumn{3}{c}{ Deg. Fahr. } \\
35 & to & 40 \\
40 & $"$ & 45 \\
45 & $"$ & 50 \\
50 & $"$ & 55 \\
55 & $"$ & 60 \\
60 & $"$ & 65
\end{tabular}} & $\begin{array}{r}6 \\
3 \\
5 \\
- \\
0\end{array}$ & $\begin{array}{r}0 \\
2 \\
2 \\
- \\
-3\end{array}$ & $\begin{array}{r}6 \\
5 \\
7 \\
- \\
3\end{array}$ & $\begin{array}{r}100 \cdot 0 \\
60 \cdot 0 \\
71.5 \\
- \\
-\end{array}$ \\
\hline & 14 & 7 & $2 I$ & $67 \cdot 2$ \\
\hline
\end{tabular}

"The influence of time is well shown by the number of specimens remaining good, even at a high temperature, when the milk had been kept only half a day. On the other hand, the influence of temperature is still more evident, for in every category the number of good specimens is almost inversely proportional to the height of the temperature. Still, it is important to keep the two factors of time and temperature in mind. What is produced in a few hours in summer may also occur in winter, when the milk has been kept a long time" (Delépine).

The converse is also true, namely, that if the temperature of milk be reduced by refrigeration the toxicity of the milk is lessened. Professor Delépine has shown that the mortality from all causes in guinea-pigs inoculated with refrigerated milk is considerably less than it is if unrefrigerated milk be inoculated :-

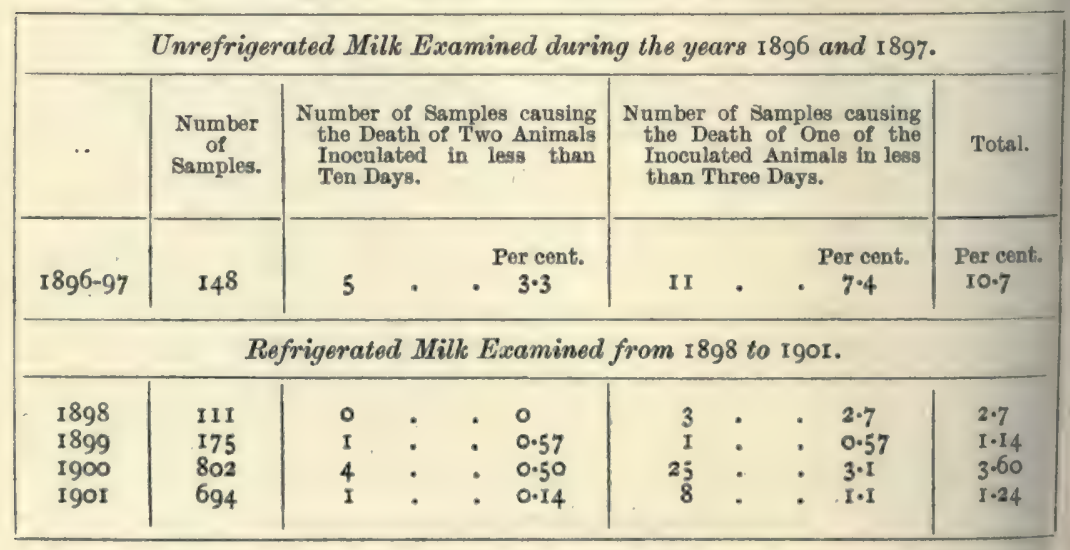


"The inference to be drawn from these gross results is clear: a certain proportion of the samples of milk contained bacteria which, under favourable circumstances, gave to the milk noxious properties, the development of which could be checked in many cases by preventing the growth of these bacteria. The difference between refrigerated and non-refrigerated milk would have been very much greater, if the milk had invariably been cooled immediately after the milking of the cows" (Delépine). It should further be noted that here also the influence of time must not be ignored, for if the milk had been refrigerated immediately after milking, the period which elapsed and during which there was multiplication of organisms and increase of toxicity would have been saved, and thus less toxic substance would have been liberated in the milk.

The practical application of the effect of temperature has been tersely expressed by Dr W. H. Park, who states that "with only moderate cleanliness, such as can be employed by any farmer without adding appreciably to his expense, namely, clean pails, straining cloths, cans or bottles, and hands, a fairly clean place for milking and a decent condition of the cow's udder and the adjacent belly, milk when first drawn will not average in hot weather over 30,000 and in cold weather not over 25,000 bacteria per c.c. Such milk, if cooled to and kept at $50^{\circ} \mathrm{F}$., will not contain at the end of twenty-four hours over 100,000 bacteria per c.c. If kept at $40^{\circ} \mathrm{F}$. the number of bacteria will not be over 100,000 after forty-eight hours." 1

By way of summary it may be said that to refrigerate milk immediately after drawing it from the cow is to reduce the number of bacteria and to diminish the potential toxicity of the milk.

\section{The Inter-relationship of Bacteria in Milk}

The third condition which controls the bacterial content of milk now calls for attention. An obvious result of a great increase in bacteria in a given quantity of milk is that the competition becomes keener owing to the struggle for existence; so that a secondary effect of time and temperature is a diminution in number. But, what is perhaps of greater importance, it appears that certain species become annihilated by the predominance of other more resistant or stronger species. Hence, at the end of twenty-four hours-and we take that period as fairly repre-

1 Jour. of Hygiene, 1901, vol. i., No. 3, p. 4 OI. 
sentative of the time elapsing between milking and human consumption of the milk-we may expect not only a commencing diminution of organisms but a survival of the fittest, that is, the most resistant. If saprophytic bacteria are present with pathogenic, there is a struggle for survival, fortunately ending generally in the survival of the saprophytic because the conditions in milk favour these rather than pathogenic bacteria. Now whilst this is in part due to competition owing to a limited food supply and an unlimited population to which reference has been made, it is also due in part to the inimical influence of one species or its products upon another species or its products. This is known as antagonism amongst bacteria, or antibiosis. ${ }^{1}$ And the antagonistic products are of two kinds. There is first the toxin, which is of the nature of a ferment, producing in many cases a local and a general effect when introduced into the body of an animal. Secondly, there is what has been termed the intracellular poison, which is a poison present in the body of the microorganism, and which may be a property common to several species. ${ }^{2}$ These products, then, may in the course of forty-eight hours be produced in milk, under favourable conditions, and greatly affect the bacteria contained therein. This seems to us to be a matter of the first importance, and one calling for a good deal more attention than it has hitherto received. But there is also another side to this question. We have little doubt in our own minds that many of those cases of milk poisoning occurring in children are due not to the effect of ordinary milk bacteria, or indeed directly to bacteria of any kind, in whatever numbers they may be present, but to the chemical and organic changes set up by the production of toxic bodies in milk which has stood for a long time since milking, and in which, though there may be but one or two surviving species present, there is a considerable amount of toxic substance accumulated.

There is another natural effect of the passing of time upon bacteria in milk which should not be lost sight of. As the struggle for survival increases, and the pabulum becomes more and more limited, and less and less favourable on account of toxic influence, there is a tendency for some of the contained organisms to sporulate, a natural process the various stages of which may be summarised as follows :-

Spore formation.-When a bacillus is about to produce spores,

1 There is some reason to suppose that symbiosis and metabiosis may also occur amongst bacteria in milk, bringing about an inter-relationship between them.

${ }^{2}$ Croonian Lectures of Royal Coll. of Phys., 1898. (Sidney Martin.) 
certain well-marked changes occur in its protoplasm. This becomes granular, and may even contain globules at an early stage. At the same time the bacillus becomes distinctly broader, and paler in substance. Meanwhile the globules increase in size, and in a few hours' time become spores. ${ }^{1}$ The agencies which bring about sporulation are not fully known. One theory obtaining considerable support may be expressed in the words of the late Professor Kanthack : "There must always be the optimum of air, warmth, and nourishment." Sporulation from this point of view is looked upon as the zenith of the individual's life history-the highest stage in the vital activity of a bacterium. In support of this theory there are certain facts. The formation of spores can be shown to take place before the exhaustion of the favourable medium. B. filamentosis is a simple case in point, for it is able to produce spores on agar or potato within tiventy-four hours, and long before the medium is exhausted or competition of other bacilli has had any effect. Another example is $B$. anthracis. This organism requires a sufficient quantity of oxygen and warmth, and the absence of such favourable conditions inhibits sporulation. For example, by continued cultivation in carbolised broth, or at an unfavourably high temperature $\left(42^{\circ} \mathrm{C}\right.$.), this bacillus may permanently lose its property of forming spores, and thus become asporogenous.

On the other hand, there is a second theory, which has the support of a large number of observers, and which holds that sporula-

1 Koch found that spore formation in $B$. anthracis occurred in six hours. The spores may be situated in the middle of the bacillus (as in $B$. anthracis, $B$. acidi butyrici, etc.), towards one end (Bacillus of Malignant (Edema) or actually terminal ( $B$. tetani). Those spores produced inside the capsule of the bacillus are termed endospores. Hueppe has described the spores of certain streptococci as arthrospores. The spores of yeast are termed ascospores. The spores of all bacillary species possess, however, certain characters in common. They are as follows. The spore is generally oval, though more spherical in the Hyphomycetes : it is bright and glistening in aspect ; it is often greater in diameter than the bacillus giving rise to it; its capsule is thicker and stronger than the capsule of the parent bacillus; and it is generally held that the contained protoplasm is more concentrated, so to speak, than that of the bacillus. These two last characters are of chief importance to us, for it is owing to them that spores possess such marked power of resistance. Cohn has suggested that the capsule of a spore is in reality a double envelope, an inner one of fatty and an outer one of gelatinous nature, and it is owing to this that its resistance to heat and desiccation is due. The protoplasm of the spore contains, of course, the essential constituents of the mother cell. It is the method by which "the continuity of germ plasm" is secured in these lowly forms of life. Under favourable circumstances this spore-protoplasm will germinate into a new bacillus. 
tion is due to the opposite of favourable or optimum conditions. In short, that when a bacillus forms a spore it does so because it finds itself amid unfavourable conditions, and sporulates in order to carry on its species by means of more resistant forms. We think there is considerable evidence in support of this theory. Not a few of the lower forms of life in both animal and vegetable kingdoms have more or less analogous conditions of sporulation; for example, some of the Protozoa and Saccharomycetes. In the latter, multiplication by budding goes on so long as they are well supplied with food. If the supply of nutriment fails they sporulate. ${ }^{1}$

It should be understood that whilst holding the view that spores are a resting stage during adverse conditions, we fully recognise that certain favouring external conditions are essential to spore formation. Of these, there are at least three of which bacteriologists have knowledge, namely, moisture, oxygen, and a certain temperature. Fluid media form an excellent nidus for sporulation so long as some oxygen can gain access to the sporulating germs. But many organisms will not sporulate if lying

1 Yeast can be effectually starved ty cultivating on a small block of plasterof-Paris kept moist under a bell jar; under these circumstances the yeast is supplied with nothing but water. In a few days the protoplasm of yeast-cells thus circumstanced becomes filled with vacuoles and fat cells. The protoplasm has been undergoing destructive metabolism, and, there being nothing to supply new material, has diminished in quantity and at the same time been partly converted into fat. Both in plants and animals fatty degeneration is a more or less constant phenomenon of starvation, and to this bacteria are no exception. After a time the protoplasm collects towards the centre of the cell, and divides simultaneously into four masses arranged like a pyramid of four billiard balls, three at the base and one above. These are the ascospores, and sooner or later they are liberated by the rupture of the mother cell wall. Certain of the Streptothrix family also "sporulate" when they find themselves, like yeast upon gypsum, surrounded by an unfavourable environment. Again, in old cultures, it will be found that when the food supply has been exhausted the bacteria have either sporulated or have died. For these reasons sporulation may be looked upon not as a method of multiplication but one of reproduction, of carrying on the species under adverse conditions. With regard to the rapid formation of spores under apparently favourable circumstances (B. flamentosus, $B$. anthracis, etc.), it must be borne in mind that the medium may not be by any means so favourable as appears to be the case (Flïgge). It is clear that the food supply immediately around many of the bacteria in a culture must soon be exhausted. Besides there is the toxic influence early at work, often as an inimical agency acting unfavourably towards the bacillus producing it. So that the appearance of spores in such a culture may still be due to conditions which are actually unfavourable. 
deep in such a medium. In moulds and yeasts oxygen is essential, and for some spore-bearing bacilli a supply of oxygen is a sine quâ non (the exceptions are strict anaërobes like B. tetani, $B$. butyricus, etc.) of sporulation. Prazmowski has pointed out that it is characteristic of these forms that they are non-motile during sporulation. B. tetani, B. butyricus, and other strict anaërobes continue to remain motile during sporing. Temperature exerts a marked influence on the process. ${ }^{1}$ In the. case of $B$. subtilis, an organism frequently present in milk, spore formation did not occur below $6^{\circ} \mathrm{C}$; ; at $18^{\circ} \mathrm{C}$. it required two days; at $22^{\circ} \mathrm{C}$. one day; and at $30^{\circ} \mathrm{C}$. only twelve hours. ${ }^{2}$

Finally, it may be noted that directly spores find themselves again in a favourable nidus they forthwith germinate into bacilli. The time required for the production of a bacillus from a spore varies with different species. Koch observed the germination of the spore of a $B$. anthracis to be completed in about sixty minutes. But there is little doubt that this change may occur in less time than that.

We venture to think that these elementary facts concerning the formation of spores furnish a matter of the first importance to the milk bacteriologist. For from these facts we learn that the lapse of a few hours may bring about very marked changes in the potentiality of a milk as a germ carrier and disease producer. Summarising what we have said we may express the result thus: That a few hours after milking the number of contained organisms is vastly greater than at milking, owing to multiplication in a favourable medium ; that in twenty-four or thirty hours, owing to this increase of bacteria, conditions are beginning to appear in the milk which encourage spore-bearing bacilli to sporulate; that sporulation may take several hours; that the spores possess a very high degree of resistance to heat, germicides, or desiccation; that the spores contain the specific protoplasm of the bacilli producing them; that the conditions in milk upon which we rely for the limitation or inhibition of bacilli may have no injurious or controlling influence upon spores, which thus pass without check;

${ }^{1}$ Koch has shown in the case of $B$. anthracis that at least $16^{\circ} \mathrm{C}$. is necessary for spore formation, and at this temperature limited formation of spores did not occur until after seven days. At $2 \mathrm{I}^{\circ} \mathrm{C}$. spores had formed after seventy-two hours, at $25^{\circ} \mathrm{C}$. after thirty-five to forty hours, and between $30^{\circ} \mathrm{C}$. and $40^{\circ} \mathrm{C}$., in about twenty-four hours ; the best and strongest cultivations were obtained from $20^{\circ}$ to $25^{\circ}$. C.

${ }^{2}$ Flugge.-Micro-organisms. Translation by W. Watson Cheyne, 1890, p. 539. 
and finally, that when these spores find themselves in a favourable environment of temperature and nutriment, like the weakened and unresisting tissues of an infant, they may in the course of an hour or two germinate, become adult bacilli, and play their specific rôle. We venture to think that the standard of milk sterilisation should be almost entirely a standard based on the temperature which will ensure the destruction of spores. For it is clear that the only efficient milk sterilisation is one which will prove germicidal to them.

This excursion into the question of spores and sporulation may appear to have led us far distant from the matter of inter-relationship among bacteria in milk. But in fact it has not done so. For it will now be apparent that the organisms in milk capable of sporulation possess a powerful protective and resistant faculty which enables them to survive a degree of competition or a shortness of pabulum to which non-sporulating organisms would succumb.

The view that fresh milk possesses some germicidal power may also be mentioned here. Some milk bacteriologists hold that there exist in milk, as in blood, certain properties which are antagonistic to the growth of organisms and that this is the explanation of a decline which occurs in the number of bacteria soon after milk is drawn. The theory seems not unreasonable, but we have not met with any substantial evidence in support of it.

\section{Bacteria in Separated Milk}

In March and April I 899 we carried out an extended series of experiments with the object of determining the quantity and quality of the bacteria present in whole milk as drawn from the cow under ordinary dairy conditions, and the constituent parts of the milk after separation and setting. The first series of experiment in respect of the set milk and set cream extended from 2 nd March when the milk was first drawn and set, to 5 th April when it became impossible to manipulate and examine the set cream Fresh flasks were inoculated on March Ist, 8th, I5th, 22nd, and 29th, and the progress of the colonies carefully observed. The dilution throughout was $I$ in 500 , and of this dilution.I c.c. was used for making the plates. (The plates throughout the experi-

${ }^{1}$ Compare the survival of sporulating organisms in sewage. See Reports to the London County Council on the Bacterial Treatment of Sewage, by H. C. Houston, M.D., 1898 and 1899 . 
PLATE 18.
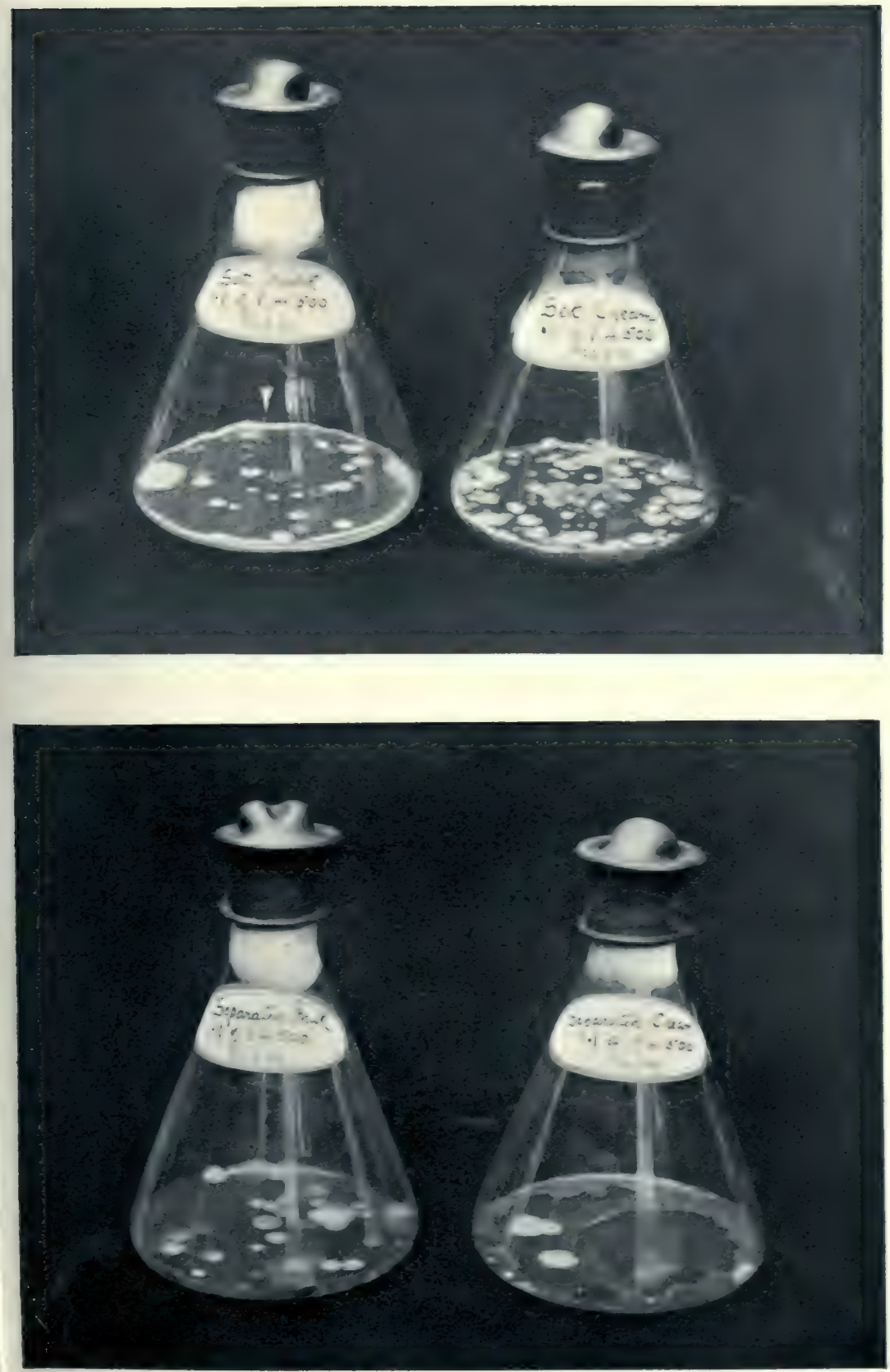

Relative distribution of organisms in milk and cream-(i.) Set milk and cream ; (ii.) Separated milk and cream. Colonies on nutrient agar. Incubation, 48 hours at $37 \mathrm{C}$. ; 7 Cays at $2 \% \mathrm{C}$. 

ment were made in flat-bottomed flasks as illustrated on Plate 18.) Without entering into all the minutiæ of the experiments, the general results may be stated as follows:-Set cream contains more bacteria than set milk from the first day till after the twentyfirst day of setting. The two chief factors contributing to the predominance of organisms in the cream are $(a)$ that as the fat cells rise through the milk to form the cream layer, they carry up with them the organisms contained in the whole milk, as on the principle of "the raft theory" of Tyndall ; and $(b)$ that the cream is exposed to the air, acquiring from it a large number of organisms and acting as a protective film over the milk. After the twenty-first day the organisms in both cream and milk became equalised in number. This change is, of course, mainly due to the fact that both cream and milk came under similar conditions on account of the milk being exposed to the air by the removal of the layer of cream from its surface in the process of creaming.

There is also a decline in the organisms of the set cream after the twenty-first day, which is probably due to competition and acidity, both of which conditions are present in much greater degree in the cream than the milk. The increase of organisms in both set cream and set milk continues to the twenty-first day or thereabouts, after which date, or between the twentieth and thirtieth days of the setting, organisms decline in number. This decline, however, is not general, and does not affect all organisms present. The chromogenic organisms and most of the bacteria derived from the air disappear from both the cream and milk between the third and eighth days, and do not reappear. The lactic acid organisms, on the other hand, present in the set cream at the beginning, increase and become predominant till between the third and fourth week. In the set milk the lactic acid organisms do not appear frequently until the third or fourth or sixth day, being kept under by the common air saprophytes, but after this period they become predominant, as in the cream. The set cream contains many more colonies than the set milk, but in both, the organisms present are of the lactic acid species, all the common air and chromogenic organisms having long since succumbed.

A comparative series of experiments were also carried out in respect of separated milk and separated cream. It is unnecessary to explain that the principle of the separator is that of centrifugalisation. The whole milk being placed in the separator, the machinery is put in motion, and the milk by centrifugal action becomes 
separated into two layers, the outside being separated milk and the inside separated cream. As the revolutions continue, there comes to be a sediment on the wall of the bowl consisting of what is sometimes termed the "slime." By means of a simple contrivance the layer of milk is conducted to an exit tube on the one side of the machine, and the layer of cream to a separate exit tube on the other side. The whole milk, therefore, which was poured in at the top of the machine has, by centrifugal action, become separated into three parts-the cream, the milk, and the "slime" left as a sediment or film on the walls of the bowl, most of which eventually falls to the bottom of the bowl. We have made many examinations of these three constituent parts, with the result that the majority of the bacteria contained in the whole milk are found eventually in the "slime" or sediment of the bowl. Such bacteria have been driven out to the periphery by centrifugalisation, first passing from the inner layer (the cream) through the second layer (the separated milk) into the third layer (the "slime"). Hence it comes about that this mechanical separation results in "filtering" the milk of many of its bacteria, and driving them into the sediment, which is discarded. The bowl sediment, therefore, contains the majority of the organisms, the separated milk being next in order, and the separated cream containing fewest organisms. These results are illustrated on Plates 18 and 19. Separated cream contains comparatively few organisms derived from the air unless some period has elapsed between the drawing of the milk and its separation. In this respect it is similar to set milk. Set cream and whole milk, on the contrary, always show a number of air and chromogenic organisms, which begin to disappear when the tide of lactic acid organisms commences.

If we compare, by means of the quantities of bacteria present, the set and separated constituent parts of milk, we find they come out in the following order, viz.: (I) the bowl sediment contains most organisms; (2) next to it, but comparatively similar to it, the set cream ; (3) the separated milk ; (4) the separated cream ; (5) the whole milk; and lastly (6), the set milk.

It may be assumed that this centrifugal action of the separation upon bacteria in milk acts in a uniform manner upon all organisms, controlled chiefly, in all probability, by two simple conditions, namely, the entanglement of the organism amongst the fat cells, and the weight and body of the organism itself. It should be said in passing that the quantity of slime is affected by the acidity of the milk and other conditions. In a general way, then, the slime or 
PLATE 1?.

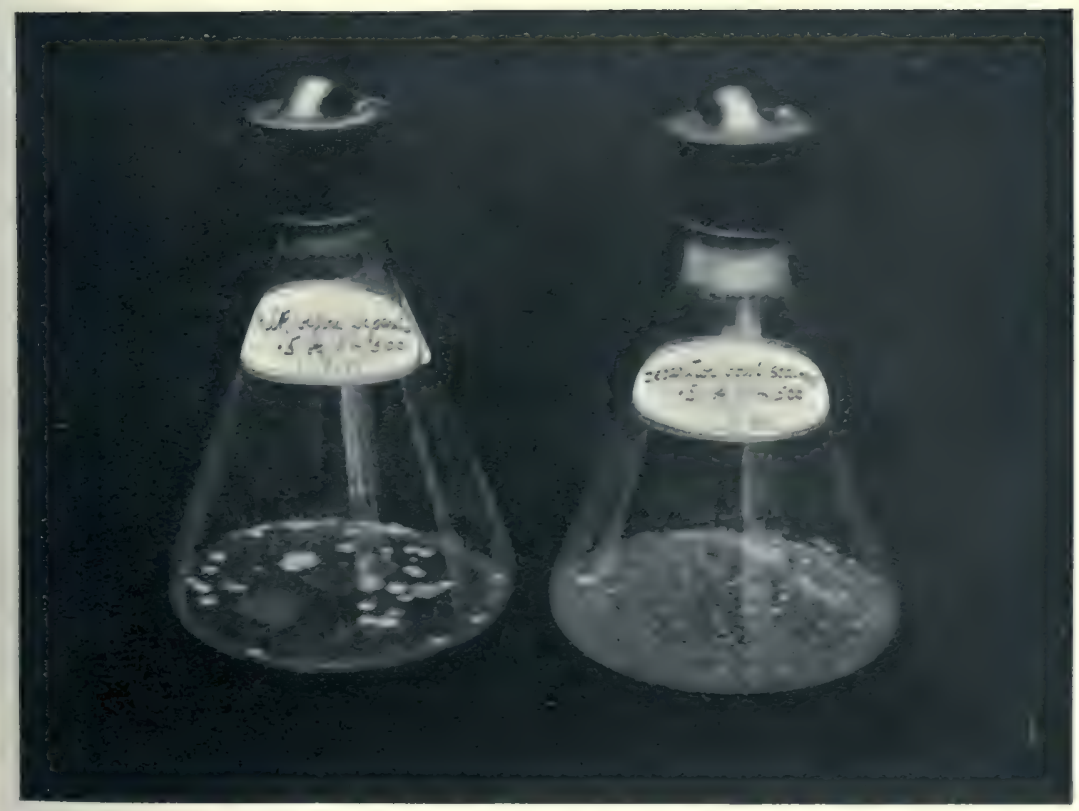

Relative distribution of organisms in milk and cream-(iti.) Milk bef re serparation, an! separ.stor bowl sediment. Colonies on nutrient agar. Incubution, ts hours at 3i C.; F Jays at a.: C. 

bowl sediment will contain the majority of the organisms contained in the whole milk, pathogenic or non-pathogenic, the next largest amount of organisms will be in the separated milk, and the smallest quantity, pathogenic or otherwise, in the separated cream. Roughly, 60 per cent. of the organisms will be found in the bowl sediment, 25 per cent. in the separated milk, and 15 per cent. in the separated cream. ${ }^{1}$ The matter is important, as it is claimed by certain makers or vendors of separators that mechanical separation "filters" milk of its organisms, and removes, in particular, its pathogenic bacteria. To a certain extent this is undoubtedly true. But it must be remembered that only about half are removed.

In 1899 , in order to test the effect of centrifugalisation upon pathogenic organisms in milk, we carried out a series of experiments with the B.tuberculosis, as follows :-Some 5 or 6 gallons of ordinary fresh country milk was taken, and to it was added a pint of human tuberculous sputum, which was thoroughly disintegrated in water and mixed with it. The whole was then poured into the pan of a Lister's "Alexandra" separator. From the pan the milk passed in the ordinary way into the bowl of the separator, which had been sterilised, and was separated. On examination of the slime, the separated milk, and the separated cream, the tubercle bacillus was chiefly found in the slime, but was also present in the milk and cream. Subsequently, butter and cheese ("skim-milk" cheese) were made from the cream and milk respectively, and in both butter and cheese the tubercle bacillus was readily found. Hence it may be taken as a fact that when milk contains the tubercle bacillus, all its products-cream, butter, cheese, whey-may also contain it.

Moore ${ }^{2}$ conducted a number of experiments to determine the effect of the separator in removing certain pathogenic germs, and found that although considerable numbers are removed with the slime, some remained in the separated milk and cream. His results and those of several European investigators are in accordance with our own.

Species of bacteria present in milk.-Probably the most con-

1 As a result of some recent experiments, Eckles and Barnes have estimated that only 47 per cent. of the organisms appear in the slime, 29 per cent. in the separated milk, and 24 per cent. in the cream. They hold that separation removes 36 per cent. of the organisms from the milk and cream. (Iowa Sta. Bulletin, Ig02, lix., 55-59.)

2 United States Department of Agriculture Year Book, 1895, p. 439. 
venient provisional classification of organisms occurring in milk is a fourfold one as follows :-

(a) Saprophytic bacteria, an indefinite group of unknown function, gaining access to milk from air, water, soil, dirt, dust, etc.

(b) Fermentation bacteria, which set up the various "diseases" of milk and the specific fermentations, such as lactic, butyric, etc.

(c) The economic bacteria, which give flavour to butter and cheese, and gain access to milk from various quarters.

(d) The pathogenic bacteria, some of which, such as the tubercle bacillus, set up slow infective processes; and others of which, as $B$. typhosus, are the causal agents in certain epidemic diseases.

The quality, like the quantity, of bacteria found in milk depends upon a variety of external circumstances. Sometimes only half a dozen species may be found in a milk, whilst at others thirty or more species may be isolated. Several hundred species of bacteria have been described as occurring in milk. The chief of them will be found recorded in a subsequent chapter, and special chapters are devoted to a consideration of the fermentation, economic, and pathogenic bacteria. 


\title{
CHAPTER VI
}

\author{
FERMENTATION IN MILK
}

General Introductory Notes. Kinds of Fermentation:-I. Lactic Acid Fermentation; 2. Butyric Acid Fermentation; 3. Alcoholic Fermentation ; 4 The Coagulative Fermentations. 5. Diseases of Milk: (1) Blue Milk, (2) Red Milk, (3) Yellow Milk, (4) Bitter Milk, (5) Soapy Milk, (6) Ropy Milk. The Prevention of Milk Anomalies.

Introductory Notes. - The story of the discovery of the vital principle in fermentation is one of the most interesting and instructive in the whole range of chemistry and physiology. Our knowledge of fermentation, even now by no means complete, has been slowly built up by nearly 250 years of careful observation and experiment, and the record forms one of the most brilliant chapters in research. For its beginnings we have to go back to the middle of the seventeenth century, when Antony von Leeuwenhoek of Delft was busily engaged in inventing the microscope and in describing what he saw through his simple lenses. He it was who first saw and described bacteria and yeast cells. Yet it was not until more than IoO years later that these "animalcula," as they were termed, were deemed to be anything more than accidentally present in any fluid or substance containing them. Plenciz of Vienna was one of the first to conceive the idea that decomposition could only take place in the presence of some of these "animalcula." This was in the middle of the eighteenth century. Another 100 years passed by before it was established beyond dispute that these micro-organisms had an intimate causal relation to the great processes of fermentation, putrefaction, and disease. This body of facts was, as we have said, the outcome of the labours of many men working in various spheres. The great question of spontaneous generation, the physiology of digestion, the study of decomposition and fermentation, and the in-

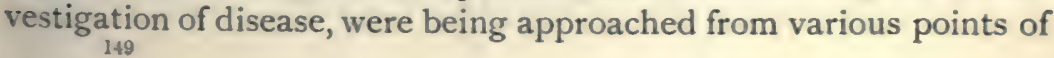


view, and proved facts in any of these directions threw light upon the other allied subjects.

In I776 Spallanzani showed that organic liquids and materials could be prevented from undergoing fermentation by being thoroughly boiled and subsequently protected from the air by sealing the flasks. If, however, the flasks were cracked, or, in any other way, air even in small quantities gained admittance, then almost invariably both organisms and fermentation appeared. This phenomenon was interpreted as due to the entrance of the oxygen of the air (Gay-Lussac). Schwann, the founder of the cell-theory, and Schultze both showed, however, that if the air gaining access to the flask were calcined or drawn through strong acid (or potash and sulphuric acid), the result was the same as if no air entered, namely, there were no organisms and there was no fermentation. It thus became evident that the origin of the fermentation was not in the atmosphere, nor yet in the oxygen of the atmosphere, but in some fermenting agent borne into the flask by the unsterilised air. To this primary contribution Schwann added two other new facts. The first was the discovery of the yeast cell and its function. The yeast itself was simultaneously seen by Cagniard-Latour. In point of fact neither of these men was first in that particular observation, for Leeuwenhoek had already seen yeast plants, but had mistaken them for crystals. Schwann, however, advanced a great step forward, for he not only rediscovered the yeast cell but learned its function. He devised a simple but striking experiment to illustrate the direct action of yeast on a solution of sugar, namely, splitting it up into carbonic acid and alcohol. In a long tube he placed a solution of sugar faintly tinged blue with litmus, and to this he added very little yeast, so that the cells had time to subside as a deposit at the foot of the tube. The red coloration of the litmus due to the liberation of the carbonic acid then began to appear at the bottom of the tube. ${ }^{1}$

The second new fact contributed by Schwann proved to be a stage in the further establishment of the hypothesis that the fermenting agent was introduced by the air. To unsterilised air he added an antiseptic substance which would destroy the fermenting agent. Arsenic was found by Schwann to possess this germicidal property. From these facts Schwann drew the conclusion that vinous fermentation was due to an agent borne in by the air, and that this agent was yeast. If, however, the air, as the

1 Some Apostles of Physiology, by William Stirling, M.D., D.Sc. (1902), p. 98 . 
vehicle of the agent, or the agent itself, were not introduced into the organic fluid, or if introduced the agent was not living, then no fermentation occurred. The work of Schwann thus laid the foundation of what finally came to be known as the truth respecting fermentation. But his conclusions were not destined to be accepted without protest. Passing over a number of the experiments and counter experiments of Turpin, Helmholtz (1843) and others, we come to the work of Liebig, who viewed the transformation of sugar into alcohol and carbonic acid simply and solely as a non-vital chemical process depending upon the dead yeast communicating its own decomposition to surrounding elements in contact with it. Liebig insisted that all albuminoid bodies were unstable, and if left to themselves would fall to pieces-i.e. ferment-without the aid of living organisms or any initiative force greater than dead yeast cells.

It was at this juncture that Pasteur intervened to dispel obscurities, and by new methods of research brought forward convincing proofs in support of the vitalistic theory of fermentation. In reply to Liebig's contention as to the natural instability of albuminoids, he abolished albuminoids altogether from his fermentations, and in solutions of pure sugar with nothing but mineral additions, he demonstrated that ordinary brewer's yeast grew and multiplied, and that its growth caused the fermentation of the sugar and the production of alcohol and carbonic acid. This is but one example of the work of Pasteur in the elucidation of the process of fermentation. Reference might here be made to his discovery of the butyric ferment and of anaërobic organisms. ${ }^{1}$ But we shall have at a later stage occasion to speak of this and of his great contribution in respect of lactic acid fermentation. In 1863 came Pasteur's discoveries in vinous fermentation $;^{2}$ in 1866 his investigations into silkworm disease; and five years later his work on beer. ${ }^{3}$ Nor is it too much to say that Pasteur's researches and discoveries in other directions were intimately related to his first work on fermentation in 1857. Out of that came, by his genius, new light upon spontaneous generation, the etiology of virulent diseases, and the cause and prevention of rabies. ${ }^{4}$ Only a small

1 Nouvel Exemple de Fermentation déterminée par des Animalcules infusoires powvant vivre sans Oxygine Libre (1863).

2 Etudes sur la Vin (1866), and Etudes sur le Vinaigre (1868).

3 Etudes sur la Bière (1876).

4 For some account of Pasteur's work, see the appreciations by Roux (Agenda du Chimiste, I896) and Duclaux (Histoire d'un Esprit), and also Life of Pasteur, by René Vallery-Radot (2 vols. transl. I902). 
part of the subject of fermentation, namely, that occurring in milk, falls to be considered in these pages, but in this brief introductory note we desire to make it clear that we look upon the fermentations which occur in milk as illustrative of the great laws of fermentation generally, wherever and however it occurs in nature.

\section{KindS OF FERMENTATION}

The process of fermentation is for convenience divided into two chief kinds, ${ }^{1}$ namely, (I) When the action is direct and the chemical changes involved in the process occur only in the presence of the cell, the ferment or cell is described as an organised ferment; (2) when the action is indirect and the changes are the result of the presence of a soluble material secreted by the cell, acting apart from the cell, this soluble substance is termed an unorganised ferment or enzyme. The organised ferments are yeasts, bacteria, or vegetable cells allied to them; the enzymes are ferments found in the secretions of highly specialised cells in plants or animals, and have their best illustrations in the digestive agents which bring about assimilation of pabulum in the human body. This function is performed in some cases by the enzyme combining with the substance on which it is acting, and then by decomposition yielding the "new" digested substance and regenerating the enzyme; in other cases the enzyme, by its own molecular movement, sets up molecular movement in the substance it is digesting, and thus changes its condition.

The unorganised ferments may be classified as follows :-

I. Amylolytic, converting starch and glycogen into sugar-e.g. ptyalin (in the saliva), diastase, and amylopsin. Diastase is developed in the process of malting. Fermi states that on albuminous media the bacilli of the subtilis group (B. anthracis, $B$. megatherium, etc.) and other species, including Micrococcus mastitidis, produce amylolytic ferments. Most of these varieties afterwards utilise the sugar further to form acid, while others, e.g. B. subtilis, do not. ${ }^{2}$

2. Proteolytic ferments, those which change proteids into proteoses and peptones-e.g. pepsin, trypsin. This fermentation is a not uncommon accompaniment of bacterial growth. The liquefaction of the glue in gelatine is a sure indication of the presence of a

1 Text-book of Physiology, vol. i., p. 312, E. A. Schäfer, F.R.S.

2 Atlas and Principles of Bacteriology, Lehmann and Neumann, 1901, part ii., p. 63. In this book will be found further matter on bacterial fermentation in general, pp. 58-92. 
proteolytic ferment (Lehmann). The production of proteolytic ferments fluctuates with many, perhaps with all, bacteria in a greater degree than might be supposed. Upon albuminous media liquefy-ing bacteria produce bitter-tasting metabolic products, as Hueppe pointed out in the case of milk.

3. Steatolytic ferments split fats into fatty acids and glycerine. Steapsin of the pancreatic juice is an example.

4 Inversive ferments are those which convert cane sugar, maltose, and lactose into glucose. In this class is invertin of the intestinal juice, and yeast. According to Fermi and Montesano, B. megatherium, B. fluorescens liquefaciens, B. vulgare, and several other species produce inversive ferments.

5. Coagulative ferments are such as convert soluble into insoluble proteids. Rennet is an example; and bodies like it, which coagulate milk of neutral or amphoteric reaction unconnected with the action of acid, are not lacking among the products of bacteria (e.g. B. prodigiosus).

Lehmann states that the presence of rennet ferment may be suspected in all varieties of bacteria which coagulate milk without being able to form lactic acid from milk sugar. Fibrin ferment and myosin ferment are other examples of coagulative fermentation.

From what has been said, it will be seen that enzymes may be the products of bacteria and yeasts as well as of highly specialised cells. ${ }^{1}$

We may now turn to what are designated organised ferments, although the term is not wholly a suitable one, and we shall confine ourselves to such fermentations set up in milk. They may be classified in five main divisions as follows :-

I. Lactic acid fermentation.

2. Butyric acid fermentation.

3. Coagulative fermentations.

4 Alcoholic fermentations.

5. Diseases of milk.

\section{Lactic Acid Fermentation : the Souring of Milk $\forall$}

As every one knows, milk readily "turns sour." When so changed it becomes semi-solid and acid. This ordinary common "souring" of milk is generally due to the lactic acid ferment. It does not occur spontaneously, nor is it a necessary and invari-

1 Buchner has proved that fermentation can be produced by adding the expressed ferment of yeast cells alone to sugar solutions. 
able change. As we point out elsewhere, milk may be drawn from the udder and kept in sealed vessels for weeks and months without any apparent change whatever. The souring of milk is therefore due to some extraneous agent or ferment capable of setting up these two chief changes when introduced into milk: first, the fermentation of the lactose with the production of lactic acid; and secondly and subsequently, the curdling of the casein present in milk, which becomes held in suspension in a semicolloidal form. If the acidity exceeds a certain percentage, the casein is precipitated and the milk becomes coagulated. ${ }^{1}$

Such is the simple process of lactic acid fermentation. Yet when we come to examine these changes closely, many points of interest arise concerning the mode of the fermentative action, the fermenting agent or agents, the constitution of the acid produced, the external conditions favouring the fermentation, and the purposes to which the fermentation may be subservient in the economy of nature. $^{2}$

The earliest chemical investigation of the souring of milk was probably that instituted in 1833 by Pelouze and Gay-Lussac, but from that date fully twenty-five years elapsed before the knowledge that this process is a manifestation of vital activity on the part of sundry micro-organisms assumed definite shape. But as early as I70I, Andry had noticed that sour milk contained living organisms, and Blondeau, in 1847 , made a microscopic examination of milk, and distinguished therein two types of micro-

${ }^{1}$ As we have already stated, milk is often acid the moment it passes from the udder. This is evidently not due to lactic fermentation and should be differentiated from it. Various text-books are responsible for the idea commonly held that milk is absolutely neutral as it passes from the udder. We examined milk on one occasion by testing with sensitive litmus paper. Twenty-six healthy cows (Shorthorns and their cross) were milked in the evening, and as the milk passed from the udder to the pail the litmus paper was used. Out of the twenty-six milks, twenty-four gave an acid reaction ( $92 \cdot 3$ per cent.).

${ }^{2}$ The equation of lactic acid fermentation is generally expressed as follows : $\mathrm{C}_{12} \mathrm{H}_{22} \mathrm{O}_{11}$ (Lactose) $+\mathrm{H}_{2} \mathrm{O}=4 \mathrm{C}_{3} \mathrm{H}_{6} \mathrm{O}_{3}$, or more simply $\mathrm{C}_{6} \mathrm{H}_{12} \mathrm{O}_{6}=2 \mathrm{C}_{3} \mathrm{H}_{6} \mathrm{O}_{3}$. Actually the process is not simple but complex, and $\mathrm{CO}_{2}$ is liberated, and alcohol and volatile acids formed. Warington has shown that the amount of acid varies greatly in different species. The organisms producing lactic acid are inimically affected if the acid produced passes above a certain slightly variable point. Variations depend on time, temperature, and the particular species of lactic bacteria. Ordinary lactic acid exists in three forms characterised by their optical properties. One turns the plane of polarised ray to the left, a second turns it to the right, and a third is inactive. The most common is dextro-lactic acid. 
organisms, a Torula and a Penicillium, which latter he thought was the cause of lactic fermentation.

Rowlandson, under the influence of Liebig, defined the preliminary conversion of lactose into lactic acid in the souring of milk as an oxidation process, and expressed the opinion that a cow that had been in active exercise (and therefore breathing rapidly before milking) would yield a milk rich in oxygen, and therefore liable to turn sour with unusual rapidity.

Pasteur, as we have already pointed out, was the first to describe an organism characteristic of lactic fermentation, and to prove that it was capable of producing acidification in a sweet, sterile milk. This organism, which Pasteur named the "ferment, or yeast, of lactic fermentation" (Levure lactique) was a bacterium. Pasteur demonstrated the difference existing between this "ferment" and that of alcoholic fermentation, by proving that in media containing sugar, the former organism always set up lactic acid fermentation, whilst the latter invariably gave rise to alcoholic fermentation. Pasteur noticed that in cases of apparenfly spontaneous souring of milk there was a greyish deposit, and that the quantity of this increased as the process advanced. By microscopic examination this deposit was found to consist of rod-like corpuscles possessing marked differences from the alcoholic ferment. Pasteur then introduced a trace of this deposit into a solution of sugar to which he had added a decoction of yeast and some chalk, and in this artificially prepared liquid he thus obtained lactic acid fermentation. From this fermenting fluid he again transferred a slight trace to a similar solution of sugar, and so on, invariably obtaining the lactic fermentation and invariably finding the same corpuscles in the deposit. Such characteristics as these were more fundamental than were morphological differences, and justified Pasteur in concluding that in this deposit he had found the specific agent of lactic fermentation. ${ }^{1}$

In I 877 Lister was able, by means of the "dilution method," to isolate from sour milk, in a form of pure culture, an organism to which he gave the name $B$. lactis, and which he believed gained access to milk from the air of dairies and similar places. ${ }^{2}$ For some time this organism was held to be causally related to lactic fermentation. But in 1884 , by means of culture on solid media, as introduced by Koch, Hueppe was able to isolate a

1 In his Etudes sur la Biere, Pasteur describes organisms found by him growing in wort or beer in which lactic fermentation was proceeding.

2 Trans. of Path. Soc., I878, p. 437. 
bacillus which he named the Bacillus acidi lactici. This, was probably identical with Lister's bacillus, and is now a term used to cover a whole family of organisms having somewhat similar characters and possessing the property of setting up lactic fermentation. ${ }^{1}$

In I894 Günther and Thierfelder published the result of their work on lactic acid fermentation, from which they concluded that Lister and Hueppe had discovered one and the same species, and that it was the causal agent of lactic acid production in Europe. Esten found a similar organism to be the cause of lactic acid fermentation in America, and Conn holds that three organisms, or rather types of species, are the chief agents in the production of lactic fermentation, namely B. acidi lactici, Nos. i. and ii., and $B$. lactis arogenes. The first named forms between 75 to 90 per cent. of the bacteria present. No. ii. is also very abundant. b. lactis arogenes is found almost universally, although never in large numbers. It is a type of a species which produces intense acid on litmus gelatine cultures, produces much gas in milk or milk sugar broth, curdles milk at high temperatures, and produces a distinctive odour in the milk, which it ferments. ${ }^{2}$ According to Escherich, the formation of lactic acid by this organism prevents fermentation in the stomach and intestines.

It was Hueppe who made the important discovery that many different species of bacteria are capable of setting up lactic fermentation, and what we have now said amply supports that view. Indeed, it has been estimated that upwards of 100 different bacteria possess this property. ${ }^{3}$ A description of the more impor-

${ }^{1}$ Hueppe isolated five forms of his lactic acid bacillus. Maddox, Beyer, Fokker, Krueger, Grotenfeld, and other workers isolated lactic acid organisms.

2 Storr's Agricultural Expt. Sta. Rep., 1899, p. 22. Others than those named are B. acidi lactici of Günther, B. acidi lactici of Leichmann, Bacillus xix. of Adametz, Bacillus a of Freudenreich, B. and $M$. acidi lavolactici of Leichmann, Grotenfeld's B. acidi lactici Nos. i. and ii., No. 8 of Eckles, and Bact. casei.

${ }^{3}$ Delbrück, Zopf, Krause, Peters, Lindner, Weigmann, Storch, and Marpmann, are amongst those who, in addition to workers we have named, have described bacteria possessing the power of setting up lactic fermentation. Only provisional classifications are possible at present, as, owing to variations in biology and terminology, it is probable that certain lactic organisms are described under several different terms. Generally, it may be said that some grow well in the presence of oxygen, and others do not. The latter group, facultative anaërobes, are perhaps the most common. They sour milk best in deep vessels, and produce a right-handed lactic acid. (They are widely distributed in nature, and may form 90 per cent. of the total bacteria in milk. Some 
tant organisms associated with lactic acid fermentation will be found at a later stage in the present volume. Here we may content ourselves with the general remark that as a rule the lactic fermentation bacteria are short rods, do not liquefy gelatine, nor do they form spores. They grow readily on gelatine at room temperature, forming as a rule small circular colonies, white or grey in colour, with sometimes a tinge of yellow, and the surface of the colony is smooth and glistening. The lactic acid organisms produce appreciable amounts of lactic acid only at somewhat elevated temperatures. If the amount of acid rises much above 2 per cent., the growth of the lactic acid bacteria is inhibited. Many other substances, as we have seen, are produced in addition to lactic acid (e.g. acetic and ferric acids, alcohol, methane, $\mathrm{CO}_{2} \mathrm{H} . \mathrm{N}$., etc.). Lactic acid organisms (as non-spore bearers) are readily killed by pasteurisation. The economic function of the lactic ferments concerns, of course, the manufacture of butter and cheese.

External conditions affecting lactic fermentation in milk.-Time and temperature are the two chief external conditions necessary for the development of fermentation, and the exact time of souring varies in relation to temperature. At $10^{\circ} \mathrm{C}$. milk becomes sour after 120 hours, and curdles on or about the seventh day. At I $5^{\circ} \mathrm{C}$. it is sour in sixty-six hours, and curdles on or about the seventh day. At $37^{\circ} \mathrm{C}$. (blood-heat) milk is sour in sixteen or eighteen hours, and curdles on the second day. At boiling point there is a diminution of the total acidity in milk, partly due to the decomposition of calcium salts having an acid reaction, and partly to the expulsion of carbonic acid. The general rule is that milk turns sour most readily at between $35^{\circ} \mathrm{C}$. and $42^{\circ} \mathrm{C}$, and when 2 per cent. of lactic acid is reached the process of further production ceases. If the milk be then neutralised with carbonate of lime, the process of fermentation will recommence until the acidity again stands at 2 per cent. Pepsin may also be added to restart the process. As will be seen elsewhere in the present volume, there is abundant evidence to show that lactic acid fermentation is most active in milk between twelve or twenty hours and fifty or sixty hours after milking. After this period of time

of the aërobic species produce gas, and are the lactic organisms which set up "gassy" changes in cheese making. Such organisms are present in milk in from 2 to 5 per cent. Some lactic organisms liquefy gelatine, and probably a few produce spores. The quantity of lactic acid produced by different species varies. 
the lactic acid organisms decrease (see p. 132). It should be added that at room temperature the type of lactic acid produced turns the plane of polarised light to the right, whereas at blood heat it may turn to the left or be inactive (Kozai).

Other outside conditions play a primary part in lactic fermentation. That this is so, has become increasingly apparent as the knowledge of milk changes has grown. Whilst the exact origin of lactic acid bacilli is not known, many bacteriologists hold that they gain entrance to the milk from the surrounding air of byre or dairy. Others maintain that some species, at any rate, are soil bacteria, and associated with certain geographical localities. Russell states that, under ordinary conditions, the organisms found in the teat of the udder are those which produce lactic fermentation. He quotes Bolley and Hall as finding twelve out of sixteen species in the teat of the udder to be lactic acid producers. ${ }^{1}$ Veranus Moore has arrived at very similar results. ${ }^{2}$ Rollin Burr has recently investigated this subject with a different result. ${ }^{3} \mathrm{He}$ finds that when milk is drawn from the cow in such a manner as to exclude from it dirt and dust from the air, the stalls, and the cow, such milk may contain none of the organisms capable of producing a normal souring of milk. This also has been our experience. The lactic acid organisms are a secondary contamination of the milk from some external source. None of the species of lactic organisms characteristic of the locality in which Burr worked, could be found in the udder. This is in accordance with the results of others who have had the opportunity of examining the udder or milk ducts for lactic bacilli. Out of 300 examinations made of fore-milks drawn directly from the udder into sterile flasks, Burr found only 2 per cent. contained ordinary lactic acid bacteria, and in these cases the origin was probably outside contamination. Conn found the acid organisms present in 5 cases out of 200 examinations, involving 75 cows. He also maintains that the origin of lactic acid bacteria is in external conditions. ${ }^{4}$ Further, there is the recognised fact which has been pointed out by Conn and Esten, and frequently met with by ourselves, namely, that lactic acid organisms are not the predominant species in freshly drawn

1 Outlines of Dairy Bacteriology, H. L. Russell, I898, p. 43.

2 Twelfth and Thirteenth Reports of the Bureau of Animal Industry, U.S.A., 1895 and 1896, p. 265.

3 Storr's Agricultural Expt. Sta. Rep. for 1900, pp. 66-81. Centralb. f. Bakt. ii. Abth., 1902, p. 236.

- Storr's Agricultural Expt. Sta. Rep., 1899, p. 23. 
milk, as they undoubtedly would be, were they organisms of the udder. Hence there can, we think, be little doubt that the origin of lactic acid organisms is to be found in some external condition or conditions.

It follows from what has been said that cleanliness of byre, dairy, and general manipulation is an important factor in the presence, both actual and in degree, of lactic acid organisms.

Some authorities have suggested that breed of cow and fodder affect the lactic acid bacteria, and that on this account the milk of certain farms contains more acid organisms than other farms. The probability is that the difference is due to other causes; but the subject has not been fully worked out.

The function of lactic acid bacteria.-Of all the fermentations occurring in milk and its products, that of lactic acid has by far the highest degree of utility. Many of the other fermentations bring about anomalous conditions in the milk, which are looked upon as "diseases." But the souring of milk and the ripening of cream, both of which are due to lactic acid organisms, may be turned to good account. Of this we shall speak in a later chapter.

\section{Butyric Acid Fermentation}

Lafar states that the first mention of the fact that butyric acid, discovered in 1814 , can also be produced by fermentation, was made by $R$. Marchand in 1840 , in connection with his researches on the composition of the milk of the South American cow-tree (Galactodendron americamum). In 1841 Noellner described, under the name of pseudo-acetic acid, a substance which he had found to result from the spontaneous decomposition (fermentation) of calcium tartrate, and which was then recognised by Berzelius as a mixture of butyric and acetic acids. Two years later Th. Pelouze and $\mathrm{A}$. Gelis observed that the lactic fermentations instituted by them did not progress satisfactorily, butyric acid and considerable quantities of hydrogen being produced.

But the discovery of the true state of the case was made in $186 \mathrm{I}$ by Pasteur, who showed that two successive processes are here involved : first, the conversion of sugar into lactic acid as calcium lactate, and afterwards the transformation of the lactate into butyrate. He demonstrated that each of these changes is due to a special ferment : in the first place the lactic ferment, in the second place the butyric ferment. But that was not all. Indeed, it was by no means the most important attribute of this newly discovered 
organism. For Pasteur, when he discovered it, discovered also the principle of anaërobic life. It was in November I 860 that, returning to his studies on fermentation in general and lactic fermentation in particular, Pasteur met with some complications which hampered the purity and progress of his lactic fermentation. In short, he found a secondary fermentation proceeding, namely, butyric. But, as previously in the lactic acid investigation, he found that in. addition to the butyric acid being present there was also present a new kind of organism. "The most constantly repeated tests," he wrote in February I86I, "have convinced me that the transformation of sugar, mannite, and lactic acid into butyric acid is due exclusively to those infusories, and they must be considered as the real butyric ferment." Further, in studying under the microscope a drop of butyric fermenting fluid, he observed that the organisms at the edge of the drop were motionless and apparently dead, whilst in the central portion of the drop the bacilli were executing those active movements which are characteristic of their vitality. To Pasteur's mind this at once suggested, what he was able later to demonstrate, namely, that these organisms at the periphery were paralysed by contact with the oxygen of the air. When he passed a stream of air through a flask containing a liquid in butyric fermentation, he observed that the process slackened and eventually ceased. "Those infusory animalcula," he wrote, "live and multiply indefinitely, without requiring the least quantity of air. And not only do they live without air, but air actually kills them. It is sufficient to send a current of atmospheric air during an hour or two through the liquor where these vibriones were multiplying, to cause them all to perish, and thus arrest the butyric fermentation, whilst a current of pure carbonic acid gas passing through the same liquor hindered them in no way. Thence this double proposition," concluded Pasteur: "the butyric ferment is an infusory; that infusory lives without free oxygen." Thus was discovered what Pasteur afterwards termed the anaërobies. ${ }^{1}$ It will now be readily understood that the aërobic ferments give rise to oxidation of certain products of decomposition; the anaërobic organisms, on the other hand, commence to grow when the aërobic have used up the available oxygen. Thus in such fermentation certain bodies (carbohydrates, fatty acids, etc.) undergo decomposition, and by oxidation carbonic acid gas is produced; the remaining bodies are "reduced" products of the whole process. Hence sometimes this is termed fermentation by reduction.

1 For methods of cultivation of anaërobic bacteria, see p. 78 . 
The chemical formula of the butyric reaction may be expressed thus :-

$$
\begin{aligned}
& \mathrm{C}_{6} \mathrm{H}_{22} \mathrm{O}_{6} \text { [by simple decomposition] }={ }_{2}^{2} \mathrm{C}_{3} \mathrm{H}_{6} \mathrm{O}_{3} \\
& \text { Or, } \quad \underset{\text { Lactoes }}{\mathrm{C}_{12} \mathrm{H}_{22} \mathrm{O}_{11}}+\mathrm{H}_{2} \mathrm{O}=\underset{\text { Lactic acid }}{{ }_{4} \mathrm{C}_{3} \mathrm{H}_{6} \mathrm{O}_{3}}
\end{aligned}
$$

which is followed by the fermentation of the lactic acid by the agency of the butyric ferment:-

$$
\underset{\text { Lactic seid }}{\mathrm{C}_{3} \mathrm{H}_{6} \mathrm{O}_{3}}=\underset{\text { Butyric seid }}{\mathrm{C}_{4} \mathrm{H}_{8} \mathrm{O}_{2}}+\underset{\begin{array}{c}
\text { Carbonie } \\
\text { acid gas }
\end{array}}{\mathrm{CO}_{2}}+\underset{\text { hydrogen }}{2 \mathrm{H}_{2}}
$$

These three bodies may be accepted as the most important, but not the only, products of butyric fermentation. Although according to Pasteur's researches the butyric acid ferment performs its functions anaërobically, many butyric organisms act in the presence of oxygen (Hueppe, Fitz), and yield somewhat different products. For example, Gruber has pointed out that one or two species produce butyl alcohol. All of them, however, ferment most actively at a temperature at or about blood heat, and the spores are able to withstand boiling for several minutes (Fitz). Hence butyric organisms may be found in sterilised milk. It will be noted that as in the lactic fermentation, so in the butyric, the results are not due to one species only. We must now consider some of the chief organisms associated with butyric fermentation.

Bacteria associated with butyric fermentation.-Previously to I 880 the only work which had been done in the elucidation of the bacterial origin of butyric fermentation had been accomplished by Prazmowski and Pasteur; the former designating the organism he found Clostridium butyricum, and the latter naming his "infusoire" Vibrion butyrique. Prazmowski emphasised the motility and resistance of the bacillus, and found that the latter was due to the spores produced by the organism. These spores were able to withstand boiling for several minutes. Fitz went so far as to say that butyric spores could resist boiling for twenty minutes. Prazmowski was unable to obtain pure cultures. Clostridium butyricum grows most readily at a temperature of about $40^{\circ} \mathrm{C}$., and is very widely distributed in nature. It is capable of dissolving cellulose, and therefore plays a part in the cellulose fermentation which is employed in various maceration industries. It is generally held that in such fermentations there is symbiotic action between the butyric bacillus and an organism incapable of causing "retting" 
by itself. The organisms discovered by Prazmowski and Pasteur were anaërobic. But Fitz and Hueppe isolated an aërobic butyric bacillus.

This fact was confirmed by Gruber, working with a pure culture in 1887 , and it was at the same time demonstrated that the Clostridium butyricum of Prazmowski consists of a number of closely allied, but distinct, species. Lafar states that nearly related to this is a ferment isolated by Liborius from old cheese, and introduced into literature under the name of Clostridium foctidum. This organism liberates foul-smelling gases, in addition to producing butyric acid, and forms one of the many connecting links between the butyric acid bacteria and the so-called "potato" bacilli. No sharply defined limit can be drawn between these two groups.

The butyric acid bacteria which produce aromatic substances are important for the ripening of cheese, and to this subject we shall refer again subsequently. Freudenreich separated from milk a Clostridium foetidum lactis, which develops, in this medium, an odour resembling that of Limburger cheese, and the same observation was made by $\mathrm{H}$. Weigmann. Winogradsky isolated the butyric Clostridium Pasteurianum; and Baier, Weigmann, Behrens, and others described other butyric-producing bacteria. Butyric acid is to be regarded in some ways as an end product of a long series of fermentative changes. It is accompanied by many byproducts, and is a complex process. There are therefore many organisms which, although not designated butyric acid bacilli, have, in fact, the capacity of producing butyric acid amongst other substances. In ordinary cream ripening it is absent, and therefore not of much utility in the dairy industry. In the ripening of cheese it may play some part, and certainly does so in the rancidity of butter.

\section{Alcoholic Fermentation in Milk}

Solutions containing sugar, if exposed to the air, are apt to undergo alcoholic fermentation, and milk is no exception to the rule. The change is, of course, due to saccharomyces of different kinds, which have the power of breaking up the lactose and producing alcohol and carbonic acid gas. At the same time, it must be said that lactose is not readily acted upon in this way, and the percentages of alcohol produced in milk are very small in comparison with those occurring after vinous fermentation. It generally occurs in two stages. The lactose is first inverted, 
and dextrose and galactase are formed; and, in the second place, these sugars are fermented, and alcohol produced, thus :-

$$
\text { (I) } \underset{\text { (Lactose) }}{\mathrm{C}_{12} \mathrm{H}_{22} \mathrm{O}_{11}}+\mathrm{H}_{2} \mathrm{O}=\underset{\text { (Dextroes) }}{\mathrm{C}_{6} \mathrm{H}_{12} \mathrm{O}_{6}}+\underset{\text { (Galactase) }}{\mathrm{C}_{6} \mathrm{H}_{12} \mathrm{O}_{6}}
$$

(2) $\underset{\text { (Dextrose) }}{\mathrm{C}_{6} \mathrm{H}_{12} \mathrm{O}_{6}}\left\{\begin{array}{c}\text { (plus the saccharomyces, } \\ \text { or fermenting agent) }\end{array}\right\}=\underset{\text { (Alcohol) }}{2 \mathrm{C}_{2} \mathrm{H}_{6} \mathrm{O}}+\mathrm{CO}_{2}$

Alcoholic fermentation of grape sugar, of cane sugar, or even of starch, is, if we may so express it, precisely the same as that which occurs here, namely, an inversion followed by a fermentation, the sugar disappears, the carbonic acid gas escapes into the air and the alcohol remains behind.

Occasionally alcohol is present in the milk of a dairy, as a sort of by-product accompanying lactic fermentation, and alcoholic fermentation may, under exceptional circumstances, cause serious trouble to the dairyman. But the chief illustrations of this fermentation in milk are the well-known examples of the artificial beverages known as koumiss (or kumiss, kumys) and kepliir (or kefyr, kefr), the former a fermentation of mare's milk, the latter of cow's milk. Matzoon ${ }^{1}$ and Leben ${ }^{2}$ are two other examples of similar changes.

Koumiss is made on the Steppes of South Western Siberia and European Russia, by nomadic Tartars. ${ }^{3}$ It is, as already stated, a fermentation of the milk of the mare. But it is not a simple process nor a single fermentation. There is first a lactic fermentation producing lactic acid, and secondly a vinous fermentation resulting in alcohol. The former is produced by bacteria, the latter by yeasts. In neither case is the process set up by a pure culture, the general method adopted being as follows: A small quantity of old koumiss is added to about ten times its volume of mare's milk. The fermentation is conducted in small casks or vats fitted with a stirring apparatus which is worked vigorously for the first few minutes. Before the fermentation is complete the fluid is bottled and an effervescent beverage thereby obtained. The object of the maker is, of course, to encourage the vinous or later fermentation, and check the lactic or earlier fermentation. Mare's milk contains less casein and fat than cow's, and its làctose favours lactic fermentation more readily than cow's milk. For this double fer-

1 Centralb. f. Bakt., 1898 , ii. Abth., iv. 418.

2 Ann. de l'Inst. Pasteur, 1902, xvi. 65.

${ }^{3}$ See works by Carrick, Reynolds Green, Dochmann, Rothschild, Rubner, etc. 
mentation, therefore, the milk of the mare is particularly suitable. The chemical changes of the process have been well summarised by Hutchison. ${ }^{1}$ He states that-

"The chemical changes which take place in the milk under the double fermentation, are not difficult to follow. The lactic ferment simply changes part of the sugar into lactic acid. The vinous ferment eats up a very small part of the proteid of the milk, and at the same time produces from the sugar a little alcohol and a good deal of carbonic acid gas. The milk thus becomes sour, it effervesces, and is weakly alcoholic. But the lactic acid causes the casein to be precipitated just as it does in the ordinary souring of milk, and the casein falls down in flocculi."

"Now, one of the essential points in the making of koumiss is that during the whole process of fermentation the milk should be kept constantly agitated by stirring. This agitation is primarily intended to permit of the access of oxygen to the fermenting fluid, but it has also the result of breaking up the precipitated casein into exceedingly fine particles, and it is to this extremely fine state of division in which the casein is found that much of the ease with which koumiss can be digested is to be attributed. As the process goes on, it would appear that a small part, at least, of the casein undergoes a sort of spontaneous digestion, and is converted into soluble products. One certainly finds that ordinary kephir contains a small amount of protone."

"These changes, of course, only go on gradually, so that at the end of twelve hours of fermentation one gets a weak koumiss which is only slightly sour, and which still looks and tastes quite milky. After twenty-four hours have elapsed some of the casein has been redissolved, with the result that the koumiss is thinner; it has also increased in sourness. This is called 'medium' koumiss. After another twenty-four hours or more most of the sugar has been destroyed, and the 'strong' koumiss which results is a thin, sour fluid which effervesces briskly. In this form it can be kept indefinitely without undergoing much further change."

"The net change which has taken place in the original milk may be summed up by saying that the sugar has been to a large extent replaced by lactic acid, alcohol, and carbonic acid gas; the casein has been partly precipitated in a state of very fine division, and partly predigested and dissolved, while the fat and salts have been left much as they were."

The total proteid in koumiss is hardly less than in mare's and cow's milk, the fat is practically the same as in mare's milk, and the sugar is reduced from about 5 per cent. to $\mathrm{I} \cdot 5$ per cent. The

1 Food and the Principles of Dietetics, by R. Hutchison, M.D., F.R.C.P., 1902, p. 136. 
amount of alcohol in koumiss is as little as $1 \cdot 7$ per cent. and there is not as much as I per cent. of lactic acid.

Kephir, the second example named, is an effervescent alcoholic sour milk prepared by inhabitants of the Caucasus from the milk of goats, sheep, and cows. The process of fermentation is a double one, and precisely parallel to that occurring in the production of koumiss. Its method of manufacture is simply to add to milk a few "kephir grains," allow the milk to stand for twenty-four hours at a temperature of $17^{\circ}$ to $19^{\circ} \mathrm{C}$., pour off the milk and mix with fresh volumes, and so on. Fermentation is complete in two or three days' time, and the resultant fluid contains about 2 per cent. of alcohol, being slightly more than in koumiss.

The kephir grains are whitish or yellowish irregular granules about the size of a walnut, and of tough gelatinous consistence. They have been carefully studied by Beyerinck, ${ }^{1} \mathrm{Mix},{ }^{2}$ Rothschild, ${ }^{3}$ and other workers and appear to contain several organisms and yeasts, the former bringing about lactic and the latter alcoholic fermentation. Kern isolated a bacillus which was named Bacterium dispora caucasia and Beyerinck states that this organism causes lactic fermentation. Two forms of streptococcus, $a$ and $\beta$, have also been found. Lastly, a kephir yeast and a true saccharomyces (Jorgensen) have been isolated from the grains. The latter was capable of fermenting milk by itself. But there can be little doubt that the kephir fermentation is a double one changing lactose into lactic acid, and subsequently to dextrose, and the dextrose into carbonic acid and alcohol. Exactly what rôle each individual fermenting agent plays it is impossible to say at present, but in all probability the bacteria perform the lactic, and the saccharomyces the alcoholic, fermentation. ${ }^{4}$

1 Arch. Neerl., 1888-89, p. 428.

5 Proc. Am. Acad. Arts and Sc., 26 (1891), p. 102.

s L'Allaitement, Paris, 1898.

4 A closely allied example of an alcoholic fermentation occurring alongside other chemical changes is obtained in the ginger beer plant investigated by Professor Marshall Ward in 1891 (see Philos. Trans. Roy. Soc., 1892, vol. 183, pp. 125-197). It does not, however, set up alcoholic fermentation in milk-sugar. Its activity depends on Sacch. pyriformis and Bacillus vermiformis. Another example of symbiotic fermentation is the fermentation of the sugar cane in Madagascar, and consisting again of a yeast and a bacterium associated. To these, as well as the alcoholic fermentations in milk, may be added Winogradsky's clostridium, and nodule bacteria in Leguminosæ. 


\section{The Coagulative Fermentations of Milk}

We now come to a series of fermentations about which there is considerable obscurity, for of all the fermentations the exact process has been least worked out in the case of these. The prominent feature of their action, however, as far as it concerns milk, is that it results in the formation of a semi-gelatinous clot or jelly, which soon after its formation undergoes a species of contraction and shrinkage similar to the coagulation of blood. Hence for convenience we have assumed the name of the "coagulative fermentations" of milk, meaning by that the clotting of the casein as distinguished from its curdling by acid. The process might perhaps equally well be termed the non-acid fermentations of milk.

We shall obtain a clearer view of these fermentations by considering for a moment two facts, one having relation to alimentary digestion and the other to bacterial products. When milk enters the stomach it becomes coagulated, owing to the effect upon it of the rennin contained in the gastric juice. ${ }^{1}$ The acid action is prevented in all probability by being neutralised by the alkaline salts contained in the milk. In this way, the rennin, and not the acid, obtains primary action on the consumed milk, and its action is that of a coagulation fermentation. If the acid predominated we should get a curdling as is obtained in lactic acid fermentation, when the casein, being dissociated from the lime salts and not soluble, would be precipitated as a flocculent curd. Here then is a simple example of the fermentations we are about to consider taking place in the stomach naturally.

The second fact to be borne in mind, is that certain bacteria produce chemical ferments allied to, or identical with, the enzymes (see p. 152). The class of bacteria in general which liquefy gelatine have been found to possess proteotytic characters like trypsin (Lehmann, Hueppe), and many of them also produce a ferment which coagulates milk without rendering it acid. Here then are two functions of bacteria analagous to the double fermentation capacity of the gastric juice, the one coagulating like rennin, the other digesting like pepsin and trypsin.

For a number of years these general principles had been held as probably the explanation of certain facts known to physiologists.

1 Gastric juice is composed in the main as follows: water, 99 per cent. ; hydrochloric acid, 0.3 ; organic ferments (pepsin and rennin), 0.3 ; and salts (including calcium chloride), 0.3 per cent. 
The matter was however demonstrated by Fermi and Conn. The former isolated from bacterial cultures a tryptic (proteolytic) ferment, and the latter isolated from milk cultures of several species of bacteria a ferment having all the essential characteristics of the rennet (coagulation) ferment. ${ }^{1}$ Both ferments are frequently produced by the same species of bacteria, although not in equal quantities. Other species only digest without previously coagulating.

We are now in a position to consider the two chief examples of these coagulation fermentations of milk. The class of organisms which produce them is abundant in the dairy, though not so abundant as the lactic acid organisms. The relationship between the two is highly variable. It is stated by some authorities that the different species favour different localities. The two examples of coagulation ferments produced by bacteria may now be briefly considered.

(i.) Rennet.-Rennet is of course well known as the agent in the production of cheese. If a small quantity is added to a relatively large quantity of milk the latter rapidly changes to a set jelly, contracts, exuding the whey, and eventually becomes the firm clot forming the basis of cheese. The element of the milk chiefly affected is the casein, which when acted upon by rennet gives rise to a body known as tyrein, the proteid constituent of the clot. The whey also contains a by-product resulting from the alteration of the casein. The clotting of rennet is not to be confused with the precipitation of casein by acid. The two processes are distinct. The curd of the latter is a soluble body; the clot of the former is a decomposition of casein with the formation of an insoluble curd, which is sweet rather than sour, and hence sometimes called "sweet curdling." The chief source of rennet is the mucous membrane of the stomach-where it occurs in the gastric juice as rennin-and is present in largest quantity in the stomach of young animals, particularly the calf. It exists also in the pancreas of various animals, including man. It may be prepared in an impure form by mincing the organ finely, and extracting the pulpy mass with glycerine water, or solution of neutral salts. ${ }^{2}$ It has also been found in the stomach of birds (Roberts), and fishes (Benger).

The salts of calcium play an important part in the action of

1 Storr's Station Report, 1892, Rep. v., p. I96.

2 The Soluble Ferments and Fermentation, J. Reynolds Green, F.R.S., I899, p. 236. 
this ferment, the most pronounced effect being produced by calcic phosphate. Indeed, it is generally held that clotting of milk will not take place in the absence of this salt. Hammarsten explains this by saying that the ferment induces the conversion of casein into tyrein, and the calcic phosphate causes the latter to separate out as a clot. The character of the clot depends also upon the source of the milk. Cow's milk coagulated by rennet yields a more solid clot than human milk, which clots loosely and with a flocculent consistence. This is due, of course, to the fact that cow's milk contains much more casein and calcium than human milk. Rennet will clot boiled milk but not so firmly, on account of the precipitation in boiled milk of the soluble calcium salts (Söldner).

On adding a small portion of such a solution of rennet to sweet, unboiled, lukewarm milk, the latter gradually curdles, the coagulum thus formed being a derivative of casein. The casein is in this case split up into two portions differing greatly in amount, viz., lacto-protein, small in quantity, soluble, and remaining in the whey, and the insoluble paracasein. The latter, therefore, forms the chief constituent of the coagulum separated ("set") in cheesemaking by the aid of rennet, and known as rennet curd (Ger. Bruch), or crude cheese (Hammarsten). Casein or paracasein, though the sole nitrogenous constituent of the coagulum produced in any of these methods, is, however, by no means its sole component, a number of other substances being precipitated at the same time. If whole milk-i.e. unskimmed milk-is set for cheese, almost the whole of the fat will be found in the curd, which will then subsequently produce rich cheese, skim milk cheese being the result in the converse case. Along with the fat, the calcium phosphate contained (in suspension) in the milk will also be thrown down only in the case of rennet coagulation, not in the curd produced by acidification (Lafar).

Lafar has also pointed out that not only are fat and calcium phosphate carried down by the coagulum, but also a large number of the organisms present in the milk, so that the clot is relatively as rich in organisms as the milk from which it was precipitated.

Acid curd differs from that produced by rennet, both in the method of production and also in composition. Being devoid of flavour, both kinds are, however, unsuitable for food; their conversion into a form in which they both stimulate the appetite and are also themselves more readily digestible, is the task of the cheese-maker's art.

But rennet is not alone a product of animal metabolism. 
Certain plants, for example, contain it. In certain parts of the country Galium verum is used to clot milk for cheese-making. In the Italian Alps Pinguicula vulgaris (butter wort) is used in a similar manner (Pfeffer). The secretion of the glands of Drosera clots milk, and so also do the juice of the papaw and the pine apple. Generally speaking it is the fruit and seed of a plant which contains rennet. The ferment has also been obtained from bacteria. In 1852 Haubner found that milk would coagulate under certain conditions without previous acidification, without lactic fermentation, and without the addition of rennet as such. Thirty years later Duclaux studied this matter and finally showed that such coagulation was due to micro-organisms which had the capacity of producing rennet themselves. Wood has isolated the proteolytic ferments produced by a variety of bacilli, and he found in many cases that rennet was also present. Vignat has shown that rennet exists in the $B$. mesentericus vulgatus side by side with four other enzymes. Conn working in 1892 , forty years after Haubner, was able to isolate rennet from bacterial ferments as follows. He clotted milk with the organisms in question, then broke the clot down and mixed it with distilled water, and filtered the whole through porcelain. Then he added sulphuric acid till the whole liquid contained - I per cent. acid, and it was then saturated with sodium chloride. A white granular scum rose to the surface which was found to consist of rennet. Rennet was most plentifully secreted when the temperature of the cultures was about $20^{\circ} \mathrm{C}$, and the proteolytic ferments preponderated at $35^{\circ} \mathrm{C}$.

Flügge has placed the peptonising bacilli of milk under a systematic classification. He differentiates four anaërobic bacteria (of the Rauschbrand group), one anaërobe with terminal spores (Tetanus group), and elevep other anaërobes whose spores, with oné exception, withstand two hours' boiling.

(ii.) Casease is the second body of this group to which brief reference must be made. Not infrequently the coagulation of casein effected by such bacteria disappears again after a short time. Duclaux ${ }^{1}$ ascertained that this new alteration is due to a second (albumin-dissolving) ferment, to which he gave the name of Casease. It acts not only on the clot but on the unchanged casein of milk, and is of the nature of trypsin. The same observer also discovered, in the case of several species of Tyrothrix isolated from Cantal cheese, certain bacteria gifted with the faculty of

1 Le Lait, 1894, p. I13. 
excreting both enzymes, the casein precipitant and the casein solvent. These species liquefy nutrient gelatine.

The ratio between these two ferments differs in the various species. A few produce the casein solvent alone, and when sown in milk do not precipitate the casein, but decompose it direct into soluble fission products, among which leucin and tyrosin have been identified. In proportion as the casein disappears the milk becomes clearer, and is finally quite transparent. The essential phenomenon of this change is digestion of the casein. Milk which has undergone lactic fermentation cannot be so digested. Whilst rennet acts only within narrow limits of temperature, casease has a wider sphere of activity, and it has been shown that some organisms produce both enzymes, at first curdling the milk and subsequently digesting it.

The constitution of casease has not yet been accurately determined, neither has any one succeeded in ascertaining in what precise respect this enzyme differs from pepsin and trypsinwhich it greatly resembles in action-nor whether the casein-dissolving enzyme produced by different species of bacteria is the same in all cases. Weigmann states that he has isolated casease from bacterial cultures, and that this substance favours and accelerates ripening when added to fresh cheese. Occasionally these non-acid coagulative fermentation organisms occur in dairies and curdle milk. They have also been found (because of their spores) to withstand sterilisation. Organisms which digest casein liquefy gelatine, and for this reason are readily demonstrable in culture. This feature of liquefaction has been commonly used as an indication of the quality of a milk. If a milk contains few liquefiers, though many bacteria, the milk, though not fresh, is probably not greatly contaminated. If, on the other hand, milk contains many organisms, most of which are liquefying, its quality is at once open to suspicion. As we have pointed out, these species are as a rule overpowered by the lactic acid organisms.

The spontaneous coagulation of milk without the co-operation of micro-organisms, the possibility of which was maintained by early workers, and finally established as a fact by Meissner, was more closely examined in 1887 by A. Levy, who found that a very faint coagulation can be detected in all milk that has been left to stand for some time. ${ }^{1}$ He pointed out that the sediment deposited

${ }_{1}$ The rapid curdling of milk so frequently observed during thunderstorms has been explained in various fantastic ways. The opinion expressed by $\mathrm{J}$. Liebig in 1890 will not bear investigation, and the assumption put forward, on 
by such milk contains, however, only a small quantity of coagulated casein, the bulk consisting of small fragments of decomposed colostrum. As the cells of this latter substance die off a slight degree of acidification ensues, which causes the precipitation of a certain quantity of casein. ${ }^{\mathrm{t}}$

\section{Diseases of Milk}

A "disease" of milk may be described as the production therein, by the action of a specific micro-organism, of such an organic change as will render it or its resultant products unfit for human alimentary purposes, although in themselves they may have neither poisonous nor pathogenic properties. Some authorities have described these conditions as "milk anomalies."

The diseases of milk, properly so called, are but few in number and they may be comprised under the following heads, viz.:I. Blue Milk. 2. Red Milk. 3. Yellow Milk. 4. Bitter Milk. 5. Soapy Milk. 6. Viscous, Ropy, or Slimy Milk.

\section{(I.) Blue Milk}

A disease characterised by blue flecks in the milk either locally on the surface or permeating the whole mass by diffusion, and appearing in from twenty-four to seventy-two hours after milking, according to temperature and climatic conditions.

The first attempt to investigate the nature of this disease, which frequently takes an epidemic form, appears to have been made by Ehrenberg and Steinhof about the year 1838 , but it was not until the year 184I that Fuchs, working in their footsteps, clearly demonstrated that the diseased condition was transmissible, and due to a specific micro-organism, which however he was unable to isolate owing to defective technique. To this-now known as the Bacillus cyanogenes of Hueppe-Fuchs gave the name of Vibrio cyanogenes and subsequently Bacterium syncyaneum. In later years it has been studied by many workers, especially by Neelsen (I880), Hueppe, Flügge, Heim, and Gessard, who showed that to it, and as far as could be ascertained to it alone, the distinctive

experimental grounds, by G. Tolomei, that it is caused by the action of ozone produced by electrical discharges, is also unsupported by evidence. The effect of thunderstorms on milk is almost certainly indirect. It is bacteria which sour milk and the climatic conditions of a thunderstorm hasten their development.

${ }^{1}$ For a discussion of fermentations allied to the coagulation processes see Centralb. f. Bakt., iii. Bd., 1897, 615 . 
coloration was due. ${ }^{1}$ In the year 1896 , however, M. Zangmeister, working on the same subject, claimed to have isolated ${ }^{2}$ a microorganism which, although resembling in many respects the Bacillus cyanogenes, presented certain morphological and cultural properties sufficient in his opinion to differentiate it from that of former workers in the same field. To this he gave the name of Bacillus cyaneo-fuorescens, a description of which will be found in a subsequent chapter ( $p .430$ ). It has been asserted that the Bacillus janthinus of Zopf has the power of colouring milk blue or bluishviolet.

The Bacillus cyanogenes (Bacterium syncyaneum of Ehrenberg) or as Hueppe originally termed it, the Bacillus lactis cyanogenus, is an anaërobic, non-liquefying, bacillus motile, bi-polar, flagellated, chromogenic, and round-ended with a varying average length of from I to $4 \mathrm{~mm}$. by $\cdot 3$ to $.5 \mathrm{~mm}$. in breadth. Spore formation has been claimed by Hueppe and others, but this is denied by Heim, who describes the so-called spores of Hueppe as involution forms only. A careful investigation by the authors, and the fact that these so-called spores cannot withstand a temperature of $80^{\circ} \mathrm{C}$. for more than one minute, would tend to confirm this view. In liquid cultures curious involution forms are often observed, which are especially noticeable if the organism is grown in mineral media, as those of Conn and Nrgeli.

The organism does not liquefy gelatine and grows freely on all the usual laboratory media at room temperature, the dark purplish blue or in some cases brownish coloration of the medium being very characteristic, but this freedom of growth becomes less as the temperature advances to $37^{\circ} \mathrm{C}$, and the cultures themselves die at $40^{\circ}$. The reaction is invariably alkaline, although the medium itself may have been in the first place acid. It stains well with all the ordinary stains but does not hold the Gram.

In milk the bluish tint would appear to be dependent upon certain conditions, and in the sterile milk used for the laboratory purposes it is not easy to obtain it "in vitro."

1 It is well here to note that a certain blue or bluish coloration of milk when freshly drawn has been attributed by certain investigators to the fact of the cow having partaken when in pasture of one of the Butomacæ (Butomus umbellatus), a not uncommon plant on water meadows and which contains a blue pigment of the nature of indigotin. The authors, however, have never been able to trace an authentic case of blue coloration of milk to this cause.

${ }^{2}$ Centralb.f. Bakt., I sec. XVIII., p. 32 I. 
These conditions appear to be:bacteria.

I. An association of other organisms-the lactic acid producing

2. The production of acid set free by these organisms in such proportion as to be readily taken up by the Bacillus cyanogenes as soon as formed, so that it never exists in excess.

The addition to sterile milk of lactic acid per se will not, in conjunction with the micro-organism, give rise to the coloration, and to make it appear under these conditions it is necessary to add to the milk some natural substance (e.g. glucose), of which the organisms can make use in producing such an amount of acid as is necessary for their existence and development, for alone they have not the power of reducing milk sugar to lactic acid. They ferment glucose with production of acid, the blue colour in this case being produced. The milk infected with the disease transmits its colouring properties to butter prepared from it.

Introduced into ordinary milk the organism produces no coagulation, and the reaction becomes fully alkaline; a bluish coloration appears on the surface at first in patches, which gradually extend themselves over the whole surface. The milk serum, however, rarely becomes affected. Grotenfeld states that its ordinary growth in milk favours the multiplication of many common milk organisms which in their turn destroy $B$. cyanogenes.

In sterile milk little or no blue tint is apparent; the upper surface, however, at times takes a greyish hue if the reaction is acid.

Cultures of the organism made in mineral media are very characteristic; the medium quickly becomes turbid, and a thin white veil appears on the surface, the medium itself taking a bluish colour. Later a green tint appears, and this gradually extends until after some twelve or fourteen days the whole of the liquid becomes coloured, the veil itself turning a greenish-grey. In older cultures the greenish tint of the liquid turns to yellow, and the reaction is strongly alkaline.

In saccharine solutions or glycerinated media the blue colour is rarely, if ever, present. The best temperature for the formation of colouring matter would appear to be from $15^{\wedge}$ to $18^{\circ} \mathrm{C}$.; it is difficult to obtain at $25^{\circ}$, and at $37^{\circ}$ loses the property entirely.

A temperature of $60^{\circ} \mathrm{C}$. for some minutes would appear entirely to destroy the activity of the organism, but it stands desiccation for a lengthened period. ${ }^{1}$ It is not pathogenic.

${ }^{1}$ According to Heim this period does not extend beyond five or seven months. 


\section{(2.) Red Milk}

The peculiar red coloration sometimes apparent in milk can be traced to several causes, two species of the sarcina group amongst others possessing this property, which is equally shared under certain climatic conditions by the Bacillus prodigiosus, ${ }^{1}$ although in this latter case it is the surface only on which the red coloration takes place.

It is of importance clearly to differentiate between milk reddened by the admixture of blood from the mammary gland, and that produced by the organism isolated and studied by Hueppe and Grotenfeld-Bacillus lactis erythrogenes - the presence of which in the milk is now looked upon as the active causation of the disease. In the former case the coloration is apparent immediately after milking, is uniform, and if the milk is allowed to rest the flocculent blood coagulum causing the coloration will gradually sink and deposit itself in the form of a precipitate at the bottom of the milk receptacle. In the latter the red spots do not appear until later, the infection of the milk is comparatively slow, and the milk serum is alone affected, the cream layer not taking the red coloration. This is probably due to the simple fact that the cream layer being on the surface is exposed to the light, which inhibits the coloration. A general coagulation of the milk takes place accompanied by a distinctive sickly sweetish odour. The red coloration will not take place if the milk is exposed to light or has an acid reaction.

The Bacillus lactis erythrogenes of Hueppe (Bacterium erythrogenes of Grotenfeld) is an aërobic, liquefying, non-motile, nonsporing, chromogenic bacillus of I to $1.5 \mathrm{~mm}$. in length by .3 to $.4 \mathrm{~mm}$. in breadth, at times attaining, especially in broth cultures, a length of 4 to $5 \mathrm{~mm}$. in the form of filaments. It takes readily all ordinary stains and holds the Gram. In sterile milk a gradual precipitation of the casein takes place with a neutral or slightly alkaline reaction of the medium. The resultant serum, in the absence of light, absorbs the red colouring matter produced by the organisms, taking a deep red tint provided the medium has no acid reaction. The coagulation by rennet of milk infected with the organism has the effect of producing a marked dirty red coloration, changing to a reddish-brown and finally to blood red.

The organism produces no red coloration in the presence of 1 According to Hueppe "mostly B. prodigiosus-rarely by Sarcinæ." 
light. The pigment is insoluble in alcohol, ether, or chloroform. It grows at room temperature on all the ordinary media, but more freely between $28^{\circ}$ and $35^{\circ} \mathrm{C}$. Upon Petri dishes of gelatine whitish-grey spherical colonies gradually turning yellow appear, the gelatine slowly liquefying in saucer shape and acquiring a pinkish colour. Upon agar a more yellowish hue is developed, with a slight yellowish red coloration of the medium in immediate proximity to the growth. In gelatine stab cultures, the culture takes a yellowish tint-liquefaction is slow and the liquefied gelatine becomes turbid and takes a pinkish colour, the welldefined colonies of the organism remaining suspended therein. On potato, development is rapid, a greyish-white layer being formed which gradually turns to sulphur yellow, the potato itself taking a dark colour which later turns to a yellowish-red.

The disease cannot be looked upon as a difficult one to combat, the ordinary precautions as to cleanliness of byres, dairy and milk utensils, usually resulting in its complete disappearance.

The power of reddening milk has also been attributed to the red sarcina, a red yeast, B. rubidus, and Micrococcus cinnabareus.

It is said that a large proportion of red madder (Rubia tinctorum) in the pastures will sometimes give rise to a distinct red coloration in milk. The authors, however, have failed to trace any authentic record of this.

\section{(3.) Yellow Milk}

This is a defect of boiled milk caused by the Bacillus synxanthus of Ehrenberg, more recently studied by Schröter under the name of Bacterium synxanthum. A short thin actively-motile bacillus producing a coloration of a deep golden yellow, which is easily soluble in water, but not in alcohol or ether. Treated with acid, the yellow colour disappears, returning on re-alkalinisation.

Boiled milk infected with this organism coagulates in from twenty-four to thirty-six hours, the yellow coloration, however, not appearing until later. Slow liquefaction of the precipitated casein then sets in, the milk becoming gradually converted into a yellowish watery liquid with strong alkaline reaction. The fact that it is boiled milk alone which is liable to infection, would tend to show that it is the lactic acid bacteria which in this case act as the inhibiting agent, until destroyed by the process of boiling.

In any case, however, the disease, if it can be so called, is of slight importance from a practical point of view. 


\section{(4.) Bitter Milk}

From an economic point of view this "milk anomaly" is perhaps the most serious with which the dairy bacteriologist may have to deal, for, once the organism gains admittance to a dairy it is somewhat difficult to eradicate, and although from the intermittent nature of the disease one would be led to suppose that its development is accelerated or retarded by certain climatic conditions, yet the frequent and continued recurrence of the condition after considerable lapses of time are often a cause of serious loss to the dairy industry. The defect is rarely observed in fresh milk, owing probably to the time required for the due development of the organism, but appears chiefly in the cream set apart for butter-making purposes, in which it causes the development of a peculiar bitter taste which makes both it and the resultant butter unfit for alimentary purposes.

In the Report of the Dairyman's Association of Ontario for I900, it is stated that during the first year the creameries in the North-West territories were working, the milk had no bad flavours, but that latterly bad and bitter flavours had developed. This was attributed by certain Canadian authorities to insanitary conditions of milk "factories," and by others to the importation of microorganisms able to set up bitterness in milk.

The disease has been attributed to several micro-organisms, as well as to irregularity of diet in the cow, and the question has been closely studied by various workers, especially by Weigmann, Hueppe, Freudenreich, and Bleisch.

Hueppe has suggested that some bitter conditions of milk are due in part at any rate to a proteid decomposition, the final products of which are bitter. ${ }^{1}$ Peptones may be demonstrated by the biuret reaction. Such substances may act like alkaloidal poisons upon animals, and are produced by bacteria from the albuminoids of milk. It is probable that these or similar conditions give rise to benzine derivatives, the most important of which is diazo-benzine or tyrotoxicon, isolated by Vaughan of Michigan. Spasmotoxine is a similar body found by Brieger in putrefied milk. Various

1 We have on more than one occasion met with milk which became bitter after sterilisation, particularly some time after sterilisation. It may be that such bitterness is due to toxic properties remaining in the milk as the result of bacterial activity previous to sterilisation, and possibly to proteid decomposition. Hueppe has met with a similar experience, and attributes the change to ineffectual sterillisation. 
workers have pointed out that certain foods and certain diseases of the udder produce bitter milk. Advanced lactation is said also to bring about this condition under certain circumstances.

There can, however, be little doubt that the chief cause of bitterness in milk is due to the growth of micro-organisms. Such bitterness appears, not immediately after milking, but only after an incubation period.

Amongst the organisms now generally recognised as prime agents in the causation of the disease, are:-

I. The bacillus von bittere milch of Weigmann.-A small, threadlike, motile, spore-bearing bacillus of 1.5 to $\mathrm{I} \cdot 8 \mathrm{~mm}$. in length.

This bacillus was found in sweet milk, and turned sterilised milk bitter in twenty-four hours. It does not digest casein, nor does it produce butyric acid. Weigmann thought the bitterness was due to the decomposition of the albuminoids of milk.

2. Conn's micrococcus of bitter milk is an aërobic coccus isolated from bitter cream, which, inoculated into nutrient gelatine, liquefies it and makes it slimy and of bitter taste. It coagulates milk, with subsequent gradual liquefaction of the precipitate, and production of an intensely bitter flavour and a slight acid reaction. The presence of butyric acid was determined in the culture, and butter made from the bitter milk soon became rancid. This organism is of particular interest as being the first microbe of bitter milk naturally experienced.

Although, as we have already said, the above two organisms may be looked upon as the principal agents in the causation of the disease, it must be noted that many other organisms, and especially those of the Hay bacillus group, almost all of which seem to have the power of peptonising casein with the production of a markedly bitter flavour, are endowed with the property of producing a bitterness in milk and its products. Amongst these may be cited:

3. The bacillus of bitter milk of Bleisch-A facultative anaërobe, motile, flagellated, spore-bearing bacillus, liquefying gelatine, and producing a whitish-grey growth upon agar and potato. It was first isolated from imperfectly sterilised milk. According to Bleisch, the spores of this organism can withstand boiling for a lengthened period. ${ }^{1}$

4. The micrococcus casei amari of Freudenreich.2-Isolated from bitter cheese, and which, inoculated into milk, produces a markedly bitter flavour both in it and the resultant cheese. This organism

1 Zeitschrift f. Hyg., Bd. xii., 1893, p. 81.

2 Ann. de Micrographia, Bd. vii., 1895, p. I. 
belongs to the class of lactic acid bacteria, coagulating milk by acid reaction, the bitter flavour becoming accentuated as the coagulation proceeds. It liquefies gelatine, grows well in broth or nutrient agar, and on potato gives rise to white colonies with yellow margins.

5. The tyrothrix geniculatus of Duclaux. ${ }^{1}$ - Isolated from Cantal cheese, and producing a bitter flavour both in milk and soft cheeses; the former, however, according to Duclaux, not proving a favourable medium for the development of the organism.

6. The bacillus albus of Löfler.-An organism resembling the Bacillus subtilis, and taking in milk the form of threads. Liquefies gelatine very rapidly, and forms a thick layer on agar. It produces peptone and butyric acid. ${ }^{2}$

7. Bacillus liquefaciens lactis amari of Freudenreich.-Isolated from bitter cream. A motile bacillus of varying length ( $\mathrm{I} \cdot 5$ to 5 or $6 \mathrm{~mm}$.) and averaging $.5 \mathrm{~mm}$. in breadth-liquefies gelatine and coagulates milk, producing an intense bitter flavour without acid reaction.

8. The bacillus pseudo-butyricus of Hueppe is a peptonising organism of the Hay bacillus type, which coagulates milk without production of lactic acid. It liquefies gelatine rapidly, and forms bluish-white growths upon agar. It will be remembered that ordinary butyric fermentation (see p. I 59) adds a bitterness to milk, as Hueppe first pointed out. In his first paper, published in Berlin in 1884 , he definitely stated that bitterness was not due to lactic acid bacteria, but to others present with them. In his second paper (I89I), Hueppe referred the bitter taste to Mesentericus vulgatus as well as to B. butyricus. He expressed the view that bitterness was due to peptones, and supported his contention by stating that all the bitter-producing organisms were peptonisers and liquefiers of gelatine.

To these must be added a series of organisms isolated and studied by Flügge, and designated by him as Bacillus lactis I., II., III., IV., V., VI., VII., VIII., IX., X., XI., and XII., a description of which will be found in the chapter on "Species."

9. Torula amara (Harrison). ${ }^{3}$-Owing to the widespread complaints as to bitterness, at Guelph in Canada, a careful investigation was made by F. C. Harrison, with the result that amongst many common organisms which were present he isolated a yeast to

1 Le Lait, Duclaux, pp. 231-233.

${ }^{2}$ Klin. Wochenschr., 1889, p. 630.

${ }^{3}$ Centralb.f. Bakt., Bd. ix., No. 6/7, 1902, p. 206. 
which he gave the name Torula amara, and which appears to have been the active agent in producing the bitterness. Harrison isolated a torula identical with the bitter organism from leaves of maples near where the milk-cans were kept. It produced bitterness in sterile milk in six hours at $37^{\circ} \mathrm{C}$. In ten days' time the milk becomes thickened and acid, and there is gas production. No butyric acid is present. The organism ferments milk and produces bitter cheese. It could not be found in the fore-milk of Ioo cows at the infected station, though it was present in all samples but two of the can washings.

In $190 \mathrm{r}$ we investigated a case of bitter milk in a country dairy in one of the home counties. The milk had slowly developed a bitter flavour. We isolated an organism of the hay bacillus group, which produced typical bitterness in milk inoculated with it. Thorough disinfection of the whole premises and dairy utensils resulted in an entire disappearance of the pest. Its exact origin remained obscure.

There can we think be little doubt that bitterness in milk is a disease set up by extraneous organisms of more than one species. Not infrequently such a species infects a farm or a dairy for months or even years, and contaminates all the milk and dairy produce. The whole subject is one of obscurity, and has by no means received the attention and careful experimentation which it deserves. We believe, however, that it is safe to assert that the origin of bitter organisms in a dairy is almost invariably conditions of uncleanliness, failure to sterilise or scald utensils, dirty byres, or unclean manipulation.

\section{(5.) Soapy Milk}

This is a defect of milk appearing within twenty-four hours of milking. It is caused by an organism (Bacillus lactis saponacei of Weigmann and Zirn) found in straw, and introduced into the milk. (to which it gives a peculiar soapy taste) through disturbance of the fodder or litter during the process of milking (Freudenreich). On gelatine plate cultures the colonies are white with a yellowish centre, the yellow coloration gradually spreading over the whole, with slow liquefaction of the gelatine. In gelatine stab cultures a funnel-shaped liquefaction takes place, at the bottom of which there is a deposit of yellowish flocculi. In agar stab cultures the growth is more luxuriant, the culture taking a yellowish hue. On potato the growth is slimy and of a waxen yellow. Milk is not coagulated, but becomes slimy and somewhat ropy, with a distinct soapy taste. 


\section{(6.) Ropy or Viscous Milk}

This is a condition of milk produced by certain micro-organisms, some of which have, however, an economic value in the preparation of certain milk products, and can therefore, scarcely be looked upon as "disease-producing." Nevertheless this condition has been traced apparently to garget in the cow. Under conditions favourable to the development of the organisms, the ropiness appears within from twelve to twenty-four hours after milking, and is so pronounced that the milk can be drawn out in long threads or strings. This is a not uncommon condition of milk in Switzerland, where it is considered specially noxious, but in Holland, since the year 1887 , it has been produced by design in the making of Edam cheese. Such cheese ripens quickly and uniformly.

It is to Schmidt-Mühlheim (I882) that we are indebted for the first investigation of this disease of milk from a bacteriological point of view, ${ }^{1}$ although the question had been studied chemically by Girardin as far back as the year 1847 , and later by Lister $(1878)^{2}$ who was the first artificially to infect milk with the disease.

With the study of the question by Duclaux, however, began the first serious attempt to differentiate the various organisms acting as causation of the disease, and these may now be classified as follows :-

I. Bacillus actinobacter (Actinobacter polymorphus) of Duclaux. -A long, thin, non-sporing, facultative anaërobic bacillus of 2 to $3 \mathrm{~mm}$. in length, isolated or appearing in pairs, a most interesting feature being the presence around each organism of an ovoid or rounded gelatinous capsule of from 5 to $6 \mathrm{~mm}$. in length, two bacilli, united end on end, at times being enclosed in the one capsule. In old cultures a shortened form of the rods is observed. The organism grows freely, at ordinary temperature, on almost all the usual laboratory media, showing however a somewhat extraordinary variety of morphological characteristics, according to the medium employed, a character clearly recognised by Duclaux, when he applied to it the nomenclature of Actinobacter polymorphus.

In bouillon there is a characteristic formation of white flocculi, which on microscopic examination are seen to be made up of

1 The organism, supposed by Schmidt-Mühlheim to be the active agent of this disease, was described by him as "an apparently motile micrococcus of I $\mathrm{mm}$. in diameter, isolated or appearing sometimes in chains." No pure culture, however, of this organism seems to have been obtained by him.

${ }^{2}$ Quarterly Journal of Microscopical Science, Oct. 1878, p. 391. 
agglomerated masses of chains of from 3 to $10 \mathrm{~mm}$. in lengththe individual elements of the chains, however, being shorter than those observed in the milk. Grown in bouillon, the organism is non-capsulated. Grown in solutions of glycerine or highly glycerinated media, the capsule reappears, although not to such an extent as in milk. In saccharine solutions, no capsule is apparent, the individual elements are shorter, and the growth gives rise to a glairous veil on the surface of the liquid. Sterile starch paste gives rise to a growth of reddish pellicles on the surface when it is introduced into milk, saccharine or glycerinated solutions, an active fermentation is set up, with the production of hydrogen and carbonic acid gas, and (in the case of milk only) small quantities of sulphuretted hydrogen.

The organism is destroyed at a temperature of $60^{\circ}$ to $65^{\circ} \mathrm{C}$.

2. The Bacillus lactis viscosus of Adametz.-A strictly aërobic, non-motile, capsulated bacillus of I to $1.5 \mathrm{~mm}$. in length, and averaging I $\mathrm{mm}$. in breadth, but found also in threads. Originally isolated by Adametz, from the water of the Liesing brook (Vienna), and afterwards discovered by him in samples of Swiss milk. Introduced into sterile milk, it produces sliminess after a period of two or three days, decided ropiness, however, not becoming apparent for some time afterwards. It does not coagulate milk or liquefy gelatine, and grows freely upon all the ordinary media of the laboratory. On gelatine plates the colonies, in the depth are small, those on the surface are of quick growth, and take the form of slimy whitish drops, with more or less crenated edges. In gelatine stab cultures, the growth is sparse along the needle track. On agar and potato the growth is more luxuriant, viscous, and of a dirty white colour. In bouillon great turbidity is produced, and the medium, like milk, becomes slimy and viscous, and can be drawn out into long threads. The organism does not ferment glucose. The reaction is alkaline. Grown upon glycerinated gelatine, the colonies are characteristic, and develop rapidly in the form of a thin, mucous, transparent, opalescent growth with more or less sinuous borders. The organism takes readily all the ordinary stains, and holds the Gram.

The authors have been unable to trace any sporulation such as described by Zimmerman, and the late investigations of Lehmann and Neumann tend to confirm their view.

The great injury to the butter industry, caused by this organism, has been well pointed out by Adametz, butter produced from cream infected by it being soft and without keeping qualities. It 
probably gains access to the milk by the cleansing of milk utensils with water impregnated with the organism.

3. Micrococcus Freudenreichii of Guillebeau.-A large aërobic and facultative anaërobic coccus of $2 \mathrm{~mm}$. or more in diameter, described by Guillebeau as endowed with motility-often arranged in small chains, especially in broth cultures. First isolated from a milk factory at Berne, which had suffered much from ropy milk, and recently held to be the cause of slimy milk in Switzerland. Cultures on gelatine give a white growth, with rapid liquefaction of the medium-colonies granular and punctiform. On agar the colonies are white. On potato the growth is of sulphur yellow, occasionally taking a brownish tint. Introduced into bouillon it produces great turbidity, which gradually clears, a flocculent precipitate forming at the bottom of the tube. In sterilised milk the organism thrives best at a temperature of $20^{\circ} \mathrm{C}$. The medium becomes rapidly acid, and so stringy that threads of a yard or more in length can be drawn out. If kept at $35^{\circ} \mathrm{C}$, however, the ropiness disappears after two days. There is no production of gas, but old cultures of the organism develop a disagreeable odour. The organism is killed at a temperature of $100^{\circ} \mathrm{C}$. in two minutes, but bears desiccation well.

4. Bacillus Hessii of Guillebeau. ${ }^{1}$-An aërobic, actively motile, non-capsulated, spore-bearing bacillus of from 3 to $5 \mathrm{~mm}$. in length by I to $\mathrm{I} \cdot 2 \mathrm{~mm}$. in breadth. First isolated by Guillebeau from ropy cream at Emmenthal in the Canton of Berne, Switzerland. It liquefies gelatine, and grows upon all the ordinary media at room temperature. On gelatinised milk or nutrient gelatine with an admixture of milk serum, there is a rapid appearance of colonies with a well-defined border, which, however, disappears as the liquefaction of the gelatine proceeds, the colonies with their broken borders remaining suspended upon the liquid surface. The liquefied gelatine is extremely viscous, and may be drawn into threads. Cultivated upon potato, the organism gives rise to a dirty white growth, turning later to a brownish hue. Introduced into bouillon, the medium becomes rapidly viscous and of an alkaline reaction. In milk, however, the organism appears to be soon crowded out by the lactic acid bacteria, when the viscosity disappears. The resultant effect, therefore, cannot be considered so serious as in the case of those organisms to which allusion has already been made.

5. Bacillus lactis pituitosi.-A bacillus described by Löffler as 1 Centralb. f. Bakt., xi. 439. 
a thick, slightly curved rod, growing rapidly at room temperature and not liquefying gelatine.

According to Flügge, the rods quickly break up into segments resembling cocci. On gelatine plates the colonies are white, with a brownish hue by transmitted light. They are of from $\cdot 2$ to $\cdot 5$ $\mathrm{mm}$. in diameter, radiating from centre, and with sharply defined contours. On agar these colonies are of a dirtyish white colour. On potato the growth is of a greyish-white, dry and somewhat granulated. Milk, infected with the organism, takes a distinctive odour and becomes sour and slimy, the ropy condition being more marked in the lower strata.

It is well to observe that although to the above described organisms, the condition known as ropy or viscous milk may under ordinary circumstances be ascribed, there are many other microorganisms which seem to be endowed with this peculiar property. Amongst these may be cited the Bacillus mesentericus vulgatus of Flügge-specially studied by W. Vignal; the Bacillus viscosus $I$ and 2 of Van Laer-isolated from ropy beer, but producing the same effect in milk; the Bacillus gummosus of Happ and others. To various common milk organisms, such as $B$. lactis aërogenes, has also been attributed the power of creating slimy milk.

According to Flügge, it is questionable whether the slimy condition of milk is not identical with the slimy fermentation of substances containing saccharose, such as the juice of the beet and other Chenopodiacia, produced by such organisms as Lenconostoc mesenteroides of Crenkowsky and Van Tieghem, and the Bacillus viscosus sacchari of Kramer.

Allusion has already been made to the fact that although in most countries, and especially in Switzerland, this peculiar condition of milk is looked upon as one of the greatest pests of the cheese-maker, and special attention is paid to its eradication, immediately it has gained admittance to a dairy, by thoroughly fumigating and cleansing both the byres and the dairy, yet in Holland it is produced by design in the manufacture of Edam cheese, by the introduction into the whey of the micro-organism specially studied by Weigmann, and known as the Streptococcus Hollandicus, which, introduced into sterile milk, causes it to become ropy and sour in twelve to fifteen hours at a temperature of from $25^{\circ}$ to $30^{\circ} \mathrm{C}$. This organism was also observed by Weigmann in the commercial products of Norway and Sweden known as Toetmoelk or Taettamoelk (thick milk) and Filmjölk (stringy milk), which are prepared by rubbing the interior of the 
milk-pails with the leaves of the Bog violet (Pingincula vulgaris). It is probable that the action is due to the infection of the milk by a micro-organism which finds its general habitat in the leaf rather than to any peculiar property of the plant itself.

Mention ought to be made whilst on the subject of Diseases of Milk, of the Bacillus lactis niger of Gorini. ${ }^{1}$ The authors have, however, been unable to trace any authentic evidence of any coloration in milk due to this organism, which would seem to be nearly allied to, if not identical with, the Bacillus aterrimus of Biel.

\section{The Prevention of Milk Anomalies}

The measures to be taken in order to check and put an end to any of the above pests of the dairy industry should present no difficulty to the dairy bacteriologist, although if the origin of the infection is traceable to the byre rather than to the dairy, drastic measures may be found necessary.

The first step should be a thorough examination of both dairy and byre-the latter at milking time and without notice to the attendants. The general state of the byre-the litter, fodder-and cleanliness or otherwise of the cows and their milkers should be carefully observed, and deductions drawn therefrom.

In the dairy, the general cleanliness both of utensils and their surroundings should be noted and, if the outbreak can be traced to no other cause, a careful examination of the water used in cleansing utensils should be made. A portion of the milk from each milking for some days, taken both at the time it leaves the byre and at an interval of some hours after its admission to the dairy, should be set aside in a sterile test-tube, and its condition and changes carefully watched. In the case of bitter cream or butter, a portion of the cream at each churning should be preserved, and should the resultant butter prove of bitter flavour, this must be examined, and the organisms therein compared with those of the butter itself. This pest in particular is of such an intermittent nature, that unless this precaution is taken great difficulty may be experienced in isolating the particular organism acting as causation of the disease.

The following measures, which are the result of the personal experience of the authors, will be rarely found to fail to eradicate completely any of the above pests, even in cases when they would appear to have become firmly established.

Byres.-These should be thoroughly cleaned out, every trace of 
fodder and litter removed, and the floors, partitions, and mangers washed down with water and then with permanganate of potash or other antiseptic solution (carbolic acid and its derivatives are objectionable on account of the facility with which milk takes up any strong odour existing in the byre at the time of milking). It may be best to use sulphur fumigation, followed by vigorous spraying of disinfectant solution (chloride of lime, I lb. to 6 gallons of water), or a hose may be used.

After the process of disinfection is complete, two days may be allowed to elapse, and then the byres should be lime-washed throughout.

Insistence should be made that for some days at all events (experience unfortunately teaches that under ordinary conditions it is almost hopeless to count upon this being done after the apparent immediate necessity for it has disappeared), the hands of the milkers should be carefully washed, and the udders, bellies, and flanks of the cows sponged over with clean water before milking, which should be carried out in the bare stall, from which all litter has been removed, as recommended above.

Dairy.-The dairy should be made as air-tight as possible by pasting up the windows and other outlets with paper, as in the case of ordinary fumigation by sulphur. Every article, utensil, and apparatus used in the dairy manipulation (including the milking pails) having been thoroughly washed out with hot water and soda and dried, should be placed in the dairy in such a position that the fumes of the antiseptic used may have free access to all parts.

If this is done after the afternoon's milking, the whole process of disinfection can be carried out with but little interference with the ordinary routine of the dairy, as the utensils will be ready for use the first thing on the morning following, after scalding out with hot water.

With regard to the special disinfectant to be employed, the authors have found that the vapour of formic aldehyde is the most efficacious, and has the advantage over the fumes of sulphur dioxide in not affecting to such a degree the metal work of the various appliances. After disinfection, thorough cleansing and lime-whiting should be carried out.

Dairy utensils are to be treated as above suggested, or thoroughly steamed by forcing steam into them. After steaming, they should be rinsed out with fresh pure water. A pure water supply is one of the most essential dairy requirements if taints are to be avoided. 
Cows.-It is important that after the occurrence of any taint in the milk, the cows should also be specially cleaned. The udders should be washed with water and soda solution ( 2 per cent.) or boracic acid solution (3-5 per cent.).

The most convenient and economical methods of producing formaldehyde gas, which is probably the best gaseous disinfectant to use, are as follows :-

(i.) By the vaporising of polymerised formic aldehyde in the form of paraform tablets placed in an old iron kettle or other utensil suspended over a lamp, or in the Alformant lamp A of the Formalin Hygienic Company.

The paraform is supplied in tablets of I gramme, the vaporisation of ten tablets being sufficient for the disinfection of 1000 cubic feet of ordinary space.

Taking the average dairy at, say, a cubic content of 2000 feet, this would mean the expenditure of twenty tablets, the expense of which is trifling. Should, however, there be any difficulty in making the dairy comparatively air-tight, some margin over the quantity should be allowed, to make good any escape of the gas through unenclosed apertures. After carrying out the measures above recommended, the tablets should be placed in the receptacle, the lamp lit, and the door closed from without and made as airtight as possible. Six hours may be taken as the minimum of time required for thorough disinfection, but a more convenient course will be for the door to be kept closed till the following morning, when the utensils can be taken out, and the dairy put in order for the work of the day.

(ii.) Autoclave under pressure.-Trillat's apparatus for the liberation of the aldehyde from formalin (a 40 per cent. solution of formic aldehyde being used, to which is added a little calcium chloride) is equally efficacious, but the expense of the autoclave is a serious consideration.

(iii.) Retort without pressure may also be used. In both these cases the gas is passed, by means of a tube, through the keyhole or some other hole in the door, the apparatus remaining outside the room.

(iv.) Lingner's apparatus has been used for stables and cowsheds with some success. It is a steam generator which vaporises formalin. ${ }^{1}$

${ }_{1}$ For particulars as to working disinfecting apparatus, etc., see $A$ Practical Guide to Disinfection, by M. J. Rosenau, M.D., and F. J. Allan, M.D., 1903, pp. 93-118; and Supplement, pp. 33-53, and 59-70. 


\section{CHAPTER VII}

\section{ECONOMIC BACTERIA IN MILK AND MILK PRODUCTS}

Cream Ripening. Bacteria in Butter-making. Control of the Ripening Process. Bacteria in Cheese-making. Number of Bacteria in Cheese. Kinds of Bacteria present in Cheese. The Use of Artificial Cultures in Cheese-making. Abnormal Cheese Ripening and Poisonous Cheese.

In the elementary and provisional classification of bacteria found in milk, one group is set apart to include the organisms which are neither pathogenic or to be classified wholly under the organisms of fermentation. It is true many of them fulfil their life function by setting up fermentation. But this is so to speak only a means to an end. The end and result of their activity is beneficial in the economy of nature and in the applications of nature to manufacture. They bring about the ripening of cream and they flavour butter and cheese. It is not unlikely that as our knowledge increases this group of "economic" bacteria in nature generally, and in milk in particular, will become larger and attract more and more interest. In them man possesses powerful allies. Therefore, we have deemed it desirable to insert in the present volume a short chapter dealing provisionally with what is at present known respecting these organisms and their function in milk, cream, butter, and cheese.

\section{CREAM RIPENING}

The number of organisms found in cream is enormous. Probably no other natural medium contains more. We have frequently examined fresh cream in the country and found it to contain more than $100,000,000$ bacteria per c.c. It is not only a favourable medium. It is the filter, so to speak, of milk. For as the cream rises the milk parts with more than 90 per cent. of its contained bacteria. Conn and Esten ${ }^{1}$ found $\mathrm{I} 10,000,000$ of bacteria per c.c. in unripened cream (average of four examinations) and $284,000,000$ in the same cream ripened (average of four examinations). Cream obtained from a creamery gave an average on eight examinations of $56,000,000$

1 Thirteenth Annual Report of the Storr's Agricultural Expt. Sta., Connecticut, I900, pp. 13-33. 
organisms per c.c. unripened, and $350,000,000$ organisms per c.c. ripened. Other examples of unripened cream averaged more than $90,000,000$, but when ripened averaged over $300,000,000$. Normally ripened cream probably averages four or five hundred millions of bacteria per c.c. which is greatly in excess of any other natural media. The number of organisms in unripened cream varies much more widely than in ripened cream, which is due to the fact that "unripened" cream is examined at different stages of the ripening process. What we have already stated as characteristic of souring milk is also true of cream. In both we have detected the "tides" of organisms. At first the ordinary miscellaneous and extraneous bacteria; and secondly, after some thirty hours, the acid producing organisms. Conn and Esten have carried out a series of experiments on cream of a parallel nature to our own experiments on milk, and the results are full of interest. They find that the number of acid-producing bacteria in unripened cream varies from 50 to 97 per cent., whereas in the ripened cream the number varies from 97 to 100 per cent. The total number of bacteria present at the maximum is independent of the number present at the minimum. The maximum is commonly reached within forty-eight hours, after which there is a decline, as occurs in milk. After a week has elapsed a few acid organisms remain, and an abundance of Oidium lactis.

The most characteristic feature of cream ripening is the growth of the acid producing organisms, chiefly $B$. acidi lactici, and the decline of the liquefying and extraneous organisms. $B$. acidi lactici is found in very small numbers in fresh milk as we have already pointed out, and also in cream. But as the ripening process proceeds, with uniform regularity, the numbers of this organism rapidly increase, until ultimately it reaches its maximum, when it furnishes 90 per cent. of all the millions of organisms present. It has been suggested that this bacillus is the cause of the ripening process. Against that suggestion it has to be remembered that cream is ripening within the first twelve hours, when the $B$. acidi lactici and other acid producing bacteria are present in small numbers, and the liquefying and miscellaneous organisms predominate. It would, therefore, appear that other bacteria in addition to the acid bacteria have some effect upon the cream ripening. Conn and Esten think that the process consists of two phases, the first comprising the first twelve hours or more of ripening, and due to the growth of miscellaneous bacteria; the second beginning after twelve hours, and due almost wholly to the growth of lactic acid 
bacteria. As to the kinds of organisms found in ripened cream, we have isolated $B$. acidi lactici, the predominating organism, and $B$. lactis aërogenes. The latter organism does not increase very much during the ripening process, and its part in the process is obscure. In three or four days the number of organisms in cream has abated, and eventually nothing remains but Oidium lactis, as in milk.

In the experiments carried out by Conn and Esten it was found that three types of lactic organisms predominated, when the period of the acid increase arrived and which indicated ripening. During the ripening $B$. acidi lactici increased uniformly, a second form also appeared to increase in proportion, whilst $B$. lactis aërogenes slightly declined. The alkaline or miscellaneous group showed a universal decline as ripening proceeded. ${ }^{1}$ These points are well illustrated in the two following tables:-

Percentages of Chief Types of Bacteria in Cream from a large Creamery.

\begin{tabular}{|c|c|c|c|c|c|c|c|c|c|c|}
\hline \multirow[b]{2}{*}{ Date. } & \multicolumn{5}{|c|}{ Unripened Cream. } & \multicolumn{5}{|c|}{ Ripened Cream. } \\
\hline & $\begin{array}{l}\text { B. ac. } \\
\text { lact. } \\
\text { No. } 1 .\end{array}$ & $\begin{array}{l}\text { B. ac. } \\
\text { lact. } \\
\text { So. } 2 .\end{array}$ & $\begin{array}{c}\text { B. } \\
\text { lact. } \\
\text { aërog. }\end{array}$ & $\begin{array}{l}\text { Total } \\
\text { deid. }\end{array}$ & $\begin{array}{l}\text { Mis- } \\
\text { celia- } \\
\text { neous. }\end{array}$ & $\begin{array}{l}\text { B. ac. } \\
\text { lact. } \\
\text { No. } 1 .\end{array}$ & $\begin{array}{l}\text { B. ac. } \\
\text { lact. } \\
\text { So. } 2 .\end{array}$ & $\begin{array}{l}\text { B. } \\
\text { lact. } \\
\text { aèrog. }\end{array}$ & $\begin{array}{l}\text { Total } \\
\text { Acid. }\end{array}$ & $\begin{array}{l}\text { Mis. } \\
\text { cella- } \\
\text { neous. }\end{array}$ \\
\hline Oct. I9. '99. & $5 I \cdot 6$ & 10.4 & I.9 & $6 f \cdot 3$ & $35-7$ & $63-6$ & $35 \cdot 6$ & 0.5 & $99 \cdot 7$ & 0.3 \\
\hline$\therefore \quad 26,99$. & 770 & $14 \cdot 3$ & $3 \cdot 5$ & $94 \cdot 8$ & $5 \cdot 2$ & 730 & $24 \cdot 0$ & 0.7 & $97 \cdot 7$ & $2 \cdot 3$ \\
\hline Nov. 2,'99. & $71 \cdot 0$ & 23.0 & $3-9$ & $97 \cdot 9$ & $2 \cdot 1$ & $77 \cdot 5$ & 19-0 & $I \cdot I$ & $97 \cdot 6$ & $2 \cdot 4$ \\
\hline Oct. $30,99$. & $50 \cdot 0$ & 350 & 10.0 & $95-0$ & 50 & 5500 & $41-0$ & $2 \cdot 5$ & $9^{8 \cdot 5}$ & $1 \cdot 5$ \\
\hline Mar.23, 00. & $44-0$ & 20 & $6-0$ & $50-0$ & 50.0 & $88-0$ & 100 & $\ldots$ & 980 & 20 \\
\hline May $4, \infty$. & $52 \cdot 0$ & $3 \cdot 3$ & $2-0$ & 870 & 13.0 & $66-0$ & $26 \cdot 0$ & 0.2 & $94-0$ & 60 \\
\hline Jan. I9. 'OI. & $33-0$ & 12.0 & 70 & 520 & $4^{8-0}$ & 770 & $17-0$ & 0.5 & $99-0$ & $I \multimap 0$ \\
\hline " 28, 'OI . & 75.0 & $\ldots$ & 90 & 840 & 14.0 & $89 \cdot 0$ & $\ldots$ & 0.2 & 910 & $9 \cdot 0$ \\
\hline Averages & 56.6 & $14 \cdot 2$ & $5 \cdot 4$ & $78 \cdot 1$ & $21 \cdot 6$ & $73 \cdot 6$ & $24 \cdot 7$ & 0.8 & 96.9 & 30 \\
\hline
\end{tabular}

It should be stated that the miscellaneous group do not produce acid, but otherwise have nothing in common. Some liquefy gelatine, others do not. Conn and Esten made a similar study of percentages in cream from private dairies with the following result :-

\begin{tabular}{|c|c|c|c|c|c|c|c|c|c|c|}
\hline \multirow{2}{*}{ Date. } & \multicolumn{5}{|c|}{ Unripened Cream. } & \multicolumn{5}{|c|}{ Ripened Cream. } \\
\hline & $\begin{array}{l}\text { B. ac. } \\
\text { lact. } \\
\text { No. } 1 .\end{array}$ & $\begin{array}{l}\text { B. ac. } \\
\text { lact. } \\
\text { No. } 2 \text {. }\end{array}$ & $\begin{array}{l}\text { B. } \\
\text { lact. } \\
\text { aêrog. }\end{array}$ & $\begin{array}{l}\text { Total } \\
\text { Acid. }\end{array}$ & $\begin{array}{l}\text { Mis- } \\
\text { cella- } \\
\text { neous. }\end{array}$ & $\begin{array}{l}\text { B. ac. } \\
\text { lact. } \\
\text { No. } 1 .\end{array}$ & $\begin{array}{l}\text { B. ac. } \\
\text { lact. } \\
\text { No. } 2 .\end{array}$ & $\begin{array}{l}\text { B. } \\
\text { lact. } \\
\text { aërog. }\end{array}$ & $\begin{array}{l}\text { Total } \\
\text { Acid. }\end{array}$ & $\begin{array}{l}\text { Mis. } \\
\text { cells. } \\
\text { neous. }\end{array}$ \\
\hline $\begin{array}{l}\text { Jan. } 4 \\
\mathrm{Feb}^{\prime \prime}{ }^{4} 4\end{array}$ & $\begin{array}{l}50 \\
9 \cdot 2 \\
100\end{array}$ & $\begin{array}{l}20 \\
\cdots \\
\cdots\end{array}$ & $\begin{array}{l}\cdots \\
\cdots \\
\cdots\end{array}$ & $\begin{array}{l}700 \\
9=0 \\
1=0\end{array}$ & $\begin{array}{l}93 \\
91 \\
99\end{array}$ & $\begin{array}{l}96 \\
86 \\
59\end{array}$ & $\begin{array}{r}1=0 \\
1200 \\
2=0\end{array}$ & $\cdots$ & $\begin{array}{l}97 \\
98 \\
62\end{array}$ & $\begin{array}{r}3 \\
2 \\
38\end{array}$ \\
\hline Averages & $5 \cdot 1$ & 2.0 & ... & $5 \cdot 7$ & 94 & 80 & 50 & $3-0$ & 85 & 14 \\
\hline
\end{tabular}

1 See also Zeit. f. Hyg., 1901, xxxviii., p. 387. Kozai finds three bacteria associated with souring, the chief one apparently identical with Esten's B.ac. lact. 
It is clear that in these last three experiments the cream was much fresher than in the creamery experiments, and the results are therefore different. This led to a series of experiments (nine in all) to show the variations in the proportions of the different types of bacteria during cream ripening. One fair example of the results is given, as follows :-

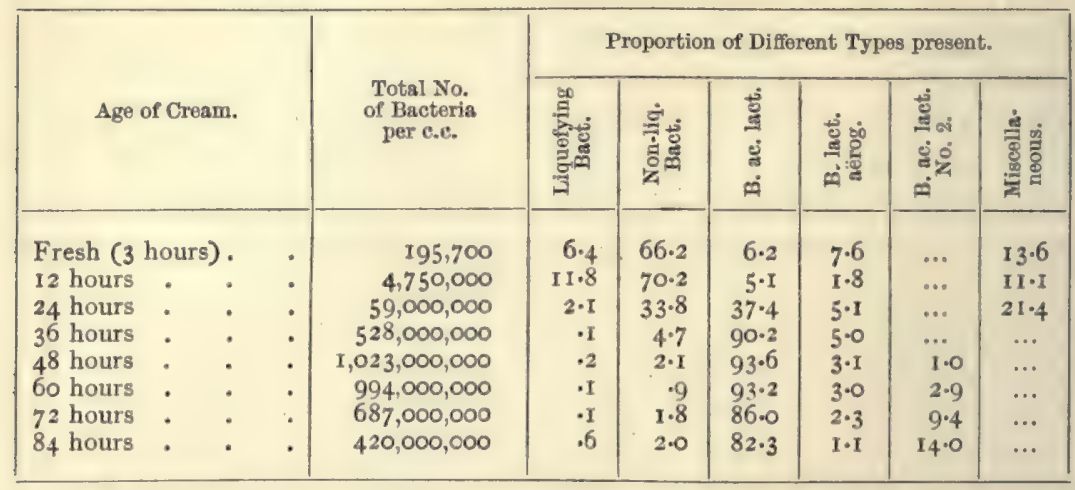

The various points to which we have made reference are here illustrated in tabular form. The parallel between the rise and fall of the acid organisms in cream and those in our own experiments in milk (see p. I30) is very striking. The rise at forty-eight hours, the prodigious number of organisms then present, and the fact that this is the exact period of the zenith of the lactic acid organisms is shown with remarkable clearness in this Table, and a careful study of it will furnish an exact appreciation of what the ripening process consists. The changes occurring in cream as it ripens are, therefore, due to the enormous multiplication of bacteria, which, by their growth and products, profoundly modify the cream. This multiplication occurs in two stages, the first due to the development of miscellaneous and peptonising species, the second due to the lactic acid species.

The source of lactic organisms. - The origin of the lactic acid organisms in milk and cream is not known. We have carried out various experiments which show-(I) that these organisms do not come from the udder of the cow; and (2) that these organisms are present in the dust, or air, or surroundings of the byre. Rollin Burr also has carried out experiments with the same object and with much the same results. ${ }^{1}$ He assumes that the three organisms

1 Thirteenth Annual Report of Storr's Agricultural Expt. Sta., Connecticut, 1900, pp. 66-8I ; also Centralb. f. Bakt., 2nd Abth., 1902, p. 236. 
selected by Conn and Esten are in fact the three chief types of lactic acid bacteria. He experimented with 70 cows, and found that in each cow if the milk was drawn under sterile conditions no lactic acid bacteria were present in the milk, whilst in the dairy experimented upon all three types were present in the air. The $B$. acidi lactici, though always present in samples of air examined, was not present in excessive numbers. None of the three lactic bacteria were found post mortem in the udders of two cows examined, although $M$. varians lactis was found in the gland part of the udder. Lastly, out of 300 tests of fore-milk only 2 per cent. contained the normal acid bacteria, and those were contaminations from outside.

\section{BACTERIA IN BUTTER-MAKING}

As a preliminary to butter-making, the general custom in most countries is to subject the cream to a process of "ripening." As we have seen, cream in ordinary dairies and creameries invariably contains some bacteria, a large number of which are in no sense injurious. Indeed, it is to these bacteria that the ripening and flavouring process are due. They are perfectly consistent with the production of the best quality of butter. The aroma of butter, as we know, controls in a large measure its price in the market. This aroma is due to the decomposing effect upon the constituents of the butter of the bacteria contained in the cream. ${ }^{1}$ In the months of May and June, the variety and number of these types of bacteria are decidedly greater than in the winter months, and this explains in part the better quality of the butter at these seasons. But butter is not a good bacterial medium on account of its deficiency in nitrogen. As a result of these ripening bacteria, the cream becomes changed and soured, and slightly curdled. Thus it is rendered more fit for butter-making, and gives to butter its pleasant taste and aroma. It is then churned, after which bacterial action is reduced to a minimum or absent altogether. Sweet-cream butter lacks the flavour of ripened or sour-cream butter. The process is really fermentation, the ripening bacteria acting on each and all of the constituents of the milk, resulting in the production of various bye-products. This fermentation is a decomposition, and, just as we found when dis-

1 Various experiments have been carried out to select good-flavouring bacteria from bad-flavouring bacteria. $B$. subtilis and $B$. mesentericus vulgatus have been found to be among the latter.-Centralb. f. Bakt., 2nd Abth., 1898, pp. 730 and 759. (Eckles.) 
cussing fermentation, so here also, the action is only beneficial if it is stopped at the right moment. If, for example, instead of being stopped on the second day, it is allowed to continue for a week, the cream may degenerate and become offensive, and the pleasant ripening aroma would be changed to the contrary. The majority of the species of organisms found in cream are indifferent in their effect upon butter. About 25 per cent. of them, perhaps, exert a favourable influence, some Io to 15 per cent. exerting a bad effect. Of the good flavour-producing bacteria, the majority belong to the acid group. But no hard and fast lines can be laid down. Some develop flavour with acid, others develop aroma with flavour, and others develop aroma without any special flavour. Many of the organisms producing a good flavour in butter are very widely distributed, especially at certain times of the year. It should be added that Babcock and Russell have pointed out that enzymes are present in milk, and take some share in the ripening of cheese. If such enzymes are present in cream, as is likely, it is possible they play some part in cream ripening. Hence cream ripening may be due to a double fermentation, organised and unorganised. ${ }^{1}$

Bacteriologists have demonstrated that butters possessing different flavours have been ripened by different species of bacteria. Occasionally one comes across a dairy which seems to be impregnated with bacteria which improve cream and give good flavour. In other cases the contrary happens, and a dairy becomes impregnated with a species having deleterious effects upon its butter. Such a species may be favoured by unclean utensils and dairying, by disease of the cow, or by a change in the cow's diet. Thus it comes about that the butter-maker is not always able to depend upon good ripening for his cream. At other times he gets ripening to occur, but the flavour is an unpleasant one, and the results correspond. It may be bitter or tainted, and just as certainly as these flavours develop in the cream, so is it certain that the butter will suffer. Fortunately the bacterial content of the cream is generally either favourable or indifferent in its action. Thus it comes about that the custom is to allow the cream simply to ripen, so to speak, of its own accord, in a vat exposed to the influence of any bacteria which may happen to exist in the vicinity. This generally proves satisfactory, but it has the great disadvantage of being indefinite and uncertain. Occasionally it turns out wholly unsatisfactory, and results in ${ }^{1}$ Centralb.f. Bakt., Bd. iii., 1897, p. 615. 
financial loss. The factors which tend to have a favourable influence on butter-making, which, though carried out with cleanliness and good management, is not scientifically controlled, are probably of a simple nature. The bacterial species, which give a good flavour, are probably common in a well-kept dairy, and are more vigorous than the members of the miscellaneous alkaline group. Moreover, the temperature used in ripening cream is found in practice to stimulate the growth of the favourable species of ripening organisms existing normally in a well-kept dairy, or in the milk ducts of a clean and healthy cow. Conn has pointed out that the effect on dairy produce of common bacteria in milk will in measure depend upon conditions, and not simply upon bacterial species. Moreover, there may be natural varieties, in a physiological sense, of one organism, by which different effects will be produced in the butter or cream. ${ }^{1}$

By way of summary, then, we may say that the part which cream-ripening plays in the production of butter may be considered as four-fold. First, ripening makes churning easier and more effectual. Conn accounts for this by suggesting that bacteria soften and dissolve the protein matter connecting the fat globules, and thus cause the fat globules to unite more freely than they do in unripened cream. Secondly, ripening increases the yield of butter, particularly in set cream, and although the increase is small, it becomes not inconsiderable in total amount on a year's working in a large creamery. Thirdly, it is generally held that butter made from properly ripened cream has better keeping properties than that made from unripened or improperly ripened cream. Fourthly, the most important purpose of cream-ripening is the production in butter of a desirable flavour and aroma. It is by this, of course, that butter is judged to be good and marketable, or bad and unmarketable.

\section{Control of the Ripening Process}

There are various means at our command for improving the ripening process. Perfect cleanliness in the entire manipulation necessary in milking and dairying, combined with freedom from disease in the milch cows, will carry us a long way on the road towards a good cream-ripening. Recently, however, a new method has been introduced, largely through the work and influence of Professor Storch in Denmark, and Weigmann in Germany, which

${ }^{1}$ Centralb. f. Bakt., Bd. v., No. 20, 1899, pp. 665-669. 
is based upon our new knowledge respecting bacterial action in cream-ripening. We refer to the artificial processes of ripening set up by the addition of pure cultures of favourable germs. If a culture of organisms possessing the faculty of producing in cream a good flavour be added to the sweet cream, it is clear that advantage will accrue. This simple plan of starting any special or desired flavour by introducing the specific micro-organism of that flavour may be adopted in two or three different ways. If cream be inoculated with a large, pure culture of some particular kind of bacteria, this species will frequently grow so well and so rapidly that it will check the growth of the other bacteria which were present in the cream at the commencement, and before the "starter" was added.

The usual method adopted is to take a small amount of milk, cool it, and add the commercial culture provided. Allow the mixture to stand, protected from dust, for twenty-four hours, and then pour into a larger volume of milk or cream to increase the number of bacteria. The whole "starter" is now ready for use. That is, perhaps, the simplest method of adding an artificial culture. But secondly, it will be apparent that if the cream is previously pasteurised at $70^{\circ} \mathrm{C}$, these competing bacteria will have been mostly or entirely destroyed, and the pure culture, or "starter," will have the field to itself. This is the method as introduced by Storch, and as now practised in Denmark, where 97 per cent. of the butter is made on this principle. Pasteurisation in Denmark is required by law, with the view of exterminating tuberculosis. ${ }^{1}$

There is a third modification, which is sometimes termed ripening by natural starters. A natural "starter" is a certain small quantity of cream taken from a favourable ripening-from a clean dairy and a good herd, and under conditions of strict cleanlinessand placed aside in a covered sterilised vessel to sour for two days until it is heavily impregnated with the specific organism which was present in the whole favourable stock of which the natural starter is but a part. It is then added to the new cream, the favourable ripening of which is desired. A "natural starter" is therefore not a pure culture, but a mixture of which 95 per cent. of the organisms are of two or three species of lactic acid bacteria.

There are at the present time more than a score of different

${ }^{1}$ See also "Pasteurisation as applied to Butter-making," Wisconsin Agricultural Expt. Sta., Bulletin 69, 1898. 
artificial cultures in use in Europe and America. They are supplied in various forms, as milk-cultures in bottles, as powders, or as pastes. Of the species which produce good flavours in butter, the majority are found to be members of the acid-producing class; but probably the flavour is not dependent upon the acid. Moreover, the aroma of good ripening is also probably independent of the acid production.

Methods of artificial ripening.-Of all the methods of ripening -natural ripening, the addition of "natural starters," the addition of pure cultures with or without pasteurisation-there can be no doubt that pure culture after pasteurisation is the most accurate and dependable. The use of "natural starters" is a method in the right direction; though being mixed cultures they are not uniform in action. In order to obtain the best results with the addition of pure cultures, Professor Russell has made the following recommendations :-

I. The dry powder of the pure culture must be added to a small amount of milk that has been first pasteurised, in order to develop an active growth from the dried material.

2. The cream to be ripened must first be pasteurised, in order to destroy the developing organisms already in it, and thus be prepared for the addition of the pure culture.

3. The addition of the developing "starter" to the pasteurised cream and the holding of the cream at such a temperature as will readily induce the best development of flavour.

4. The propagation of the "starter" from day to day. A fresh lot of pasteurised milk should be inoculated daily with some of the pure culture. In this way the purity of the starter is maintained for a considerable length of time. Those starters are best which grow rapidly at a comparatively low temperature $\left(60^{\circ}-75^{\circ} \mathrm{F}\right.$.), which produce a good flavour, and which increase the keeping qualities of butter. Now, whilst it is true that the practice of using pure cultures in this way is becoming more general, very few species have been isolated which fulfil all the desirable qualities above mentioned. In America starters are preferred which yield a " high" flavour, whereas in Danish butter a mild aroma is desired. In this country as yet very little has been done, and that on an experimental scale rather than a commercial one. In I89I it appears that only 4 per cent. of the butter exhibited at the Danish butter exhibitions was made from pasteurised cream plus a culture starter; but in 1895,86 per cent. of the butter was so made, and now upwards of 95 per cent. is so made. Moreover, such 
butter has obtained the prizes awarded for first-class butter. Different cultures will, of course, yield differently flavoured butter. If we desire, say, a Danish butter, then some species like "Hansen's Danish Starter" would be added; if we desire an American butter, we should use a species like that known as "Conn's Bacillus, No. 4I." But whilst these are two common types, they are not the only suitable and effective starters. On many farms in England there are equally good cultures, which, placed under favourable temperatures in new cream, would immediately commence active ripening.

Jensen makes the following demands regarding a lactic acid culture for creamery use-I. That it will sour the cream rather strongly in a comparatively short time; 2 . That it will thrive at a comparatively low temperature; 3 . That it will coagulate the cream to a uniform homogeneous mixture, and give it a slightly sour taste and odour; and 4, That it will produce an agreeable aromatic taste and flavour in the butter. ${ }^{1}$

Professor H. W. Conn, who, with Professor Russell, has done so much in America for the advancement of dairy bacteriology, reports a year's experience with the bacillus to which reference has been made, and which is termed No. $41 .^{2}$ It was originally obtained from a specimen of milk from Uruguay, South America, which was exhibited at the World's Fair in Chicago, and proved the most successful flavouring and ripening agent among a number of cultures that were tried. The conclusions arrived at after a considerable period of testing and experimentation appear to be on the whole satisfactory. A frequent method of testing has been to divide a certain quantity of cream into two parts: one part inoculated with the culture, and the other part left uninoculated. Both have then been ripened under similar conditions, and churned in the same way; the differences have then been noted. It is interesting to know that, as a result of experience, creameries have been able to command a price varying from half a cent. to two cents. a pound more for the "culture" butters than for the uninoculated butters. The method advised in using this pure culture is to pasteurise (by heating at $155^{\circ} \mathrm{F}$.) six quarts of cream, and after cooling to dissolve in this cream the pellet containing bacillus No. 41. The cream is then set in a warm place $\left(70^{\circ} \mathrm{F}\right.$.), and the bacillus is allowed to grow for two days, and the whole is added

1 Copenhagen Expt. Sta., Bulletin No. 22.

2 Storr's Agricultural Expt. Sta., Connecticut, U.S.A., Eighth Annual Report, 1895 . 
to twenty-five gallons of ordinary cream. This is allowed to ripen as usual, and is then used as an infecting culture, or "starter," in the large cream vats in the proportion of I gallon of infecting culture to 25 gallons of cream, and the whole is ripened at a temperature of about $68^{\circ} \mathrm{F}$. for one day. The cream ripened by this organism needs to be churned at a little lower temperature (say $52^{\circ}-54^{\circ} \mathrm{F}$.), but to be ripened at a little higher temperature than ordinary cream to produce the best results. Cream ripened with No. 4I has its keeping power much increased, and the body or grain of the butter is not affected. But it must be borne in mind that the use of starters will not make good butter out of poor cream. More than 200 creameries in America used this culture during 1895 ; which proves that its use for the production of flavour in butter is feasible in ordinary creameries and in the hands of ordinary butter-makers provided they will use proper methods and discretion. ${ }^{1}$ More recently, however, pasteurisation has fallen into abeyance, on account of the added expense, and the use of artificial cultures is said to have declined in America. Nevertheless the principles involved in the cultural process have remained, and as a consequence there has been increased attention paid to cleanly dairying and cream ripening. ${ }^{2}$

In England, with a few exceptions, practically nothing has been done in a commercial way in the direction of artificial starters. But this development elsewhere has doubtless had a beneficial effect upon dairying generally, and in particular upon the teaching of the various dairy colleges and institutes. For example, one of the chief systems now laid down and practised with great success lays stress upon the hygiene and cleanliness of cows and clean milking, in order to avoid the presence of bad-flavouring bacteria in the cream ripening.

The number of bacteria in butter varies considerably according to time of examination. If the butter is just made, as many as thirty to fifty million organisms may be found in one gramme. But if some three or four days old, from 50,000 to two or three million only will be present. This marked decline is an almost invariable characteristic of the bacterial content of butter. Many of the cream organisms have been eliminated in the butter-

1 See also University of Wisconsin Agricultural Expt. Sta., Twelfth Annual Report, 1895, p. 174, and Bulletin No. 48, 1896.

2 Fifteenth Anmual Report of Bureau of Animal Industry, Washington, 1898, p. 85. 


\section{8}

milk, others are inhibited by salting, and yet others find an unfavourable nidus in butter. Reference will be made subsequently (see p. 220) to the presence of B. tuberculosis and its allies in butter. Various pathogenic organisms have been found but only rarely. Butyric fermentation and rancidity are more common. The latter condition is probably due to micro-organisms (possibly Oidium lactis and $B$. fluorescens liquefaciens and its peptonising allies). Putrid butter is caused by various putrefactive bacteria, including B. foetidus lactis (Jensen). Lardy and oily butters have been investigated by Storch and Jensen, and traced to bacteria. Bitter butter is due to fermentative changes.

\section{BACTERIA IN CHEESE-MAKING}

Cheese is made by the precipitation of casein, in the form of a curd. This is usually done by adding rennet to milk; but the precipitation may also be accomplished by allowing acid to develop in the milk, such lactic acid producing curdling. The former method is that usually adopted, though some sour milk or cottage cheeses are made by the latter process. After the curd is produced it is collected in moulds for shaping and pressing, and is eventually set aside for ripening. It is the ripening process which gives to cheese its characteristic flavour, and upon which depends its marketable value. It has been suggested that bacteria, contained in the rennet, cause the ripening, and that thus rennet is responsible for the primary precipitation as well as the secondary fermentation of ripening. That bacteria perform the major part of the ripening process, and are essential to it, is proved by the fact that when they are either removed or opposed, the curing changes immediately cease. If, for example, the milk itself be sterilised (Freudenreich), or if antiseptics, such as thymol, be added (Adametz), the results are negative. Rennet exerts a digestive effect on casein in cheese, due to the presence of peptic enzymes contained in rennet extracts, the action of which is intensified by the development of acid in the curd. But as far back as $1875, \mathrm{~F}$. Cohn held that the ripening of cheese was a true fermentation, due to organisms present in the rennet (termed "lab bacilli"), and several years later Duclaux isolated from Cantal cheese a number of species of the Schizomycetes which he classified under the generic term of Tyrothrix, and associated with that group of bacilli of which the type is B. subtilis. Since Duclaux's work many investigations have been made. 
Adamet $z^{1}$ was one of the first to isolate the cheese bacteria. Wider investigation led the same worker to the conclusion that these bacteria operated on the ripening of milk, not only through the peptonisation of casein, but also through the arrest of the fermentation of milk-sugar. The fermentation of milk-sugar leading to formation of acid and coagulation, can be obtained by different bacteria (Staphylococci, Sarcina, B. coli, and members of the B. subtilis group). Spontaneous coagulation of milk is chiefly caused by $B$. arogenes, and other closely related bacteria -occasionally also by $B$. coli immobilis (Flügge).

At present it is generally held that the ripening changes consist of two processes, namely, chemical changes set up by ferments, affecting the solubility of the curd, and bacterial changes, producing the flavour of the cheese. Freshly precipitated casein, as we have pointed out elsewhere, is insoluble and difficult of digestion. During ripening it becomes converted into soluble bodies, the change being brought about in part by unorganised ferments (enzymes), such as galactase (Babcock and Russell), and casease (Weigmann, and others), and in part by bacteria. It is with the latter that we are concerned, though it should not be forgotten that both are essential to cheese-making. In the second place there is the ripening, and that is probably wholly due to bacteria. ${ }^{2}$

\section{The Number of Bacteria in Cheese}

The number of bacteria in cheese is naturally less than that present in milk or cream. The closer texture and consistence of cheese, coupled with the lessened degree of moisture are sufficient factors to account for this. Nevertheless cheese contains a considerable number of organisms. Adametz found that freshly precipitated curd, moulded in the press and freed from excess of whey, contained between 90,000 and 140,000 micro-organisms per gramme, a comparatively large number of them having the power of liquefying gelatine, or in other words they possessed a peptonising ferment. During the period of ripening the bacterial content of the cheese gradually rose to 850,000 in Emmenthaler cheese, and 5,600,000 per gramme, in a soft household cheese. ${ }^{3}$ Only a small percentage

1 Land. Jahrb., 1889.

See also Centralb.f. Bakt., 1901, vii., p. 187.

${ }^{3}$ Soft cheeses (Camenbert, Brie, etc.) are made by moulding the curd but not subjecting it to great pressure or heat. They ripen rapidly and decay readily. Hard cheeses, on the other hand, are cut in the curd to release the superfluous whey and subsequently subjected to $110^{\circ} \mathrm{F}$. and heavy pressure. They keep well and form the chief cheeses of commerce. 
of this great increase was found to be composed of the peptonising species. Russell has pointed out that "tides" of organisms occur in cheese-ripening much in the same way as we have seen to occur in milk and cream. He points out that there is always at first a marked increase in the number of micro-organisms which is soon followed by a more gradual decline. While the casein-digesting and gas-producing species suffer a more or less rapid decline, the lactic acid species develop to an enormous extent, from which fact it would appear that cheese offers favourable conditions for the development of the latter, and the latter by their acid production, create unfavourable conditions for the former. Russell divides the ripening process, as regards bacterial content, into the three following divisions : ${ }^{1}$

(I) Period of initial bacterial decline in cheese.-Where the green cheeses were examined immediately after removal from the press, it was usually found that a diminution in numbers had taken place. This period of decline lasts but a short time, not beyond the second day. Lower temperature (than that present in the milk) and expulsion of the whey would account for this general decline in all species of bacteria.

(2) Period of bacterial increase.-Soon after the cheese is removed from the press a most noteworthy change takes place in green cheese. A very rapid increase of bacteria occurs, confined almost exclusively to the lactic acid group. This commences in green cheese about the eighth day and continues more or less for twenty days. In Cheddar cheese it commences about the fifth day, reaches its maximum about the twentieth day, declines rapidly to the thirtieth day, and gradually for a hundred following days. During the first forty days of this period the casein-digesting and gas-producing organisms are present and at first increasing, but relatively to only a slight degree. With the increase in the number of micro-organisms the curd begins to lose its elastic texture, and before the maximum number of bacteria is reached the curing is far advanced. Freudenreich has shown, as we have already stated, that acid inhibits the growth of the casein-digesting microbes; and in cheese as in milk it is found that the maximum development of the lactic acid bacteria marks the practical elimination of the peptonising organisms although small traces may persist for some time.

(3) Period of final bacterial decline.-The maximum development

1 Thirteenth Annual Report of Agricultural Expt. Sta., Univ. of Wisconsin, 1896, p. 105. 
of bacteria in cheese is soon followed by a period of decline, at first rapid and ultimately slow. The cause of this decline can only be conjectured, but it is highly probable that is due to the general principle to which we have before referred, namely, that after a certain time the further growth of any species of bacteria is prevented by the action of its own products. It should be noted that the gas-producing bacteria in Cheddar cheese last much longer than the peptonising organisms, being still present up to eighty days.

Two examples illustrating these general principles may be given. The first is the result of an examination of a Cheddar cheese in September:-

\begin{tabular}{|c|c|c|c|c|c|}
\hline $\begin{array}{c}\text { Days of } \\
\text { Examination. }\end{array}$ & $\begin{array}{c}\text { Total No. of } \\
\text { Bacteris per gr. }\end{array}$ & $\begin{array}{l}\text { Lactic Acid } \\
\text { Bacteris. }\end{array}$ & $\begin{array}{l}\text { Gas-Producing } \\
\text { Bacteria. }\end{array}$ & $\begin{array}{l}\text { Casein-Digest- } \\
\text { ing Bacteria. }\end{array}$ & $\begin{array}{l}\text { Percentage } \\
\text { of Lactic } \\
\text { Acid Bsct. }\end{array}$ \\
\hline $\begin{array}{l}\text { Milk } \\
\text { 4th day } \\
\text { Ioth " } \\
\text { I8th " } \\
\text { 3oth " } \\
53 \text { rd " } \\
86 \text { th " } \\
\text { I08th " }\end{array}$ & $\begin{array}{r}26,200,000 \\
\text { I I } 5,400,000 \\
64,350,000 \\
43,264,000 \\
36,887,000 \\
5,304,000 \\
\text { I } 5,223,000 \\
7,084,000\end{array}$ & $\begin{array}{r}5,240,000 \\
\text { II } 5, \text { I } 30,000 \\
64,286,000 \\
43,259,000 \\
36,882,000 \\
5,299,000 \\
\text { I } 5,210,000 \\
7,080,000\end{array}$ & $\begin{array}{r}13,100,000 \\
271,000 \\
64,000 \\
5,000 \\
5,000 \\
5,000 \\
\ldots \\
\ldots\end{array}$ & $\begin{array}{c}7,860,000 \\
\ldots \\
\ldots \\
\ldots \\
\ldots \\
\ldots \\
\ldots \\
\ldots\end{array}$ & $\begin{array}{l}20 \cdot \\
99 \cdot 8 \\
99 \cdot 9 \\
99 \cdot 9 \\
99 \cdot 9 \\
99 \cdot 9 \\
99 \cdot 9 \\
99 \cdot 9\end{array}$ \\
\hline
\end{tabular}

The second is the record respecting a Cheddar cheese examined in February :-

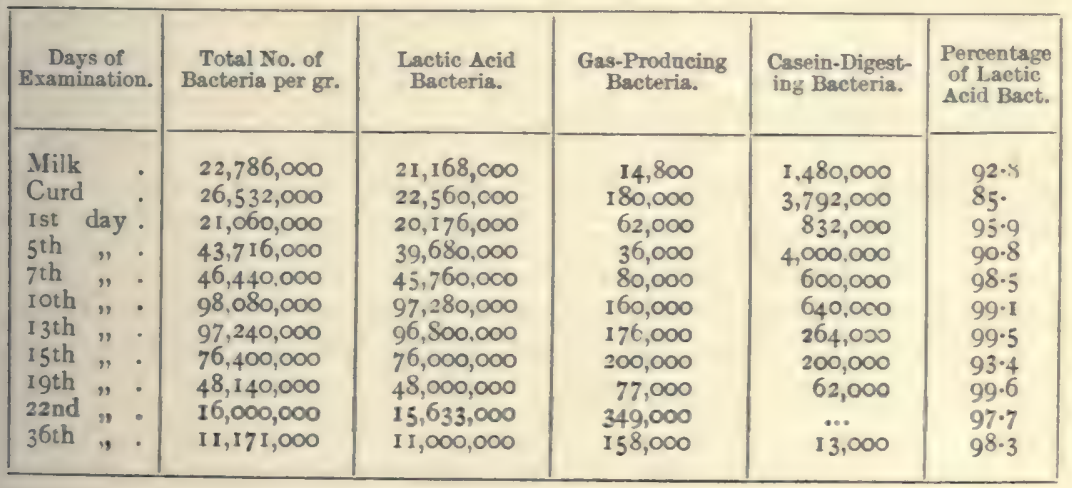

\section{Kinds of Bacteria present in Cheese}

As far as at present known, the micro-organisms present in cheese may be divided into four chief groups :-

(I) The lactic acid bacteria form the largest group. The feature 
common to all members of the group is the production of lactic acid fermentation. At present it is impossible to say that ripening of cheese is solely due to lactic acid species, but there is no doubt that they play an important part in the ripening, and form more than 95 per cent. of all organisms present during the ripening process. When the cheese is partially ripened the species begins to decline.

(2) The casein-digesting bacteria probably assist in the peptonising process of breaking down the curd, but do not take much, if any, part in flavouring.

(3) The gas-producing bacteria give to cheese its honeycombed appearance.

(4) A miscellaneous group of organisms occurring in the milk at the outset of cheese-making, or as intruders from air, rennet, or the various utensils employed in cheese-making, also appear in cheese. In this group are included those "disease" organisms which set up abnormal ripening, and which yet cannot be classified under the three previous heads. ${ }^{1}$

In addition to bacteria, moulds, and possibly yeasts, should be added as agents in the ripening process, particularly of soft cheeses. Conn divides the soft cheeses into three classes according to the relation of bacteria and moulds respectively: (a) soft cheeses in which bacteria alone are concerned in the ripening, the cheeses ripening from the outside (Limburger cheese, Backstein, etc.); (b) soft cheeses in which moulds contribute to the ripening, the moulds growing on the surface and extending to the interior (Brie, Camembert, and others); (c) soft cheeses in which moulds play an important part in ripening, and grow throughout the entire mass. These latter include the Roquefort, Gorgonzola, and Stilton cheeses. In imitations of these cheeses the curd is inoculated on the outside, and planted on the inside with mould spores, and in some cases the process of ripening is facilitated by piercing the cheese with special appliances in order to admit of the entrance of oxygen into the interior, to enable the moulds to grow. After inoculation the cheese is placed under suitable conditions of temperature and moisture. These moulds grow rapidly, and soon

1 One of the classifications of cheese fermentation bacteria suggested in 1896 was as follows :-Sour milk and cheese fermentation bacteria : (1) Bacillus acidi lactici of Hueppe ; (2) Bacillus acidi lactici of Grotenfeld ; (3) Bacillus lacticus of von Günther and Thierfelder; (4) Bacillus acidi lactici of Marpmann; (5) Bacillus acidi lactici of Peters; (6) Bacillus No. I9 of Adametz; (7) Bacillus pallescens of Henrici; (8) Bacillus Schafferi of Freudenreich ; (9) Bacilli $a, b$, and $c$ of Guillebeau. 
HYPHOMYCETES (MOLLDS).

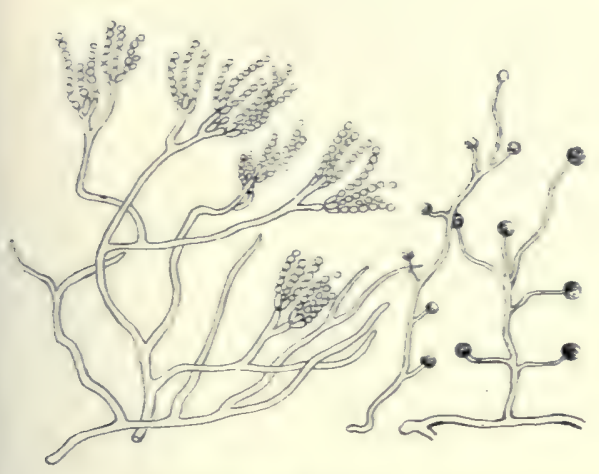

Gammelost cheese ripening moulds.

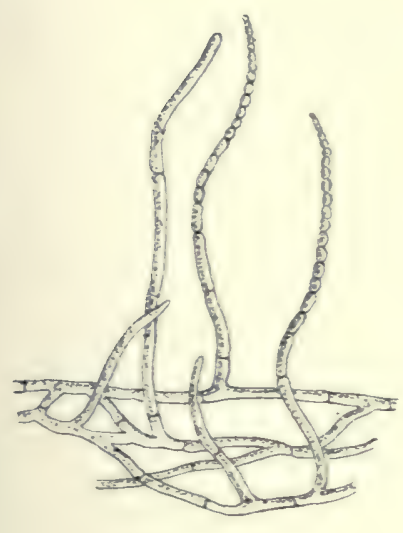

Oidium lactis.

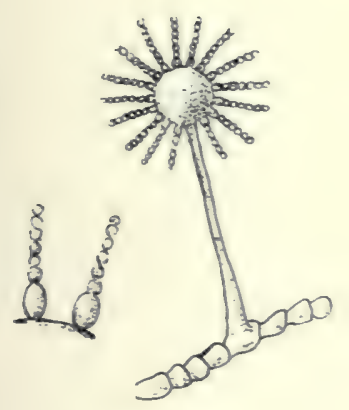

Asperyillas.

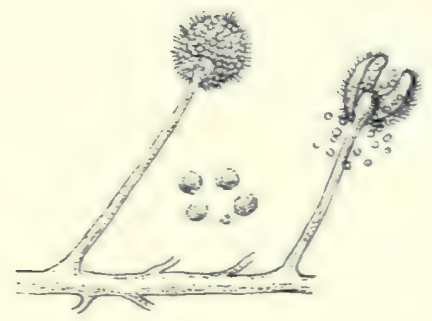

Sueor mucelo.

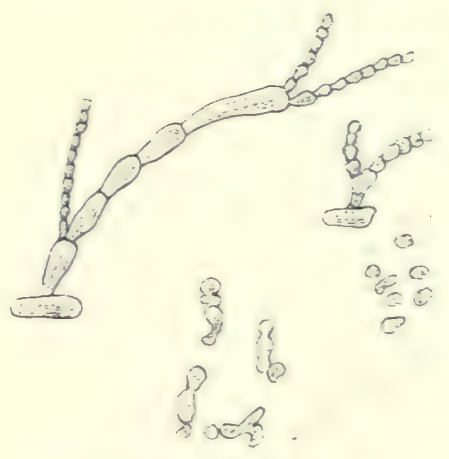

Uidium lactis (mogrifiol)

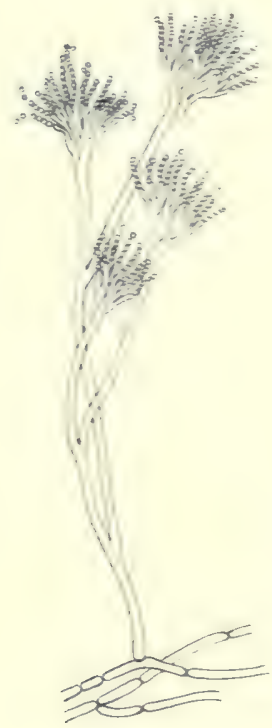

Pencillium glancum. 

cover the cheese, which in due time is placed in a maturing "cellar" at a constant, low temperature, where the-growth of mould is stopped or retarded, and bacterial growth becomes rapid. Thus new products are formed which give to the cheese its fully ripened flavour. If the bacterial ripening is carried too far, or progresses at too high a temperature, the result is an ill-flavoured cheese. Undesirable bacteria such as are found in cheese seem unable to grow at a temperature of $38^{\circ} \mathrm{F}$., and consequently bad flavours in cheese, caused by bacteria, do not increase in coldstorage. The long life of lactic acid bacteria in cheese seems to have an important bearing on the question of ripening, in checking the development of bacteria which produce bad or undesirable flavours. The exact temperature at which cheese will best cure has yet to be ascertained. It is obvious that in such a manufacture there is ample scope for many and wide variations in detail, which in fact take place and give rise to the different types of soft cheeses. The differences are based upon empirical knowledge and general experience. The scientific rationale is not at present understood, nor do we know the various species of moulds and bacteria which give the different flavours. Even less is known as to the exact bacteriology of hard cheeses. Moulds, however, play no part in their ripening, which is due to bacteria. Some have held that the peptonising or casein-digesting organisms ripen hard cheeses, but the majority of dairy bacteriologists look upon the lactic acid species as that chiefly responsible.

Freudenreich and Marchal have shown that in certain Swiss and Belgian soft cheeses the principal part in the ripening is due to Oidium lactis; various budding fungi also seem to aid in the process. Another example of the soft cheeses, the Roquefort, is ripened, at least in part, by the Penicillium glaucum, which filamentous fungus occupies all the cracks and fissures which abundantly intersect the cheese. There it consumes the acid produced by the lactic acid bacteria, and retards the development of the albumindigesting organisms. The penicillium flavour has become so much appreciated that the mould is now often sown in the first curd, and aërating holes are pierced in each cylinder of cheese. The coatings of mould appearing in Gorgonzola, Brie, and Stilton cheese have a similar action. Edam cheese, on the other hand, appears to be ripened by Streptococcus Hollandicus, an urganism which possesses the property of making milk or whey ropy. It is employed by adding 2 per cent. by volume of ropy whey to the milk to be set for cheese. 


\section{The Use of Artificial Cultures in Cheese-making}

The practicability of employing pure cultures as "starters" in cheese-making in a similar manner to that employed in buttermaking has been tested. Upon the belief that lactic acid bacteria are the main agents in the curing of cheese, this species has been suggested as a "starter" under the following conditions, viz.: (a) that the organism should be a pure lactic acid producing germ incapable of producing gaseous products; $(b)$ that it should be free from any undesirable aroma; and $(c)$ that it should be specially adapted for vigorous development in milk. Such a "starter" might be propagated in pasteurised or sterilised milk although it has been found in practice that such milk does not make good cheese. The advantages accruing from the use of such a "starter" are that with sweet milk it saves time in the process of manufacture, that with tainted milk in which acid develops imperfectly, it is an aid to the development of a proper amount of acid for a typical Cheddar cheese, and that the flavour and quality of such cheese is preferable to that of cheese which has not been thus produced. Professor Russell is of opinion that the lactic acid organisms are to be credited with greater ripening powers than the casein digesting organisms, and Lloyd holds that "the greater the number of lactic acid bacilli in the milk the greater the chance of a good curd." Still, in his opinion, "this organism alone will not produce that nutty flavour which is so much sought after as being the essential characteristic of an excellent Cheddar cheese." 1 Lloyd concludes, however, that by the growth of this species and the resulting production of lactic acid, most of the organisms which are present in the milk due to air contamination are gradually destroyed, while even those which are not destroyed are frequently checked in their growth, and thus taints in the milk are diminished; that up to the time of vatting the curd all the operations in the manufacture of the cheese are dependent upon and subservient to the growth of the lactic acid bacillus in the curd; and that subsequent ripening of the cheese is also dependent upon this organism.

Three difficulties have, up to the present, prevented any wide application of the method of artificial starters. The first is that of not being able to use pasteurised milk, which is uncoagulable by rennet (Lloyd). The use of ordinary milk means some degree of contamination, but it contains the enzymes which may be

1 Board of Agriculture, Report on Cheddar Cheese-making (F. J. Lloyd), 1899, p. I64. 
injured or destroyed by sterilisation. Secondly, the continual supply of pure culture is by no means always an easy matter. Thirdly, the maintenance of a low temperature cellar to prevent the rapid multiplication of extraneous bacteria will in some localities be a serious difficulty. These difficulties have, however, not proved insurmountable, and various workers have produced cheese by artificial culture. Adametz, for example, has used one of the peptonising species in this way with some success. JohanOlsen has made Gammelost cheese by artificial starting with a mould. Edam cheese has also been made in Holland by using, as artificial starter, whey advanced in slimy fermentation. This last is not a pure culture, but a mixture of organisms of which the chief is a micrococcus possessing the power of setting up slimy fermentation. Cheese made in this way ripens in a uniform manner, and irregular taints are less likely to occur. The whole subject is at present, however, only in the experimental stage. What is required is the ideal combination of pure bacterial culture plus enzymes, and this at present seems difficult to obtain. ${ }^{1}$

\section{Abnormal Cheese Ripening}

Unfortunately, from one cause or another, faulty fermentations and changes are not infrequently set up in cheese. Reference will now be made to the chief of these.

(a) Inflated cheese (other terms used to describe this condition are "swelled " cheese, "puffy," "gassy," "blown," or "spongy" cheese). In this abnormality the substance of the cheese is split up into innumerable cavities and holes containing various gases. Russell has maintained that the real cause of the difficulty is to be traced to the action of certain bacteria that gain access to the milk. Most of these belong, he thinks, to the lactic acid producing bacteria. The cultures form enough acid to curdle the milk, with an abundant evolution of gas. The gas is formed by the decomposition of the milk sugar, and is in most cases $\mathrm{CO}_{2}$ and an inflammable gas which is mainly hydrogen. Most gas-producing micro-organisms give also an unpleasant flavour. They do not ferment the casein to any considerable extent. They are readily susceptible to heat: $140^{\circ} \mathrm{F}$. for fifteen minutes is fatal to most

1 Whilst the above chapter was passing through the press, a most useful summary of this subject for the dairy student appeared in a small book by Professor H. W. Conn, entitled Bacteria in Milk and its Products (1903). See pp. $179-250$. 
of them. They do not form spores. Nor will they grow in the presence of 2 per cent. acid. Most of them occur in winter milk, but the temperature does not favour their increase. ${ }^{1}$ In ordinary sound curd there is lactose present, which in due time is decomposed by various organisms involved in ripening. In some degree we get lactic acid produced; but we also get gas liberated, and in consequence smaller or larger holes are produced. Occasionally this process takes place in an extreme degree, and the whole cheese becomes swollen and inflated. Such cheese is termed "spongy," or "puffy," or "blown." It has been found that some 25 species of bacteria have the power of producing this abnormality, and on occasion it may be so marked as wholly to ruin the cheese. Freudenreich found three species of organism, which he termed Bacillus Guillebeau, $a, b$, and $c$, which produced inflated cheese when inoculated into fresh curd. Bad flavour was also present. In the holes produced by $B$. Guillebeau, $a, \mathrm{CO}_{2}$ and gases were found in some abundance. $B$. coli communis (Lloyd), $B$. lactis aërogenes, and $B$. Schafferi have also been isolated from such inflated cheese. Adametz also separated an organism termed Micrococcus Sornthalii from a Sornthal dairy which was able to produce gassy cheese. Baumann believed that this abnormality was due to organisms ordinarily capable of producing normal pitting in cheese, but which if present in excessive numbers brought about the abnormal pitting. $\mathrm{He}$ isolated $B$. diatrypeticus casei, a facultative anaërobe, non-motile, and a liberator of $\mathrm{CO}_{2}$ and $\mathrm{H}$ in substances containing sugar, with the formation of alcohol and lactic acid. As a matter of fact, there is evidence to show that it is not the exclusive property of one or two organisms to inflate cheese. Gas-producing bacteria in large numbers in milk are capable of doing this, and the abnormality is widespread. It may be prevented by not using milk containing gas-producing bacteria, ${ }^{2}$ or by the addition of 3 per cent. of sodium chloride, or acid, to the freshly precipitated curd, or by obtaining milk from perfectly healthy udders and keeping it free from all dust and uncleanness. There is evidence to show that bacteria from sore teats, or dirty water, or dust and manure, may set up spongy cheese. Lloyd states that when a cheese shows signs of sponginess it is well to take special precautions to ensure obtaining

1 Twelfth Annual Report of Agricultural Expt. Sta., Wisconsin, I 895.

2 These organisms can be detected by the fermentation test, or by placing suspected milk in flasks at $90^{\circ} \mathrm{F}$., when gas-producing bacteria, if present, will make themselves obvious. 
sufficient acidity, and not on any account to hasten the ripening. He claims that at least five organisms are capable of producing spongy curd in Cheddar cheese, and that one of these produces a taint also.

It may be noted that the character of the gas holes in cheese is not of import in the differentiation of species. If few gas bacteria are present, the holes will probably be large and few in number (e.g. Gruyère); if many, the holes will be small but very numerous. Swiss cheese having this characteristic in limited measure is known as Nissler cheese.

It should be added that many of these gas producers belong to the lactic acid group, and are susceptible to heat. A temperature of $140^{\circ} \mathrm{F}$. maintained for fifteen minutes is fatal to most of them, largely because they do not form spores. The sources of these gas-producing organisms and of other bacteria producing abnormal cheese are varied. In addition to the organisms brought to the cheese factory in the milk, others may be added from the vats and apparatus, from the rennet, from the water, and from dust and dirt.

(b) Tainted cheese may be due partly or wholly to putrefaction in the curd, especially in soft cheeses.

Lloyd describes what he calls a "vinegar taint" present in Cheddar cheese curd in the early part of the year, caused by a micrococcus described by him. ${ }^{1}$ The origin appears to be in stall manure. The practical remedy is a higher scald to obtain a drier curd, and the production in the curd of less acid than usual prior to grinding. Fæcal and other taints, sometimes localised in certain districts, have also been described.

(c) Bitter cheese was first attributed to bacteria by Nägeli, and since then many species have been found to possess this property. Tyrothrix geniculatus (Duclaux) produces a bitter taste in Cantal cheese, and Micrococcus casci amari was isolated by Freudenreich from bitter, hard Swiss cheese. The bitterness generally makes its appearance at the stage of semi-ripeness. Not all the organisms producing bitter milk have been found to produce bitter cheese.

(d) Spotted and coloured cheese.-The following chromogenic conditions may occur in cheese :-Red spots may occur, particularly on the surface or in the open spaces and interstices of the cheese. These spots and patches generally appear one to three weeks after the curd is pressed, and mostly in hot weather (July and August). W. T. Connell isolated in 1896 an organism termed B. rudensis,

${ }^{1}$ Board of Agriculture, Report on Cheddar Cheese-making, pp. 176-180. 
which he believed was the cause; others have found a micrococcus. Blue cheese is produced, according to Vries, by a bacillus, and black cheese is produced by a copious growth of fungi.

(e) Poisonous cheese.-In I 883 and I884 there occurred in Michigan, U.S.A., an outbreak of cheese-poisoning. Three hundred persons in all were affected, and the illness was traced by Professor V. C. Vaughan to a poisonous ptomaine present in the cheese, and to which he gave the name tyro-toxicon. It is not improbable that this ptomaine is a product of bacterial fermentation. It is one of a large class of substances said to be formed by the action of bacteria upon nitrogenous compounds. It is unstable, and easily destroyed by the action of heat and moisture, and even by exposure to the air. Being present in small quantities only, it has never been isolated in sufficiently large quantities to allow of its composition being definitely determined. Tyro-toxicon has been proved to be a violent poison both to man and the lower animals. A minute portion consumed by a child produced sickness and diarrhœe in a manner almost identical to cholera infantum (Vaughan). Similar symptoms were obtained with cats and dogs. Vaughan found that three months are required for the formation of tyro-toxicon in milk kept in tightly-stoppered bottles; but under certain circumstances, and in the presence of butyric fermentation in milk, the poison is produced in about eight or ten days. Similar, and possibly identical, poisons occasionally occur in cream, rancid butter and milk (lacto-toxicon and diazo-benzol). They have the same poisonous effects. Vaughan has isolated a microbe growing readily on ordinary culture media and upon fruit and vegetables. This micro-organism, it is considered, may be the agent producing tyrotoxicon, but the bacteriology of the subject has not been worked out.

The writer investigated a similar outbreak due to tyro-toxiconjin Dutch cheese in London in I901. ${ }^{1}$ Seventeen persons were affected. The symptoms of illness in all these I7 cases occurred in from two to eight hours after eating the cheese in question, which came from the same consignment. Moreover, the symptoms were similar, namely, epigastric pain, rigors, vomiting, diarrhœe, prostration, and some fever. The degree of sickness does not appear to have depended upon the amount of cheese eaten. There was no death attributed to the poisoning, and in general the symptoms appear to have passed off in the course of forty-eight hours. A

1 Report on the Public Health of Finsbury, 1901, pp. I10-116. 
short incubation period suggests that the poison was "available" in the cheese, as a product of previous changes, possibly bacterial, set up therein. A long incubation period between eating the food and symptoms of poisoning would suggest that the persons affected had consumed, not the products of bacteria, but the bacteria themselves, which had then taken some little time to produce their injurious effects in the persons eating the food. In the present case the incubation period was comparatively short, and the acuteness of the symptoms appeared to have no direct relationship to the amount of cheese eaten. ${ }^{1}$

${ }^{1}$ For a general discussion and bibliography of this subject, see Die Milch und ihre Bedeutung für Volkswirtschaft und Volksgesundheit, Hamburg, 1903, pp. 345-357. 


\section{CHAPTER VIII}

\section{PATHOGENIC BACTERIA IN MILK. TUBERCULOSIS AS A TYPE}

Infective Diseases of the Cow communicable to Man by Milk. Pathogenic Bacteria in Milk. Clinical Evidence; Bacteriological Evidence. The Tubercle Bacillus in Milk and Butter. Streptococcus in Milk. Tuberculosis as a Type. The Specificity of Tuberculosis; The Biology of the Bacillus Tuberculosis; Tubercle Bacilli of Bovine and Human Origin compared; Polymorphism; Pseudo-Tuberculosis; Bovine Tuberculosis. The Entrance of Tubercle Bacilli into Milk. Virulence of Milk containing Tubercle Bacilli. Powers of Resistance of Tubercle Bacillus. The Acid-Fast Bacilli allied to the Tubercle Bacillus.

WE propose to deal with the disease-producing power of milk in several chapters. In the present chapter general reference to the subject will be made, and in particular the conveyance of tuberculosis by means of milk. The succeeding chapters will treat of the chief epidemic infective diseases spread by infected milk.

\section{Infective Diseases of the Cow communicable to Man by Milk}

Broadly speaking, the diseases of the cow which may give rise to some form of disease in man are such as directly affect the udder, or indirectly the milk. The chief of such diseases are tuberculosis, mastitis, enteritis, and possibly foot-and-mouth disease. Our views on the communicability of tuberculosis will be stated subsequently, and the matter need not be discussed here. It will be sufficient to say that we hold that the communicability of this disease is possible, and may occur, between the bovine species and man through the channel of milk. The disease may not be chiefly, nor greatly, spread in this way, but that it may be thus communicated, we do not doubt. Some facts will be furnished to show the existence of the active agent of tuberculosis in cows and their milk.

In regard to mastitis, there is no doubt that the immense majority of cases of what are called ordinary inflammation of the 
udder are caused by bacteria, which may set up diseased conditions in the bodies of persons consuming the milk. The specific organism of mastitis is a diplococcus or streptococcus, and it may be readily found in the milk of animals whose udders are so affected. The causal bacteria gain access to the interior of the udder, in all probability, by way of the milk duct in the teat, and the disease may therefore be conveyed from one cow to another by the hands of the milker. The relationship between mastitis and disease in persons drinking the milk, is at present obscure. Many abnormal conditions, including some epidemic infective diseases and outbreaks of sore throat, have been attributed to mastitis in the cows supplying the incriminated milk. But little is known of the exact conditions set up. ${ }^{1}$ Streptococci are frequently found in milk derived from presumably healthy udders, and in such cases have apparently gained access from the air, or as a result of unclean handling or manipulation. Under certain circumstances, these organisms set up marked changes in the milk containing them.

The organisms, setting up enteritis in the milch cow, may be responsible for setting up diarrhcea in man. In any case there is evidence of the capacity which milk has, under certain circumstances, of causing gastric and intestinal disturbance in persons consuming it.

Foot-and-mouth Disease in milch cows is occasionally accompanied by vesicles on the udder, especially at the opening of the milk duct. This condition often leads to sores and crusts being formed, preventing the ready flow of milk, and leading to a marked pollution therein. The infection of the disease may be conveyed for long distances by persons and things which have come into contact with the diseased animals. Dr Thorne was one of the first to investigate some apparent cases of transmission of the disease to man by means of infected milk. ${ }^{2} \mathrm{He}$ concluded "that a disease appears sometimes to have been produced in the human subject when the milk of cows suffering from Foot-and-mouth Disease has been freely used without being boiled. There is no evidence to show whether this affection is of a specific nature or not." But Dr Thorne had to acknowledge "that in a very large

${ }^{1}$ Jour. of Comp. Path., vol. xv., p. 163. Report of Roy. Vet. Coll., 1903, pp. 19-21.

${ }^{2}$ Report of Medical Officer of the Privy Council, 1869, p. 294. See also record of outbreak affecting fourteen persons reported by Siegel, Jour. of State Med., 1898, p. 178. 
number of cases the milk of cows undoubtedly affected has been used without producing any noticeable morbid effects." Sir John Simon came to somewhat similar conclusions seven years earlier. ${ }^{1}$ The conditions occurring in persons who had consumed the milk, and which was attributed to Foot-and-mouth Disease, were, mainly, alimentary disturbance, some fever, and an herpetic eruption about the lips and mucous membrane of the mouth. Against this suggestion of communicability must be set the broad fact that Footand-mouth Disease used to be extremely prevalent, affecting hundreds of thousands of cattle, and if it had been a disease communicable to man through the milk supply, outbreaks due to it would have been very frequent and extensive, which they certainly were not. It may, therefore, be assumed that this disease is not, as a rule, communicable to man through milk. ${ }^{2}$ It should, however, be noted that the milk of cows suffering from the disease is highly poisonous to calves and lambs, in the latter soon proving fatal. But the communicated disease is not Foot-and-mouth Disease.

\section{Pathogenic Bacteria in Milk}

When we ask ourselves what is the evidence as to the existence and amount of disease in milch cows, and what pathogenic bacteria have actually been found in milk (viewing the matter, of course, solely from the standpoint of communicability of disease to man through milk), we are met by a very large body of facts. Reference can only be made to a few typical series of such facts in this place.

Clinical evidence.-In the first place we may take London as an example. From 1899 to June 1903, the London County Council have carried out periodic veterinary inspections of the milch cows stabled in the metropolis. The cows numbered between 4000 and $5000 .{ }^{3}$ Thirteen examinations have been made with the following result :-

1 Fifth Report of Medical Officer of Privy Council, 1862, p. 31.

2 In Allbutt's System of Medicine, vol. ii., pp. 691, 692, McFadyean enumerates various instances of transmission to man through infected milk.

${ }^{3}$ Compiled from Reports of Medical Officer of Health to the London County Council, 1899-1901. For the returns since I901 we are indebted to the courtesy of Mr Shirley F. Murphy, the Medical Officer of Health of the London County Council, who has kindly furnished us with the figures before they have appeared in his Annual Reports for 1902-3. The "first examination" was conducted by Mr P. J. Simpson, the subsequent examinations were made by Mr W. F. Shaw. 


\begin{tabular}{|c|c|c|c|c|c|c|c|c|c|}
\hline 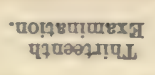 & $\widehat{\stackrel{8}{8}}$ & ळ̊ & & m & นกูก & œ & "ా & $\stackrel{2}{2}$ & $\stackrel{\infty}{\stackrel{8}{\dddot{0}}}$ \\
\hline 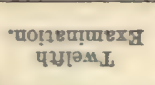 & $\widehat{\widehat{\Phi}}$ & $\tilde{o}$ & & $m$ & $+p^{\circ}$ in & 욿 & 0 & $\stackrel{n}{\pi}$ & $\stackrel{\circ}{\dot{0}}$ \\
\hline 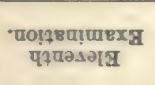 & $\widehat{\widehat{\Phi}}$ & के & & $m$ & m & $\dddot{2}$ & $m^{\circ}=$ & \% & $\overrightarrow{\dot{n}} \stackrel{m}{0}$ \\
\hline 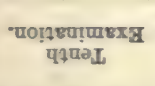 & $\widehat{\widehat{\Xi}}$ & ळे & & en & $-\stackrel{\circ}{\circ}$ & 욧 & mo min & $\stackrel{q}{q}$ & $\stackrel{\infty}{m} \frac{n}{\dot{0}}$ \\
\hline 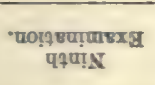 & ङ્ & $\stackrel{n}{\circ}$ & & + & $m_{\infty}^{\infty}$ & 10 & $\approx 0 \cong \infty$ & 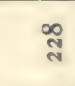 & $\stackrel{0}{\circ}$ \\
\hline 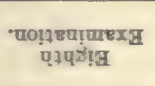 & बิ용 & ్ㅗํ & & N & 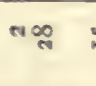 & $=\infty$ & 유 mం유 & के & in o \\
\hline 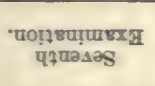 & 厄: & $\stackrel{0}{\square}$ & & + & $m$ & in & $\operatorname{TH}^{ \pm}$ & $\underset{\sim}{\infty}$ & $\vec{b}$ \\
\hline 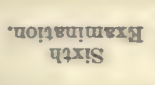 & $\widehat{\bar{\Xi}}$ & $\stackrel{\circ}{\circ}$ & & 0 & $+\infty$ & 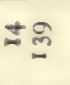 & $\hat{n}^{\infty} a$ & ภู & $\stackrel{-1}{0}$ \\
\hline 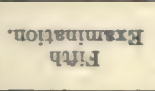 & $\widehat{\bar{\Xi}}$ & 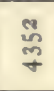 & & + & $\infty=$ & $=8$ & อ " बूल & $\approx n$ & $\stackrel{\infty}{\text { i }}$ \\
\hline 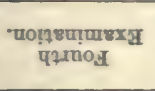 & $\widehat{\bar{\Xi}}$ & $\stackrel{m}{+}$ & & 0 & 2 虽 & $\infty$ & $\mathrm{N}^{-2} \cong$ & 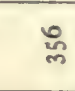 & $\ddot{\infty}$ वे \\
\hline 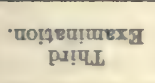 & $\widehat{\widehat{े}}$ & $\stackrel{0}{\pi}$ & & $\infty$ & $0 \mathrm{~m}:$ & $\stackrel{\infty}{\infty}$ & $m-\infty$ & $\underset{\infty}{\infty}$ & $\ddot{b} \ddot{0}$ \\
\hline 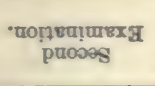 & 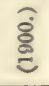 & 움 & & 0 & $\div$ & ¿ & 이문 & $\underset{\infty}{\infty}$ & $\underset{\infty}{\infty} \overline{0}$ \\
\hline \multirow[t]{2}{*}{ 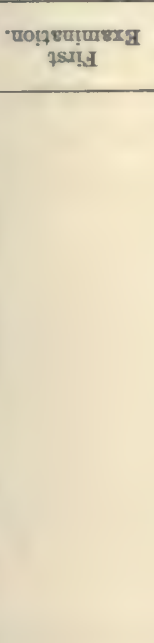 } & 厄. & 市 & & $n=$ & $n \infty$ & $\mathscr{\varepsilon}$ & 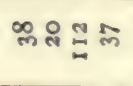 & 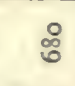 & $\stackrel{\tilde{D}}{\sim}$ \\
\hline & & 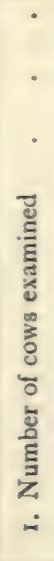 & 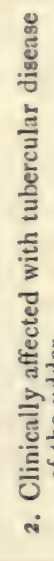 & 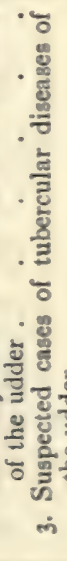 & 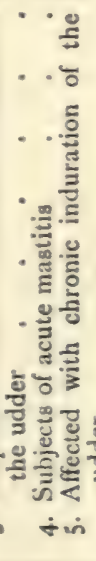 & 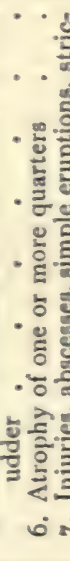 & 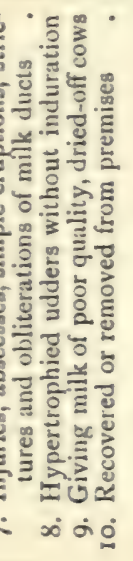 & 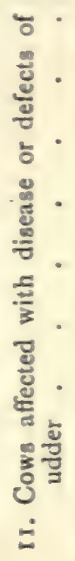 & 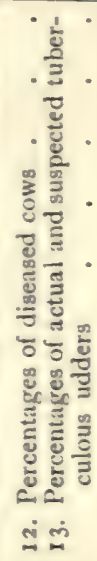 \\
\hline
\end{tabular}


From these returns we see that the number of cows having any kind of udder disease varied from 3.8 per cent. to $\mathrm{I} 3.2$ per cent. These figures included cases of recently recovered udder disease, and a few cases of cows removed by owners to avoid infection. The presence of tuberculosis, as judged by clinical examination, was found to be very small. The chief pathological conditions met with were chronic induration or atrophy of the udder, and a yield of milk of poor quality. It should be noted that the diseased conditions included in Numbers 5, 6, 7, and 8 are of a nature not likely to cause the milk yielded to be injurious or dangerous to persons consuming it. It will be seen that whilst there has been a decline in the number of cows stabled in the metropolis equal to 22 per cent., the decline in diseased udders has fallen from 13.2 per cent. in 1899 to 4.8 in June 1903 . If it be suggested that these figures might depend on differences in veterinary opinion as to what should, and what should not, be considered "disease" of the udder, the returns of Mr Shaw only may be considered (viz., those from 1900 to 1903) when it will be found that the number of cows with diseased or defective udders has fallen from 387 to 195 , and from 8.3 per cent. to 4.8 (having been as low as 3.8 in the third quarter of 1902). This marked decline in the number of cows found to have diseased or defective udders clearly proves, in the opinion of $\mathrm{Mr}$ Shaw, that there has been a gradual tendency on the part of the owners not only to purchase animals with sound udders but also not to retain in their sheds for any length of time cows which have unsound udders. ${ }^{1}$

Secondly, we may take Manchester, where, out of 1839 cows examined in IgoI in the city cowsheds, only I was found to be suffering from tuberculosis of the udder. ${ }^{2}$ Inspection was also made in the same year of I44I cows in country cowsheds whose milk was consumed in Manchester. The number of cows found with diseased conditions of the udder vas 67 , giving an average of 4.65 per cent. Of these the number which proved to be tuberculous was I 5 , equal to a percentage of $\mathrm{I} \cdot 04$. The percentage, of course, varies in different districts, and from time to time in the same district. Adding together the results of a series of clinical inspections of cows and bacteriological examinations of milk carried on in Manchester up to I9OI, it was found that out of 4031 cows (town and country) 0.36 per cent. were tuberculous in regard of the town cows, and 0.48 per cent. in regard of the country cows. Or taking

1 See Appendix R, p. 592.

${ }^{2}$ Report on the Health of the City of Manchester, I901, pp. 238 and 248. 
the whole total of 9576 cows, the milk of which was tested for tuberculosis, or among which cows with tuberculous udders were detected by clinical examination, it was found that a total of 52 had tuberculous udders, which gives an average percentage of 0.54 . This percentage, if uniform throughout England, would yield on the total number of cows and heifers in milk (approximately 2,000,000) some 10,000 tuberculous udders. ${ }^{1}$ Professor McFadyean, in agreement with many other authorities, is of opinion "that about 2 per cent. of the cows in the milking herds in this country are thus affected," " (i.e. have tuberculous udders). Not confining ourselves to udder disease, it may be said about 25 to 30 per cent. of all milch cows in this country suffer from tuberculosis. So much for the clinical examination of the cow. ${ }^{3}$

Bacteriological evidence.-If we now study the available returns in relation to this subject as obtained by bacteriological examination of milk as placed on the market, we arrive at much the same conclusions, except, of course, that we find, owing to obvious reasons, the percentages of diseased milk are much higher than those of diseased cows. At the time of the first clinical examination of cows in London cowsheds (1899), only one cow was found in the district of Hackney to have a tuberculous udder. In the same district 77 samples of milk were taken, 17 of which (or 22 per cent.) on bacteriological examination were found to contain the tubercle bacillus. ${ }^{*}$ Such a result might, of course, conceivably be due to the milk of one infected udder being widely distributed. But it was subsequently shown that I 3 out of these I7 tuberculous samples of milk were derived from milk shops supplied from country farms and not from Hackney cowsheds, and even the other 4 samples were obtained at shops receiving part of their supply from country sources. This discrepancy is but an illustration of the marked difference which has been found to exist between town and country milks. For example, in the seven years I 896-I 902 in the city of Liverpool, I I I 9 samples of town milk were examined bacteriologically, and 23 samples contained the tubercle bacillus, giving a percentage of $2 \cdot 0$. In the same period

1 Transactions of the British Congress on Tuberculosis, 1901, vol. ii., p. 294. In 1901 there were $1,887,414$ milch cows in England, 4, 102,061 in the United Kingdom, and $18,000,000$ in the United States of America.

${ }^{2}$ Ibid., vol. i., p. 84 .

${ }^{3}$ For clinical methods, see Rep. Roy. Com. on Tuberculosis, 1896, part iii., pp. I-8.

4 Report of Medical Officer to London County Council, 1899, p. 52. 
I 124 country milks were examined, of which 84 (or $7 \cdot 4$ per cent.) were tuberculous. Nor does this difference hold only in respect to $B$. tuberculosis. In the years 1900 and 1901, 509 samples of town milk were examined for $B$. coli and $B$. enteritidis sporogenes (two organisms associated with contaminated disease-producing milk) with the result that I 26 samples (or 24.7 per cent.) contained $B$. coli, and 30 samples out of the 509 (or 5.8 per cent.) contained $B$. enteritidis sporogenes. During the same two years $49 \mathrm{I}$ samples of country milks were similarly examined, with the result that 2 I 8 (or 44.4 per cent.) contained $B$. coli, and 47 (or 9.6 per cent.) samples contained $B$. enteritidis sporogenes. It will be seen, therefore, that of all the milks examined in Liverpool, more than twice the number of tuberculous samples were found in the country milks than in the town milks, and nearly twice the number of contaminated samples, when judged by the standard of the presence of $B$. coli or $B$. enteritidis sporogenes. The table of results ${ }^{1}$ is as follows :-

\begin{tabular}{|c|c|c|c|c|c|c|c|c|c|c|c|c|c|c|}
\hline & \multirow{3}{*}{ 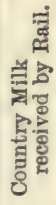 } & \multirow{3}{*}{ 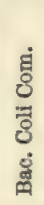 } & \multirow{3}{*}{ 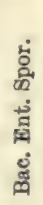 } & \multirow[b]{3}{*}{ है } & \multirow{3}{*}{ 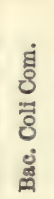 } & \multirow{3}{*}{ 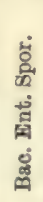 } & \multirow{3}{*}{ 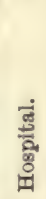 } & \multirow{3}{*}{ 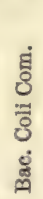 } & \multirow{3}{*}{ 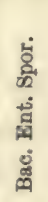 } & \multirow{3}{*}{ 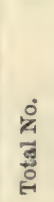 } & \multicolumn{4}{|c|}{ Tubercular. * } \\
\hline & & & & & & & & & & & \multicolumn{2}{|c|}{ Rail. } & \multicolumn{2}{|c|}{ Town. } \\
\hline & & & & & & & & & & & No. & $\begin{array}{l}\text { Per } \\
\text { cent. }\end{array}$ & No. & $\begin{array}{l}\text { Per } \\
\text { cent. }\end{array}$ \\
\hline I896 & 36 & $\ldots$ & .. & $8_{3}$ & ... & ... & $\ldots$ & ... & .. & I I9 & 5 & 14.0 & 4 & 4.8 \\
\hline $18 g$ & 87 & $\ldots$ & & 63 & .. &. & $\ldots$ & .. & . & 150 & 5 & $5: 7$ & 4 & 6.3 \\
\hline 1898 & 28 & $\ldots$ & $\ldots$ & 84 & ... & $\ldots$ & $\ldots$ & $\ldots$ & ... & II 2 & 5 & 17.8 & 7 & $8 \cdot 3$ \\
\hline I 899 & 185 & $\ldots$ & ... & 167 & $\ldots$ & $\ldots$ & $\ldots$ & $\cdots$ & $\ldots$ & 352 & 15 & $8 \cdot 1$ & I & 0.6 \\
\hline Igoo† & 245 & 40 & 42 & 255 & I5 & 26 & 60 & 7 & 4 & 560 & 5 & 1.6 & 4 & 1.5 \\
\hline Igort & 246 & 178 & . & 254 & III & 4 & 66 & 43 & 4 & 566 & 20 & $8 \cdot 1$ & 2 & 0.7 \\
\hline $1902 \dagger$ & 297 & 216 & 27 & 213 & $4^{6}$ & I & 85 & 59 & 3 & 595 & 29 & $9 \cdot 7$ & I & 0.4 \\
\hline
\end{tabular}

* These returns and percentages are calculated on the total numbers of rail and town milks examined. No account is taken of the comparatively fow cases in which the test was incomplete.

+ In 1900 out of the total of 560 samples, $B$. coli occurred alone in 47 cases $(0.8 \%)$, B. enteritidis sporogenes alone in 59 cases $(10.5 \%)$, and both organisms occurred together in 14 instances $(2.5 \%)$. In 1901 out of the total of 566 samples, $B$. coli was found alone in 335 cases $(59 \cdot 1 \%)$, $B$. enteritidis sporogenes alone in 6 cases $(1.0 \%)$, and the two organisms together in 7 eases $(1 \cdot 2 \%)$.

In an investigation into the distribution of $B$. coli communis made by Harriette Chick in the Thompson-Yates Laboratory at Liverpool, this bacillus was found in 17 out of 239 samples of new milk. Its presence was explained by contamination occurring in the cowsheds. ${ }^{2} \quad$ Balfour Stewart carried out a parallel investiga-

1 Reports of the Medical Officer of Health of the City of Liverpool, 18981902.

2 Report of Thompson-Yates Laboratories (Liverpool, 1900), vol. iii., part i., p. 29. 
tion in regard to the distribution of $B$. enteritidis sporogenes, which he found to be present either alone or with $B$. coli in 49 samples out of 213 . Out of these 49 samples containing $B$. enteritidis sporogenes the organism proved to be non-pathogenic in II cases, and pathogenic in 38. In eighteen instances it produced a fatal result in the guinea-pigs inoculated. ${ }^{1}$

As a result of the veterinary inspection of the cows in London cowsheds to which reference has been made, a bacteriological examination was instituted and entrusted to Dr Klein, who thereupon made a careful study of 100 milks derived from various milk-shops in London. His findings may be quoted:-(I) seven per cent. of the samples of "country" milk sold in London produced typical true tuberculosis in the guinea-pig; (2) eight per cent. of the samples of "country" milk produced typical pseudo-tuberculosis (non-acid-fast bacillus pseudo-tuberculosis of Pfeiffer); (3) one per cent. of milk samples yielded the typical true $B$. diphtheria; (4) one per cent. contained a pathogenic yeast; and (5) out of the secretions of the cow's udder two pyogenic microbes were obtained, B. diphtheroides and Streptococcus radiatus (pyogenes). ${ }^{2}$

In the same place Klein summarises the general knowledge concerning pathogenic bacteria in milk as it was in 190I. He points out that milk healthy in appearance may contain "bacteria which belong to various species, and some of which when grown separately in sterile milk cause rapid changes and alter profoundly the character of the milk, e.g. B. lactis, Proteus vulgaris, $B$. coli, $B$. mesentericus, spores of $B$.enteritidis, etc. If allowed to stand, the milk containing the above mixture of bacteria exhibits, even at ordinary temperatures (but in a more marked degree at temperatures of $70^{\circ} \mathrm{F}$. and above), those profound changes which are popularly expressed as 'going bad,' changes caused by the rapid multiplication of one or other of the above microbes. Thus, for instance, if different samples of milk received and brought in a sterile vessel from a shop be placed in an incubator at $37^{\circ} \mathrm{C}$., the next day, or at the latest after two days, the milk may be completely clotted and sour, due to the growth and activity of $B$. coli; or it may be decomposed by Proteus vulgaris or B. mesentericus; or it may be full of gas, clotted, and with a large amount of clear whey, caused by the growth of the anaërobic $B$. enteritidis sporogenesp. 37 .

1 Report of Thompson-Yates Laboratories (Liverpool, 1900), vol. iii., part i.,

2 Jour. of Hygiene, Igor, vol. i., p. 95. 
the layer of cream on the top of the milk ensuring something approaching anaërobiosis." 1

The tuberele bacillus in milk.-Illustrations may be taken from Manchester and Liverpool in respect to bacteriological examination especially as concerns tuberculosis. ${ }^{2}$ During I9OI, 3 IO samples were taken at Manchester railway stations from the milkcans belonging to 272 farmers. Of these farmers 172 resided in Cheshire, and 18 of them (10.46 per cent.) sent milk found to contain the tubercle bacillus on bacteriological examination; 65 lived in Derbyshire, and 6 of them ( 9.23 per cent.) sent tuberculous milk; 25 lived in Staffordshire, and 2 of them ( 8 per cent.) sent tuberculous milk. It will thus be seen that of the milk sent by rail to Manchester from 272 farms (the milk of which was examined by Professor Delépine), that from 26 of them ( 9.5 per cent.) was tuberculous. ${ }^{3}$ Since inspection under the Manchester milk clauses was instituted, the percentage of tuberculous samples has fallen from 18.5 to 9.5 per cent. ${ }^{4}$

The experience of the city of Liverpool in regard to tuberculous milk is also instructive. ${ }^{5}$ It appears that the daily consumption of milk in this city amounts to about 26,000 gallons. One half of this quantity comes from about 6000 cows kept within the city, the other half being derived from cows kept in the country, and sent in by rail. Within recent years, that part of the milk supply derived from the town cows has become practically free from tuberculosis. This result has been brought about by the sanitation of the cowsheds, adequacy of air, light, and cleanliness, systematic and frequent inspection of the cows by qualified inspectors with veterinary assistance, and by frequent bacteriological analyses of the milk (Hope). The country milk, on the other hand, is more or less frequently tuberculous, which is a serious reflection upon the sanitation of the cowsheds and the supervision and management of the cows. In a general way, it may be said of Liverpool that tubercle bacillus is found more than twice as often in samples of milk from the country than from the town. The returns, from 1896 to 1902 inclusive, to which refer-

1 Jour. of Hygiene, 1901, vol. i., pp. 78, 79. On pp. 392-45 I of the present volume will be found a description of the species of bacteria, including those which are pathogenic, which have from time to time been isolated from milk.

${ }^{2}$ For latest returns, see Appendix R, p. 592.

${ }^{3}$ Report on Health of City of Manchester, 1901, p. 238.

4 Trans. of the Brit. Congress on Tuberculosis, I901, vol. ii., p. 292.

${ }^{5}$ Reports on the Health of the City of Liverpool, 1898, I899, 1900, 1901, 1902, by E. W. Hope, M.D., D.Sc. 
ence has already been made, may be classified separately for convenience as follows :-

Table showing the Total Number of Milks Examined Bacteriologically FOR TUBERCLE BACILli FROM AUGUST I896 TO 3IST DECEMBER 1902.

(Liverpool.)

\begin{tabular}{|c|c|c|c|c|c|c|c|}
\hline \multirow{2}{*}{ Xear. } & \multirow{2}{*}{$\begin{array}{c}\text { Total } \\
\text { number } \\
\text { of Samples } \\
\text { taken. }\end{array}$} & \multicolumn{3}{|c|}{ Town Samples. } & \multicolumn{3}{|c|}{ Country Samples. } \\
\hline & & $\begin{array}{l}\text { Namber } \\
\text { taken. }\end{array}$ & Tubereular. & $\begin{array}{l}\text { Percentage } \\
\text { Tubercular. }\end{array}$ & $\begin{array}{l}\text { Number } \\
\text { taken. }\end{array}$ & Tubercular. & $\begin{array}{l}\text { Percentage } \\
\text { Tubercular. }\end{array}$ \\
\hline 1846 & 119 & 83 & 4 & 4.8 & 36 & 5 & $\mathrm{I}_{4} \cdot \mathrm{O}$ \\
\hline 1897 & 150 & 63 & 4 & $6 \cdot 3$ & 87 & 5 & $5 \cdot 7$ \\
\hline 1898 & I 12 & 84 & 7 & $8 \cdot 3$ & 28 & 5 & $17 \cdot 9$ \\
\hline 1899 & 352 & 167 & I & 0.6 & 185 & 15 & $8 \cdot I$ \\
\hline 1900 & 560 & 255 & 4 & I.5 & 305 & 5 & $I \cdot 6$ \\
\hline I901 & 500 & 254 & 2 & 0.7 & 246 & 20 & $8 \cdot I$ \\
\hline 1902 & 510 & 213 & I & 0.4 & 297 & 29 & $9 \cdot 7$ \\
\hline
\end{tabular}

Obermüller, Rabinowitsch, Jäger, Petri, Bollinger, Martin, Peters, Hamilton, Klein, Woodhead, McFadyean, Delépine, and many other workers have found the tubercle bacillus in milk in dairies or in the market. It has been found in almost all countries and in almost every species of cow. It has been found in very few instances, and scantily present, and it has been found in a high percentage of cases, and present in large numbers. These variations depend upon a variety of circumstances and conditions. In some cases of marked tuberculosis of the udder, it may be impossible to detect any bacilli at all, and yet the milk is without doubt infective. More commonly, the bacilli are present in udder disease, in such large numbers, that they may be found on microscopical examination in almost every cover slip prepared from the milk.

From the large number of examinations of milk for the tubercle bacillus now on record, we may quote a few examples, all of which are based upon inoculation tests and not upon microscopical examination only. Bang found true tubercle bacilli present in 14 per cent. of the cases he investigated; Ernst 25 per cent.; Hirschberger over 50 per cent. ; Klein 7 per cent. in country milks ; and Boyce 6 to 8 per cent. in town milks, and 17 per cent in country milks. Kanthack and Sladen, in a model piece of investigation, found the tubercle bacillus present in the milk of nine out of sixteen dairies examined at Cambridge. ${ }^{1}$ In the same year 1 i 8 milks were examined in Islington, with the result that 14.4 per cent. were found to contain the tubercle bacillus. ${ }^{2}$ In Hackney, as

1 Lancet, 1899, i. 74.

2 Report on the Health of Islington (Dr Harris), I899, p. 224. 
we have seen, 22 per cent. of the milks examined contained the bacillus. In Croydon 164 milks were examined, with the result that 6.7 per cent. were found to contain $B$. tuberculosis. ${ }^{1}$ In 1897 Obermüller found 38 per cent. of the milks examined in Berlin contained the tubercle bacillus; in the same year Rabinowitsch found the bacillus in 28 per cent.; and in 1898 Petri, also in Berlin milk, found it in 14 per cent.. As early as $1884 \mathrm{H}$. Martin found 33 per cent. of the milks examined in Paris yielded the tubercle bacillus; in 1893, Sacharbekow found 5 per cent. of St Petersburg milks positive; and in I 898 Beuge obtained 30 per cent. in Halle milks. Numerous other records might be quoted, but the above will amply suffice to illustrate the occurrence of this organism in milk as it is actually placed on the market.

The tubercle bacillus in butter.-The products of milk also become infected, as we have already pointed out. Grœning found that 47 per cent. of butter samples apparently contained tubercle bacilli. Again, Obermüller found virulent tubercle bacilli in each of fourteen samples of butter, while Rabinowitsch, in eighty samples, found no tubercle bacilli, but organisms similar in appearance in 28.7 per cent. On another occasion she found $\mathrm{I} 3$ per cent. of butters examined contained the tubercle bacillus. Petri found 32 per cent. of true tubercle bacilli in butter. Korn found the true tubercle bacillus in 23 per cent. of market butters in Freiburg. Hormann and Morgenroth melted butter at $37^{\circ}$, and injected 4 to 5 c.c. into each of four guinea-pigs intraperitoneally. In some experiments the butter was centrifugalised after melting, and the fat-free sediment injected, with the object of introducing the infection as free as possible from fat. With each sample four guinea-pigs were injected direct with the fluid butter, four with a portion which had been incubated at $37^{\circ}$ for twenty-four hours, and two with the sediment obtained on centrifugalising water in which the butter had been washed. Of ten samples treated in this way three gave entirely negative results, three disclosed the tubercular organism which could be cultivated from the organs of the test animals, and four gave results which were ambiguous. Organisms, similar to the tubercle bacillus, were recognised in several cases. So far as the experiments went, they showed no advantage in centrifugalising the butter or in incubating it before injection. Glycerine agar failed in every instance as a culture medium, blood serum with 5 per cent. glycerine being the only medium which turned out to be satisfactory, and the growth on this was in every case small.

1 Report on the Health of Croydon (Dr Richards), I90I, p. 753. 
The identity of the organism was thoroughly verified by the postmortem examination of the test animals. ${ }^{1}$

We have summarised a number of these investigations into butter in tabular form as follows :-2

\begin{tabular}{|c|c|c|c|c|c|}
\hline \multicolumn{6}{|c|}{ Tubercle Bacillus in Market Butter. } \\
\hline \multicolumn{2}{|l|}{ Investigator. } & $\begin{array}{l}\text { No. of } \\
\text { Samples } \\
\text { Tosted. }\end{array}$ & $\begin{array}{l}\text { No. in which } \\
\text { the Tubercle } \\
\text { Bacillus } \\
\text { was found. }\end{array}$ & Percentage. & Remarks. \\
\hline Brusafferro & . & 9 & 1 & II & ... \\
\hline Roth . & . & 20 & 2 & I0 & $\ldots$ \\
\hline Schuchardt & . & 42 & 0 & 0 & $\ldots$ \\
\hline Obermüller & . & 14 & 14 & 100 & obtained from I source. \\
\hline Groening . & . & 17 & 8 & 47 & $\ldots$ \\
\hline Petri . & - & 102 & 33 & 32 & from Berlin and München. \\
\hline Rabinowitsch & - & 80 & $\circ$ & of & $\begin{array}{l}30 \text { samples from Berlin. } \\
50 \text { samples from Philadelphia. }\end{array}$ \\
\hline Hormann and & Mor- & & & & \\
\hline $\begin{array}{l}\text { genroth } \\
\text { Rabinowitsch }\end{array}$ & $: \quad:$ & I0 & $\begin{array}{l}3 \\
2\end{array}$ & 30 & obtained from ${ }^{14}$ shops. \\
\hline Korn & $\therefore$ & $\begin{array}{l}15 \\
17\end{array}$ & $\begin{array}{l}2 \\
4\end{array}$ & $\begin{array}{l}13 \cdot 3 \\
23\end{array}$ & $\begin{array}{c}\text { obtained from I4 shops. } \\
\ldots\end{array}$ \\
\hline Ascher : & - & 27 & 2 & 7 & obtained from 22 shops. \\
\hline Weisenfeld & . & 32 & 3 & 9 & $\ldots$ \\
\hline Hellsträu . & . & 12 & I & 8 & $\ldots$ \\
\hline Bonhoff - & - & 39 & 0 & 0 & $\ldots$ \\
\hline Markl . & . & 45 & 0 & 0 & \\
\hline Augeszkky & & 17 & 3 & 17 & $\begin{array}{l}\text { from different sources in } \\
\text { Budapest. }\end{array}$ \\
\hline Totals & . & 498 & 76 & $15 \cdot 2$ & \\
\hline
\end{tabular}

It will, of course, be understood that the above table is merely for convenient classification. Doubtless there were many differences in manipulation, and it is highly probable that some of these investigators were dealing with acid-fast bacilli allied to the tubercle bacillus. The returns, however, are stated to be only of the true tubercle bacillus.

Streptococeus in milk.-Another pathogenic organism frequently present in milk, the significance of which has been much debated, is the Streptococcus. On the one hand, it has been associated with inflammatory conditions of the cow's udder and even with a form of scarlet fever in the cow; and on the other, it has been held to be the cause of suppurative condi-

1 Hygienische Rundschau, viii., 5 .

2 Extracted from Die Milch und ihre Bedeutung für Volkswirtschaft, Hamburg, I903, pp. 358-366 (with bibliography). 
tions, gastro-intestinal disease, and scarlet fever in persons consuming the milk. Moore has also found streptococci in relation to a number of diseases of cattle, sheep, and horses, ${ }^{1}$ as well as in conditions of health. Klein, Kurth, Mervyn Gordon, ${ }^{2}$ and others, have made attempts to work out a satisfactory classification of streptococci, but up to the present it cannot be said that this is yet accomplished. There is, however, a good deal of evidence in support of the view that there is some causal relationship between streptococcus and infectious mammitis. ${ }^{3}$ This affection of the udder is, as is well known, an insidious condition, and gradual in onset, sometimes becoming acute and epidemic, and at other times of such mild degree as to be scarcely recognisable. Symptoms may be marked or absent, and general or local, or both. Consequently it is often impossible to diagnose clinically. The commonest signs are some congestion, enlargement, and induration of the udder, and the appearance of white casein masses in the milk. A microscopic examination is generally necessary for diagnosis, and results in finding pus cells and streptococci. Eastes found pus cells in 30 per cent. of the milks which he examined, muco-pus in 48.7 per cent., and streptococci in 75.2 per cent. ${ }^{4}$ An investigation of milk in St Pancras in 1899 yielded 24 per cent. of samples containing pus cells. Such milk generally has an acid reaction. Eastes, Holst, Niven, Stokes, Bergey, Hirsch, and others, have drawn attention to the ill effects which streptococcal milk has upon persons consuming it. In the main these are twofold, namely, gastrointestinal diseases and sore throats.

The evidence implicating streptococcal milk is empirical and circumstantial, and yet it appears to be growing in force and volume. On the other hand, streptococcus has been found in the fresh milk derived from healthy udders. Reed and $\operatorname{Ward}^{5}$ have recently

1 Moore, "Observations concerning the significance of Streptococci in Comparative Pathology," American Vet. Rev., 1900, vol. xxiii.

2 See Reports of Medical Officer to Local Government Board, 1898-1899 and 1899-1900.

${ }^{3}$ Nocard and Mollereau, Archiv. Vet., 1884; Moore, "The Morphology, Biology, and Pathogenic Properties of twenty-eight Streptococci found in Investigation of Animal Diseases," Bureau of Animal Industry, Washington, 1893, Bull. No. 3. Hess, Landw. Jahrb. der Schweiz, 2 Band, I888; Nencki, Ibid., 5 Band, I89I; von Freudenreich, 1bid., 7 Band, 1890; Guillebeau, 1bid., 4 Band, 1890,5 Band, 1891 , and 8 Band, 1894.

4 Brit. Med. Jour., 1899, ii., 1342.

${ }^{5}$ H. C. Reed and A. R. Ward, "Streptococci in Market Milk," American Medicine, 14th February 1903, p. 257. 


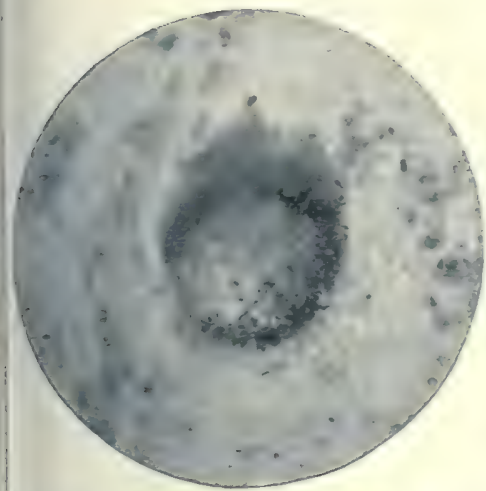

Is. tulwevelusis (bacilli in giant cell). staine 1 fiehl-Seelsen. $\times 1000$.

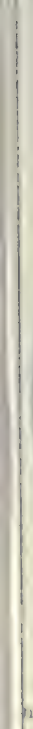

Staphlyluesecus isolater frum milk of cow with fithued uhder (1903). Staine 1 carbol-fuchsin dil. $\times 1000$.

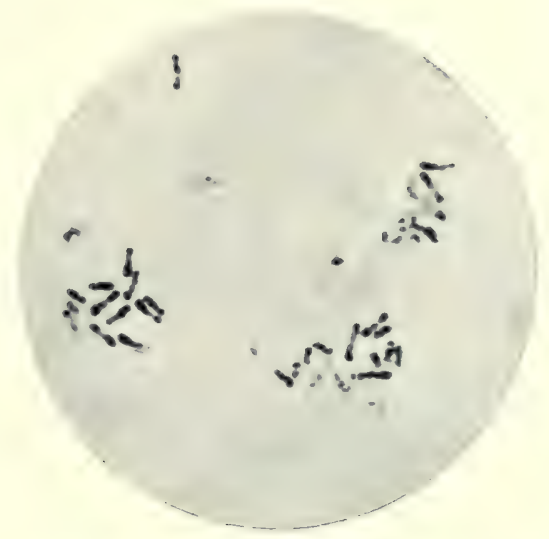

Bacilli from a case of b vine tuberculosis (Perlisucht), 1903. Stained Ziehl-Neelsen. $\times 1000$.

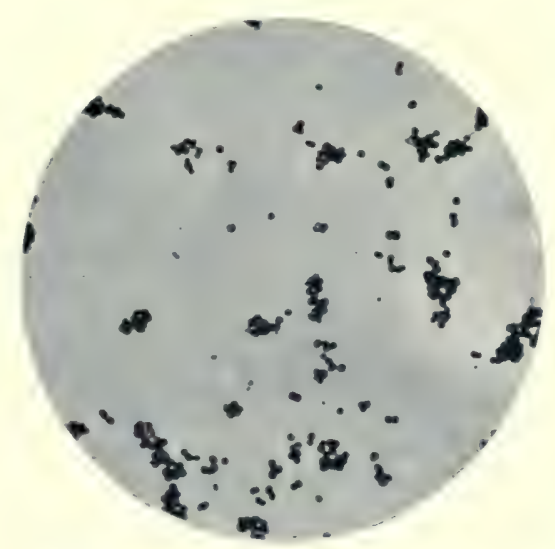

Dipleserents is lateel frum milk of enw with

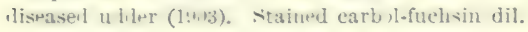
$\times 1000$. 

repeated an interesting study of such a streptococcus present in the healthy udder of a cow from 1897 to 1900 . After death the glandular tissue of the udder was also examined, with the result that abundant quantities of streptococcus, possessing some degree of virulence, were found in the substance of the gland. It was not possible to differentiate this organism from streptococci derived from udders with marked mammitis, set up by infection or by some slight local irritation. It is probable that streptococci exist in the healthy udder much more frequently than is generally supposed, and on this account it is possible that its effect upon persons consuming the milk is slight or nil, for if it were otherwise more illness might be expected to follow milk consumption. Bergey found that of 40 samples of market milk 36 (or 90 per cent.) yielded micrococci, and 20 (or 50 per cent.) yielded streptococci. Streptococci were found somewhat more frequently in the milk of cows kept under insanitary conditions, but the difference was not very marked. ${ }^{1}$ Bergey agrees with Beck, who repeated a number of experiments in 1900 , that it is most probable that these organisms are not infrequently the cause of serious gastro-intestinal disorders in infants, and probably far more frequently than is generally supposed.

We may now consider in some detail a typical disease of man contracted through the channel of the milk supply, and reserve to a subsequent chapter a consideration of epidemic infective diseases so transmitted. The type is tuberculosis, and we propose to make reference to it only as bearing upon its relationship to the milk supply.

\section{Tuberculosis and the Milk Supply}

We have selected tuberculosis as a type of disease intimately related to milk for two simple reasons. Its bacteriology and pathology are more fully worked out than that of any other disease related to milk, and it is a disease the agents of which may gain entrance to the milk from either the bovine or human side. It is, moreover, a disease which is claiming a large amount of study and consideration at the present juncture, on account of the many and wide practical issues raised by the new knowledge acquired respecting it during the last twenty years.

The specificity of tubereulosis.-There is now a large body of evidence which goes to prove that tuberculosis is one and the same

1 American Medicine, 190I, 20 th April, p. 122. 
disease in man and animals. ${ }^{1}$ Villemin (I865) was the first to maintain this identity on the results of inoculation of bovine and human tubercular matter into small animals. Chauveau (I868) carried out similar experiments upon cattle. ${ }^{2}$ Both workers were successful in transmitting the disease, which produced similar effects in the inoculated animals. Many other workers have obtained like results, including Gerlach, ${ }^{3}$ Bollinger, ${ }^{4}$ Klebs, ${ }^{5}$ Kitt, ${ }^{6}$ Crookshank, Martin, ${ }^{8}$ Thomassen, ${ }^{9}$ and Nocard. ${ }^{10}$

It is unnecessary to describe in detail the experiments of these various workers. The results obtained were more or less uniformly in support of the view that the identity of bovine and human tuberculosis was a thing to be accepted as a proved and fundamental proposition. Not only have the various workers named arrived individually at that conclusion, but the conclusions of the Royal Commission on Tuberculosis, 1895, included the following words:- "We find the present to be a convenient occasion for stating explicitly that we regard the disease as being the same disease in man and the food animals, no matter though there are differences in the one and the other in their manifestations of the disease; and that we consider the bacilli of tubercle to form an integral part of the disease in each, and (whatever be its origin) to be transmissible from man to animals, and from animals to animals. Of such transmissions there exists a quantity of evidence, altogether conclusive, derived from experiment." ${ }^{11}$

Whilst there was up to I90I almost entire unanimity of opinion amongst various workers in respect to this identity, it should not be supposed that there was unanimity in respect to the degree of pathogenicity. It was, in fact, conceded on all hands that tuberculosis was a more virulent disease in animals than in man, and that the

1 Kruse, Pansini, Fischel, Johne, etc. See also Twelfth and Thirteenth Annual Reports of the Bureau of Animal Industry, Washington, 1895-96. (Theobald Smith.)

${ }^{2}$ Congrès pour Pétude de la Tuberculose, Paris, 1888.

${ }^{3}$ Cornil's Les Bactéries, 1885 , pp. 630-633.

4 Münch. Med. Woch., 1894, No. 5.

5 Virchow's Archives, Bd. xli. and xlix.

' Strauss' La Tuberculose et son Bacille.

7 Transactions Pathological Society of London, 1891.

8 Report of Royal Commission, 1895, part iii., pp. 18, 19.

9 Trans. British Congress on Tuberculosis, 190I, vol, iv., pp. 21-27.

${ }^{10}$ La Revue Veterinaire, 1902, p. 49.

11 Report of Royal Commission on Tuberculosis, 1895, part i., p. 10, par. 23. This report was signed by the late Sir George Buchanan, Sir George Brown, Dr J. F. Payne, and Sir J. Burdon Sanderson. 
bacillus in the two species differed in various respects as to morphological, biological, and pathological properties (Theobald Smith, Dinwiddie, Frothingham). In I901, however, Dr Koch expressed the opinion that "human tuberculosis differs from bovine, and cannot be transmitted to cattle," ${ }^{1}$ and that bovine tuberculosis was scarcely, if at all, transmissible to man. On the same occasion counter evidence was produced by McFadyean, ${ }^{2}$ Ravenel ${ }^{3}$ Crookshank, ${ }^{4}$ and many others. Various experiments are now in process of being carried out to elucidate this question, including Government commissions in Great Britain and Germany.

The evidence furnished by Dr Koch for the conclusion that human tuberculosis is not communicable to animals was briefly this:Nineteen young cattle which had stood the tuberculin test (and were therefore presumably free from tuberculosis) were treated as follows:-Six were fed with tubercular human sputum almost daily for seven or eight months. Four repeatedly inhaled great quantities of bacilli which were distributed in water and scattered with it in the form of spray. The remainder (nine) were infected in various ways with pure cultures of tubercle bacilli taken from human tuberculosis, or tubercular sputum direct from consumptive patients. In some cases the bacilli or sputum were injected under the skin, in others into the peritoneal cavity, and in others into the jugular vein. None of these nineteen cattle showed any symptoms of disease. After six to eight months they were killed, and in their internal organs not a trace of tuberculosis was found. The result was entirely different, however, when the same experiment was made on cattle free from tuberculosis with tubercle bacilli from boine sources. In this case virulent tuberculosis rapidly supervened. Further, an almost equally striking distinction between human and bovine tuberculosis was brought to light by a feeding experiment with swine. Six young swine were fed daily for three months with the tubercular sputum of consumptive patients. Six other swine received bacilli of bovine tuberculosis with their food daily for the same period. The animals that were fed with sputum remained healthy and grew lustily, whereas those that were fed with the bacilli of bovine tuberculosis soon became sickly, were stunted in their growth, and half of them died. After three months

1 Trans. British Congress on Tuberculosis, 1901, vol. i., p. 29.

2 Ibid., vol. i., p. 79.

3 Ibid., vol. i., p. 9I, and vol. iii., p. 553.

4 Ibid., vol. i., p. 92. 
and a half the surviving swine were all killed and examined. Among the animals that had been fed with sputum no trace of tuberculosis was found, except here and there little nodules in the lymphatic glands of the neck, and in one case a few grey nodules in the lungs. The animals, on the other hand, which had eaten bacilli of bovine tuberculosis had, without exception (just as in the cattle experiment), severe tubercular disease, especially tubercular infiltration of the greatly enlarged lymphatic glands of the neck and of the mesenteric glands, and also extensive tuberculosis of the lungs and the spleen. The difference between human and bovine tuberculosis appeared not less strikingly in a similar experiment with asses, sheep, and goats, into whose vascular system the two kinds of tubercle bacilli were injected. Dr Koch also stated that other experiments in former times, and recently in America, had led to the same result.

In support of his second contention, namely, that bovine tuberculosis is not transmissible to man, Dr Koch points out that the direct experiment upon human beings is, of course, out of the question, and hence it is necessary to rely upon indirect evidence. Dr Koch, therefore, reasons as follows: Tuberculosis, caused by meat or milk, can be assumed with certainty only when the intestine suffers first, i.e. when a so-called "primary tuberculosis" of the intestine is found. If bovine tubercle bacilli are capable of causing disease in man there are abundant opportunities for the transference of the bacilli from one species to the other, and cases of primary intestinal tuberculosis from consumption of tuberculous milk ought therefore to be of common occurrence. "But such cases," he maintains, "are extremely rare." In support of this view Dr Koch stated that he had only seen two cases; that only ten cases had been met with in the Charité Hospital in Berlin; and that out of 3104 post-mortems of tubercular children, Biedert observed only 16 cases. $^{1}$ Reference was also made to other similar evidence.

At the end of 1902, Dr Koch again referred at length to his views on the transference of bovine tuberculosis to man at the

1 The statistics set forth by Dr Koch with regard to primary intestinal tuberculosis cannot be accepted as representing universal experience. For example, in two separate reports from children's hospitals in London and Edinburgh dealing with 547 cases of death from tuberculosis in children, it appears that in 29.1 per cent. and 28.1 per cent. of the cases respectively, primary infection appeared to have taken place through the intestine (see also p. 244). 
International Conference on Tuberculosis in Berlin. ${ }^{1}$ He again emphasised the comparative rarity of primary intestinal tuberculosis in the human being, and the local, as distinguished from the general, infective nature of accidental bovine inoculation of man (tuberculosis verrucosa cutis). In isolated cases the nearest lymph glands might become affected, but the disease remained nevertheless a local one. Dr Koch further expressed the view that if bovine tuberculosis was transmissible to man by means of the milk of cows with tuberculous udders, it would be reasonable to suppose that "groups of illnesses" would occur, in a manner analogous to other infective diseases, though the circumstances would differ owing to the different length of the incubation periods. By way of illustrating the non-infectivity of bovine tubercle bacilli conveyed by milk, Koch points out $(a)$ that bovine tubercle bacilli must be taken into the numan system very frequently, as I to 2 per cent. of all milch cows suffer from tuberculous udders; $(b)$ that in addition to being drunk in considerable quantity and for long periods, such milk is also widely distributed; $(c)$ that domestic sterilisation of milk does not occur to any appreciable extent; $(d)$ that the same may be said of the large dairies; and finally $(c)$ that if milk under such circumstances is dangerous, the butter derived from it will also be dangerous. For these reasons he maintained that any resulting disease must be widespread. Yet Koch has found "instead of the countless cases," which we ought to expect, "two groups of illnesses and 28 isolated cases of illness." On examination he finds most of these recorded cases not free from objection. To carry conviction as to milk-borne tuberculosis, Koch maintains, that the following conditions must be fulfilled:-(i.) Certain proof of tubercle in the person affected; (ii.) exclusion of other sources of infection; (iii.) the condition of all the consumers of the suspected milk; (iv.) the exact source of the suspected milk, particularly in respect to the disease of the udder of the cow yielding the milk. Finally, he concludes that all that can be said at present is that the injurious effects of milk infected with bovine tuberculosis and its products are not proven.

On the other hand, it must be said that a number of investigators have already brought forward fresh evidence in support of the view previously held that human tuberculosis is transmissible to animals, and, prima facie, the reverse proposition, that bovine tuberculosis is transmissible to man. Among the workers who have thus by later experiment, already thrown doubt upon 1 Brit. Med. Jour., 1902, vol. ii. (20th December), p. 1885 et seq. 
Koch's conclusions are Delépine, ${ }^{1}$ De Jong, ${ }^{2}$ Arloing,, Ravenel, ${ }^{4}$ Fibiger and Jensen, ${ }^{5}$ Spronck and Hofnagel, ${ }^{6}{ }^{6}$ Max Wolff, ${ }^{7}$ and Hamilton. ${ }^{8}$ These experiments included a variety of inoculations and some feeding experiments. In all, thirty-nine positive results were obtained, that is, in thirty-nine instances human tubercle inoculated into cattle set up a greater or lesser degree of tuberculosis. None of these inoculations were of artificial cultures of B. tuberculosis, but of virulent bacilli direct from human sources. Arloing's thirty-three positive results are not included, as they were obtained by inoculation with cultures. There can, we think, be no doubt of the authenticity of these experiments, and the only points of criticism which arise in relation to them are: (a) that the human tuberculosis may have been, in point of fact, of bovine origin, and the bacillus in some or all of the cases of the bovine species; or $(b)$ that the resulting tuberculosis was in some of the cases of a limited degree, or so strictly localised as not to be of a positive nature. The first criticism may be set on one side. It cannot be proved, nor can it be contested. If it be true, it establishes the proposition that bovine tuberculosis is communicable to man. If it be false, the proposition that human tuberculosis is communicable to animals is proved. The validity of the second criticism must depend upon definitions of what positive result means. If the tubercle bacillus which is inoculated is growing, and reproducing itself, and is setting up tissue changes, and such affected tissues if inoculated in guinea-pigs set up the disease once more, we hold strongly that the result should be considered positive, irrespective of the degree or particular organ affected. ${ }^{9}$

${ }^{1}$ Brit. Med.Jour., 1901, ii., 1224. ${ }^{2}$ La Semaine Medicale, 1902 (I 5 th Jan.).

3 Bull. Acad. de Med., Dec. 1901, and Jour. de Med. Veterinaire, May 1903.

4 Intercommunicability of Human and Bovine Tuberculosis, Philadelphia, 1902 ; Univ. of Pennsylvania, Med. Bull., May 1902.

- Berliner Klin. Woch., 1902, No. 38.

- La Semaine Medicale, 15 th October 1902.

7 Deutsche Med. Woch., 1902, No. 32.

8 Trans. of the Highland and Agricultural Society of Scotland, 1903.

${ }^{9}$ Whilst the present volume was passing through the press the result of further experiments was announced from Berlin. From these it appears that Kossel reports that out of the experiments conducted by the Imperial Department of Health human bacilli had in two cases only affected the animals under experiment. Orth, on the contrary, reported Io per cent. of his inoculations on calves as positive, and inducing progressive tuberculosis. Some of these were subcutaneous injections. Behring also reports positive results. An elaborate review of recent knowledge by Kober appears in Amer. Jour. Med. Sc., Oct. 1903,pp. 684-705. 
One of the most interesting points brought out by the recent experiments of Hamilton has relation to the absence of intestinal tuberculosis in the animals fed on tubercular sputum, although the mesenteric and other glands showed abundant signs of tuberculosis. Koch had maintained that it can only be assumed with certainty that a case of tuberculosis has been caused by alimenta when the intestine suffers first, and in a general way it may be said that wherever a discharge containing the tubercle bacillus passes over a surface provided with absorbents like the mucous membrane of the intestine, there is liability of the surface becoming secondarily tubercular. Such auto-infection is not, however, necessary, unless it can be proved that the tubercle bacillus cannot pass through the intestinal mucous membrane to the abdominal glands without causing an intestinal lesion. According to Hamilton, such passage may occur. Whether that be so or not, his experiments show that tuberculosis may be set up in calves by feeding on human tubercular sputum, and yet the intestine remain unaffected. He concludes that "when administered by the mouth tubercular sputum induces an abdominal lymph-gland tuberculosis without necessarily the intestine being in any way involved." 1

This point is one of considerable importance and so closely related to the transmission of tuberculosis by milk that it may be desirable to make reference to the researches of Sidney Martin for the Royal Commission on Tuberculosis.. ${ }^{2}$

I. Four pigs were fed upon bovine tuberculous material (meat and milk) and contracted tuberculosis. In commenting upon the post-mortem signs Dr Martin says :- "In the tuberculosis resulting from feeding pigs with the meat and milk of tuberculous cows, the absence of any lesion (abrasion, nodule, or other mark of disease) in the alimentary tract is very noticeable, so that the disease is first shown in its course from the alimentary tract by an affection of the lymph-glands in connection with the different parts of that tract." And again:- "The chief points to be noticed in these results as they bear on tuberculosis in the human being is that by feeding with tuberculous material there may be no lesion in the alimentary tract; that there may be absorption only through the pharynx and tonsil, producing tuberculosis of the connected lymphgland, so that this condition of things exactly tallies with the

I Trans. of the Highland and Agricultural Society of Scotland, 1903, p. 47.

2 Report of the Royal Commission appointed to inquire into the Effect of Food derived from Tuberculous Animals on Human Health, 1896, part iii., Appendix pp. 18 and 19. 
'strumous' glands of children and young adults. Lastly, from feeding there may be affection of the lymphatic glands alone."

2. Four calves received at one meal, mixed with their food, about I kilogramme of bovine tuberculous material. They all contracted tuberculosis. Dr Martin further pointed out that although "from feeding calves with bovine tuberculous material a lesion in the form of nodules is produced in the intestine, it may not proceed to ulceration but remain stationary. The intestine is affected in the form of isolated nodules as early as four weeks after feeding. . . . The mesenteric glands are in all cases affected to a greater or less degree. From the intestinal lesion the disease in calves spreads to the lymphatic glands in the thorax and the lungs or pleura, or both of these."

3. Four calves received at one meal, with their food, 70 c.c. of sputum (containing a large number of tubercle bacilli) from two cases of human consumption. Three of these calves contracted tuberculosis, but in none of them was there any sign of disease except nodules in the intestine, and in the fourth calf every organ and gland was healthy. Two calves received 440 c.c. of human sputum containing a large number of tubercle bacilli. One calf contracted tuberculosis of the intestine and mesenteric glands. The other remained perfectly healthy. Dr Martin concludes that "it is evident, therefore, that in the case of human tuberculous sputum we are dealing with material which is less infectious to calves than bovine tuberculous material. This lessened infectivity is possibly not merely a question of dosage, but one of diminished activity of the tubercle bacilli in sputum as compared to its activity in the tuberculous lesion of the cow." 1

1 Various circumstances have in all probability contributed to render unsuccessful or irregular in result the numerous feeding experiments which have been made. The tissues of animals differ greatly in susceptibility to tuberculosis; and the virulence of the infective material itself varies enormously, as does the virulence between different generations or races of tubercle bacilli. Hence it comes about that one animal may eat with its food a certain amount of tuberculous material and yet not develop tuberculosis, whilst another animal of the same species might quickly develop the disease, which would in all probability show itself at the animal's weakest point, and not always necessarily in the intestine. Again, there is the question as to what constitutes a positive effect of inoculation and as to whether or not there has been made a microscopical study of the viscera and glands. Further, there is another point which should not be overlooked, namely, the subsequent treatment of the inoculated animal. Whilst it is essential to prove that the animal to be inoculated is free from tuberculosis, it should be remembered that in taking very healthy animals for experiment, and in subsequently treating them in what 
Here, then, are the main facts in the evidence for both sides of the question under discussion. The latter evidence tending to show the transmission of tuberculosis to man by means of milk and meat, is part of that upon which the Royal Commission relied when it reported, "We cannot refuse to apply, and we do not hesitate to apply, to the case of the human subject the evidence (of transmission of the disease) thus obtained from a variety of animals that differ widely in their habits of feeding-herbivora, carnivora, omnivora. As regards man we must believe that any person who takes tuberculous matter into the body as food incurs some risk of acquiring tuberculous disease." 1 And again, "We have obtained ample evidence that food derived from tuberculous animals can produce tuberculosis in healthy animals. In the absence of direct experiments on human subjects we infer that man also can acquire tuberculosis by feeding upon materials derived from tuberculous food-animals." ${ }^{2}$ Because the tubercle bacillus derived from bovine sources is, either by inoculation or ingestion as food, admittedly very virulent and dangerous for such diverse species of animals as the rabbit, horse, dog, pig, sheep, and cow, it is highly probable that it is also dangerous to man. ${ }^{3}$ For it is well known that the majority of disease-producing bacteria are harmful to only one or two species of animals, but

may be termed an "ideal" fashion, some of the very conditions essential to the production of the disease in ordinary life are removed. As in men, so in cattle and other animals, it may be presumed that abundance of good food and fresh air, and, in general, an ideal environment, tend to counteract the effect of the inoculated or communicated virus. Thus such experiments as those stated above may not always fairly represent the modes of transmission of the disease as they occur in ordinary life. It is not the "very healthy" animal of a herd, well-housed and fed, which contracts tuberculosis. It is essential to bear in mind the relationship existing between the seed (the tubercle bacillus) and the soil (the tissues of man or animal).

I Report of Royal Commission, 1895, part i., p. 10, par. 22.

2 Ibid., p. 20, par. 77.

${ }^{3}$ See the researches of Villemin (1865), Klebs, Chauveau (1868), Gerlach, Günther and Harms (1870-1873), Bollinger, and others. Further, Friedberger and Fröhner state in their Veterinary Pathology that Wesener compiled reports up to 1884 of 369 feeding experiments, the positive and negative results of which were about equal in number. From this compilation it appears that (a) 71 animals, among which guinea-pigs and swine proved most susceptible, were experimented upon with human tubercular matter; (b) 180 experiments were made with tubercular matter from cattle ; $(c)$ the flesh of tuberculous cattle was given on 32 occasions as food, with the result that pigs were found to be more susceptible than other animals, and that dogs were unaffected; and $(d)$ the milk of tuberculous cows was given as food in 86 cases. From these experi- 
those disease-producing bacteria that are common to all the domesticated animals are also able to produce disease in man.

We cannot pursue the subject further in this place, particularly as the whole matter is sub judice until the report of the larger experimental work of the Royal Commission is published.

The provisional attitude we take up at the present is the same as that which we have adopted throughout our work on the bacteriology of milk, namely, that tuberculosis in all animals is generically one and the same disease, but that it differs in various ways in different animals, and according to the strain and virulence of the infecting bacillus. That human tuberculosis can be transmitted under certain circumstances to animals, we do not doubt. There is also prima facie evidence to show that the reverse proposition is true, namely, that under certain conditions bovine tuberculosis is transmissible to man. We therefore look upon the two diseases as different species or varieties of one and the same generic disease, and intercommunicable. Whilst we hold this view in respect to the communicability of tubercle, we do not for one moment suppose that its transmission through milk is very frequent or very wide spread. The great field of infection in tuberculosis is from animal to animal and from man to man, and cross-infection is probably less common than is generally supposed.

\section{The Biology of the Bacillus Tuberculosis}

The comparative biology of the Bacillus tuberculosis and its allies becomes every year more complex. As early as I884 Koch pointed out that it was not improbable that in time other bacteria would be discovered possessing the same tinctorial properties as the tubercle bacillus. This was but the first step in the study of its allies, which have now been found to be numerous. Secondly, the tubercle bacillus itself has been proved to exist in a variety of polymorphisms. In the third place, certain pathological conditions have been found to simulate in a marked degree the "tubercles" having their origin in the growth of the specific tubercle bacillus. For some time these allied conditions were known as "pseudo-tuberculosis." It is in the main these

ments it was found that in the scale of comparative racial susceptibility the herbivora (cattle, sheep, goats) proved highest, then swine, and after these guinea-pigs and rabbits. Carnivorous animals were little affected. Bovine tubercular matter was found to possess the greatest power of infection, then came the sputum of tuberculous men, then the milk of tuberculous animals, and lastly, tuberculous flesh. 
three facts which have complicated our knowledge of the biology of the B. tuberculosis. Before discussing this subject in so far as it affects the bacteriology of milk, it may be desirable to state briefly the chief biological facts known respecting the true tubercle bacillus. ${ }^{1}$

The $B$. tuberculosis of artificial culture is usually an unbranched, slender, immotile rod, $\mathrm{I} \cdot 5$ to $4 \mu$ long and $\cdot 4 \mu$ broad, often slightly bent. In sputum and tissues the bacillus may appear branched and in thread forms. The protoplasm of the bacillus consists of fat and wax (26 per cent.), protamin (24 per cent.), nucleo-proteid (23 per cent.), mucleic acid ( 8 per cent.), and the remainder of mineral and proteinoid (chitin) substances. The protoplasm is frequently vacuolated and irregularly segmented, and this becomes particularly obvious after staining. As to staining, the bacillus is acid-proof, and stains well with Ziehl-Neelsen or Gram. Growth does not occur in the absence of oxygen, is most favoured by a temperature varying from $29^{\circ} \mathrm{C}$. to $42^{\circ} \mathrm{C}$, and is at all times slow on artificial media. The common media used are such as agar, blood-serum, potato, and fluid media. The addition of glycerine (4 to 6 per cent.) favours growth. The organism is resistant in a high degree to various kinds of physical agencies. It is retentive of vitality. Its distribution outside the body is probably wide. All kinds of animals seem liable to its infection, and the bacillus has been isolated from dust, milk, etc. As one result of so wide a distribution, it will be understood that man is very liable to infection. It is said that of apparently healthy men, about 30 per cent. are infected at some time or other in their lives by this organism in a greater or lesser degree. The virulence of the human tubercle bacillus for animals is much less than for man, and different strains of the human bacillus may differ from each other very considerably in their degree of virulence. Various external conditions also affect the virulence, which is greatly reduced by continued cultivation on artificial media.

The tubercle bacillus may attack almost any organ of the body. The lymphatic system is constantly involved, and as a rule the organs most affected are those in primary contact or relationship to the site of introduction. When tuberculosis from a human source has been ingrafted upon a calf, it gains enormously in virulence by being reinoculated upon a second calf (Hamilton).

There is an important biological fact which may be mentioned

1 An account of the organism will be found in the chapter describing the various species of bacteria found in milk, see p. 432 . 
here as it bears directly upon the virulence of tubercle bacilli passing through the alimentary canal. It is to the effect that experiment shows that the tubercle bacillus can withstand the action of the gastric juice. Falk and Wesener both. worked at this subject, placing human tubercular sputum with artificially prepared gastric juice, with the result that the bacillus was not affected. Wesener took the additional precaution of keeping the emulsion at blood-heat for a number of hours. Cadeac and Bournay, and Strauss and Wurtz, have also investigated this subject, the two latter in a most thorough manner. They employed natural gastric juice, and tested its effect on pure cultures of the bacillus on glycerine-agar. After six hours the bacilli were found on inoculation to be uninjured. From these facts it may be concluded that tubercle bacilli voided in the excreta of cows having intestinal tuberculosis have not lost their virulence owing to any supposed germicidal effect of the gastric juice.

Tubercle bacilli of bovine and human origin compared.The morphology of the bacilli in cultures of bovine origin is more uniform and constant than in cultures from man. The borine bacilli are thicker, straight, and short, seldom more than $2 \mu$ in length, and averaging less (Theobald Smith). In the early generations many individuals are seen which are oval, their length not more than double their breadth. They are less granular than those from a human source. They stain evenly and deeply with carbol-fuchsin, beading being almost always absent from young cultures, and often from old ones.

The human bacilli are, on the other hand, much longer, thinner, and tend to increase in length in sub-cultures. They are generally more or less curved, sometimes showing S-shaped forms. They stain less intensely with carbol-fuchsin, but beading is generally seen, even in early growths, and is often very well marked.

The above characteristics are most evident and persistent in cultures grown on blood-serum. On glycerine-agar, glycerinebouillon, and glycerine-potato, bovine and human tubercle bacilli approach each other in cultural features and morphology much more closely, and by continued cultivation the differences tend to become obliterated. Bovine cultures are more difficult to isolate than human, are apt to grow as discrete colonies in the first culture, and for several generations grow in a thin layer which somewhat resembles ground glass. The optimum temperature and the thermal death-point are practically the same in both forms. 
. 


\section{ACID-FAST BACILLI.}

Mature colonies on glycerine-agar. Actual size.
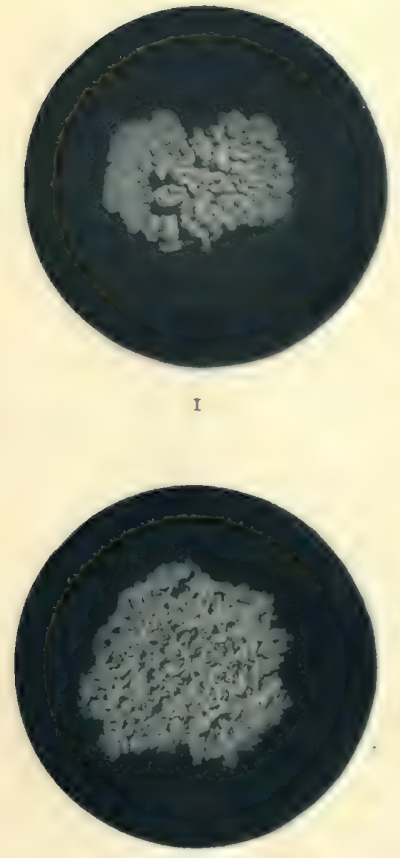

3

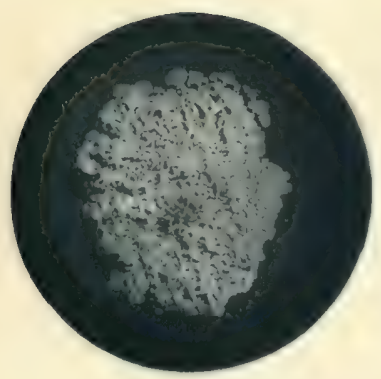

5

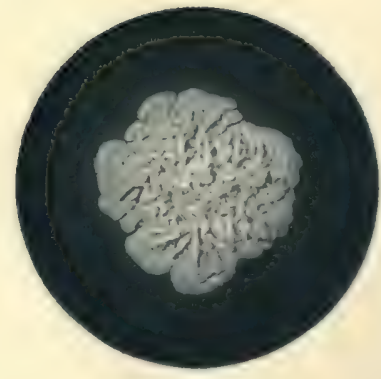

2

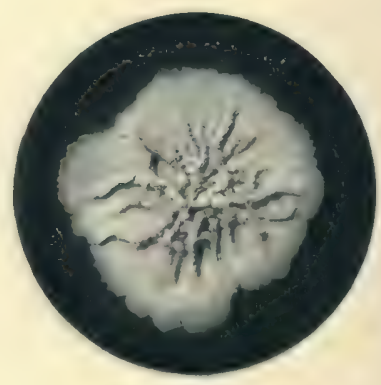

4

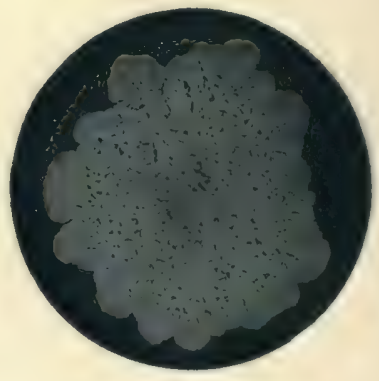

6
1. Bacillirs tuberculosis-source, human.

3. Butter bacillus $\rightarrow$ Rabinowitsch.

5. Bacillus Friburgensis I,-Korn.
2. Bacillus tuberculosis-source, bovine.

4. Butter bacillus-Grassberger.

6. Baclllus Friburgensis II.-Korn. 


\section{ACID-FAST BACILLI.}

Mature colonies on glycerine-agar. Actual size.

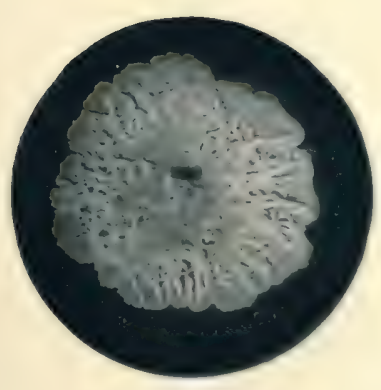

I

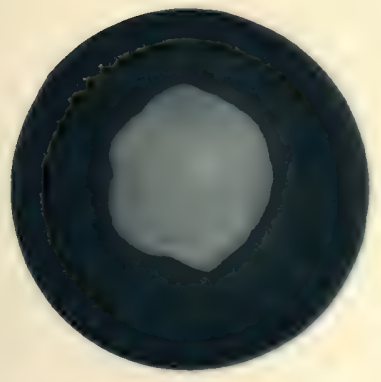

3

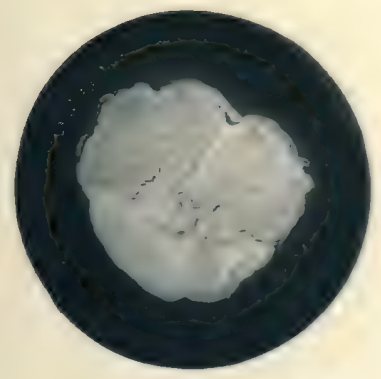

5

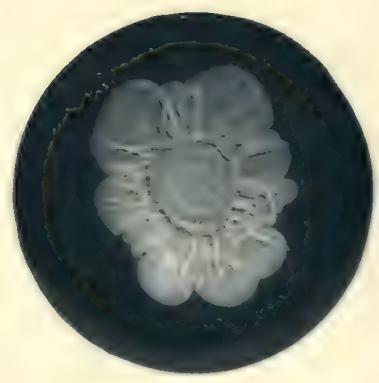

2

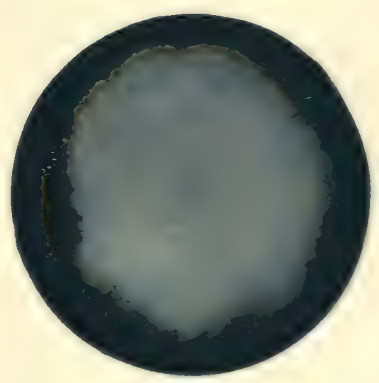

$+$

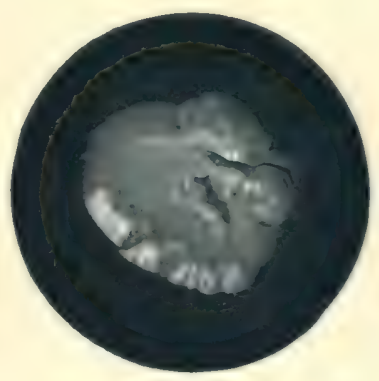

6
1. Butter bacillus-Minot.

3. Timothy-grass bacillus-Moeller.

5. Marpman's bacillus.
2. Milch bacillus-Moeller.

4. Grass bacillus II.-Moeller

6. Manure bacillus-Moeller. 

The human bacillus, as a rule, grows somewhat more easily and abundantly from the first, and will grow well on glycerine-agar in sub-cultures made directly from the original growth on bloodserum. All attempts to obtain a like result with the bovine organisms have failed. In artificial culture the human bacillus rapidly loses virulence. The bovine bacillus grows as a film on blood-serum, whereas the human bacillus produces warty growths.

The morphological distinctions tend to disappear also in the tissues of susceptible animals. We may inoculate a typical bovine culture, and in a short time obtain from the various organs long and much beaded bacilli simulating the human variety (Hueppe).

The most striking dissimilarity is, however, seen in the action of the bacilli from the two sources on animals. By whatever method of inoculation, the bovine bacillus, as a rule, possesses a much greater pathogenic power than the human bacillus for all animals on which it has been tried (Villemin), the only exceptions being possibly those animals, like guinea-pigs, which are so extremely susceptible to both types that it is difficult to draw any distinction between them.

Polymorphism.- Another matter which claims consideration in connection with the biology of the tubercle bacillus is its own polymorphism. As this feature does not directly affect the relation of the bacillus to milk, it will be unnecessary to do more than refer to it in passing. A number of workers since 1882 have described aberrant forms, threads, filaments, branched forms, clubs, dumb-bell shaped forms, diplococcal forms, etc. (Babes, Nocard, Metchnikoff, Fischel, Bruns, Coppen-Jones, Friedrich, Schultze, Schweinitz, Dorset, Moeller, Lubarsch, etc.). Recently it has been suggested that these various forms may be different stages in a single lifehistory, and that upon morphological grounds the tubercle bacillus may be classified among the group of higher fungi like the actinomyces. This latter parasite is able, like the tubercle parasite, to set up specific inflammatory new growths of a granulomatous type.

Pseudo-tuberculosis.-Another characteristic which makes our knowledge of the biology of tubercle complex is that appertaining to what is generally called pseudo-tuberculosis. In I899 the Pathological Society of London urged that this term should be discarded, and it is admittedly an unsatisfactory term, especially when applied to various pathological conditions bearing only a semblance to the tuberculous process. On the other hand, it is a term of convenience, if not suitability, to describe that group of bacilli not identical with tubercle bacillus and not acid-fast, and 
yet capable of producing in certain animals lesions similar to those of the tubercle bacillus. ${ }^{1}$ The chief organism coming within such a category is the B. pseudo-tuberculosis of Pfeiffer. ${ }^{2}$ But other workers have described very similar organisms. Klein found this bacillus present in 2 out of 5 samples of London milk, ${ }^{3}$ and in 8 out of 100 samples of country milk delivered in London. ${ }^{4}$ Delépine found that out of 450 samples of milk, lesions produced by pseudotubercle bacilli were met with four times. It seems not unlikely that this group of bacilli includes several varieties bearing a close general resemblance to each other, but possessing slightly different properties. They gain access to milk in all probability by some accidental contamination. The milk itself remains unaltered in appearance, though it becomes alkaline. As regards differential diagnosis, it may be said that the pseudo-tubercle bacillus is not acid-fast, nor is it similar to $B$. tuberculosis in morphological or cultural characters. The pathological changes set up by it, and which form its chief claim to be considered as in any way related to tuberculosis, differ from that disease in showing an absence of giant cells in the nodules, absence of the true tubercle bacilli, copious presence of the pseudo-tubercle bacilli, and a more rapid development of disease.

\section{Bovine Tuberculosis}

Without entering fully into the conditions and characteristics of bovine tuberculosis (which may be found in any standard work of veterinary pathology), we may say here that the chief anatomical changes are commonly found in the lungs and in the serous membranes. In about one half of all cases, the lungs and the serous membranes become simultaneously affected; in about one third the lungs alone, and in about one fifth the serous membranes alone (Friedberger and Fröhner). Tuberculosis of the pleura and peritoneum produces the well-known "grape disease" (perlsucht). Generally speaking, there is a marked tendency to calcification

1 It is perhaps unnecessary to add that under this term of pseudo-tubercle we do not include the ordinary tuberculosis of animals, protozoal infections, or parasitical disease set up by echinococcus or strongylus in the lungs of cattle.

2 The pathology and etiology of pseudo-tuberculosis is fully treated of by Klein in the Supplement to the Twenty-Ninth Annual Report of the Local Government Board, 1899-1900, pp. 355-384. See also Annales de IInstitut Pasteur, I894, No. 4, and Jour. of Path. and Bact., I898, pp. I60-I 8 I (Muir).

3 Report of Local Government Board, I899-1900, p. 360, and 1900-190 I, p. 332.

4 Jour. of Hygiene, 1901, vol. i., p. 83. 
and calcareous degeneration as compared with caseation in tuberculosis of man.

Tuberculosis of the udder is comparatively rare. Out of 100 tuberculous cows not more than 3 or 4 have tuberculosis of the udder (Bang). The disease occurs as a diffuse, slightly hard, enlargement, generally unaccompanied by fever or tenderness of the organ. Usually only one quarter is attacked, and that generally a posterior quarter. The gland lobules become hypertrophied, and the larger milk-ducts contain yellowish caseous masses, full of bacilli. As the condition advances, there is a considerable increase of the interlobular connective tissue (interstitial mastitis) of the nature of a sclerosis, and firm tubercles of various sizes begin to appear. Consequent upon these changes the udder becomes nodular, and hard and tough. Miliary tubercles appear in the walls of acini, and enormous deposits of bacilli may be found in the udder. Simultaneously with these changes, the mammary lymphatic glands (pudic glands) lying above the posterior region of the udder became enlarged, indurated, and caseous. The disease may advance slowly or with great rapidity. But finally the condition is such that the glandular tissue of the udder is, as it were, smothered by the hypertrophy and fibrous transformation of the interstitial connective tissue. The large excretory ducts become blocked by granulations or fibrous growth outside them, or by caseous masses inside. This stage inevitably leads to milk suppression.

It should not be forgotten that tuberculosis of the udder is associated with tuberculosis of the internal organs. It is almost invariably secondary. It may exist with mild or advanced disease of the internal organs. Its diagnosis is all the more difficult owing to the fact that there may be no symptoms. Generally, opinion must be guided by the local condition of the udder coupled with the condition of the milk. It may occur as a slow, painless growth only evident when advanced, or it may increase with extraordinary rapidity. This latter fact makes it desirable that every animal suffering from tuberculosis of however mild a character should be strictly eliminated from dairy stock. The three points usually emphasised for diagnosis of tuberculous udder disease are-(a) abnormal milk from one quarter, generally a posterior quarter; (b) some hardness, toughness or irregularity of the udder; and (c) enlargment of supra-mammary glands. ${ }^{1}$ The best diagnostic of general tuberculosis is the tuberculin reaction.

I See also Report of Royal Commission on Tuberculosis., r896, part iii., pp. 4I, 42. 
The milk in udder disease.-In the early stages of udder disease the milk remains to all appearances quite normal. One of the first signs of abnormality is the diminution in the yield. Previously to this it is said there is an actual increase in the quantity of milk. As soon as the disease begins to have effect, there is a definite decline in the yield. For example, a cow which in health gave, say, fifteen litres of milk, falls to one half or one quarter of that amount. The milk also changes in consistence, becoming thin, watery, and serous. At the same time the colour may turn to yellow, and the flocculi and flakes which occur in milk from a healthy udder are present in larger size. As the yield diminishes, the consistence of the fluid becomes more and more irregular, the flocculi predominating. If such milk be allowed to stand in a vessel, a deposit of solid matter, composed of these fragments, settles down, leaving a superficial layer of thin fluid at the top. Finally, the consistence becomes sero-purulent and then purulent. Hence, previously to suppression we get a thick yellow purulent fluid, having an alkaline reaction, coagulated casein, and diminution of lactose. As a rule, tubercle bacilli are readily found, and whether that is so or not the milk is highly infective. $^{1}$

\section{The Entrance of Tubercle Bacilli into Milk}

There are two main sources of the tubercle bacilli found in milk, namely, a bovine source and a human source. The two common channels respectively are a tuberculous udder and a phthisical lung. From the former, milk may derive a direct and abundant supply of tubercle bacilli, from the latter, milk may become indirectly contaminated by the particulate matter of dried sputum.

Tuberculosis may be introduced into healthy cows in a variety of ways. The most common method is by means of a tuberculous animal, from the excretions and discharges of which infection may be conveyed to soil, water, air, fodder, and general surroundings. In this way not only other animals cohabiting with a tuberculous animal become infected, but premises, stables, and utensils may also become infected. The milk of a tuberculous animal may also be consumed by other animals on the farm, and so a vicious circle of infection is completed. Ravenel has shown that by the cough of a tuberculous cow tubercle bacilli may be distributed. Of thirtyfour examinations carried out on five tuberculous cows tubercle

\footnotetext{
1 See also Report of Royal Commission on Tuberculosis, 1896, part iii., p. 142.
} 
bacilli were detected on twenty occasions. One of the cows constantly coughed up a tenacious mucus containing large numbers of tubercle bacilli. During a period of time extending over fortythree days mucus from two cows was collected and inoculated into forty-five guinea-pigs, twenty-two of which, escaping peritonitis, were available for post-mortem examination for tuberculosis. Eleven, or fifty per cent. of them, were found to be markedly tubercular. ${ }^{1}$ The saliva as well as the bronchial mucus of tuberculous cows has been found to contain abundant bacilli, and by licking her udder it is possible for a tuberculous cow to convey tubercle bacilli to its exterior surface.

The excreta also are infective when lesions are located in the alimentary canal. In tuberculosis affecting the alimentary canal of the cow (one per cent. of the cases), it is thus possible to get contamination of the milk, indirectly, from the excreta. The mucous membrane of the intestine, especially the colon, sometimes shows tubercular ulcers, which are less frequently observed in the abomasum. Tubercles may also develop under the mucous membrane and serosa of the stomach and intestines. In these ways arises a condition of intestinal tuberculosis, which in its acute or ulcerating stage will cause the excreta to be loaded with tubercle bacilli. Any one familiar with a cowshed will at once recognise how readily milk might become infected under such circumstances, which, though undoubtedly exceptional, must not be overlooked. ${ }^{2}$ As we have seen, Cadeac and Bournay, Strauss and Wurtz, and other investigators have shown that tuberculous material is not rendered innocuous by the gastric juice, and there is therefore no doubt that true virulent tubercle bacilli can pass through the alimentary canal of the cow and be found in manure, straw, and stable refuse. In these ways stalls may become infected and transmit the disease to fresh herds stabled in such premises. Nor are herds unstabled always free from tuberculosis, as has been recently stated. A number of observers have shown that whilst it is true that ill-ventilated, dark, damp cowsheds predispose to infection, milch cows living entirely in the open do not, on that account, escape the disease. ${ }^{3}$ It

1 Commonwealth of Pennsylvania, Bulletin 75 (Pearson and Ravenel), I90I, p. 82.

${ }^{2}$ Trans. British Congress on Tuberculosis, 190I, vol. iii., p. 664 (Boinet and Heron).

${ }^{3}$ Report on Bovine Tuberculosis, Government of New Zealand, Ig00 (Gilruth). 
depends upon infection in the herd, that is upon contagion. But it is probable that, as a channel for the conveyance of infection, the udder is more common than any other. When the udder is affected, the milk invariably contains large numbers of bacilli, and it will be understood that when one cow in a herd is so diseased, the entire volume of mixed milk from the herd will be contaminated. The presence of the bacilli in the milk is not always proportionate to the extent of the disease in the animal, especially when diagnosed clinically. The reason of this is the difficulty of clinical diagnosis between chronic interstitial mastitis and tuberculous udder. There can, however, be little doubt that the chief source of tubercle bacilli in milk is the tuberculous udder.

Finally, milkers affected with phthisis may readily infect the milk, either by the repulsive habit of spitting on their hands prior to milking, or by dried expectoration in cowshed, dairy, or milkshop. After distribution, milk is exposed in a variety of ways to dust, and it cannot be doubted that such dust does at times contain particulate matter derived from dried tubercular expectoration, and that therefore in this way also it is possible for milk to become infected.

The requirements for infection by tuberculosis may be summarised very simply as follows:-First, the causa causans, without which there can be no tuberculosis, is the tubercle bacillus of Koch. Secondly, the conveyance of tubercular infection from man to man and from animal to animal in an infected fluid or dried infective discharge ground into fine dust. Thirdly, an important condition of transference and of site of disease is that the part of the body of man or animal in which the infective germs begin to grow must, as a general rule, have been in some way weakened or injured. And lastly, to gain foothold and produce disease, the infection itself must be of some degree of intensity, or have been in operation for some period of time. It is these conditions which are the controlling factors in the limitation, such as it is, of the ravages of the disease.

The possibility of infected milk without clinical udder disease. - It was believed by many, and is still held by some, that the milk of tuberculous cows is infective only when the udder is affected. Ernst, Theobald Smith, Bang, and Hirschberger have, however, shown that cows having generalised tuberculosis but apparently unaffected udders may, in fact, yield a small percentage of cases of tuberculous milk. The last named found this percentage to rise as 
high as 55. A series of experiments were made in the laboratory of the State Live Stock Sanitary Board of Pennsylvania in 1896 and 1897 , from which it was concluded (a) that the number of bacilli in the milk of tuberculous animals varies from day to day; and (b) that the tubercle bacillus may pass into the milk of cows having generalised tuberculosis, but whose udders are perfectly healthy so far as the most careful examination during life and post-mortem could show. ${ }^{1}$ Again, Rabinowitsch and Kempner inoculated the milk of fifteen cows into guinea-pigs. The cows had reacted to tuberculin, but had no tuberculous disease which could be detected by clinical examination, and showed no sign of udder disease. The results showed that $7 \mathrm{I} \cdot 4$ per cent. of the animals yielded milk containing virulent tubercle bacilli.

Lastly, an elaborate series of experiments on this matter have recently been conducted by J. R Mohler, of the Bureau of Animal Industry at Washington. He inoculated and fed guinea-pigs upon the centrifugalised sediment of milk and cream from a number of cows which had reacted to tuberculin and yet revealed no clinical signs of udder disease. Subsequent post-mortem examinations of the cows were made. One hundred and twelve cows were used, 56 of which reacted to tuberculin. The combined results of the ingestion and inoculation experiments show that the milk of 12 out of the 56 reacting cows, or 21.4 per cent., contained virulent tubercle bacilli. Dr Mohler arrives at the following important conclusions:-(I) That the tubercle bacillus may be demonstated in milk from tuberculous cows when the udders show no perceptible evidence of disease either macroscopically or microscopically; (2) that the bacillus of tuberculosis may be excreted from such an udder in sufficient numbers to produce infection in experimental animals, both by ingestion and inoculation; (3) that in cows suffering from tuberculosis the udder may, therefore, become affected at any moment; (4) that the presence of the tubercle bacillus in the milk of tuberculous cows is not constant, but varies from day to day ; (5) that cows secreting virulent milk may be affected with tuberculosis to a degree that can be detected only by the tuberculin test; (6) that the physical examination or general appearance of the animal cannot foretell the infectiveness of the milk; (7) that the milk of all cows which have reacted to the tuberculin test should be considered as suspicious and should be subjected to sterilisation before using; and ( 8 ) that it would be better still that tuberculous cows should not be used for general

1 Commonwealth of Pennsylvania, Bulletin 75, pp. 66-68. 
dairy purposes. ${ }^{1}$ We know of no experiments on this subject which have been so thorough as Dr Mohler's work, and we consider his findings to be of great importance. Further, it should be remembered that his work does not stand alone, but is only confirmatory of many other similar experiments. Either the udder is affected in a much larger proportion of cases than has been hitherto admitted, or many cows with apparently sound udders excrete tubercle bacilli in their milk. In any case, if these findings be accepted, clinical examination of the udder cannot be admitted as a reliable test of the presence or absence of tuberculous infectivity. ${ }^{2}$ At the same time we must add that we believe the subject requires careful and repeated experiment before absolutely final conclusions can be drawn. ${ }^{3}$

\section{The Virulence of Milk containing Tubercle Bacilli}

In considering this question, it should be remembered that "virulence" is only a relative term. There is no exact standard of virulence. One strain of bacillus tuberculosis is highly virulent, and a second strain the reverse. Hence it is only possible to deal in a broad way with the virulence which milk may obtain from tubercle bacilli.

The virulence of milk from tuberculous udders was first insisted upon by Klencke in 1846 , and confirmed by Gerlach in 1869 . Koch, Bang, McFadyean, Woodhead, Hirschberger, Nocard, Crookshank, and many others obtained evidence in support of the view which is now universally accepted, namely, that milk drawn from a tuberculous udder is highly virulent to guinea-pigs and other animals. Most observers go a step further, and assume that on this account it is also virulent, in some measure, for man.

1 Bureau of Animal Industry, Washington, Bulletin 44. "Infectiveness of Milk of Cows which have reacted to the Tuberculin Test," John R. Mohler, 1903, p. 93.

2 See also Eighteenth Annual Report of Bureau of Animal Industry, Washington, 1901, p. 263.

${ }^{3}$ Only recently Ostertag experimented on Io cows which reacted to tuberculin and yet showed no signs of clinical tuberculosis. Inoculation of the milk failed to set up any tuberculosis in guinea-pigs, and feeding calves and pigs with the milk for many weeks also failed to set up disease. From these results it was concluded that the milk of cows which simply reacted to tuberculin and had no udder disease, does not contain tubercle bacilli (Exp. Sta. Rec., Oct. 1902). J. H. Young of Aberdeen has also made some recent experiments on 14 cows free from udder disease and yet reacting to tuberculin. Inoculations into guinea-pigs proved negative (Brit. Med. Jour., 1903, i., p. 816). 
Martin reported, as a result of his experiments, that "the milk of cows with tuberculosis of the udder possesses a virulence which can only be described as extraordinary." 1 All the animals he inoculated with milk from tuberculous cows showed tuberculosis in its most rapid form, such as is seen after inoculation with a recent tubercular lesion. In experiments for the same Commission, Woodhead records similar conclusions. "Raw milk from tubercular udders," he says, "is in all cases possessed of the power of setting up tubercular infection, even when mixed with considerable quantities of sound milk, whilst in some cases it possesses this power in a most remarkable degree." 2

Nor is the virulence wholly a question of species. When sterilised milk is inoculated with tubercle bacilli derived from a culture on serum, or from the tubercular deposit of the organs of a guinea-pig, it shows after fourteen or twenty days at $37^{\circ} \mathrm{C}$. a growth of tubercle bacilli in the deeper layers, the milk remaining unchanged. Such growth consists of typical clumps of bacilli. After six weeks the number of such clumps is greatly increased. But more than this, for it is found that the virulence of such milk is more exalted than the virulence of the original culture (Klein). Old non-pathogenic cultures of tubercle bacillus or glycerine-agar may thus be changed into virulent agents with marked pathogenic action. The same increase in virulence follows sub-culturing from milk to milk. Similar experiments with cheese or cream gave negative results. ${ }^{3}$ It is not suggested, it need scarcely be added, that this increase of virulence in sterilised milk cultivation is of a high degree; indeed, there is evidence to the contrary; nor is it to be supposed that an exactly similar condition appertains in the case of ordinary milk which becomes infected with tubercle bacillus. The two cases are not similar, for in the latter instance there is, as we have seen, the enormous increase of other milk bacteria, whose rapid growth might exert an inimical effect upon a small infection with the tubercle bacillus.

On the other hand it has been shown that dilution of tuberculous milk with water or sound milk lessens the degree of its virulence (Bollinger and others), and there may be other conditions in milk, at present unknown, which diminish the virulence

${ }^{1}$ Royal Commission on Tuberculosis Report, 1896, Appendix, Inquiry ii., par. 148.

2 Ibid, Inquiry iii., par. 120.

3 Twenty-Ninth Annual Report of the Local Government Board. Medical Offer's Supplement, 1899-1900, pp. 577-585. 
of the tubercle bacillus. The whole matter of virulence is one very difficult to measure, or form precise opinion upon, owing to the wide variations in specific virulence of the tubercle bacillus and the impossibility of estimating the effect of dosage. From a practical point of view it may be said that milk of a tuberculous cow is most virulent when the udder is diseased and undiluted milk from such udder is consumed in fairly large and continuous quantities.

It is now generally admitted that the bovine tubercle bacillus possesses greater pathogenic power than the human bacillus. Yet this, of course, is a variable quantity, and is a factor relative to the point at issue. Exaltation of virulence for one species of animal does not necessarily prove an increased power for other species, although such would be the general rule. There is evidence of direct infection of man by accidental inoculation with the bovine bacillus, and of indirect infection by means of infected milk. It is with the latter only that we are concerned. Apart from the question as to how much primary intestinal tuberculosis exists in the human, and what percentage of it is due to cow's milk, there are certain facts giving evidence of the virulence of milk which must be mentioned. ${ }^{1}$ In the first place, after all deductions are made,

1 We do not consider that the widespread discussion as to the inoculability of the bovine bacillus upon man, or the degree of primary intestinal tuberculosis in man, has up to the present yielded sufficiently definite results for inclusion in the present volume. These are, and must remain for the present, matters rather for clinical investigation and inquiry. Here we only have space to refer very briefly to the question.

As to the evidence of direct inoculation of the bovine bacillus, it must be said that a number of cases are now on record appearing to prove that under certain circumstances man may so contract tuberculosis (Tscherning, Pfeiffer, Hartzell, Mueller, De Jong, Lesser, Joseph, Trantmann, Ravenel, etc.). Such cases seem to prove the possibility of inoculation, but it is well known that this is not a common mode of contracting the disease, and Koch believes that in many of these cases there were other sources of infection.

The evidence as to the indirect conveyance of tuberculosis from the bovine race to man by means of infected milk is of a nature which leaves little doubt that such may occur. But in all probability it is neither as serious in degree or wide in extent as has been supposed. Koch has laid down the view that tuberculosis caused by aliments can only be assumed with certainty where the intestine suffers first, that is, when a so-called primary tuberculosis of the intestine is found. This condition, he states, is extremely rare, and therefore there is no considerable infection by means of milk. But primary intestinal tuberculosis cannot be considered as the only criterion of tubercular infection by means of food, and even if that were so, there is evidence to show that whilst primary infection of the abdomen is by no means so common as primary infection of the respiratory tract, nevertheless it does occur. Many observers 
a considerable amount of alimentary tuberculosis undoubtedly occurs. This may be measured partly by the returns of the Registrar-General, and partly by the results of special inquiry. The Registrar-General's returns for the last thirty years have shown a marked decrease in deaths due to phthisis, and a less

(including Stang, Demme, Gosse, Ollivier, Law, Ebers, Bang, Von Ruck, Nocard, Klebs, and Rievel) have recorded cases which appeared to show that infection had been derived from milk. Carr, Guthrie, Still, Shennan, Holt, Northrup, Bovaird, Kossel, and others have reported on the results of necropsies. Broadly, it may be said that there are two conclusions to be drawn from these post-mortem examinations, namely, (i.) tuberculosis is a common disease of children; and (ii.) the primary lesion is in more than half the cases in the lungs. We may quote as illustration of this latter statement, the percentage returns in the post-mortem examinations of tuberculous cases by Carr, Guthrie, and Still as follows :-

\begin{tabular}{|c|c|c|c|c|c|c|c|c|c|}
\hline & & & & & $\begin{array}{l}\text { No. of } \\
\text { Cases. }\end{array}$ & $\begin{array}{l}\text { Primary } \\
\text { Lesion in } \\
\text { Lang. }\end{array}$ & Percentage. & $\begin{array}{l}\text { Primary } \\
\text { Lesion in } \\
\text { Intestine. }\end{array}$ & Percentage. \\
\hline Carr $^{1}$. & - & . & . & - & 120 & 77 & $64 \cdot I$ & 20 & I6.6 \\
\hline Guthrie 2 & * & . & . & . & 77 & 42 & $54 \cdot 5$ & 19 & $24 \cdot 6$ \\
\hline Still ${ }^{3}$. & . & . & . & . & 269 & I 38 & $51 \cdot 3$ & 63 & $23 \cdot 4$ \\
\hline
\end{tabular}

The frequency with which intestinal tuberculosis is met with in children varies in different places. It is recorded as occurring in Tubingen in 14.7 per cent. of the cases, in Kiel in 31 per cent. (Simmonds), and a later record gives it at $4 \mathrm{I}^{\cdot} 3$ per cent. (Bolz). In Munich, also, it has twice been estimated, once at $3^{I}$ per cent., and a second time at $3^{8}$ per cent. Widerhofer, in an examination of 418 cases found the total cases of intestinal tuberculosis to be ror. Shennan, working in Edinburgh, found 27.8 per cent. of alimentary tuberculosis ; Batten in London found 13 per cent. ; and Northrup in New York met with 3 in 125 (2.4 per cent.) cases of infant death from tuberculosis. In many of these inquiries, however, sufficient care has not been taken, in our opinion, to differentiate between whether the intestinal tuberculosis was prinary or secondary in origin. Amongst 269 tuberculous children under twelve years of age whom Dr Still examined post-mortem, he found it possible to determine the channel of infection with some degree of certainty in 216 cases. In 138 ( 63.8 per cent.) infection entered through the lung, in 63 (29.I per cent.) primary infection occurred in all probability through the intestine. Of children up to two years of age he found 65 per cent. contracted infection through the lung, and 22 per cent. through the intestine. In infants under one year of age apparently only I 3 per cent. contracted tuberculosis through the intestine. ${ }^{4}$

It is recognised that, owing to the great tendency to generalisation of

Lancet, 1894, vol. i., p. 1177.

2 Ibid., 1899, vol. i., p. 286.
${ }^{3}$ Brit. Med. Jour., 1899, vol. ii., p. 457.

4 Practitioner, Igor (July), p. 94. 
marked decrease in deaths due to Tabes mesenterica, and in children under one year a notable increase of deaths due to this latter disease. $^{1}$ Deaths from phthisis occur mostly between the ages of twenty and forty years, and from Tabes mesenterica in children under two years. Such returns admittedly vary according to variations in diagnosis, post-mortem examinations, medical opinion, and other unknown quantities. There is also the difficulty of forming correct opinions as to whether or not the condition was primary or secondary, and due to alimentary or other infection. In this connection it should be remembered that it is comparatively rare to find the mesenteric glands affected in phthisis. The result of recent special inquiries by post-mortem examination by a number of workers has crystallised the deductions to be drawn from the reports of the Registrar-General, and has added emphasis to the view that a considerable amount of alimentary tuberculosis tuberculosis in children, it is a matter of extreme difficulty to determine which was, in fact, the primary channel of infection, and this must be taken into consideration in estimating the significance of the frequency in the above figures. Further, owing to the fact that children swallow their pulmonary expectoration, secondary infection of the intestine may rapidly follow primary infection of the lungs. Hence it comes about that, in many cases, the intestine and mesenteric glands are affected, and yet such a condition cannot be taken as evidence of the infection by food. Dr Still concludes that $(a)$ the commonest channel of infection with tuberculosis in childhood is through the lung; $(b)$ infection through the intestine is less common in infancy than in later childhood; (c) milk, therefore, is not the usual source of tuberculosis in infancy; and (d) inhalation is much the commonest mode of infection in the tuberculosis of childhood and especially in infancy. Dr Still has placed on record five cases of tuberculous ulcer of the stomach in children.

It would seem, therefore, that the evidence in support of the theory that children commonly contract their tuberculosis from milk is not conclusive, but rather the reverse. The first Royal Commission on Tuberculosis and Sir Richard Thorne ${ }^{2}$ may be said to have laid the chief emphasis upon the milk theory of infantile tuberculosis. But later researches have thrown some doubt upon some of the conclusions of the Commission, and with others we have, for long, thought that the facts emphasised by Sir Richard Thorne bear, in part, another interpretation than that to which he drew attention. Further exact research and careful inquiry is needed to obtain necessary data upon which to base any reliable conclusions respecting this matter. But we are satisfied that, in any event, there is evidence to show that tuberculosis can be conveyed to man by means of milk, and that milk for sale containing the bacilli of tubercle is being sold, not alone to the prejudice, but also to the risk, of the purchaser.

1 Reports of Registrar-General, Somerset House ; The Administrative Control of Tuberculosis (Sir Richard Thorne), 1899, pp. 5-9 and 28-32.

2 The Administrative Control of Tuberculosis (Harben Lectures), 1899, pp. 5-7, and 28-32. 
is primary in origin. Probably it would be correct to say that of all tuberculosis in children in this country about 25 per cent. is alimentary in origin, ${ }^{1}$ and in 60 or 70 per cent. of the cases the mesenteric glands are affected. ${ }^{2}$ Both these sources of information deal with deaths only, but no doubt a number of cases of alimentary tuberculosis recover, the infection having been a mild one (Raw).

In the second place, as we have already pointed out, some 2 or 3 per cent. of all milch cows have tuberculous udders, and yield virulent milk. There can, therefore, be no doubt that a certain amount of tuberculous milk is being daily consumed, and mostly by children, the least resistant of whom contract alimentary tuberculosis, which in a certain percentage of cases proves fatal.

Dr Nathan Raw has recently made the ingenious suggestion that the human body is susceptible to both human and bovine tuberculosis (which he admits are different species of the same disease), but that they are antagonistic to each other, and do not attack the body simultaneously. Further, he holds that the bovine species of tubercle, conveyed by milk, attacks the body (the alimentary tract) in the early periods of life, setting up Tabes mesenterica and abdominal tuberculosis, which is bovine in origin; and that the human species, conveyed commonly by contact with dried infective matter, attacks the body (the respiratory tract) in the later periods of life, setting up pulmonary tuberculosis. He agrees with Martin, Woodhead, and others that if tuberculous milk be absorbed through the tonsils and pharynx, it causes tuberculosis in the cervical glands in the same way as in the pig. ${ }^{3}$

Taking a broad view of the facts set out above, we hold that whilst tuberculosis is not chiefly spread by means of milk, there is unmistakable evidence, derived from pathological and clinical experience, proving that tuberculous milk can, and does, set up some form of tuberculosis (bovine or human) in the bodies of man and other animals consuming the milk.

\section{Powers of Resistance of the Tuberele Bacillus}

In this sub-section we propose to deal briefly with the effect of high temperatures upon the tubercle bacillus, as it concerns the

1 Practitioner, August 1903, pp. 201, 216 (Price-Jones).

2 Ibid., also Brit. Med. Jour., 1903, p. 596 (Raw).

3 Report of Royal Commission on Tuberculosis, 1895, part iii., pp. 18, 19 ; Human and Bovine Tuberculosis, by Nathan Raw, M.D., F.R.S.E., I903, pp. 4,8 , and 12 . 
milk question indirectly. Mention will also be made of the effect of other physical agencies.

(i.) High temperatures. - A large number of experiments have been made, with the object of establishing the thermal death-point of the $B$.tuberculosis. There has been considerable discrepancy in the results that have been obtained. The reason for this is due in part to the varied degrees of virulence of different strains of tubercle bacillus, and in part to the fact that there has been little or no uniformity in the carrying-out of the experiments. In some instances tuberculous tissue has been used, in others cheesy pus, in others pure cultures of more or less lengthened artificial cultivation, and, in others, sputum. Again, different media have been used in which to test the thermal death-point. The difficulty of growing the bacillus on artificial media may also have affected the experiments in some cases.

As far back as I884 Schill and Fischer made a number of experiments to determine the thermal death-point, and they found that an exposure at $100^{\circ} \mathrm{C}$. for five minutes destroyed the tubercle bacillus in sputum. Three years later Sternberg inoculated guinea-pigs with tuberculous sputum subjected for ten minutes to the following temperatures, $50^{\circ}, 60^{\circ}, 70^{\circ}, 80^{\circ}, 90^{\circ} \mathrm{C}$. That exposed at and above $60^{\circ} \mathrm{C}$. was rendered innocuous. Yersin found a temperature between $65^{\circ}$ and $70^{\circ} \mathrm{C}$. for ten minutes to be the deathpoint. ${ }^{1}$ Many other workers obtained somewhat similar results (Grancher, Lebard, Bonhoff, Beck, Hewlett, etc.).

In 1893 de $\mathrm{Man}^{2}{ }^{2}$ working with broken-down, semi-fluid cheesy matter derived from tuberculous udders, obtained the following results, which have been generally accepted as the standard quoted in text-books :-

\begin{tabular}{|c|c|c|}
\hline \multicolumn{3}{|c|}{ Thermal Death Limits of B. tuberculosis from the Udder. } \\
\hline Temperature. & & Time of Exposure. \\
\hline $\begin{array}{l}\text { At } 55^{\circ} \mathrm{C} .\left(131^{\circ} \mathrm{F} .\right) \\
" 60^{\circ} \mathrm{C}\left(140^{\circ} \mathrm{F} .\right) \\
" 65^{\circ} \mathrm{C} .\left(149^{\circ} \mathrm{F}\right) \\
" 70^{\circ} \mathrm{C}\left(158^{\circ} \mathrm{F} .\right) \\
" 80^{\circ} \mathrm{C}\left(176^{\circ} \mathrm{F} .\right) \\
" 90^{\circ} \mathrm{C}\left(194^{\circ} \mathrm{F}\right) \\
" 95^{\circ} \mathrm{C}\left(203^{\circ} \mathrm{F}\right)\end{array}$ & $\begin{array}{l}: \\
:\end{array}:$ & $\begin{array}{l}4 \text { hours. } \\
\text { I hour. } \\
15 \text { minutes. } \\
10 \text { minutes. } \\
5 \text { minutes. } \\
2 \text { minutes. } \\
1 \text { minute. }\end{array}$ \\
\hline
\end{tabular}

1 Ann. de Inst. Pasteur, 1888, ii., 60.

2 Archiv. für Hygiene, 1893, xviii., 133. 
Woodhead ${ }^{1}$ fed six guinea-pigs with milk from a tuberculous udder, which had been exposed for fifteen minutes to $60^{\circ} \mathrm{C}$., without effect. Similarly, milk heated for thirty minutes at $60^{\circ} \mathrm{C}$. had no effect on guinea-pigs fed with it. On the other hand, milk heated fifteen minutes at $60^{\circ} \mathrm{C}$., and injected into the peritoneal cavity produced tuberculosis in two of three guinea-pigs. Of three guinea-pigs inoculated in the same way with milk heated at $60^{\circ} \mathrm{C}$. thirty minutes one became tuberculous. In another series of experiments with milk from a tuberculous udder no disease resulted from milk heated twenty-five or more minutes. Milk heated for shorter periods was not injected. Experiments were also made with milk to which ground and minced tuberculous tissue had been added. In such milk, artificially infected, the tubercle bacillus was in some instances still alive after two hours exposure to $60^{\circ} \mathrm{C} .^{2}$

The next series of experiments to which reference must be made were those by Theobald Smith in $1897 .{ }^{3}$ The basis of his experiments was artificial cultures and caseous material from tuberculous cattle. Milk was at first used exclusively as the suspending fluid, but at a later stage it became necessary to use simpler fluids, such as bouillon, distilled water, and physiological salt solution to clear up the discordant results obtained with milk. The manipulations were modified from one test to another, either to remove some supposed defect of former methods, or to answer certain new questions which former tests had raised. Blood serum from the $\operatorname{dog}$ set at $75^{\circ}$ to $76^{\circ} \mathrm{C}$. was used exclusively as the culture medium. The suspensions were made by rubbing masses of bacilli against the inner surface of sterile test-tubes near the bottom until a fairly homogeneous coating had been formed. The rubbing was done with a heavy platinum wire beaten into the form of a slender spatula. The suspending fluid was then poured into the tube and thoroughly stirred. The resulting suspension contained many clumps of bacilli made up of a few to thirty or more rods. The tubes thus prepared were exposed in a waterbath, or else other tubes were used to which definite quantities

1 Report of the Royal Commission on Tuberculosis, 1895, p. 145. The whole of the Inquiry iii. is full of instruction respecting this matter, especially pars. 103 et seq.

2 See also Galtier's experiments, Comp. Rend. de la Soc. de Biologie, 1900. Bang, Brit. Congr. on Tuberculosis, Igor, vol. iii., p. 592. Also Delépine, Ibid., vol. iii., p. 602 .

3 Journal of Experimental Medicine, 1899, vol. iv., p. 217 et seq. See also vol. iii., 1898, p. 45 I. 
of the original suspension had been added. In all cases the suspensions were injected directly into the peritoneal cavity of guinea-pigs in order to give the bacilli the best opportunity for multiplication.

The experiments strengthened the supposition that the pellicle which forms on milk in test-tubes during heating is responsible for the increased resistance of milk suspensions of tubercle bacilli, and the irregular results hitherto obtained. (This pellicle, which is familiar to all who have scalded milk, is a feebly cohesive mass, easily washed out in patches for microscopic examination by diluting the heated milk in water. The patches consist of fat globules and an amorphous, cohesive substance of slight refrangibility, by which they are held together.)

The experiments demonstrate that tubercle bacilli are no more resistant to heat than many other bacilli not producing spores, and that at $60^{\circ} \mathrm{C}$. destruction is complete in fifteen to twenty minutes. Even after exposures lasting ten minutes the bacilli were dead in most instances. After five minutes' exposures the disease produced by inoculation in guinea-pigs was greatly retarded, even though three times the control dose was injected. When, however, milk is used as the suspending fluid, the formation of the surface pellicle into which bacilli are carried by fat globules shields them from the effect of the heat, so that they may survive an exposure of sixty-five minutes. The peculiarly irregular results obtained by the Royal Commission are probably to be explained in the same way. The importance of a clear understanding of this phenomenon in the pasteurisation of milk is obvious, and it remains to be seen how far bottled milk may be freed from tubercle bacilli without resorting to the higher temperature of $68^{\circ} \mathrm{C}$. now generally employed. Probably a complete immersion, or else a complete filling of the receptacle, may furnish the conditions desired.

The different results obtained by de Man, already quoted and now generally accepted, are probably due to the fact that he used caseous material or else tuberculous tissue, which he ground up in a mortar and only diluted with salt solution "when necessary." Such dense suspensions cannot be used to determine the thermal death-point to be compared with that of other bacilli, nor can they be regarded as imitating the conditions under which tubercle bacilli appear in milk.

The method of using narrow sealed tubes has shown itself superior to that which employs ordinary test-tubes so far as milk 
suspensions are concerned. When other fluids were used the results were unequivocal and in harmony with the other method, excepting perhaps in the last experiment, in which an increased resistance of the tubercle bacilli in water is manifest.

The two chief conclusions drawn by Theobald Smith from his experiments are :-

I. Tubercle bacilli when suspended in distilled water, normal salt solution, bouillon, and milk are destroyed at $60^{\circ} \mathrm{C}$ in fifteen to twenty minutes. The larger number are destroyed in five to ten minutes.

2. When tubercle bacilli are suspended in milk, the pellicle which forms during the exposure at $60^{\circ} \mathrm{C}$. may contain living bacilli after sixty minutes.

The last experiments in relation to thermal death-point which need be quoted are those of Russell and Hastings. ${ }^{1}$ This series of experiments was of a very thorough character.

The standards previously used in pasteurisation had been $150^{\prime}$ to $155^{\circ} \mathrm{F}$. $\left(65^{\circ}\right.$ to $68^{\circ} \mathrm{C}$.), for fifteen to twenty minutes. Under these conditions the consistency of milk is materially changed. The cream does not rise readily in milk so treated, and the consistency or "body" of cream itself is much lessened. If the exposure is made at the temperature of $140^{\circ} \mathrm{F}$. $\left(60^{\circ} \mathrm{C}\right.$.) or below, this diminution in consistency does not occur. In fact milk or cream heated no higher than this cannot be distinguished from the normal product. Hence these experiments were undertaken, in the first instance, to check the work of Theobald Smith quoted above.

If it is possible effectually to destroy the tubercle bacillus at this temperature $\left(140^{\circ} \mathrm{F}\right.$.) by an exposure for a brief period of time, a new standard for pasteurisation may be made, that will enable one to overcome the only objection that has been raised against the use of this product.

The results of the experiments of Russell and Hastings are as follows :-

1. An exposure of tuberculous milk to $140^{\circ} \mathrm{F}$. $\left(60^{\circ} \mathrm{C}\right.$.) in a tightly closed commercial pasteuriser for a period of ten minutes, destroyed in every case the tubercle bacillus as determined by the inoculation of such heated milk into susceptible animals like guinea-pigs.

2. Where milk was exposed under conditions that would enable a pellicle or membrane to form on the surface, the tubercle

1 Seventeenth Annual Report of the Wisconsin Agricultural Expt. Sta., I900, pp. $147-170$. 
organism is able to resist the action of heat at $140^{\circ} \mathrm{F}$. $\left(60^{\circ} \mathrm{C}\right.$.) for considerably longer periods of time.

3. Efficient pasteurisation can be more readily accomplished in a closed receptacle, such as is most frequently used in the commercial treatment of milk, than where the milk is heated in open bottles or open vats.

4. It is recommended in order thoroughly to pasteurise milk so as to destroy any tubercle bacilli which it may contain, without in any way injuring its creaming properties or consistency, to heat the same in closed pasteurisers for a period of not less than twenty minutes at $140^{\circ} \mathrm{F}$. $\left(60^{\circ} \mathrm{C}\right.$.).

Under these conditions one may be certain that disease bacteria, such as the tubercle bacillus, will be destroyed without the milk or cream being injured in any way. For over a year this new standard has been in constant use in the University of Wisconsin Creamery, and the results, from a purely practical point of view, reported last year by Farrington and Russell, ${ }^{1}$ have been abundantly confirmed.

Whilst these results seem definite and clear, it should not be forgotten that other recent workers have failed to obtain such satisfactory results, and many authorities still hold that the only practicable standard upon which absolute reliance can be placed is to raise the milk to the boiling point.

(ii.) Low temperatures.-Low temperatures per se appear to have little or no effect upon the bacillus tuberculosis. Cadeac and Malet kept portions of a tuberculous lung in a frozen condition for four months, and found that, at the end of this time, the substance was still virulent. Experiments carried out by Swithinbank in the year $1900^{2}$ are of interest upon this point (see Plate 23). Virulent cultures of the organism were exposed to the temperature of liquid air $\left(-193^{\circ} \mathrm{C}\right.$.) for continuous periods varying from six hours to forty-two days, with the result that there was no apparent modification of either vitality or virulence. It was shown, however, that successive alternations of extreme cold and normal temperatures had a decidedly destructive effect.

(iii.) Light.-Pure cultures of the tubercle bacillus are very susceptible to the effect of direct sunlight, a comparatively short exposure being sufficient to destroy their virulence (Koch, Ransome,

1 Sixteenth Annual Report of the Wisconsin Agricultural Expt. Sta., 1899, p. 129.

2 Proc. of Royal Society, 1901, p. 498, and Trans. Brit. Cong. on Tuberculosis, rgor, vol. iii., p. 657 . 
PLATE 23.

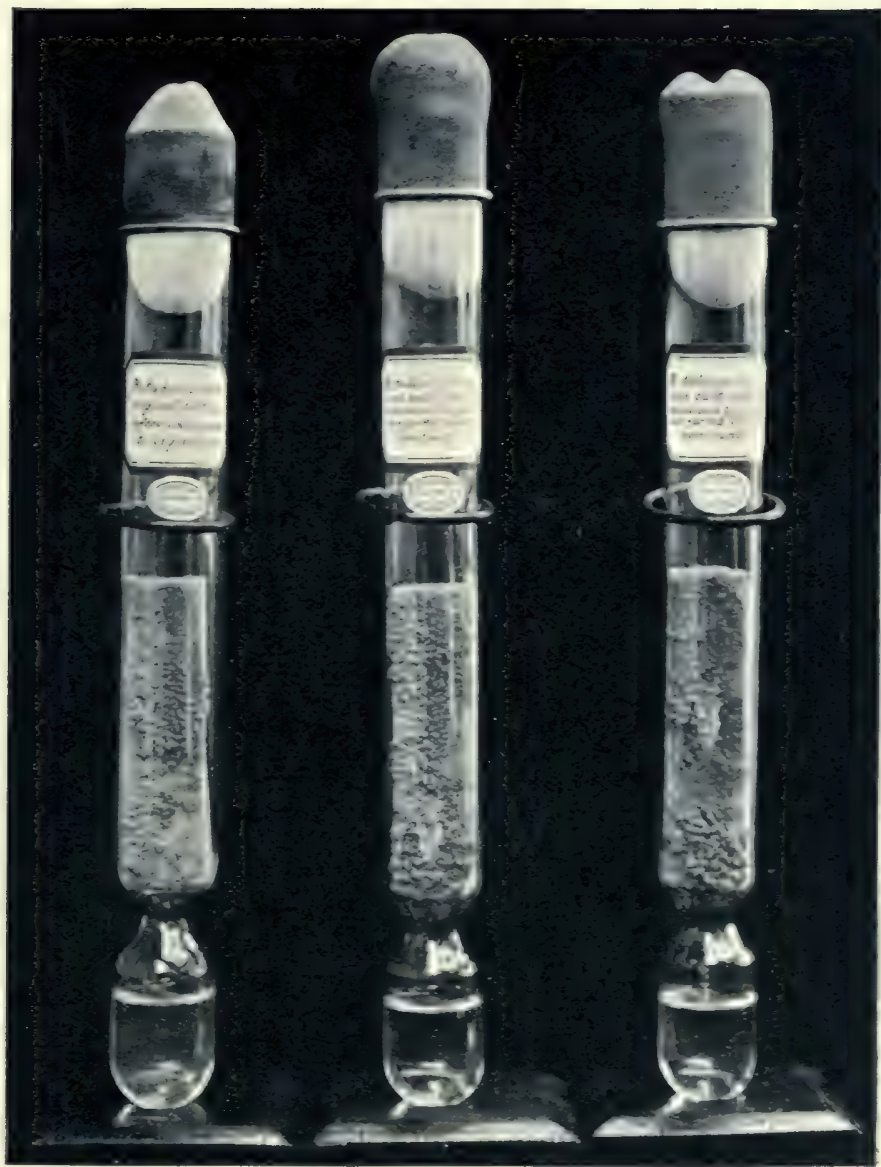

u.

b.

e.

Powers of resistance of the B. tuberulosis.

c. A culture of the organism on glyeerine-potato befure exposure to liquiı air.

b. A sub-culture made after imm retion of the oranism in liyliil air fur a cuntintious period of 42 days.

c. A sub-culture made after exposure of the orzanism to the trmueroten, of linuid air for a continuous period of 42 days.

Note.-The temperature of liquid air nay te taken as $-193 \mathrm{C}$. 

and others). Diffuse daylight has the same effect but in a less degree. Obviously, however, these results are not obtained when the bacillus is protected by the medium in which it is present, as in the case of milk for example.

(iv.) Desiecation.-In ordinary dust, Cornet was the first to demonstrate that the tubercle bacillus might retain its vitality and virulence for long periods of time. Sawizky put this at two and a half months, but there is little doubt that tubercular sputum in a dried form may remain virulent for many months (Schill and Fisher, Galtier, Swithinbank, and others).

(v.) Chemical substances.-Koch, Yersin, Crookshank, and many others have demonstrated the germicidal effect of carbolic acid on tubercle bacilli. It is generally accepted that these bacilli are killed by a 5 per cent. solution in one minute. Absolute alcohol kills them in five minutes; mercuric chloride (I-I000) in ten minutes; and tricresol (I per cent.), formalin ( 5 per cent.), lysol ( 2 per cent.), and chloride of lime ( 2 per cent.) are also held to be germicidal to the tubercle bacillus.

\section{ACid-Fast Bacilli allied to the Tubercle Bacillus}

We are now in a position to consider the group of organisms morphologically and tinctorially similar to the bacillus tuberculosis. This group is known as that of the acid-fast bacilli on account of the fact that in staining by the Ziehl-Neelsen method (see p. 6o) these organisms possess, like the tubercle bacillus, the power of resisting decolorisation by the acid following the red stain. ${ }^{1}$ In England such bacilli are termed acid-fast, in Germany saurefeste, and in France acidophile. The group is one of great importance, partly on account of the ease with which its members may be mistaken for the "true" tubercle bacillus, and partly on account of the relationship which appears to exist between them and the tubercle bacillus. Some bacteriologists hold that possibly these acid-fast bacilli represent a saprophytic stage in the life-history of the true tubercle bacillus.

Classification of acid-fast bacilli.-These bacilli may be divided provisionally and for convenience into four chief subdivisions :-

(a) The acid-fast bacilli of other diseases or conditions affecting

1 Acid-fastness is due, in all probability, not to fat in the bacillus, but to a substance of the nature of wax, which can be extracted by acid-alcohol, ether, or other wax solvents. For a discussion of this subject see Trans. British Congress of Tuberculosis, 1901, vol. iii., pp. 498-502 (Bulloch). 
man (e.g. B. lepre, B. smegmatis, Bacillus of syphilis of Lustgarten, etc.). Other non-tubercular acid-fast bacilli have been found in lung gangrene, in the nasal cavities, in excreta, and in certain chronic eye diseases, etc.

(b) The acid-fast bacilli occurring in other animals (e.g. $B$. tuberculosis avium of Maffucci; the B. tuberculosis piscium of Dubard, Bataillon, Terre; B. tuberculosis ranicola of Lubarsch; $B$. tuberculosis anguicola of Moeller, etc.).

(c) The acid-fast bacteria of butter and milk (e.g. B. laticola planus, perrugosum, Friburgense, etc.), of Petri, Moeller, Rabinowitsch, Binot, Markl, Coggi, Tobler (Nos. i.-v.), Grassberger, and Korn (Nos. i. and ii.).

(d) The acid-fast bacilli of grass (e.g. B. phlei or Timothy bacillus, and Grass bacillus, No. ii., of Moeller), the "manurebacillus" of Moeller, ${ }^{1}$ the urine bacillus of Marpmann.

All these various organisms are morphologically and in staining properties allied to the $B$. tuberculosis. The chief characteristics known concerning those found in milk and butter will be described at a subsequent page (see p. 392). It is obvious that groups ( $a$ ) and $(b)$ have little or nothing to do with the bacteriology of milk, and for particulars concerning these organisms the ordinary textbooks may be consulted. It should, however, be remarked in passing that in all probability it is right to regard tuberculosis in birds and in cold-blooded animals as a modification under different conditions of tuberculosis in mammals.

Acid-fast bacilli in butter and milk.-Several years after Koch's discovery of the tubercle bacillus, species of bacteria were found possessing acid-fast properties, but it was not until I896 that Koch and Petri isolated such organisms from the milk and butter of Berlin, and in the following year Lydia Rabinowitsch carried out her research on the same subject. In 1899, Korn discovered two bacilli in the butter of Freiburg, Binot a bacillus in the butter of Paris, and Coggi a bacillus in the butter of Milan, all four of which were acid-fast. In the same year Grassberger published a statement upon acid-fast organisms occurring in butter and margarine. In 1900, Beck and Santori met with similar organisms in milk, and in I90I, Maria Tobler, Markl, and Moeller and Jong isolated acid-fast bacilli from both butter and milk. All

1 Since the above classification was drawn up by us, our attention has been drawn to the classification by Courmont and Potet, Arch. de Med. Exp. et $d^{\prime}$ Anat. Path., t. xv., 1903, p. 87. This paper also contains a most complete bibliography on the whole subject. 


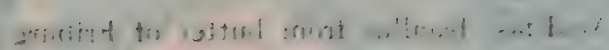

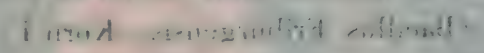

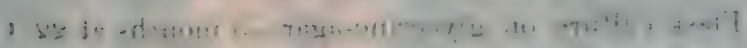

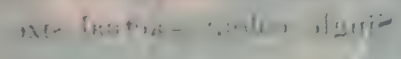

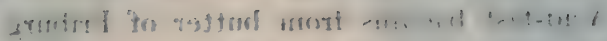

i.li my+A

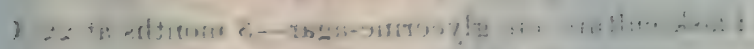

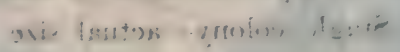


PLATE 24.

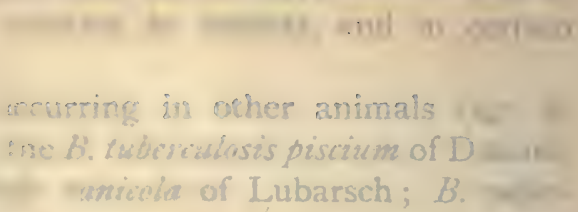

Acid-fast bacillus from butter of Friburg

(Bacillus Friburgensis-Korn I.)

Flask culture on glycerine-agar- 3 months at $22^{\circ} \mathrm{C}$. Single colony-actual size.

Acid-fast bacillus from butter of Friburg . He Hahimente ans (1. B. phlei or Timothy if. of Mueller), the "manure-

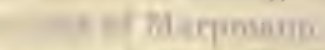

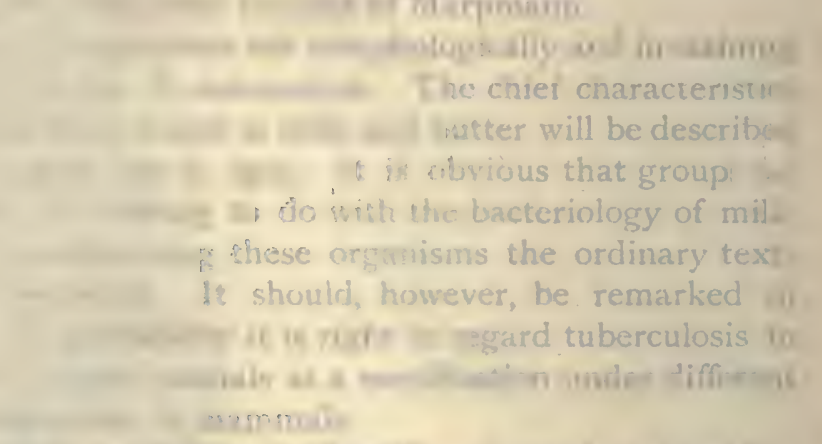

and milk

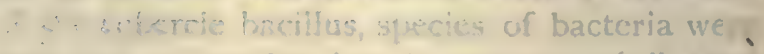
- arcepereies, but it was not until (Korn II.) : . . In $1899, \mathrm{~K}$. Flask culture on glycerine-agar- 3 months at $22^{\circ} \mathrm{C}$.

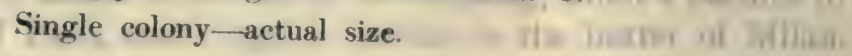

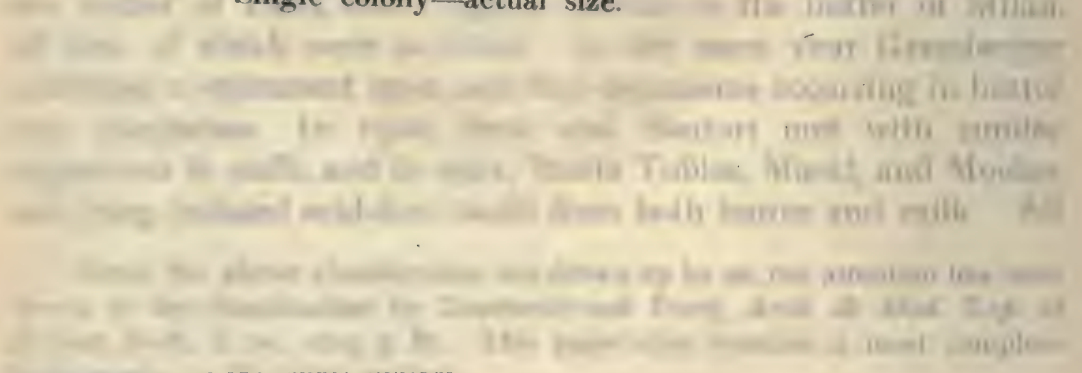




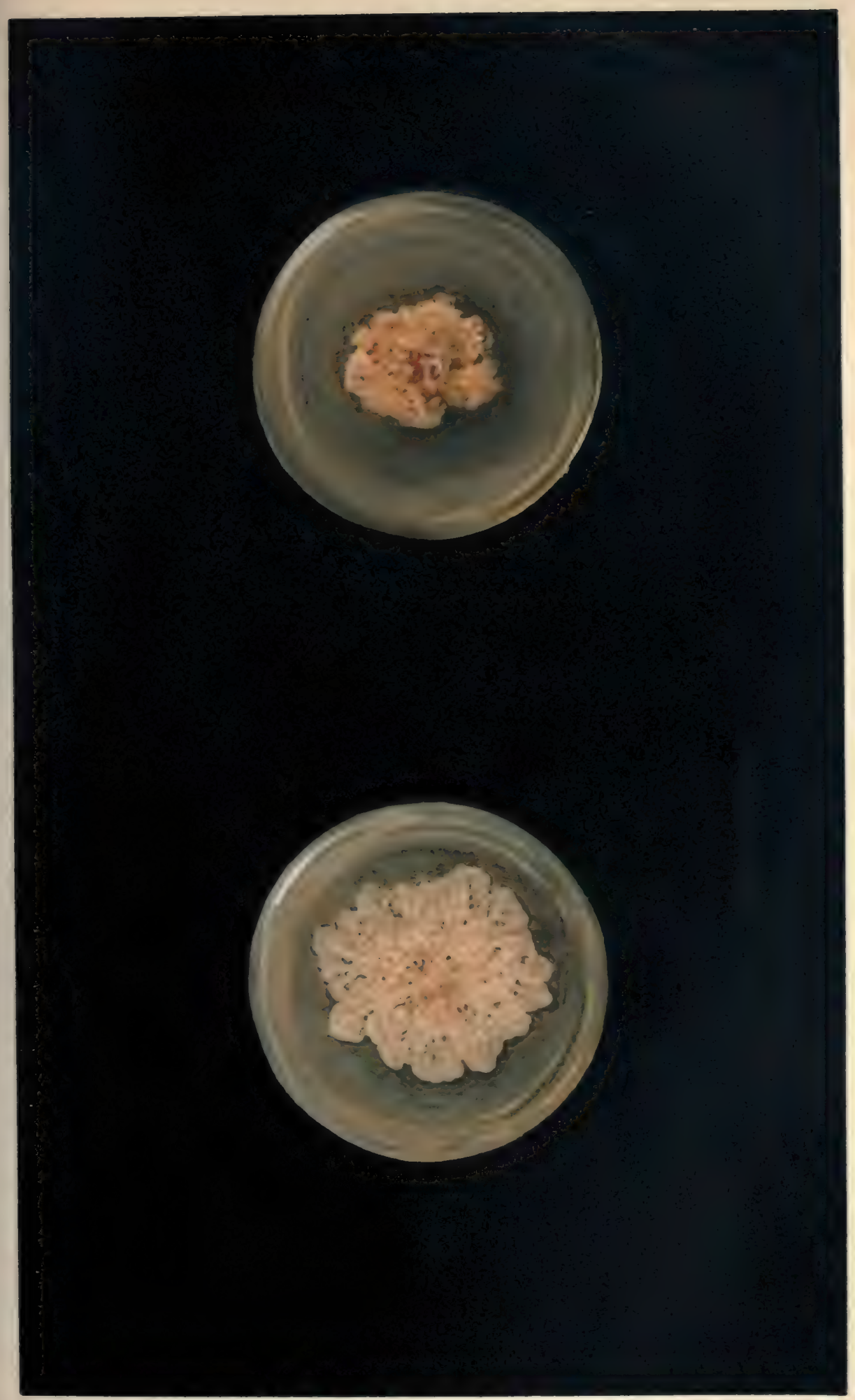

FRON WATEA COLOUN DRAWINES BY A $C$ M 

these organisms were bacilli, but they showed much variation in form and polymorphism, some appearing to be like $B$. diphtheria, and others like actinomyces. The staining properties were, in all cases, those of the true bacillus of Koch, except that the power of resistance to decoloration by acid was rather less. ${ }^{1}$ We have cultured many of these organisms upon different media and have found them to show various modifications in form, chromogenicity, vitality, polymorphism, etc. (Plate 22, between pp. 234 and 235, from a series of photographs of cultures, shows the various characters of ten of these acid-fast species compared with the human and bovine tubercle bacillus, all the cultures having been grown on the same media, for approximately the same length of time, under precisely the same conditions. The illustrations are of the exact size of the cultures, which were in each case composed of one colony only.) These acid-fast bacilli live and develop on all ordinary media at room temperature and blood heat, preferably under aërobic conditions. They do not form indol or liquefy gelatine, nor do they possess much pathogenic action. Whilst the acid-fast bacilli found in milk and butter are described in detail in a subsequent chapter, a few general notes may be made here respecting the type, namely, the organism obtained from butter by Rabinowitsch.

The butter bacillus of Petri-Rabinowitsch.-Morphologically, this organism is like the ordinary tubercle bacillus, though somewhat shorter and thicker. It stains in the same manner, but grows readily at room temperature and rapidly at blood-heat. The cultures appear as moist, thick, creamy, wrinkled layers of growth on the surface of the medium. The organism possessed less virulence when inoculated in pure culture. But when inoculated with, or without, butter, it has clearly defined effects. Giant cells, nests of epithelioid cells, and typical tuberculous caseation are, according to Rabinowitsch, never to be found in the foci of disease set up by this bacillus. None of the animals injected with this bacillus reacted to tuberculin. The intraperitoneal injection of pure cultures often produces a formation of nodules in the abdominal organs which frequently heal. If, however, the animals are killed in three or four weeks, the following characteristics are found, namely, slightly distended abdomen, more or less severe peritonitis, nodules on mesentery and beneath the intestinal serosa, mesenteric glands enlarged, and liver, spleen, and kidneys showing small nodules with yellowish exudation. When the butter itself containing the organisms is used, a fatal result often follows

${ }^{1}$ Report of Medical Officer of Local Government Board, 1900-1, pp. 33I-3. 
the injection after three to fifteen days. Similar changes to the above have occurred, but of a more intense degree. Rabinowitsch found rabbits insusceptible in contrast to guinea-pigs. It is not known whether this bacillus is in any degree pathogenic for man. But probably such is not the case. It appears to be widely distributed in nature, as 60 per cent. of butter samples in Berlin were found to contain it. The only satisfactory way to differentiate this bacillus from the true bacillus is by inoculation of animals.

Obermüller, Moeller, Korn, and others have isolated very similar organisms to the bacillus of Rabinowitsch from milk or milk products. Korn's bacillus of butter is said to differ in morphology, cultivation, and pathogenic action from the PetriRabinowitsch bacillus. All these acid-resistant bacilli obtained from milk or its derivatives show a close resemblance to another group of organisms to which brief reference must be made. Some authorities, indeed, maintain that the two groups are really varieties of a common species.

Acid-fast bacilli in grass, hay, and manure.-This second group of acid-fast bacilli associated with milk (marked $(d)$ in the classification above) is often designated as the grass bacilli. They were first cultured on Timothy grass (Phleum pratense), which is much valued for feeding cattle. ${ }^{1}$ Since then, however, this grass bacillus has been found in various places, and it or its allies have been isolated from cattle fodder, hay, hay-dust, manure, milk, and its derivatives. Morphologically, this bacillus is similar to the tubercle bacillus, slender and slightly curved. It contains highly stained granules; oval, clear spaces; often grows in threads; and is branched, and sometimes has club swellings at one end. It is acid-fast in staining, and grows best at incubation temperatures on the ordinary culture media. The colonies become visible in thirtysix hours, are scale-like and greyish-white in colour. Under certain conditions its growth in artificial media is very similar to the tubercle bacillus, which however does not thrive at room temperature. As regards pathogenic properties, the grass bacillus is almost identical with the Petri-Rabinowitsch butter bacillus in its effects on guinea-pigs. It has somewhat different effect on rabbits, producing a condition difficult to distinguish from true tuberculosis

1 Cats-tail or Timothy grass (Phleum pratense). Flowers, June to August. Spikelets, one-flowered, and arranged in spicate manner. Although well known to the British grower this grass is more extensively cultivated in the United States, where it was introduced from Britain, nearly a century ago, by $\mathrm{Mr}$ Timothy Hanson, after whom it is named Timothy grass. 


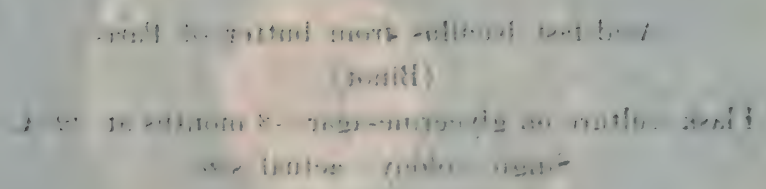


PLATE 25.

vorts to be widel

uamples in Berlin wr.

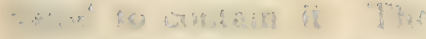

Acid-fast bacillus from butter of Paris

inoculation of animals.

(Binot).

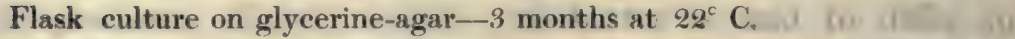
Single colony-actual size.

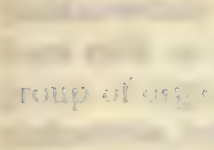

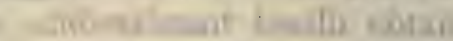

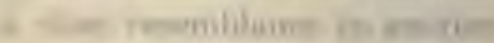
$\because$ reference must be made.

$\sin (10)$ ..: ahe two groups are really farith
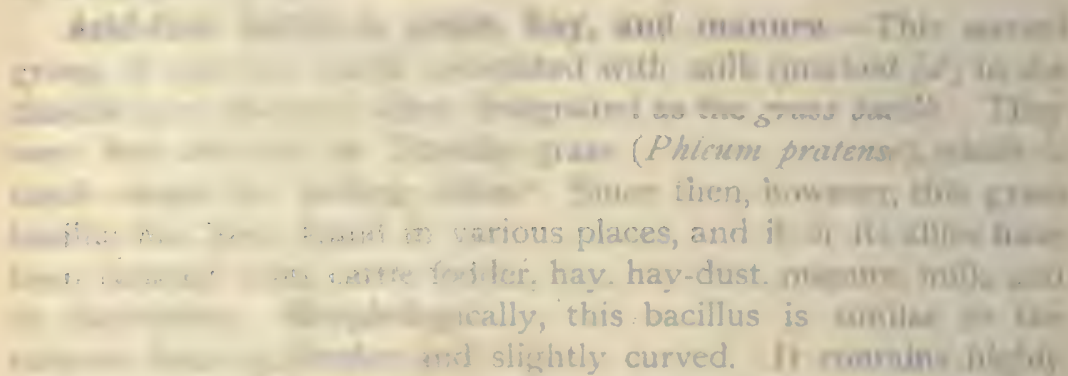

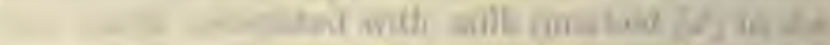

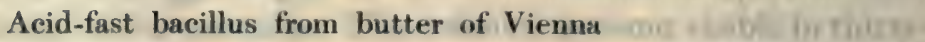
(Grassberger), h-w hite: in colour. If in w Flask eulture on glycerine-agar-3 months at $22^{\circ} \mathrm{C}$. 3 mintin wh Single colony-actual size. 


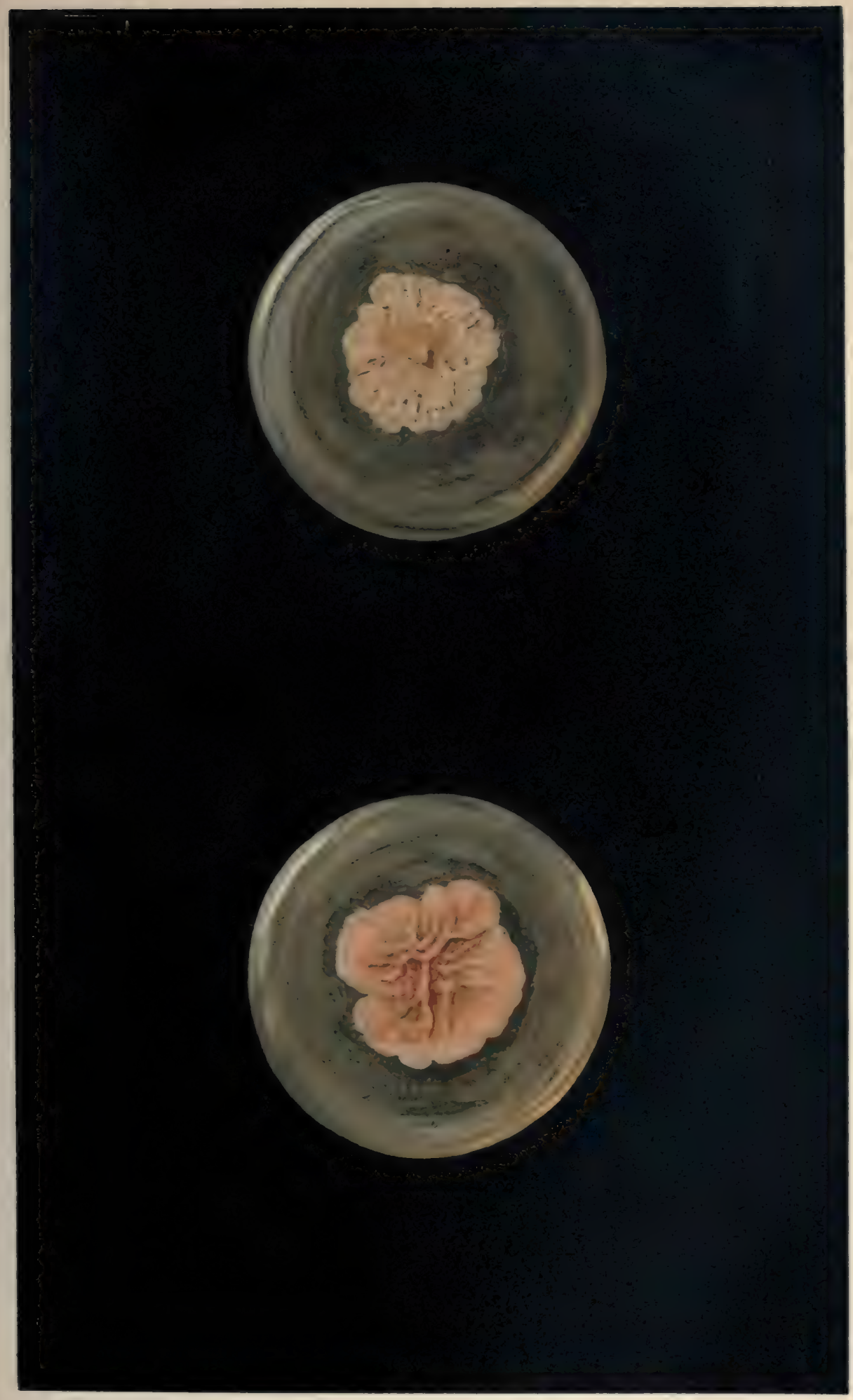

FROM WATER COLOUR DRAWINGS BY A.C.W 

(Lubarsch). Giant cells, epithelioid cells, and caseation are all said to occur. In all animals injected with the grass bacillus a negative reaction to tuberculin is obtained. Moeller has isolated a grass bacillus, No. ii. (from the dust of a hay-loft), which he considers essentially different from the Timothy bacillus. The colonies are moist and sticky, become confluent, and are yellow in colour. It loses its acid-fast properties in old cultures. Its pathogenic properties are most marked when cultured in milk. It frequently shows marked polymorphism. In culture it is like the butter bacilli. Freymuth has shown that the changes this organism sets up in cold-blooded animals are indistinguishable from true tuberculosis. An acid-fast bacillus similar to grass bacillus No. ii. has recently been isolated by Moeller from milk. A variety of the grass bacillus has also been found by Moeller in the excreta of animals, and is therefore termed the manure bacillus (mist bacillus). This acid-fast bacillus has been isolated from the fresh excreta of cattle, and bears a morphological and tinctorial resemblance to the Timothy bacillus, whilst in cultures it is like grass bacillus No. ii. It has certain pathogenic properties. It is possible that most of the acid-fast bacilli found in milk and butter have their origin in the soil or vegetation.

Differential diagnosis. - This brief record of the acid-fast bacilli is enough to show that there exist a large number of bacilli, which on occasion may be present in milk or milk products, having characters which ally them closely to the tubercle bacillus. Moeller holds that the primitive form is the grass bacillus, and that the butter bacillus, manure bacillus, etc., are varieties thereof. The main points of distinction between this group and the true tubercle bacillus are five.

First, the tubercle bacillus shows a fairly uniform manner of growth.

Secondly, it requires incubation temperature.

Thirdly, it is unique with respect to its excessively slow growth. Fourthly, it is as regards growth and propagation a parasite.

Fifthly, on inoculation it produces pathological cellular changes distinct from the nodular new growths following inoculation of acid-fast bacilli. In particular this is true, as far as is known at present, in regard to the human organism.

In a sentence, the acid-fast bacilli differ from the tubercle bacillus in three main particulars, viz.: morphology, conditions of development (chromogenicity, rapidity of growth, and wide 
range, of temperature within which they flourish), and their feebler pathogenic properties. From these facts it follows that however great the degree of similarity between these various acid-fast bacilli, and however much it is possible by artificial cultivation to modify the morphology of the various forms, there is sufficient difference to enable a differential diagnosis to be made if all the biological characters are ascertained, and most of all the pathogenic properties. Hence the importance of the inoculation test being applied to all acid-fast and tubercle-like organisms detected in milk or butter.

The pathological differences from Koch's bacillus are that inoculation with acid-fast bacilli gives rise to no "giant cells," no epithelioid cell clusters, and no tuberculous caseation. Nodular lesions occur suggestive of tubercle, but according. to Potet, ${ }^{1}$ and Abbot and Gildersleive $^{2}$ : (a) they constitute a localised lesion only, having no tendency to dissemination, metastasis, or progressive destruction of tissue by caseation; $(b)$ they tend to terminate in suppuration like ordinary abscesses; $(c)$ when occurring as result of intravenous inoculation they appear in the kidney, rarely in the lung and other organs; $(d)$ the form of granuloma set up is similar to actinomyces.

This group of organisms is one of considerable importance to the milk bacteriologist, and in all investigations dealing with the tubercle bacillus, or with milk and its products, it is essential that the bacilli met with should be clearly differentiated from the tubercle bacillus. Insufficient care has been taken in this respect up to the present. Any such organism found should be compared in cultural and pathogenic properties with the human tubercle bacillus, the bovine bacillus of pseudo-tuberculosis, and the various acid-fast organisms, and not simply accepted on tinctorial properties as a tubercle bacillus.

1 Etude sur les Bactéries dites Acidophiles (Potet, Paris, 1902), pp. 188-194.

2 The University of Pennsylvania Medical Bulletin, June, 1902. 


\section{CHAPTER IX}

\section{PATHOGENIC BACTERIA IN MILK (continued)}

Introductory Note. Signs and Characteristics of Milk-borne Epidemics:-(1) Special Incidences, (a) Local, $(b)$ as regards Social Position of those attacked, (c) Incidence on Milk-drinkers, $(d)$ Age and Sex Incidence ; (2) Incubation; (3) Sudden Onset and Rapid Decline; (4) Clinical Characters; (5) Mortality-rate in Milk-borne Disease.

\section{The Characteristics of Milk-Borne Epidemics}

IT has been known for some time past that the materies morbi of certain epidemic diseases may be conveyed by means of drinking infective milk. As far back as I857, Dr Michael Taylor of Penrith was able to demonstrate in a clear manner such conveyance. In $188 \mathrm{I}$ it was possible to record more than seventy outbreaks which had been traced to the milk supply. ${ }^{1}$ Some fifty of these were epidemics of typhoid fever, more than a dozen were of scarlet fever, and several were outbreaks of diphtheria. In I897 a further search among the records revealed nearly one hundred more such epidemics. ${ }^{2}$ Neither of these lists was, or professed to be, complete. Other compilations, including one by R. G. Freeman of New York, ${ }^{3}$ have added to the already ample chronicles of such outbreaks. Every year brings confirmatory evidence. So that the authors have been able to collect and study the main facts respecting some two hundred and fifty epidemics due to an infected milk supply. ${ }^{4}$ Brief abstracts of the more typical outbreaks will be found in the present chapter, but in order to avoid a repetition of these or similar details which would become wearisome and serve no useful purpose, it has been thought sufficient if in the remaining cases the names of the places and the dates of occurrence are stated. Lastly, it must be remembered that no records, how-

1 Trans. of International Medical Congress, 1881, vol. iv. (E. Hart).

2 Brit. Med. Jour., 1897, vol. i., pp. 1167, 1229, 1293.

3 Medical Record, 1896 (28th March).

4 In this task they desire to acknowledge the convenient assistance they have derived from the bibliographical references attached to the lists of Hart, Freeman, and others. 
ever perfect, can adequately recount the history of milk epidemics. There can be no doubt that the unrecorded and unrecognised cases in which milk has acted as the vehicle of contagion, are legion.

It is only since 1857 that attention has been drawn to this channel of infection. In that year, Dr Michael Taylor of Penrith, in the ordinary course of his practice, came across a number of cases of typhoid fever which had only one thing in common, namely, a community of milk supply. Briefly, it appears that about the beginning of September I857, a young servant girl, E. O., returned home to Penrith from Liverpool suffering from typhoid fever. The family of which she was a member consisted of father, mother, and five children, of whom she was the eldest. The cottage in which they lived consisted of two ill-ventilated and ill-lighted rooms, a kitchen or sitting-room, and a bedroom opening out of it. The father possessed three cows, and carried on a small milk business dealing with some fourteen families. The mother milked the cows, and the milk was brought into the kitchen direct from the byre, and in due course distributed in tin measures amongst the customers. After her return home the girl continued ill for about a fortnight, during which period she was nursed by her mother in the kitchen or common sitting-room. At the end of the fourth week in September she was convalescent, and began to help at once in the distribution of the milk. Two other children of the family sickened and passed through the fever. The mother nursed all three patients, and continued to milk the cows and attend to the distribution of the milk. In October and November some thirteen cases of typhoid fever occurred in seven families dealing with the infected cottage, and from these primary cases a large number of persons, over a wide area, were infected by contact. By most careful observation and reasoning, Dr Taylor arrived at the conclusion that the milk became contaminated in the kitchen of this cottage, from the typhoid patients there being nursed. ${ }^{1}$

Dr Taylor's views were accepted with considerable hesitation and reserve, and no further milk-borne epidemics of note are recorded for ten years. But in $\mathrm{I} 867$ a second outbreak occurred in Penrith, and, fortunately, Dr Taylor was again able to carry out the necessary investigations. On this occasion the disease was scarlet fever. In April of that year there were a few cases, amongst them a child of J. C., aged four months. After an illness of two or three weeks the child died during the period of desquamation. In the cottage where the child was nursed and where it died a small milk

1 Edin. Med. Jour., 1858, pp. 993-1004. 
business was carried on. J. C. kept four cows, which were generally milked by his wife, who also nursed the child suffering from scarlet fever. The milk was brought direct from the byre to the back kitchen of the cottage, where it was strained in the usual way, and then distributed to some fourteen families. Fifteen cases of scarlet fever followed in six of the families supplied by the milk, and a number of secondary infections also occurred. Here, again, Dr Taylor was able, after minute inquiry, to discover that the milk itself became in some way infected in the cottage, and so conveyed the disease. ${ }^{1}$

Such then, in a few words, is the history of the two first, comparatively small, outbreaks of which we have record, one of typhoid fever and one of scarlet fever, traceable to milk. The views of the investigator as to the relationship borne between a specific disease, such as typhoid fever, and the contamination of milk or water, became a matter of grave import. Finally, he concluded that specific contamination of milk "may occur either from the soiling of the hands, or from direct admixture of the exuviæ or discharges with liquids, or vessels used by the patient, by the drying up of these discharges, or the dissemination of their poisonous elements either in the form of dust, or by particles of dust already existing in the apartment acting as carriers of the infected germs, which, when absorbed by liquids, by adhering to clothes, or by currents of air, might be carried to distant quarters." These words were written before the days of bacteriology, and even before the date of the publication of Tyndall's researches on floating matter in the air ; and yet some of the most modern ideas of infection are foreshadowed in them, as indeed they were likewise foreshadowed in the work of Budd, Simon, Burdon-Sanderson, and others.

Dr Michael Taylor's investigations at Penrith form then the opening page in a chapter of public health, which has proved to be one not only of exceptional interest, but of vital importance. For not only has much been learned in the observance of the behaviour of zymotic diseases conveyed by milk, but not a little also of the potentiality of milk, and of the milk trade where not properly conducted, to carry disease. This record of nearly half a century's progress in a single department of research and inquiry, naturally divides itself into two main periods in correspondence with two main steps in the record. First, there was the accumulated evidence of milk conveyance, that is,' there was evidence that milk might, and did on occasion, obtain infective properties from human sources. This knowledge was derived not from any discovery in

1 Brit. Med. Jour., 1870, vol. ii., pp. 623-625. 
the milk of the specific bacillus of any human disease, but from the incidence of the outbreaks amongst those having a community of milk supply, and from the more or less direct evidence of the contamination of that milk supply by patients suffering from the disease in question. Secondly, since $188 \mathrm{I}$ there has been the added inquiry as to the communicability of zymotic disease from the cow itself. About these two matters, and particularly about the second, there has been not a little controversy. To the different views which have had their supporters, reference will be made in the present chapter. It will, however, be necessary to restrict our review in this connection to scarlet fever and diphtheria, and these matters will be treated of under the diseases in question.

Finally, it must not be forgotten that in the first two decennial periods of milk epidemiology, investigators lacked two powerful instruments of inquiry which we possess to-day, namely, Notification and Bacteriology. With the former, it is practically impossible to conceal disease on a large scale. Not only has notification given a more exact record of cases of infectious disease, but it has also advanced the accuracy of diagnosis. By the latter, bacteriology, we have learned something of the etiology of bacterial diseases and of the flora of milk.

\section{Chief Characteristics of Milk-Borne Epidemics}

Owing to the variety of circumstances and conditions which affect the question, it is not possible to formulate any general laws, the fulfilment of which may be recognised, on all occasions, when disease is conveyed by milk. The milk trade itself is becoming more and more complex, especially in towns, and hence it happens that simple outbreaks of disease, derived from, say a single milk supply, are rare. Still more complex are the circumstances and conditions surrounding the consumer. In every case there are manifold idiosyncrasies and susceptibilities in addition to a bewildering number of trifling external differences between individuals even in the same families or households, and the manner and degree in which they respond to various infectious diseases. From this it follows that no two epidemics are alike. To this has further to be added, the disability due to an incomplete and, what may be called provisional, knowledge both of the bacteriology of milk and of the ways and means by which it conveys disease. Hence, we can lay down no clear and definite signs which never vary, no hard and fast laws of pathognomic significance. We desire it, therefore, to be understood, that what follows is of the 
nature of a collection of signs and characteristics which have, up to the present, been observed in milk-borne disease. For convenience we shall state them briefly and in some sort of tabular form :-

\section{Special incidences of disease when conveyed by milk}

(a) Local Incidence.-Milk-borne infection follows the milk cart. - In almost every epidemic traceable to milk on record, as far as we know, the conveyed disease has appeared in the main along the lines of distribution. In some cases outbreaks have followed with remarkable precision the exact rounds of the milkman's list of calls. Certain streets, certain houses, certain tenements in houses become infected, always in some measure, and in certain instances in a remarkable measure, because they deal with the particular milkman whose milk is implicated. These sections have sometimes been whole villages, at other times a group of houses in a restricted local area, having absolutely nothing in common but the milk supply. On more than one occasion a house at a distance, and altogether out of the district, becomes infected. On inquiry it is found, that by some means or other implicated milk was consumed there. Even in towns the incidence is plain. In the epidemic at Macclesfield in I889 Dr Parsons found that 77 per cent. of the houses being served with the suspected milk were invaded with illness. In other outbreaks the number of houses served and houses attacked are almost equal.

It is this characteristic of localisation in milk epidemics which affords the primary clue as to causation. It is, of course, in some degree universally present, although it frequently happens that it remains obscure at the time of the outbreak, and is only revealed after nuch careful inquiry. We might choose illustrations from a number of cases, but we could scarcely select a simpler one than the record of the Wimbledon outbreak of scarlet fever and sore throat at Christmas 1886:-

\begin{tabular}{|c|c|c|c|c|c|c|}
\hline \multirow{2}{*}{ Customers. } & \multicolumn{5}{|c|}{$\begin{array}{l}\text { Number of Persons Attacked by Scarlet Fever } \\
\text { and Sore Throst during weeks ending }\end{array}$} & \multirow{2}{*}{$\begin{array}{c}\text { Attacks in whole } \\
\text { period, } \\
\text { December 19, } 1886 \text {, } \\
\text { to } \\
\text { January } 15,188 \% \text {. }\end{array}$} \\
\hline & Dec. 25. & Jan. 1. & Jan, 8. & Jan. 15. & Jan. 22 . & \\
\hline $\begin{array}{l}\text { The particular dairy . } \\
\text { (1400-1500 in number) }\end{array}$ & 4 & 425 & 62 & 2 & $\ldots$ & 493 \\
\hline $\begin{array}{l}\text { Other dairies } \\
(14,000-15,000 \text { in num ber) }\end{array}$ & 6 & 6 & 29 & II & $\ldots$ & 52 \\
\hline Totals & 10 & 431 & $9 \mathrm{I}$ & 13 & ... & 545 \\
\hline
\end{tabular}


We may say, then, that in all cases of milk-borne epidemics there is some local community of milk supply.

(b) Incidence as regards social position.-In not a few epidemics a particularly large measure of disease has fallen upon persons in good social position. This, of course, is due to the fact that such persons consume in one way or another more milk than do the poorer classes. The poor take their milk only in small quantities in tea, coffee, or cocoa. The middle and upper classes consume in some households large quantities of milk, often in an uncooked condition. Particularly is this so with regard to children. Again, in such households, the opportunities for milk consumption are numerous, sometimes as frequently as four times a day. There is yet another condition characteristic in the milk consumption of well-to-do people, and that is that in their households milk is stored more than in the homes of the poor. The poor purchase what little milk they require, and drink it at once. But in betterclass houses milk is set for cream to rise, or stored for the following day, or put aside for various culinary purposes. Hence there is more opportunity not only for exposure to poison, but for organisms to multiply and form products in the milk. These are all minor matters, but they distinctly affect the quantity of milk consumed by different social classes.

In Marylebone in 1872 an epidemic particularly affected the wealthier classes, and the same occurred in a more marked degree at Wimbledon in the sudden epidemic, referred to above, which occurred there at Christmas time in I886. In this case, fortunately, a careful record was drawn up for the Local Sanitary authority respecting the incidence of scarlet fever upon houses of certain rental, with the following interesting result:-

\begin{tabular}{|c|c|c|c|c|c|c|c|}
\hline \multicolumn{8}{|c|}{ Houses in Wimbledon supplied from particular Dairy, rated at } \\
\hline \multicolumn{2}{|c|}{610 to 615} & \multicolumn{2}{|c|}{616 to $\$ 30$} & \multicolumn{2}{|c|}{631 to $£ 40$} & \multicolumn{2}{|c|}{$\oint_{41}$ and upwards } \\
\hline $\begin{array}{c}\text { Total } \\
\text { Houses. }\end{array}$ & $\begin{array}{c}\text { No. } \\
\text { Invaded. }\end{array}$ & $\begin{array}{c}\text { Total } \\
\text { Houses. }\end{array}$ & $\begin{array}{c}\text { No. } \\
\text { Invaded. }\end{array}$ & $\begin{array}{c}\text { Total } \\
\text { Houses. }\end{array}$ & $\begin{array}{c}\text { No. } \\
\text { Invaded. }\end{array}$ & $\begin{array}{c}\text { Total } \\
\text { Houses. }\end{array}$ & $\begin{array}{c}\text { No. } \\
\text { Invaded. }\end{array}$ \\
\hline 44 & 17 & I 20 & 73 & $5^{8}$ & 40 & 55 & 42 \\
\hline \multicolumn{2}{|c|}{$=38.6 \%$} & \multicolumn{2}{|c|}{$=60.8 \%$} & \multicolumn{2}{|c|}{$=68.9 \%$} & \multicolumn{2}{|c|}{$=78.2 \%$} \\
\hline
\end{tabular}


$\mathrm{Mr}$ Power was also able to furnish returns throwing light upon the same point in the diphtheria epidemic which he investigated in York Town and Camberley in I886. He divided the cases into those belonging to the better class and those of cottagers, small tradesfolk and the like, with the following very interesting results :-

\begin{tabular}{|c|c|c|c|c|c|c|c|c|c|}
\hline \multirow{3}{*}{ Class of Customers. } & \multicolumn{3}{|c|}{ Persons. } & \multicolumn{3}{|c|}{ Adults. } & \multicolumn{3}{|c|}{ Children $(0-15)$} \\
\hline & \multirow[b]{2}{*}{ Total. } & \multicolumn{2}{|c|}{ Attacked. } & \multirow[b]{2}{*}{ Total. } & \multicolumn{2}{|c|}{ Attreked. } & \multirow[b]{2}{*}{ Total. } & \multicolumn{2}{|c|}{ Attacked. } \\
\hline & & $\begin{array}{l}\text { Num- } \\
\text { ber. }\end{array}$ & $\begin{array}{l}\text { Per } \\
\text { cent. of } \\
\text { Total. }\end{array}$ & & $\begin{array}{c}\text { Num- } \\
\text { ber. }\end{array}$ & $\begin{array}{l}\text { Pex } \\
\text { cent. of } \\
\text { Total. }\end{array}$ & & $\begin{array}{c}\text { Num- } \\
\text { ber. }\end{array}$ & $\begin{array}{l}\text { Per } \\
\text { cent. of } \\
\text { Total. }\end{array}$ \\
\hline Better Class & 317 & 105 & $33 \cdot 1$ & 269 & 79 & $29 \cdot 3$ & 48 & 26 & $54 \cdot I$ \\
\hline Other. & 239 & 15 & 6.3 & 145 & 9 & 6.2 & 94 & 6 & 6.4 \\
\hline
\end{tabular}

The same incidence was also illustrated in the milk-borne epidemic of typhoid fever investigated by Dr Davies at Bristol in I897.

(c) Incidence on milk-drinkers. - A third characteristic is the obvious one that milk-borne disease affects in highest degree those individuals who drink most milk. With almost incredible accuracy the disease attacks persons in a family who are the chief milkdrinkers. When this occurs it is one of the surest signs of milkinfection. In 1893 there was an outbreak in a school at Hastings. There were twenty boys, only two of whom took the implicated milk-they alone were attacked. In the same outbreak a man isolated for twelve days on account of influenza drank the milk and was attacked. In Canterbury in I 886 diphtheria picked out one member from a family, and it turned out that he was the only one who had consumed the infected milk in his tea at a friend's house. In 1882, out of fifteen persons in a family only one consumed the suspected milk raw. He was attacked. There are several instances on record of how a casual glass of milk at the farm gave rise to disease in persons not residing in the infected locality. In one of the typhoid outbreaks recorded, only one person was affected in a certain house. He was the butler who "saved his beer money," and contracted enteric fever. In an epidemic at the Iowa State College the attack rate was 8.8 per cent., except among the football players, who were served with a double allowance of milk, and amongst them the attack rate was over 50 per cent. In the Clifton typhoid epidemic in 1897 in one of the College Houses containing fifty-five persons, thirty-nine were attacked. In the scarlet fever outbreak in North London in 
I 882, the milk supply was derived from a farm south of London, and was conveyed straight through to North London. But at Charing Cross Station 2 or 3 gallons of the milk were removed from the churns for local consumption. Thirteen persons amongst the railway men or their families were attacked. At Eagley in I876, of fifty-nine families supplied with implicated milk, 96 per cent. were attacked with typhoid; of two hundred and sixty-one families not so supplied, 5 per cent. were attacked. Particularly instructive are cases which occur at a distance from the infected locality but in which those attacked have consumed the implicated milk.

In the outbreak of diphtheria of 1886 in York Town and Camberley, of the fifty-seven houses invaded, forty-eight (or 84 per cent.) took the implicated milk. Of the one hundred and forty individuals attacked, 88 per cent. were consumers of the milk, and there was evidence to show that the amount of milk consumed determined the severity of the disease. Eighty-four per cent. of the houses of the better class consuming this milk were invaded, and only 22 per cent. of the poorer class; and further, while 33 per cent. of the consumers of the better class contracted diphtheria, only 6 per cent. of the poorer class acquired it. "Though uniformly infective on the farm," wrote Mr Power, "the milk had a very different effect in causing diphtheria, according as it was distributed to one and another class of consumers. This difference of effect had to do with difference of amount (and related difference of use and difference of conservation) of milk distributed in the two classes. The diphtheria among the better class was, as regards amount and fatality, so conspicuous that its relation to the milk service was readily seen; but among poor class consumers it was so inconspicuous in amount and fatality that the fact of its relation to the milk service might readily have been overlooked. It is altogether doubtful, therefore, whether, in the absence of the test of the milk which was furnished by the behaviour of the epidemic among customers of the better class, the ability of this milk service to cause diphtheria would have been detected." 1 In this outbreak households of the better class, which suffered so exceptionally, had averaged 5.2 pints of the implicated milk per day; whereas, among the cottagers and tradespeople, it had averaged only 0.8 pints daily.

In that same year Mr Power investigated an outbreak of scarlet fever at Wimbledon, to which reference has been already made. As we have seen, the disease attacked in particular the classes

${ }^{1}$ Local Government Board Medical Officer's Supplement, 1886, pp. 31 I-326. 
inhabiting houses of higher rental. But the incidence upon the milk-drinkers in proportion to the amount of milk they consumed is even more striking :-

\begin{tabular}{|c|c|c|c|c|c|c|}
\hline \multicolumn{6}{|c|}{$\begin{array}{l}\text { Relation of Amount of Particular Milk consumed to incidence of Epidemic } \\
\text { (Wimbledon and Merton, 1886-7). }\end{array}$} & \multirow[b]{2}{*}{$\begin{array}{l}\text { In raded } \\
\text { per cent. of } \\
\text { Total. }\end{array}$} \\
\hline $\begin{array}{l}\text { Amount of Particular } \\
\text { Milk consumed daily } \\
\text { per honsehold. }\end{array}$ & $\begin{array}{c}\text { Total } \\
\text { Houses. }\end{array}$ & $\begin{array}{l}\text { No. } \\
\text { Invaded. }\end{array}$ & $\begin{array}{c}\text { Invaded } \\
\text { per cent. of } \\
\text { Total. }\end{array}$ & $\begin{array}{c}\text { Total } \\
\text { Persons. }\end{array}$ & $\begin{array}{l}\text { No. } \\
\text { Inraded. }\end{array}$ & \\
\hline Less than I pint. & $8 \mathrm{I}$ & 39 & $48 \cdot 1$ & 3 IS & 68 & $2 \mathrm{I} \cdot 6$ \\
\hline I to I $\frac{1}{3}$ pints & 102 & 62 & $6 I \cdot 7$ & 472 & 162 & $34 \cdot 3$ \\
\hline 2 to 21 pints & 43 & 32 & 76.7 & 207 & 87 & 420 \\
\hline 3 or more pints. & 48 & 39 & $8 \mathrm{I} \cdot 2$ & 362 & 169 & $46 \cdot 6$ \\
\hline
\end{tabular}

It is but rarely that it is practicable to draw up a return which throws such a clear light upon the incidence in proportion to consumption as this. But three years later (in 1889) Dr Parsons, who was then investigating an epidemic of scarlatina at Upton and Macclesfield, found opportunity for a similar return, which is as follows :-

\begin{tabular}{|c|c|c|c|c|c|c|c|c|c|}
\hline \multirow[b]{2}{*}{$\begin{array}{l}\text { Average daily } \\
\text { amount of Milk } \\
\text { used per head. }\end{array}$} & \multicolumn{2}{|c|}{ Households. } & \multicolumn{2}{|c|}{ Inmates. } & \multicolumn{3}{|c|}{ Cases. } & \multicolumn{2}{|c|}{$\begin{array}{l}\text { Percentage } \\
\text { Attacked. }\end{array}$} \\
\hline & Total. & 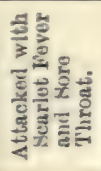 & 爮 & 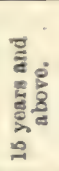 & 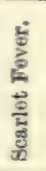 & $\frac{\text { 总 }}{\underline{\underline{E}}}$ & 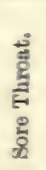 & 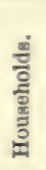 & 密 \\
\hline $\begin{array}{l}\text { I pint and up- } \\
\text { wards }\end{array}$ & 9 & 8 & 10 & 30 & 8 & $\cdots$ & I2 & 89 & 50 \\
\hline $\begin{array}{l}\frac{1}{3} \text { under I pint. } \\
\text { pint and }\end{array}$ & 16 & 12 & 35 & 62 & II & I & $2 \mathrm{I}$ & 75 & 34 \\
\hline under $\frac{2}{3}$ pint. & 37 & $2 \mathrm{I}$ & 37 & 123 & 10 & I & 34 & 56 & 28 \\
\hline Under $\frac{1}{2}$ pint & 38 & 17 & 68 & 127 & 9 & ... & 16 & 45 & 12 \\
\hline Total & 100 & $5^{8}$ & I50 & 342 & 38 & 2 & 83 & $5^{8}$ & 25 \\
\hline
\end{tabular}

Whilst there is some slight divergence in the percentages of invaded houses and persons in these two tables, there is, taking all things into consideration, a large measure of similarity. Such figures must not be pressed too far, for it is evident that questions of infection incidence are infinitely too complex to be reducible accurately to mathematical proportion. In I 892 there was a double outbreak of scarlet fever and sore throat in North-East London (Upper Clapton), traceable to the milk supply, though the exact method by which it became infected was not discovered. 
Dr King Warry, the medical officer of health, investigated this point of incidence according to consumption, with the following result :-

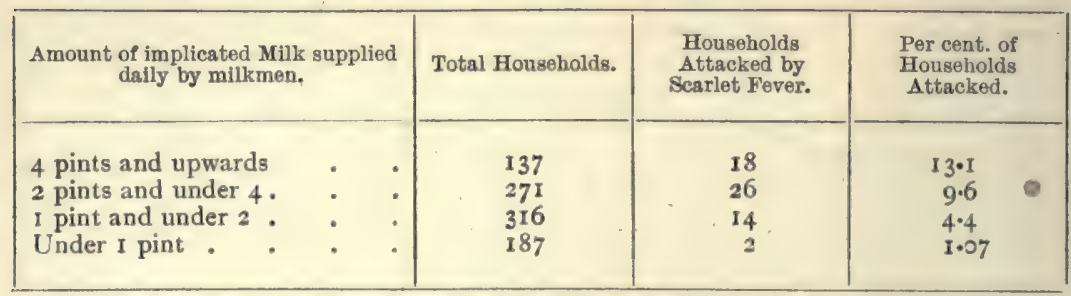

Here, again, the same clear result is demonstrated, and we think it may be set down as a principle almost always present in milk-borne epidemics, though not aiways discoverable. And it may be added that the amount of infected milk consumed bears a direct relation to the length of the incubation period, the attack rate, and the severity of the attack. Therefore it is related also to the mortality rate.

There are some further facts which emerge from a careful study of the records of the milk-borne epidemics dealt with in the present chapter in respect to incidence of infection on milkdrinkers. These facts have reference to the numbers of persons who took the suspected milk in relation to the total number of cases, and the number of families attacked in relation to the number of families supplied by the suspected milkman. We have made a careful study of the available records, and we find that of the persons attacked, the lowest percentage of those who took the implicated milk was :-in scarlet fever 64 -per cent., in typhoid fever 66 per cent., and in diphtheria 57 per cent. The highest in each case was 100 per cent. The average percentage of persons in all the epidemics who took implicated milk out of the total cases was :in scarlet fever 92.5 per cent., in typhoid 92.4 per cent, and in diphtheria 86.6 per cent. That is to say, if in each disease one hundred persons were attacked, ninety-two, ninety-two, and eightysix respectively were the numbers of persons who had taken the implicated milk. Or, if we study the incidence on families supplied, we also get instructive returns. In scarlet fever outbreaks the lowest percentage of the families supplied which were invaded with disease was 6 , the highest was 100 ; in typhoid, the lowest incidence of which we have reliable figures was I I, and the highest 100 ; in diphtheria, the lowest incidence is represented by 7 per cent., and the highest by 59. That is to say, of one hundred families supplied by the suspected milkman, seven and fiftynine were attacked, respectively. Or the averages for the same 
facts may be taken as follows: In scarlet fever 49 per cent. of the families supplied were invaded; in typhoid fever 59 per cent. were invaded; and in diphtheria outbreaks 32 per cent. The average percentages may be tabulated as follows :-

\begin{tabular}{|c|c|c|c|}
\hline \multicolumn{2}{|l|}{ Diseases. } & \multicolumn{2}{|c|}{ Percentages. } \\
\hline & & $\begin{array}{l}\text { Of Persons who } \\
\text { drank implicated } \\
\text { Milk, out of total } \\
\text { persons attseked. }\end{array}$ & $\begin{array}{l}\text { Of Families affected } \\
\text { out of total } \\
\text { hamilies supplied by } \\
\text { milkman. }\end{array}$ \\
\hline Scarlet Fever outbreaks & & $92 \cdot 5$ & $48 \cdot 9$ \\
\hline Typhoid outbreaks & . . & 92.4 & $59 \cdot 4$ \\
\hline Diphtheria outbreaks . & . & 86.6 & $32 \div 4$ \\
\hline
\end{tabular}

These figures raise various points of interest, into which we cannot now enter. Briefly, it may be said that the chief reasons of the comparatively low percentages of families invaded out of families supplied are social and economical. Dairymen have various supplies, some of which are mixed, and some of which are unmixed, and various persons handle the milk. Hence, though a milkman supplies one hundred families, only forty-nine, or fiftynine, or thirty-two become affected with the disease, partly owing to distribution, and partly owing to conditions of domestic economy.

The subject of distribution is an important one. It is a general custom for purveyors of milk to obtain their daily supply, not from a single source, either farm or dealer, but from several. Hence it may come about that a milkman supplies, say, one hundred customers, and yet only a dozen contract infectious disease. From this it is argued that milk cannot have conveyed the disease, for if it had done so, a higher percentage would have been attacked. Of course the fallacy is obvious. To arrive at a correct conclusion it is necessary to trace each source of the milkman's supply, and its particular distribution. This factor will frequently be found to be the explanation of a low percentage of families invaded. In regard to individuals attacked, idiosyncrasy and individual powers of resistance, of course, also play their part.

(d) Age and sex incidence.-It generally happens that women and children are the heaviest milk-drinkers, and for this reason it follows that the female sex and children of tender years are more affected in milk epidemics than are men. For some time in the earlier investigations of milk-borne disease this general principle was so strongly held that if an outbreak occurred including a large 
percentage of women and children it was provisionally suspected that milk had been the channel of infection. Nor is this principle likely to prove a fallacy if school infection be disproved or unlikely. Diphtheria and scarlet fever are more commonly conveyed by school infection than by milk; but in typhoid fever, school infection is of course rare. Hence other circumstances may be present in an outbreak which account for a marked incidence on children other than the consumption of milk, and these should receive due consideration. In this connection it is well to remember that at the present time children, especially those of the poor, are fed in a large measure on "condensed milk" and other similar preparations. Still it remains true in a general way that milk outbreaks commonly affect in a marked degree women and children.

Dr Davies found that in the milk-borne typhoid epidemic at Clifton in 1897 the disease proved to have a heavier incidence upon females than males, which of itself is a characteristic of milk-borne infection. His table, which includes occupations and social position, is as follows :-

\begin{tabular}{|c|c|c|c|c|c|c|c|}
\hline \multirow{2}{*}{ Occupation. } & \multicolumn{3}{|c|}{ Attracks. } & \multirow{2}{*}{$\begin{array}{c}\text { Fatality } \\
\text { in each } \\
\text { class } \\
\text { por cont. }\end{array}$} & \multicolumn{3}{|c|}{ Deaths. } \\
\hline & M. & F. & $\begin{array}{c}\text { Total } \\
\text { Attacks. }\end{array}$ & & $\begin{array}{c}\text { Total } \\
\text { Deaths. }\end{array}$ & M. & F. \\
\hline $\begin{array}{l}\text { Householders and } \\
\text { families }\end{array}$ & 13 & 37 & 50 & 16.0 & 8 & 4 & 4 \\
\hline Masters and gover- & & & & & & & \\
\hline $\begin{array}{l}\text { nesses } \\
\text { Boys and girls at }\end{array}$ & 4 & 4 & 8 & $25 \cdot 0$ & 2 & I & I \\
\hline school (ages 3-20) & 58 & 52 & IIO & $4 \cdot 5$ & 5 & I & 4 \\
\hline $\begin{array}{l}\text { Domestic servants } \\
\text { Medical students, }\end{array}$ & 3 & 62 & 65 & $2 \mathrm{I} \cdot 5$ & 14 & 2 & 12 \\
\hline $\begin{array}{l}\text { nurses } \\
\text { Others : Shop assist- }\end{array}$ & I & 3 & 4 & $25 \cdot 0$ & $x$ & ... & $\mathbf{I}$ \\
\hline ants. & $\mathbf{I}$ & 6 & 7 & $14 \cdot 2$ & I & $\cdots$ & I \\
\hline Totals . & 80 & 164 & 244 & $12 \cdot 7$ & 31 & 8 & 23 \\
\hline
\end{tabular}

From these figures it is clear that two-thirds of the total number of attacks were in females. The occupations of those attacked in this instance is not of particular interest except in regard to the greater or lesser incidence of the disease upon persons habitually consuming much or little milk.

On the other hand, several observers have emphasised the incidence of milk-borne disease upon adults, and particularly men. Quite recently Sir Henry Littlejohn of Edinburgh, in recording a 
scarlet fever outbreak in $1899^{1}$ in that city, suggested that a very important guide in determining whether an outbreak of scarlet fever is due to milk infection is to be found in the proportion of persons above 15 years of age who are attacked, more especially if of the male sex. In the Edinburgh outbreak it appears that four adults aged $22,26,29$, and $4 \mathrm{I}$ respectively, were attacked with scarlet fever whilst residing in one block of a large institution, previously free from scarlet fever and constantly kept under supervision. "The chance of four adults becoming infected by ordinary means," writes Sir Henry Littlejohn, "and all developing the same disease on the same day is very unlikely, but, on the other hand, the circumstance is quite consistent with a poisonous dose of the infection having been conveyed by milk." In this particular outbreak nineteen out of forty-nine persons affected were above 15 years of age.

In the 1901 outbreak of milk-borne scarlet fever in London there were in Bethnal Green and Shoreditch a proportionately large number of adults attacked.

Hence, whilst age incidence may be useful as a provisional indicator, it should be considered in relation to other circumstances, and amongst these circumstances there are two in particular to which due weight should be given, namely, the incidence upon the persons living in the houses supplied with the implicated milk, and the specific disease in question. The following tables are significant in this relation. They refer to the outbreak of milk-borne enteric fever in Clifton in $1897:^{2}-$

\begin{tabular}{|c|c|c|c|c|c|c|}
\hline \multirow{2}{*}{\multicolumn{3}{|c|}{ Ages. }} & \multicolumn{3}{|c|}{ Attacks. } & \multirow{2}{*}{$\begin{array}{l}\text { Fatality in } \\
\text { each class } \\
\text { per cent. }\end{array}$} \\
\hline & & & Males. & Femsles. & $\begin{array}{c}\text { Total } \\
\text { Attacks. }\end{array}$ & \\
\hline $0-5$ & . & . & 3 & 7 & ro & 10.0 \\
\hline $5-10$ & . & . & 10 & 18 & 28 & $7 \cdot 0$ \\
\hline $10-15$ & - & - & 14 & 22 & 36 & $5 \cdot 5$ \\
\hline $15-20$ & . & . & 38 & 28 & 66 & 6.0 \\
\hline $20-25$ & - & . & 2 & 27 & 29 & 310 \\
\hline $25-30$ & . &. & 2 & 22 & 24 & 16.0 \\
\hline $30-35$ & - & . & 5 & $I_{4}$ & 19 & 10.5 \\
\hline $35-40$ & . & - & 2 & 7 & 9 & $\mathrm{II} \cdot \mathrm{I}$ \\
\hline $4^{c}-45$ & . & - & 0 & 9 & 9 & $22 \cdot 2$ \\
\hline $45-50$ & - & - & 2 & 3 & 5 & 20.0 \\
\hline $50+$ & - & . & 2 & 7 & 9 & $33 \cdot 3$ \\
\hline \multicolumn{2}{|c|}{ All ages. } & & 80 & 164 & 244 & I 2.7 \\
\hline
\end{tabular}

1 Anmual Report of the Medical Officer of Health of the City of Edinburgh, 1899, p. 40.

${ }_{2}^{2}$ Iransactions of the Epidemiological Society of London, New Series, vol. xvii., 1897-98, pp. 93 and 96 (Dr D. S. Davies.) 
"The incidence of the attacks by age," writes Dr Davies, " is in this outbreak very much controlled by the special circumstances of the case, and especially by the fact of the milk having been distributed and used amongst large school boarding-houses. As the school age here extends up to 20 years, there is a notably large number of attacks at ages 15 to 20 . But at all school ages from 5 upwards the attacks are significantly high." $\mathrm{He}$ further adds, "The excess in attacks upon younger children is significant in conjunction with the heavy incidence upon females and upon domestic servants of the communication of the infection by milk." 1

In this same outbreak Dr Davies was able to show very clearly the age incidence of attack in relation to the persons coming directly in the way of infection by living in the houses supplied with the implicated milk:-

\begin{tabular}{|c|c|c|c|c|c|c|c|c|c|c|}
\hline \multicolumn{11}{|c|}{ Rates per 100 living at each age-group. } \\
\hline & & & $0-5$. & 5-10. & 10-15. & $16-20$. & $20-30$ & $30-40$. & $40+$. & Totals. \\
\hline $\begin{array}{l}\text { Total persons } \\
\text { Total attacks }\end{array}$ & : & : & $\begin{array}{r}14 \\
5\end{array}$ & $\begin{array}{l}33 \\
15\end{array}$ & $\begin{array}{l}53 \\
27\end{array}$ & $\begin{array}{r}202 \\
57\end{array}$ & $\begin{array}{r}309 \\
40\end{array}$ & $\begin{array}{l}99 \\
\mathbf{2 3}\end{array}$ & $\begin{array}{r}205 \\
17\end{array}$ & $\begin{array}{l}915 \\
184\end{array}$ \\
\hline Rate per cent. . & . & & $35 \cdot 7$ & $45 \cdot 4$ & 50.9 & $28 \cdot 2$ & I 2.9 & $23 \cdot 2$ & $8 \cdot 2$ & $20 \cdot 1$ \\
\hline
\end{tabular}

So much for external circumstances affecting age incidence. There is also demanding consideration the specific disease in question. Scarlet fever and diphtheria are, in the main, children's diseases; typhoid fever is, in the main, a disease of young adults. An exception to either of these common standards would, of course, raise a prima facie suspicion of milk infection. The above is an excellent example. Another occurred at Barrowford in Lancashire in 1876 , when, out of fifty-seven persons attacked with milk-borne typhoid, as many as twenty-five were under ro years of age.

\section{Incubation Period}

It is well known that, between the time of infection and the time of invasion, or symptoms of disease, there is a longer or shorter period of incubation. A man comes in the way of infection, say, of smallpox or typhoid fever. But a period of about fourteen days passes before the disease commences to show itself. In scarlet

\footnotetext{
1 Loc. cit., pp. 93 and 94.
} 
fever or diphtheria this incubation is a short one of three to five days. Now, whilst there is no hard-and-fast line strictly delimiting the period of incubation for any of the infectious diseases, it has been observed that, when such diseases are conveyed by milk, the incubation period is shorter than the normal. There are not a few epidemics which show that scarlet fever and diphtheria may commence to reveal their presence in a few hours, and typhoid fever in a few days. Yet this varies, and no two epidemics are precisely the same. In the Clifton typhoid epidemic of 1897 , to which reference has been made, there were two cases of girls who had each only consumed the implicated milk on one occasion, and hence the incubation period was ascertained with fair precision. In both cases it was under seven days. At Great Harwood in Lancashire in 1895 the incubation period in typhoid was, in some cases, as short as two days (Sarjeant). In Marylebone in 1873 a girl is said to have drunk two pints of the infected milk once, and sickened within five days. In contrast to this was a case observed by Power in which the attack of typhoid fever followed a single glass of milk after an interval of three weeks.

Scarlet fever carried by milk may have almost no incubation period at all. In the Fallowfield epidemic, near Manchester, in 1899 , Dr Airy found that in some cases attacked the incubation period was less than twenty-four hours. In a recent London epidemic of scarlet fever, one of the writers traced several cases where the incubation period was under twenty-four hours.

Where the incubation period is abnormally short, the symptoms which follow resemble an "intoxication" rather than an infection. In this particular, milk outbreaks follow the behaviour of food poisoning outbreaks. We may digress for a moment to note the chain of events. An individual in perfect health partakes of some poisonous food, and shows signs of illness immediately thereafter. There is no incubation period. On investigation the chemist succeeds in separating, from the poisonous article of food, an alkaloidal poison, a ptomaine, i.e a product of organismal action. The condition has been one of intoxication. Another individual, in perfect health, also partakes of some poisonous food, and shows signs of illness some hours or even some days thereafter. There is an incubation period, during which the infective agent is forming its poisonous products in the blood and tissues of the patient. On investigation, if within a sufficiently short period of time, the bacteriologist isolates from the poisonous article of food an organism, a vegetable cell, acting as an infective agent. This was present, 
perhaps in enormous numbers, in the food, untasteable, invisible, and imperceptible. But when introduced into the blood and tissues of the body, under favourable conditions of pabulum, moisture, and temperature, it proceeds at once, with greater or less rapidity, to multiply and pass throughout the body, producing its toxins. It is incubating, and the condition is an infection. Now, in milk epidemics, we may get one or other of these modes of invasion. In some cases the disease follows almost immediately after the introduction of the causal agents-the moment of infection-with such rapidity that the condition is allied to an intoxication. In other circumstances the events take an ordinary course, and an infection follows with a normal length of incubation period.

Various attempts have been made to explain the phenomenon of a short incubation period in milk-borne disease. Some have attributed the condition to the ready attachment of milk to the mucous membrane, and the early absorption of the milk globules into the lymph-stream, or the easy digestibility of milk. Others have considered the human element as playing a large part. Women and children, it is urged, are readily susceptible. But, in fact, neither of these hypotheses is adequate to the phenomenon. It has also been suggested that fatigue plays an important part in shortening the incubation period.

As we have seen, those diseases having a very short incubation period, such as diphtheria or scarlet fever, almost take the form of intoxications. That is to say, the symptoms produced are largely due to products of organismal activity. Hence a seemingly more reasonable explanation is surely to be found in the theory that the pathogenetic organisms present in the milk have found in it a favourable nidus for development and maturation. Milk is, as we have seen, an almost perfect pabulum ; there is also moisture, and in freshly drawn milk a favourable temperature. We know as a fact that bacteria multiply with almost incredible rapidity in fresh milk. Is it not possible that, under these circumstances, individuals consuming such milk imbibe not only organisms but toxins and other products already produced by them? Such a condition of things would tend to shorten the incubation period.

There is another point to be borne in mind in this relation. We have already seen that persons become infected in proportion to the amount of the contaminated milk which they drink. But we may now go a step further. In 1897 an outbreak of typhoid fever was traced to the milk supply. Amongst other things it was found that the attack rate was highest among the people who had 
received unmixed milk from the infected dairy. Two other milk vendors received a portion of their supply from the infected dairy. This portion was mixed, as is the custom in general vogue, with good milk derived from sources beyond suspicion. The houses served by these two vendors with mixed milk were attacked at the rate of $4^{1}$ and $3^{8}$ per cent. respectively as against 54 per cent. for the unmixed milk. Now it is not unreasonable to suppose that we should find the length of the incubation period to depend in some measure upon the degree of poisoning received.

We venture to think that it is along these two lines, namely, the development of the organisms in the milk and the degree of poisoning, that inquiry should proceed in future epidemics attributable to milk, and in which there is evidence that the incubation period is abnormally short.

\section{Sudden Onset and Rapid Decline}

Infectious diseases spreading by contact do not, commonly, appear suddenly. In milk-borne and water-borne disease the onset is sudden. Particularly is this so in milk infection. The evident reason for this is that the cause commences to operate suddenly. A cursory glance at the abstracts of various outbreaks printed in subsequent chapters will illustrate again and again that the majority of the cases occurred within a few days of each other. Then, after allowing for the incubation period to elapse, it will be found that the epidemic as suddenly ceases, immediately the supply of poisoned milk is withdrawn or the milk itself boiled. These two very simple features are almost pathognomic of milk-borne disease. An exception to the rule occurs in those milk-carried epidemics which are due, or are said to be due, to disease in the cow. In these cases one may get an outbreak lasting for weeks, and apparently attributable to no human source. Such an example was the epidemic in North London in $1883-84$, traced to the milk supply of a farm at Hendon, and to be referred to later on. In outbreaks originating in this way neither the onset nor the cessation is necessarily sudden, owing to the course of the disease.

Under this heading must be mentioned another very important and closely allied characteristic of milk infection. On making investigation into the circumstances of such an outbreak it will be found that multiple cases of the disease occur in the same household simultaneously and at the commencement of the outbreak rather than later on. An epidemic spread by contact not only 
drags over a longer period of time, but is characterised by the constantly recurring cases following on at the incubation period. In milk epidemics the multiple cases occur at once and simultaneously, and to a much lesser degree are they due to contact. Indeed, it is interesting to notice that many milk-borne diseases do not appear to spread, even at a later date, by contact. As a rule, they disappear as soon as the exciting cause is removed. We have noticed repeatedly how marked and clearly defined is this feature of multiple cases occurring early on in the history of an outbreak, and we think it is one of the most important signs of milk conveyance.

\section{Clinical Characters of Milk-borne Disease}

As we have already seen in passing, milk-borne disease, as a rule, does not carry with it a heavy fatality rate. The outbreaks are, as a rule, mild from the point of view of clinical signs and symptoms, and also from that of mortality. The points observed by various investigators may be very briefly stated in relation to the three chief diseases conveyed by this channel.

Scarlet Fever in not a few milk-epidemics has shown certain modifications of a more or less marked character. The disease is generally mild, and simultaneously with an outbreak of the specific disease due to milk, there will not infrequently be found a large number of "ordinary sore throats." Even in the scarlatinal cases the disease has a tendency to remain localised to the throat (Power). The rash may be evanescent, and the desquamation is scanty (Parsons). There is also a marked absence of postscarlatinal nephritis or any other kidney complication (Parsons, Buchanan, and others). A characteristic which has been frequently noted, and is readily to be understood, is the frequency of vomiting and diarrhoa, rather particularly at the commencement of the disease (the Fallowfield epidemic, I 879, is an illustration). These signs of alimentary irritation or poisoning have been observed by various authorities. It is more than possible that they are due to poisonous organismal products contained in the milk. On more than one occasion they have led to an appearance of intoxication rather than infection. Finally, there is a clinical feature, to which reference has already been made, and which may bear a significance not at first appreciated, namely, the comparative indisposition of the disease to spread by contagion. This may be attributable to the mildness of the disease, to the small amount 
of skin eruption and desquamation commonly present, and possibly to the fact that the poisonous properties of the milk are to a certain extent eliminated from the system by the vomiting and purging. Every clinical sign which has been noted leads to the conclusion that the disease as conveyed by milk is frequently mild, and therefore has both a small mortality and no tendency to spread by contact. There is one other point deserving of mention. Sir George Buchanan noticed, in a scarlet fever epidemic with which he had to deal, that in persons who had had scarlet fever at some previous time, and who drank the implicated milk, almost the only symptom of ill-health which they presented was a sore throat. There was no rash, no vomiting, no pyrexia, although other members of the family under precisely similar circumstances suffered from typical scarlet fever. Many other workers have confirmed this observation.

In outbreaks of diphtheria due to milk, the disease appears to take its ordinary course, except that it is less severe in most cases, and less contagious. Accompanying such outbreaks there is not infrequently an epidemic of "ordinary sore throat."

Enteric fever does not show any peculiar modifications except, again, these two of mildness and non-contagiousness. Multiple cases occur simultaneously or in quick succession. One investigator holds that in this disease, when conveyed by milk, adult and elderly persons are attacked earlier and somewhat more severely than children. But this observation has not been confirmed.

\section{Mortality Rate in Milk-borne Disease}

Finally, a note may be added respecting the mortality rates in outbreaks spread by means of infected milk. Unfortunately, out of some 260 records of epidemics the particulars of which we have studied and which we consider authentic, only a small number contain particulars as to deaths. If there were no deaths it should be so stated, and in each epidemic investigated it is important to record the fatalities. In the records of the outbreaks of scarlet fever, in particular, these facts are often absent. The highest mortality rate we have come across in scarlet fever was 23.5 per cent; the lowest nil. The average works out at about 12 per cent. In outbreaks of milk-borne typhoid the highest was 25.7 , and the lowest nil. The average of such outbreaks is about II per cent. Diphtheria works out at a higher fatality than the other two diseases. The highest mortality percentage which we have 
met with is 28 , and the lowest nil. The general average is 18.9 per cent. The returns may be tabulated as follows :-

\begin{tabular}{|l|c|c|}
\hline & \multicolumn{2}{|c|}{ Mortality Percentages. } \\
\cline { 2 - 3 } & Milk-Borne Outbreaks. & Not Milk-Borne. \\
\hline & & \\
\hline Scarlet Fever & I2.0 & 16.2 \\
Typhoid Fever & 11.0 & $17 \cdot 4$ \\
Diphtheria & I 8.9 & 30.3 \\
\hline
\end{tabular}

The mortality percentages in outbreaks not milk-borne, quoted in the above table, have been taken from a representative work on infectious diseases. ${ }^{1}$ General average returns have been selected. Obviously, mortality percentages differ widely in accordance with age incidence and other circumstances. Scarlet fever, for example, at all ages gives a mortality of 8.0 per cent. ( 81,350 cases), but in children under five years of age the mortality is 16.2 per cent. ( 17,310 cases). It is, of course, commonly a disease of children. The typhoid fever return of 17.4 was calculated on 9223 cases at all ages, and the diphtheria mortality percentage of 30.3 on I I,598 cases at all ages. In the last-named disease, age incidence has a marked effect upon mortality. The percentage of 30.3 refers to cases treated at a period prior to antitoxin inoculation. The mortality percentage of diphtheria in the Metropolitan Asylums Board hospitals in 1894 (without antitoxin) was 29.6 per cent.; in 1901 it had fallen to 12.5 per cent.; and in 1902 was I I.O per cent.

It will be seen that in each of three diseases the mortality percentages in milk-borne outbreaks, including many thousands of cases, is lower than the ordinary mortality from these same diseases.

1 A Manual of Infectious Diseases, by E. W. Goodall and J. W. Washbourn, I896. For obvious reasons such returns will be lower if only hospital cases are considered. The Metropolitan Asylums Board returns for 1902, for example, show mortality percentage for scarlet fever, 3.4 ; for typhoid, 15.4 ; and for diphtheria, II·O. See Metropolitan Asylums Report, 1902, p. $212 b$. 


\section{CHAPTER X}

\section{PATHOGENIC BACTERIA IN MILK (continued)}

Scarlet Fever. The Hendon Outbreak. Channels of Infection. Abstracts of Typical Epidemics. Typhoid Fever, the Vehicle of Infection, Bacteriology, and Channels of Infection. Abstracts of Typical Epidemics Diphtheria : the Question of Bovine Diphtheria. The Bacillus of Diphtheria and Milk : Channels of Infection. Abstracts of Typical Epidemics. Throat Illnesses spread by Milk. Epidemic Diarrhcea and Milk. The Bacteriology and Conditions of Diarrhœa. The Relationship of Milk to Epidemic Diarrhœea. Preventive Measures. Cholera.

\section{EPIDEMiC Diseases CONVEyed By Milk}

THE three main zymotic diseases conveyed by milk are then scarlet fever, typhoid fever, and diphtheria. Cholera must also be named as an epidemic disease in which milk has acted as carrier, and Zymotic enteritis has undoubtedly frequently been caused by milk consumption, and so have a variety of throat illnesses. Of these diseases we propose to speak in the present chapter.

In passing, it should be again clearly stated, that evidence of the fact that the diseases named have been conveyed by milk rests upon special incidence and collateral circumstance, and not upon bacteriological demonstration. Even in diseases whose bacterial etiology is known, such bacteriological demonstration would be found generally to be impracticable for the same reason as in waterborne disease, namely, that the disease occupies a certain period of incubation before it reveals itself, and therefore the infective milk is rarely obtainable.

\section{A. Scarlet Fever}

That the essential cause of scarlet fever is a micro-organism there can be little doubt. But up to the present time no organism has been definitely isolated, which fulfils the postulates of Koch in respect to specificity of bacteria. Various organisms have, how- 
ever, been described as associated with the disease. Edington, Fränkel, Freudenberg, Klein, Kurth, Gordon, Baginsky, Class, and others have described organisms which they believed to be etiologically related to the disease. At present, however, it can only be said that although these bacteria have been found associated with scarlet fever, they are not yet proved to be its cause. ${ }^{1}$

As regards dissemination it has long been known that scarlet fever, like small-pox, is most commonly spread by direct infection through the medium of infected clothing and other articles, or materials handled by the patient. The means by which infection has thus been carried are manifold, and need not claim our attention here. As we have seen, in 1870 a wider field of conveyance of scarlet fever was revealed by the investigations of $\mathrm{Dr}$ M. W. Taylor of Penrith. While studying an outbreak of scarlet fever he observed that the main incidence of the disease fell upon customers of a certain milk-shop where scarlet fever was existent. Since that date abundant evidence has been forthcoming to show that to the channels of infection previously recognised, that of conveyance by milk must be added.

In $\mathrm{I} 882, \mathrm{MrW}$. H. Power, then a medical inspector under the Local Government Board, investigated an extensive outbreak of scarlet fever in certain districts of North London. The ordinary human origins of the disease in milk-borne outbreaks appeared to be absent. True, one of the milkmen and three of his children contracted the disease, but only simultaneously with other persons suffering during the epidemic. There was, further, a peculiar incidence upon persons who derived their milk direct from the farm, thereby showing that the milk obtained its infective property from the farm and not in London. At the farm in Surrey the sanitary conditions were satisfactory, but one of the cows which had recently come into milk had been suffering from some ailment, apparently from the time of her calving. The symptoms were loss

1 We cannot here enter into a discussion as to the bacteriology of the various infective diseases conveyed by milk. The matter can only be referred to in its direct relation to the milk supply. For general information respecting the bacteriology of scarlet fever, typhoid fever, diphtheria, etc., the ordinary textbooks should be consulted (Muir and Ritchie, Crookshank, Hewlett, Macé, Lehmann and Neumann, etc.). We may, however, add a reference to the more recent bacteriology of scarlet fever which will be found to be dealt with in the Reports of the Medical Officer of the Local Government Board, 1886-87 (Klein); 1898-99, pp. 480-493; I899-I900, pp. 385-457, and 1900-or, pp. 353-404 (Gordon); Brit. Med. Jour., 1902, vol. ii., p. 445; Berl. Klin. Woch., 1900, Nos. 27 and 28 ; Chicago Medical Recorder, 1889 ; Lancet, 1900, 29th September. 
of hair in patches, and a general condition of ill-health. After careful consideration, $\mathrm{Mr}$ Power arrived at the conclusion that this cow had been the exciting cause of the outbreak, and thereby opened up a question upon which there has been considerable controversy. ${ }^{1}$ Into this we cannot enter fully, but for various reasons it is important that the matter should not be wholly avoided. We shall therefore attempt a brief aild impartial account of the subject. It will be understood that our aim is a statement of fact or theory and not an argument. Our object is historical and not advocacy. At the outset then, we may say that $\mathrm{Mr}$ Power's investigation in 1882 led him to write as follows. His remarks on the matter are of sufficient importance to quote in some detail :-

"I satisfied myself," he writes, "that it was practically out of the question that the milk at the farm had become infected in any of the commonly-believed ways that require a human subject as the source of infection: and I think it altogether unlikely that commonplace fouling of dairy utensils by polluted water or the like, can have here occurred. In the end, I found myself as on a former occasion, called upon to face the question whether or not actual cow conditions might have been competent for the results observed, and in considering the question I came to see that a hypothesis of cow causation would fit the facts that needed explanation as well as, or even better than, any other hypothesis. If only it could be believed that a single cow of this herd of 60 to 70 cows had been for a limited time capable of affording specifically infected milk, there was nothing in the routine of the farm dairy arrangements inconsistent with the milk of such single cow having been distributed among the churns consigned from the farm to London; and this in such a manner as to infect simultaneously the milk service of each London dairy supplied from the farm, and during a certain limited period a considerable proportion of that service.... On reference to his (the farmer's) books we found that one of his cows due to calve on the 27 th December had calved subsequently to, but within ten days of. that date, and had come into milking for business purposes three or four days after calving. . . . Upon examination of this cow on the IIth February I noted that she had here and there lost portions of her coat, and that her buttocks and posterior udder were fouled and stained by excremental matter, and perhaps by

${ }^{1}$ It must not be supposed that this was the first occasion on which it had been suggested that disease could be conveyed to man through milk from diseased animals. In 1862, Gamgee, in reporting to the Privy Council on the relation of cattle disease to the meat and milk supply, concluded that "the cause at present operating most actively to deteriorate the milk of cows in this country is the prevalence of Epizootic aphtha. This disease attacks the human subject and many cases of communication from cattle to man have been observed either from the virus penetrating a wound or passing into the system with the milk." This opinion received the support of Sir John Simon. Nor was this the first occasion on which milk had been shown to be the infective medium. 
vaginal discharge as well. In this respect she presented a rather strong contrast to most of the other cows. ...

"Few physicians will be disposed to deny a relation between scarlatina and certain forms of febrile puerperal diseases in women. The contagion of scarlatina introduced into the lying-in room has often and with reason been held responsible for puerperal fever.

"Now if it be true that there is one sort of relation between scarlatina and accidents of the puerperal state, another sort of relation becomes comparatively easy of belief, while there would seem nothing improbable in the further suggestion that all such relations would be qualified by the passage of a common contagion through the system of another animal.

"If scarlatina in man have other animal source than human source, it may be that one such source is the cow that has recently calved, a cow either not at all ill (except for her parturition) or not so obviously ill as to prevent her milk being used for human consumption. Milk of a recently delivered cow might become infective in more than one way. Either it might as a secretion of the cow contain infective matter that was circulating in the system of the cow ; or else uterine or other discharges of the cow fouling her udder, might, by a careless milker, be mixed with her milk in the act of milking." 1

Such is Mr Power's account of his views concerning the scarlet fever outbreak of I882. It may be added that at the time Dr Klein was consulted, and he made certain preliminary inoculation experiments with the object of learning whether or not human scarlet fever was inoculable into the cow. In the main the results were negative, though the inoculation of muco-purulent throat discharge gave rise to abcess formation in the inoculated cows. Sir George Buchanan wrote:- "He [Dr Klein] has found that a cow, having recently calved, when inoculated experimentally with human scarlatina (muco-purulent throat discharge from a scarlatina patient) is affected by an ailment which is transmissible after the manner of an acute specific disease, to dogs; that this ailment in the cow is not accompanied by any marked fever, does not take her off her food, and does not alter the quantity or visible characters of her milk. Its symptoms are not such as would be recognised as forbidding the use of the cow for dairy purposes." 2

\section{The Hendon Outbreak}

A second opportunity for the investigation of the entire problem occurred at the end of 1885 , when another outbreak of scarlet fever, traceable to the milk supply, appeared in North London. In this instance the implicated farm was at Hendon, and the necessary investigations were again entrusted to $\mathrm{Mr}$ Power.

1 Report of Local Government Board, 1882 (Medical Supplement), p. 65.

2 Report to Local Government Board, 1883, p. vii. 
The main facts respecting the investigation are printed in the Abstracts at the end of the present section. Briefly, they show that the incidence of the scarlet fever appeared to follow with remarkable accuracy the distribution of milk from three particular cows (and others infected by them) which had newly calved, and which were suffering from some specific disease. As it presented itself at Hendon, this disease, according to Dr Klein, consisted in the presence of sores and scurfiness in different parts of the skin, with loss of hair in patches, an eruption leading to the formation of a sore covered with dark brown scab or crust on the udder and teats, and a visceral disease of the lungs, liver, kidney, and spleen, which, although milder in character, much resembled, in his opinion, the visceral lesions occurring in cases of human scarlet fever. By experiment, Dr Klein found that the matter of the eruption on the udder of these Hendon cows was inoculable into calves, and when inoculated under their skin set up a similar eruption. At the time of these experiments, Dr Klein held that the milk of the cow did not itself contain the germ of this "Hendon disease," but that the germ obtained entrance to the milk from the outer surface of the udder at the time of milking, and that in milk it found a favourable nidus for its growth and multiplication.

"In short," Mr Power reported, "what had been seen to be a succession of probabilities, if the scarlatina in London districts were indeed the outcome of the milk distributed from the Hendon farm, was now established as a succession of facts. We had thus reached the point of excluding external scarlatina, of associating the importation of particular cows into the Hendon farm with presence of scarlatina in London districts, and of connecting, by a series of parallel events, the milk furnished by those cows and by related cows, with the peculiarities of scarlatina prevalence among consumers of the Hendon farmer's milk. Under these circumstances it was not judged necessary to go beyond the Hendon farm and to inquire at the two other farms which also sent milk into the London districts of South Marylebone, Hampstead, and St Pancras, in search of the cause of scarlatina in those districts. Henceforward, until anything to the contrary should appear, an influence, competent to produce scarlatina among the consumers of the milk, was held to have operated from those cows which were received into the Hendon farm on 15 th November and the further concern of the inquiry was with the nature of such influence."

Sir George Buchanan, on behalf of the Local Government 
Board, summarised his interpretation of these facts in the following words :-

"By Dr Klein's researches, together with the evidence as to scarlatina collected by Dr Power and reported by me last year, we are now in a position to state as follows concerning the disease producible by this micrococcus in bovine animals and in man:-

"(I) The disease in man and in the cow alike is characterised by closely similar anatomical features.

"(2) From the diseased tissues and organs of man and cow alike the same micrococcus can be separated, and artificial subcultures be made from it.

“(3) These subcultures, no matter whether established from man or cow, have the property, when inoculated into calves, of producing in them every manifestation of the Hendon disease, except sores on the teats and udders, no doubt for the reason that the milk apparatus is not yet developed in calves.

"(4) But-and this I learn from Dr Klein's later observations while this report is in preparation-the subcultures made from human scarlatina and inoculated into recently calved cows can produce in those cows, along with other manifestations of the Hendon disease, the characteristic ulcers on the teats-ulcers identical in character with those observed at the Hendon farm.

"(5) The subcultures, established either from the human or the cow disease, have an identical property of producing, in various rodents, a disease similar in its pathological manifestations to the Hendon disease of cows and to scarlatina in the human subject.

"(6) Calves fed on subcultures, established from human scarlatina, obtain the Hendon disease.

" ( 7 ) Children fed on milk from cows suffering under the Hendon disease obtain scarlatina.

"The above combine, I think, to form a mass of evidence to show that the Hendon disease is a form, occurring in the cow, of the very disease that we call scarlatina when it occurs in the human subject.

" $\mathrm{He}$ [Dr Klein] takes such animals and inoculates them subcutaneously at the root of the ear with the material of scarlatina. Sometimes he has derived the material direct from the human subject, sometimes after it has passed through the system of a calf. From whichever source the material may be derived, its inoculation results in ulcers on the teats of the cows. He finds these ulcers to be among the very earliest evidences of disease in the animal. They occur indifferently whether the cow is 
being milked by hand or is suckling her calf. The teat sores show themselves after an incubation period of from four to nine days from the inoculation, and subsequently a more general affection of the skin is found, accompanied by more or less febrile disturbance, sometimes by pulmonary symptoms. In the disease thus induced a number of changes are found after death distributed among various organs in the fashion of the changes of an acute specific disease, and exhibiting so much of constancy in their own manifestation as to make the whole of them characteristic of cow scarlatina; and the post-mortem appearances found in animals affected by this disease bear much resemblance in essentials to those found in human subjects dying of scarlatina. As regards the particular phenomena on the skin of animals thus affected, it is interesting to note that the sores on the teats appear to be with difficulty, if at all, transmissible by direct inoculation from the infected animal to man. This circumstance was hardly perhaps to be anticipated, seeing that other sore-teat diseases are so communicable, and how readily cow scarlatina at Hendon reproduced itself as human scarlatina in the consumers of milk from infected cows." 1

Such were the views entertained upon this matter by Dr Klein and Mr Power as representative of the Local Government Board, and with a large number of persons these views found acceptance. The veterinary profession and certain pathologists, however, were not prepared to accept the matter as proved. Accordingly, further investigations were instituted by the authorities of the Agricultural Department of the Privy Council (now merged in Board of Agriculture), and as a result certain further propositions were formulated which placed a different interpretation upon the causes of the outbreak. These we must briefly consider.

At the outset it may be remarked that two cardinal facts were accepted both by the Local Government Board and by the Agricultural Department, namely, first, that the epidemic of scarlet fever was due to contamination of the milk supply; and second, that some of the cows on the Hendon farms were suffering from disease. As to these two facts there was no controversy. Discussion, however, arose as to the relation between the cow disease and the milk-borne scarlet fever, it being contended by the Agricultural Department that no relationship was proved between these two events, and that, in point of fact, reliable evidence was not

${ }^{1}$ Seventeenth Annual Report of the Local Government Board, 1887-88 (Medical Officer's Supplement), pp. xiii. and xiv. 
forthcoming to establish the proposition that this cow disease was cow scarlatina.

In a special report, Sir George Brown, at that time Head of the Agricultural Department of the Privy Council, clearly stated the grounds upon which the chief dissension to the cowscarlatina hypothesis was based. ${ }^{1}$

The first criticism was that the Hendon cow disease of $1885-6$ was not an isolated outbreak confined to the particular farm at Hendon, but was going on at the same time with several other outbreaks, all of which were due to the introduction of cows from a Derby dealer's herd, from which were, admittedly, derived the Hendon disease cows. The 3 cows to which the implicated milk had been traced by $\mathrm{Mr}$ Power were, it appears, part of a lot of 30 cows bought in Derby market, and of which several were suffering from sore teats, and others contracted the disease from their comrades before being disposed of. "From the first day to the last, while the milk from these cows was being drunk, no case of scarlatina was heard of among the customers of the dairy. Of the 30 cows 8 were sold to $\mathrm{Mr}$ T. K., a dairyman at Merton, 3 were sold to $\mathrm{Mr} \mathrm{C}$. of Hendon, and 3 to the late $\mathrm{Mr} \mathrm{P}$. (the unfortunate Hendon farmer to whose farm the milk epidemic was traced). The remainder were disposed of in ones and twos to various metropolitan and provincial dairymen" (Axe). It is stated that the eruptive disease in the Derby cows which passed into the possession of $\mathrm{Mr} \mathrm{T}$. K. of Merton, spread in the herds which they joined, and although nearly five hundred households were supplied with their milk, no case of scarlatina occurred. Several cases of hand eruption occurred in the milkers coming into contact with the diseased cows, and this eruption was diagnosed as of the nature of vaccinia (Axe). Of the 3 Derby cows which were sold to $\mathrm{MrC}$. of Hendon, one was suffering from the udder disease, which soon affected upwards of a dozen cows belonging to $\mathrm{Mr} \mathrm{C}$. The milk from these animals was disposed of in the usual way during the entire period of the ailment, but without complaint being heard from any of the customers, or a case of scarlatina being known to exist among them (Axe). To the 3 Derby cows, which apparently suffered from the same udder disease, which passed into the herd of $\mathrm{Mr} \mathrm{P}$. of Hendon it is alleged the scarlet fever epidemic in North London was due.

1 Report on Eruptive Diseases of the Teats and Udders of Cows in relation to Scarlet Fever in Man, by Professor Brown, C.B., Agricultural Department, Privy Council Office, 1888 (with appendix and plates). 
The second proposition set up by the Agricultural Department was to the effect that in the outbreak of scarlet fever, traceable to Mr P.'s farm at Hendon, there was a possible source of infection of the milk by human agency. In an inquiry made by Professor Wortley Axe some time after the Hendon outbreak, it transpired that scarlet fever was present at Hendon, in the Mead, at the time of the outbreak in London and previously. The Mead, a low-lying and ill-conditioned street, ran immediately alongside and formed one of the boundary lines of the implicated farm. The dwellings in which scarlet fever existed stood within 600 yards of the sheds in which the cows were housed and the dairy business carried on. Of the fourteen men employed in and about the dairy, six resided in the Mead and passed infected houses several times daily. Moreover, the father of one of the patients suffering from scarlet fever in November was on intimate terms with the bailiff at the farm, and her brother constantly frequented the dairy. Professor Axe considered that whilst there was no demonstration that the milk became contaminated from this human source, the possibility of such an event might not unreasonably be expected. It should not, however, in this connection be forgotten that the scarlet fever epidemic in London lasted some weeks, and could not apparently have been due to a passing contamination of the milk.

In the third place, the Agricultural Department considered that the failure of Mr Power to visit and examine the two other farms supplying the scarlatina-infected districts in London, introduced a somewhat serious fallacy into the reasoning which referred the outbreak to the Hendon farm alone.

Lastly, Professor Brown maintained that the Hendon disease was not rare in its appearance, but comparatively common in milch cows.

Indeed a cow disease apparently identical in general character with the Hendon cow disease of $1885-6$, according to the testimony of experienced observers who saw the original Hendon outbreak, appeared in 1887-8 at Hendon. Strangely enough the affection was introduced by cows bought of the same dealer and from the same market (Derby) as the cows which are said to have occasioned the outbreak of Hendon disease in 1885 . The disease affected 24 cows out of 45 in the herd. During the whole period of the outbreak the milk of all the affected cows was used in the ordinary way by the customers of the farm, yet " no complaint was ever made of it causing illness or inconvenience, and the most diligent inquiry failed to discover any outbreak of scarlatina among the many 
families supplied with milk from this contaminated source" (Villar). Nevertheless, not only did the cows show the same symptoms as in the Hendon disease, but it was proved that the affection was readily communicable to cows by the hands of the milkers, and that calves sucking the diseased cows became ill and suffered from an eruption on the skin of the nose and mucous membrane of the mouth.

Nor was this the only outbreak having certain parallel characters with the Hendon outbreak. There was, for example, the Wiltshire disease investigated by Crookshank, ${ }^{1}$ the Edinburgh disease investigated by Woodhead and Cotterill, and other similar outbreaks in various parts of the country. The Agricultural Department declared that all these contagious eruptions were of the nature of so-called cow-pox and that they were not causally related to scarlet fever in man. Klein pointed out that in his opinion the Hendon disease differed in certain essential characters from cow-pox. It was more rapid in its course, the corium was more infiltrated, the period of encrustation was of shorter duration, and the progress of healing was quicker. Inoculation of calves gave, he held, different results; and in the case of the Hendon disease there was distinct visceral disease in the affected cows. We need not pursue the matter further than to say that Dr Klein continues to maintain that whether those other outbreaks were or were not identical with the "Hendon disease" of I 885, that specific disease was, in fact, scarlet fever of the cow and gave rise to the outbreak of scarlet fever in man in the epidemic occurring in North London in I 885-6.

For our part we are of opinion that the exact origin of the London epidemic at that time has not yet been, and now probably never will be, demonstrated. Even at the present time the specific micro-organism which is the causal agent of human scarlet fever is not fully established or proved. That is to say, no microorganism has yet been isolated in human scarlet fever which fulfils the postulates of Koch. Much less was this the case sixteen years ago when bacteriological methods were less perfect than they are even to-day. From this it follows that the vera causa was obscure, and yet without this link it was impossible to complete the chain of evidence by which it could be definitely known that any disease of the cow was responsible for the epidemic. The probabilities might be strong or weak, but proof was wanting. The inoculation

1 Report to Agricultural Department of Privy Council, 1887; see also Professor Crookshank's Bacteriology and Infective Diseases, I896, pp. 265-282. 
experiments in so far as they yielded positive results, were also open to the same unreliability. Unfortunately too there was, on the other hand, circumstantial evidence of various kinds, which, while it proved little, opened up a variety of possibilities by which the milk consumed in London might have become infected.

Such are the main facts on both sides of this matter. We have quoted the main facts respecting this epidemic, not on account of its conclusions, but because it was one which contains so many, and so varied, instructive lessons on the whole question of milk epidemiology. It opened up new ground. It brought into prominence the intimate relationship existing between the infectious diseases of animals and similar diseases in man. But after an examination of the evidence on both sides of the question we are of opinion that the case for cow scarlatina was not proved.

\section{Channels of Infection in Scarlet Fever}

Scarlet fever is disseminated in many ways from person to person, and also by the vehicle of "fomites." The virus is not diffusible, but is evidently tenacious of life. Infected garments that have been put aside for months have been known to originate an outbreak of the disease. Linen has been known on many occasions to infect laundresses. There is no evidence that the virus can be conveyed by water. As a rule, probably the infection of scarlet fever is not greatly spread by aerial connection, but by articles (toys, books, bed-clothes, letters, etc.), and such infected articles if set aside in stagnant air, at a moderate temperature, and in the absence of daylight, may retain the infection, like garments, for months.

Infectivity begins at the earliest stage of the attack, but is probably greatest when the fever is at its highest. In most cases the patient is free from infection at the end of six weeks. There is now strong evidence that at least the later desquamation is not irfective. Probably the infection lingers longest in the nasal, tonsillar, buccal, and pharyngeal mucus, and especially in any chronic discharge from those mucous membranes. Discharges from the ear may retain infection for months. ${ }^{1}$

It is most probable that milk obtains its infection of scarlet fever from being brought into contact with persons suffering, as a rule, from the early and acute stages of the disease, when the skin

1 See also Report to Metropolitan Asylum Board on Return Cases of Scarlet Fever, by W. J. Simpson, M.D., 1901, p. 24 ; also Brit. Med. Jour., 1902, vol. ii., p. 445 (M. H. Gordon). 
itself is infective. Doubtless, also, a not inconsiderable portion of milk infection is derived from infected garments and other articles, which, as stated above, may retain their infectivity for long periods.

\section{Abstracts of Typical Milk-Borne Outbreaks of SCARLET FEVER}

The following abstracts of milk-borne outbreaks of scarlet fever are inserted with a view of placing on record, in comparatively brief compass, an historical and epidemiological account of some of the more marked instances of the conveyance of the disease by the consumption of milk. A perusal of them cannot fail to furnish the reader with a more or less comprehensive view of the ways and means of infection. They are placed in chronological order and are stated in as brief a manner as possible. This note applies also to the abstracts of typhoid and diphtheria epidemics appearing later:-

Handsworth, 1876 (July).

Total number of cases

\section{Deaths}

Number of cases amongst drinkers of suspected milk .

Percentage on total cases . $\quad$ roo

Number of families, supplied by milkman, invaded .

Circumstances implicating the milk supply.-Three cases of scarlatina in dairyman's family. Milk-cans kept in house, and the dairy itself in direct communication by a doorway with the living-rooms in the house. Disease followed course of this milk supply.

Probable exciting cause.-Human source.

Reporter and reference-Dr T. B. Welch (Med. Off. of Health). Brit. Med. Jour., 1876, vol. ii., p. 225.

\section{Fallowfield, Manchester, 1879 (August).}

Total number of cases -35 in 18 families.

Number of cases amongst drinkers of suspected milk .
Percentage on total cases . . 100 Number of families supplied by milkman . . 60-70 Number of such families invaded . . . . 18

Percentage. . . . 27

Circumstances implicating the milk supply. - One of the milkers came from an infected house, and is believed to have conveyed the infection. Twenty-four of the cases were attacked within the space of 36 hours. The disease exactly followed the course of this milk supply. There was nothing else common to the houses attacked. In some cases it appears that the disease commenced within 24 hours of drinking the milk. The families invaded belong to the better class. Diarrhœa and alimentary irritation were prominent symptoms.

Probable exciting cause.-Human source.

Reporter and reference.-Dr Airy (Loc. Gov. Bd.). Brit. Med. Jour., I880, vol. i., p. 107 ; Rep. of Medical Officer of Lac. Gov. Bd., 1879. 
Paddington and Bayswater, 1880

(July and September).

Total number of families invaded

Number of polluted milk sources

Number of milkmen . . - many

Circumstances implicating the milk supply.-Many cases of scarlet fever among the families of milkers on 2 out of 8 farms furnishing the supply. Outbreak divisible into two portions, both of which arose on the track of this milk supply.

Probable exciting cause.-Human source.

Reporter and reference.-Dr J. Stevenson (Med. Off. of Health). Brit. Med. Jour., 1880, vol. ii., pp. 596, 632.

Hallfax, 1881 (Janwary).

Total number of cases

510

Deaths

Number of families supplied by

milkman .

Number of such families invaded

Percentage.

53

Circumstances implicating the milk supply. - Farmer's man who milked the cows and brought the milk to the customers had 4 children ill of scarlet fever. Having 6 children (one a baby), the mother could not attend to all her household duties and nurse the sick as well; probably, therefore, the man did his share of nursing, and he placed his clothes on the children's bed at night for extra covering. Four other children were attacked before 14 th January. Straight from his home, with his person and clothing alike infected, he went to milk the cows, and also assisted in distribution of milk.

In December 1880 there were more than a dozen cases in the district, but in the beginning of January there was a marked and sudden increase (comprising about 400 cases).
On the first three days of January 50 cases commenced. Abundant evidence to show that the milk carried the infection. In the families escaping there were in 70 per cent. no children, and in other cases various degrees of protection. Some of the cases commenced illness within six hours of having partaken of the milk. Dr Ballard suggested that there were in all probability other agencies at work besides the infected milk.

Probable exciting cause.-Human source.

Reporter and reference.-Drs Ainley and Ballard. Brit. Med. Jour., I88I, vol. i., p. 255 , and vol. ii., p. 485 ; Loc. Gov. Bd. Rep., I88I, p. 60.

Greenock, 1882 (January and Febrwary).

Total number of cases . 20

Number of cases amongst

drinkers of suspected milk . 20

Percentage . . . . 100

Number of families supplied by

milkman . . . . . II

Number of polluted milk sources I

Number of milkmen . . . I

Cincumstances implicating the milk supply.-Scarlet fever in dairyman's family ; 4 cases, initial case dating from Igth January. No cases occurred after milk supply stopped.

There occurred another similar outbreak affecting 47 persons in October and November of the same year, which was traced, in part, to unrecognised cases of scarlet fever in the milk dealer and his child.

Probable exciting cause-Human source.

Reporter and reference.-Dr Wallace (Med. Off. of Health). Brit. Med. Jour., 1882, vol. i., p. 437 , and vol. ii., p. 1325.

North London, 1882 (January).

Total number of cases - "extensive outburst." 
Number of cases amongst drinkers of suspected milk-almost wholly.

Percentage on total cases-practically 100.

\section{Circumstances implicating the milk} supply.-This epidemic occurred in the districts of St Giles, St Pancras, Marylebone, etc. One of the milkmen and 3 of his children had scarlet fever, but apparently only simultaneously with the epidemic ; moreover, some of the consumers who derived their milk direct from the farm suffered from scarlet fever, thereby showing that the milk was infected at the farm and not in London. This view was abundantly confirmed, for milk from the farm was also sent direct to Camberwell, where 39 cases of scarlet fever followed. Hence the investigation was limited to the farm. The sanitary conditions of farm and dairy were satisfactory. But one cow recently calved was looked upon by $\mathrm{Mr}$ Power as the exciting cause. She was out of health. Persons were affected in various parts of London, and certain families supplied with the milk en route from London also contracted scarlet fever. Of 6 families belonging to railway servants purchasing some two or three imperial gallons daily of the milk on its reaching Charing Cross Station, 13 members fell ill during the same epidemic.

Apparent exciting cause.-(?) Cow disease.

Reporter and reference.-W. $\mathrm{H}$. Power, F.R.S. Loc. Gov. Bd. Rep., I882, p. 63 .

\section{Oxford, 1882.}

\section{Total number of cases}

Circumstances implicating the milk supply.-Nine houses were affected containing 85 persons, 2 of whom contracted diphtheria, Io scarlet fever, and 18 sore throat. All the houses were exclusively supplied from the same milk source except 3. The supply came from 3 cows. In the house of the man tending these cows there was a case of diphtheria, and the child of the cowman had scarlet fever. The illness in the houses was connected with the case of scarlet fever in the cowman's cottage.

Probable exciting cause.-Human source.

Reporter and reference.-Dr S. D. Darbishire. St Barth. Hosp. Rep., vol. xx., pp. 93-100.

\section{Dundee, 1888 (November).}

Total number of cases _.. . 17

Deaths . . . . . 4

Number of cases amongst drinkers of suspected milk . 17

Percentage on total cases . . I00

Number of families supplied by

milkman . . . $\quad 12$

Circumstances implicating the milk supply. - The dairy formed part of a dwelling-house, the means of ventilation, cleansing, drainage, and watersupply not being such as to ensure protection of milk against contamination. The milk was stored in the kitchen where a boy was lying ill of scarlet fever, and the milk business was carried on by the dairyman's wife and servant after recent contact with the patient. All the cases notified occurred within 4 days in consumers of the infected milk.

Probable exciting cause.-Human source.

Reference.-Brit. Med. Jour., $188_{3}$, vol. ii., p. 839 .

\section{North London and Hendion, 1885 \\ (November and December).}

Circumstances implicating the milk supply.-This outbreak affected St Pancras, Hampstead, South Marylebone and Hendon. After careful preliminary inquiries, it was presumed that the scarlatina occurring in these 
districts followed the consumption of milk from a particular farm at Hendon. Further, in these four districts wherein scarlatina had shown an extravagant incidence upon the milkman's customers, the disease had begun its peculiar incidence about the end of November or beginning of December. In one of those districts (South Marylebone), scarlatina continued day by day and with increasing force up to the date of the inquiry to attack the customers of the retail business. In two other districts (Hampstead and St Pancras), after attacking in some numbers, for a few days at the end of November and beginning of December, the customers of the business, the disease showed no fresh attacks for about 10 days (a short but clearly defined intermission), and then about the middle of December attacked them again in larger numbers, and continued to do so up to date of inquiry.

The chief facts concerning the distribution of the milk may be set out as follows: (a) The Marylebone customers suffered at the end of November and up till the end of the third week in December. (b) The Hampstead cases occurred in two groups, one small group at the end of November and a larger group in the latter part of December. (c) The St Pancras customers suffered like the Hampstead ones, but in a less degree. They obtained milk from the same vendors. - (d) The St John's Wood customers did not suffer until after the end of the year. (e) The few persons affected at Hendon suffered early in December, having consumed milk which had been returned from Marylebone, and at the same time new cases of scarlet fever ceased to occur in Marylebone. Examination was then made to ascertain if there had been any possible infection of the milk to explain this incidence and intermission.
When Mr Power came to inquire as to the movements of the cows he learned that on I5th November three newly calved cows arrived at the Hendon farm from Derbyshire, this event shortly preceding the first attack of scarlatina. It happened that these three animals were placed in a shed by themselves and their milk was distributed in part to South Marylebone, Hampstead, and St Pancras, immediately preceding the outbreak of scarlatina in those districts. On examination it was found that the implicated cows were suffering from some kind of disease of the udders, which had spread to other cows in the herd. It would appear that the diseased condition, whatever it was, had been introduced by one of the Derbyshire cows, and had then spread through various sheds at the Hendon farm. Mr Power was able by the most minute inquiry to trace the movements of those cows and the various sheds in which they were placed from time to time, and he held that the various recrudescences of the outbreak in North London corresponded with the movements of the affected cows.

The exciting cause then of this outbreak was believed by Mr Power to be a condition of certain milch cows which had for its outward manifestation an eruption on teats and udders and which was communicable from cow to cow. Subcultures of the ulcerous discharges of the affected animals inoculated into calves produced a disease having unmistakable affinities, under some conditions, with the disease in the milch cows, and under other conditions with scarlet fever in the human subject. Now, it must be added, that scarlet fever appeared simultaneously in all but one of the five localities to which the milk was distributed. The exception received none of the milk from the 
affected cows until later, when the disease also appeared in this district, owing to some of the milk from the affected cows being sent there. When the sale of the milk was prohibited in London, some of it was clandestinely distributed amongst the poor of Hendon. Amongst those served, half a dozen families were invaded by scarlatina at a time when the disease had ceased to exert its influence in the London districts.

The intermission which had occurred in the scarlatina in Hampstead and St Pancras during the Io days referred to above, was at the time when the infective cows had been moved into a shed sending milk only to Marylebone. A few days later they were again moved into a shed from which milk was distributed to the two former districts.

Thus the investigation showed the Hendon farm to be the main source, and, as far as could be judged, the cows referred to, the particular source of the implicated milk, for the disease followed the distribution of their milk. The further inquiry was with regard to the nature of the disease or influence appertaining to these cows.

Apparent exciting cause.-(?) Cow disease.-(?) Human source.

Reporter and reference.-W. $\mathrm{H}$. Power, F.R.S. Loc. Gov. Bd. Rep., 1885, pp. 73-III.

Wimbledon and Merton, 1888-7 (December ana January).

Total number of cases

Number of cases amongst drinkers of suspected milk . Percentage on total cases . . Number of families supplied by milkman .

Number of such families invaded Percentage

Circumstances implicating the wilk supply.-No fewer than $43 \mathrm{I}$ attacks occurred in a single week at end of
December. In this epidemic week consumers of the suspected milk suffered 700 times as much as consumers of milk from other sources, and throughout the entire period, in the proportion of 100 to $I$. The outbreak followed the delivery track of the milk. The disease fell especially on well-to-do people, and attacked most severely those who consumed most milk. Incubation period varied from 30 hours to 5 days. The sanitary conditions of farm and dairy were satisfactory. Some of the cows however, "appeared to be recovering from an affection of the skin and udder, very similar to the malady reported on by Dr Klein" at Hendon.

Apparent exciting cause.-(?) Cow disease.

Reporter and reference.-. W. $\mathrm{H}$. Power, F.R.S. Loc. Gov. Bd. Rep., 1886, pp. 327-338.

\section{Glasgow, 1888 (October).}

Total number of cases . . 56

Number of cases amongst

drinkers of suspected milk - 43

Percentage on total cases . . 77

Number of families supplied by

milkman . . . . 363

Number of such families invaded 29

Percentage . . . 8

Number of polluted milk sources I

Number of milkmen . . . I

Circumstances implicating the milk supply.-Members of the families of two dairymen ill from throat affection following on scarlet fever in other members at one of the shops. Family relations of the two businesses were interlaced. Forty cases out of the 56 occurred in 4 days, limited to consumers of the milk, and of well-to-do classes. The amount of milk consumed bore an exact relation to the severity and extent of the disease.

Probable exciting cause.-Human source. 
Reporter and neference.-Dr J. B. Russell (Med. Off. of Health). Lancet, I888, vol. ii., p. 1079 .

\section{Pollokshields, Glasgow, 1888 (Jue).}

Total number of cases . . 7o

Number of cases amongst drinkers of suspected milk - 70

Percentage on total cases . . 100 Number of polluted milk sources I Number of milkmen . . . I

Circumstances implicating the milk supply.-Cases of scarlet fever had occurred in the persons of the servant and children of the farm supplying the milk.

Probable exciting cause.-Human source.

Reporter and reference.-Dr Carmichael. Brit. Med. Jour., 1888, vol. ii, p. 32 .

\section{Newcastle-on-Tyne, 1888 (June).}

Total number of cases . 74

Number of cases amongst

drinkers of suspected milk . 6I Percentage on total cases . $\quad$. 82 Number of polluted milk sources I Number of milkmen . . . 1

Circumstances implicating the milk supply. - Three children in the family of one man attending cows suffered from an illness not to be differentiated from scarlatina. All the 61 cases occurred in the space of 13 days.

Probable exciting cause.-Human source.

Reporter and reference.-Dr H. Armstrong (Med. Off. of Health). Public Health, 1888-89, p. I56.

\section{Macolesfleld, 1889 (Jamary).}

Total number of Scarlet fever 38 cases $\left\{\begin{array}{l}\text { Diphtheria } \\ \text { Sore throat }\end{array}\right.$.

Number of families supplied by milkman .

Number of such families invaded
Cincumstances implicating the milk supply.-The scarlet fever was accompanied by cases of severe sore throat, the more serious cases having a diphtheritic tendency. The incidence of attack on persons drinking most milk was well shown by Dr Parsons.

Larger proportion of milk indicates that more is imbibed, occasions of exposure to any poison in the milk are more numerous, and a larger proportion of what is taken has not undergone any process, as of cooking, which would destroy or impair its activity. About an equal number of persons were attacked at years above and below 15. The severity of illness depended on the amount of milk consumed as a rule. The scarlatina showed little tendency to spread in the households into which it had been introduced, the rash was ill-defined, desquamation was scanty, kidney complications were rare, and alimentary disturbance was well marked.

Probable exciting cause,-Undetermined.

Reporter and reference.-Dr Parsons. Loc. Gov. Bd. Rep., 1889, pp. 89-III.

Upton and Macelesfield, 1889 (February).

Total number of cases $\quad$ - 129

Number of cases amongst

drinkers of suspected milk . 123

Percentage on total cases . . 93

Number of families supplied by milkman . . . . I00 Number of such families invaded $\quad 58$ Percentage. . . . . $5^{8}$ Number of polluted milk sources I

Cincumstances implicating the milk supply.-Well water at farm polluted with sewage. Milk-cans rinsed out with cold water from this well. Dr Parsons thinks, however, that the outbreak was due to the milk of a cow which had recently calved and later on developed vesicular sores on the teats, and became emaciated. Of 
households using the milk in Upton, 77 per cent. were invaded. The outbreak was sudden after long-continued freedom from scarlet fever.

Apparent exciting cause.-(?) Cow disease.

Reporter and reference.-Dr H. F. Parsons. Loc. Gov. Bd. Rep., 1889, p. 89 .

Sutton Coldfleld, 1891 (February-April).

Total number of cases . . 40

Deaths . . . . 5

Cases amongst drinkers of sus-

pected milk-"almost without

exception."

Circumstances implicating the milk supply.-One cow presented characteristics similar to those at Hendon -recent calving, emaciation, ulceration on teats, eruption and desquamation. A second cow slight similar symptoms. All the 17 houses first invaded took the implicated milk. The outbreak consisted of two "explosions," coinciding in time with the ulceration and eruptive conditions of the first cow. After sale of milk had been stopped, outbreak ceased.

Apparent exciting cause.-(?) Cow disease.

Reporter and reference.-Dr Bostock Hill (Med. Off. of Health). Brit. Med. Jour., 1891, vol. ii., p. I36.

\section{Bush Hill Park and Enfleld, 1891}

$$
\text { (November). }
$$

Total number of cases

Number of cases amongst drinkers of suspected milk - 33

Percentage on total cases . . IOO Number of families invaded $\quad 23$

Circumstances implicating the milk supply.-Sanitary conditions of dairy and farm good. The exciting cause was not definitely ascertained, but some of the cows had abraded teats, boils on the udder, or vesicular scabs. Dr Klein failed to discover any strepto- coccus corresponding with that in the case of the Hendon disease.

This outbreak was limited to 8 days and to two sanitary districts, every one of the cases being customers of the one dairyman. The disease was more largely incident on better class houses taking most milk. Confirmatory evidence implicating the milk came from the Essex village where the dairy-farm was situated. Here the only two houses invaded were two to which the milk in question was delivered-except that one girl fell ill who had opportunities of helping herself to the milk. In dairyman's own family the infant fed on "nursery" milk from his own cow escaped; his little daughter drinking the Essex milk sickened with scarlet fever.

Apparent exciting cause.-(?) Cow disease.

Reporter.-Dr Copeman (Loc. Gov. Bd.).

\section{Leyton, 1892.}

Total number of cases . . $5 \mathrm{I}$ Number of polluted milk sources I Number of milkmen . . several

Circumstances implicating the milk supply.-The farmer supplying the milk and several members of his family suffered from scarlet fever. One dealer had 39 cases amongst his customers.

Probable exciting cause.-Human source.

Reference.-Brit. Med. Jour., 1892, vol. ii., p. 704 .

\section{Charlton, 1892 (March and April).}

Total number of cases - . 57

Number of cases amongst drinkers of suspected milk - 57 Percentage on total cases . . 100 Number of polluted milk sources I Number of milkmen . . : : I

Circumstances implicating the milk supply.-Dr Hamer attributes this 
outbreak to a cow that was ill, with scabs on udder and teats, and yielding ropy milk. Epidemic affected seven different areas, and began within a day or two of commencement of cow's illness. Fresh cases ceased to occur, except in two localities to which alone this cow's mill was distributed in the second week of the illness; cases entirely ceased after the cow was totally excluded from the dairy business. Dr Hamer pointed out that the 6 days on which this cow suffered from "a cold" and was "off her food" and yielded ropy milk, and yet during which time her milk was being distributed, were the 6 days of maximum infective power of the milk supplied by the implicated vendor. After this cow's milk was excluded from distribution, the outbreak declined. A large number of children were affected in Plumstead, owing to the distribution being in the immediate vicinity of 4 schools. The disease afterwards spread from child to child "by means of slight undetected cases," and from them to other children in the district.

Dr Hamer's report is full of interest and suggestion respecting the investigation of milk-borne disease. One of the points which he brings out clearly is the incidence of disease on drinkers of the milk from a particular shed. From 12th to 25th March, Io per cent. (normal); from 26th March to 1st April, 9I per cent.; and for the period of two weeks in April, 24 per cent. He resolves the matter therefore into three periods in relationship to the cow disease- (a) cow began to be ill, but its milk was distributed - epidemic began within 2 to 3 days; (b) cow under treatment, its milk sometimes distributed and sometimes discarded, but only in Greenwich and Deptford. Cases attributable to milk ceased, except in Greenwich and Deptford; (c) all suspicious milk excluded--complete cessation of disease. Incubation period was demonstrated to be very short, about 24 hours. Mortality was slight.

Apparent exciting cause.-(?) Cow disease.

Reporter and reference.-Dr Hamer (Rep. of Medical Officer of London County Concil), 1892, p. 17; also Public Health, 1891-92, p. 366; and Special Rep. to London County Council, by Dr Hamer.

\section{Langham, Dssex, 1892 (October).}

Total number of cases . 5

Number of cases amongst drinkers of suspected milk . 5

Percentage on total cases . $\quad$ I00

Number of families supplied by milkman . . . . 2

Number of such families invaded . . . . 2

Percentage. . . . . 100

Number of polluted milk sources I

Number of milkmen - . . I

Circumstances implicating the milk supply.-Suspicious udder eruptions on two cows at farm. The milk was used only at this farm and another dwelling. Both houses were invaded, 4 cases occurring in I day. A visitor at one of the houses stayed one night, partook of the milk, and afterwards developed scarlet fever.

Apparent exciting cause.-(?) Cow disease.

Reporter and reference.-Dr J. Cook (Med. Off. of Health). Report of Medical Officer.

\section{Upper Clapton, 1892}

$\begin{array}{crr}\text { No. of } & \text { persons }\end{array}$ attacked $\left\{\begin{array}{cr}\text { scarlet fever } & \text { I78 } \\ \text { drank implicated milk } & 78 \\ \text { sore throats. } & 155 \\ \text { drank implicated milk } & 145\end{array}\right.$

Circumstances implicating the milk supply. -A sudden outbreak of scarlet fever occurred in a localised area of Upper Clapton amongst houses of good class, supplied most largely by 
Mr X., a milkman. Most cases occurred where most milk consumed, and the houses supplied by $\mathrm{Mr} \mathrm{X}$. suffered 12 times more than the whole of the households supplied by the whole of the remaining dairymen. A child of one of the milkmen was taken ill with scarlet fever on Ist April, and appeared to be one of the first cases of the outbreak. Several children of one of the cowmen also had sore throat during April. Of the Iog cows supplying the milk none appeared to have any eruption condition, though there were cracks and abraisions on the teats.

Apparent exciting cause-Milk infected from human source.

Reporter and reference.-Dr King Warry (Med. Off. of Health). Practitioner, I892, vol. ii., p. 63.

\section{Glasgow, 1892 (August).}

Total number of cases . . 236

Deaths . . . . . II

Number of cases amongst drinkers of suspected milk . 236

Percentage on total cases . . I00

Number of families supplied by milkman .

Number of such families invaded 152 Number of polluted milk sources Number of milkmen . . .

Circumstances implicating the milk supply.-Sanitary conditions of dairy and farm satisfactory. Ulcerative eruption on udders and teats of cattle, vesicular, semi-pustular, crusted, with blackened centre ; communicable from cow to cow, and shed to shed. Sores on the hands of some of the milkers. Dr Klein found the cow disease in this case to be allied in some points to the Hendon disease.

The incidence of the disease was confined to the milk service of one dairy getting milk from two farms. Milk from the implicated farm mostly used on first morning round (57 families),
9I individuals being attacked out of 193 families supplied on this round. The mixed milk always used on second round, and 59 individuals in 37 (out of I66) families attacked thereon. The counter trade was mostly with the implicated milk, 86 persons in 58 families being attacked. Some of the unsuspected milk was transferred each day to another dairy during part of the epidemic period. No cases of scarlet fever occurred in the customers of the dairy so served. The suspected milk was stopped on 7 th August. No cases traceable directly to the agency of the milk occurred later than 2oth August. In one street 2 I families (more than half of those supplied with the milk) yielded 29 cases of disease. As some streets taking the milk suffered no attacks, the active principle of contagion was apparently not uniformly distributed.

Apparent exciting cause.-(?) Cow disease.

Reporter and reference.-Drs Russell and Chalmers. Special Rep. to Corporation of Glasgow.

\section{St Pancras, 1898.}

Number of persons attacked 28

Circumstances implicating the milk supply.-Outbreak began at end of October among customers of a certain milk seller in Highgate. He received milk from his own cows, from Finsbury'Park, and from Hendon. Of the customers supplied from his own cows, none were attacked. The other milk was distributed in three rounds. On two rounds the customers received Finsbury Park and Hendon milk in the morning and afternoon respectively. Customers on the third round received Hendon milk both morning and afternoon. The customers on the first two rounds numbered 191 , and 8 of them or 4.2 per cent. were attacked. The customers on the third round 
numbered 100 , and 20 , or 20 per cent., were attacked. Dr Sykes found that some of the Hendon milk had been distributed by two vendors, among whose customers 3 cases of scarlet fever had occurred, and on 2ist October a milkman on the Hendon farm was found to be suffering from scarlet fever. A veterinary inspector had also found 5 cows at the farm with "sore teats and inflammatory symptoms."

Apparent exciting cause.-Milk infected from human or bovine sources.

Reporter and reference.-Dr Sykes. Rep. of Medical Officer of St Pancras, 1893.

Glasgow, 1893 (December).

Total number of cases

Number of cases amongst drinkers of suspected milk.

Percentage on total cases . .

Number of families supplied by milkman .

Number of such families invaded

Percentage.

Number of polluted milk sources

(farms A and B)

Cincumstances implicating the milk supply. - Dr Chalmers traced the first attack to a milk boy at farm A, recently come there, who suffered from sore throat early in December. Other farm servants fell ill with similar symptoms, the disease proving to be scarlet fever. The fever attacked persons along the delivery track of the cart from this farm, which was in charge at different times of 4 persons, 3 of whom had scarlet fever in December. Seventeen cases of scarlet fever obtained their milk direct from this cart.

On farm B, 3 girls, occupying the same bedroom, fell ill of scarlet fever in December, one of them keeping at work for two days thereafter. From this source 4 households became invaded. The two farms were on terms of familiar intercourse.
Probable exciting cause.-Human source.

Reporter and reference. - Dr Chalmers (Med. Off. of Health). Brit. Med. Jour., r894, vol. i., p. 426.

Hastings, 1898 (November).

Total number of cases . . . 40

Deaths . . . . I

Number of cases amongst drinkers of suspected milk - 30

Percentage on total cases . $\quad$. 75

Number of families supplied by milkman .

Number of such families invaded

Percentage .

20

Cincumstances implicating the milk supply. - Cowsheds sanitarily defective -ill-paved, undrained, and unventilated. Cows in a febrile condition. Three quarters of the houses invaded dealt with one dairyman obtaining milk from this farm. The exciting cause of the pollution of the milk was not ascertained.

Many of the patients were large milk consumers. In one school of 20 boys, only 2 consumed the suspected milk, and they contracted scarlet fever. In another case one member of a family alone drank it unboiled, and was infected. In a third case a patient, a large milk drinker, who had been isolated for influenza for 12 days, drank the milk and caught the disease in this interval. Seventy per cent. of the houses supplied with the implicated milk were attacked.

Probable exciting cause.-Unascertained.

Reporter and reference.-Dr $\mathrm{S}$. Wilson (Med. Off. of Health). Annual Report, 1893.

\section{Richmond, Surrey, 1894 (Felnagy).}

Total number of cases . . 55

Number of cases amongst drinkers of suspected milk . 52

Percentage on total cases : $\quad$ - 95 
Number of families supplied by milkman .

Number of such families invaded

Number of polluted milk sourcesseveral.

Number of milkmen

Circumstances implicating the milk supply.-Prevalence of scarlet fever in the vicinity of the farms supplying the milk to the dairy. The outbreak was sudden and declined in 9 days. All the cases save three, consumed the implicated milk.

Probable exciting cause.-Human source.

Reporter and reference. - Dr Rowland. Annual Report for Richmond, I894.

Stroud Green, 1895 (February).

Total number of cases . . 233

Circumstances implicating the milk supply. - Of the first 200 cases all but 13 got their milk from the same dairy, while of 60 patients in the local infectious hospital 58 got their milk from this source. Cows healthy, but milk came from 2 villages-in I of which scarlet fever was prevalent, and in the other case scarlet fever actually existed in the farm supplying the Stroud Green milk. When milk from these sources was stopped the epidemic declined.

\section{Probable exciting cause.-Human source.}

Reporterand reference.-DrClothier. Brit. Med. Jour., I895, vol. i., p. 549.

\section{Glasgow, 1899 (August).}

Total number of cases . . Number of cases amongst drinkers of suspected milk . Number of polluted milk sources Number of milkmen, $\mathrm{r}$, who had milk from 3 other farms.

Circumstances implicating the milk supply.-At the farm in question there were 3 unmistakable cases of scarlet fever (and one death) and 2 illnesses, both accompanied by sore throat. The mother of one of the scarlet fever cases was a milker at the farm. There was also evidence to show that the domestic washing of infected clothes was carried on in immediate proximity to the scalding of the milk vessels.

Probable exciting cause.-Human source.

Reference.-Rep. of Medical Officer of Health of the City of Glasgow, 18991900.

\section{Walmer, 1899 ( January).}

Total number of cases $\quad . \quad 34$

Number of families invaded . 2 I Number of polluted milk sources I Number of milkmen . . . 2

Circumstances implicating the milk supply.-Two milk-retailers obtained their milk supply from a certain farm in the Easting Rural District. On this farm a milker was attacked at end of December 1898 with suspicious scarlet fever symptoms.

Probable exciting cause.-Human.

Reporter and reference.-Loc. Gov. Bd. Rep., 1899-1900, p. 50 (Dr Sweeting).

Edinburgh, 1899 (February).

Total number of cases . . II Number of polluted milk sources I Number of milkmen . . . I I

Circumstances implicating the milk supply. - The dairy and farm were clean and satisfactory, the cows healthy. But a girl who had milked the cows and attended in the shop during February was found to have suffered from sore throat. Sir Henry Littlejohn adds that though there was no direct and authentic evidence of the contamination of the milk, he believes that the milk did, in fact, convey the disease.

Probable exciting cause.-Human source. 
Reporter and reference.-Sir H. D. Littlejohn, M.D. (Med. Off. of Health). Anmual Rep. for City of Edinburgh, 1899, p. 34 .

\section{Edinburgh, 1899 (September).}

Total number of cases- 18 in 15 families.

Number of polluted milk sources Number of milkmen . .

Circumstances implicating the milk supply. - In this case scarlet fever occurred in the daughter and maidservant of the farmer who supplied the milk.

Probable exciting cause.-Human source.

Reporter and reference.-Sir H. D. Littlejohn, M.D. (Med. Off. of Health). Anmual Rep., 1899-1900, p. 36.

Edinburgh, 1809 (December).

Total number of cases a . 42

Circumstances implicating the milk supply. - A girl who was employed in milking at the farm supplying the milk suffered from an illness not to be differentiated from scarlet fever, and a young man engaged in the dairy suffered from sore throat. The outbreak in Edinburgh arose several days after the first symptoms in the girl.

Probable exciting cause.-Human source.

Reporter and reference.-Sir H. D. Littlejohn, M.D. (Med. Off, of Health). Annual Rep., 1899-1900, pp. 38, 39.

\section{Bristol, 1900.}

Total number of cases

Three distributors supplied 269 houses, 42 houses attacked, one house in every 6.4 .

Number of polluted milk sources

Number of milkmen

Circumstances implicating the milk supply. - On the dairy farm supplying the milk to the three distributors there was at the time of the outbreak a boy, having access to the milk vessels, who was suffering from an unrecognised illness which was compatible with a mild (ambulant) attack of scarlet fever, and within a week 2 brothers sickened with well-marked scarlet fever. The cows were healthy.

Probable exciting cause.-Human source.

Reporter and reference.-Dr Davies (Med. Off. of Health). Jour. of Hygiene, vol. i., No. 3, p. 388 .

\section{Buffalo, U.S.A., 1899.}

Total number of cases _ . 57

Number of families supplied by milkman . . . 26 Number of such families invaded $\quad 26$ Percentage. . . . . I00

Circumstances implicating the milk supply. - Two outbreaks of scarlet fever occurring in 1899 in Buffalo were traced to an infected milk supply. The first outbreak was that of 57 children, the second was less severe, and numbered only 20 cases. The channel of infection was well traced out in the former, and it was found that on the implicated dairy premises there were 2 cases of desquamating scarlet fever. One was a child aged 9 , and the other a young man aged I9 who did the milking, and in other ways assisted in the dairy during his illness. The 57 cases were almost simultaneous in occurrence, and the severity of the attack appeared to depend upon the quantity of the implicated milk consumed.

Probable source of infection.-Human. Reporter and reference.-Jour. Ann. Med. Assoc., 1900, vol. i., p. I5I (Dr E. Wende).

\section{Cheadle and Gatley, Cheshire, 1800}

(April and May).

Total number of cases . $\quad 52$

Number of polluted milk sources I

Number of milkmen . . . 2 
Circumstances implicating the milk supply.-The occupier of one of the dairy farms supplying the milk was suffering from scarlet fever. Although the cases were scattered over a wide district they had this common milk supply. Besides the cases diagnosed there were many cases of sore throat in infected families and otherwise. The medical officer thinks these were of scarlatinal origin. Throughout the epidemic adults' and children over school age were chiefly attacked, and the disease was of an extremely mild character.

Probable exciting cause.-Human source.

Reporter and reference.-Dr J. H. Godson (Med. Off. of Health). Annual Rep., 1900.

\section{Crewe, 1900 (July).}

Total number of cases . .

Number of cases amongst drinkers of suspected milk .

Number of polluted milk sources Number of milkmen . . . . .

Circumstances implicating the milk supply.-At the farm supplying the milk used by the retailer it was found that a lad employed as a milker had a sore throat a month previously and did not stay away from his work. Five members of this family had mild attacks of scarlet fever, and contaminated the milk supply which infected the cases. On the cessation of this milk supply the outbreak ceased.

Probable exciting cause--Human source.

Reporter and referente.-Dr Greenwood (Med. Off. of Health). Annual Rep., 1900.

London, 1901 (25th April-14th May).

(Bethnal Green, Shoreditch, FinsBURY, IsLINGTON, ST PANCRAS.)

Total number of cases _ . 293

Number of polluted milk sources I

Number of milkmen a large number
Circumstances implicating the milk supply.-This outbreak occurred on delivery course of a certain contractor in London. He obtained milk from 38 farms. On one of these farms there were 4 cases of scarlet fever, the farmer, his wife, son, and daughter. The cows (29) were healthy and there was no marked evidence of cow disease (Klein).

The cases were chiefly in Bethnal Green and Shoreditch, but there were two dozen in Finsbury, and several in Islington and St Pancras-all traceable to milk from the churns arriving from the implicated farm. Even in cases where milk from these churns became subdivided, or delivered a day or two later, scarlet fever occurred. When the implicated milk was stopped the epidemic ceased. The incubation period was in many cases very short. A large number of adults were attacked, which marked out the epidemic as different from ordinary outbreaks of scarlet fever in Shoreditch and Bethnal Green. After the ordinary distribution had been stopped the contractor sent the milk to other customers, who then contracted scarlet fever.

Probable exciting cause.-Human source.

Reporter and reference.-Dr Shirley F. Murphy and the Medical Officers of the districts involved (Bate, Byrett, -Newman). London County Council Special Rep., No. 548, 190 I.

\section{Beverley and Salem, 1801.}

Number of cases . . . 60 Number of deaths . . . II Number of implicated farms $\quad . \quad$ I

Circumstances implicating the milk snpply. - The dairyman in this outbreak produced 5 cans of milk himself daily, and obtained 14 other cans from three neighbours. At one of these 
3 farms 3 children had been ill ; a girl of 16 had sore throat, another girl of 12 , and a boy of 14 followed a few days later with a similar affection, namely, swelling of the glands in the neck, and general malaise. One of them had a slight rash. After the recovery from acute condition they assisted in handling of milk. The outbreak was very sudden, and suddenly declined from a time dating a few days after the milk supply from this farm was stopped. The fatality was high, as many as II deaths occurring. The distribution of the cases of scarlet fever was localised to the distribution of the milk in question.

Probable exciting cause.-Milk infected from human sources.

Reporter aud reference.-Dr Morse. State Board of Health Rep., Massachusetts, p. 56I, 1901 .

\section{Stroud, 1901.}

\section{Total number of cases}

Number of polluted milk sources

Number of milkmen

Circumstances implicating the milk supply.-From I 5 th November I900, to 24th January 190r, there were 14 cases of scarlet fever. Between 14th and 2 Ist February there were 13 , all of whom got their milk from one farmer. At this particular farm there was a boy who helped to milk the cows in the evening. $\mathrm{He}$ had a sore throat and enlarged glands in the neck. No desquamation was detected. The cows were healthy although one had recently calved; "chaps and warts" were present on many of the udders. The 13 cases occurred in 9 (or 15 per cent.) of the houses supplied by this farmer. The incubation period was very short, being apparently 24 hours or even less. Although the illness of the boy was so slight it appears that it started the outbreak.

Probable exciting cause.-Human infection of milk.

Reporter and reference.-Dr J. M. Martin (Med. Off. of Health), Stroud, R.D.C. Public Health, 1901-02, pp. 138-142.

\section{Sunderland, 1808 (Aprit).}

Total number of cases _ . II2

Number of families affected $\quad 70$

Number of families supplied by

implicated farm . . 329

Percentage of infected families . 21:2

Cincumstances implicating the milk supply. - The notification of scarlet fever in Sunderland during six weeks is given in table at foot of page.

During the week ending 28th March an unusual number of cases of scarlet fever were noticed among the families supplied with milk by $\mathrm{Mr} \mathrm{M}$. of

Inquiries were at once instituted, and it was found that there had been no cases of scarlet fever occurring in the families of the persons who had to do with the handling of the milk. There are 9 or 10 milkers, and 3 men who deliver the milk, and they and all the persons in their households, were found to be free from any ailment or symptom suggesting scarlet fever. A child was, however, discovered with its hands peeling, in the family of one of

\begin{tabular}{|c|c|c|c|c|c|}
\hline $\begin{array}{c}\text { Previous } \\
\text { weelss' av. }\end{array}$ & $\begin{array}{c}\text { Week ending } \\
\text { 2Sth March. }\end{array}$ & $\begin{array}{c}\text { Week ending } \\
\text { 4th April. }\end{array}$ & $\begin{array}{c}\text { Week ending } \\
\text { 1lth April. }\end{array}$ & $\begin{array}{c}\text { Week ending } \\
\text { 18th April. }\end{array}$ & $\begin{array}{c}\text { Week ending } \\
\text { 25th April. }\end{array}$ \\
\hline \multirow{2}{*}{20} & 33 & 85 & 50 & 30 & 29 \\
\hline
\end{tabular}


the outside farm workers, and two other children in the family had discharging ears. There is no doubt that this family had suffered from scarlet fever, and that the attack in the case of the girl who was peeling, began previous to the time when they were discovered.

Supposed that the infection of the milk arose from the child in question going into the byre, and possibly carrying milk pails while her hands were peeling. The milk would appear to have been principally infected during the latter part of the week ending 28th March, because the bulk of the cases notified were on 29th March and the three succeeding days. In most cases of Offerton customers who were attacked by scarlet fever at a date later than would have been consistent with the supposition that the disease was caused by milk consumed before 28 th March, the explanation was that the disease began in the households by scarlatinal sore throats, developing in the last two or three days in March or the first one or two days of April, and that these scarlatinal sore throats infected the other members of the family and gave rise to true attacks of scarlet fever.

Probable exciting cause.-Milk infected from human source.

Reporter and reference.-Dr Harold Scurfield. Special Rep., April 1903.

Milk-borne outbreaks of scarlet fever have also occurred at the following places in the years stated :-

Penrith, 1867; St Andrews, 1867 and 1869; South Kensington, 1875; New Barnet, 1877 ; High Ashurst and Headley, 1878; Newcastle-on-Tyne, I879; Dundee, I88o; Bromley, Kent, I88I ; Keswick, I88I ; Wolverhampton, I88I ; Greenock, I88I-82 ; Sunderland, I882 ; Kingston-upon-Hull, I882; Wolborough, 1883; Dundee, r883; Stapleton, 1887 ; Jesmond, Newcastle- on Tyne, 1888 ; Bristol, 1890 ; Lowell and Lawrence, I89I ; Cardworth, I89I ; Chicopee Falls, 1892; Aston Manor and Handsworth, 1892; Richmond, I894; Freemantle, I894; Blackheath, I894; Wolverhampton, 1894; Shirley, Southampton, 1894; Halifax, 1897; Kidderminster, 1902 ; Epsom, 1902.

\section{B. Typhoid Fever}

The first epidemic of typhoid fever which was traced to infected milk was one occurring at Penrith in 1858 (Taylor). Since that date there have been a large number of similar outbreaks traced to milk. Yet but little of material importance has been added to our knowledge of the mode of conveyance. It was believed then, as now, that the materia morbi were contained in the alvine discharges of typhoid patients, and that an outbreak of the disease implied some contact, directly or indirectly, with this poison. That, substantially, is the view still held, although we are able in the light of modern bacteriology to define with more exactness the real agents of the disease. (For description of the Bacillus typhosus of Eberth-Gaffky, see p. 434). 
Typhoid fever is an acute infectious disease characterised clinically by continuous fever, with diarrhoea and other symptoms, and anatomatically by a more or less extensive ulceration of the Peyer's patches in the intestine (ileum), with swelling of the mesenteric glands and enlargement of the spleen. The lesion of importance to us at the moment is the ulceration of the bowel, partly on account of its origin, partly on account of its result. The subject of the pathogenetic action of the bacilli is obscure, but there can be no doubt that the ulcers in the intestine are directly or indirectly the result of the specific bacillus of typhoid fever. When the bacilli reach the intestine they multiply and, penetrating the mucous and submucous coats, set up the changes, which lead, first to hyperemia, then to infiltration, and finally to ulceration of Peyer's patches. Some of the bacilli pass into the blood, collecting in the spleen and other glands. Whether in the bowels or in the organs of the body, the bacilli produce their toxins, and as a result of their action, inflammation and fever follow. The inflammation in the intestine leads, in conjunction with the irritation produced by the ulcers, to increased peristalsis, and therefore diarrhøa. Hence the excreta of a typhoid patient have two characteristics. They are usually abundant and frequent : and they are charged with large numbers of the bacilli of typhoid fever. It is, however, necessary to guard against the idea that typhoid fever is a local disease of the intestine, or even chiefly so. In ordinary cases, it is true, the intestinal lesions form the starting point of the disease, but the bacilli rapidly become generalised and are found in the most varied parts of the body, and not uncommonly in the blood itself. Such a state of things leads to a condition not remote from septicæmia, and this may occur with little or no local lesion in the intestinal tract. The reason why the bacilli of typhoid are not found in greater number in the blood, is probably in part due to the fact that in ordinary cases the blood is not a favourable medium for their growth, and in part to the fact that they are rapidly eliminated or excreted. "Any conception of the disease," writes Dr Horton-Smith, "which regards it merely as affecting the alimentary canal can no longer be maintained. On the contrary, so far from considering it an intestinal disease, pure and simple, we should rather look upon it as a modified form of septicæmia. It is septicæmia in that always, and in all cases, the bacilli pass into the blood and then into the various organs, and in that the symptoms, excepting so far as they are intestinal, are referable to the poisons there produced. It is a modified form, however, in that 
in nearly all cases there is a definite local and primary disease whence the secondary dissemination of the micro-organism takes place." 1

\section{The Vehicle of Infection}

From these facts it follows not only that typhoid fever is a general disease, but that the alvine discharges are not alone the vehicle for the elimination of the specific agent causing the disease. Indeed, there are four excretions which have been described as infectious, with more or less frequency, in typhoid fever-the sweat, the expectoration, the freses, and the urine. The first two need not be discussed in connection with the subject under consideration. The latter two demand somewhat careful attention.

The faces, or alvine discharges, have long been known to harbour large numbers of the bacilli, and for the reasons already stated. But in recent years our knowledge has become more precise and delimited, with the result that it is now held that only in certain stages of the disease are the excreta heavily charged with bacilli. They are not likely to be largely present, for example, in the very early stages of the disease.

Reed, Vaughan, and Shakespeare in their Report on the Origin and Spread of Typhoid Fever in the United States army military camps during the Spanish War of 1898 , state that the elimination of the typhoid bacillus in the stools probably begins soon after its introduction through the mouth. "Typhoid bacilli are most abundant," they add, "in the stools of patients suffering from this disease where there is sloughing of the intestinal ulceration. However, it should be borne in mind that typhoid stools are infectious often before the individual shows any evidence of the disease. In other words, the stools of a man in the incubation period of typhoid fever may be laden with the bacilli of this disease. On the other hand, the stools may continue to be infectious long after convalescence has set in." 2

Typhoid bacilli can, however, be demonstrated in the stoolsthough only with great difficulty owing to extraneous organismsat the end of the first and during the second weeks, and at the beginning of the third week. If there be a relapse, the bacilli can be found in the stools during the early part of the relapse. Karlinski names the ninth day as the time of their earliest appear-

1 Lectures on Typhoid Fever. (Goulstonian Lectures.) P. Horton-Smith, M.D., F.R.C.P. (J. \& A. Churchill, 1900.)

${ }^{2}$ American War Department: Official Report, 1900, p. 203. 
ance. Most observers state that they disappear totally at the commencement of convalescence, but certain authorities state that they have found the bacilli in the stools nine days and fifteen days after the temperature had become normal (Stepolansky and Stroganoff). The reason for this divergence of view is the great difficulty of detection of the bacilli in the fæces by our present methods. Hence it is not possible to lay down any hard and fast limitations. It may, however, be said that the fæces are highly infectious soon after the commencement of the disease, and that later they become less dangerous. During the infectious period it is certain that the stools are enormously charged with typhoid bacilli.

While referring to this matter of elimination of the bacillus in the intestinal excreta it should be remarked, that there is considerable evidence to show that an individual may become the bearer and distributor of the infecting agent of typhoid fever, without developing the disease himself. It would appear that just as typhoid fever may develop and death result without intestinal ulceration, so also the bacillus may gain access to, and develop in, the intestine, and yet be voided without having produced the disease. It has been suggested that such an occurrence may be due to the presence in the intestine of irritative saprophytic germs, causing a profuse diarrhoea capable of sweeping out of the alimentary canal any typhoid bacilli. Much the same series of events would occur in cases, if such are possible, of immunity of certain individuals. It is within the range of possibility that the specific germ of typhoid may be transported from place to place, and from person to person, and eventually to a milk supply by an immune man. This, or something akin to it, may be the explanation of certain epidemics which have appeared to originate de novo.

The urine is the other chief excretion by which the bacilli of typhoid fever are voided from the body. Horton-Smith has demonstrated that the urine of typhoid patients contains the bacilli of the disease in the proportion of $\mathrm{I}$ in every 4 cases. He has also shown that as a rule it is towards the end of the disease, or during convalescence, that this condition occurs. Further, whilst it is always difficult to find the bacilli in the stools, in the urine it is always easy, for when they are present they are nearly always in pure culture, and not uncommonly they are present in such extraordinary numbers that one cubic centimetre may contain many thousands of micro-organisms (Horton-Smith). 
In London, Horton-Smith found typhoid bacilli present in the urine in 25 per cent. of all cases examined. Working in Boston, Richardson obtained a positive result in 22.5 per cent. of the cases examined. Both investigators found the bacilli present, in certain cases, in such large numbers that the urine was rendered turbid by their presence. Nor are such cases rare. Out of the cases in which the specific bacillus was present in the urine, in as many as 12 it was present to the degree of turbidity, and in only 2 was the urine described as "clear" (Horton-Smith). Referring to the stage of the disease in which the bacilli appear in the urine, they have been found as early as the thirteenth day from the commencement, and as late as the fourteenth day of convalescence (Horton-Smith). Karlinski is reported to have found them as early as the third day of the fever. Speaking generally, the condition is rare before the third week of the disease. The duration of this specific bacilluria also varies. The shortest duration recorded by Horton-Smith was eight days, but in four other cases it had not disappeared until after the lapse of twenty-one days, twenty-five days, thirty days, and seventy days. But even that is not the limit, for the presence of the bacilli in the urine may persist, not for months, but even for so long as five years (Gwynn). Various explanations have been suggested for this phenomenon of typhoid bacilli in the urine, but that which seems most adequate to the facts is that which assumes that one or more bacilli, but probably extremely few, find their way into the bladder and there commence rapid growth in the urine within the bladder, which medium is by no means unfavourable to the multiplication of the bacillus (Horton-Smith). This question of typhoid bacilluria is in our judgment one of such importance, when it is considered that the urine of typhoid patients may so remain infective for weeks or even months, that we make no apology for inserting here the record of a small epidemic of typhoid fever apparently traced to such infection. In the village of Long Orton in Huntingdonshire, there were, in 1900, two houses obtaining their water supply from the same well. The water was highly contaminated with sewage. No one, however, suffered in consequence until after the arrival of a trooper invalided home from South Africa, convalescent from typhoid fever. $\mathrm{He}$ had been in hospital from 23rd May to Ist August and arrived home at Long Orton on 19th August. Immediately after his arrival the well became infected with the typhoid fever bacillus, as shown by the fact that between $4^{\text {th }}$ September and 17 th September, twelve in- 
dividuals were laid up with typhoid fever, the only feature common to them all being that each one of them had partaken of the water from the polluted well. Other wells from the same village were analysed and found to contain sewage, but no case of typhoid fever resulted from the use of the water of these wells. No other possible source was found for the pollution of the infected well except the returned trooper. The sanitary conditions were such as to render the well liable to pollution from the soil pipe. ${ }^{1}$

We have entered into matters which may at first sight appear to belong rather especially to clinical medicine for the reason that they bear, as we shall see, a very important relation to the epidemiology of the disease. Moreover, there are several other characteristics of the bacillus which may be mentioned as having some importance in the spread of the disease. Two points respecting the toxins of the typhoid bacillus must be named, one, that the toxins are for the most part intracellular, contained within the bacillus itself, and are chiefly set free when the latter is destroyed; and the other, that the toxin is a comparatively feeble one as compared with that of other pathogenic organisms, and that to account for the clinical conditions of the disease the number of bacilli present in the infected body would have to be exceptionally large. This fact, coupled with the varying virulence of the bacillus, is all the more remarkable when it is remembered that not a few of the epidemics of the disease have arisen from a dose of poison, so excessively minute in itself, and so enormously diluted, as to appear out of all proportion to the number of persons attacked. It is possible that a few organisms introduced into the human body are able, under certain conditions, to multiply rapidly, and so bring about the same results as large dosage. Again and again it has been shown that considerable epidemics have arisen from a pollution of water so slight as to escape detection by any methods of chemical or bacteriological analysis at present known. ${ }^{2}$ Precisely the same condition of things applies to pollution of milk, though possibly in an even more marked degree. It is not unlikely that when specific typhoid matter is introduced into water or milk, under ordinary circumstances, that a rapid multiplication at once takes place, whereby large volumes of such water and milk become

1 Brit. Med. Jour., Igoo, vol. ii., p. 1494. (Dr Walker, Senior Surgeon, Peterborough Infirmary.) For a general review of the question of the typhoid bacillus in faces and urine as it affects hygiene see the New York Medical Neres I Ith May Igor (Hiss).

2 See also Report of the Medical Officer to the Local Government Board, 1881. 
poisoned. In all probability such rapid multiplication occurs in the human body immediately such water or milk is consumed.

Another point which must not be wholly passed over, has reference to the saprophytic faculties of the B. typhosus. Some authorities consider that this bacillus can remain alive and virulent for months outside the human body. In sterilised stools it has been found, for example, that the bacillus can retain vitality for months. On threads soaked in cultures, they have remained alive for a year, on potato for two years, in sterilised garden soil $2 \mathrm{I}$ days, on wood 30 days, on sterilised linen 60 days, and on woollen cloth for 80 days. ${ }^{1}$ In sterilised distilled water, they have been found alive 196 days after introduction. When dried in various ways they have lived several months Such facts as these have been taken as indication of some degree of saprophytic existence being attributable to the $B$. typhosus. Moreover, this theory has gained strength from experiments recently conducted for the Local Government Board by Dr Sidney Martin, upon the vitality of this bacillus in soils. Martin found that samples of soil polluted with organic matter formed a favourable environment for living bacilli of typhoid fever for so long as 404 days, whereas in sterilised soil, without organic matter, these organisms lived only 23 days. When, however, the bacillus is placed in unsterilised natural soil containing ordinary soil bacteria, the $B$. typhosus ceased to exist within 24 hours. $^{2}$ Robertson, Klein, and others, have also obtained results with the bacillus in soil. But these various records must be accepted with some reserve, not alone on account of the fact that Eberth's bacillus has not been fully proved to be the specific bacillus of typhoid fever, but because we have as yet only an elementary knowledge of bacteria in the soil, and the entire question of the saprophytism of pathogenic bacteria is an abstruse and obscure problem. It seems difficult to believe that a non-sporulating bacillus of comparatively mild resisting powers can remain dormant and yet alive and virulent for long periods outside the body. But increasing evidence is forthcoming to show that it may be so.

The most recent experiments in regard to this matter were made by Firth and Horrocks, and in some ways they appear to be the most reliable. They have shown that the typhoid bacillus can survive in ordinary earth for more than two months, whether such soil be virgin or polluted with sewage, or indeed frozen hard. It

1 See also Report to Clinical Society of London, vol. xxv., 1892.

2 Twenty-Eighth Annual Report of the Local Government Board, 1898-99 (Medical Officer's Supplement). 
would appear that excess or deficiency of moisture in soils is the dominant factor affecting the chances of survival of the typhoid bacillus in, or at least the possibility of recovering it from, soil. Presence or absence of organic matter, upon which previous workers have laid great stress, is apparently of less importance to the issue. From these facts Firth and Horrocks conclude that the typhoid bacillus is able to assume a vegetative or saprophytic existence for considerable periods outside the body. ${ }^{1}$ lt is evident soil may receive very massive pollution from typhoid dejecta, and thus set up for a long period of time a sort of potential infection, which by rain or surface washings may readily gain access to a water supply, and thus to milk or its utensils.

Summary.-We may now briefly epitomise the meaning of the above facts and their relation to typhoid fever epidemics arising from milk pollution. We have seen that the disease is commonly spread by polluted excreta, in its early stages by alvine discharges, in its later stages and during convalescence, in a certain number of cases, by the urine; that the bacillus may remain in the body for long periods after convalescence, and hence apparently healthy persons may carry about and possibly disseminate the seeds of the disease for months or even years; that the bacillus may be voided in countless numbers during such periods; that under certain circumstances such discharges, or the dried dust resulting ultimately therefrom, may contaminate water or milk; that when this accident occurs, a comparatively small pollution may lead to a wide-spread epidemic among persons consuming such water or milk; and finally, that there is some evidence to suppose that the virus of the disease may remain dormant but alive for long periods of time in a saprophytic stage of existence. But little reflection will be necessary to convince any careful observer that an intelligent appreciation of these facts is of essential importance in considering the relationship of polluted milk to typhoid fever, or as, in part, explanatory of spasmodic or apparently spontaneous or repeated outbreaks.

\section{The Bacillus of Typhoid Fever}

Turning now to consider some of the more important points in relation to milk-borne epidemics of typhoid fever, we are met at the outset by the as yet unproved specificity of Eberth's B. typhosus. This organism has not fulfilled Koch's postulates, is not always

${ }^{1}$ Brit. Med. Jour., 1902, vol. ii., p. 941. 
detectable in cases of clinical typhoid fever, and does not produce the specific disease in animals.

The evidence that Eberth's bacillus is the cause of typhoid fever consists in the main of three parts. In the first place, the bacillus is found, with almost invariable regularity, in the spleen of persons dying of typhoid fever, when an adequate bacteriological examination is made. Secondly, Eberth's bacillus elaborates specific toxins. Thirdly, the blood serum of individuals suffering from typhoid fever has a specific agglutinative action upon the Eberth bacillus, similar to that observed in the blood serum of animals, rendered immune to this germ (compare also Pfeiffer's reaction). ${ }^{1}$ And whilst there is no evidence to suppose that animals, including the cow, suffer from typhoid fever, as the disease occurs in man, there is evidence to show that under certain conditions, a diseáse, not unlike enteric fever, can be produced by inoculation of the B.typhosus into guineapigs, mice, rabbits, etc. (Fränkel and Simmonds). Klein has also recently demonstrated by inoculation, that the bacillus is able to multiply and develop in the lymph glands of the calf.

For all practical purposes, therefore, the B.typhosus of Eberth is now generally accepted as the causal agent in typhoid fever. It lives in milk and in sewage, but in both only for a comparatively brief period, unless the medium is freshly diluted. The reason for this is, of course, the enormous competition created by the vast numbers of saprophytic organisms present both in milk and in sewage. In the struggle which ensues the $B$. typhosus succumbs. It is not unlikely that the bacillus is widely distributed in nature, and yet it is only under certain conditions and circumstances that it gains access to the human body in such numbers, or in such a degree of virulence, as to create disease. It certainly has powers of rapid multiplication, and in a few hours, some scores of bacilli introduced into a water supply will have reached many millions. Great dilution to which it may be subjected has no ill effect upon its potency. Lastly, certain organisms often associated with it are antagonistic to its growth, whilst others are favourable and appear to increase its virulence.

Therefore accepting Eberth's bacillus provisionally as the etiological agent in typhoid, we find ourselves at once in possession of an agent of extreme variability, both as regards its presence in milk and its virulence in the human body. It may be this factor of varia-

1 Compare also sensibility to agglutination of certain varieties of $B$. coli isolated from typhoid stools and note the value such a phenomenon may be in the detection of typhoid dejecta in water or milk. See Jour. of Hyg., 1902, p. 213 (W. H. Horrocks). 
bility which in part explains the large number of very mild, and often unrecognised, cases of the disease. Unquestionably such cases, as in most other infectious diseases, are potent of mischief in the spread of the complaint. Variability in bacillus (e.g. paratyphoid bacilli) may be the explanation also of conditions simulating typhoid fever, a species of anomalous typhoid, which appears occasionally.

How milk becomes typhoid infected.-On examination of those milk-borne epidemics of typhoid fever which may be looked upon as fully proved to be thus conveyed, we shall find the modes of infection of the milk to be various. Amongst the most common is watered milk. It is now an axiom that typhoid pollution is most frequently conveyed by water. Water is readily contaminated with typhoid matter. The ways in which infected water finds its way into milk are numerous. It may be added to milk, deliberately, in adulteration; or it may gain access accidentally, as in a case which actually occurred, where some typhoid infected water had been left in the bottom of the churn, and the milk was added without previously emptying the churn. Milk-churns, milkmeasures, and other dairying utensils have on more than one occasion been "cleaned" with water to which typhoid excreta had obtained access. Again and again, repeated ad nauseam, the primary infection has arisen through such unclean dairying. In the remarkable epidemic at Springfield, Massachusetts, it is reported that the contents of a privy in which the stools of a patient were deposited were spread over a field in which certain farm labourers were working. Their boots became clogged with this manure, which was thus conveyed to some boards covering the surface of a well, and so into the well-water beneath. The milk from the farm was cooled by submerging it in this well. The cans used in this submergence were stoppered by wooden plugs. Around these wooden plugs, it was afterwards found, in four cans out of nine there was leakage, and in this way the water from the well entered the cans.

Another frequent mode of infection is that the patient or the nurse of the patient has continued to assist in the dairy work whilst able to convey infection; or infected clothing is washed at the implicated farm; ${ }^{1}$ or the udders of the cows are washed

1 The interesting researches of Firth and Horrocks into the dissemination of enteric infection by means of fabrics show that the typhoid bacillus may be recovered from khaki drill fabrics 74 days after contamination, from khaki serge after 87 days, and from ordinary blue serge after 78 days. The whole of this research is full of suggestion and throws an important light upon typhoid infection generally. See Brit. Med. Jour., 1902, vol. ii., pp. 936-941. 
with infected water; or contaminated dust obtains access to the milk.

Numerous observers have declared their belief that flies may disseminate the germs of typhoid fever. Some of the most recent evidence on this point comes from the American Encampments in the Spanish War of 5898 (Reed, Vaughan, and Shakespeare ${ }^{1}$ ), and the British Army in South Africa, I900-I90I (Tooth ${ }^{2}$ ), and there can be no doubt that flies may carry infected matter from person to person, and from infected persons to food and milk. It is possible for the fly to carry the typhoid bacillus in two ways. First and more commonly fæcal matter containing the typhoid germ may adhere to the fly's head, mandibles, legs, wings, or body, and be mechanically transported. Secondly, it is possible that the typhoid bacillus may be carried in the digestive organs of the fly and be deposited with its excrement, but this has not been proved. However actually conveyed, there can be little doubt of the readiness with which the fly may carry germs from infective excreta to milk. Ficker has recently made some interesting experiments entirely confirmatory of these facts. ${ }^{3}$

In the same way the typhoid bacillus may be air-borne from dried excreta to milk. Partially dried typhoid stools on the floor may be sufficiently comminuted to form an infective dust, which may readily float through the air and be deposited in milk. In a general way it may be said that epidemics set up by flies, and by dissemination of the typhoid germ in the form of dust, are more likely to occur during late summer and autumn.

Finally, it should be remembered that the typhoid bacillus will retain its vitality in milk for thirty days or longer, or until it is destroyed by the growth of other organisms. The presence of lactic fermentation or of the Oidium lactis does not interfere with the growth of the typhoid bacillus. It flourishes in milk at $20^{\circ} \mathrm{C}$. or at $37^{\circ} \mathrm{C}$. and does not alter the appearance of the milk. ${ }^{4}$ A given sample of milk may become infected after it has been boiled or sterilised, and still afford a suitable nidus for the rapid and abundant growth of the bacillus. Care must therefore be taken to protect

1 American War Department: Official Report, 1900; see also "A contribution to the Study of the Insect Fauna of Human Excrement," by L. O. Howard. -Proc. Washington Academy of Science, vol. ii., pp. 541-604.

${ }^{2}$ Brit. Med. Jour., I901, vol. i., pp. 642, 770 et seq.; and Ibid., 1902, vol. ii., pp. 936-94I (Firth and Horrocks).

${ }^{3}$ Archiv. f. Hyg., t. xlvi., 1903, pp. 274-283.

4 Local Government Board Report, 1896-97, pp. 243-254 (E. Cautley); Ibid., 1899-1900 (Klein). 
milk from flies, dust, or other contamination after it has been sterilised.

The specific germ of typhoid fever will not only grow in milk, but in milk products. It will live in butter for many days, and in cheese for a shorter period. ${ }^{1}$

It has been suggested that the milk of cows drinking infected water may contain the B. typhosus. This is not possible, although the germ might well be eliminated by the alimentary canal, and then indirectly obtain access to the milk.

These diverse modes of primary pollution are well illustrated in the abstracts which follow. Before proceeding to the abstracts it is well to remember that milk-borne epidemics of typhoid are, of course, not so common nor so clearly proved in certain ways as water-borne epidemics. Few people drink milk as compared with the many who have opportunity of coming within the infective area when the water supply is polluted. Dr Cooper-Pattin of Norwich has pointed out that the number of persons drinking raw milk varies from about $28-30$ per cent. ${ }^{2}$ The following table is derived from 656 cases of typhoid fever notified at Norwich during the years 1895,1896 , and 1897 :-

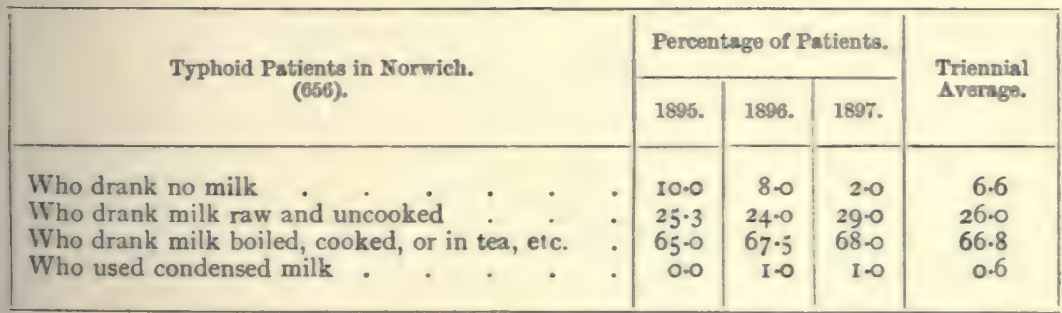

Schuder has recently published a statement based on a study of 638 epidemics of typhoid fever occurring in different countries, from which he finds that 70.8 per cent. of such epidemics are spread by drinking infected water, 17.0 per cent. of them by drinking infected milk, and 3.5 per cent. by other forms of food. The remaining 9 per cent. are caused by clothes, etc., worn by typhoid patients, latrines, dust, etc. Of epidemics spread by infected milk, he finds that in 29 per cent. the utensils used in dairying had been washed with infected water. Schuder holds that dejecta and urine are the media in which the typhoid germ remains for long periods virulent. ${ }^{3}$

1 See also La fievre typhoide, by MM. Brouardel and Thoinot. Paris, 1895.

2 "Enteric Fever at Norwich. A triennium of Typhoid."-Trans. Epidemio. logical Society of London, vol. xvii. (1898), p. I1 2.

${ }^{3}$ Zeit.f. Hyg. u. Infect., Igo2, vol. xxxviii., p. 343. 
In the milk-borne epidemics of typhoid which we have studied, numbering not far short of 200 , and of which about 160 are in our opinion sufficiently proved cases, we have arrived at certain figures respecting this matter of exact channel of infection. Unfortunately in many cases sufficient investigation has not been made for any reliance to be placed upon it. But in between 70 and 80 typhoid epidemics, it has been possible to obtain the likely channel of infection. The results are as follow.

Milk-borne typhoid epidemics probably started :-

(a) By cases of typhoid at the farm or milk-shop . $=70$ per cent.

(b) By cases of typhoid at the farm - . - $=40$ "

(c) By cases of typhoid at the milk-shop . $\quad=30 \quad "$

(d) By using polluted water for dairy purposes, method of pollution unknown . . .

(e) By insanitation at the farm or milk-shop and miscellaneous

$$
=20 \quad,
$$$$
=10 \quad,
$$

It should be understood that many of the cases included under divisions $(a)(b)$ and $(c)$ were really infections conveyed by water, or in "cleansing" the milk utensils. In one of the first outbreaks recorded in this country (namely at Islington in 1870), Dr Ballard showed that the water used for washing the milk-cans was the infected medium. In this epidemic, of $\mathrm{I}_{4} 2$ families obtaining their milk supply from a certain milk dealer, 70 (or 50 per cent.) contracted typhoid fever, of whom about 54 families took the same supply regularly, whilst the others took it at the time of infection. The whole of the infection, though attributable to previous cases of typhoid, came, as far as could be proved, by using infected water for cleansing the milk-cans.

\section{Abstracts of Typical Milk-borne Outbreaks of TYPHOID FEVER. ${ }^{1}$}

Armley, 1872 ( July).

Total number of cases . . I07

Deaths . . . . . II

Number of families invaded supplied by milkmen $37 \cdot 5$ per cent.

Number of polluted milk-sources I

Number of milkmen . . . I

Circumstances implicating the milk supply.-Adults affected 59; persons aged 5-14 years, 36 ; Under 5 years, 1o. Thirty-seven per cent. of the families supplied by farmers having implicated milk were attacked : 5 per cent. of the families supplied by 18 other milkmen or not taking milk at all. The fever picked out the customers of a certain dairyman in a remarkable manner, and the largest consumers of the suspected milk were amongst the earliest, and the smallest consumers among the latest, attacked. 
Milk became infected probably through water-pollution owing to dairyman and subsequently two of his children suffering from typhoid fever.

Probable exciting cause,-Human source through polluted water contaminating the milk.

Reporter and reference-Dr Ballard. Loc. Gov. Bd. Rep., 1874, p. 79.

\section{Moseley and Balsall Heath, 1873.}

Total number of cases-96 in 50 families.

Deaths

Number of families invaded supplied by milkman . .

Number of polluted milk sources Number of milkmen . . . 4

Cincumstances implicating the milk supply.-Boy at milkman's house ill of typhoid fever, suffered there for a fortnight and died. Two wells polluted from a privy into which typhoid excreta had been thrown. The water of the well was added accidentally or intentionally to the milk. Dr Ballard summed up his view of the causation in this outbreak as follows:-(r) Two wells upon adjoining premises occupied by milk sellers became infected early in November with the infectious matter or virus of enteric fever, through the soakage from a privy into them of excremental matters containing that matter of infection. (2) Through the medium of water drawn from these wells the milk supplied by these milk sellers became infected, and many of their regular customers who drank the milk suffered from the disease. (3) The same infected milk having been sold to two other milk purveyors, some of the persons using the milk supplied by these milkmen also suffered in a similar manner. (4) There is no evidence that the disease spread in these districts in any other way than through the consumption of the infected milks.

Probable exciting cause--Human source.

Reporter and reference.-Dr Ballard. Loc. Gov. Bd. Rep., 1874, p. 92.

Marylebone, 1878 (Twly and A wgust).

Total number of cases-244 in 143 families.

Number of cases amongst drinkers of suspected milk . 218 Percentage on total cases . $\quad 90$ Number of polluted milk sources I Number of milkmen . . . I

Circumstances implicating the milk supply.-It was found that, with one exception, wherever the milk of this supply was distributed there enteric fever was distributed. The epidemic was almost exclusively amongst wealthy people or their servants. The disease not only picked out certain houses, but also picked out the milk drinkers in those houses, and more particularly those who drank cold milk in large quantities.

The milk of six of the farms supplying the dairy was free from suspicion, but the seventh was not so. This farm was situated in Buckinghamshire. On 8th June the farmer died from typhoid fever. (On r2th August a son of the farmer also had typhoid.) The undisinfected excreta were deposited in an ash-heap, from which they found their way by soakage into the water used for dairy purposes.

Whilst these are the bare facts, it is important to note that they were by no means obvious at the time of the inquiry. On 8 th June the farmer died suddenly in the fourth week of an attack of ambulant typhoid fever. Owing to the suddenness of fatality and to the presence of some heart disease the typhoid was overlooked, and the death certified as due to heart 
disease. During the illness the medical attendant had ordered that all discharges from the bowel and bladder should be kept out of the common privy of the farm-house and buried somewhere outside the premises. This was done, and the discharges, without disinfection, were buried in the ash-heap near the pigsty. On 12th August the son had typhoid. At the inquiry special attention was paid to discover whether it was possible for soakage from the privy and drains of the farm-house to obtain access to the dairy well, and it was proved by excavations that no such soakage could occur. Yet soakage was found to exist in the well wall, and accordingly other sites of soakage were investigated, and it was eventually proved that soakage could only come from the pigsty, along the foundation of the yard wall, a distance of 25 feet. But so effectually had this taken place that the clay through which the soakage had occurred was of the consistence of soft paste. Against the yard wall near the pigsty had been deposited the undisinfected bowel evacuations and chamber slops from Ist June to 8th June. Hence the two primary difficulties, viz.: that the patient did not suffer from typhoid fever, and the drainage of the farm had no connection with the well, were surmounted, and it was proved that the very precautions taken had set up the pollution of the well. The outbreak was proved most clearly to be due to (I) infected milk (2) from this farm, and (3) by means of a pollution through the water supply.

Probable exciting cause.-Human source.

Reporter and reference.-Messrs Radcliffe and Power. Loc. Gov. Bd. Rep., 1874, pp. 103-131. (The student of milk epidemiology will find this report one of the most illuminating on record.)
Great Coggeshall, 1876 (November).

Total number of cases . . . $\quad 28$

Number of cases amongst

drinkers of suspected milk - 28

Percentage on total cases . . I00

Number of polluted milk sources I

Number of milkmen . . . I

Circumstances affecting the milk supply.-A young woman came home from London with what turned out to be typhoid fever. The slop water (including the washings of the patient's bed-linen which was saturated with bowel discharges) were poured, without disinfection, into a drain emptying into the brook from which water was regularly taken for dairying purposes, a few yards from where the drain emptied into it.

Probable exciting cause.-Human source-polluted water used for dairying purposes.

Reporter.-Sir R. Thorne Thorne, F.R.S. (Loc. Gov. Bd.).

Bolton, 1876.

Total number of cases . . I44

Deaths . . . 8

Number of families supplied by

milkman . . . 50

Number of such families invaded 47

Percentage . . . . . 94

Circumstances implicating the milk supply.-The disease followed unceasingly the track of a certain milk supply which had produced disease at Eagley. Not one household to which the milk was traced remained free from disease. The outbreak was due to the drinking of milk to which water which was contaminated with fæcal matter had been added. The question was also raised as to the possibility of the milk having obtained its infection from some disease of the cow.

Probable exciting cause.-Polluted water used for dairying purposes.

Reporter and reference.-W. $\mathrm{H}$. Power, F.R.S. and E. Serjeant. Brit. 
Med. Jour., $1876 ;$ Med. Times and Gazette, 1876.

Bagley, 1876 (Jamuary and February).

Total number of cases _ . 195

Deaths . . . . . 13

Number of families supplied by

milkman .

Number of such families invaded $\quad 55$

Percentage .

Circumstances implicating the milk supply. - No community of drainage or water supply, but a very large proportion of the families invaded were customers of a particular dairy. Of 59 families thus supplied 96 per cent. were attacked with typhoid ; of 26r not supplied, 5 per cent. were attacked. It appears not unlikely that the channel of pollution of the milk was water from a brook which had been befouled with human excrement.

Probable exciting cause.-Polluted water used for dairying purposes.

Reporter and reference.-W. $\mathrm{H}$. Power, F.R.S. Brit. Med.Jour., 1876, vol. i., pp. 209, 233, 273, etc.

\section{Barrowford, Lancashire, 1876-7} (December to Jamary).

Total number of cases

Deaths

Number of cases amongst

drinkers of suspected milk .

Percentage on total cases :

Number of families supplied by milkman .

Number of such families invaded Percentage .

Number of polluted milk sources

Number of milkmen . . .

Circumstances implicating the milk supply.-This was a very clear case. The farmer had had typhoid fever in his house for two or three weeks before the outbreak, and no precautions had been taken to prevent the spread of the disease. The milk was left for some time in the farm-house before being sold. The milk-tins were wiped with the same "dish cloth" as that used among the fever patients. The farmer himself nursed his children, and then went immediately without disinfection amongst his cattle and milked them in the same clothes he had worn whilst nursing his children. The cases occurred within a very short space of time, and every one of them without exception drank the milk from this farm. Twenty-five of the patients were under ro years of age. There was no other typhoid in the district.

Probable exciting cause-Human source.

Reporter and reference.-Dr T. Dean (Med. Off. of Health). Med. Times and Gazette, 1877, vol. i., p. 72.

Salford, 1876 (December).

Total number of cases . . $I_{3}$

Number of cases amongst

drinkers of suspected milk . 13

Percentage on total cases . . 100

Number of polluted milk sources I

Number of milkmen . . . I

Circumstances implicating the milk supply. - Typhoid fever had been frequent at a certain farm ( 16 cases in 20 years). There were various sanitary defects at the farm. The milk supply went to houses of well-to-do people, and there was no intercommunication between the families. Those members of the various families who largely partook of the milk in its unmixed form were alone affected.

Probable exciting cause,-Human source.

Reporter and reference.-Dr G. Tatham (Med. Off. of Health). Annual Rep, 1875-76.

\section{Greenock, 1876 (February).}

Total number of cases . 20

Number of polluted milk sources I Number of milkmen . . . I

Circumstances implicating the milk supply.-A farmer's family and servants 
became infected with typhoid fever by contact with a case of that disease. Upwards of 20 cases occurred among the persons using the milk from this farm.

Probable exciting cause-Human source.

Reporter and reference.-Dr $\mathrm{J}$. Wallace (Med. Off. of Health). Sanitary Record, vol. iv., p. 234.

St Pancras, London, 1877 (February).

Total number of cases . . 35

Deaths . . . . . 2

Number of cases amongst drinkers of suspected milk . . . 30

Percentage on total cases . . 85

Number of families supplied by milkman .

Number of such families invaded 400

Percentage .

Number of polluted milk sources Number of milkmen .

Circumstances implicating the milk supply. - Of the 35 cases of typhoid fever, 30 had used the suspected milk. During five days no less than 12 fresh cases appeared in eleven separate houses and all the persons attacked had used the suspected milk. Moreover, the epidemic was conterminous with the walk of a particular milk. vendor from the suspected milk-shop. There were adequate circumstances explaining the origin of the disease in the 5 cases which did not consume the suspected milk. The supply was drawn from three farms, in two of which the water supply was contaminated. The large number of families supplied and the comparatively few infected may have been due to the three sources from which the dealer obtained his milk supply.

Probable exciting cause.-Dr Stevenson adds, "How the milk became the vehicle of typhoid is at present and will remain a mystery."

Reporter and reference. - Dr
Stevenson (Med. Off. of Health). Med. Times and Gazette, 1877, vol. G., p. 653 .

The Gurnes, Ystalyfera, 1877.

Total number of cases ․ 7

Number of casesamongst drinkers of suspected milk : . . 7

Percentage on total cases . . 100 Number of polluted milk sources I Number of milkmen . . . I

Circumstances implicating the milk supply. - The milkman lived in a small overcrowded house and stored his milk, previously to sale, in a badly ventilated pantry leading out of the living-room of the family. At the time of the outbreak the milkman's son was lying in the house ill of typhoid fever. All the cases were attacked simultaneously and all were in the habit of using milk procured from this dealer.

Probable exciting cause. - Human source.

Reporter. - H. F. Parsons, M.D. (Loc. Gov. Bd.).

\section{Glasgow and Hillhead, 1877-8} (December and January).

Total number of cases _. . 163 Deaths . . . . . 15 Number of polluted milk sources I Number of milkmen . . . 1

Circumstances implicating the milk supply.-At one of the farms supplying the milk the sanitary arrangements were defective and the well water was contaminated. On Ist December a son of the farmer sickened with typhoid fever, a servant girl on 2oth December, and another boy on 27th December. The work of the dairy was carried on by persons who attended the patients, and the excreta was thrown into the byre drain. The epidemic was sudden in onset, and attacked only the drinkers of the milk from two dairies supplied by the infected farm. The disease picked out the milk-drinkers in a very marked manner. 
Probable exciting cause. - Human source.

Reporter and reference.-Dr T. B. Russell (Med. Off. of Health). Brit. Med. Jour., 1878, vol. i., p. Ior. "Special Report" by Dr Russell.

\section{Moss Side, Manchester, 1878}

$$
\text { (Uanuary-March). }
$$

Total number of cases

Deaths

Number of the cases amongst drinkers of suspected milk . Percentage on total cases . Number of polluted milk sources Number of milkmen .

Circumstances implicating the milk supply. - The dairyman was supplied with two supplies, one being from Cheshire. It was only the customers consuming this Cheshire milk, with one exception, that contracted typhoid. Farm water contaminated with sewage. Two deaths from typhoid feveroccurred at the farm. The outbreak was checked as soon as the milk from the infected farm was stopped.

Reporter and reference.-Dr A. E. Sutcliffe (Med. Off. of Health). Med. Times and Gazette, 1878, vol. i., p. 517.

Bristol, 1878 (July and August).

Total number of cases . . ${ }^{131}$

Deaths . . . . 12

Number of cases amongst drinkers of suspected milk . . . I3I

Percentage on total cases . - I00

Number of polluted milk sources I

Number of milkmen . . several

Circumstances implicating the milk supply. - In June a lady convalescing after typhoid fever resided at the farm. The excretions passed into the common privy, and from thence into a common drain to the cess-pit. This latter overflowed and the contents were traced by a recurrent course to the well, 25 feet distant. The milk was kept in the common living-rooms, and the well water was used for all dairying pur- poses. It was the only water supply at the farm. On Ist August one of the farm servants sickened with typhoid fever.

District of Bristol in which outbreak occurred was sanitarily good, and the outbreak wasstrictly confined to persons taking milk from the retailers supplied from this farm. In one family 4 drank milk, only one of whom drank it unboiled; he contracted typhoid and the others did not.

Probable exciting cause. - Human source.

Reporter and reference.-Dr Davis (Med Off, of Health). Sanitary Record, 1878, vol ii., pp. 100 and 166. Annual Rep. of Medical Officer of Health, 1878.

Portsmouth, 1878 (Seplember).

Total number of cases : . 153

Number of cases amongst

drinkers of suspected milk $\quad 78$

Cincumstances implicating the milk supply.-Farmer's children suffering from typhoid fever and there is little doubt they poisoned the well. Two children passing the well, drank from it ; and contracted typhoid. Sixty per cent. of all the cases of typhoid in Portsmouth in the third quarter were supplied from this farm. A large number of cases fell ill on the same day. The farmer also supplied 20 neighbours and eight contracted typhoid. He also supplied milkmen who retailed to Eastney Barracks, which was also invaded by the disease.

Probable exciting cause.-Human source.

Reporter and neference.-Dr Turner (Med. Off. of Health). Brit. Med. Jour., 1879, vol. ii., p. 625.

Colston, near Glasgow, 1878 (September).

Total number of cases . - 40

Number of cases amongst drinkers of suspected milk . 40

Percentage on total cases . . 100

Number of families invaded $\quad 12$ 
Circumstances implicating the milk - supply.-Sanitary conditions good. A bundle of body clothing used by a person who had died from enteric fever was brought to dairy-farm to wash. A fortnight later typhoid fever broke out in a very small community using milk from this farm. In one family eight were attacked almost simultaneously, in another seven, and another six. Persons escaping the disease in nearly every instance had not consumed this milk.

\section{Probable exciting cause,-Human source.}

Reporter and reference.-Dr J. Christie. Sanitary Record, vol. iv., p. 342 .

\section{Dublin, 1878 (December).}

Total number of cases . .

Number of cases amongst drinkers of suspected milk .

Percentage on total cases . .

Number of families supplied by milkman . . .

Number of such families invaded . . . .

Percentage . . .

Number of polluted milk sources Number of milkmen . . .

Circumstances implicating the milk supply. - Dairyman and child had an illness which was undoubtedly typhoid fever. There were also sanitary defects, premises small and illarranged, the family alone managing the business. Houses attacked were of good class. Every infected house consumed milk from infected dairy and the disease picked out the milk drinkers of each family with remarkable accuracy.

Probable exciting cause.-Human source.

Reporter and reference.-Sir C. A. Cameron, M.D. (Med. Off. of Health). Dublin Jour. of Med. Science, July I879, p. I.
Ch1chester, 1879 (February).

Total number of cases . . 50

Deaths . . . . . 6

Number of families supplied by milkman . . . . 59

Number of such families invaded 26

Percentage . . . . 43

Circumstances implicating the milk supply.-Dr Airy believed infection was derived by washing the udders of the milch cows with water from a brook polluted with typhoid excreta.

Probable exciting cause.-Human source.

Reporter and reference.-Dr Airy (Loc. Gov. Bd.) ; also Brit. Med. Jour., I879, vol. ii., p. 475 .

\section{Millbrook, Cornwall, 1880}

(July-September).

Total number of cases . . $9 \mathrm{r}$

Number of cases amongst

drinkers of-suspected milk . 91

Percentage on total cases . . 100

Number of polluted milk sources I

Number of milkmen . . . I

Circumstances implicating the milk supply.-Part of a slaughter-house not used as such but as a wash-house was boarded off to constitute a dairy. On a shelf of this dairy the milk was habitually set in pans, exposed to the air. In one corner of the slaughterhouse, nearest the dairy, was a badly trapped and very offensive drain inlet. Close to this inlet ran the wooden partition between the slaughter-house and the dairy, which near the inlet had been long broken away. The drain was in communication with an old square drain which had received typhoid excreta, so that the infected sewer air from the inlet had free access to the dairy and the exposed milk which stood in the dairy. Six cases of typhoid occurred in the butcher's family. There was evidence to show that the drain was in a "waterless" condition. 
Reporter and reference.-Dr Ballard (Loc Gov. Bd.). Brit. Med. Jour., I881, vol. i., p. 20.

\section{Glasgow, 1880 (April).}

Total number of cases . . 508

Deaths . . . . 69

Number of cases amongst drinkers of suspected milk $\quad 373$ Percentage of total cases . .

Number of families supplied by milkman invaded

Number of polluted milk sources Number of milkmen

Cincumstances implicating the milk supply._Dairyman got milk from 30 farms, in one only of which was there defect. Here the milk-house, washing-house, kitchen, and living-rooms were all en suite. Four cases of enteric fever occurred at the farm, one being the dairy-maid. She was nursed above the milk-house and suffered severely. The excreta were thrown over dung heap and the soiled linen, etc., was washed at dip-well, which was also used occasionally for dairy purposes.

Exactly I4 days after first cases at farm typhoid broke out in area of Glasgow supplied with milk from this farm. Of 40 milk-shops supplied by dairyman, 30 were infected. Entire body of trade was tainted. It was associated with fever in dairyman's own family, in persons served direct from his cart. It poisoned the families of the dealers through whose shops it passed to the public, and it poisoned the customers who dealt at those shops. After the milk supply was stopped the epidemic died out. At Possil Park Dr Christie reports that there were 92 other cases, 97 per cent. of which obtained their milk from implicated milk.

Probable exciting cause.-Human source.

Reporter and reference.-Dr J. B. Russell (Med. Oft. of Health). Brit.
Med. Jour., 1880, vol. i., pp. 985 and 864 .

Rochdale, 1880 (September).

Total number of cases - . 35

Deaths - . . . 9

Number of cases amongst

drinkers of suspected milk - 26

Percentage on total cases . : 74

Circumstances implicating the milk supply.-Defective well supplied water for dairy purposes. The excreta from a case of typhoid was thrown into a leaky cess-pool, and from this cesspool the dip of the soil inclined towards the farm well. Incidence of disease followed drinkers of milk from this farm a fortnight after the case of typhoid. Evidence to show that not improbably the milk was also adulterated with water from this well.

Probable exciting cause,-Human source.

Reporter and reference.- Dr J. Henry (Med. Off. of Health). Brit. Med. Jour., 1880, vol. ii., P. 597.

Penzance, 1880 (Jaruary).

Total number of cases . . 26

Deaths . . . . . 4

Number of cases amongst drinkers of suspected milk . 26 Percentage on total cases . . I00

Cincumstances implicating the milk supply. - Three cases of typhoid, one of them fatal, at the farm supplying the milk. The same person who milked the cows and did the dairying work nursed the patients and washed their clothes for several weeks. All the cases of typhoid were drinkers of milk from this farm. Members of families who escaped for one reason or another did not drink implicated milk.

Probable exciting cause.-Human source.

Reporter and reference.--Dr G. B. Millett (Med. Off. of Health). Brit. Med. Jour, I880, vol. ii., p. 37. 


\section{Portsmouth (Cambridge Barracks), 1880 (September and October).}

Total number of cases

Number of cases amongst drinkers of suspected milk - 7 Percentage on total cases . . 100

Circumstances implicating the milk supply.-A back kitchen used as a dairy. Son of milkman had been suffering from fever with typhoid symptoms for several weeks, excreta, etc., carried through back kitchen; attendant milked the cows. Infection through milk-absorption in back kitchen or by milker. Sanitary conditions of barracks good. All the members of the officer's family attacked were in the habit of taking a glass of milk before going to bed. The officer and two children took milk in tea very sparingly and escaped.

Reporter and reference.-Surg.-Maj. Jameson. Brit. Med. Jour., I889, vol. i., p. 61 .

\section{Bridlington, 1880 (October).}

Total number of cases . . 48

Deaths . . . . . 8

Number of cases amongst drinkers of suspected milk . 48

Percentage on total cases . . I00

Number of families supplied by milkman .

Number of such families invaded Percentage

Number of polluted milk sources Number of milkmen

Circumstances implicating the milk supply. - Defective well, insanitarily placed; water on analysis "evidently largely contaminated by sewage." Dairyman's son's wife had typhoid in August. Outbreak chiefly followed milk walk of dairyman, and showed particular incidence on milk drinkers.

Probable exciting cause.-Human source.

Reporter and reference.-Dr J.
Allison (Med. Off. of Health). Brit. Med. Jour., 188o, vol. ii., p. 786.

Worthing, 1880 (October and November).

Total number of cases . . 44

Deaths " . . . . 8

Number of cases amongst drinkers of suspected milk . 44

Percentage on total cases . . I00 Number of polluted milk sources I Number of milkmen . . . I

Circumstances implicating the milk supply. - Shallow well used for dairy purposes, especially for washing out milk-cans, near a defective drain. Clear evidence of soakage from the drain into well. Case of typhoid in house to which drain belonged, excreta of patient being thrown down drain.

Evidence that milk became infective after it was placed in cans of dairyman, as other persons and milk sellers supplied from farm whence supply was derived had no fever amongst their customers.

Those who habitually drank milk suffered most. Two teetotallers drinking half a pint night and morning were both attacked. A boy at school ordered to have a pint daily, attacked. The poor who drank little or no milk escaped.

Probable exciting cause.-Human source.

Reporter and reference.-Dr C. Kelly (Med. Off. of Health). Brit. Med. Jour., r88o, vol. ii., p. 934.

Mid-Warwickshire, 1888.

Total number of cases _. $\quad 12$

Deaths . . . . . I

Number of cases amongst drinkers of suspected milk . 12

Percentage on total cases . . 100

Number of polluted milk sources I

Number of milkmen . . . I

Circumstances implicating the milk supply.-Well polluted by leaky cesspool. Dairyman and son contracted 
typhoid which may have been directly conveyed to milk or indirectly through cess-pool, well, and washing of utensils, if not adulteration of milk with wellwater. The outbreak at once ceased after implicated milk stopped.

Probable exciting cause.-Human source.

Reporter and reference.-Dr Geo. Wilson (Med. Off. of Health). Brit. Med. Jour., r883, vol. i., p. I1 36.

\section{Dundee, 1888 (October).}

Total number of cases . . 118

Number of cases amongst drinkers of suspected milk . 82

Percentage on total cases . . 7o

Number of polluted milk sources I Number of milkmen . . . I

Circumstances implicating the milk supply.-Several members of dairyman's family suffered from typhoid fever. The cases all occurred within three weeks.

Probable exciting casse.-Human source.

Reference.-Brit. Med. Jour, $188_{3}$, vol. ii., p. 839 .

\section{Gateshead, 1888 (February).}

Total number of cases

Deaths

Number of cases amongst drinkers of suspected milk .

Percentage on total cases .

Number of families supplied by milkman invaded

Circumstances implicating the milk supply.-Dairy utensils kept in dirty scullery. Premises defective, and enteric fever among the children at the farm. All the cases contracted their disease from the implicated milk.

Probable exciting cause.-Human source.

Reporter and reference.-Dr C. Green (Med. Off. of Health). Lancet, 1883 , vol. ii., p. 986.
Port Jervis, U.S.A., 1884 (October).

Total number of cases . . 148

Number of cases amongst

drinkers of suspected milk . I28

Number of polluted milk sources I

Percentage. . . . 86

Circumstances implicating the milk supply. - Typhoid fever at dairy in August and September, 3 cases. These patients were nursed by the same person who attended to the dairy work.

Probable exciting cause.-Human source.

Reporter and reference.-Dr Curtis. Rep. New York Bd. of Health, 1884, p. 185 .

\section{Aberdeen, 1888 (December).}

Total number of cases $\quad$ - 25

Number of cases amongst drinkers of suspected milk . 25

Percentage on total cases . . 100 Number of polluted milk sources I Number of milkmen . . . I

Circumstances implicating the milk supply.-Sanitary conditions extremely bad. Foul watercourse flowing down a ditch used for the dairying purposes. This watercourse was found to be polluted with typhoid excreta. All the cases consumed the one milk supply. Of 52 persons residing in 13 invaded houses, 25 were attacked. In some instances the disease only attacked the one member of the family who used the implicated milk.

Probable exciting cause.-Human source.

Reporter and reference.-DrSimpson (Med. Off. of Health). Lancet, I884, vol. i., p. 487.

Derby, 1884 (September and Nowember).

Total number of cases . . 289

Deaths . . . . 3I

Number of cases amongst

drinkers of suspected milk - 258

Percentage on total cases . $\quad . \quad 89$ 
Number of families supplied by milkman .

Number of such families invaded Percentage .

Number of polluted milk sources

Ist November the son was convalescent and able to assist his father in work on the farm. A few days after Ist November the privy vaults were cleared out, and the contents (including the undisinfected typhoid excreta), were thrown out on the ground near by and covered with earth. On 18th November the epidemic broke out. Contamination of milk by means of water, dust, or milker's hands. Community of milk supply was clearly proved.

Reporter and reference,-Dr C. Harrington. Boston Med. and Surg. Jour., vol. ii., p. 49.

\section{Nottingham, 1887.}

Number of cases

Circumstances implicating the milk supply.-The interest of this outbreak was that the customers were partly supplied by cart and partly by can from a certain farm, the former by the farmer, the latter by 3 milk vendors. A disproportionate number of the attacks were among the households supplied by the cart, 19 .exclusively by the cart, and 3 exclusively by the can. In households supplied by the 3 milk dealers, there was a marked absence of cases of typhoid. Concluded that the milk was infected by person or persons engaged in its distribution by the cart - most likely a particular employé from the Union.

Apparent cause.-Human infection.

Reporter and reference.-Dr B. A. Whitelegge. Medical Officer's Rep., I88I.

\section{Stirling, 1889.}

Total number of cases . 40-50 Deaths . . . . . 4 Number of cases amongst drinkers of suspected milk - all Percentage on total cases . . I00

Circumstances implicating the milk supply.-Water supply polluted. Cases of typhoid at the farm-house by which 
the storeroom probably became contaminated, and hence the milk.

Probable exciting cause.-Human source.

Reporter and reference. - Dr M'Fadyen (Med. Off. of Health). Brit. Med. Jour., 1889, vol. i., p. 1250.

Edinburgh, 1890 (October).

Total number of cases _ . $\quad 63$

Deaths . . . . 3

Number of cases amongst drinkers of suspected milk - $\quad 56$

Percentage on total cases . $\quad$ - 89

Number of polluted milk sources I

Number of milkmen . . . 7

Circumstances implicating the milk supply.-Serious sanitary defects at farm. Well water of a dangerous character (analysis). Probable channel of infection was through unclean cans. As a rule large milk drinkers were affected. Various collateral evidence implicating milk.

Probable exciting cause.-Undetermined.

Reporter and reference.-Dr Harvey Littlejohn. Trans. Edin. Med. Chir. Sac., I89I.

Shawland, Glasgow, 1891 (A ugust).

Total number of cases . . 42

Deaths . . . . . 6

Number of cases amongst

drinkers of suspected milk . 37

Percentage on total cases . . 88

Number of polluted milk sources 1

Number of milkmen . . : I

Cincumestances implicating the milk supply.-Bad sanitary conditions existed at farm; drainage liable to pollute business water service. Farmer's daughter had typhoid, and excreta thrown into dung-pit in hot weather. All first 39 cases scattered over wide area were drinkers of implicating milk.
Probable exciting cause:-Human source.

Reporter and reference. - Dr Campbell Munro (County Med. Off. of Health). Public Health, 1891-92, p. 275 .

\section{Dundee, 1892 (Angust and September).}

Total number of cases $\quad . \quad 73$

Deaths - . . . . II

Number of cases amongst drinkers of suspected milk . 43

Percentage on total cases . $\quad 59$

Number of families supplied by milkman invaded . . . 32

Number of polluted milk sources I

Number of milkmen . . . I

Cincumstances implicating the milk supply.-Buildings old and dilapidated, badly kept. Well water used for dairying purposes. Typhoid fever case on dairyman's premises in August. Milk supply stopped on 28th August.

Probable exciting cause.-Human source.

Reporter and reference.-Dr Anderson (Med. Off, of Health). Brit. Med. Jour., 1892, vol. ii., pp. 598, 902, 915.

\section{Plymouth, 1892 (Spring).}

Total number of cases $\quad . \quad 12$ Deaths . . . . . 1

Number of cases amongst drinkers of suspected milk . 12 Percentage on total cases . . I00

Cincumstances implicating the milk. supply.-Well leaky, within 40 feet of cess-pit, polluted with typhoid excreta. Typhoid fever in farmer's familyfarmer and wife carrying on dairy work including milking the cows whilst they were also nursing the sick. The fatal case was of a young man who had been ordered a milk diet.

Reporter and reference.-Dr F. M. Williams (Med. Off. of Health). Brit. Med. Jour., 1892, vol. i., p. II 57. 
Springfleld, Mass., U.S.A., 1892

$$
\text { (August). }
$$

Total number of cases

Deaths

Number of cases amongst drinkers of suspected milk . I35

Percentage . . . . 90

Number of polluted milk sources I

Number of milkmen . . . . I

Circumstances implicating the milk supply.-Upon the farm supplying the implicated milk there was one, and probably more than one, case of typhoid fever. The farmer submerged his sealed milk cans when full of milk, in the well adjoining the cow yards with the object of keeping the milk cool. The water in this was polluted, and it was found that 4 of 9 milk cans leaked, when inverted. Hence it became evident that water could gain access if the cans were submerged as they had been. The investigators suggest as an hypothesis that as the typhoid excreta of the patient were placed, undisinfected, in the privy, and the conterits of the latter spread over the tobacco field, the germs of typhoid may have gained access to the well by dirt from the labourer's boots who both worked in the field and at the milk. Coliform organisms were found in the well water.

Probable exciting cause.-Human source.

Reporter and reference.-Drs W. T. Sedgwick and W. C. Chapin. Boston Med. and Surg. Jour., I893, vol. ii., p. 485 .

Somerville, Mass., U.S.A., 1892 (A ugust).

Total number of cases

Number of cases amongst drinkers of suspected milk. Percentage . . . . Number of polluted milk sources Number of milkmen . . .

Circumstances implicating the milk supply. - Farm sanitary. Infection of milk at milk-shop where the utensils had been cleaned by the son of the milkman whilst he was suffering from typhoid fever. He continued his work during the acute stages and died from intestinal hæmorrhage a few days later. There was abundant evidence to show that this young man assisted in all the dairy work whilst suffering from typhoid. When he was too ill to work and withdrew altogether from the dairy, the outbreak (allowing for incubation period) ceased.

Probable exciting cause.-Human source.

Reporter and reference,-Dr W. T. Sedgwick. Boston Med, and Surg. Jour., r893, vol. ii., p. 489 .

\section{Shildon and Flast Thickley, County} Durham, 1893 (July-September).

Total number of cases . . 45

Number of cases amongst drinkers of suspected milk . 45

Percentage on total cases , , 100

Number of families supplied by milkman . . . . 147 Number of such families invaded 26 Percentage . . . $\mathbf{1 7} 6$

Circumstances implicating the milk supply.-Room used for storing milk had one large window opening on to three uncovered privy middens, the furthest of which was 33 feet away, the nearest 23 feet. Also 13 feet away was an imperfectly trapped gully in yard communicating with sewer. Inside storeroom a sink-pipe passed direct to sewer. Three undoubted cases of typhoid occurred on the dairy premises in July and August. The milk was stored all night in room exposed to emanations from sewer, which ventilated directly into room. The room was also open to infection from privy midden on which were cast the untreated bowel discharges of the dairy fever patients throughout their illness. 
Probable exciting cause.-Human source.

Reporter-Dr Bruce Low (Loc. Gov. Bd. Rep.).

\section{Stockport, 1898 ( July).}

Total number of cases

Deaths

Number of cases amongst drinkers of suspected milk

Percentage on total cases.

Number of families supplied by

milkman invaded .

Number of polluted milk sources

Cincumstances implicating the milk supply.-Probably infection of milk arose on the vendor's premises by dried dust from a specifically infected privy-pit. Among an extensive round of customers of the milk in question taken direct from the farms to their houses no cases occurred. The attacks were all at the houses of customers buying milk over the counter of the vendor's shop.

Probable exciting cause.-Human source.

Reporter and reference.-Dr Porter (Med. Off, of Health). Public Health, December 1893, and January 1894.

Kelso, 1898 (May-A sgust).

Total number of cases

Deaths

Number of cases amongst drinkers of suspected milk .

Percentage on total cases . .

Number of families supplied by milkman .

Number of such families invaded Percentage.

Number of polluted milk sources Number of milkmen .

Cincunstances implicating the milk supply. - Well water greatly contaminated with farm sewage. Well water used for dairying purposes. Two cases of illness on farm premises diagnosed as enteric fever only after the occurrence of a third case in June. Of the first 18 cases only one was of a non-consumer of the implicated milk. Of 48 households taking the milk only three escaped invasion by enteric fever. Three irregular customers had one drink of implicated milk and contracted the disease.

Probable exciting cause.-Human source.

Reporter and reference.-Dr Oliver (County Med. Off. of Health). Annual Rep., I893.

Bacup, 1804 (April).

Total number of cases . . 33

Deaths . . . . . 5

Number of cases amongst drinkers of suspected milk . 30

Percentage on total cases . $\quad 91$

Number of families supplied by milkmen . . . 30

Number of such families invaded 13 Percentage . . . . 43 Number of polluted milk sources I Number of milkmen . . . I

Circumstances implicating the milk supply.-Dairy farmer, who milked the cows, also acted as night-soil scavenger, and probably conveyed the germs of the disease into his own household. The supply was stopped and the outbreak ceased.

Reporter and reference.-Dr Brown (Med. Off. of Health). Anmual Rep., I894.

Bufralo, U.S.A., 1894, 1895, 1896.

Total number of cases . . 5I

Circumstances implicating the milk supply.-These were three small epidemics of typhoid fever occurring in 1894,1895 , and 1896 , and affecting respectively 19 patients, 18 patients, and 14 patients. In each of the three outbreaks the implication of the milk was definitely traced, and the milk was contaminated in each case from human sources, namely, persons suffering from enteric fever. 


\section{Probable exciting cause.-Human origin.}

Reporter and reference.-Dr E. Wende. American Jour. Med. Assoc., I900, vol. i., p. I 5 I. Welply reports six typhoid epidemics due to "creameries" in the States. Lancet, 1894 , vol, i., p. 992.

\section{Great Harwood, Lancashire, 1895} (January).

Total number of cases

Deaths

Number of cases amongst drinkers of suspected milk

Percentage on total cases

Number of families supplied by milkman

Number of such families invaded

Percentage

Number of polluted milk sources Number of milkmen

Circumstances implicating the milk supply.-Dairy-maid, who milked the cows and washed the utensils, suffered from ambulatory enteric fever. No case occurred outside the particular milk supply. The outbreak ceased after the removal of the dairy-maid. Drinkers of raw milk attacked most virulently. The incubation period from 2 to 14 days.

Probable exciting cause.-Human source.

Reporter and reference.-Dr Sargeant (County Med. Off. of Health). Brit. Med. Jour., I895, vol. i., pp. I I IO-I I I I.

Providence, U.S.A., 1895 (November).

Total number of cases . . 30

Deaths . . . . . 3

Number of cases amongst drinkers of suspected milk
Percentage on total cases 100 Number of polluted milk sources I Number of milkmen . . . I

Circumstances implicating the milk supply. - A boy working at dairy, who had recently driven a night-soil waggon, became sick with typhoid fever in middle of October. A sister of the boy was taken ill ro days later. Their mother nursed them both and also tended the dairy, washing the milk utensils, including a cloth strainer.

Reporter and reference.-Dr Swarts. Monthly Bulletin R. 1. Bd. of Health, December 1895 .

\section{Shettleston, Lanarkshire, 1895} (October).

Total number of cases _. . 35

Deaths . . . . 2

Circumstances implicating the milk supply. - The incidence was greater in the new tenement houses than in the old cottage houses. In 15 cases in 8 families it was found that the milk came from one dairy. On inquiry it was found that a case of typhoid had been notified there on 28 th September, but the patient had been ill since I 4 th September, and had been nursed by one of the family who also took part in the dairy work. In the main the disease was of a mild type.

Probable exciting cause.-Human source.

Reporter and reference.-Dr J. T. Wilson (Med. Off. for Lanarkshire). Brit. Med. Jour., I895, vol. ii., p. 1204.

Liverpool, 1897 (September).

Total number of cases . . 27

Circumstances implicating the milk

1 This outbreak was apparently due to infected ice cream. Such outbreaks have appeared in various places, including a number in the metropolis. Special reports and records of such epidemics have been made by Murphy, Hope, Collingridge, Newsholme, Newman, and others. Klein, Foulerton, Delépine, Wilkinson, Pakes, etc., have reported bacteriological investigations (Loc. Govt. Bd. Reports, Medical Officer's Reports, Brit. Med. Jour., 1897 ; Practitioner, 1892, etc.). 
supply.-On 6th and 7 th September, 25 of the cases of enteric, which were all those of children, had partaken of ice cream sold by an Italian vendor at a fair. The two remaining sufferers were believed to have eaten "chippotatoes" purchased from the same vendor at the same time. The vendor in question was found to have an ice cream factory in a low district of the city of Liverpool, and there was at the time of the fair a case of typhoid fever in his house.

Probable exciting cause.-Human source.

Reporter and reference-Dr E. W. Hope (Med. Off. of Health). Annual Rep. on Health of Livenpool, 1897.

Clifton, Bristol, 1897 (October-December).

Total number of cases . . . 244

Deaths . . . * . $3 \mathrm{I}$

Number of cases amongst drinkers of suspected milk . 234

Percentage on total cases $\quad 95.9$

Number of families supplied by milkman .

Number of polluted milk sources

Number of milkmen .

139

I

3

Circumstances implicating the milk supply. - This epidemic was distinctly traceable to 3 different milk deliveries, which had become polluted by the contamination of the main source. At the farm from which the suspected milk was derived, the necessary water for dairying purposes was obtained from a pump which drew water from a shallow well situated only a few feet from a stream known as Ashton Brook, which flowed through the farm and close to the buildings. This brook appears to have become contaminated with the excreta of a workman who suffered about the middle of September from some kind of illness not to be differentiated from typhoid fever. His blood gave a distinctive Widal reaction (Klein), and other symptoms were similar to those of typhoid. The outbreak began early in October, and was distributed along the track of three milk deliveries which had received portions of the farm in question. There was an excess of attack among children under 15 years of age. (See chart, p. $3^{81}$.)

Probable exciting cause.-Human source, through polluted water used for dairying purposes.

Reporter and reference.-Dr D. S. Davies (Med. Off. of Health). Trans. Epidemic Soc. of London, vol. xvii., pp. 78-103.

Glasgow, 1898 (November).

Total number of cases . . 43

Number of polluted milk sources I

Number of milkmen . . . $\quad$ I

Circumstances implicating the milk supply. - The sister of the shopgirl of the milkman suffered from unrecognised typhoid fever in the middle of September. The shopgirl contracted the disease from her sister or from the milk itself, which it is possible that she may have infected. Milk from the same farm and delivered by the same van-driver, but not passing into consumption through the dairy in question, caused no illness. The 43 cases arising from the infected dairy occurred in two main groups, within a fortnight.

Probable exciting cause.-Indirectly from buman source.

Reporter and reference.-Dr A. K. Chalmers (Med. Off. of Health). Report, 1898 .

Fdinburgh, 1899 (Jure ana July).

Total number of cases-14 in II families.

Number of families supplied by milkman invaded.-Only a small proportion.

Number of polluted milk sources I Number of milkmen . $\quad$ - $\quad$. 2

Cincumstances implicating the milk supply. - The only common link 
between all the cases was the milk supply. The conditions existing at the dairy and cow-house were extremely bad, being dirty and having a very offensive manure pit and privy. The young son of the dairyman was stated to have suffered from inflammation of the bowels about the middle of June. Although there was no direct or authentic evidence of the contamination of the milk supply, Sir H. D. Littlejohn believes that the milk was the means of carrying the disease.

Probable exciting cause.-Human source.

Reporter and reference.-Sir H. D. Littlejohn M.D. (Med. Off. of Health). Annual Rep., 1899-1900, p. 35.

Salford, 1900 (November).

Total number of cases . .

Number of cases amongst drinkers of suspected milk . 18

Percentage .

Number of polluted milk sources

Number of milkmen in Manchester

Circumstances implicating the milk supply. - The dairyman had suffered from a disease which appeared to Dr Niven to lrave been typhoid fever, although it had been treated as uncomplicated pneumonia.

Probable exciting cause.-Human source.

Reporter and reference.-Dr Niven (Med. Off. of Health). Annual Rep., I900, p. 79.

\section{Glasgow, 1900 (July).}

Total number of cases . .

Number of polluted milk sources Number of milkmen . . .

Cincumstances implicating the milk supply. - The milk distribution was the only circumstance common to all the patients. Each member of the dairyman's family and all the milk distributors were examined and found well. On examining the farm from which the milk was chiefly obtained, it was found that the water supply had recently been changed, and was open to criticism. Several relatives of the farmer were known to have suffered from typhoid fever.

Probable exciting cause.-Human source, affecting the water supply.

Reporter and reference.-Report of Med. Off. of Health of Glasgon, 1899-1900, p. 45.

Elkton, Maryland, U.S.A., 1900 (October).

Total number of cases . . 64

Deaths . . . . . 2

Number of cases amongst

drinkers of suspected milk . 6I

Percentage on total cases . . 95.3

Number of families supplied by milkman . . . $\quad 39$

Number of such families invaded 39

Percentage. . . . I00

Number of polluted milk sources I

Number of milkmen . . . I

Circumstances implicating the milk supply.-Mrs A., wife of the dairyman, assisted in nursing a case of typhoid in an adjoining farm. Mrs $\mathrm{A}$. and her son, who did the dairy work, were also "ill with fever." Cows (22) were healthy. Contaminated water supply was used for dairying purposes.

Probable exciting cause.-Human source.

Reporter and reference.-Dr John S. Fulton (Sec. State Bd. of Health, Maryland, U.S.A.). Jour. of Hygiene, vol. i., No. 4, p. 422-429.

Hamar, Lake Mjosen, Norway, 1900.

Total number of cases . . 53

Circumstances implicating the milk supply.-Sudden outbreak traced to dairy where dairyman and his wife were both suffering from typhoid fever. The woman was affected first, but continued to work in the milk-shop. Out of the 53 persons affected, 42 contracted 
the disease directly through the milk, 6 by secondary personal infection, and 4 indirectly. The premises were old and insanitary, and were probably infected from previous cases which had occurred long before. But the month before the dairyman's wife became ill, the cess-pit which had been closed was cleared out, and the contents were put in the dustbin, and left until June when the outbreak occurred.

Probable exciting cause.-Human infection of milk.

Reference.-Public Health, 1901-02, p. 403 .

Wallasey, Cheshire, 1801 (1) ( July).

Total number of cases . . 26

Number of cases amongst

drinkers of suspected milk .

Number of polluted milk sources

Number of milkmen . . . 2

Cincumstances implicating the milk supply.-Grave sanitary defects found at farm whence the milk supply came, including bad water supply. Slop water and cess-pool contents could gain access to water supply. $B$. coli was found in the milk.

Wallasey, Cheshire, 1001 (2) (September).

Total number of cases

Total number of cases amongst drinkers of suspected milk . 50

Percentage on total cases . $\quad$. 55.5

Grave sanitary defects at farm supplying milk, and water contained many $B$. coli. Stoppage of the milk supply checked the outbreak.

[The medical officer issued the following circular to all the milk dealers in the district :-

"With a view to preventing the spread of infectious disease, especially typhoid fever, by means of contaminated milk, it is most desirable that all milk purveyors should ascertain for themselves the sanitary condition of the farms from which their milk is procured, both in the interests of the public and to protect themselves. In deciding upon a milk supply, the purveyors should consider not only the cleanliness of the farmer's premises generally, and the care taken to keep all milk vessels pure, but above all he should ask about the water supply. It is best that this should be from a public supply, laid on in pipes direct to the farm with a tap in the dairy, and communicating also with the refrigerator. The purveyor ought to look with suspicion on all wells, whether open or connected by pumps, especially if the wells are close to the house or farmyard, so as to be open to contamination by soakage into the well. It is also most desirable to find out what care is taken by the milkers to keep their hands clean, and to wash the udders of the cows, otherwise the milk is sure to be more or less contaminated."]

Probable exciting cause.-Contaminated water.

Reporter and reference.-Dr A. Craigmile. Annual Report of Medical Officer of Wallasey, rgor.

Melbourne, 1901.

Total number of cases $\quad$. 22

Number of cases amongst drinkers of suspected milk . 22

Percentage on total cases . . $\quad$ roo

Number of families supplied by milkman . . . . I54

Number of such families invaded 22

Percentage. . . . . 14

Number of polluted milk sources I

Number of milkmen . . . I

Cincumstances implicating the milk supply. - The keeper of the dairy was removed to hospital on 6th December 1900, suffering from enteric. He remained in hospital till 15 th January rgor. He went home from the hospital, and declared that he did not work 
about the dairy for several weeks. On I Ith March, the outbreak commenced. No other possible source of infection was discovered except the keeper himself; fully three months elapsed between the beginning of his illness and the infection of the other cases.

Probable exciting cause. - Human infection during convalescence.

Reporter and reference. - James Jamieson, M.D. (Med. Off. of Health, Melbourne). The Australasian Med. Gazette, 2 Ist April Igo2.

\section{Clydach, Glamorgan, 1901.}

Total number of cases . .

\section{Deaths}

Number of families supplied by milkmen.

Number of such families invaded Percentage.

Number of polluted milk sources Number of milkmen

2 I

Circumstances implicating the milk supply. - The eldest son of the farmer supplying the milk had enteric fever and died. From 12 th October to $25^{\text {th }}$ October, 16 cases of typhoid occurred among persons using the milk from this farm either directly or through two other milkmen ( 7 cases). No evidence to show how the milk became infected, but it must have been in the house.

Reporter and reference. - Dr Williams (Med. Off. of Glamorganshire). Public Health, IgoI-02, pp. 650-654.

Adams, Mass., U.S.A., 1901.

Number of persons affected .

Number of persons affected who drank common milk supply of a particular dealer . Percentage of cases to drinkers . I00

Circumstances implicating the milk supply.-Upon examination it was found that all the cases of typhoid had obtained milk from one dealer, who obtained his milk ( 27 cans) from 2 farms. The dealer fetched the milk every afternoon and distributed it the following morning. During the night it remained in his house. On 5th November, he became ill, and continued to work till IIth November, when he was too ill, and he remained away from work till and December, returning then only to have to give up again on 8th December. His illness was described as "influenza," but simulated typhoid, and his blood gave a positive Widal reaction. His illness began $5^{\text {th }}$ November. His customers began to be ill on I 5 th November.

Probable exciting cause.-Milk infected from human sources.

Reporter and reference.-Dr Morse (State Bd, of Health, Massachusetts). Report, I901, p. 559.

\section{Beverley and Salem, Mass., U.S.A.,} 1901.

Total number of cases . . 26

Number of cases amongst

drinkers of suspected milk . 22

Percentage on total cases . $\quad 85$

Circumstances implicating the milk supply.-The milk dealer implicated supplied most of the milk from his own cows, but supplemented it by small quantities from two other sources, the whole of the supply being mixed before distribution. It was found that the farmer's son who had assisted in the milking and the distribution of the customers, was ill with typhoid fever, and at one of the farms supplying the milk there were 7 cases of typhoid, probably due to a polluted spring and well water. When the milk supply was controlled the outbreak ceased.

Probable exciting cause.-Human.

Reference.-Thirty-second Ann. Rep. of State Bd. of Health, Massachusetts, I9OI. 
Brockton, Mass., U.S.A., 1901.

Total number of cases 2I-II of whom were children.

Number of cases amongst drinkers of suspected milk.

Percentage on total cases . . I00

Cincumstances implicating the milk supply. - Though both millk dealer and his son distributed the milk, all the cases but two were on the son's route. The son obtained his portion of the supply from four sources, in one of which it was found that typhoid fever was present. The son himself drank this milk, because he thought it better quality than that of the other three sources. Healso contracted the typhoid fever. It was believed that originally the milk became infected by washing the cans with polluted well water.

Probable exciting cause.-Human.

Reference.-Thirty-second Ann. Rep. State Bd. of Massachusetts, IgoI.

Norwood, Mass., U.S.A., 1901.

Total number of cases- -6 and 2 I.

Circumstances implicating the milk supply.-The first outbreak involved only 6 cases, the second 21. All cases were traceable to one dealer, who obtained his milk from 17 farms, mixing it and distributing it himself. Inquiry at his depot and amongst his employees, and at the 17 farms, yielded no result. He was told, however, not to mix the 17 supplies. This was done on $4^{\text {th }}$ January. On I 4 th January 2 fresh cases occurred in persons who had taken unmixed milk from the same farm. Here it was found that the well water used for washing the cans was polluted. The use of the well was stopped, and the cans sterilised, and no further cases occurred. Later in the year, however, typhoid again broke out, and was traced to this identical farm, where the farmer had neglected the order, and used the well water for dairy purposes.
Probable exciting cause-Milk infected through well water.

Reference.-Thirty-second Ann. Rep. of State Bd. of Massachusetts, 1901 .

Milk-borne outbreaks of typhoid fever also occurred at the following places in the years stated:-

Penrith, 1857; Islington, 1870; Leeds, 1870; Parkhead (Glasgow), 1873; Ascot, 1873-77; Moseley, 1873 ; Brighouse, 1873; Brierley (Lancashire), 1874; Taunton, 1874; Queensberry, 1874; Dundee, I874; Crosshill, 1875 ; Jarrow, 1875; Glasgow, 1875 ; Churwell and Morley, 1876; Greenock, I877; Edinburgh, I877; Tunbridge Wells, 1877 ; Croydon, 1878; Perth, I878; Huncoat, I878; Bristol, 1879 ; Marylebone, I880; Southport, I88I ; Hawick, 188I ; Christ Church (New Zealand), I881 ; Grangemouth, 1882; Leicester, 1882 ; Glasgow (2), 1882; Clapham, 1882 ; Shelf, 1882 ; Exeter, 1883; Cologne, 1883; St Pancras, 1883; Cardiff, 1883; Upsala, 1883 ; Bannockburn, 1883 ; St Albans, 1884 ; Aberdeen, 1884 ; Lower Sherringham, 1885 ; Lancing College, 1886; Swanage, 1886; Carlisle, 1886-7; Leichhardt, New South Wales, 1886; Bandon, 1887 and 1893 ; Spilsby, 1888 ; Providence, U.S.A., I888; Spennymoor, Durham, I888; Dundee, I889; Melbourne, 1889; Leeds, 1889; Belgrade, 1889; Svarteborg, 1889; Waterbury, U.S.A., 1890; Nottingham, 1890 ; Randwick, N.S.W., 1890 ; Whitchurch, 1891 ; Sutton Coldfield, 1891 ; Edinburgh, r891 ; Clermont-Ferrand, I891 ; Torquay, 1892 and 1894; Greenwich, 1892 ; Louisville, U.S.A., 1893 ; Paisley, 1893; Lambeth, I894; Castle Island (Ireland), I894; Mont-Clair, U.S.A. (cream), I894; Marlborough, Mass., U.S.A., I894; Bay Head, U.S.A., I894; Fintry, I895; Helmsley, I895; Stamford, 1895; Plumstead, I895; St Helens, 1895; Folkestone, 1896; 
Dublin, 1896 ; Kirkcaldy, 1896 ; Reuland, I897; Auro, 1898; Aberdeen, I898; South Witham, I899; Bleialf, 1899; Coleford, 1899; Market Harborough, 1899; Büllingen, 1899;
Coleford and West Dean, I900; Folkestone, I900; Salford, I900; Plymouth, I900; Gildersome, Yorks, I90I; Northampton, I902; Porth, South Wales, 1903; Ealing, 1903.

\section{Diphtheria}

It was not until 1878 that evidence was forthcoming in support of the view that diphtheria, like scarlet fever and typhoid fever, might on occasion be spread by means of milk. In that year, $\mathrm{Mr}$ W. H. Power made an inquiry into an outbreak of diphtheria in North London, chiefly in Kilburn and St John's Wood. There were as many as 264 persons attacked and 38 died. The infection invaded some 118 different households.

The epidemic was most severe in May (first four weeks), when about I90 cases occurred. The outbreak terminated abruptly. The area infected, and time of infection, clearly showed that there was some factor at work over a circumscribed area, and operative during a limited time. There was no antecedent throat illness, and no school infection or contact contagion traceable. The houses were sanitarily good, and had a good water supply. With the exception of a "special sewage area," there was but one thing common to most of the cases, and this was the milk supply. It was found that within the area of the greatest prevalence of throat illness, about one-fifth of the households were supplied by a common milk supply. The "special sewage area" was also found to be supplied in the main from the same milk supply. The incidence of the disease fell, actually and relatively, upon consumers of the suspected milk. Further, in regard of each business (the milkman in question owning two, one at Kilburn and one at Muswell Hill), the above relation of milk service to throat illness was observed for a limited period only, that period beginning for one business only about the time that it ceased for the other.

Inquiry into the milk supply elicited no evidence of human disease pollution, nor contamination by water or air. Nor was there any definite disease of cows present at the time as far as could be judged. But by a process of exclusion, $\mathrm{Mr}$ Power suggested that "there may have been risk of specific fouling of milk by particular cows suffering, whether recognised or not, from specific disease." The affirmation that the cows had not been ill, 
does not exclude occurrence among cows of "cold or other minor ailments." 1

This was the first occasion on which milk was shown to be definitely implicated in the spread of diphtheria, and although the fons et origo was not discovered, the inquiry drew attention to the possibility of such a channel of infection.

Between 1878 and 1882 , there were several epidemics of diphtheria traceable, more or less, to the milk supply. The small outbreak at Little Horton, and the large one at Rugby School, may be taken as fair illustrations. In the former, the origin of the infection was probably human; in the latter, the origin was supposed by the investigator to be bovine. But there was little progress in the matter until 1882 . In that year, Dr Parsons investigated an outbreak at Devonport, in which there was some evidence of milk conveyance of infection. ${ }^{2}$ In the same year, $\mathrm{Mr}$ Power inquired into a "sore throat" and diphtheria epidemic affecting more than sixty persons at Hendon. Six of the patients died. In the first ten days there were fifteen households affected, thirteen of which obtained milk from a particular dairy. Multiple cases occurred in these households. Mr Power, after full inquiry, finally set aside as unlikely, various channels of infection, and thought that the "possible explanation of infectiveness of the milk" was by "causes operating through the cow herself, and enabling her to furnish milk capable of inducing diphtheria or throat illness in persons consuming it." The milk itself was ropy or stringy, especially so four or five days before the throat illness broke out. No cause of the ropiness was detected. The cows were apparently well so far as the farmer could remember. "But," adds Mr Power, "ailments of animals so trivial as to be disregarded or even unnoticed by people about them, may have larger concern with occurrence of specific disease in the human subject than has heretofore been thought likely." 3

It may be here remarked, that in 1883 Klebs discovered the specific bacillus of diphtheria, and in the following year Löfler isolated it in separate culture. Hence on the occurrence of the next epidemic of diphtheria traceable to milk, the problem came more within the region of scientific tests. The first epidemic following the discovery of the Klebs-Löffler bacillus occurred in 1886, in York Town and Camberley. ${ }^{4}$ It was investigated by $\mathrm{Mr}$

1 Local Government Board Report for 1878 (W. H. Power).

2 Local Government Board Report, 1883-84, pp. 42-48.

3 Ibid., p. 49.

Ibid., 1886, pp. 31 I-326. 
Power, and also, experimentally, by Dr Klein. The facts elicited and theories propounded, brought the whole question to the front. Mr Power found that 84 per cent. of the invaded houses used the implicated milk, and 88.5 per cent. of the total number of persons attacked (140) were consumers of the milk. Ninety-three per cent. of the 140 were attacked within the same ten days, and the severity of the attacks appeared to be determined by the amount of milk consumed. The outbreak mostly affected better-class persons.

\section{The Question of Bovine Diphtheria}

The investigation, however, was remarkable not only for its collected evidence of milk implication, but for the further fact that $\mathrm{Mr}$ Power found that two of the cows from which the infective milk had been derived showed some slight signs of "scabs " and "chaps" on the teats. There was evidence that these abnormal conditions of the teats had occurred both at a time anterior to, and simultaneous with, the outbreak of diphtheria. Somewhat similar evidence was forthcoming in other subsequent outbreaks. The question therefore arose as to whether the cows could suffer from diphtheria or some modified form of that disease, and by means of their milk convey the disease to man.

In this connection, Dr Klein undertook some experiments to ascertain whether or not diphtheria was inoculable into cows. He took for the experiment two healthy milch cows which had calved some 'three or four weeks previously. One c.c. of broth culture of $B$. diphtherice (derived from human diphtheritic membrane) was injected under the skin into the subcutaneous tissue of the left shoulder in each of the two cows. Two or three days after the inoculation (a) the temperature rose to $40.6^{\circ} \mathrm{C}$., and the animals suffered from slight general malaise. On the third day (b) a tumour appeared at the site of inoculation, which steadily increased in size to the seventh day. On the fifth day (c) papular eruption appeared on the udder and hind teat. In addition to the papules there were half a dozen vesicles, and some round patches covered with brown crusts. The process of eruption was mature by the eighth day. In the lymph of the vesicles and pustules the $B$. diphtherice could be demonstrated, according to Klein, both microscopically and by culture. He therefore concluded that the $B$. diphtheria, as such, inoculated into the shoulder of the cow, was received into the general system of the cow, and produced its effects, not in the viscera, but on the udder as a specific erup- 
tion, and that before the end of five days after inoculation, was finally excreted in the cow's milk. "The presence of the diphtheria bacillus," he wrote, "could with certainty, by microscopic and culture observations, be demonstrated in the milk of the cow collected under all precautions; the number of bacilli present on that day in the milk amounted to 32 per c.c." Scrapings from vesicles on the sixth day were inoculated into two calves, which then suffered from a like disease. ${ }^{1}$

During 1890 and 1891 , Dr Klein repeated these experiments on milch cows, and in two further instances, out of six cows, an eruption was produced on the udder and teats, and in one of these positive cases the $B$. diphtheria was found in the milk about a week after inoculation.

It must be admitted that positive results did not always follow these experimental researches; there was not always eruption, nor was the bacillus uniformly isolated from the milk. Löffler, Abbott, Ritter, and others, including many veterinarians, have criticised the experiment, and hold that there is no evidence at present that diphtheria is a bovine disease. Since the time of the York Town epidemic, some twenty milk-diphtheria outbreaks have been investigated, with the result that, with one or two exceptions, the infectivity of the milk was certainly derived from human sources and not from bovine. In the Croydon outbreak in 1890 , an udder eruption was discovered on the cows yielding the suspected milk, and Klein reported that this eruption was similar to those met with in diphtheria outbreaks traced to milk of diseased cows. At Worcester in the following year, and at Glasgow in 1892, similar evidence was obtained.

Up to the present, however, it may be said that the evidence forthcoming points in the direction of human rather than bovine infection as the origin of the diphtheria bacillus in milk.

An interesting investigation has recently been made by Dean and Todd, respecting a small outbreak of diphtheria occurring in $1901{ }^{2}$ In this outbreak several individuals suffered from diphtheria, and several others in the same households suffered from sore throat, probably diphtheritic. These individuals obtained their

1 See A Treatise on Hygiene and Public Health (Stevenson \& Murphy), vol. ii., pp. I6I-I64 (Klein). Also Local Government Board Report, I889, p. 167 et seq.

${ }^{2}$ Jour. of Hygiene, April 1902 (vol. ii., No. 2, p. 194). Experiments on the relation of the Cow to Milk Diphtheria, by George Dean, M.B., and Charles Todd, M.D. 
milk supply from two cows. Members of one household who did not drink milk, or who used it only after sterilisation, escaped infection. The cows yielding the milk were found to be suffering from an eruptive disease of the udder, and both from the lesions and from the milk, cultures of virulent diphtheria bacilli were isolated. The pathological condition of the cows preceded by a short interval of several days the onset of the disease in the patients, and had the following characters:-On the udders and teats of both cows there were present papules and ulcers, covered by dark brown scabs. The papules were about the size of a pea, and had an indurated base which extended into the subcutaneous tissue. The majority of the lesions were in the form of ulcers covered with dry brown crusts, and were of the size of a sixpence to a shilling. On removing the crust from one of these there was exposed a slightly moist, fairly smooth surface, with an elevated, puckered, cicatricial-looking margin. The largest lesion, measuring 2 inches by $I$ inch, was evidently formed by the running together of smaller ulcers. In this case the ulcerative process was considerable. In one of the cows there was no mammitis, and abundant secretion of apparently normal milk. In the other, there was, in addition to the ulcerative lesions, mammitis affecting a posterior quarter of the udder, and the milk was scanty, ropy, semi-purulent looking, and slightly tinged with blood. Drs Dean and Todd made a bacteriological examination of the material from the ulcers and from the milk, and bacilli indistinguishable from those of the Klebs-Löffler bacillus were isolated. The authors add that " brushings were made from the teats of thirteen apparently healthy cows at a small dairy farm. In two cases, the cultures on solidified blood-serum showed diphtheria-like bacilli, but these on isolation were proved not to be the $B$. diphtheria." Experiments were also made to determine the comparative virulence of the bacilli isolated from the cows, the milk, and from the patient's throat. These were each found to be virulent for guinea-pigs, and diphtheria antitoxin given simultaneously, or twenty-four hours before inoculation, completely protected the experimental animals. The toxins from these three races were also found to show a remarkable correspondence in toxicity.

The disease affecting these two cows, was by further experiment shown to be readily contagious for calves. In the resulting lesions of this animal, whether in the vesicular or ulcerative stages, the diphtheria bacillus could not be found. Moreover, diphtheria antitoxin (10,000 units) did not protect against infection in the calf. 
Hence the authors conclude that "there was present a specific contagious eruptive condition apart from the diphtheritic infection."

Such then is the present position of the bovine diphtheria question. It is obviously a matter sub judice.

\section{The Bacillus of Diphtheria and Milk}

There can, however, be no doubt as to the conveyance by milk, on occasion, of the infective matter of diphtheria. In relation to this fact, there are three further pcints to which it is necessary to refer. They have to do with the behaviour of the $B$. diplitherice in milk, the cases in which the bacillus has been found in the milk supply, and the relationship of diphtheria in the human body and the milk supply. The first point may be dismissed at once by saying that milk is a favourable medium for the $B$. diphtheria. The organism both lives and multiplies in ordinary sterilised milk; it thrives better in unsterilised milk at comparatively low temperatures than at $37^{\circ} \mathrm{C}$. In ordinary milk unsterilised and unprepared, the commoner organisms multiply much more rapidly, and so the diphtheria bacillus is in all probability soon crowded out.

The cases in which the $B$. diphtherice has been actually isolated from milk are extremely few. In the outbreak of diphtheria at Senghenydd in South Wales, in 1899 , Bowhill ${ }^{1}$ isolated a diphtheria bacillus from the suspected milk. The culture of the bacillus in broth proved fatal to a guinea-pig in two days. In the same year, Eyre ${ }^{2}$ isolated a virulent diphtheria bacillus from some milk implicated in an outbreak of diphtheria in a school. In I900, Klein ${ }^{3}$ also reported the isolation of a genuine diphtheria bacillus in an examination of 100 samples of milk in London. Lastly, Dean and Todd, as we have seen, isolated the $B$. diphtheria from cow's milk in $1901 .{ }^{4}$ These are the only four authentic cases of actual detection of the $B$. diphtherice in ordinary milk with which we have met.

The method which proved successful in the hands of these bacteriologists may be briefly described. Bowhill obtained two sample bottles of the suspected milk. These were well shaken before being opened, in order that any suspended organisms might be uniformly distributed. Five sterilised tubes of Löffler's serum mixture were utilised for each sample, and a series of

1 Veterinary Record, 8th April 1899, No. 561. Jour. of State Medicine, I899, pp. 705-710.

2 Brit. Med. Jour., 1899, vol. ii., p. 586.

3 Jour. of Hygiene, Igor, vol. i., p. 85.

4 Ibid., I902, vol, ii., pp. 194-205. 
cultures instituted in the following manner:-A stout platinum loop, previously sterilised, was dipped into the centre of the milk, and three parallel streaks made upon the oblique surface of the medium in one tube. Then without redipping the loop in the milk, similar streaks were made consecutively on the oblique surface of the media in four other tubes. The tubes were incubated at $37^{\circ} \mathrm{C}$. The following day a growth was observed in Tube 2, resembling a typical diphtheria colony. A subculture was prepared in Löffler serum, and cover glass specimens made. Subcultures were also made, and a pure culture of the organism suspended in broth was injected beneath the skin of a guinea-pig ( 563 grammes). The biological characters and staining properties, as well as the post-mortem signs, all indicated a true diphtheria bacillus. Bowhill also centrifugalised samples of the milk and made cultures from the sediment. But the result was negative.

Eyre also, by shaking the sample bottles, uniformly distributed the suspended particulate matter of the milk. He then plated out and enumerated 10,960 micro-organisms per c.c. The milk was also very thoroughly centrifugalised (roughly 20,000 revolutions). On removing the tubes from the centrifuge, the milk in each was seen to have separated into three distinct layers. The uppermost, consisting of cream, formed practically one quarter of the column of fluid; below this came the separated milk: whilst the rounded portion of the lower extremity of the tube was filled with sediment. The contents of one tube were used for microscopical examination. The cream in other tubes was used for culturing on blood-serum, after the manner adopted by Bowhill. The centrifugalised sediment in the remaining tubes was similarly planted on blood-serum. All the cultures were incubated at $37^{\circ} \mathrm{C}$, and showed a growth next day. In fourteen out of the twenty cultures made, organisms morphologically resembling the $B$. diphtherice were present. Every "cream" culture possessed them, but only three out of five "sediment" cultures appeared to do so. These organisms, on being isolated and tested in the usual way, were found to be bacilli of diphtheria.

Klein relied upon animal inoculation. The milk to be tested was injected subcutaneously into the groin of a guinea-pig. By the fifth day, the inguinal glands were found to be swollen to about the size of a filbert, and surrounded by soft œedematous tissue. Film specimens were made from the juice of the incised gland and stained. They showed numerous bacilli resembling the diphtheria bacillus in size and shape. Cultures made on agar and ascitic agar 
brought forth numerous colonies of a pure culture of $B$. diphtheria. A broth culture was made from one of these colonies, and after forty-eight hours incubation at $37^{\circ} \mathrm{C}$. showed the characters of a diphtheria culture, with formation of acid. One quarter of a c.c. was injected subcutaneously into the groin of a guinea-pig, with the result that the animal died in thirty-six hours with a hæmorrhagic tumour in the groin, and deep congestion of the viscera. Films and cultures made from the fluid of the tumour showed the diphtheria bacilli in pure culture. Stained by Neisser's method, the bacilli gave a positive result. It was also tested by inoculation into a guinea-pig with $\frac{1}{10}$ c.c. of diphtheria antitoxin. The animal remained alive and well.

Dean and Todd centrifugalised the milk which they examined, and blood-serum tubes were inoculated from the deposit. In the cultures, a few diphtheria-like bacilli were demonstrated, and by further plating, pure cultures were obtained. Morphologically, the bacilli were found to be typical Klebs-Löffler bacilli of medium size. Their virulence was tested as follows:-A twenty-four hours' culture of the bacillus in alkaline broth, was inoculated into five guinea-pigs, thus :-
1. G.P. weighing $25^{8}$ grs. 2 c.c. of culture .
dead in 48 hours.
2. G.P
4 c.c. of culture.
3. G.P. "
258 "
258 "\{ 4 c.c. of culture $\begin{aligned} & \text { I c.c. diph. antitoxin } \\ & \text {. }\end{aligned}$
ived, very slight
4. G.P. " 248 , "
5 c.c. diph. antitoxin, and 24 hours later 2 c.c. of culture .
5. G.P. "
250 "
5 c.c. diph. antitoxin, and 24 hours later 4 c.c. of culture .

The diphtheria antitoxin employed in these experiments contained 300 units per c.c. (Ehrlich). The toxins of the organisms were also similarly tested, thus:-A pure culture of the bacillus was placed in nine tubes of 10 c.c. of alkaline broth in an incubator at $36^{\circ} \mathrm{C}$. for ten days. The total mixture of the nine tubes was then filtered through a Berkefeld filter. The toxins thus obtained were tested as follows :-

\begin{tabular}{|c|c|c|c|c|c|c|c|}
\hline \multirow{2}{*}{ G.P. } & \multirow{2}{*}{$\begin{array}{c}\text { Weight in } \\
\text { grammes. }\end{array}$} & $\begin{array}{c}\text { Wose of } \\
\text { toxin. }\end{array}$ & \multicolumn{5}{|c|}{ Weight of G.P. on successive days. } \\
\cline { 4 - 8 } & & 1 & 2 & 3 & 4 & 5 \\
\hline 1 & 275 & 1.0 & 255 & Dead & $\ldots$ & $\ldots$ & $\ldots$ \\
2 & 258 & 0.5 & 250 & Dead & $\ldots$ & $\ldots$ & $\ldots$ \\
3 & 278 & 0.2 & 265 & Dead & $\ldots$ & $\ldots$ & $\ldots$ \\
4 & 270 & 0.1 & 255 & 252 & 245 & 245 & Dead \\
5 & 255 & 0.05 & 240 & 235 & 225 & 225 & 230 \\
\hline
\end{tabular}


Lastly, the virulence of toxin-free cultures of the bacillus, obtained by growing the bacilli for twenty-four hours in broth containing I per cent. of glucose was tested on a guinea-pig weighing 250 grammes, with the result that it killed at 0.2 c.c.

\section{Channels of Infection in Diphtheria}

What is the relationship of diphtheria in man to the milk supply? How does the milk supply become infected? These questions it is not possible at present to answer with any measure of exactness. But a consideration of their subject-matter raises several points concerning diphtheria, to which reference must here be made. In the first place, it is now generally held that the $B$. diphtherice has a comparatively wide distribution in nature. Whilst it appears not to be conveyed by water, it is believed that certain conditions of soil favour its growth as a saprophyte. But this is not proved. Eyre states that several groups of bacilli, having similarities to the diphtheria bacillus, may be present in milk, and the only way in which they are to be differentiated is by biological and pathogenetic characters. ${ }^{1}$ In the second place, it has been proved that persons suffering from diphtheria are foci of infection. The exact channels of infection differ under varying circumstances; but, in general, the source of infection is the throat and mouth of the patient. Anything which comes into contact with the mucous membrane becomes infected. Thus handkerchiefs, sweets, children's toys, etc., may act as the vehicles of contagion. The mucus and saliva may also be infective, and in speaking, kissing, coughing, or expectorating, such mucus (probably rich in bacilli) may be disseminated in very fine particles, and so carry the disease. It is by such means that the disease is spread. Richardière and Tollemer ${ }^{2}$ and others have proved that the dust floating in the air of a diphtheria ward may contain large numbers of diphtheria bacilli, and in this way milk and other foods may become contaminated.

There remains another characteristic which it is not unlikely plays a significant part in the propagation of the disease from person to person, and therefore may at any moment convey the contagion to milk, and that is the long period during which the human throat may remain infective. Professor Sims Woodhead has recently stated that "the persistence of the diphtheria bacillus for periods up to eight weeks is of very common occurrence whether antitoxin be given or not; indeed, the majority of cases

1 Brit. Med. Jour., 1900, vol. ii., p. 427.

${ }^{2}$ Gasette des Maladies Infantiles, 1899, No. ro. 
appear to retain bacilli in the throat for from two to nine weeks." 1 After the ninth week, the number falls off very rapidly, but not infrequently the bacillus remains in the throat for 100 days, and it has been known to remain more than 200 days. This persistence of diphtheria bacilli in the throat may play an important part in determining the spread of the disease by means of such cases which are supposed to be no longer infective. "For it is now a matter of common experience that so long as these diphtheria bacilli, even the less virulent forms, remain in the crypts of the tonsils, etc., so long is the patient a centre of infection, the diphtheria bacilli present resuming, under favourable conditions, their more virulent form " (Woodhead). In this way diphtheria bacilli can be readily transmitted by patients who are apparently no longer suffering from the effects of the disease, to those who have weak or ulcerated throats. In precisely a similar manner, may the bacillus be conveyed to articles of attire and articles of food, such as milk (as in outbreak at Leeds, 1903).

Further, it has now been shown that in apparently healthy persons who have not suffered from diphtheria, the $B$. diphtherice may be present. Löffler found diphtheria bacilli in the throats of 4 out of I 60 healthy children, and Park and Beebe found similar virulent bacilli in 8 out of 330 "healthy" throats. Hewlett and Murray found 15 per cent. of the children in a general hospital had diphtheria bacilli in their throats. ${ }^{2}$ Kober $^{3}$ examined diphtheria cultures from two series of healthy persons. The first series comprised 128 individuals known to have been in recent contact with patients suffering from diphtheria. The diphtheria bacillus was found in the throat of 8 per cent of these. The second series comprised 600 individuals who had not recently come into contact with any diphtheria cases-from five of these a bacillus similar to that of diphtheria was isolated. It was rather short with swollen ends, and was not pathogenic to guinea-pigs.

It is probable that, as a rule, "healthy" throats do not yield the true $B$. diphtheria unless the persons have been in contact with infected persons. ${ }^{4}$

1 Report on the Bacteriological diagnosis and Antitoxic Serum Treatment of cases admitted to the hospitals of the Metropolitan Asylums Board, I895-1896, by Professor Sims Woodhead, Sect. 2, I902, pp. I4, 28, 3 I.

2 Brit. Med. Jour., Igor, vol. i., p. 1474.

3 Revue des Maladies de I Enfance, Juillet, 1900.

- Since the above was written a paper on the distribution of the diphtheria bacillus, by Graham-Smith, has appeared in the Jour. of Hygiene, 1903, pp. 216-257, which should be consulted. 


\section{Abstracts of Typical Milk-BORne OUtbreaks OF DIPHTHERIA ${ }^{1}$}

Little Horton, Bradford, 1879 (September).

Total number of cases

Deaths

Number of cases amongst drinkers of suspected milk .

Percentage on total cases .

Number of polluted milk sources Number of milkmen .

Circumstances implicating the milk supply.-At the farm supplying the milk a child had been ill with sore throat commencing on 18th August. Another child was ill in bed with unmistakable signs of diphtheria, commencing 3oth August. The milkcans were dirty and were kept in a scullery in which was a sink with untrapped pipe. Near the milk was a wash-tub, half full of dirty water, and resting on the milk-cans were two bundles of dirty linen from the bed and person of infected child. Houses attacked were not insanitary, and there had been no communication between the families, who were strangers to each other. But they all obtained their milk supply from this source.

Probable exciting cause.-Human source.

Reporter and reference.-Dr $\mathrm{H}$. Butterfield (Med. Off. of Health). Brit. Med. Jour., I88o, vol. i., p. 953.

Kulburm and St John's Wood, 1879.

Total number of cases . . 264 Deaths . . . . 38 Number of families invaded . 116

Circumstances implicating the milk supply. - In an area one and a half miles in diameter, there occurred between 2nd March and 15th June, 264 cases of diphtheria ; 78 households showed their first attacks during four weeks ending 25th May. Cases most numerous in central part of area, where in four weeks there occurred 175 cases in 70 households. It was found that one man $(\mathrm{X})$ owned two dairies, one at Kilburn and one at Muswell Hill. The milk from these two dairies was not mixed, but the cows were interchanged sometimes. $\mathrm{X}$ supplied two dairies, A 49 gallons daily and B 24 gallons daily. A study of the households in the most infected area showed that one-fifth of them obtained milk from A and B. Of 236 households supplied by A, 37 were invaded or $\mathbf{1 5}$ per cent. Of 237 households supplied by $\mathrm{B}, 3 \mathrm{I}$ were invaded or 13 per cent. Of 2227 households supplied by other milkmen 30 were invaded, or $\mathrm{I} \cdot 3$ per cent. The occurrence of the disease was especially marked among children, as will be seen in the results of inquiry into 233 of the 264 cases.

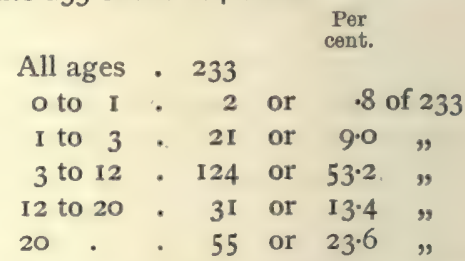

Exciting cause. - Undetermined. (?) Cow disease.

Reporter and reference. - W. $\mathrm{H}$. Power. Loc. Gov. Bd. Rep., 1879.

\section{Rugby, 1881 (March).}

Total number of cases . . 100

Number of cases amongst drinkers of suspected milk : I00 Percentage on total cases . . 100 Number of families supplied by milkman . . . . 40 
Number of such families invaded

Percentage .

Number of polluted milk sources

Circumstances implicating the milk supply.-This outbreak occurred at Rugby School, three houses being attacked with some 30 cases in each house. These houses alone were served with the infected milk. The milk of a cow suffering from "garget" was mixed with milk from healthy cows.

Apparent exciting cause.-(?) Cow disease.

Reporter and reference.-G. Wilson, M.D. (Med. Off. of Health). Brit. Med. Jour., I881, vol. ii., p. 415.

\section{Hendon, 1882.}

Total number of cases

Deaths

Number of cases amongst drinkers of suspected milk

Percentage on total cases .

Number of families supplied

by milkman . . . .

Number of such families invaded

Percentage .

Number of polluted milk sources

Number of milkmen

Circumstances implicating the milk supply.-Sewage gained access to dairy pond, the water of which was used for washing dairy utensils. Certain families habitually using the milk in a boiled state wholly escaped invasion. Disease specially in evidence where taken in large quantities and used in an unboiled state. Evidence that immediately before outbreak milk from particular dairy was "ropy or stringy." In some cases it was returned by the customers on account of this feature. Some attributed this to unclean utensils, others to cow disease of some kind. The farmer himself adopted the latter theory. No "garget" was found, or any udder disease in any of the herd supplying the milk.
Probable exciting cause-Contaminated water supply.

Reporter and reference.-W. H. Power, F.R.S. Loc. Gov. Bd. Rep., 1883-84, pp. 42-48.

Devonport, 1882 (December).

Total number of cases . . . $3^{1}$

Deaths . . . . 5

Number of cases amongst drinkers of suspected milk . 27

Percentage on total cases . $\quad$. 87

Number of families supplied by milkman . . . 256

Number of such families invaded 18 Percentage . . . 7

Circumstances implicating the milk supply.-Dr Parsons formed the opinion that the facts of this outbreak were consistent with contamination of milk by infective material of partial amount, and of occasional occurrence. Cause of infection may have been some condition of farm or dairy premises, or by milk-cans being wiped out with cloths which hung up in dairy yard and which might have contracted impurities from the atmosphere. The outbreak was limited to persons in good social position. Distributing milk-shop had a case of diphtheria next door, the yards to the two houses being closed in by high walls, and the air being thus stagnated.

This outbreak affected 18 households, in 8 of which there were multiple cases. All the patients were of good social position. Twenty-seven of the $3^{1}$ patients derived their milk supply from a particular dairy. The dairy supplied about 500 families, so that those attacked with diphtheria formed a small percentage of the whole. Twenty of the $3^{1}$ cases were above the age of childhood.

Probable exciting cause.-Indirectly from human source.

Reporter and reference. $-\mathrm{H}$. $\mathrm{F}$. Parsons, M.D. Loc. Gov. Bd. Rep., 
1883-84, p. 49 ; Brit. Med. Jour., 1883, vol, i., p. 876 .

\section{Canterbury, 1888 ( /uly).}

Total number of cases

Number of families supplied by

milkmen .

Number of such families invaded

Percentage .

Circumstances implicating the milk supply.-Milkman and family suffered from "sore throat." Of 422 houses canvassed in one locality 196 were supplied wholly ( 160 ) or partly (36) by the suspected milk, Of these 196 households II 5 were invaded by diphtheria in a period of 8 days, and only 5 of the remaining 226 so invaded. One patient had the milk only at a friend's house in tea.

Probable exciting cause,-Indirectly from human source.

Reporter and reference.-Dr Washer (Med. Off. of Health). Brit. Med. Jour., 1886, vol. ii., p. 397.

\section{Yorktown and Camberley, Surrey, 1888 (October).}

Total number of cases . . 140

Deaths . . . . . 16

Number of cases amongst drinkers of suspected milk . 124

Percentage on total cases . $\quad . \quad 89$

Number of families supplied by milkmen .

Number of such families invaded

Percentage . . . . . $5^{\mathrm{I}}$

Number of polluted milk sources I

Number of milkmen . . several

Circumstances implicating the milk supply.-Human agency and polluted water for washing purposes were both supposed by some to have brought about the epidemic. But at a time immediately preceding the outbreak the milk of two cows which had just calved was added to the general supply. Of the 57 houses invaded 48 (or 84 per cent.) took the implicated milk.
Of the 140 individuals attacked 124 (or 88.5 per cent.) were consumers of the milk, and 93 per cent. of the 140 were attacked within the same ten days, 8th to 17 th October. There was also evidence to show that the amount of milk consumed determined the severity of the disease. Persons of the better class had averaged 5.2 pints per household daily, whereas among the cottagers and tradesfolk it had averaged only 0.8 pint daily. The disease affected various classes, both rich and poor, but 84 per cent. of the milkman's better class customers were attacked compared with 22 per cent. of the poorer class. A broad distinction between the two classes was found in the amount of milk which they were respectively in the habit of taking. Of children under 15 years of age 54 per cent. were attacked in the better class families, and 6 per cent. in the poorer class.

Probable exciting cause.-Undetermined.

Reporter and reference. - W. $\mathrm{H}$. Power, F.R.S. Loc. Gov. Bd. Rep., I 886, pp. 31 1 -326.

\section{Melrose and Malden, Mass., U.S.A.,} 1887.

Total number of cases : . 50 Deaths . . . . . I4 Number of polluted milk sources I

Circumstances implicating the milk supply. - The disease occurred in 30 families, 24 of which derived their milk directly from milkmen having diphtheria in their families.

Reporter and reference.-Dr J. S. Clark. Boston Med. and Surg. Jour., I887, vol. ii., p. I00.

Fnfleld, 1887 (November and December).

Total number of cases . . $5 \mathrm{I}$

Deaths . : . . . 12

Number of cases amongst

drinkers of suspected milk . 42 
Percentage on total cases

Number of families supplied by milkman .

Number of such families invaded

Percentage -

Number of polluted milk sources Number of milkmen

Circumstances implicating the milk supply.-Dr Bruce Low concluded that this recrudescence of diphtheria (following a "school" epidemic) was due to a pollution of the milk supply probably from human sources. These 5I cases were limited alike in locality and social status of sufferers, the latter being of better class altogether and quite unlike the earlier epidemic. All the cases at the end of November and in the first three weeks of December, and all the deaths in this period, occurred in persons consuming milk from one source.

Probable exciting cause.-Indirectly from human source.

Reporter and reference.-R. B. Low, M.D. Loc. Gov. Bd. Rep., I888, pp. 123-I 32.

Devonport, 1888.

Total number of cases . . 61 Deaths . . . . . 17

Circumstances implicating the milk supply. - Of the $6 \mathrm{I}$ cases of diphtheria it was possible to get particulars as to milk supply from $50: 47$ of this number obtained their milk from a common source. The first 35 of the 61 cases obtained their milk from this source. Eight of the cows at the implicated dairy showed signs of "cow-pox"small scabs and ulcers at the base of the teat.

Probable exciting canse.-Undetermined.

Reporter and reference.-Quoted by Thorne Thorne in his Milnoy Lectures in $1891, p .162$. The investigation was made by the Medical Officer of Health, Dr May.

\section{Croydon, 1890 (October).}

Total number of cases $\quad . \quad$. 19I

Deaths . . . . . 38

Number of cases amongst drinkers of suspected milk . 123

Percentage on total cases . . 65

Number of families supplied by milkman . . . . . 124

Percentage . . . . 40

Number of polluted milk sources I

Number of milkmen . . . 2

Circumstances implicating the milk supply.-In this case teat eruptions were discovered upon the cows yielding the milk supply. Dr Klein reported these eruptions to be similar to those met with in diphtheria outbreaks traced to milk of diseased cows. The milk was from one source supplied by one dairy, the customers of which suffered to the extent of 40 per cent. of households supplied. Same milk formed one-seventh of the supply of another dairy, the customers of which suffered in to per cent. of households taking it. Cases ceased to arise when the implicated milk was stopped.

Apparent exciting cause.-(?) Cow disease.

Reporter and reference.-Dr Philpot (Med. Off. of Health). Rep. of Medical Officer of Health, and Brit. Med. Jour., I89I, vol. i., p. 47 o.

\section{Worcester, 1891 (November).}

Total number of cases . . 6

Deaths . . . . . I

Number of cases amongst drinkers of suspected milk . 6

Percentage on total cases . . 100

Number of families supplied by milkman .

Number of such families invaded

Percentage. . . . . I00

Number of polluted milk sources I

Number of milkmen . . . I

Circumstances implicating the milk supply.-Cows yielding supply had a pustular eruption of the teat, and were 
in a febrile condition. The pustules in some cases broke during the act of milking. Five children and the mother in the farmer's family were affected. One child died. The mother and son who milked the cows were first attacked. There was no diphtheria elsewhere in the locality.

$$
\begin{aligned}
& \text { Apparent exciting cause.-(?) Cow } \\
& \text { disease. }
\end{aligned}
$$

Reporter and reference.-Dr Thursfield (Med. Off. of Health). Public Health, I89 I-92, p. 130.

\section{Surbiton, 1891 (December).}

Total number of cases . . 27

Deaths . . . . . 3

Number of cases amongst drinkers of suspected milk . 27

Percentage on total cases . . I00

Circumstances implicating the milk supply. - The inmates of the farm supplying the milk suffered from diphtheria, and all the persons infected consumed milk from this farm.

Probable exciting cause. - From human source.

Reporterand reference.-Dr Coleman (Med. Off. of Health). Public Health, I89I-92, p. 158.

Glasgow, 1892 (August).

Total number of cases $\quad . \quad 224$

Circumstances implicating the milk supply.-Dairy and farm sanitarily good. Nearly all of a herd of 53 cattle suffered from teat-eruptions leading to ulceration, in some cases all the teats were affected. The "Hendon streptococcus" was isolated from the lymph. Two varieties of eruption in question shown by experiment on calves, one being "true vaccinia," the other a non-vesicular eruption like that of the Camberwell outbreak. No desquamation as in Hendon disease and at Camberwell. Farmer and three other milkers had eruptive sores on hands, one being com- pelled to stop work. Between 6th and 8th August from $60-80$ cases notified. Milk supply stopped on 8th, and disease ceased on 12th.

Apparent exciting cause.-(?) Cow disease.

Reporter and reference.-Dr Russell (Med. Off. of Health). Brit. Med. Jour., I897, vol. ii., pp. 432, 666.

Limekilns, Fifeshire, 1892 (August-December).

Total number of cases . . 28

Deaths . . . . . 4

Number of cases amongst drinkers of suspected milk . 16 Percentage on total cases . $\quad 57$ Number of families supplied by milkman . . . . . I Number of such families invaded $\quad 14$ Number of polluted milk sources I Number of milkmen . . . I

Circumstances implicating the milk supply.-A patient suffering from diphtheria was walking about dairy premises and byres after notification. Also direct communication between those attending the patient and those managing the dairy. The disease broke out amongst a large proportion of the families using the milk from this particular dairy.

Probable exciting cause. - From human source.

Reporter and reference. - Dr Nasmyth (Med. Off. of Health). Annual Rep., 1892.

\section{Ashtabula, Lake Errie, 1894.}

Total number of cases . . I00

Deaths . . . . 24

Number of families supplied by

milkman invaded about one-third Number of polluted milk sources I Number of milkmen . . . I

Circumstances implicating the milk supply. - Out of the 100 cases, 64 occurred between $5^{\text {th }}$ and $13^{\text {th }}$ December. The remainder occurred at different dates up to 3 rst December. 
The 49 houses in which the 64 cases occurred were widely separated and situated in districts generally free from the disease. $B$. diphtheria was isolated, and there can be little doubt that "all the cases reported as diphtheria were true diphtheria, and that some mild cases had been overlooked."

The town received its milk from one of two sources, $\mathrm{X}$ and $\mathrm{Y}$. The cases of diphtheria were entirely confined to houses taking $\mathrm{Y}$ milk. On inquiry at the $\mathrm{Y}$ dairy farm it was found there had been a case of bad "sore throat" with painful swallowing, reddened fauces, congested tonsils, and enlarged throat. The dairy was wellordered. The patient had assisted at the dairy work whilst suffering acutely from sore throat.

The proportion of milk drinkers affected was much greater than in individuals not taking milk, or only drinking it in tea or coffee. Out of the 49 households it was practicable to investigate 44 . It was found that 32 of these received their milk directly from the patient having the sore throat, the remaining 12 being served from dairy, but by a different milkman. No evidence of cow disease.

Probable exciting cause.-Human infection.

Reporter and reference.-Dr W. T. Howard, Jr. American Jour. of Med. Sciences, December 1897.

\section{Senghenydd, South Wales, 1888} (December).

Total number of cases

Number of cases amongst drinkers of suspected milk . Number of polluted milk sources Number of milkmen . . .
Circumstances implicating the milk supply. - This outbreak was evidently due to milk, but there is no evidence to show how the milk became infected. " $B$. diphtherice was isolated from the suspected milk."

Reporter and reference. - J. W. Thomas, M.R.C.S. (Med. Off. of Health). Jour. of State Medicine, November 1899, p. 705.

\section{Edinburgh, 1900 (June).}

Total number of cases

Number of polluted milk sources I Number of milkmen . . : 5

Circumstances implicating the milk supply. - There appeared to be a community of milk supply in this outbreak, and on visiting the dairy situated at Liberton, it was found that a young man assisting in the business was suffering from unrecognised diphtheria. Diphtheria was also somewhat prevalent in Liberton. Sixty cases were traced directly to milkshops supplied from this dairy. A considerable proportion of the patients were adults over 20 years of age. After the stoppage of the suspected milk the cases ceased.

Probable exciting cause.-Human source.

Reporter and reference.-Sir H. D. Littlejohn, M.D. (Med. Off. of Health). Annual Rep., I900, Pp. 39, 40.

Other diphtheria outbreaks traced to milk.-Sutton, 1877 ; Kilburn and St John's Wood, 1878 (see p. 336) ; Weybridge, 1878; Leatherhead, 1878; Addlestone, 1878; Surbiton, 1880; Aberdeen, 1881 ; Cardiff, 1883; Ealing, 1887; Barking, I888; Hendon, 1889; Finchley, 1889 and 1894; Highstown, U.S.A., I893; Germantown, Philadelphia, I898; Leeds, I903. 


\section{Throat Illnesses}

In several outbreaks of disease due to an infected milk supply it has appeared that the only condition present has been "sore throat." Under the heading of "throat illness" we propose to make brief reference to such outbreaks which were apparently not diphtheria on the one hand, or scarlet fever on the other. Although it is true that records of such outbreaks are few and far between, it must not be supposed that they are rare. We think in all probability they are comparatively common. But as the condition is not notifiable, it is but seldom that a record is obtained of the cases occurring. We think it safe to assume that a year never goes by in which there are not outbreaks of sore throat or tonsillitis due to milk or cream. There is some evidence to show that the latter is more a vehicle in such epidemics than the former.

Signs and symptoms. - The usual symptoms are congestion of the tonsils and mucous membrane of the throat, with sometimes ulceration, enlargement of the cervical glands, and some pyrexia, and general malaise. In not a few instances there has been observed various kinds of rash which have generally been of an evanescent character. Where the throat illnesses have occurred contemporaneously with outbreaks of scarlet fever or diphtheria, it is not unlikely that the condition was in reality one or other of these diseases. In South Kensington, in 1875 , there was an outbreak of disease which attracted much attention at the time, and was officially investigated. ${ }^{1}$ The illness was traced to some cream consumed at a dinner party, and in all twenty persons suffered, some of whom had scarlet fever, and others only sore throat. But the investigation showed that in the district from which the cream was obtained I I 9 cases of sore throat had occurred. Dr Darbishire described an outbreak of 18 cases of sore throat at Oxford in I882, the condition being characterised by marked severity of throat symptoms and a disproportionate amount of constitutional symptoms. $^{2}$

In 1892 there was an extensive outbreak of sore throat in Upper Clapton. Dr King Warry, describing the symptoms in the Practitioner, at the time held that the pathological condition was scarlet fever in a mild form. His reasons for this view were three : (a) scarlet fever attacked one member of a family, and the sore throat disease other members who had previously had scarlet

1 Report of Medical Officer of Local Government Board, 1875, vol. vii., p. 80.

2 St Bartholomew's Hospital Reports, vol. xx. 
fever; $(b)$ both scarlet fever and sore throat patients were isolated together, but those suffering from sore throats did not contract the scarlet fever; and $(c)$ some of the patients suffering from sore throat had at the same time certain symptoms simulating scarlet fever, such as desquamation of the skin, kidney disease, and rheumatic symptoms. With this view of the specificity of throat illness under similar circumstances Dr Parsons agrees. ${ }^{1}$ In the Upton and Macclesfield scarlet fever outbreak of 1889 , there were 40 cases of sore throat which were apparently related to scarlet fever for the following reasons:- (a) The sore throat occurred in older persons, in whom rashes are less prone to occur, and who had had scarlet fever; $(b)$ in some cases there was skin desquamation; $(c)$ when the children suffered from scarlet fever the adults in the same house suffered from sore throat; $(d)$ two cases of diphtheria at the same period showed symptoms simulating scarlet fever; and (e) pyrexia and delirium were present in the more severe cases of sore throat. In 1894 Dr Kenwood reported on an outbreak of sore throat at Finchley, with symptoms showing possible alliance to diphtheria. The disease attacked persons in good social position, and particularly adults. ${ }^{2}$

It is impossible to generalise upon a few outbreaks, but these facts are of a nature impossible to discard, and point clearly to some relationship. Nor are such common features exceptional in ordinary scarlatinal outbreaks.

It may be said in passing that the incidence of throat illness in certain milk supplies, is, so far as has been investigated, similar to milk-borne scarlet fever. The record of the Upper Clapton outbreak, for example, is as follows :-

\begin{tabular}{|c|c|c|c|c|c|}
\hline & & & Totals. & $\begin{array}{l}\text { Supplied by } \\
\text { the implicated } \\
\text { Farm. }\end{array}$ & $\begin{array}{l}\text { Supplied by } \\
\text { other } \\
\text { Dairymen. }\end{array}$ \\
\hline $\begin{array}{l}\text { Households } \\
\text { Sore throat households } \\
\text { Percentage attacked by } \\
\text { Sore throat cases. }\end{array}$ & sore throat: & $: \quad:$ & $\begin{array}{l}170 \\
54 \\
31-7 \\
84\end{array}$ & $\begin{array}{l}99 \\
45 \\
45 \cdot 5 \\
73\end{array}$ & $\begin{array}{l}71 \\
9 \\
12.6 \\
11\end{array}$ \\
\hline
\end{tabular}

Many other illustrations might be cited of similar incidence upon drinkers of implicated milk (such, for example, as occurred at Macclesfield, Hackney, and other places).

1 Report of Medical Officer of Local Government Board, 1889.

2 Brit. Med. Jour., I895, vol. i., p. 1167, and Medical Officer's Annual Report, 1894. 
We may now consider briefly several typical sore throat outbreaks, which practically stand alone, and have a less intimate relationship to scarlet fever.

\section{Milk-borne Sore Throat Outbreaks}

I. In the oft-quoted Aberdeen outbreak of sore throat traceable to milk which occurred in $188 \mathrm{I}$, about 300 individuals were affected and some 90 out of the I ro families supplied with the implicated milk. No case occurred in any family not supplied with this milk. The disease commenced with severe rigors, which were followed by high fever. The throat and tonsils were mainly affected, though there was no false membrane produced. Three deaths occurred. It was believed that the virus gained access to the milk from the water supply.

2. In the same year, Dr Wilson reported a similar epidemic among the boys at Rugby School. Some 90 boys were attacked in three school-houses supplied by one milkman, who did not supply any other houses in the school. But he supplied houses in the town, and of these nearly 50 per cent. were attacked with sore throat. Inquiry showed that some of the milk used had been obtained from a cow suffering from mastitis, and Dr Wilson attributed the outbreak to this cause. ${ }^{1}$ A similar outbreak took place in Edinburgh in I888, and was investigated by Cotterill and Woodhead.

3. At Dover in 1884 there was a sudden and severe outbreak of sore throat in a localised area of good-class houses, affecting 205 persons, who all obtained milk from one particular farm. The chief symptoms were local inflammation of the throat, enlargement of lymphatic glands in neck, and vesicular eruptions preceding and accompanying the inflammation. In 19 streets out of 42 invaded every house supplied with the implicated milk was attacked. There was generally an exact correspondence between the milk supply and the houses invaded by the disease. The number of persons attacked in each invaded house averaged $2 \cdot 5$. In one house the servants had the implicated milk, and all suffered, whilst the family had a special supply from the same dairyman, and did not suffer. The "nursery milk" from the same dairyman was also free from disease, and children in certain families having this special supply were free from the infection, whilst the adults using the mixed supply were attacked. The dairyman obtained his supply from 12 cows of his own, and from three farms in the country.

1 Brit. Med. Jour., 188r, vol. i., p. 657 ; vol. ii., p. 4 15. 
On one of these latter apthous fever had broken out, and it was from this farm that the dairyman obtained his implicated milk and cream. Moreover, when the supply from this farm was diverted temporarily it set up a simultaneous second outbreak of sore throat elsewhere. ${ }^{1}$

4. In I 890 there occurred an epidemic of sore throat at Craigmore which was referred to milk infection. The number of cases was 80 . The disease manifested itself chiefly in the form of severe sore throat, but in a number of the cases erysipelas developed in addition. The milk appears to have been infected by two milkmaids who were suffering from sore throat. Those attacked most severely had drunk most of the implicated milk. The period of incubation was three or four days. The disease was chiefly confined to the mouth and throat; in most of the cases there was high fever, general constitutional disturbance, rigors, vomiting, and muscular pains. The disease was differentiated from either diphtheria or scarlet fever. A dog and cat which had a good deal of the milk became very ill with "severe inflammation of the throat." 2

5. Two comparatively small milk-borne outbreaks of "follicular tonsillitis" were reported in 1897, one in Anglesey $^{3}$ and the other at Surbiton. ${ }^{4}$ In the Anglesey outbreak the total number of cases was 15, and there were no deaths. They all partook of a common milk supply implicating one dairy. Several persons and children in the affected families who consumed the same milk as the patients, but in a boiled state, escaped the tonsillitis. All the patients had the same milk supply. The milk was bacteriologically examined, and Staplylococcus pyogenes and Streptococcus pyogenes were found, but no $B$. diphtherie. Bacteriological examinations of the patients' throats yielded precisely similar results. The total number of cases at Surbiton was 30 , and the milk supply was derived from one milkman. A man whose business it was to milk the cows was found to be out of health, with well-marked tonsillitis, and suppurating whitlows on both hands. Dr Coleman was able to trace more than 30 cases, some very severe, and he assumed that in all probability there were others. No other cause except milk was traceable.

6. Outbreak at Hackney, 1900.-In April and May 1900, an outbreak of septic sore throat occurred in North Hackney. The

1 Practitioner, 1884, vol. i., p. 467 (Dr M. K. Robertson).

2 Glasgow Med. Jour., 1890, vol. xxxiv., pp. $241-258$.

3 Brit. Med. Jour., 1897, vol. ii., p. 339 (Dr C. Grey-Edwards of Beaumauris).

+ Annual Report of Medical Officer of Health, 1897 (Dr. Coleman). 
medical man in whose practice the first cases appeared, informed the Medical Officer of Health (Dr King Warry), who, in due course, made a full investigation and reported to his Authority. ${ }^{1}$ The leading symptoms of the illness were as follow :-

Symptoms.-(a) In every case there was tonsillitis (not follicular). (b) In two cases there was in addition superficial ulceration of the tonsil. (c) In every case there was considerable swelling of the cervical lymphatic glands, more marked on the one side than the other-in two cases the swelling was very great. (d) In one case the lymphadenitis proceeded to suppuration. (e) In every case the temperature was raised, in a few cases it was $105^{\circ} \mathrm{F}$, and there were rigors. In all the cases the temperature persisted after the tonsillitis had disappeared, lasting in nearly all for at least a fortnight. $(f)$ The temperature assumed the remittent type, and there was in every case profuse sweating at nights. $(g)$ There was great prostration in most cases, and considerable cachexia in all. (h) In one case acute septicæmia supervened, followed by acute septic pneumonia and death. (i) In two cases in the same family acute nephritis with hæmaturia came on about a week after the onset of symptoms. ( $j$ ) In one case a child had an attack of purpura hæmorrhagica. ( $k$ ) In all cases convalescence was protracted. In addition there was a marked tendency for multiple cases to occur in families, and while no age or sex was exempt, a very large number of the sufferers were children; these features helped to direct suspicion to the milk.

The medical officer addressed himself to obtaining information from the medical men in the district, a bacteriological examination of milk from the implicated dairy, and a house-to-house visitation in the affected area. Septic and infectious sore throat being not notifiable, special inquiries were necessary to discover the prevalence of the disease.

No less than $15 \mathrm{I}$ cases of sore throat illness in 88 households occurred, with very few exceptions, in Upper Clapton and Stamford Hill. Of these totals, 138 cases occurred in 75 households, all of which were supplied with milk by one particular milkman who shall be termed $\mathrm{X}$, the remaining $\mathrm{I} 3$ cases occurring in 13 households supplied with milk by seven other dealers. That is to say, over 85 per cent. of the households in which sore throat illness occurred were supplied with milk by $X$, and less than 15 per cent. by other dealers. One or two remarkable instances occurred

1 Annual Report of Medical Officer of Health for Hackney, 1900, pp. $60-69$ (J. King Warry, M.D.). 
in distant parts of the parish in households supplied by $\mathrm{X}$, but where his milk was not expected to be supplied. In some of the families where sore throats existed, the milk was boiled before use, and where this practice obtained the sore throat disease was milder or absent.

When the above returns were analysed, they showed that sore throat illness had occurred amongst the customers of $\mathrm{X}$ in undue proportions compared with other vendors, which could only be attributed to some infective properties in the milk supplied, except upon the hypothesis that X supplied 85 per cent. of the households in the area from which these replies were received; but this was not so. Dr Warry divided the affected district into two areas, $\mathrm{A}$ and $\mathrm{B}$, and the result of his inquiries in these two sub-districts was as follows :-

(I) AREA "A."

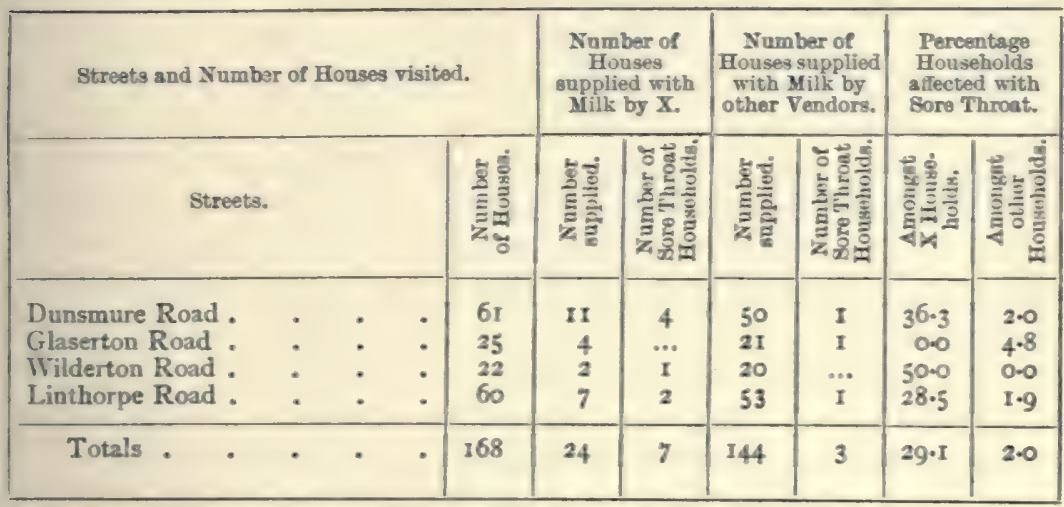

(2) ARea "B."

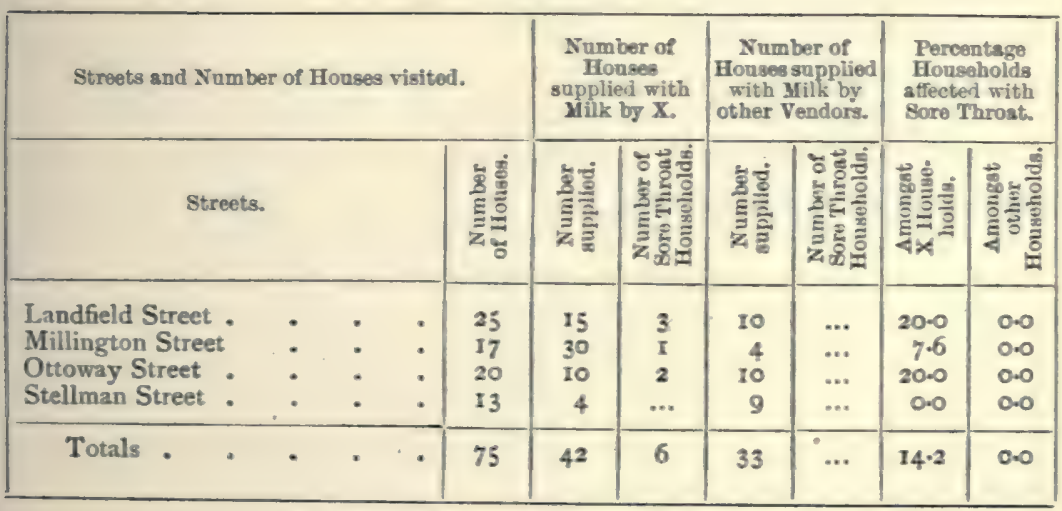


Table "A" shows in the first place that the percentage of households supplied by $\mathrm{X}$ in that area falls very far short of 85 per cent. of the total, being a little over I 4 per cent, and even if allowance is made for considerable losses of customers the incidence of disease would be out of all proportion to his supplies. Further, area " A" shows that while the incidence of throat illness is 29 per cent. amongst X's customers, it is only 2 per cent. amongst all the remaining dealers. In area " $\mathrm{B}$ " the respective percentages of throat illness are:-X's $14 \cdot 2$, others $0 \cdot 0$. It should be added, that the bacteriological examination of milk from X's dairy threw no light on the subject. Dr Warry concludes from a consideration of the whole of the circumstances:-(I) That sore throat disease of a septic character existed almost exclusively in the northern part of Hackney. (2) That this disease differed greatly from ordinary catarrhal sore throat or follicular tonsillitis, being of a septic character, and suggestive of being caused by some article of diet. (3) That nearly all the cases of sore throat in question had occurred in the households supplied by a particular milk vendor. (4) That this milk supply was "X's," and that in some way his milk was the cause of the septic sore throat disease.

7. A sore throat outbreak at Brighton in November I9OI was investigated by $\mathrm{Dr}$ Newsholme. It was complicated with a simultaneous infection of scarlet fever, but reference will only be made here to the sore throat illness. In all there were 18 persons affected, I4 of whom were girls under 17 years of age, scholars at a private school. The remaining 4 were teachers and servants. Out of a total of 29 persons in the school, 18 were affected. The chief symptoms were high temperature, rapid pulse, tonsillitis with fibrinous exudation locally, except on the soft palate. In two cases there was an evanescent rash lasting only a few hours and without desquamation. There were no deaths. The illness was not as far as could be judged scarlet fever, and diphtheria was negatived both bacteriologically and clinically. Dr Newsholme was able after minute inquiry to trace the cases at the school to one of their number, who had come into the way of infection derived from a milk supply contaminated by infectious disease in three families connected with the dairy. One of the points of particular interest in this outbreak is the relationship between simultaneous outbreaks of scarlet fever and sore throat, and the secondary infections arising. ${ }^{1}$

1 Jour. of Hygiene, 1902, vol. ii., pp. 150-169. Annual Report of Medical Officer of Health of Brighton, I9or. 
8. In May I902 an outbreak occurred at Lincoln, affecting a large number of persons. ${ }^{1}$ Dr Brook had seventy-five cases in his own practice. The chief symptoms were erythema of the face, and sore throat. In many cases a drab-coloured fur covered the tonsils. A roseolous, papular eruption, in some cases appearing to be urticarial, occurred in two-thirds of the cases. There was no marked fever, except in cases having complications. The pulse rate was not increased, and no albuminuria occurred. The onset was sudden, and in no case out of the seventy-five investigated by $\mathrm{Dr}$ Brook was infection communicated to others by contact. Nearly all the patients were adults, and well advanced in years. The complication most commonly met with was swelling and tenderness of the cervical glands. With one doubtful exception, all the patients had had milk from the same dairy. Boiling the milk appeared to prevent persons from taking the complaint. The poison seemed to be present particularly in the cream. The differences between the disease and scarlet fever were very marked. ${ }^{2}$ Dr Klein isolated a special organism from swabs taken from the throats of the patients.

9. A sudden outbreak of a severe form of "septic sore throat" occurred at Bedford at the end of June 1902. On 27 th June, the first case occurred; on 29th June, 4 cases occurred; 30th June, I 5 cases occurred ; Ist July, I 3 cases ; 2nd July, 3 cases ; 3 rd July, 2 cases ; and 4 th July to 8 th July, 4 cases-making a total of 42 cases in 22 families. The symptoms included redness, swelling of the throat, fauces, palate, and uvula, with numerous spots, patches of exudation, and in some cases ulcers. The general symptoms consisted of severe headache, giddiness, backache, and pains in the limbs, very much like an attack of influenza. The temperature was about $102^{\circ}$ $103^{\circ} \mathrm{F}$, but in a few cases was higher. In some cases there was gastric and intestinal disturbance. Great weakness was also present.

In every case the milk supply was obtained from the same dairy. On Sunday, 3oth June, many persons consumed cream with fruit, and these included nearly all the worst cases. In some families children who drank boiled milk escaped, whilst

1 Report of Medical Officer of Health, Lincoln, 1902.

2 Lancet, 1902, vol. ii., p. I391. Dr Savill compares this outbreak with one occurring in certain metropolitan infirmaries in 1891 (see Trans. of Medical Society of London, 1892, vol. xv., pp. 103-129), and Mr Lunn states that a disease of this kind has recurred every autumn for six years, with one year's intermission. He has seen several such outbreaks, and believes them to be due to milk. 
parents who consumed unboiled milk or cream were attacked. One man took cream in the form of ice cream, and had a severe attack. ${ }^{1}$

$\mathrm{Mr}$ Gifford Nash made inquiries of the dairy farmer and visited the farm. The cows and milkmen were healthy, the water supply good, the cowsheds clean, and the milk-cans were said to be scalded with boiling water. The arrangements appeared to $\mathrm{Mr}$ Nash to be excellent. Specimens of milk were examined bacteriologically a week or so later, and no disease germs were found at that time. Such probably existed at the time of the outbreak.

On inquiry as to what was done with milk from cows with sore udders, $\mathrm{Mr}$ Nash was informed that it was passed through the separator, as this was stated by the manufacturers "to filter milk, removing tubercle and other disease germs." Mr Nash adds :-

"Outbreaks like this show the weak points in our sanitary system. It is nobody's duty to investigate the cause of an outbreak of septic sore throat, as the disease is not notifiable and therefore the Medical Officer of Health does not hear of it. I think that every case of septic sore throat should be notifiable. Then outbreaks due to defective drains, water, or milk supply, would become known to the Medical Officer of Health and could be investigated by him. The difficulties of tracing an outbreak like to this to its origin in the dairy can only be successfully carried out by a sanitary expert with the aid of a skilled bacteriologist." 2

The age and sex incidence of the cases was as follows:-

\begin{tabular}{|c|c|c|c|c|c|c|c|c|}
\hline & & 0 to 10. & 10 to 20. & 20 to 30 . & 30 to 40 . & 40 to 50. & 50. & Totals. \\
\hline $\begin{array}{l}\text { Males } \\
\text { Females }\end{array}$ & : : & $\begin{array}{l}\cdots \\
\cdots\end{array}$ & $\begin{array}{l}10 \\
12\end{array}$ & $\ldots$ & $\begin{array}{l}2 \\
7\end{array}$ & $\begin{array}{l}1 \\
4\end{array}$ & $\begin{array}{l}\mathrm{I} \\
\ldots\end{array}$ & $\begin{array}{l}14 \\
28\end{array}$ \\
\hline Totals. & . & .. & 22 & 5 & 9 & 5 & I & 42 \\
\hline
\end{tabular}

$\mathrm{Mr}$ Nash believes that the incidence of the disease follows closely the consumption of infective cream, all of which came from the one implicated dairy. "Milk which was boiled," he writes, "did

1 The particular infectivity of cream in milk epidemics is referred to by Sir R. Thorne Thorne in his Milroy Lectures, 1891, p. 171 I. $60-62$.

2 Anmual Report of Medical Officer, Bedfordshire County Council, 1902, pp. 
not produce the disease, but cream which could not be boiled produced the disease." There were a number of other cases beyond the 42 of which record was not kept. In several of the families affected, the disease only attacked persons who had consumed some of the cream, and left out those who had not.

These nine sore throat outbreaks will suffice as illustrations of throat illnesses the infection of which was conveyed by milk.

\section{E. Epidemic Diarrhøa}

Definition of epidemic diarrhœa.-The term diarrhœa is obviously only the name of a symptom arising in relation with many diseased conditions. By "epidemic diarrhoea," however (zymotic or epidemic enteritis), is meant a specific disease, which for brevity and convenience may be defined as an acute infective disease, affecting chiefly children under two years of age, occurring during the summer months in epidemic form, and characterised by the occurrence of diarrhœa, vomiting, and wasting, accompanied in severe cases by toxæmia and collapse, which generally end fatally. This disease is a large contributor to the bills of infant mortality, and in many urban districts it is the most serious of all infant diseases, if measured by fatality. It is now believed that there is an intimate relationship between epidemic diarrhoea and the milk supply.

\section{The Cause of Diarrhøa}

The exact cause of epidemic diarrhoa is not at present known. In 1887 , Ballard formulated certain propositions which have obtained general acceptance. They are as follow :-

"That the essential cause of diarrhœea resides ordinarily in the superficial layers of the earth, where it is ultimately associated in the life processes of some micro-organism not yet detected or isolated.

"That the vital manifestations of such organism are dependent among other things, perhaps principally, upon conditions of season and on the presence of dead organic matter which is its pabulum.

"That on occasion such micro-organism is capable of getting abroad from its primary habitat, the earth, and having become air-borne, obtained an opportunity for fastening on non-living organic material, and of using such organic material both as 
nidus and as pabulum in undergoing various phases of its life history.

"That in food, inside as well as outside the human body, such micro-organism finds, especially at certain seasons, nidus and pabulum convenient for its development, multiplication, or evolution.

"That from food, as also from the contained organic matter of particular soils, such micro-organism can manufacture by the chemical changes wrought therein through certain of its life processes a substance which is a virulent chemical poison; and that this chemical poison is, in the human body, the material cause of epidemic diarrhœa." ${ }^{1}$

It will be observed that the three causal agents which $\mathrm{Dr}$ Ballard mentions as playing a large part in the production of this disease are the soil, season, and food-and the causa causans is "some micro-organism not yet detected or isolated." It must be said that we have not got much further than this during the last fifteen years. We do not yet know what is the "virulent chemical poison" which produces the chemical and morbid conditions of the disease. If anything, however, additional knowledge lays added emphasis upon the relationship of food, and more particularly milk, as the main channels of infection. To this matter reference will subsequently be made.

Bacteriology of diarphœa.-In I 885, Escherich published his classical researches on $B$. coli communis. 'He pointed out that the meconium of the newly-born infant is free from bacteria, but by the second day they are present in large numbers, and in the ordinary excreta of healthy infants he found chiefly two organisms, $B$. lactis arogenes and $B$. coli communis. Of these the former was the more abundant in the upper part of the small intestine, and the latter in the lower part and in the colon, so that in the excreta $B$. coli was abundant, and $B$. lactis comparatively scarce. Booker, working in I 886 and onwards, found that the constant bacteria of the healthy excreta of the infant ( $B$. coli and B. lactis arogenes) do not disappear in the excreta of diarrhœa. $B$. coli, however, does not predominate in the same degree, and $B$. lactis is present generally in greater numbers than in the healthy excreta. Booker examined the excreta of $3 \mathrm{I}$ children, and isolated 33 different species of bacteria. Many varieties of bacteria are sometimes found in individual cases of diarrhoea. The greatest number were found in cases of cholera infantum, and a larger number in catarrhal enteritis than in

1 Supplement to the Report of the Medical Officer of the Local Government Board, 1887 . 
dysenteric enteritis. The actual number of individual bacteria were, he found, as great in the healthy excreta as in the diarrhœal excreta. Proteus vulgaris was found very generally, and in the most serious cases. No chromogenic bacteria were isolated, and cultures from a large number of green stools failed to develop green colonies. All the varieties of bacteria isolated from diarrhoal excreta were found to be capable of thriving in milk. ${ }^{1}$ From these facts Booker concluded "that not one specific organism but many different varieties of bacteria are concerned in the etiology of the summer diarrhœas of children." From I889-I895 Booker continued his studies, isolating bacteria from the rectum in 92 infants affected with epidemic diarrhøe, and also from the organs of 33 infants who died from this disease. He found the conditions for the development of bacteria in the intestine of infants affected with summer diarrhœa, different from those in the healthy intestine of milk-fed infants, in that they favoured more varied bacterial vegetation, a richer growth of the inconstant species of intestinal bacteria, and a more uniform distribution through the intestine of the two constant varieties of healthy excreta bacteria ( $B$. coli communis and $B$. lactis arogenes). The first step in the pathological process, Booker believes to be a direct injury to the epithelium from abnormal or excessive fermentation and from toxic products of bacteria; and secondly, that a general intoxication may be brought about indirectly through the production of soluble poisons. He holds that bacteriologically and anatomically three principal forms of summer diarrhœe of infants may be provisionally distinguished, viz.: dyspeptic or non-inflammatory diarrhœa, streptococcus gastro-enteritis, and bacillary gastro-enteritis. $^{2}$ As a result of his extended researches, Booker came to a general conclusion which he expressed as follows:- "No single micro-organism is found to be the specific exciter of the summer diarrhœe of infants, but the affection is generally to be attributed to the result of the activity of a number of varieties of bacteria, some of which belong to well-known species, and are of ordinary occurrence and wide distribution, the most important being

1 Trans. Ninth International Medical Congress, vol. iii. Archives of Pediatrics, February 1890 .

2 Johns Hopkins Hospital Reports, 1896, vol. vi., p. 253. See also a paper "On the Growth of Bacteria in the Intestine," by Lorrain Smith and Tennant.Brit. Med. Jour., 1902, vol. ii., p. 1941. Also Jeffries, Trans. American Pediatrics Society, vol. i., 1889; and Baginsky, Archiv f. Kinderheilkunde, xii., Nos. 1 and 2 ; and Berliner klin. Woch., 1889. 
the streptococcus [enteritidis] and Proteus vulgaris." The streptococcus termed $S$. enteritidis varies in morphology, and seems to be associated with two classes of cases, one of which simulates cholera, the other typical enteric fever. "Micrococci are present in all of the cases, in immense numbers in some, being few in others." 1 It may be added that Cumston, Holst, Escherich, and Hirsch, have also laid emphasis upon the causal relationship of certain streptococci and diarrhœa.

Klein was one of the first workers to isolate an anaërobic organism from cases of epidemic diarrhœea. This organism, which he named $B$. enteritidis sporogenes, was found in three successive outbreaks of diarrhœa occurring among patients in St Bartholomew's Hospital. In the first two outbreaks the milk was evidently the channel of infection, in the third it was some rice pudding. The bacillus was carefully studied and the main facts respecting it will be found elsewhere in the present volume. Here it may be said that it is a widely distributed organism and occurs in normal and typhoid excreta, in sewage, manure, soil, dust, and milk. ${ }^{2}$ The etiological relationship between this bacillus and epidemic diarrhoea has been called in question, and it is, of course, not proved that the organism is the cause of the disease. On the other hand, it has been very frequently found in the mucous flakes of the dejecta in patients suffering from the disease, and, in the outbreak produced by the consumption of cooked rice pudding, it is difficult to understand how any organism except an anaërobe of highly resistant qualities could have produced the condition. It will be apparent, moreover, that B. enteritidis sporogenes fulfils in a somewhat exceptional degree the requirements suggested by Ballard.

That epidemic diarrhœe is caused by the $B$. coli either alone or in conjunction with other organisms, has been held by a number of authorities. Cumston, who investigated 13 cases of the disease, concluded that $B$. coli associated with Streptococcus pyogenes was the chief pathogenic agent concerned, and he claims that the virulence of $B$. coli is exalted by the association. ${ }^{3}$ Lesage also formed the opinion that the disease was due to $B$. coli, and investigated the agglutinative properties of the serum of children suffering from epidemic diarrhœa on $B$. coli isolated from the intestine. $\mathrm{He}$ obtained a positive result in 40 out of 50 cases, and the serum of each of these 40 cases, also agglutinated samples of $B$. coli from 39

1 Johns Hopkins Hospital Reports, I896, vol. vi., p. 251.

2 Report of Medical Officer of Local Government Board, 1897-98, pp. $210-251$.

3 International Medical Magazine, February 1897. 
other children seized with the same disease. ${ }^{1}$ Some of the most recent work on the relationship existing between $B$. coli and epidemic diarrhœa has been done by Delépine, who examined milk in the outbreak of epidemic diarrhœa which occurred in Manchester in 1894 (vide infra), and has also examined a large number of town and country milks. His conclusion is that:-

"Epidemic diarrhœa of the common type occurring in this country is apparently, in the great majority of instances, the result of infection of food by bacilli belonging to the colon group of bacilli, and which are present at times in fæcal matter. It appears that this infection of food does not generally lead to serious consequences, unless the infection is massive from the first, or the food is kept for a sufficient length of time, and under conditions of temperature favouring the multiplication of these bacilli.

"Milk, which is the most common cause of epidemic diarrhœea in infants, is usually infected at the farm, or (through vessels) in transit. Of the bacilli of the colon group which are capable of rendering the milk infectious, those which do not produce a large amount of acid, and do not coagulate milk, are the most virulent, and are probably the essential cause of epidemic diarrhoea." 2

Flugge and Lubert ${ }^{3}$ believe that cows' milk contains at certain seasons aërobic spore-bearing bacilli of the subtilis type, which have the power of decomposing and peptonising milk, producing metabolic products, which, when swallowed by animals, cause diarrhœea, etc., and which may therefore produce diarrhoea in infants.

It is evident that our knowledge of the bacteriology of diarrhoea is not sufficiently established to permit of any very definite conclusion on the matter. It may be that the whole group of choleraic, enteric, and diarrhœal diseases are caused by a group of micro-organisms having many similarities and relationships to each other; or it may be that different forms of diarrhœea have their own specific causal organism; or, lastly, it may be a question of association of organisms which brings about the disease. In any event, there is abundant evidence to prove that epidemic diarrhœa is a bacterial disease in the same way as typhoid fever.

Conditions favourable to epidemic diarrhor-The provisional results of Ballard's inquiry into the causation of epidemic diarrhœe may be stated as follows:-

"The summer rise of diarrhoeal mortality does not commence until the mean temperature recorded by the four-foot earth ther-

1 La Semaine Med., October 1897.

2 Jour. of Hygiene, 1903, vol. iii., No. 1, p. 90.

3 Zeitschrift f. Hyg., xvii., p. 272 ; and xxii., p. I. 
mometer has attained somewhere about $56^{\circ} \mathrm{F}$., no matter what may have been the temperature previously attained by the atmosphere or recorded by the one-foot earth thermometer. The maximum diarrhœal mortality of the year is usually observed in the week in which the temperature recorded by the four-foot earth thermometer attains its mean weekly maximum. The decline of the diarrhœal mortality is in this connection not less instructive, perhaps more so, than its rise. It coincides with the decline of the temperature recorded by the four-foot earth thermometer, which temperature declines very much more slowly than the atmospheric temperature, or than that recorded by the one-foot thermometer; so that the epidemic mortality may continue (although declining) long after the last-mentioned temperatures have fallen greatly, and may extend some way into the fourth quarter of the year. The atmospheric temperature and the temperature of the more superficial layers of the earth, is little if at all apparent until the temperature recorded by the four-foot earth thermometer has risen as stated above; then their influence is apparent but it is a subsidiary one."

In addition to these conditions of soil, Ballard and other workers have concluded that insanitation in the widest sense of the term favours epidemic diarrhœe. Density of population, or houses, upon an area, unclean soil, dusty surfaces, bad light, absence of ventilation, maternal neglect, etc., all have a share in creating an environment favourable to the disease. As we have seen, Delépine, like Ballard, attributes the disease in large measure to milk. Ballard believed that milk gained its infection by unsuitable storage and by the mode in which it was used. He found that "infants fed solely from the breast are remarkably exempt from fatal diarrhœa; that infants fed in whatever way with artificial food to the exclusion of breast milk are those which suffer most heavily from fatal diarrhœa; that children fed partially at the breast and partially with other kinds of food, suffer to a considerable extent from fatal diarrhoea, but very much less than those brought up altogether by hand; and that, as respects the use of 'the bottle,' it is decidedly more dangerous than artificial feeding without the use of the bottle."

Dr Newsholme of Brighton, has published an interesting paper on the causation of epidemic diarrhœe. Some of his chief conclusions, which are now widely accepted, may be added :-

"( I) Epidemic diarrhœa is chiefly a disease of urban life. (2) Epidemic diarrhœa as a fatal disease, is a disease of the artisan and still more of the lower labouring classes to a preponderant 
extent. (3) Towns having the water-carriage system of sewage have as a rule much less diarrhœea than those retaining other methods of removal of excrement. (4) Towns with the most perfect scavenging arrangements have the least epidemic diarrhœa. (5) The influence of the soil is a decided one. Where the dwellinghouses of a place have as their foundation solid rock with little or no superincumbent loose material, the diarrhœeal mortality is, notwithstanding many other unfavourable conditions and surroundings, low. On the other hand, a loose soil is a soil in which diarrhœeal mortality is apt to be high. (6) Given two towns equally placed, so far as social and sanitary conditions are concerned, their relative diarrhoeal mortality is proportional to the height of the temperature and the deficiency of rainfall of each town, particularly the temperature and rainfall of the third quarter of the year."

Dr Newsholme concludes that "the fundamental condition favouring epidemic diarrhcea is an unclean soil, the particulate poison from which infests the air and is swallowed, most commonly with food, especially milk." In other words, epidemic diarrhœea is a so-called "filth-disease" preventable by improved sanitation in the broadest meaning of the term. ${ }^{1}$

Summary.-From the facts and suggestions quoted above, and they are but representative of many other similar views receiving the general support of epidemiologists, it will be evident that at the present time the cause of epidemic diarrhœa is to be found in four conditions, which may be expressed shortly as two propositions, thus: (I) Epidemic diarrhœa is a bacterial disease; (2) Its occurrence depends, wholly or partly, upon surrounding temperature, deficiency of rainfall, and pollution of food, chiefly milk. The exact relationship which these conditions have to each other is not known. Some authorities hold that a certain temperature affects food, conducing towards creating in it injurious properties. Others believe that it is a question of pollution of milk by dust, which carries to the milk the causal micro-organisms, and that deficient rainfall favours this contamination, and increased temperature favours the growth and multiplication of the bacteria thus conveyed to the milk. As Dr Newsholme says, "Whatever be its mode of operation, a frequent fall of rain during the summer weeks, even though its total amount be not great, is one of the most effectual means of keeping down the diarrhœal death rate" ; 2 and whilst he considers temperature conditions of great import-

1 Public Health, 1899-1900, vol. xii., pp. I39-2 I3.

' Annual Report on Health of Brighton, 1902, p. 48. 
ance, "rainfall is more important than temperature in relation to epidemic diarrhœe." Rain washes the air, if the expression may be allowed, and carries to the surface aërial dust. It, of course, also washes the surface of the soil and removes surface pollution, and with it micro-organisms capable of infecting infants, usually by food. Thus the relationship between these meteorological conditions and milk, though an open question, may be an essential one to the origin of the disease. In any event, it seems clear that milk is probably the common vehicle of infection (Ballard, Delépine, Newsholme), and this probability must now be referred to.

The relationship of milk to epidemic diarrhœa.-Most of our knowledge respecting the part played by milk in the production of epidemic diarrhœa is derived from cases of the disease traceable to milk infection. These cases have occurred in outbreaks or sporadically.

In $1892 \mathrm{Gaff}^{1}{ }^{1}$ recorded an instance in which three men connected with the Hygienic Institute at Giessen were suddenly taken ill. They were the assistant, the chemist, and the janitor. They had chills, fever, diarrhoea, and general symptoms. The only article of diet of which they had all partaken was milk, which was traced to a cow suffering from enteritis. The milk of this cow as it left the udder contained no bacteria. But bacteria gained access during the milking from the dried particles of frcal matter on the posterior portion of the udder. In these particles was found a bacillus which proved very pathogenic for mice and guinea-pigs, and which corresponded to an organism isolated from the stools of the patients.

In I894 an outbreak occurred at Manchester, ${ }^{2}$ characterised by diarrhoa, sickness, and abdominal pains. The cases numbered 160 in 47 houses, or just 50 per cent. of the houses served by one and the same milk seller. Raw-milk drinkers were the chief sufferers, and those not drinking the implicated milk did not suffer. Dr Niven visited the farm whence the milk came, and found that it was the milk from the farm itself, and not the added milk from a more distant farm which supplemented the farmer's stock, that had caused the epidemic, the home farm milk only being sent into the affected district. Near the farm were 40,000 tons of privy-midden refuse. Two streams ran near the farm, meeting below, one fouled by the drainage of the "tip," and the other being

1 Deut. Med. Woch., vol. xviii., p. I4.

2 Annual Report of Medical Officer of Health of Manchester, I894 (Dr Niven). 
contaminated with sewage and with matter from a tripe-boiling place. The water used for washing the milk-pails was tepid and kept in a foul cistern. The cows drank from a pool which received the drainage from the cowshed midden. The stored milk could be readily contaminated from emanations from the cowshed. Professor Delépine examined the milk and found $B$. coli communis abundant in the milk, and Dr Niven elicited the fact that a cow affected with inflamed udder ("garget") had been removed from the farm and slaughtered. The outbreak was attributed to milk in any case, and to the probable infection of it by the diseased cow. But Delépine has pointed out that it is more probable that the milk was contaminated with frecal pollution rather than infectious disease of the cow. ${ }^{1}$

In $1895^{2}$ and $1898^{3}$ three outbreaks of epidemic diarrhœa occurred amongst the patients at St Bartholomew's Hospital, London, traceable in the first two instances to milk, and in the third to rice pudding made with milk. ${ }^{4}$ On Sunday night, 27 th October I 895, an outbreak of diarrhoea affected 59 in-patients, all of whom had recently taken milk, and from the evacuations the spores of B.enteritidis sporogenes was isolated by Klein. The patients suffered quite irrespective of whether or not the milk had been boiled. Some milk also, derived from the same source as the milk which had caused the poisoning, was examined by Klein and found to contain the spores of the same organism. On Sunday, 6th March I898, a second outbreak of severe diarrhœea occurred in this hospital affecting 146 patients, and there was evidence on this occasion also that the medium of infection had been milk. On $5^{\text {th }}$ August I898, a third outbreak affecting 84 patients and 2 nurses took place at the same hospital, the vehicle of infection in this instance being some rice pudding made with milk, also said to contain an organism similar or identical with the $B$. enteritidis sporogenes. There can be no doubt that milk was the agent of infection in each of these three outbreaks. It was in these outbreaks that the $B$. enteritidis sporogenes of Klein was isolated and held to be the specific organism, and Dr Klein has produced evidence in behalf of this bacillus being the true cause of epidemic diarrhœea. ${ }^{5}$

1 Jour. of Hygiene, 1903, vol. iii., No. I, pp. 76, 77.

2 Report of the Medical Officer of Local Government Board, 1895-96, pp. $197-204$.

1bid., $1897-98$, p. 235.

4 Ibid., I898-99, p. 336. Lancet, 7th January 1899.

Beports of Medical Officer of Local Government Board, 1895-96, 1896-97, I897-98, $1898-99$. 
Stokes and Wegefarth reported an outbreak of epidemic diarrhœa in 20 girls at a girls' school in Baltimore, which was traced to the milk supply. The investigation revealed a condition of mastitis in one of the cows yielding the implicated milk. The milk itself was found to be "almost entirely pus cells, together with numerous streptococci," and to this was attributed the outbreak of diarrhøea. ${ }^{1}$

In I899, Dr Zammit of Malta traced an outbreak of sickness to milk. Five families of 12 persons were taken ill with vomiting, diarrhœa, cramps, etc. Two children out of the 12 died. The poisoning was sudden and severe. Another series of cases occurred ten days later. Five houses were implicated, and I7 persons attacked. The symptoms were similar to the first series, and included acute pains in the abdomen and cramps in the limbs. The attack followed three hours after consumption of the milk. Several persons in one of the families who had boiled the milk before use did not suffer. B.enteritidis sporogenes was isolated from the cans in which the milk had been placed, and $\mathrm{Dr}$ Zammit attributed these illnesses to that organism. ${ }^{2}$ Cases of "milk poisoning," allied to outbreaks of this character, have been very frequent, if we are to judge by the records of such.

In addition to the evidence obtainable from outbreaks of diarrhœea of which the above kinds may be taken as types, there is also much reason to suppose that a large number of sporadic cases of diarrhca are produced every year by milk. Bacteriological examination is, naturally, made in very few sporadic cases of diarrhœa, but there is available evidence of other kinds.

In Brighton in 1900-1902 there were 226 fatal cases of diarrhœea, in I9I of which it was possible to trace the milk supply. Of these I 8 (or 9.4 per cent.) were "breast fed," 84 (or 44 per cent.) were fed on condensed milk, and 89 (or 47 per cent.) were fed on cow's milk. $^{3}$ In the Metropolitan Borough of Finsbury in $190 \mathrm{I}$ and 1902 it was possible to trace the milk supply in II 5 deaths of infants from diarrhœe. Of these, 24 (or 20 per cent.) were fed with human milk, 39 (or 35 per cent.) were fed on condensed milk, and 52 (or 45 per cent.) were fed on cow's milk. ${ }^{4}$

At Croydon, Dr Richards has shown that of 253 deaths of

1 Jour. of State Medicine, 1897, p. $44 \mathrm{r}$.

2 Brit. Med. Jour., 1900, vol. i., p. I1 5 I.

${ }^{3}$ Report on Health of Brighton, 1902, p. 50.

4 Report on Health of Finsbury, 1902, p. 78. 
infants under six months from diarrhœea, fourteen per cent. were fed on human milk, thirty-three per cent. were fed on condensed milk, and forty-eight per cent. on cow's milk. Between the ages of six and twelve months, eleven per cent. were fed on human milk, twenty-nine per cent. on condensed milk, and fifty-four per cent. on cows' milk. ${ }^{1}$ These percentages may be tabulated thus :- ${ }^{2}$

\begin{tabular}{|l|c|c|c|}
\hline & Breast Fed. & Condensed Milk. & Cows' Milk. \\
\hline Brighton : & 9 & 44 & 47 \\
Finsbury : : & 20 & 35 & 45 \\
Croydon : & 12 & 31 & 51 \\
\hline
\end{tabular}

From these facts there is one evident deduction, namely, that most of the deaths of infants from diarrhcea occur in children who have been hand-fed (according to Still, 90 per cent.). Further, there is no substantial evidence that condensed milk, ${ }^{3}$ which may be presumed to be, though not germ-free, at least free from the chances of the active pollution of cows' milk, contains infective matter before it is opened. Therefore, any infectivity it possesses is obtained by contamination in the home. In the homes of

1 Jour. of Hyg., 1903, p. 329.

2 See also work of Ashby and Wright, H. R. Jones, Still, Niven, Hope, etc.

${ }^{3}$ It is impossible to enter into the question of condensed milk and its effect upon the consumer, as that is now almost a subject by itself. It may, however, be stated that the trade in condensed milk has enormously increased in recent years, and there can be little doubt that the consumption of this substitute for milk is having very injurious effects upon infants. But this is due to its sugar and deficiency in fat, and not to infective properties. Hutchison says :-

"There can be no doubt that an immense amount of harm is done to infants by the indiscriminate use of such milks. Babies fed on them may look fat enough, but they are pale and flabby, and often suffer from rickets; for fatness produced by abundance of sugar in the milk is by no means a sure indication of health, and the pictures of such fat but flabby infants so freely spread abroad by the makers of condensed milks are very deceptive." (Robert Hutchison, M.D., F.R.C.P., Food and Dietetics, 1902, p. 444.)

An examination of twenty-two condensed milks in Liverpool showed excessive quantities of sugar (Hope), and the same condition was still more marked in Finsbury (Colwell). Dr Hope of Liverpool, speaking of a bacteriological examination of fifty condensed milks, says, that "the great majority were not sterile." He adds :-

"There can be no doubt that condensed milk is a most unsatisfactory product. Bacteria are usually present, their products are masked by the large quantity of sugar present, but their irritant properties are not destroyed." Reports on Health of Liverpool, 1901 and 1902.

A large number of medical officers of health have borne similar testimony. 
the poor, such contamination from flies and dirt is inevitable. Probably in large measure the same applies to cow's milk. Hence it appears that most, if not all, of the eighty per cent. of the milks used in these fatal cases of diarrhœa obtained their injurious properties at the home of the consumer.

Delépine has urged that milk is infected at the farm or in transit, as much of that which he examined and proved to be virulent, had not been exposed to any influence attributable to a consumer's home, but was in fact infective before it reached the consumer. ${ }^{1}$ He considers the injurious properties of such milk is due to fæcal pollution and the action of $B$. coli. Newsholme considers such contamination may be responsible for setting up epidemics of diarrhœa occurring in connection with a particular milk supply, as in the analogous case of epidemics of infectious diseases, such as typhoid. But he holds that the ordinary sporadic cases of diarrhœa, which carry off single children in large numbers in urban districts, are due "chiefly to domestic infection of milk or other foods, or to direct swallowing of infective dust." 2 With this view we agree. Further, a study of the bacterial contents of milk, from town and country, within twelve hours of its production, will, we think, lend support to this view. We have a double pollution of milk in actual practice, one originating at the farm, one brought about subsequently. The latter may be produced by flies, or from manure heaps (Waldo), or from dust in roads and yards of towns (Richards), or from the generally filthy manipulation of the milk from the time when it becomes the property of the milk seller to the moment of consumption. It should not be forgotten in this relation that stale milk contains toxic properties altogether apart from, and in addition to, actual bacteria. It is possible that the products of organismal action have a much greater effect in the causation of diarrhœa than is generally supposed.

\section{Preventive Measures}

1. Among the preventive measures which these conclusions indicate, cleanliness of cows, dairy-hands, and milk-cans or other milk vessels, stands first, refrigeration of the milk second in importance. Refrigeration of the milk, being more easily obtained than cleanliness, should be insisted upon without delay. Similar measures are also needed with regard to all things or persons coming in

2 Report on Health of Brighton, 1902, p. 50. 
contact with the milk, manufactured or not. Absolute cleanness is most difficult to obtain, if not practically impossible. Occasional infection must, therefore, occur.

2. To guard against the effects of accidental frcal infection, milk should be consumed fresh, when possible. When it cannot be consumed fresh it should be refrigerated, i.e. kept at a temperature below $4^{\circ} \mathrm{C}$. When it cannot be used fresh or refrigerated, it should be sterilised by heat.

3. Greater domestic and municipal c!eanliness is an essential requirement. This must include the cleanly preparation of food, and particularly the handling and storage of milk; the cleansing of milk-bottles; reduction of dust in houses, courts, and streets; impervious roads and paving; and the substitution of wet cleansing for dry cleansing, and frequent cleansing for infrequent.

4 "A crusade against the domestic fly, which is most numerous at the seasons and in the years when epidemic diarrhoea is most prevalent, and probably plays a large part in spreading infection" (Newsholme).

\section{F. Cholera}

Cholera is rarely spread by milk, but as several outbreaks of the disease have been traced to milk, a note may be added on the subject.

The cholera bacillus is unable to live long in an acid medium, hence its life in milk is a limited one, and generally depends upon some alkaline change in the milk. Heim found that cholera germs would live in raw milk from one to four days, depending upon the temperature. D. D. Cunningham, from the results of a large number of investigations in India, concludes that the rapidly developing acid fermentations normally or usually setting in, connected with the rapid multiplication of other common bacteria and moulds, tend to arrest the multiplication of cholera bacilli, and eventually to destroy their vitality. Boiling milk appears, on the contrary, to increase the suitability of milk as a nidus for cholera bacilli, partly by its germicidal effect upon the acid-producing microbes, and partly that it removes from the milk the enormous numbers of common bacteria, which in raw milk cause such keen competition that the cholera bacillus finds existence impossible.

Professor W. J. Simpson has placed on record an interesting series of cholera cases on board the Ardenclutha, in the port of Calcutta, which arose from drinking milk which had been polluted with onequarter of its volume of cholera-infected water. This water came 
from a tank into which some cholera dejecta had passed. Of the ten men who drank the milk four died, five were severely ill, and one, who drank but very little of the milk, was only slightly ill. There was no illness whatever amongst those who did not drink the milk. ${ }^{1}$

In July I 894 an epidemic of cholera occurred in the Gaya Jail, affecting twenty-six persons, half of whom had consumed some milk which had become infected by flies carrying the virus of cholera from some cholera stools (Macrae). ${ }^{2}$ Comma bacilli were actually found in the implicated milk by Haffkine and Simpson.

1 Practitioner, vol. xxxix., p. I44.

2 Indian Med. Gazette, 1894, pp. 407-412. 


\section{CHAPTER XI}

\section{THE INVESTIGATION AND PREVENTION OF MILK-BORNE EPIDEMICS}

The Investigation of Milk-borne Epidemics. Methods of Prevention. Modern

Bacteriological Methods applied to Milk-borne Disease: Diagnosis and Preventive Inoculation.

THE investigation and prevention of milk-borne disease depends upon exact knowledge of the disease, and an intimate knowledge of the milk trade. The former has already been treated of; the latter will be considered in the chapters dealing with the control of the milk supply. The present chapter will be concerned with some of the methods to be adopted in investigating milk-borne outbreaks.

\section{The Investigation of Milk-Borne Epidemics}

In these days everyone is aware in a general way of the machinery by which epidemics of infectious disease are controlled. It has now been in vogue in England for more than a decade. In the main, this machinery may be described in three words-notification, isolation, disinfection. The first of these (notification) is the only one which can be considered as auxiliary to the methods of investigating outbreaks of infectious disease. The Infectious Diseases (Notification) Act, I889, was a compulsory Act in London after the expiration of two months from its passing. But in urban and rural districts outside the metropolitan area it was an adoptive Act, and until its adoption in a locality did not become law. Fortunately its adoption soon became fairly general, for sanitary authorities found that without it they were unable to obtain the data of infectious epidemic disease necessary to its efficient control. So satisfactory did notification work during the first ten years, that on Ist January 1900 the provisions of the 1889 Act were enforced everywhere by means 
of the Infectious Diseases (Notification) Extension Act of 1899. In London the notification provisions are incorporated in the Public Health (London) Act of I89I. Thus it happens that at the present time notification of certain infectious diseases (including diphtheria, scarlet fever, and enteric or typhoid fever) is compulsory. The onus of notifying is cast upon the medical attendant, or in his absence the head of the family. The notification is made to the Local Authority in whose district the infectious disease occurs, and in general it is made direct to the Medical Officer of Health of the district. This then is the first step in the investigation of milkborne, or otherwise conveyed, outbreaks of infectious disease, and by the receipt of a number of such certificates the medical officer becomes aware of the existence of an epidemic.

The second step is the investigation of each case of disease and of the cases as a whole. In the former it is customary for the medical officer or his inspectors to make a minute inquiry as to the individual. The precise method of inquiry differs of course in different districts. The same form would not be equally suitable in a rural as in an urban district. On the opposite page is given a copy of such a form as is used daily in one of the metropolitan areas.

By means of such facts the medical officer becomes aware of the most likely channels of infection and of some at least of the most likely persons to contract the infection from the original case. He obtains moreover a large amount of valuable information respecting the disease under consideration and the sanitation surrounding his cases. It cannot be doubted that in the long run such records must prove of the utmost value in showing the causes of disease and the channels of infection. To ascertain the milk and water supply, the school or place of work, and the date of the onset of the disease are of immense value in an endeavour to search out the cause or to check the spread of the disease. It is unnecessary here to discuss in detail each item of the said form. But a word may be said upon the importance of knowing the exact date of the onset of the symptoms of illness. This date is of greater importance than the date of notification. For example, in a recent inquiry conducted by the writer on the occasion of a small outbreak of milk-borne scarlet fever, I9 notifications were received extending over nine days. In a large population such a series would scarcely constitute an outbreak. But on making careful inquiry in the case of each of the 19 persons suffering from the scarlet fever, it was found that all these 19 persons commenced 


\section{ENTERIC FEVER.}

No, in Notification Register

Name
Age and Sex

Occupation

Dr

Date of Onset and Symptoms

Notific. recd. Date of Removal to Hosp.

Date of Disinfection

Day School_- Sunday School___
Work

No. of Persons in House

Age, occupation, and sex of each Person in House, and whether had Enteric Fever. If so, when?

(1)
(घ)
(8)
(4)

\section{Overcrowding}

Water Supply

Washing done at Milk $\int$ Supply

Sanitary condition of House, Yard, and Surroundings ; (clean, dry, ventilated, W.C., ashpit, soil-pipes, gullies, sinks, interceptors, baths, cisterns, animals).

Drainage

\section{Notes on Case.}

Possible sources of Infection ( 2 to 4 weeks previously):-(1) Visits; (2) Visitors; (3) Place of work; (t) Food, milk, ice cream, shellfish, watercress, etc. - what, when, where purchased, condition; (5) Water; (6) Previous cases of Sick Headache or Diarrhœea in the house or neighbourhood; (7) School, including Sundays; (8) Drain Nuisances, at or near house ; (9) Tailoring, washing, mangling.

[If the case was one of Diphtheria, infection from Cats or Birds might be inquired into, and previous cases of Diphtheria or Sore Throat or Scarlet Fever noted.] 
illness on the first two days of the nine days over which the notifications were received. Here then was an outbreak not occurring during nine days, but during two days. Consequently the source of the epidemic had to be sought previously to those two days, and approximately the length of the incubation period before the two days. Contamination of the milk supply was detected. But if the cases had been traced by the date of their notification and not the onset of the symptoms, it is probable that the infected milk would not have been discovered, or at all events not so rapidly.

The incubation period and approximate infectivity of diphtheria, scarlet fever, and typhoid fever, are (according to Goodall and Washbourn) as follow :-

\begin{tabular}{|c|c|c|c|c|c|}
\hline Disease. & $\begin{array}{l}\text { Length of } \\
\text { Incubation } \\
\text { Period. }\end{array}$ & $\begin{array}{l}\text { Day of Disease } \\
\text { upon which } \\
\text { Sruption } \\
\text { Appears. }\end{array}$ & $\begin{array}{l}\text { Length of Time of } \\
\text { Isolation of Patient. }\end{array}$ & $\begin{array}{l}\text { Length of } \\
\text { Quarantine } \\
\text { Period. }\end{array}$ & $\begin{array}{l}\text { Protection } \\
\text { Afforded. }\end{array}$ \\
\hline Diphtheria . & 2 days $(1-5)$ & No eruption . & $\begin{array}{l}\text { For } 3 \text { weeks from dis- } \\
\text { appearance of exuda- } \\
\text { tion ; or as long as } \\
\text { diphtheria bacilli are } \\
\text { present in throat }\end{array}$ & 7 days & $\begin{array}{l}\text { Relapses and } \\
\text { second attacks } \\
\text { not uncom- } \\
\text { mon. }\end{array}$ \\
\hline Scarlet fever . & $\begin{array}{l}8 \text { days (fow } \\
\text { hours to } 5 \\
\text { days) }\end{array}$ & $\begin{array}{l}\text { Usually within } \\
24 \text { hours }\end{array}$ & $\begin{array}{l}\text { For at least } 6 \text { weeks } \\
\text { from appearance of } \\
\text { rash; or until desqua- } \\
\text { mation has entirely } \\
\text { finished or chronic dis. } \\
\text { charge has ceased }\end{array}$ & 7 days & $\begin{array}{l}\text { Relapses un. } \\
\text { e o m m o n } \\
\text { second attacks } \\
\text { not uncom. } \\
\text { mon. }\end{array}$ \\
\hline Enteric fever. & 14 days & 4th-7th day & .... & .. & $\begin{array}{l}\text { Rolapses com- } \\
\text { mon; second } \\
\text { attacks rare. }\end{array}$ \\
\hline
\end{tabular}

Note.-By Quarantine Period is meant the length of time during which a susceptible person, who has been exposed to infection, is to be isolated.

The importance of a careful inquiry into each individual case in an investigation into milk-borne outbreaks cannot well be exaggerated. Not only do the facts elicited frequently reveal the exact channel of infection, but ways and means of preventing further spread are thus discerned.

In the investigation of suspected milk-borne outbreaks of disease the third step is certainly of the nature of an inquiry into the relationship of the milk supply : in short as to whether or not there is a community of the milk supply. Suspicions of such community may first be aroused by a detection of some of the characteristics of milk outbreaks (see p. 262). There are various ways in which the matter may be followed up. The essential fact, in this connection, as to each patient is recorded on the investigation form discussed above. The relationship of a particular dairy or milk-round and the occurrence of cases of the disease will be obtained by a considera- 
tion of the cases as a whole. Sometimes this may be done by a "spot-map," on which each case of the disease is marked. By such a simple plan groups of cases, if such groups exist, at once become evident. Or lists of the customers of the suspected dairy can generally be obtained and compared with a list of the persons suffering from the disease. Charts of one kind or another have proved very serviceable in tracing out a community of the milk supply. One of the most effective and graphic of these methods was devised by Dr Davies, the Medical Officer of the City of Bristol. In 1897 there occurred in Bristol an epidemic of enteric fever attacking more than 240 persons. This was traced by $\mathrm{Dr}$ Davies to three milk-rounds of polluted milk coming in to Clifton from the country. The facts which he was able to discover and the graphic manner in which he presented these facts are so full of instruction that we may quote his words at some length. Not only is the "graphic method" recommended by Dr Davies one very useful for adoption, but the method of inquiry and the kind of fact elicited, as revealed in the following paragraphs, provide an admirable illustration of how to investigate milk-borne disease.

Speaking of the sources of the infected milk, he writes :- 1

"I. The milk from the farm X, situate in the county of Somerset, was consigned to a distributor A whose man B met it on its entry into Clifton, over the Suspension Bridge, and distributed it directly from the churn to the customers without its going to any local dairy. Any milk not used on the round was, however, returned to the branch dairy and sold to casual customers. Before the 26th September, when the big schools reopened, the supply was sufficient to supply two districts, A, the lower level, and B, the higher level: but after this date the supply was confined to the high level district (see diagram). Some of the cases seem to have developed on the low level round at the beginning of October, while the incidence on the high level round was more marked in the second week of October.

"On the low-level round:-

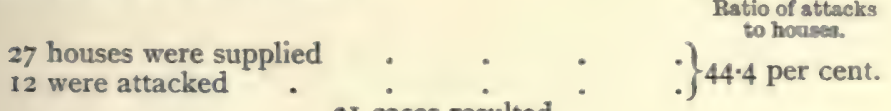

31 cases resulted.

"On the high-level round:-

29 houses were supplied 19 were attacked

83 cases resulted. . 65.5 per cent.

1 Trans. of Epidemiological Society of London, 1898, vol. xvii., p. 82. 
"Or taken together:-

56 houses were supplied
3I were attacked
I I 4 cases resulted, or more than 2 cases per house.

The inmates of these houses numbered 453 , so that $25 \cdot$ I per cent. were attacked, or just a quarter of the whole number.

" 2. Milkman Y started with a pure supply from Ashton in the county of Somerset, but at Bower Ashton he met the X cart and occasionally took a supplementary supply from the churn consigned to Clifton; after receiving which he supplied milk, yielding two cases of typhoid, at Bower Ashton, proceeded to Leigh Woods, where six more cases appeared in three houses supplied, and then entered Clifton.

"On his rounds in Clifton:-

4o houses were supplied

18 were attacked

Ratio of attacks to houses.

- 48 cases resulted, or more than I case per house.

The inmates of these houses numbered 308 , so that 15.5 per cent. were attacked, or about one-sixth.

" 3 . The third and last supply known to be implicated $(Z)$ came from a farm at Westbury, and entered the city from the north, at a point remote from the Suspension Bridge over which supplies $\mathrm{X}$ and $\mathrm{Y}$ came. The milk from this farm is distributed in Clifton on three rounds. Two of these rounds were entirely innocent of cases, and the third round was (with one exception, a servant who lived close to, and admits to frequently obtaining casual supplies from the branch dairy to which unused $\mathrm{X}$ milk was returned) also innocent of cases up to a certain point, but beyond this point cases began to occur with marked frequency (see diagram).

"On comparing the routes of the three dairymen, which we had plotted upon a map, it was at once discovered that, at the very corner where this change from freedom to infection occurred, Z's round met that of the $\mathrm{X}$ supply, in charge of the man $\mathrm{B}$; and $\mathrm{Z}$ admitted that he was in the habit, when running short, of obtaining at this point supplementary supplies from B. After this, cases commenced at the very next house he supplied, and continued with considerable frequency along his route:-

Before adding the X milk $\left\{\begin{array}{c}\text { II houses were supplied } \\ \text { I house attacked }\end{array}\right\} \begin{gathered}\text { Ratio of attacks } \\ \text { to houses. }\end{gathered}$
$9 \cdot 0$ per cent.
I case resulted (obtaining casual supplies from X shop).

After adding the $\mathrm{X}$ milk. $\left\{\begin{array}{c}16 \text { houses were supplied } \\ 8 \text { were attacked }\end{array}\right\} 50 \cdot 0$ per cent. 22 cases resulted. 
"In the aggregate we find up to I8th December, the close of the outbreak :-

On regular milk rounds .

On regular rounds after i 7 th October

Obtaining casual supplies from shop to which $\mathrm{X}$ milk was returned

Obtaining milk from shop on low-level round supplied with $\mathrm{X}$ milk

\begin{tabular}{|c|c|}
\hline $\begin{array}{c}\text { Houses } \\
\text { attacked. }\end{array}$ & Cases. \\
\hline 57 & 184 \\
\hline I & 2 \\
\hline 21 & 35 \\
\hline \multirow[t]{2}{*}{6} & 9 \\
\hline & 230 \\
\hline 13 & I4 \\
\hline 98 & $244^{\prime}$ \\
\hline
\end{tabular}

Houses attacked obtaining milk from dairymen not known to obtain infected supplies

Cases.

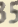

9

30

244.

Dr Davies found the same methods of inquiry and statement applicable in tracing the distribution of milk-carried scarlet fever in an outbreak in Clifton in Igoo. He writes :- 1

"The graphic method, which I first used in connection with the outbreak of milk-carried enteric fever which occurred in Clifton in 1897 , is here applied to a similar outbreak of milk-carried scarlet fever.

"The dairy farm $\mathrm{X}$ is regarded as the source of infection. On this farm at the time of the outbreak a boy who had access to the milk vessels was suffering from an unrecognised illness which was compatible with a mild (ambulant) attack of scarlet fever. The veterinary surgeon found no evidence of disease among the cows, and no other persons connected with the dairy farm showed signs of illness.

"The dairy farm $\mathrm{X}$ supplied milk to two Clifton distributors $\mathrm{Y}$ and $\mathrm{Z}$, and also distributed milk in the city direct from the farm; no other retailers obtained any milk from $\mathrm{X}$.

"These three distributors receiving milk supplied 269 houses, of which 42 were attacked, furnishing 66 cases; that is, one in every 6.4 houses was attacked. During the same period 85 other distributors not receiving $X$ milk supplied 6922 houses; and 9 cases occurred in as many houses, that is, I in every 769 houses was attacked, an incidence not indicating any unusual prevalence of scarlet fever in a large city. (Clifton has a population of $47,30 \mathrm{I}$. It is a registration sub-district of Bristol, which has a population of 324,973 .)

"Y obtained a part of his milk from a dairy farm A, but as A also supplied the retailers D and E, whose rounds were absolutely free from scarlet fever, this farm is at once cleared from any suspicion.

" $\mathrm{Z}$ also obtained some of his supply from farmers B and C ; but

1 Jour. of Hygiene, Igor, vol, i., p. 388. 
no cases were traced to their supplies except on rounds associated with the $\mathrm{X}$ supply.

\section{"Distribution of the milk."}

"The dealer X, bringing in only milk from his own farm, distributed it first outside the city, with a record of two cases; the milk then entered Clifton:-

$$
\left.\begin{array}{llr}
\text { X. Houses supplied } & \bullet & 23 \\
\text { Houses attacked } & - & 5
\end{array}\right\} 2 \mathrm{I} \cdot 0 \text { per cent. Cases, } 8 .
$$

"The distributor $\mathrm{Y}$, receiving milk in churns from farms $\mathrm{X}$ and A, affirmed his habit of mixing it before delivery, a statement borne out by the uniform implication of his three delivery rounds :-

Yr. Houses supplied Houses attacked

Y2. Houses supplied Houses attacked

Y3. Houses supplied Houses attacked

Y. Total houses supplied . Total houses attacked .

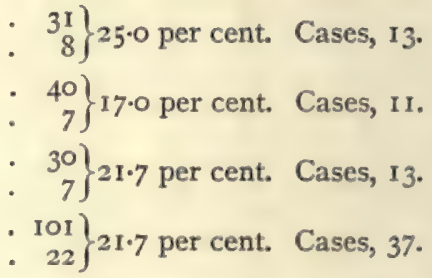

"The distribution of $\mathrm{Z}$ milk, over an extensive round, is more complicated.

"The supply from farm X, about 50 quarts, was usually reserved for the supply of the district $W$, the chief incidence of the disease was upon two out of four streets in this district.

"The milk was always distributed in this order :-

WI. The hand-can (holding) about 2 gallons) filled by dipping from the Houses supplied 1 I $)$ churn - usually X Houses attacked I\} $9 \%$ per cent. Cases, 2. milk, occasionally B

W2. The hand-can filled a

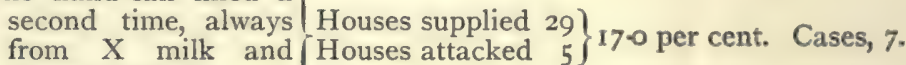
carried round.

W3. The hand-can filled a $\left.\left.\begin{array}{l}\text { third time, always } \\ \text { from } \mathrm{X} \text { milk . }\end{array}\right\} \begin{array}{l}\text { Houses supplied } 24 \\ \text { Houses attacked } \\ 5\end{array}\right\} 20 \cdot 0$ per cent. Cases, 6 .

W4. The hand-can filled a fourth time, usually from $\mathrm{X}$ milk, but when this ran short from $\mathrm{B}$ or $\mathrm{C}$.

"The immunity of $\mathrm{W}_{4}$ street seemed at first remarkable, as it was on the supply from X churn, but as this street is invariably dealt with last, and as the infection probably occurred upon a 
single occasion, all the cases sickening within a few days, absolute exemption of $\mathrm{W}_{4}$ street was quite likely to occur in the circumstance of the X supply running short on that particular day, which the distributor $Z$ believes may have happened on or about Saturday or Sunday the I3th or I4th of October.

"The WI round was amongst a different class of houses limited in number, and admittedly supplied on occasion from $B$; whereas $\mathrm{W}_{2}$ and $\mathrm{W}_{3}$ streets got no chance of exemption from the $\mathrm{X}$ supply, and suffered heavily.

"The general rounds ( $\mathrm{C}_{1}, \mathrm{C}_{2}, \mathrm{C}_{3}$ ) of this distributor in Clifton, were as a matter of routine practice restricted to the milk supplied from farms $B$ and $C$, but in order to make up quantity small portions of $\mathrm{X}$ milk were occasionally added :-

Houses supplied

Houses attacked

$\left.\begin{array}{r}52 \\ 4\end{array}\right\} 7 \cdot 6$ per cent. Cases, 6 .

"This milk was sent out on three distinct rounds, one of which ( $\left.C_{I}\right)$ remained exempt, the second had one case, and the third showed three infected houses and five cases."

These two examples set forth step by step the kind of investigations necessary in milk-borne epidemics. No fact is too trivial to be neglected, as the reader of Mr Power's reports well knows. Only by great patience and by following up every hint and suggestion or possible channel can the ultimate truth be arrived at. It is perhaps unnecessary to remark, that in London or any great city such investigations are much more complex than in the country. This is largely owing to the complications of the dairy trade. It is not simply a question of the farmer and his customers. The London milk supply is derived from various parts of the provinces, even to a distance of 200 miles from the metropolis. The farmer collects his milk from several farms and places it on the railway. It is received by a London contractor, say in the early hours of the morning, and is sorted by him at the railway terminus or at his own depôt, frequently the former. The vans are loaded and the milk is distributed over various parts of London to milkshops and dairies. These milk-shops supply smaller retail shops or street vendors or customers. To trace a small quantity of infected milk from a farmstead in, say, Staffordshire, to a small tenement in a London back street or court, is therefore a matter of considerable intricacy. But it may be said that if the problem is attacked in the way set out above in the two charts of Dr Davies, there is some hope of ultimate success. But success also depends upon an intimate knowledge of the milk trade. (See Control of Milk Supply, pp. 46I-468.) 
Methods of prevention in milk-borne epidemics.-Preventive methods in milk-borne outbreaks will be much the same as for epidemics spread in other ways. Nevertheless it is desirable that a few words should be added on this subject. (I) As the first step in the investigation of outbreaks proved to be a system of notification common to infectious diseases, by which exact data could be acquired, so in any attempt at prevention the usual first line of defence, namely, isolation of the patient and disinfection of the premises, must be considered as essential. The problem of isolation, is not in every way a simple one, and its methods and results in certain directions remain for the present sub judice. But this does not affect the elementary principle at the back of the method. To isolate an infectious person from amongst a susceptible community, whether large or small, is an obvious step in the direction of limiting the opportunities afforded for the spread of the disease. (2) In the second place, thorough and efficient disinfection of the patient's belongings and the premises he has occupied, equally stands to reason, when infectious disease is considered in the light either of the old doctrine of materies morbi or the new doctrines of modern bacteriology. (3) To these two methods of delimiting the spread of infectious illnesses may be added the third matter for consideration in all such outbreaks, namely, general sanitation. In diseases such as cholera, plague, or yellow fever, the question of sanitary environment, is the immediate necessity. So great a part do insanitary conditions and uncleanliness play in these diseases, that they have been termed "filth" diseases. No attempt at prevention could afford to neglect in such cases the immediate remedy of sanitary defects. In the diseases which we have taken as typically milk-borne, namely, scarlet fever, diphtheria, and enteric fever, the same remarks apply only in a less degree. Diphtheria and typhoid fever have a somewhat intimate relationship to insanitation in relation to milk, which therefore demands immediate and adequate consideration in any preventive methods which may be attempted.

These three cardinal principles of prevention which are applied in all epidemic outbreaks will not be forgotten or set aside, simply because an outbreak happens to have had its origin in infected milk supply. True, the one thing required is the control of the milk supply, but this should not be accepted as an indication that isolation, disinfection, and remedial sanitation are not required as much as under any other circumstances. They have their place, and although in the mind of the responsible sanitary official the 
question of the milk supply is predominant, ordinary methods must be allowed their right exercise.

We propose to treat of the control of the milk supply in succeeding chapters. The matter is a large one, and cannot be treated in the present chapter. Before, however, turning to that subject we desire to refer briefly to the relationship of bacteriology to the investigation and prevention of outbreaks of infectious disease. Such methods have only become available in late years, but they are in every way applicable to milk-borne outbreaks, and, indeed, have already been applied in such cases.

\section{Modern Bacteriological Methods and the Investigation and Prevention of Infectious Diseases}

The rapid growth of our knowledge of bacteria, particularly the pathogenic species, has afforded many additional advantages in the investigation and prevention of outbreaks of infectious disease. In a general way the new agencies are either of advantage as aids to diagnosis or as auxiliary to prophylaxis.

\section{Bacteriological Diagnosis}

(a) The bacteriological "swab" examination of the human throat has proved of the greatest utility in all throat affections, principally in diphtheria. It may prove serviceable in epidemics or threatened epidemics. Briefly, its advantage is that an outbreak can be checked at an early stage owing to the possibility of making a much earlier diagnosis than was formerly the case. It is now well known that the diphtheria bacillus may exist in the throats of healthy persons, in sore throats, and in the throats of persons suffering clinically from scarlet fever. Meade Bolton found that of 214 healthy persons who had some time been exposed to infection 45.5 per cent. showed Klebs-Löffler bacilli in their throats. ${ }^{1}$ Parke and Beebe examined the throats of 48 children apparently in good health, in 14 families in which diphtheria was present, and in 50 per cent. found the $B$. diphtherice. ${ }^{2}$ Muller examined bacteriologically 100 children in the general wards of the Charite Hospital in Berlin. Of these six, on entry, had the bacillus in their throats without exhibiting any symptoms of the disease. Two days later,

1 Med. and Surg. Reporter, 1896, vol. Ixxiv., p. 799.

${ }^{2}$ New York Medical Record, 1894, vol. xlvi., p. 396, also p. 385. 
the bacillus had infected 14 others. ${ }^{1}$ Hewlett and Murray examined the throats of 385 children in a general hospital, and found the pseudo-diphtheria bacillus in 92 (or 24 per cent.), and the Klebs-Löffler bacillus in 58 (or 15 per cent.). ${ }^{2}$ It has been estimated that of all persons exposed to infection of diphtheria about 33 per cent. get the bacillus in their throats. ${ }^{3}$. From these facts the evidence of experience is not surprising. Such evidence is twofold. In the first place it is now accepted that the continuance of an epidemic of diphtheria, particularly in isolated communities such as schools, depends in large measure upon the bacilli carried in the apparently healthy throats. This latter point has been well illustrated, and the inestimable advantage of bacteriological examination in such epidemics has been demonstrated, in the Poplar Union Schools at Forest Gate in I 898-I 899 (Goadby) ${ }^{4}$; the London Orphan Asylum at Watford $1898,{ }^{5}$ and in an outbreak at Cambridge, Chesterton, and Colchester in 1900 and 1901 (Cobbett). ${ }^{6}$ So serviceable did bacteriological diagnosis prove in the last-named outbreak that Dr Cobbett concluded that "the principal means of combating diphtheria are, after the isolation of persons actually sick, the detection of persons who go about apparently in good health carrying with them the diphtheria bacillus, and the isolation of such persons and of convalescents from the disease until diphtheria bacilli can no longer be cultivated from them." If actual isolation is impossible such infectious persons should be warned that they are a danger to others, and instructed to take certain precautions.

(b) The serum diagnosis of typhoid fever is a second example of the application of bacteriology to the investigation of epidemics. An account of the method as generally used will be found below. After five years' most careful investigation and experience of this reaction, it is safe to assume that the test is generally reliable. In 400 hospital cases the serum diagnosis was supported

1 Jahrbuch für Kindeheilkunde, 1896, B. xiii., Heft I.

${ }_{2}$ Brit. Med. Jour., Igor, vol. i., p. 1474 .

3 Trans. of the Epidemiological Society of London, 1900, vol. xix., p. 94. For further evidence on this subject see Jour. of Hygiene, I903, vol. iii., pp. 217-223 (Graham-Smith).

4 Trans. of the Epidemiological Society of London, Ig00, vol. xix., pp. 87-1 16.

I Ibid., pp. I17-122.

6 Jour. of Hygiene, I90I, vol. i., pp. 228-259 and 485-1499. See also vol. ii., 1902, pp. 170-194, and vol. iii., 1903, pp. 216-253 (Graham-Smith). 
by the course observed clinically, and all post-mortem examinations of fatal cases confirmed the result of the test. ${ }^{1}$ The true value of Widal's test can indeed only be estimated by comparing in this way the number of cases in which the test and clinical observations at an early stage prove to be correct, by the subsequent course and termination of the disease. Negative reactions before the seventh day are not always reliable, and this, in some degree, limits the utility of the test. But Delépine has maintained that of 6000 cases diagnosed in his laboratory 97 to 98 per cent. of the tests proved correct. In certain sets of cases it rose to 99 per cent. ${ }^{2}$ But it may be said that in general it is now conceded that from 95 to 97 per cent. of all cases of typhoid fever give the reaction at some time or other in the course of the disease. ${ }^{3}$ The improvements recently effected in the diagnosis test have served to make it more accurate, and therefore more reliable. Its application to individual cases of typhoid fever is now a common practice, and in most well equipped Public Health Departments in this country arrangements are made for prompt serum diagnosis in doubtful cases of enteric. But we may go even further and say that the applicability of the Widal reaction in milk-borne epidemics has been proved. One of the best known instances in which this diagnostic test was used was in the milk-borne outbreak of enteric fever in Clifton in 1897 . So graphic and explicit is the statement of Dr Davies, the Medical Officer of Health, who applied the test in this outbreak, that we may quote his own words :- ${ }^{4}$

"Between the dates 19th and 2ist October 1897 I found myself in this position: I knew definitely of five cases of undoubted typhoid, all apparently in the second week, if not later, of their illness; at the same time I had heard of a very wide prevalence of reputed 'Influenza,' the distribution of which, as it were, in 'selected' houses, struck me as but little characteristic of this disease. For instance, one college house was seriously affected, whereas adjacent houses of the same class on each side were absolutely untouched.

"I was also aware of the indeterminate nature of the onset of 'typhoid' fever, in which, as Newsholme has shown, nearly 8c percent. of the cases are not notified until the second week or later.

"In addition, the habitual exemption of Clifton from prevalence

1 Jour. Sanitary Institute, 1898, No. I.

2 Brit. Med. Jour., rgor, vol. i., p. 832.

3 Ibid., Igor, vol. ii., p. 596.

4 Trans. of the Epidemiological Society of Loncion, vol. xvii., 1898, pp. 97-101. 
of this disease was, I felt sure, liable to mislead the clinical observer in dealing with the early stages of a widespread prevalence of disease accompanied by obscure feverish symptoms.

"At the same time it became most essential in the public interest to clear up, in as short a time as possible, any doubts as to the actuality of a typhoid outbreak, so that the cause might be ascertained and removed. Now the Widal reaction will give results as early as the fourth or fifth day in cases of typhoid, and I at once determined upon the wide application of this test.

"The use of the Widal reaction in this outbreak is, I think, deserving of some attention; for, so far as I am aware, this is the first occasion upon which it has been used on a large scale and has proved successful, not only for confirming selected doubtful cases, but in 'establishing' a diagnosis amongst diverse cases with feverish symptoms, where clinical observation wås as yet at fault.

"My reliance upon the test seems to be fully justified by the results, a summary of which I submit:-

"In the case of 192 examinations made during the course of the outbreak, between I9th October and 20th December, I have been able to collate the Widal and clinical results; I find (using a dilution of in most cases $1-10$ or IO-15, but in no case exceeding I-20) that I 2 I gave a 'complete' reaction within an hour.

"All these positive results were clinically confirmed with two exceptions in which the clinical development of cases did not support the diagnosis of typhoid; so that, allowing these not to have been typhoid, the error did not amount to 2 per cent.

"In the case of I 5 specimens examined, some definite clumping was observed only after a period extending much over an hour and considerable motility persisted. Of these doubtful cases six developed as clinical typhoid, and nine proved not to be cases of this disease.

"In the case of 39 other specimens examined, negative results were obtained, of which four developed clinical typhoid; in two of these latter an opportunity of a second examination was afforded and gave then a positive result; in the other two, a second examination was not made. The other cases all proved to be not typhoid fever, thus agreeing with the Widal reaction. I think I am justified in considering these results satisfactory, especially as the examinations were made under great pressure.

"In a large number of cases the reaction I obtained was practically 'immediate,' complete clumping and entire loss of motility taking place almost at once.

"I use a hanging drop upon a cut-out moist blotting-paper cell, and in the edge-region of the drop characteristic phenomena seem to occur.

"In these 'immediate' reactions, the edge-track, which is the scene of the busiest activity before the addition of the serum, 
becomes quite deserted, and for some time the bacilli seem to have disappeared from the entire field, until found in definite 'spellican' masses in various parts of the field.

"Many other cases showed a reaction not quite so immediate, but 'complete' within five or ten minutes: in these I noticed often a peculiar 'spinning' motion of individual bacilli, to be seen frequently in the tangle of a forming clump: the bacilli seeming as if entangled by one or more flagella, and spinning rapidly round in the effort to get free, this seems a somewhat definite indication of typhoid clumping in progress.

"The varieties of false clumping which occur do not seem likely to cause confusion with a little practice; but in certain cases of scarlet fever upon which I tried the reaction, I observed in one no reaction in half an hour, marked clumping in one hour and ten minutes, but the clumps loosely aggregated, flat, and floating freely in the fluid, the free bacilli still active; after $2 \frac{1}{2}$ hours the masses ' unclumped ' and in three hours the bacilli were quite dissociated, and as active as in the original broth culture.

"The observations during the epidemic were made, as I have stated, with low dilutions, mostly $\mathrm{I}-\mathrm{IO}$ and never exceeding I-20; but I now examine a low dilution, I-IO or I-I 5, and a high dilution, I-40 or I-50; but if the low dilution gives a negative result, the higher dilution is of course unnecessary. I find generally that, in the blood from a case of actual typhoid, the reaction in the case of the high dilution is very little less rapid and marked than in low dilution.

"The results obtained show, I imagine, that considerable reliance can be placed upon the reaction when 'complete,' i.e. as to aggregation and loss of motility, within a short time limit, even when I-IO or I-I 5 dilution alone is used. I do not think this time should exceed an hour even for I-50 dilution.

"I used two cultures, one an agar subculture from one supplied to me by Dr Klein; the other an agar subculture from the spleen of a fatal case during the outbreak; broth cultures are made from one or another of these daily; they are both extremely active, especially the older one; for measurement, drop measurement on a watch-glass seems sufficiently accurate, and is handier, especially with small quantities of blood. I prefer blood collected in small centre bulb-glasses: vaccine tubes are vexatious to blow out, and apt to get over heated in the sealing-which accounts, I imagine, for some of my negative results that proved wrong, as they occurred under these conditions of collection. Zeiss D or an English $\frac{1}{6}$-inch objective appear the most convenient for observation.

"Comparison of results. - Of the Widal examinations, 36 were of cases admitted to the Royal Infirmary, Bristol. Of the 36 cases, 26 gave a positive result: of these 14 acted almost immediately, clumping and loss of motility being established in a few minutes; 9 acted not quite so rapidly, the reaction being complete within 
fifteen minutes; the remaining 3 showed a complete reaction within thirty minutes. The dilution varied from I in IO to $I$ in 20 , but did not exceed this. The clinical course of every one of these 26 cases determined them to be typhoid."

Thursfield ${ }^{1}$ has shown that the Widal test is as reliable in children as in adults.

Preventive inoculations.-As we have seen bacteriology has materially assisted in the checking of epidemics partly by diagnosis and partly by preventive inoculation. The latter is illustrated in various diseases such as small-pox (vaccination), rabies (Pasteur's treatment), cholera, typhoid, and plague (inoculations), and diphtheria (antitoxic serum). It will be sufficient if typhoid fever and diphtheria, the two diseases more particularly conveyed by milk, are alone referred to in this place, and that only in so far as applicable to the prevention of epidemics.

The vaccine employed in the anti-typhoid inoculation was first made by Professor Wright of Netley. ${ }^{2}$ It has been used to a large extent among soldiers in India and South Africa with some measure of success, and it has been applied in an epidemic of typhoid fever. ${ }^{3}$

The antitoxin of diphtheria has been used on various recent occasions as a prophylactic in outbreaks of the disease, and it is now considered as one of the practicable means for controlling an epidemic. Antitoxin inoculation played a greater or lesser part in the checking of diphtheria outbreaks at Cambridge, ${ }^{4}$ Colchester, ${ }^{5}$ Kempston, ${ }^{6}$ and other places. In the Cambridge outbreak antitoxin was supplied free for prophylactic use in the case of those who had come into contact with actual cases of diphtheria, or where those who, not being ill, were known by bacteriological examination of the throat to be harbouring the diphtheria bacillus. Thus free bacteriological examination of the throat of suspected or known "contacts" was first carried out. In the cases yielding positive results antitoxin was injected. At Cambridge 500 units of antitoxin were given in such contact persons; at Kempston 1000 units was the dose. It is unnecessary to dogmatise as to

1 Brit. Med. Jour., I9or, vol. ii., p. 596.

2 For the methods which have been employed in the preparation of antityphoid vaccine, see Brit. Med. Jour., I900, vol. i., pp. 122-129.

${ }^{3}$ Brit. Med. Jour., 1901, vol. ii., p. 1226.

4 Jour. of Hygiene, 1901, vol. i., pp. 228 and 487.

S1bid., 1902, vol. ii., p. 170.

- Report on an Outbreak of Diphtheria at Kempston, p. 2I (Newman). 
the exact size of the dose. The two chief points to be borne in mind are early administration (following at once on the bacteriological positive diagnosis), and as large a dose as is practicable. $^{1}$

1 The benefit of antitoxin inoculation is now generally admitted. Its value is clearly demonstrated in the returns of the Metropolitan Asylums Board. In 1894, 3042 patients of all ages were treated for diphtheria in the Board's hospitals without antitoxin ; 902 died, yielding a mortality of 29.6 per cent. on all cases of diphtheria treated. In 1895 the antitoxic serum treatment was inaugurated; 3529 cases of diphtheria were treated and 729 died, yielding a mortality of 22.5 per cent. Hence in the first year there was a fall in mortality of $7 \cdot 1$ per cent. In $1900,727 \mathrm{I}$ patients suffering from diphtheria were treated with antitoxin in the Board's hospitals; 936 died, yielding a mortality of 12.8 per cent. That is to say, during six years the mortality has fallen 17 per cent., or in other words, more than I 200 lives were saved in London in 1900, which would not have been saved, other things being equal, had the mortality rate been what it was in 1894 without antitoxin treatment. In 1902,7707 cases of diphtheria were treated with antitoxin, and 739 died, yielding a mortality of $\mathrm{I}$ I.04 per cent. (as compared with 29.6 per cent. in 1894). The value is particularly noticeable among children. Amongst cases in the first year of life the rate has fallen from $6 \mathrm{r} \cdot 8$ to 37.8 ; in the second year from 63.1 to 35.4 ; in the third year from 55.1 to 26.4 ; in the fourth year from 48.3 to 22.9 ; and in the fifth year from 39.6 to 20.7 . At the Metropolitan Asylums Board Brook Hospital in 1901, 723 cases of diphtheria were treated with antitoxin, and 78 died, yielding a mortality per cent. of 10.79. The antitoxin treatment was applied in each of these cases, but in some it was possible to begin on the first day of the disease, in others on the second, and so on. The paramount importance of administration at the earliest possible moment is seen in the result. The mortality percentage of the first day cases was 0.0 ; of the second day, $4 \cdot 1$; of the third day $11 \cdot 9$; of the fourth day; I2.4; and of the fifth and subsequent days, 16-6. For five consecutive years there has not been a death at this hospital among the cases that came under treatment on the first day of the disease. Even more striking evidence of exactly the same nature has just been reported in Massachusetts as the result of seven years' experience with antitoxin (see Report of Metropolitan Asylums Board, 1901, 1902). For the most complete account of diphtheria antitoxin and its effects see Report on the Bacteriological Diagnosis and Antitoxic Serum Treatment of cases admitted to the Hospitals of the Metropolitan Asylums Board, 1895-96, by Professor Sims Woodhead, M.D. 


\section{CHAPTER XII}

\section{DESCRIPTION OF SOME SPECIES OF MILK BACTERIA}

THE method of description and differentiation adopted in the following pages follows the recommendations of the Bacteriological Committee of the American Public Health Association (I 897), in so far as it was found practicable to apply them to species already described. Each organism, as far as facts were obtainable, is therefore described on the same basis. The list is of milk bacteria or their allies. Organisms of water, soil, etc., are not included. ${ }^{1}$ Descriptions are given only of organisms, for which the claim has been made that they have a particular and intimate relationship to milk, butter, or cheese. Of course the list is provisional only. Present knowledge of the various species of bacteria belonging to milk is very limited, and what there is remains at present in a condition of extreme chaos. Nomenclature technique vary widely. Milk also varies widely. Hence at present confusion reigns, except in regard to a few well-known species. The following list is an attempt to rescue from the chaos some of the more reliable descriptions of organisms. They are placed here for the guidance of milk bacteriologists, and, although imperfect, form, we believe, the most complete record yet published. We recognise, however, that it is very far from satisfactory. As far as possible, the author's own description of each species has been adopted. No attempt has been made at classification. ${ }^{2}$ The species are placed in alphabetical order.

1 For descriptions of such organisms the works of Sternberg, Flügge, Lehmann, and Neumann, Macé, or Horrocks may be consulted.

2 Flügge, Migula, Conn, and others have made attempts to classify milk bacteria, and the last named has probably carried the matter to the most successful issue. But all such classification is premature at present. See Storr's Agric. Sta. Twelfth Report, 1899, p. I3. 
AROMA BACTERIUM (Weigmann).

Source and habitat-Milk and its products.

Morphology-Bacillus. Thick rods of varying length, at times curved, isolated, or in groups.

Staining reaction-Ordinary aniline stains.

Spore formation-Present.

Biology : cultural characters (including biochemical features).

Bowillon-Becomes cloudy after 24 hours; a thin veil forms on the surface of the medium; later, the medium clears with formation of thick, surface veil; no deposit.

Gelatine-In stab-cultures a thin beaded growth along the needle track, with slow liquefaction of gelatine in "stocking" form, the growth forming a creamy deposit as the liquefaction proceeds. In stroke cultures a similar growth.

Agar - Stab - cultures, sparse growth along the needle track, with hob-nail formation on surface, of a creamy nature and yellowish tint. On stroke cultures a creamyyellowish growth along. needle track. On glycerine-agar, growth more rapid and of similar nature to above, but of a drier nature and slightly wrinkled with age.

Potato-At first a smooth, white growth, darkening slightly with age, and showing fine granulation.

Milk-No coagulation. Deposit formed at bottom of tube.

Aërobic.

Non-pathogenic.

\section{BACHLUS ACIDI LACTICI}

(Hüppe).

Sounce and habitat-Sour milk.

Morphology-Oval rods; 0.6 to $2 \mu$ long, 0.4 to $0.6 \mu$ broad; usually in pairs, rarely in chains.
Staining reaction - Ordinary stains and slightly by Gram's method.

Motility-No flagella; non-motile.

Spore formation-Absent.

Biology: cultural characters (including biochemical features)-Good growth at room temperature and blood heat.

Bowillon - Diffuse turbidity ; abundant sediment.

Gelatine plates and tubes-Colonies similar to $B$. coli; small, smooth, round, white.

Agar plates and tubes-Colonies similar to $B$. coli; small, smooth, white growths, moist and shiny.

Potato-A wavy, smooth-edged growth, elevated; greyish-white or yellowish-white in colour, sometimes turning brown.

Milk-Solid coagulation, leaving clear fluid; occasionally some gas bubbles. Lactic and acetic acids are produced. Powers of acid coagulation of milk are gradually lost after long cultivation upon gelatine or agar.

Anaërobic or aërobic-Grows well aèrobically, and if sugar present in medium anaërobically also.

Non-pathogenic.

\section{BACILLUS ACTINOBACTER}

(Duclaux).

Source and habitat-Widespread in nature and common in milk, the most favourable medium for its growth.

Bacillus-In milk, fine rods from 2 to $3 \mu$ long. Isolated or united in pairs.

Capsule; motility-In milk the organism, either singly or in pairs, endon-end, is surrounded with an ovoid or rounded gelatinous capsule of 5 to $6 \mu$ broad. The organism is non-motile.

Spore formation-None observed. 
Biology: cultural characters - Milk becomes gelatinous and very viscous.

Bouillon-Formation of white flocons composed of chains of 8 to $10 \mu$ in length, the elements of which are shorter than those observed in milk. No capsule formation in bouillon. In solutions containing sugar, the elements are shorter. A slimy veil appears on the surface of the liquid.

In milk, and in solutions containing sugar or glycerine, the organism sets up an active fermentation, with disengagement of carbonic acid and hydrogen, and (in the case of milk only) small quantities of sulphuretted hydrogen. There is production of ethylic alcohol and a little acetic acid. In a solution of lactate of lime, lactic acid is obtained, but no alcohol.

Non-pathogenic.

BACILLUS AIRPYUS (Adametz).

Source and habitat-Isolated from water, and found in milk.

Morphology_Slender bacilli, straight or slightly bent. $I \cdot 5 \mu$ to $4 \mu$ long by $0.5 \mu$ broad. The majority lie parallel to one another in groups; sometimes occur in long threads.

Staining reaction-Ordinary aniline stains.

Motility-Feebly motile.

Spore formation-None.

Biology: cultural characters (including biochemical features).

Gelatine plates-The colonies develop slowly, and are of various shapes. After 8 days the larger number only appear as small white dots, which, however, become later of a bright yellow colour, and finally of a chrome yellow tint. No liquefaction of gelatine.

Potato-Anirregular, dark brown- yellow growth, becoming reddishbrown in old cultures.

Non-pathogenic.

BACILIUS, No. 14 (Adametz).

Source and habitat-Milk.

Morphology-Straight bacillus; I to $4 \mu$ long by 1 to $\mathrm{I} \cdot 2 \mu$ broad. Sometimes in threads.

Staining reaction-Ordinary aniline dyes.

Motility-Non-motile.

Spore formation-Oval spores are present.

Biology: cultural characters (including biochemical features).

Gelatine plates-Slimy membrane; rapidly liquefying the medium. Colonies with sinuous margins, granular and grey.

Agar plates-Produces a thickly folded, dirty-white skin, later becoming reddish-yellow in colour.

Milk-At $37^{\circ}$ C. a flaky precipitation occurs, with alkaline reaction and peptonisation.

Non-pathogenic.

BACILLUS, No. 15 (Adametz).

Source and habitat-Milk.

Morphology - Straight rod ; involution forms occur; 3.5 to $5 \mu$ long by I. 2 to $\mathrm{I} \cdot 4 \mu$ broad. Sometimes in threads.

Staining reaction-Ordinary aniline stains.

Motility-Non-motile.

Spore formation-Oval spores are present.

Biology: cultural characters (including biochemical features).

Gelatine plates and tubesColonies granular, showing in the middle a dark grey cloudy mass, but having a clear grey margin. Stab-cultures are rapidly liquefied, with slight surface membrane. Old 


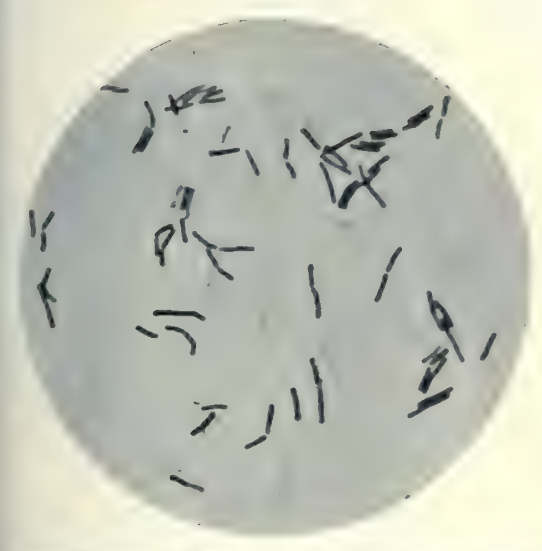

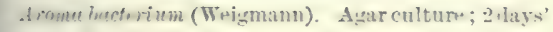
gruwth at $37^{\circ} \mathrm{C}$. Stained carbol-fachsin dil. $\times 1000$.

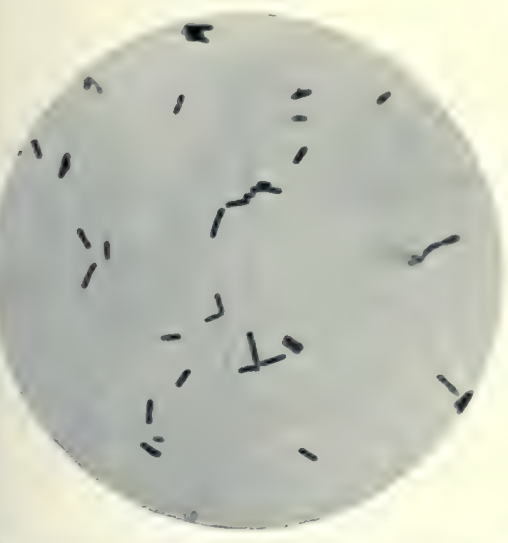

L. cyomonenes (Fliigge). Agar culture ; 2 days' crowth at 37 C. Stained carbol-fuchsin dil, $\times 1000$.

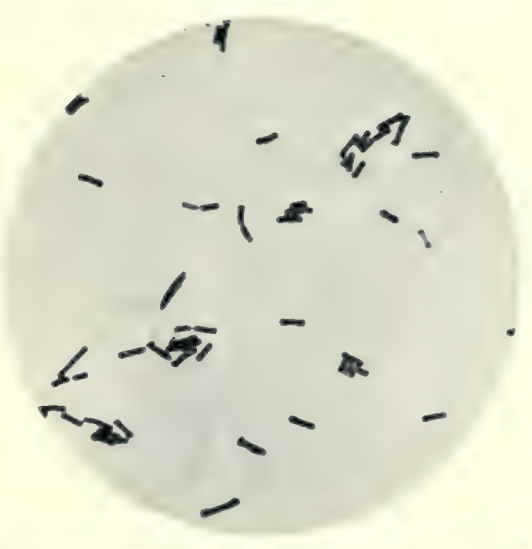

L. Imetyricus (Huepun). Agar culture; 2lays grouth at 3 i C. Stained carbol-fuchsin dil. $\times 1000$.

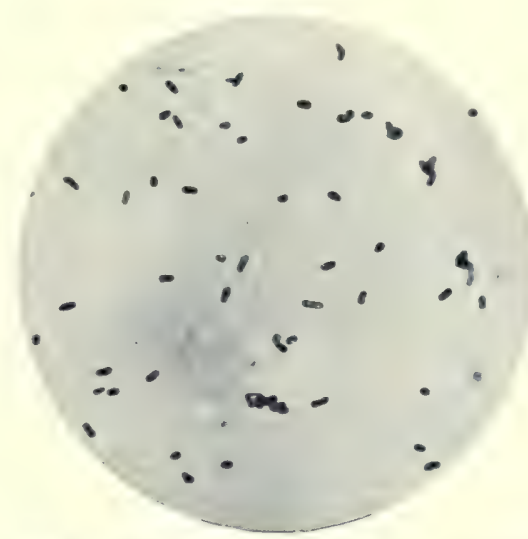

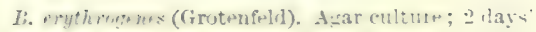
growth at $3 r^{\circ} \mathrm{C}$. Stained carbol-fuchsin dil. $\times 1000$. 

cultures have a smell of butyric acid.

Milk-At $37^{\circ} \mathrm{C}$. becomes slimy and flaky, with weak acid reaction, and a smell of butyric acid. Peptonisation.

\section{Non-pathogenic.}

BACILLUS, No. 16 (Adametz).

Source and habitat-Milk.

Morphology-Straight rod ; involution forms; 3.5 to $5 \mu$ long by $I$ to $2 \mu$ broad. Growing sometimes into long threads.

Staining reaction-Ordinary aniline stains.

Motility-Non-motile.

Spore formation-Present.

Biology : cultural characters (including biochemical features).

Gelatine plates-Radiate and arborescent, rapidly - liquefying colonies. In stab-cultures a pellicle appears on the surface of the liquefied medium.

Agar plates-Show a thin, wrinkled growth. In agar stab there are ramifications of the growth into the medium; arborescent colonies.

Milk-At $37^{\circ} \mathrm{C}$. rapid gelatinous precipitation occurs through formation of acid, with agreeable smell of cheese. Does not ferment milksugar.

Non-pathogenic.

BACILLUS, No. 17 (Adametz).

Source and habitat-Milk.

Morphology -3 to $4 \mu$ long by 1 to $1 \cdot 2 \mu$ broad. Frequently forming threads.

Staining reaction-Ordinary aniline stains.

Motility-Non-motile.

Spore formation-Present.
Biology : cultural characters (including biochemical features).

Gelatine plates-Membraneous, white, irregularly bounded colonies, which rapidly liquefy the medium.

Agar plates-A wrinkled, slimy layer.

Milk-At $37^{\circ} \mathrm{C}$. becomes rapidly a plastic mass, which changes with shaking into a slimy syrup, with strong acidification and a smell of butyric acid. Does not ferment milk-sugar.

Non-pathogenic.

BACILLUS ARBORESCENS LACTIS (Conn).

Source and habitat-Milk.

Morphology- $\mathrm{r} \cdot 8 \mu$ by $3 \mu$; grows in long chains.

Staining reaction-Ordinary aniline stains.

Spore formation-Large spores ( $1 \cdot 2 \mu$ by $2 \mu)$; occurring in centre of bacillus and causing it to swell very much.

Biology: cultural characters (including biochemical features).

Gelatine plates and tubes-Large "felted ground glass" colony which sinks slowly in a shallow pit of liquefied medium. In stab-cultures a thick, ground-glass surface growth which eventually floats on liquefied medium as a tenacious folded scum.

Agar plates and tubes-A remarkably spreading branching mass covering the surface and even growing under the surface.

Potato - Almost snow-white growth which extends into the potato. The surface is raised into folded mounds.

Milk - Is rapidly curdled at $20^{\circ} \mathrm{C}$ and $35^{\circ} \mathrm{C}$, and digested into a cloudy or amber coloured liquid with alkaline reaction. Butter made 
from cream ripened with this organism has an unpleasant flavour and aroma.

Aërobic.

Non-pathogenic.

BACILLUS ARBORESCENS LACTIS, No. 2 (Conn).

Source and habitat-Milk.

Morphology $\longrightarrow 8 \mu$ by $4 \mu$; occasionally two or three rods together, but no chains.

Staining reaction-Ordinary aniline stains.

Spore formation-Spores at one end of bacillus (tetanus type); size of spores I $\mu$ by I.2 $\mu$.

Biology: cultural characters (including biochemical features) - In bouillon a tough pellicle is formed which sinks if disturbed and forms a flocculent sediment.

Gelatine plates and tubes-Colonies show radiating fibres strewn with knots; fibres fine and branching, growing mostly under surface. In stab-cultures an arborescent growth occurs; fibres ending in knots. Liquefaction; dense, cloudy liquid.

Agar plates and tubes-Thin; hardly visible growth.

Potato-Thin and scanty growth. Milk-No effect produced in milk.

Non-pathogenic.

\section{BACILLUS ANANA (Conn).}

Source and habitat-Milk.

Morphology - $5 \mu$ broad, I to $\mathrm{I} \cdot 2 \mu$ long.

Staining reaction-Ordinary aniline stains.

Biology: cultural characters (including biochemical features).

Gelatine plates and tubes-Round opaque granular colonies breaking up to form a pit covered with mottled granular masses. Liquefaction. In stab-cultures a narrow pit is produced containing a granular liquid. The pit broadens at the surface and contains very cloudy liquid.

Agar plates and tubes-A moist white abundant growth.

Potato - A very thick, white, abundant growth, having the odour of pine-apple.

Milk-Curdles at $20^{\circ} \mathrm{C}$, into a soft curd. No digestion noticeable. No curdling at $36^{\circ} \mathrm{C}$.

Aërobic.

Non-pathogenic.

\section{BACILLUS AURANTIACUS.}

Source and habitat-From deep well water obtained from the chalk; thence to milk.

Morphology-Short thick bacilli ; I.7 $\mu$ long by $0.6 \mu$ broad. Singly, in pairs, or in threads.

Staining reaction-Ordinary aniline dyes.

Motility-Present.

Spore formation-None.

Biology: cultural characters (including biochemical features).

Bouillon - Remains clear; growth occurs as a slight orange deposit. There is a thin pellicle exhibiting here and there bright orange spots.

Gelatine plates-There slowly appears a bright orange punctiform growth, and there is no liquefaction.

Agar tubes-The growth shows itself along the track of the needle. It is a bright orange colour.

Potato - Thick growth of a brilliant orange-red colour.

Aërobic.

Non-pathogenic. 
BACILLUS AUREUS ACIDI (Conn).

Sounce and habitat-Milk.

Morphology-A bacillus; $6 \mu$ broad, $.7 \mu$ long (occasionally longer).

Staining reaction-Ordinary stains.

Biology: cultural characters (including biochemical features).

Gelatine plates and tubes-Round yellow colonies; opaque if deep ; transparent if on surface; of an orange colour; non-liquefying ; in stab-culture produces a dry pit lined with an orange-yellow skin. Agar plates and tubes; potatoOrange-yellow growth.

Milk-Curdled in 2 to 4 weeks into moderately hard curd which is acid, showing no whey and no peptonisation.

Aërobic.

Non-pathogenic.

\section{BACILUUS AUREUS LACTIS, NOS. 1} and 2 (Conn).

Source and habitat-Milk.

Morphology - Bacillus, round ends ; $.5 \mu$ broad, $\cdot 7$ or I $\mu$ long. No chains, but two or three may adhere together.

Staining reaction-Ordinary aniline stains.

Biology : cultural characters (including biochemical features).

Bouillon-No. I produces an orange-yellow tenacious scum on clear bouillon. No. 2, a thick tenacious scum which sinks in flakes and produces a sediment.

Gelatine plates and tubes-Yellow colonies, dark or depressed centre. No liquefaction. In stab-culture, No. I, good growth with yellow surface growth, No. 2, slight growth with white surface growth.

Agar plates and tubes-No. I produces a moist, thick, yellow growth. No. 2, a smooth, white growth, turning lemon colour.
Potato - Abundant growth ; yellow, or commencing white and becoming yellow.

Milk-No effect except formation of a scum in both varieties.

Aërobic.

Non-pathogenic.

\section{BACILLUS AURHUS MTNU. TISSIMUS (Conn).}

Source and habitat-Milk.

Morphology-Rod; $4 \mu$ broad and I. $6 \mu$ long. Three or four may be united together in threads (especially in bouillon).

Staining reaction-Ordinary aniline stains.

Capsule.

Biology: cultural characters (including biochemical features).

Bouillon-A slight scum on uniformly clouded fluid. A yellow sediment in a few weeks.

Gelatine plates and tubes-Thin irregular branching colonies. Liquefaction in 2 days, with yellow centre and irregular processes extending into medium. In stab-culture a deep, narrow funnel with bright yellow sediment and scum.

Agar plates and tubes - An orange-yellow growth, spreading over whole surface.

Potato-A dark orange growth of deep colour.

Milk-Becomes pasty and dark coloured at $20^{\circ} \mathrm{C}$. Slight slimy change. Reaction alkaline.

Aërobic.

Non-pathogenic.

\section{BACILLUS BERNENSIS.}

Source and habitat - Emmenthaler cheese, producing aroma.

Morphology-Thick rods.

Staining reaction-Ordinary aniline stains. 
Motility -Feebly motile.

Spore formation-Oval spores in presence of oxygen.

Biology: cultural characters (including biochemical features).

Bouillon-Bouillon becomes cloudy with formation of surface pellicle.

Gelatine tubes; agar tubes-Stabcultures resemble those of the hay bacillus.

Potato-Growth moist, smooth, without lustre and without wrinkle.

Milk-Is coagulated in about 24 hours, the coagulum being subsequently dissolved after about 43 hours. There is a strong, genuine odour of Emmenthaler cheese, and this also occurs upon sterilised casein precipitated by rennet.

Facultative anaërobe.

Non-pathogenic.

\section{BACILLUS BUTYRICUS (Botkin).}

Source and habitat-Widely distributed in milk, water, soil, dust.

Morphology—Rods, I to $3 \mu$ long, 0.5 $\mu$ thick. Sometimes in threads, sometimes in chains.

Staining reaction-Stains by Gram's method.

Motility-Motile.

Spore formation-Spores in middle or at end of bacillus; about $\mathrm{I} \mu$ thick (provisional); sporulation not proved (Botkin).

Biology: cultural characters (including biochemical features)-Favourable temperature $37^{\circ} \mathrm{C}$. ; organism contains starch granules.

Bouillon-Slight growth; at $18^{\circ}$ C. involution forms may occur. Vigorous growth if glucose present, with opaque turbidity.

Gelatine plates and tubes-Round or oval colonies with sinuous edges ; medium is rapidly liquefied; gas development; slight undulating colonies as if consisting of mass of felted threads. No odour.

Agar plates and tubes-A luxuriant growth with gas development and ramifications in medium. Odour present.

Potato - Growth extends into potato substance; smell of alcohol.

Milk-At $37^{\circ}$ C., after 15 hours casein precipitated and butyric acid is formed without intermediate formation of lactic acid. Coagulum eventually dissolves; before that stage the appearance is very characteristic; there is a spongy fatty layer on surface, then clear fluid, and then a white deposit. The presence of this organism is readily proved in almost all milk. Fill a half litre flask with milk, and steam at $100^{\circ} \mathrm{C}$. for half an hour. Incubate at $37^{\circ} \mathrm{C}$. In less than 24 hours the characteristic changes will occur, with strong odour of butyric acid. Care must be taken that the gas pressure does not burst the flask. There is a marked odour of butyric acid. Other acids present are acetic, formic, and lactic.

Anaërobic oraërobic-Facultative anaërobe.

Non-pathogenic - It has been suggested that the $B$. enteritidis sporogenes of Klein is a pathogenic form of this bacillus.

\section{BACILLUS BUTYRICUS (Hüppe).}

Source and habitat-Milk.

Morphology-Slender rods; I.2 to 4 $\mu$ long, $0.5 \mu$ thick; round ends. May grow into filaments; rods slightly bent; $2.1 \mu$ long, $0.3 \mu$ broad.

Staining reaction-Stains by Gram's method.

Flagella; motility-Many flagella; actively motile. 
Spore formation-Oval spores at $37^{\circ}$ C. ; mesially situated.

Biology : cultural characters (including biochemical features).

Bouillon-A pellicle is formed; bouillon remains clear. No indol.

Gelatine plates and tubes-Small whitish-yellow colonies with crater shaped depression; liquefaction; whitish grey wrinkled pellicle produced in liquid cultures in tubes; liquefied medium cloudy and yellowish in colour.

Agar plates and tubes-A thin yellow layer, similar to $B$. mesentericus.

Potato-A fawn coloured transparent layer, sometimes wrinkled. Somewhat similar to $B$. megatherium.

Milk - Is coagulated. Precipitated casein subsequently dissolved. Bitter taste. Butyric acid produced from salts of lactic acid; also from milk-sugar when it is previously hydrated.

Facultative anaërobe.

Non-pathogevic.

BACILLUS BUTYRICUS (Pasteur). (Vibrion Butyrique.)

Source and habitat-Air, and thence to milk.

Monphology - Cylindrical rods with rounded extremities; $3 \mu$ to $5 \mu$ long by $.6 \mu$ to $.8 \mu$ broad. Isolated or in chains; at times in long filaments indistinctly articulated.

Staining reaction-Ordinary aniline stains.

Motility - Feebly motile ; motility ceases at once in presence of free oxygen.

Spore formation-Ovoid spores.

Biology : cultural characters (including biochemical features).

Bouillon-Grows freely under strictly anaërobic conditions in bouillon containing lactate of lime. Agar plates and tubes-In agar "shake" cultures free from oxygen the medium becomes clouded in the lower portion, and is soon broken up, with copious gas formation accompanied by strong smell of butyric acid.

Gelatine plates and tubes-As upon agar, but in a less degree, the medium liquefying in the neighbourhood of the forming colonies.

Anaërobic.

Non-pathogenic.

\section{BACILLUS BUTYRICUS}

(Prazmowski).

\section{Source and habitat-Milk.}

Morphology - Rods; sometimes appear as unjointed threads; sometimes bent like vibrios ; 3 to ro $\mu$ long by I $\mu$ broad. Sometimes in groups in zoogloea.

Staining reaction-Ordinary aniline stains.

Motility-Actively motile.

Spore formation-Present ; rods sivell and become spindle-shaped or ellipsoidal.

Biology: cultural characters (including biochemical features)-Grows best between $35^{\circ}-40^{\circ} \mathrm{C}$.

Gelatine plates and tubes-Liquefaction of gelatine; a scum forms on the surface.

Milk-Butyric fermentation set up.

Non-pathogenic.

Several other butyric acid organisms have been isolated, of which a few notes may be added:-

Bacillus acidi butyriei-(Kedrowski's Butyric acid bacillus). Anaërobic. Kedrowski (Z. I6. 3) has isolated from mixtures of sugar solution 
with bad cheese or rancid butter which has been placed in the incubator, two organisms which only show small deviations from one another. ( $C f$. the $B$. saccharo butyricus of Klencki from cheese.) Kedrowski's $B$. acidi butyrici is a motile bacillus, which towards one end produces ellipsoidal spores. The staining of the spores is readily accomplished. The colonies in gelatine show rays-those in agar partly reticulated-and interlaced spurs. Liquefaction of gelatine is more or less marked. Milk is coagulated with separation of serum on the surface (acid reaction). There is gradual peptonisation and simultaneous gas development.

Gruber's Butyric acid bacillus, No. 1, isolated by Gruber. Anaërobic. Bacilli of 3 to $5 \mu$ long by 0.6 to $0.8 \mu$ broad; sometimes joined together in threads. Before sporulation they become swollen to the thickness of $2 \mu$. The spores appear generally nearer to one end, they are readily double-stained. Colonies in gelatine are blackbrown. The organism produces butyric acid and "Butylalkohol."

Gruber's Butyric acid baclllus, No. 2 Anaërobic. Bacilli 2 to $8 \mu$ long by $\cdot 5 \mu$ broad ; curved in comma or sigma shape. Before forming spores they become enlarged in all dimensions. Spores $1.5 \mu$ long by $.8 \mu$ to $\mathrm{I} .0 \mu$ broad. The colonies are round or slightly uneven, at first faintly yellowish, then yellow-brown and roughly granulated. Gas production.

Gruber's Butyric actd baclllus, No. 3A facultative anaërobe, differing from the bacillus pseudo-butyricus of Huippe, in that it is spore-bearing-bacillus $3 \mu$ to $5 \mu$ long by 0.6 $\mu$ to $0.8 \mu$ broad. During sporula- tion the bacilli swell up to twice or thrice their breadth, in spindle and citron shape-medial spores of variable size are formed (maximum I $2 \mu$ to $2 \mu$ ). Dwarf spindles with spores are frequent. In gelatine colonies round, resembling a ball. Gelatine liquefied. Fermentations same as in Nos. I and 2 of same worker.

\section{BUTTER BACILLUS OF RABINOWITSCH}

(Petri-Rabinowitsch), 1897.

Source and habitat-Butter.

Morphology-Same form as tubercle bacillus, but thicker and often clubbed. Isolated, or ranged parallel to one another-at times in forms of long non-ramified filaments.

Staining reaction-Ziehl-Neelsen, or method of Ehrlich, and remains coloured by Gram's method.

Motility-Non-motile.

Biology: cultural characters (including biochemical features).

Bouillon-Renders bouillon turbid and acid eventually, but at first the bouillon remains clear; produces trace indol (Rabinowitsch); thick wrinkled pellicle formed ; disagreeable ammoniacal odour. In glucose bouillon the pellicle is very marked. $\mathrm{H}_{2} \mathrm{~S}$ is produced in bouillon.

Gelatine plates-Slow growth; small granular colonies ; transparent periphery, becoming opaque later ; non-liquefying.

Agar plates and tubes-Grows very well, especially on glycerineagar. Golden or copper coloured film, much wrinkled, moist, thick, creamy (see frontispiece); glistening appearance; somewhat unpleasant odour. Aftermuch subculturing becomes dry, and loses its creamy and moist appearances. On magnification granularity visible. Agar 


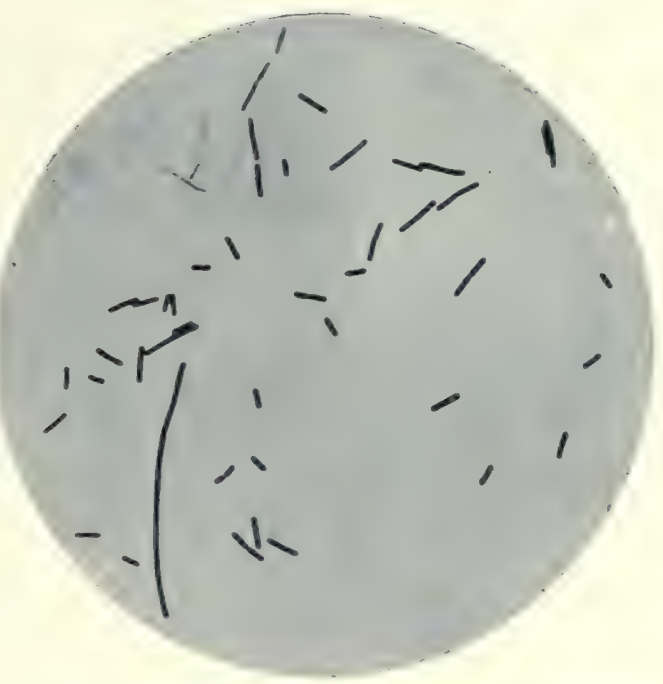

Acid-fast bacillus from butter (Petri-Ralinowitsch). Agar culture; 2 days" growth at $37^{\circ}$ C. Stained Ziehl-Xeelsen. $\times 1000$.

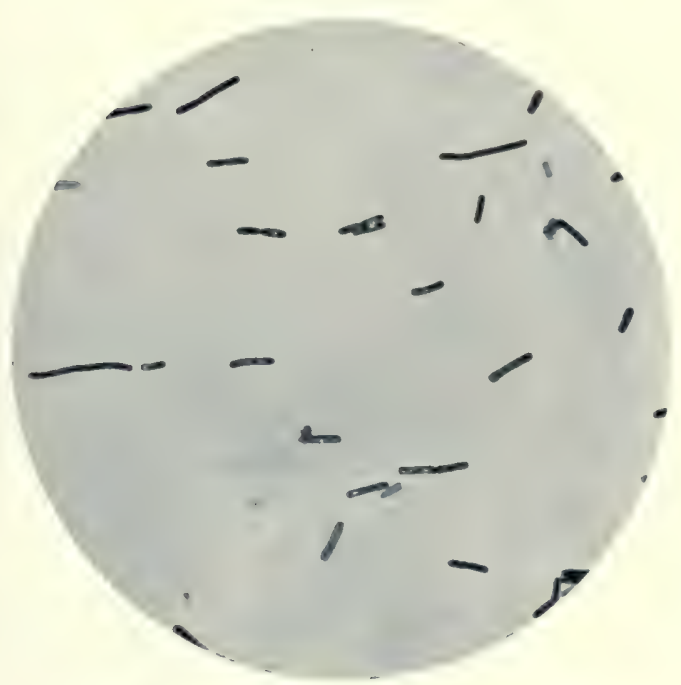

B. lact is niger (Gorini). $\Delta$ gar culture; 2 days" growth at $37^{\circ} \mathrm{C}$. Stained carbol-fuchsin dil. $\times 1 \mathrm{c} 00$. 

smeared with butter-small,-dry, white colonies, soon assuming an orange tint and becoming confluent.

Milk-No coagulation; formation of reddish-yellow surface ring.

Potato-Whitish to bright orange growth with some elevation; dull, dry, wrinkled, not glistening.

Blood serum-Poor growth; a thin greyish layer after a month.

\section{Aerrobic.}

Cultures contain a fatty substance. Treated with Flemming's solution, they take a black tint.

Pathogenesis-Rabbits and white mice are immune. In guinea-pigs nodules are found in peritoneum, mesentery, liver, and spleen. Mesenteric glands though swollen do not usually become caseous. The bacillus is found to be most numerous in glands and nodules, but it is scarce in the blood. Pure cultures are less virulent than butter containing the bacillus. The lesions have a tendency to heal. Giant cells, epithelioid cells, and caseation are generally absent.

BUTTHR BACILLUS (Binot).

Source and habitat-Butter.

Morphology-Bacillus; like tubercle bacillus.

Grouping - Like the tubercle bacillus. Staining reaction-Acid-fast. ZiehlNeelsen and Ehrlich stains.

Motility - No motility observed.

Spore formation-None observed.

Biology: cultural characters (including biochemical features)-Grows at ordinary room temperature, but more rapidly at $37^{\circ} \mathrm{C}$.

Bouillon-A thin friable veil is formed of a pale yellowish colour ; no deposit. Very slight turbidity : thick viscous creamy veil is produced in glycerine bouillon. Stalac- tite growth in the bouillon; becomes orange colour in a few weeks.

Gelatine plates and tubes-Grey white colonies; thin, opaque, and creamy; surface irregular. There is no liquefaction of the gelatine.

Glycerine-agar plates and tubes -White colonies becoming strawcoloured, finally orange. May attain diameter of two franc-piece or larger; bright glistening surface ; very adherent to medium ; no growth in depth of medium; surface soon becomes wrinkled, with scalloped edges (Plate 25, p. 256). Chromogenic characters more marked if growth exposed to air and light. Stroke cultures show same character. On ordinary agar colonies similar, but less luxuriant.

Potato-Scanty growth, at first moist ; clear yellow colour, becoming "farineuse" with age. On glycerine potato an abundant homogeneous growth occurs; opaque, yellow, turning to orange; irregular nodosities appear on the colony.

\section{Aërobic.}

Vitality-Considerable.

Pathogenesis-Binot inoculated a guinea-pig in March 1899 with the butter bacillus. The animal died spontaneously, slightly emaciated, at the end of 29 days, having the following post-mortem characters : - On post-mortem examination the viscera of this animal were found to be of normal size and aspect, with the exception of the spleen, which was slightly enlarged. The liver and the spleen were, however, found to be studded throughout with fine miliary granulations of the size of a small pin's head, and of a yellowish-white colour, recalling vaguely those found in cocco-bacilliary tuberculosis, In the peritoneum 
could be seen numerous miliary granulations and a few voluminous masses with caseous contents forming as a whole a mass more considerable than the small quantity of butter inoculated (2 centimetres cube). Four of these masses, in fact, were larger than a fair-sized nut. The lungs and kidneys, otherwise normal in appearance, showed a few miliary granulations of a similar nature. Both in these miliary granulations and in the caseous masses, of which no detailed histological study was made, were found numerous bacilli presenting all the characters of bacillus tuberculosis, and in particular when stained by Ehrlich's method (Binot).

\section{BUTTER BACILLUS (Markl).}

Source and habitat-Vienna butter.

Morphology - Bacilli forming filaments, short or long, and resembling streptothrix.

Staining properties - Is not very resistant to decolorisation by acids or alcohol. Remains coloured by Gram's method.

Biology : cultural characteristics.

Bouillon - Thick surface veil, clear liquid, light precipitate.

Gelatine plates-Rare, very small, pin-head like colonies. Nonliquefying.

Agar-On agar plates small colonies of dry appearance and a rose colour.

Agar stroke - A sparse, dry growth of a rose tint.

Potato-Rapid growth, dry, and of a rose colour.

Milk-Surface formation of rose coloured veil. No coagulation.

Aërobic.

Pathogenic properties - Pure cultures inoculated intraperitoneally into guinea-pigs give rise to subacute hæmorrhagic peritonitis. Subcutaneous inoculation of a second animal from the lesions of the first, gives rise to an abscess at the point of inoculation.

\section{BUTTFR BACILLUS (Coggi).}

Source-Milan butter. Coggi met with these bacilli in 17.89 per cent. of samples of Milan butter, while $2 \cdot 12$ per cent. only contained true bacilli of tuberculosis.

Morphology-Small fine rods closely resembling the bacillus of tuberculosis, always isolated, sometimes a little curved.

Motility-Absent.

Staining properties-They resist decolorisation by acids, and remain coloured by Gram's method.

Biology : cultural characters.

Bouillon-Formation of a surface veil after 48 hours growth. The liquid remains clear with a thread-like precipitate at the bottom of the tube. No indol.

Gelatine-Surface growth, humid, and of a whitish tint. Round granular colonies, of a yellowish tint,with indented margins. Non-liquefying.

Agar-Soft creamy - white growth, turning to a yellowish tint in old cultures.

Glycerine serum-As on agar, but with the coloration more marked.

Potato-A moist growth of a greyish tint.

Milk-A yellowish pellicle forms on the surface of the medium. No coagulation.

Pathogenic properties - Pure cultures are pathogenic for guineapigs when inoculated intraperitoneally, but the lesions have a very marked tendency to spon- 
taneous cure. Animals sacrificed after from 12 to 16 days inoculation, present lesions more accentuated than those sacrificed after 37 and $3^{8}$ days. Inoculated subcutaneously, pure cultures give rise to small abscess which quickly cures. The organism is non-pathogenic for rabbits and pigeons.

BUTTER BACILLUS (Grassberger).

Sounce and habitat-Butter.

Morphology - Form, dimensions, manner of grouping. Resembles $B$. tuberculosis.

Staining reaction-Ziehl-Neelsen, but loses acid-fast property after some days in culture.

Biology: cultural characters (including biochemical features).

Bovillon-At $37^{\circ} \mathrm{C}$. the liquid becomes cloudy after 24 hours, a veil forms on the surface, later the medium clears, a deposit falls to the bottom of the tube, the veil remains. At $22^{\circ} \mathrm{C}$. the medium clouds, no surface veil, a deposit at the bottom of the liquid. The growth is less than at $37^{\circ} \mathrm{C}$.

Glycerine bovillon-This medium is less clouded than ordinary nutrient bouillon.

Gelatine-Gelatine stab cultures, a faint growth along the needle track. At point of inoculation the growth is dry, wrinkled and of a deep rose colour. Gelatine stroke cultures present a dry, raised, wrinkled growth which does not extend over the surface of the medium; colour deep rose. No liquefaction.

Nutrient agar - In stab and stroke cultures, the growth is dry, slightly wrinkled, and extends over the surface of the medium. Rose colour, but lighter than upon gelatine.
Glycerine-agar-Growth is more granular than upon gelatine, dry, and spreads over the surface of the medium. Colour is not so deep as upon gelatine (Plate 25, p. 256).

Milk-Milk is coloured throughout by the organism, but not coagulated; thick surface growth of a deep rose colour, with deposit of same tint.

Potato - The growth is raised, granular and of a deep rose colour. Apart from colour, resembles the growth of $B$. tuberculosis on potato.

BUTTER BACILLUS, No. 1

(Maria Tobler).

Source and habital-Zurich butter.

Monphology - Form, dimensions, manner of grouping. Morphologically resembles $B$. tuberculosis; straight or slightly curved, of varying length. Club-like swellings are of frequent occurrence, and more rarely ramified flaments. The bacilli are often found lying parallel the one to the other, and, especially in bouillon cultures, groups of filaments, ramified or not, and forming the figure 5 are found.

Staining reaction-The bacillus stains well with ordinary aniline stains, and holds the Gram. It resists decolorisation by acids even in old cultures, but there is a tendency on the part of the filaments to decolorise. Long subjection to the action of alcohol renders it less resistent to decolorisation by acid.

Biology: cultural characters - The organism grows well upon all ordinary media. At $37^{\circ} \mathrm{C}$. growth appears in from $I$ to 3 days, at ordinary room temperature in from 6 to ro days.

Bovillon - On the second day appears a yellow filamentous deposit, with light veil on the surface but no turbidity of medium. 
In old cultures the deposit becomes more voluminous and, when the tube is shaken at times, rises in the form of column-like filaments. The veil becomes thicker, wrinkled, of a yellowish colour, and creeps along the walls of the tube. The liquid remains clear and without odour.

Agar stab-Growth abundant; yellow and wrinkled on the surface of the medium; very rare growth on the needle track.

Glycerine-agar stroke cultureOn the second day fine white growth commences on the needle track; after from 4 to 6 days the culture becomes of a deep yellow colour, moist, fatty, shining, with raised edges and much wrinkling on the surface. At the end of from I to 2 months the tint passes to orange, the folds or wrinkles become more marked and especially more numerous; the culture remains moist and is well raised above the surface of the medium. In the rare cases in which the chromogenic property is lacking, the isolated colonies are round and dull in the centre with clear edges; the centre of the colony is umbilical.

Gelatine-Growth very slow and poor. No wrinkling; yellowish white colour which turns to a brownish tint in old cultures. No liquefaction.

Potato-A greyish white humid growth turning later to a yellowish orange and becoming wrinkled.

Milk-Is not coagulated.

Blood serum - A yellowish humid growth with fine edges and no wrinkling, it does not extend beyond the surface of the medium.

Pathogenic properties-The intraperitoneal injection of a large quantity of pure culture leads, at the end of a few days, to a generalised infection, with invasion of the bacilli into the blood and organs, followed rapidly by death. Subcutaneous injection of a pure culture gives rise to local abscess, with passage of the bacilli into adjacent glands. A simultaneous injection of pure culture and sterilised butter gives rise to peritonitis, and a strong general reaction, with invasion of the bacilli into the blood, and death within from 4 to 6 days.

Maria Tobler has never found giant cells in any of the lesions caused by this organism. She is of opinion that it presents a strong general resemblance to the Grassbacillus, No. 2, of Möeller, but she has never observed the ramifications which Möeller gives as characteristic of his bacillus.

BUTTER BACILLUS, No. 2 (Tobier).

Source and habitat-Zurich butter.

Morphology - Morphologically resembles $B$. tuberculosis. The bacilli are of variable length and breadth. In cultures upon agar of from 2 to 3 weeks growth filaments found, but the filaments are not ramified; the organism is present also in club form.

Staining reaction-The organism is less resistant to decolorisation by acids than the bacillus, No. I, when stained by the method of ZiehlNeelsen. In sections its resistance to acids is very feeble. Stains by Gram's method.

Spores and motility-Absent.

Biology : cultural characters-Growth is rapid and abundant upon all ordinary media at $37^{\circ} \mathrm{C}$, cultures becoming visible at the end of 24 hours; it grows at room temperature and more quickly than the bacillus, No. $I$.

A Agr-A wrinkled humid growth which becomes dry at the end of a few weeks, and takes a brownish tint. 
Bowillon-A white wrinkled veil, turning to a rose brown. The liquid becomes slightly turbid and emits an unpleasant odour. Indol.

Gelatine-Growth sparse, nonchromogenic and without wrinkling. No liquefaction.

Agan-There is but little growth in the medium.

Potato-A dry brownish yellow growth with very slight wrinkling.

Milk-Formation of a whitish surface veil.

Pathogevic properties - This organism, according to Madame Tobler, gives rise in the guineapig and the white mouse to a fibrino-purulent peritonitis which is rapidly mortal. The bacilli are found both in the blood and in the glands.

BUTHFR BACILLUS, No. 3 (Tobler).

Source and habitat-Zurich butter.

Monphology - Morphologically resembles $B$. tuberculosis; ramified filaments or club forms are rare.

Staining reaction-This bacillus has only proved acid-fast when stained by the Ziehl-Neelsen method within the first 2 days of its cultural growth.

Motility - No motility observed.

Biology : cultural characters.

Bovillon-The liquid becomes turbid throughout with surface formation of a pellicle of a pale rose colour which becomes wrinkled and mounts up the sides of the tube. Later a granular deposit forms at the bottom of the tube and the liquid clears itself completely.

Gelatine - Isolated colonies possess a finely granular centre with a clear bordering zone extending in delicate ramifications into the surrounding medium.
Glycerine-agar stroke culture-A humid growth turning to a red lead colour at the end of a few days.

Potato-Humid growth of red lead colour, and feebly wrinkled surface.

Glycerine potato - In its early stages the growth is creamy, shining, and of a dirty brown colour with no deposit in the liquid, at the end of 6 weeks the culture is still of a dirty brown tint, shining, and very little raised above the surface of the medium. At times there is a very abundant deposit of a flocculent nature at the bottom of the tube.

Milk-Milk is not coagulated, but there is surface formation of a pellicle of pale rose colour.

Blood serum-Growth is similar to that upon agar but little less abundant, and the colour is not so deep a red.

Pathogevic properties-Is pathogenic for guinea-pigs, which, injected subcutaneously or intraperitoneally, die within from 2 to 3 weeks, with lesions which are more characteristic when a mixture of the culture with sterilised butter has been injected than when the injection has been with pure culture alone.

BUTTER BACHLUU, No. 4 (Tobler).

Source and habitat-Zurich butter.

Monphology - Thelength of this bacillus is very variable, it is often met with in filaments with ramifications, and in club form.

Staining reaction-It resists well decolorisation by acids even in cultures of 3 weeks growth.

Motility-Non-motile.

Biology : cultural characters-Accord-

ing to Madame Tobler cultures resemble very much those of No. 
2, except upon agar. Those upon this latter medium are well developed at the end of $\mathbf{2 4}$ hours, and about the seventh day appear a few granulations of a pale rose colour; and about the eighteenth day the culture is found to be dry, thin, lightly wrinkled, and of a pale rose colour. At the end of 2 months the culture is of the same aspect, and there is but little exuberant growth.

Pathogenic properties - Inoculated into guinea-pigs, either intraperitoneally or subcutaneously, the animal dies in from ro days to a fortnight with general congestion and local lesions, from which pure cultures of the organism can be obtained upon glycerine-agar after incubation for 7 or 8 days. The colonies are of greyish-rose colour, shining, and of fatty appearance, and will be found to be constituted of bacilli strongly resistant to acids.

BUTTHER BACILLUS, No. 5 (Tobler).

Source and habitat-Zurich butter.

Morphology - The bacilli, which ordinarily will be found arranged paralled the one to the other without ramifications, are of varying lengths, but of more or less stumpy appearance.

Staining reaction-The power of resistance to acids disappears almost completely after 2 days growth in culture.

Biology : cultural characters-Growth is rapid at $37^{\circ} \mathrm{C}$., and fairly so at ordinary room temperature.

Bouillon-Formation of a thick veil of granular nature and pale rose tint which creeps up the walls of the tube. A deposit falls to the bottom of the liquid.

Gelatine-Granular growth of pale rose colour. No liquefaction.
Agar stab-Surface growth of pale rose colour.

Glycerine-agar. - Stroke cultures; granular colonies of a dull yellowish-rose colour.

Potato-Cultures of a pale rose colour ; dry and granular.

Milk-A surface veil of a pale rose tint. No coagulation.

Pathogenic properties - Inoculated into guinea-pigs the organism gives rise to general congestion, and the formation of local abscesses from which small bacilli resistant to acid can be isolated without difficulty. A tube of glycerineagar inoculated on the surface from the lesions, presents at the end of 8 days an abundant, wrinkled, dry, and colourless growth of the organism.

\section{bacillus caUcasium (Kern).} (Dispora caucasia.)

Source and habitat-The Kephyr organism.

Morphology-Rods ; 5-6 $\mu$ long, I $\mu$ broad. Often connected in chains, with gelatinous membrane.

Staining reaction-Ordinary stains.

Motility-Slightly motile.

Spore formation-Bacilli often possess small clear spaces which are probably not spores.

Biology: cultural characters (including biochemical features)-Growth at 22 is feeble, $37^{\circ} \mathrm{C}$. more favourable, grows well if milk-sugar added.

Bouillon-Grows well in milksugar bouillon.

Gelatine plates and tubes-Fresh cultures do not grow well on gelatine; old cultures do.

Agar plates and tubes-Whitishgrey colonies on milk-agar. Dentate margins.

Milk-Is not coagulated, little 
gas formation. Able partly to liquefy (peptonise) coagulated casein.

Freudenreich obtained Kephyr in sterile milk most often by mixing the Kephyr yeast with 2 streptococci isolated from Kephyr and the $B$. caucasium.

Non-pathogenic.

\section{BACILUUS CIRCULANS, No. 2} (Jordan).

Source and habitat-From milk by Conn.

Morphology $\longrightarrow 6 \mu$ broad by $\mathrm{x} \cdot 5 \mu$ long ; long chains are produced in bouillon.

Staining reaction-Ordinary aniline dyes.

Motility-Motile.

Spore formation - Found in most media by Jordan, not by Conn.

Biology : cultural characters (including biochemical features).

Gelatine plates and tubes-A granular colony sinking into a dry pit, the pit liquefies and the bacilli can be seen circulating in the liquid. In stab-cultures a deep narrow funnel is produced with a white sediment.

Agar plates and tubes-An abundant yellowish growth, which is not characteristic.

Potato-A thin, watery, transparent growth.

Milk-Is not curdled but digests into a weakly alkaline liquid which is cloudy and gives off unpleasant odours. Gives butter a good flavour, the putrefactive odour present in milk and cream is not present in butter.

Aërobic.

Non-pathogenic.

\section{BACILLUS CITREUS ACIDI (Conn).}

Source and habitat-Milk.

Morphology-Rod; $.8 \mu$ long by $\cdot 4 \mu$ broad; singly. No chains or groups.
Staining reaction-Ordinary aniline stains.

Biology : cultural characters (including biochemical features).

Bouillon-A slight scum which eventually sinks as abundant sediment.

Gelatine plates and tubes-Large white opaque colony turning yellow. In stab-cultures a good needle growth with spreading surface, depressed centre, raised edge, becoming lemon yellow.

Agar plates and tubes-An abundant spreading growth, lemon yellow in colour.

Potato-A thick white, slightly transparent growth; centre becomes yellow and eventually whole growth.

Milk-Curdled at ordinary temperatures in 6 to 9 days with clear hard curd; acid reaction. Same change occurs in cream with gas production; produces a good butter.

Aërobic.

Non-pathogenic.

\section{BACIILUS CITREUS ARBOR- HSCENS (Conn).}

Source and habitat-Milk.

Monphology-A rod $4 \mu$ long by $8 \mu$ broad. Two or three may adhere together, but no long chains.

Staining reaction-Ordinary aniline stains.

$A$ capsule which does not stain.

Biology: cultural characters (including biochemical features).

Gelatine platesand tubes-Widely spread colonies with fine radiating rods growing from centre outward over medium. These fibres have frequent nodules. Looks like a mould to the naked eye. In stabcultures a slight needle growth, but a thick ground-glass surface growth occurs. 
Agar plates and tubes-White, moist, irregular colonies spreading in streaks over the medium.

Potato - Dry, thin growth ; lemon-yellow in colour.

Milk-No effect, except alkaline reaction.

Aërobic.

Non-pathogenic.

\section{BACILLUS CITREUS LACTIS,}

Nos. 1 and 2 (Conn).

Source and habitat-Milk.

Morphology-Rod I $\mu$ long by $\cdot 7 \mu$ broad; with round ends.

Staining reaction-Ordinary aniline stain.

Capsule-A slimy capsule connects the rods when grown on potato and agar.

Biology: cultural characters (including biochemical features).

Gelatine plates and tubesSmall raised lemon-yellow colonies, smooth and clear. (No. 2 only liquefies gelatine.) In stab-culture a rough surface growth, thick, but transparent. Moderate needle growth.

Agar plates and tubes-A thin, moist, transparent, lemon-yellow growth.

Potato-A thick lemon-yellow growth.

Milk-No effect is produced by No. I on milk or cream. No. 2 curdles milk, producing a weak, alkaline curd at room temperature.

Aërobic.

Non-pathogenic.

\section{BACILLUS COLI COMMUNIS}

(Escherich).

Source and habitat - An organism of wide distribution, normally present in the excreta of man and animals. Abundant in crude sewage $(100,000$ per c.c. in London sewage, Houston). In polluted water, milk, soil, etc.

Monphology-A short rod with round ends; size and shape may vary in same colony; polymorphism, depending upon age of culture, products of culture, composition of medium, etc. 2 to $3 \mu$ long, 0.5 to $0.6 \mu$ broad; sometimes oval, hardly longer than broad. Usually single, but occasionally in pairs, bundles, or even chains and threads.

Staining reaction-Ordinary aniline dyes. Decolorised by Gram. (Schmidt stated that $B$. coli from fatty stools of infants holds the Gram.)

Capsule-Present.

Flagella -3 or 4 in number, fragile, short, and not wavy. Sometimes only a terminal one; sometimes several long ones; but polar staining and vacuolation frequently present in old cultures, or cultures grown under unfavourable conditions.

Motility-Present, especially in young cultures, but not, as a rule, so active as $B$. typhosus; oscillatory rather than progressive.

Spore formation-None.

Biology: cultural characters (including biochemical features)-Grows best at $37^{\circ} \mathrm{C}$, but will also grow at room temperature. Gordon showed that many varieties of $B$. coli exist, with many minor modifications (Jour. of Path. and Bact., 1897).

In gelatine plate cultures the colonies appear generally within 24 hours at $20^{\circ} \mathrm{C}$. The deep colonies appear as small white dots, the surface colonies as delicate, slightly granular films of an irregularly circular shape. They are bluish-white by reflected, and amber colour by transmitted 
light. The diameter of the colony is $I$ to $2 \mathrm{~mm}$. The colonies are transparent, and sometimes iridescent, especially towards the periphery, but at the centre and over the entire surface in old cultures an opacity due to a greater thickness of the bacterial growth is observed.

It has been observed that species derived from water grow in transparent colonies, whereas those from the alimentary canal or excreta may show opacity of the colony, which characteristic disappears if the culture be passed through milk. About the second or third day the surface colonies attain a diameter of 5 to $6 \mathrm{~mm}$, and become marked by concentric, or radiating, or irregular markings. The surrounding gelatine very frequently acquires a dull, cloudy, faded appearance, and the edges of the colony become more crenated and thinner. The whiteness of the colony turns to yellow. There is no liquefaction of the gelatine.

In gelatine stab-cultures the organism grows rapidly. On the surface, in 24 hours, the growth is often 2 to $3 \mathrm{~mm}$. in diameter, and closely resembles a surface colony in a plate culture, though more luxuriant. A thick white growth extends along the whole length of the track of the needle, and not infrequently gas bubbles or fissures appear. The gelatine is not liquefied, even in old cultures.

In gelatine streak cultures growth is also abundant. In 24 hours the elongated milky surface colony may be $5 \mathrm{~mm}$. in diameter. It consists of a delicate faintly-granular film with transparent and irregular margins.
Down the centre longitudinally the growth is thicker and therefore more opaque. Irregular thickenings, foldings, and corrugations may occur in old cultures. Sometimes the film shows iridescence, and the medium, though not liquefied, becomes clouded. The growth, as on the plate cultures, is bluishwhite by reflected, and yellowishamber in colour by transmitted light.

In 25 per cent. gelatine at $37^{\circ} \mathrm{C}$. In 48 hours the melted gelatine remains clear, but a thick pellicle forms on the surface (Klein).

Gelative shake cultunes become turbid, and within 24 hours at $20^{\circ} \mathrm{C}$. are riddled with bubbles of gas, which are generally more numerous and larger towards the foot of the tube. They increase in size by the second day, sometimes even forming fissures. The gas is mainly carbonic acid. The presence of a small per cent. of fermentable sugar in the medium increases the gas production.

On potato-gelatine the colonies of $\boldsymbol{B}$. coli are similar in appearance to those occurring on ordinary gelatine, except that they grow more slowly, are more circumscribed, and of a characteristic brown colour (Houston).

On carbol-gelatine (.05 per cent. of phenol) the growth does not differ from ordinary gelatine cultures except that it is delayed.

Bouillon-In less than 12 hours at $37^{\circ} \mathrm{C}$. the medium becomes uniformly turbid. It may be very pronounced. Frequently there is also at a later stage a marked amorphous flocculent sediment consisting of bacteria. Only a faint film forms on the surface, which rarely becomes a pellicle. There is a foetid odour, and sometimes 
gas formation. In glucose, lactose, or saccharose bouillon ( 2 per cent.) the growth is abundant, and gas is produced. In phenolated bouillon (.05 per cent. of phenol), and in bouillon containing formalin ( $\mathrm{I}$ to $7000)$ there is also growth.

On agar at $37^{\circ} \mathrm{C}$. the organism grows rapidly, producing thin, moist, translucent, creamy, greyish-white colonies of irregular shape and size. The colonies grow more rapidly on the surface than in the depth of the medium. The same appearances occur on agar at $20^{\circ} \mathrm{C}$., except that the growth is delayed. Gas bubbles frequently occur in the condensation fluid.

Sugar litmus agar-The medium is turned red in 24 hours, and the surface growth becomes tinged slightly with the reddened litmus. Numerous gas bubbles are produced in the medium.

On potato at $37^{\circ} \mathrm{C}$. there is produced in 24 hours a thick, moist, yellowish-grey growth, becoming brown in old cultures. The colour varies widely in degree, sometimes being richer than at other times. The potato becomes changed in colour near the growth. If the potato is not fresh, or its reaction has been made alkaline, the growth of $B$. coli may be almost colourless. There are, of course, a very large number of bacteria which produce a growth on potato not readily distinguishable from $B$. coli. On "Dutch" potatoes the culture may be colourless like $B$. typhosus (Perré).

Litmus milk-Usually an acid curdling of the milk occurs in 24 hours at $37^{\circ} \mathrm{C}$., though sometimes slightly delayed. The bluish-purple colour changes to pink, then the whole of the milk is turned into a solid compact coagulum, the milk itself becoming white. Later the redness extends from the surface downwards until the whole contents of the tube are bright red in colour.

On wort-gelatine-B. coli produces an abundant growth of thick consistency and creamy appearance.

Lactose gelatine or lactose agar (2 per cent.) plus a little litmusa red-rose shade is produced.

On blood serum at $37^{\circ} \mathrm{C}$. an abundant white glistening layer is rapidly developed, somewhat similar to the growth on agar. There is no liquefaction.

Indol is produced in bouillon fluid cultures (or in peptone water). The reaction is frequently obtainable in 48 hours at $37^{\circ} \mathrm{C}$., but in any case is generally well-marked in bouillon cultures kept at $37^{\circ} \mathrm{C}$. for 5 days. The "red reaction" may be obtained by adding to such a culture I c.c. of a 0.02 per cent. solution of potassium nitrite, and 0.5 c.c. of strong sulphuric acid. If the colour (due to nitroso-indol) does not appear at once, the culture may be incubated for a brief period.

Reduction of nitrate- $B$. coli is a vigorous denitrifying organism. In 24 hours at $37^{\circ} \mathrm{C}$. the reduction of nitrates to nitrites is well marked. (Bouillon 5 per cent., $\mathrm{KNO}_{8}$ O.I per cent., water 94.9 per cent.) In fresh urine no $\mathrm{NH}_{3}$ is produced, but slightly in solutions of urea which are peptonised.

Widal reaction-Serum from the blood of a typhoid patient gives a negative result (i.e. agglutination and immotility are not produced).

Whey-When grown in whey $B$. coli gives rise to an acid reaction by the fermentation of the lactose. The reaction, however, is only tem- 
porary, and is succeeded by alkalinity (Petruschky).

Aërobic or facultative anaërobic.

Vitality-Thermal death-point, $60^{\circ} \mathrm{C}$. in 10 minutes, more hardy than $B$. typhosus but not highly resistant.

Pathogenesis - B. coli may be pathogenic in association with other bacteria ; it has been held to be the cause of epidemic diarrhoea, and plays a part in pneumonia, suppuration, etc.

Remarks-Whilst the above description applies to the normal type of $B$. coli, it should be clearly understood that a large number of bacilli have been described which possess some, but not all, of the above characters. Refik has described (Ann. de l'Inst. Pasteur, x., 1896, 242), 5 varying types very similar to the normal $B$. coli, but differing in one or more characters. Almost all forms, however, have some features in common, e.g. motility, few flagella, and characteristic growth on potato. Moreover, there are a group of organisms allied to $B$. coli, and often associated with it. Like it also they are related etiologically or otherwise to similar pathological processes. Refik's types are briefly as follows:-

A. Fermentslactose, coagulates milk, but no indol reaction.

B. Ferments lactose, does not coagulate milk, gives indol reaction.
C. Ferments lactose, does not coagulate milk, does not give indol reaction.

D. Does not ferment lactose, coagulates milk, does not give indol reaction.

E. Does not ferment lactose, does not coagulate milk, does not give indol reaction.

Roux, Rodet, and others have stated that $\boldsymbol{B}$. colb, under certain circumstances, may assume a character not distinguishable from $B$. typhosus, both in its biological and cultural characteristics, and in its pathogenic properties. Klein and other bacteriologists, as the result of numerous experiments, have been unable up to the present to effect any transformation of one form into the other. Each organism has retained unimpaired its differential characters.

The virulence of $B$. col $i$ is affected by :-

1. The pathological condition of intestinal canal.

2. Increased by passing through a guinea-pig.

3. Attenuated by age.

The chemical properties of its products are among its most striking features.

The following are the differences between $B$. coli and $B$. typhosus usually relied upon for diagnostic purposes :-

\section{Comparative Features of B. coli and B. typhosus.}

\section{B. typhosus.}

Morphology-Bacillus of unequal lengths; some filaments.

Flagella-Long, wavy, spiral, numerous ( 9 to 18); movement very active.

On gelatine and agar - Angular, irregular, slightly raised colonies; slow growth; medium remains clear.

In gelatine-In ordinary gelatine and in lactose gelatine no gas is produced (at $20^{\circ} \mathrm{C}$.).

\section{B. coli.}

Bacillus shorter and thicker; filaments rare.

Shorter, stiffer, few (average 3) movement less active, and sometimes almost absent.

Colonies with even margin, homogeneous, much larger and quicker growth, medium becomes turbid or coloured

Under the same circumstances abundant gas is produced. 


\section{Comparative Features-Continued.}

\section{B. typhosus.}

Milk-Not curdled by the bacillus (at $37^{\circ} \mathrm{C}$.). No acid production.

Indol-In bouillon and Witte's peptone water, no production of indol.

Bouillon containing 0.3 per cent. Phenol, or Formalin $(\boldsymbol{\tau}: 7000)-$ No growth.

Lactose-bouillon at $37^{\circ} \mathrm{C}$. - No gas production.

Neutral-red ghucose-agar-No change.

Glucose or lactose media, shake cultures-No gas production.

Potato-An "invisible growth" if the potato is acid in reaction.

25 per cent. gelatine at $37^{\circ} \mathrm{C}$.-Strongly and uniformly turbid (Klein). No pellicle.

Elsner's iodised potato-gelatine-Slow growth; small transparent colonies.

Proskaner and Capaldi's Medium, No. 1-No growth ; no change in reaction.

Widal's reaction-Bacilli became motionless and agglutinated when suspended in blood serum from a typhoid patient. (See Appendix.)

Thermal death-point $-62^{\circ} \mathrm{C}$. for five minutes (Klein).

Vitality in water or sewage-B. typhosus soon ceases to multipiy and more or less readily dies.

Pfeiffer's inoculation test with anti-typhoid serum -Negative result.

\section{BACILLUS COMMUNIS LACTIS,} Nos. 1 and 2 (Conn).

Source and habitat-Milk.

Morphology-.6 to I $\mu$ long. May occur as chains (bouillon).

Staining reaction-Ordinary aniline stains.

Biology: cultural characters (including biochemical features).

Gelatine plates and tubes Smooth, round, white colonies (like $B$. coli) raised centre; moist and glistening. Needle growth abundant in stab-cultures with white, flat, glistening growth on surface.

\section{B. coli.}

Milk is curdled, within 24 to 48 hours at $37^{\circ} \mathrm{C}$. Abundant acid production.

Indol is present.

Grows well and uniformly throughout medium.

Gas production occurs.

Marked green fluorescence.

Marked gas production.

Thick, yellowish-white growth, later becoming brown in colour.

Gelatine remains clear within 48 hours, but a thick pellicle forms on the surface.

Rapid growth ; large brown colonies.

Growth ; acid reaction.

B. coli remains motile and not agglutinated.

$66^{\circ} \mathrm{C}$. for five minutes (Klein).

$B$. coli retains vitality and power of self-multiplication.

Positive result, variable symptoms according to virulence of bacillus.

Agar plates and tubes-No. I not characteristic; No. 2 moderately thick, moist smooth transparent growth.

Potato-No. I a slight growth having a lemon tinge; No. 2 white moist thick growth.

Milk-Nos. I and 2 no growth.

Aërobic.

Non-pathogenic.

\section{BACILIUS DIPHTHERI AD}

(Klebs-Löffler).

Source and kabitat-Throat, nose, larynx, trachea, more rarely 
stomach and other parts of patient suffering from the disease. May be present in healthy throats of persons in proximity to diphtheria patients. Generally somewhat localised to membrane, but may be found in blood and internal organs. Secondarily, infects articles of clothing, milk, etc.

Morphology - Slightly curved rods with one end commonly rounded or club shaped, the opposite end commonly pointed; occasionally in dumb-bell form; polymorphism in old cultures and in some media. Escherich classifies diphtheria bacilli into three main groups, (a) short, wedge-shaped, commashaped ; (b) long, slender, cylindrical form; (c) involution forms, club shaped. The forms vary however very widely and irregularly, especially in old cultures. Some authorities hold that the wedgeshaped and spindle forms are the more virulent ; and the short ovoid forms are less so. $1 \cdot 2$ to $2 \mu$ long, 0.3 to $0.5 \mu$ broad.

Manner of grouping-In cover-glass preparation from typical and pure culture the diphtheria bacilli may occur singly but more commonly in groups of two, three, or four, parallel with each other or at angles resembling a $\mathrm{V}$ or a circumflex accent. They never form long chains, Frequently grouped like cruciform characters or Chinese letters. Paucity of interlacing and club forms and predominance of parallel forms points to the presence of pseudo-diphtheria bacillus (Escherich). Crossing and interlacing of the longer forms is supposed to be due to a formation of new bacilli by the outgrowth of chromatic granules in a direction at right angles to the parent bacillus (Escherich, Neisser, Shattock, etc.).
Staining reaction - Aniline dyes; Gram's method (if decolorising not too prolonged); Löffler's stain (brings out striped appearance); Nicolle's stain.

Capsule-Present; no flagella; nonmotile.

Spore formation-None.

Biology: cultural characters (including biochemical features)-Grows best at $37^{\circ} \mathrm{C}$.

Bovillon--Dust-like granules, a cloud at foot of tube ; pellicle usually present ; indol, acid, and nitrites are produced.

Gelatine plates and tubes-Slow growth at temperature 22 to $24^{\circ} \mathrm{C}$., not characteristic, non-liquefying ; surface growth yellowish-white, and slightly elevated.

Agar plates and tubes-Characteristic growth in 24 hours, circular, round, white elevated colonies; smooth border; moist appearance. Luxuriant growth in glycerine-agar tubes. Growth not so luxuriant as on blood serum; the pseudo-bacillus grows well on agar.

Potato-Upon acid potato little or no growth ; upon alkaline potato scanty veil-like growth in several days.

Milk-Abundant growth; no coagulation ; amphoteric reaction.

Blood serum (or Löffler's medium) -Rapid growth at $37^{\circ} \mathrm{C}$, characteristic by twelfth hour (thus getting the start of other organisms); round, raised, greyish-white colonies, with a yellowish tinge, translucent if very young ; surface moist and margin irregular; by transmitted light the colonies show an opaque and thickened centre; in older colonies this centre may appear even more obvious. If the colonies are few and widely separated they may grow to a considerable size ( 4 to $5 \mathrm{~mm}$.), but if 
numerous they remain small, discrete, and non-confluent. In older growths colonies become flattened, the central opacity more marked, the crenation of the margins more distinct. On the surface, which has its moist shiny appearance and becomes dull, radial striation appears. Growth is generally vigorous for the first 48 hours, after which, particularly if the serum becomes dry, growth is much less. Stroke cultures, a grayish streak along the needle track; central part opaque and thicker than margins, which are crenated and more developed at the lower end of streak; at the upper part of the streak the growth thins off and is frequently broken up into isolated colonies.

Other media-Abundant growth is obtained on hen's eggs. Glycerineascitic-agar has been used with much success.

Aërobic.

Vitality - Loses virulence by long subculture; vitality retained for weeks and even months in throat; in the incubator cultures die in 2 or 3 months. Considerable resistance to drying, even in the state of pulverisation; rapidly killed at $60^{\circ} \mathrm{C}$.

Pathogenesis-Sets up diphtheria in man and many animals (including cats, dogs, birds, guinea-pigs, rabbits, monkeys, etc.); white mice and rats are immune. Some authorities claim that true diphtheria can be set up in the cow (see p. 338). Animals can be immunised against diphtheria.

\section{BACILLUS DISPORA LACTIS}

$$
\text { (Conn). }
$$

Source and habitat-Milk.

Morphology - Long chains of bacilli are formed with rounded ends, "like a string of sausages."
Staining reaction-Ordinary aniline stains.

Spore formation-Spores (I $\mu$ to I.5 $\mu$ ) variable in size, situated at both ends of the bacillus. (Double spore formation, Conn.)

Biology: cultural characters (including biochemical features).

Gelatine plates and tubes-A rough tough colony, yellow in colour, easily removed intact by a platinum needle. Sinks into a slowly liquefying pit. In stabculture a shallow funnel is produced and liquefication becomes complete.

Agarplates-Colonies in isolated clumps rather than in a uniform layer.

Milk-Is curdled at $20^{\circ} \mathrm{C}$. and rendered amphoteric or alkaline. A slight digestion is apparent.

Non-pathogenic.

\section{BACIILUS FHNERITIDIS SPORO-} GHNES (Klein).

Source and habitat-Milk, sewage, soil, dust, excreta.

Morphology-Rods, $\mathbf{I} \cdot 6$ to $4 \cdot 8 \mu$ long, and $0.8 \mu$ broad. May occur in chains, in morphology somewhat resembles $B$. anthracis.

Staining reaction-Ordinary aniline stains; also by Gram's method.

Motility-Present.

Spore formation-Present, not seen in milk cultures; oval, I. $6 \mu$ long by I. $2 \mu$ broad. Occur generally near one end of bacillus.

Biology: cultural characters (including biochemical features).

Gelatine plates and tubes-In glucose gelatine grows well; gas production; liquefaction. In gelatine stab there are no lateral offshoots.

Agar plates and tubes-Grows well in glucose agar; marked gas 
formation; colonies in depth are round, white by reflected light, brown and granular in transmitted light. On surface of agar plates flat, circular, moist, grey colonies appear in 24 to 48 hours; thicker in centre than at margin, and showing granularity.

Milk-Grows well in milk, producing the "enteritidis change." After 36 hours of anaërobic incubation at $37^{\circ} \mathrm{C}$. in milk, the cream is torn or dissociated by development of gas, so that the surface of the medium is covered with stringy, pinkish-white masses of coagulated casein enclosing a number of gas bubbles. The main portion of the tube of milk contains a colourless, thin, watery whey, with a few casein lumps here and there adhering to the sides of the tube. The whey has a smell of butyric acid, and is acid in reaction. It contains many bacilli.

\section{Anaërobic.}

Vitality-Considerable.

Pathogenesis-If I c.c. of milk whey containing the bacillus be injected into a guinea-pig ( 200 to 300 grammes), a swelling appears in 6 hours, extending over abdomen and thigh, and death occurs in 18 to 24 hours. Post-mortem : subcutaneous gangrene with much sanguineous exudation, in which bacilli and spores will be found. Klein considers this organism the cause of epidemic diarrhoea.

\section{BACILLUS FILIFORMIS LACTIS} (Conn).

Source and habitat-Milk.

Morphology-Delicate rods.

Staining reaction-Ordinary aniline stains.

Capsule-A thick capsule with central staining matter.
Spore formation-Spores $(I \cdot 2 \mu$ by $1.8 \mu)$.

Biology: cultural characters (including biochemical features).

Gelatine plates and tubes-Colony with fine granular centre, breaking at its margin and surrounded with clear liquefying zone. Later the margin shows contorted lacing threads. In stab-cultures a narrow funnel of liquefaction is produced ; much gas is produced, appearing as bubbles in liquid and in gelatine. Liquefaction becomes complete and a dense serum and sediment is formed.

Agar plates and tubes-A thick spreading branching growth is generally produced, sometimes thin and dry.

Potato-A moist yellowish, shiny mass grows over surface.

Milk-Is curdled in 2 days with little change in reaction. Cream is rendered slightly acid with a sour cream taste, and butter made therefrom has a good flavour but no aroma.

Aërobic and facultative anaërobic.

Non-pathogevic.

Henrici describes another organism under this term.

\section{BACILUS FLUORFSCENS ITQUE- FACIFNS.}

Source and habitat-Water and air; thence to milk.

Morphology; form, dimensions, and manner of grouping-Short rods, about $-4 \mu$ in breadth and about I. $5 \mu$ in length; on solid media the length is sometimes as much as $3 \cdot 2 \mu$; in media unfavourable for its growth many involution forms appear, curved, in form of cross, or spiral.

Staining reaction-Ordinary stains. By Gram's method decoloration 
is irregular, certain elements holding the stain.

Motility-Motile.

Spore formation - No sporulation observed.

Biology: cultural characters (including biochemical features) - Development and fluorescence takes place as low as $14^{\circ} \mathrm{C}$., optimum temperature for production of pigment from $20^{\circ} \mathrm{C}$. to $22^{\circ} \mathrm{C}$., for growth, $28^{\circ} \mathrm{C}$. At $32^{\circ} \mathrm{C}$. there is no fluorescence; growth will take place up to $42^{\circ} \mathrm{C}$. but without fluorescence.

Bouillon - Medium becomes turbid after I2 hours; no surface veil, or if any, very slight. Heavy precipitate at bottom of the tube. Fluorescence in 24 to 48 hours; the liquid becomes yellow by transmitted light, light green by reflected light - and remains turbid for lengthened period. When it clears, in very old cultures, the green fluorescence has diminished and the bouillon assumes a slightly brownish tint.

Gelatine plates and tubes-The gelatine is rapidly liquefied, the liquid assuming a green tint in the upper portion. When liquefaction has reached to the bottom of the tube a thick whitish sediment is deposited, the green tint of the liquid disappears or is replaced by a brownish coloration.

Agar-A thick mucous surface growth, viscous and of a greyishyellow colour. The medium is often coloured green in its upper portion.

Potato-A thin glistening growth of a dirty yellow colour.

Milk-Slow viscous coagulation with tardy fluorescence. The liquid is alkaline.

Aerobic.

Non-pathogenic.
BACILLUS FLUORESCENS NONLIQUEFACIENS.

(B. fucorescens putriaus, Flügge).

Source and habitat-Air and water; thence to milk.

Morphology; form and dimensionsMotile rods $4 \mu$ broad by 2 to $2 \cdot 2 \mu$ long.

Biology: cultural characters (including biochemical features).

Bouillon-The liquid becomes soon turbid at $20^{\circ} \mathrm{C}$.; in 3 or 4 days formation of a fragile veil, easily torn; abundant whitish precipitate at bottom of tube. The liquid presents a general fluorescence, but much less pronounced than in the case of $B$. fluorescens liquefaciens.

Gelatine plates-In plate cultures colonies appear as small transparent slightly yellowish discs. Those which reach the surface develop quickly and in a few days appear as hyaline pellicles with sinuous borders and irregular surface, resembling slightly the colonies of $B$. typhosus, but flatter and much larger. The surrounding medium assumes a green tint and the plate gives off a strong urine-like odour.

Agar-A greyish mucous-like growth; the medium assumes a greenish tint.

Potato.-Cultures somewhat resemble that of $B$. typhosus in its early stages.

Almost all cultures, but especially those upon potato or in bouillon develop an odour, at times very strong, resembling that of putrified urine. At times, however, those upon gelatine and agar may be almost odourless.

Aërobic.

Non-pathogenic. 


\section{BACILLUS FLUORESCENS SCHUYLKILLIFNSIS}

(Wright).

Source and habitat-Milk.

Momphology-2 $\mu$ long, $.8 \mu$ broad; sometimes occurs in chains.

Staining reaction - Ordinary aniline stains.

Biology : cultural characters (including biochemical features).

Gelatine plates and tubes-Large liquefying colonies. Green and granular. Later becoming surrounded by a clear space. In tubes slow growth. At first a shallow pit and later a horizontal liquid layer with a scum and a precipitate.

Agar tubes-A thin white moist growth. The agar becomes green.

Potato - Thin moist brown spreading growth.

Milk-Curdles in 3 days at $20^{\circ} \mathrm{C}$. Sometimes fails to curdle. Makes the milk alkaline.

Aërobic.

BACILUUS, No. 41 (Conn).

Source and habitat - Milk from Uruguay.

Monphology-A bacillus; I-I $\mu$ by $6 \mu$ (longer on potato than agar). Sometimes in twos or in chains.

Staining reaction--Ordinary aniline stains.

Spore formation-None.

Biology: cultural characters (including biochemical features)-Grows best at $20^{\circ}$ to $23^{\circ} \mathrm{C}$. ; only slightly at $35^{\circ}$; killed at $60^{\circ} \mathrm{C}$. in Io minutes.

\section{Bouillon-}

Gelative plates and tubes - A smooth, round colony under surface; grey bead-like colony on surface ; non-liquefying; nonchromogenic. In stab-culture a slight "needle growth"; spreads over surface as a moist, white, thick mound or nail growth.

Agar plates and tubes - An abundant white, smooth, glistening growth.

Potato-Raised thick white layer. If very moist, whiter; if dry, yellower. Grows profusely. A pleasant aromatic odour produced.

Milk-Does not curdle at $20^{\circ}$ or $35^{\circ} \mathrm{C}$.; becomes translucent and brownish in 2 to 3 weeks. Reaction slightly acid. Produces a pleasant aroma. Much used for cream ripening and butter making.

Aërobic.

Non-pathogenic.

BACHLUS FRIBURGFNSIS, NO. 1 (Korn).

Source and habitat-Butter of Friburg.

Morphology; form, dimensions, and manner of grouping - In preparations made from the organs of an inoculated animal, the bacilli very much resemble in shape and size the $B$. tuberculosis of Koch. In bouillon, the shape very much resembles the $B$. coli. It is, however, a little longer and slightly curved. On agar the bacilli are slightly thinner. In old cultures upon agar and serum, they assume the aspect of "Coccothrix." Upon potato they appear under form of cocci, diplococci, and specially of short, stout bacilli slightly curved. Upon cooked beetroot at the end of 3 or 4 days the organisms resemble staphylococci. They are shorter when grown at ordinary temperature than when grown at $37^{\circ} \mathrm{C}$.

Staining-Stains well by the method of Ziehl-Neelsen, but is a little less resistant to acids than the bacillus tuberculosis. It does not stain well with ordinary aniline stains. 
Motility-Non-motile.

Spore formation-Absent.

Biology: cultural characters (characters of cultures).

Bouillon-Upon bouillon a light yellowish veil with deposit at the bottom of the tube of a viscous nature and yellowish tint.

Glycerine bouillon-In glycerine bouillon a thick veil, dry, white, and folded. The liquid remains clear with an abundant white deposit.

Gelatine-Abundant culture of a creamish-white colour ; no wrinkling. The medium is not liquefied.

Agar-Upon agar stab-culture, a round greyish growth with raised edges and umbilical centre. Stroke cultures upon agar give after from 24 to 36 hours a smooth, creamy, glistening growth, slightly greyish, and non-wrinkled. The culture changes but little with age.

Glycerine-agar-U pon glycerineagar a thick, white, brilliant growth with deposit in the water of condensation, which is soon covered with a veil adherent to the walls of the tube. Later on the growth becomes slightly folded. At the age of about 3 weeks the culture is creamy, presenting a few, light folds, and of yellow-orange colour. In older cultures the growth is very abundant, raised considerably above the surface, and irregularly folded and convoluted, the colour varying in depth from light to dark orange or even red brick.

Glycerine potato-Culture at first of a dull, white appearance, becoming later creamy and glistening, with wrinkling on surface as growth proceeds, the liquid in the bulb of tube is covered with a surface veil, and there is deposit in the bottom of the tube.
Milk-Is not coagulated, but at the end of 3 weeks has a dirty yellowish-brown appearance.

Blood serum-Small, shining, transparent colonies : no coloration.

Anaërobic or aërobic-It is a facultative anaërobic, but the growth in hydrogen is slight.

Temperature of developmentOptimum temperature of development $37^{\circ} \mathrm{C}$.

Pathogenesis-Subcutaneous or intraperitoneal injection of pure cultures produce only an abscess at site. If injected with butter itself in white mice, granulations are produced in thoracic and abdominal viscera, showing no giant cells, but commencing caseation (see Plate 24, p. 254).

BACILLUS FRIBURGENSIS, NO. 2 (Korn).

Source and habitat-Butter of Friburg. Morphology -Small rods, one to three times longer than broad, often of irregular form and clubbed, but without ramifications.

Staining-Stains well after the method of Ziehl-Neelsen, and is much less sensible to decolorisation by sulphuric acid than Korn No. I. Stains fairly well with ordinary aniline dyes and holds the Gram.

Spore formation - No spore formation observed.

Motility-Non-motile.

Biology: cultural characters-Compared with otheracid-fast organisms, this organism grows but poorly upon ordinary laboratory media, and gives scarcely any growth at all at room temperature.

Bouillon-The liquid becomes troubled and of muddy appearance, and there is a slight brownish deposit at the bottom of the tube. No indol. 


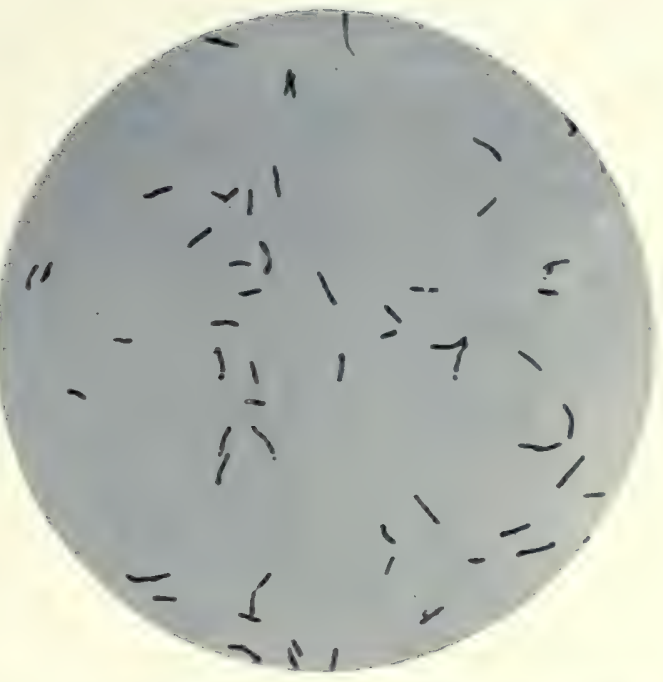

b. friburgensis, I. (Korn). Acid-fast bacillus from butter. Agar culture; 2 day's' yrowth at $37^{\circ} \mathrm{C}$. Stained Ziehl-Neelsel. $\times 1000$.

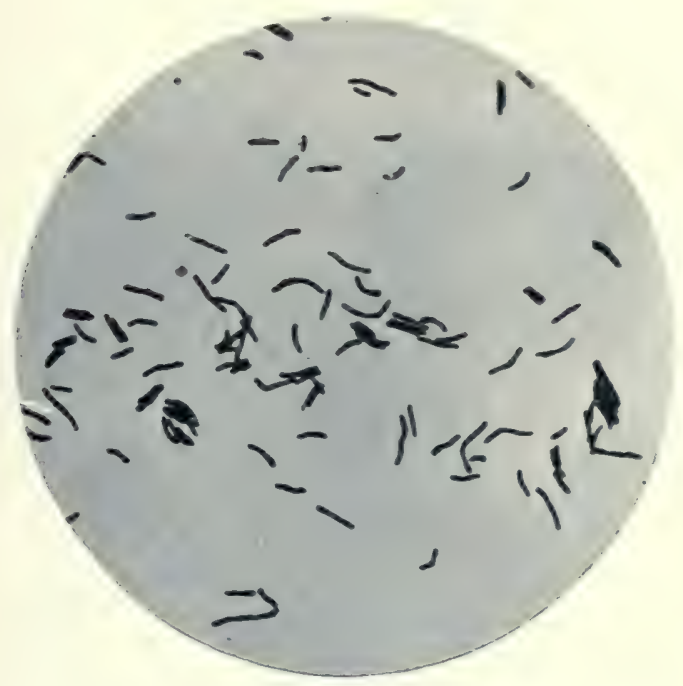

B. friburgensis, II. (Korn). Acil-fast bacillus from butter. Agar culture; 2 days growth at $37^{\circ} \mathrm{C}$. Stained ZiehlNeelsen. $\times 1000$. 

Glycerine bouillon-In glycerine bouillon, the liquid remains clear, with slight surface veil and fine whitish deposit at the bottom of the tube.

Gelatine - Very scanty growth with dull wrinkled surface. Growth does not increase with age.

Agar-Upon agar the growth appears in about 24 hours. The culture is creamy, shining, and of a yellowish to deep yellow tint.

Glycerine-agar-On glycerineagar as upon agar.

Glycerine potato - Very little growth, a few yellowish colonies are occasionally observed.

Milk-Is not coagulated. After about 3 weeks, the medium becomes of a diry rose colour, and a crust forms on the surface of the liquid which later on becomes of a dirty yellowish colour.

Blood serum-Upon blood serum a brilliant culture of light orange colour.

Temperature of developmentOptimum temperature $37^{\circ} \mathrm{C}$.

Pathogenesis - White mice, chickens, pigeons, and guinea-pigs not affected by inoculating pure culture. Rabbits are not refractory. Lesions appear similar to tuberculosis (see Plate 24, p. 254).

\section{GRAsS BACIIUUS, No. 2 (Moeller).}

Sounce and habitat - On certain grasses, hay-dust, etc. Thence to milk.

Morphology-Rods; 1 to $5 \mu$ long, $\cdot 2$ to $.4 \mu$ broad; variable forms, filaments, club-shaped forms, branching.

Stuining Reaction.-Acid-fast; ZiehlNeelsen; also Gram.

Motility-Absent.
Biology : cultural characters (including biochemical features).

Bowillon-In 3 to 4 days at $22^{\circ} \mathrm{C}$. a flaky deposit and a surface membrane; no general turbidity and no odour ; more abundant growth in glycerine bouillon.

Gelatine-In 4 to 5 days thick, white growth, becoming yellow in older parts of culture. No liquefaction.

Agar-On glycerine-agar, rapid growth; small colonies becoming confluent, yellow; slightly raised.

Potato - Abundant growth at $37^{\circ} \mathrm{C}$. ; thick, moist, white.

Milk-Rapid growth, changing medium to brown-red colour, slight pellicle. No coagulation. Milk becomes acid in 2 to 3 days.

Blood serum - Small, meagre colonies; undeveloped.

\section{Aërobic.}

Pathogenesis-Similar to Timothy grass bacillus. Moeller states: "The bacillus produces the same lesions as the butter bacillus; the cultures in milk are the most virulent."

\section{BACILII OF GUHLLEBEAU.}

Baclllus " $\mathrm{a}$ " of Gutllebeau-Found in the milk of cows suffering from mastitis; motile; $\mathbf{I} \cdot \mathbf{2} \mu$ long by I $\mu$ broad. Resembles in growth the $B$. arogenes. Does not stain by Gram's method. Causation, according to Freudenreich, of abnormal blowing in cheese. Rapid coagulation of milk, and fermentation of lactose. Gelatine liquefied. Colonies spherical and granular. In gelatine, growth along track of needle and white patch on surface. On agar, growth is white, and on potato yellowish and viscid. 
Baclllus " $b$ " of Guillebeau-Source and characteristics as " $\mathrm{a}$," differing only in that liquefaction of gelatine is very slow, and the colonies are viscid and sticky.

Bacillus "c" of Gulllebeau-Source and characteristics as "a" and " $b$," but does not liquefy gelatine. Cultures very tough and slimy, and colonies adhere to medium, and are coarsely granular. Milk becomes slimy until coagulation begins.

BACILLUS GUMMOSUS (Happ).

Source and habitat - Isolated from viscous vegetable infusion.

Morphology-Bacillus, from 5 to $7 \cdot 5 \mu$ long by 6 to $2 \mu$ broad.

Motility-Feebly motile.

Spore formation-Ovoid spores.

Biology: cultural characters (including biochemical features).

Gelatine - Gelatine is rapidly liquefied. The viscous matter which is produced at the expense of the sugar is soluble in water, but insoluble in alcohol or ether; with it are found, as fermentation subproducts, mannite, and lactic, and butyric acids. This organism is probably related to $B$. vulgatus (Flügge).

Non-pathogenic.

BACTLLUS HBSSII (Guillebeau).

Source and habitat-Milk.

Morphology-Belongs to group of hay bacilli ; small rod.

Staining reaction-Ordinary aniline stains.

Capsule; motility-Actively motile; no capsule.

Spore formation - Spores are not described.

Biology: cultural characters (including biochemical features).

Bouillon-Becomes slimy.
Potato-Dull white growth which later becomes brown in colour.

Milk - Coagulated into slimy masses; the stringy characteristic of this slimy milk disappears after 2 days at $35^{\circ} \mathrm{C}$.

Non-pathogenic.

\section{BACILLUS LACTIS NIGFR.}

Source and habitat-Milk.

Morphology - Rods with rounded ends ; $.8 \mu$ broad, 2.8 to $2.6 \mu$ long. Isolated or united in pairs.

Staining reaction-Ordinary aniline stains and by Gram's method.

Motility-Actively motile.

Spore formation-Mesially situated.

Biology: cultural characters (including biochemical features).

Bouillon-A yellowish veil; the liquid quickly clears.

Gelatine plates-Young colonies are grey, granular, and with undulating prolongations into the medium. As growth proceeds they darken in colour and penetrate into the medium, which liquefies with great rapidity.

Agar-A brownish yellow pellicle, the medium assumes a darkbrown colour especially upon glucose-agar.

Potato - A humid brownish growth, turning to brownish black, the medium assumes a blackish tint.

Milk-Is coagulated in from 24 to 36 hours at $37^{\circ} \mathrm{C}$., casein subsequently redissolves almost completely, the liquid remaining clear.

Non-pathogenic.

BACILLUS LACTIS PITUITOSI

(Loffler).

Source and habitat-Milk.

Monphology - Slightly bent rods. 
Staining reaction-Ordinary aniline stains.

Biology: cultural characters (including biochemical features).

Gelatine plates and tubes-Small circular greyish-white colonies.

On agar-Greyish-white growth occurs.

On potato - A greyish-white growth.

Milk-Make it viscid and shiny.

Non-pathogenic.

\section{BACILLUS LACTIS SAPONACEI}

(Weigmann and Zirn).

Source and habitat-Soapy milk.

Monphology-Short bacillus.

Staining reaction-Ordinary aniline stains.

Motility - Non-motile.

Spore formation-None.

Biology: cultural characters (including biochemical features) - The most favourable temperature is $10^{\circ} \mathrm{C}$.

Gelatine plates and tubes-White colonies with yellowish centres, later becoming yellow throughout but without special markings. Slow liquefaction. In gelatine stabcultures a funnel forms, at the bottom of which lie the yellow flocculi.

Agar plates and tubes-In agar stab-cultures, Iuxuriant growth, yellow in centre, later over whole growth.

Potato-A waxy yellow shiny growth.

Milk-Is not coagulated but becomes shiny and tenacious. The culture gives off an odour of soap.

Aerobe-Facultative anaërobe.

Non-pathogenic.

\section{BACILLUS LACTIS VISCOSUS}

(Adametz).

Source and habitat-Butter and slimy cream ; in water (Zimmerman).

Morphology-Short rods with round ends.

Staining reaction-By Gram's method. Capsule; motility-Non-motile ; capsule stains.

Biology: cultural ckaracters (including biochemical features).

Bovillon-Becomes very cloudy ; viscous, shining, and may be drawn out in threads.

Gelatine plates and tubes-Colonies like elevated droplets. In stabcultures wide-spread growth but not luxuriant.

Potato-Abundant, white, tenacious growth.

Milk-Is not coagulated ; feebly alkaline; becomes viscous, shiny, and may be drawn out in threads; makes cream shiny. Capsule of bacillus more marked in milk cultures.

Non-pathogenic.

\section{BACILIUS LACTERICUS}

(Adametz).

Source and habitat-Milk, air, etc.

Morphology-Short rods pointed at both ends; 0.8 to $1.6 \mu$ long, 0.4 to o-6 $\mu$ thick.

Staining reaction-Stains by Gram's method.

Motility - Non-motile.

Biology : cultural characters (including biochemical features).

Bouillon-remains clear; slight indol production.

Gelatine plates and tubesRound, reddish-brown colonies, opaque, smooth edges. No liquefaction. In gelatine stab-cultures a reddish-brown growth.

Agar plates and tubes-Reddish- 
brown growth on agar streak cultures - on agar plates round granular colonies.

Potato-Very slow scantygrowth. Milk-Is not coagulated. No acid or gas is formed.

Aërobe-Facultative anaërobe.

Non-pathogenic.

BACILLUS IACTICUS (Kruse); B. GUNTHERI, B. LACTIS ACIDI (Leichmann); B. ACIDI PARALACTICI (Kozai).

Source and habitat-Isolated from sour milk.

Morphology - Short thick rods, pointed at the ends; I $\mu$ long, $0.6 \mu$ thick; in pairs or chains.

Staining reaction-Stains by Gram's method.

Motility - Non-motile.

Biology: cultural characters (including biochemical features).

Bouillon-Slight turbidity when no sugar is present; marked if sugar present.

Gelatine plates and tubesPunctiform colonies, $.5 \mu$ in diameter-if sugar present colonies are larger - always delicate in structure. Non-liquefying. In stab-cultures the growth only occurs in depth.

Agar plates and tubes-Delicate transparent growth on agar plates.

Potato-A scanty limited growth.

Milk-Coagulated with strongly acid reaction. From milk sugar pure dextro-rotatory lactic acid is produced. No gas.

Facultative anaërobe.

Non-pathogenic.

Leichmann describes eleven lactic acid bacilli, each of which shows some differences. Esten describes a $B$. acidi lacti, No. I, which Conn considers identical with above.

\section{BACILLUS I.ACTIS OF FLUGGE.}

A series of milk organisms described by numbers. A short account of each will be given as follows :-

Bacillus lactis, No. 1 (Flügge).Thick, short, actively motile bacilli with terminal spores. Gelatine cultures: colonies indentated. Rapid liquefaction of gelatine and also of blood serum. On agar and potato greyish-white growth. In bouillon a general turbidity with flocculent deposit. Milk is rapidly coagulated. A facultative anaërobe; highly poisonous (Flügge).

Baclllus lactis, No. 2 (Flügge).Short, plump, actively motile bacilli with medial spores. Gelatine cultures: rapid liquefaction, slow liquefaction of blood serum. On agar and potato a whitish, wrinkled and strongly viscous coating. Bouillon: the only appearance is the formation of a surface membrane. Milk: flocculent coagulation with slow peptonisation. Very widely distributed in nature (c.f. $B$. mesentericus vulgatus).

Bactllus lactis, No. 8 (Flügge).Short, fine, sporing bacilli. Slow liquefaction of gelatine. On agar a delicate, and on potato a luxuriant slimy cream-coloured growth. Gas development in lactose-agar stab-cultures. In bouillon isolated flocons. Milk rapidly coagulates with development of gas and labsmell. Slow peptonisation of milk. Spores are killed in $\mathrm{I}$ hour at $100^{\circ}$. Highly poisonous (Flügge).

Bacillus lactis, No, 4 (Flügge).Short, fine, actively motile bacilli. Spore formation. Gelatine cultures: colonies indentated. Marked liquefaction both of gelatine and blood serum. On 
agar and potato a yellowish wrinkled growth. Bouillon remains clear with surface veil. Slow peptonisation of milk; very widely spread in nature (c.f. $B$. mesentericus fuscus).

Baclllus lactis, No. 5 (Flügge).Long, thin, spore-bearing bacilli. Gelatine cultures : colonies indentated. Rapid liquefaction. On agar transparent smooth growth. On potato a clear yellow, dry, and later wrinkled growth. Bouillon clear with surface veil. On blood serum, a wrinkled growth with subsequent liquefaction. Milk is quickly peptonised.

Bacillus lactis, No. 6 (Fligge)-Thin actively motile bacilli with terminal or medial spores. Gelatine cultures; colonies with fine, interwoven processes ; slow liquefaction. On blood serum a dry growth occurs. On agar a whitish wrinkled growth. On potato a diffused and flakey culture. Bouillon made turbid by fine small flocons with formation of thin veil. Milk; finely granular coagulation and slow peptonisation.

Bacillus lactis, No. 7 (Flügge).-Long, actively motile bacilli with medial spores. Gelatine cultures ; proteuslike colonies. Rapid liquefaction. On blood serum, a wrinkled growth with slow liquefaction. On agar and potato, a markedly wrinkled grey and later brownish growth. Bouillon becomes turbid with formation of a thin surface veil. Milk is quickly peptonised. Highly poisonous (Flügge).

Bacillus lactis, No. 8 (Flügge). Moderately thick, actively motile bacilli, with oval or medial spores. Rapid liquefaction both of gelatine and serum. On agar a thick, white, smooth, and shiny coating. On potato a whitish and, later, yellow to brown, wrinkled, viscous growth. Bouillon remains clear with thick surface coating. Milk is quickly peptonised.

Bacillus lactis, No. 9 (Flügge).-Long, actively-motile bacilli with medial spores. Gelatine culture, indentated colonies with raised processes. Slow liquefaction of gelatine. On agar a wrinkled wax-like coating. On potato a finely wrinkled growth at first white, and later chamoiscoloured. Bouillon turbid with thick wrinkled surface membrane. Rapid peptonisation of milk.

Bacillus lactis, No. 10 (Flügge)Small motile bacilli often forming threads, and with medial spores. Gelatine cultures; rayed colonies with stellate processes. Slow liquefaction. On agar a white leatherylike coating. On potato, at first a waxy sugar-like crust with finally a clear grey coating with scanty thick folds. On bouillon a white, thick surface membrane. Rapid peptonisation of milk.

Bacillus lactis, No. 11 (Flügge).Very slender, actively-motile bacilli, with oval spores situated near to the end. Gelatine colonies, irregular and of mycelial type. In stabcultures an arborescent growth. Slow liquefaction of gelatine. Agar, a leather-like, deeply furrowed, and faintly dead - white growth occurs. Potato, a delicate and clear, reddish, crumb-like growth which turns later to a clear brown colour; finely granular; aromatic smell. Bouillon, a flocculent suspension with surface membrane which sinks down to the bottom of the tube; fresh surface growth appearing in its place. 
Milk, slow peptonisation, finally flocculent coagulation of milk; develops an aromatic smell. If the milk is warmed a coagulation of tough consistency results through free rennet fermentation.

\section{BACILLUS LACTIS AROGENES}

(Escherich and Kruse).

Source and habitat-Milk; milk stools of infants (Escherich).

Morphology-Short, thick rods with round ends. .5 to $\cdot 8 \mu$ broad, I to 2 $\mu$ long ; usually in pairs or irregular heaps.

Staining reaction-Does not stain by Gram's method.

Flagella; motility - No flagella ; nonmotile.

Spore formation-None.

Biology: cultural characters (including biochemical features) - Grows best at $37^{\circ} \mathrm{C}$.

Gelatine plates and tubes-Luxuriant shiny growth upon surface in gelatine stab-culture; growth like $B$. coli; raised, white, shining moist colonies; porcelain-like ; an abundant nail-shaped growth in stab-cultures. No liquefaction.

Potato-White raised colonies appear which coalesce and form a creamy layer. Gas bubbles.

Blood serum-A raised, moist, shining white growth.

Other media - In milk sugar and grape solution it produces gas.

Pathogenic - For rabbits and guinea-pigs.

BACILLUS LACTIS ALBUS (Loffler).

Source and habitat-Milk.

Morphology - This is amongst the largest of known bacilli, resembling the $B$. subtilis. Rods, $4 \mu$ long, 0.9 to I $\mu$ broad; forms long translucent threads, especially in milk.
Gelatine-Rapid liquefaction without formation of surface membrane.

Agar-A thick growth.

Potato-Flat, dry, white colonies with ill-defined edges.

Milk-Coagulated by lab fermentation with subsequent peptonisation and the setting free of lactic acid salts with production of butyric acid. Produces bitter milk.

Non-pathogenic.

\section{BACILLUS LACTIS HFRYTHRO- GFNES (Grotenfeld).}

Source and habitat-Milk.

Morphology - Rods and sometimes filaments. $0.8 \mu$ to $3 \mu$ long, .5 to I $\mu$ broad.

Staining reaction-Stains by Gram's method.

Motility-Non-motile.

Spore formation-Absent.

Biology: cultural characters (including biochemical features).

Bouillon - Produces abundant indol, and turbidity of medium.

Gelatine plates and tubes-Small circular greyish-yellow colonies, sinking on liquefaction. At first resemble $B$. coli, after liquefaction border appears hairy and irregular. Degree and rapidity of liquefaction varies. A sulphur yellow growth becoming red in stab-culture; liquefaction cylindrical, turbid and pink.

Agar plates and tubes-Moist yellow growth, medium becomes rose-red in diffuse daylight. Grotenfelt states that the pigment presents two lines between $\mathrm{D}$, and $\mathrm{E}$. and one in blue part of spectrum.

Potato-Sulphur yellow; elevated growth.

Milk-The cream is separated and casein forms a flocculent pre- 
cipitate with alkaline reaction. The clear serum becomes rose-red.

Aèrobic.

Non-pathogenic.

Conn describes a variety in the form of a large coccus.

\section{BACILLUS MEGATHFRIUM}

(De Bary).

Source and habitat-Decomposing cabbage leaves (De Bary).

Morphology-A bacillus with blunt ends 1.6 to $5 \mu$ long, .6 to $.8 \mu$ broad; De Bary described the bacillus as $3 \mu$ thick; often united in strings.

Staining reaction-Ordinary stains and by Gram's method.

Flagella; motility - Slow motility; many flagella.

Biology : cultural characters (including biochemical features).

Bowillon - Cloudy growth with pellicle. No indol, Formation of $\mathrm{H}_{2} \mathrm{~S}$.

Gelative plates and tubesGreyish opaque colonies resembling $B$. subtilis. Liquefaction. In stab-cultures tube or sack shaped liquefaction takes place, the liquid is turbid and fiocculi of deposit occur. No pellicle. The colonies on gelatine plate form no twisted bands or curls.

Agar plates and tubes-White to whitish-grey, elevated, moist colonies, with hairy outgrowths.

Potato-Thick, yellow-coloured growth.

Milk - Is alkaline and fully acid, becomes coagulated and later dissolved.

\section{Facultative anaërobe.}

Non-pathogenic.
BACTLIUS MESTENTERICUS FUSCUS (Flügge).

Source and habitat-Soil, dust, etc.

Monphology - Slender rods with round ends; .8 to $2.4 \mu \mathrm{long}, \cdot 7$ to $.9 \mu$ broad. Sometimes forms chains.

Staining reaction - Ordinary stains and Gram's method.

Flagella; motility-Motile ; many flagella.

Spore formation-Round spores.

Biology : cultural characters (including biochemical features).

Bowillon - Growth medium cloudy and makes pellicle.

Gelatine plates and tubes-Small round grey-white colonies which soon sink in the liquefied medium. Colonies are like those of $B$. subtilis. In stab-culture colony sinks into saucer-like depression within 24 hours, the contents of the funnel of liquefaction are cloudy and a whitish-grey film appears on the surface.

Agar plates and tubes-Round grey colonies, thin and veil-like. Wavy, moist, shining, yellow-brown growth in streak cultures, condensation water cloudy with growth; a yellow precipitate; and a pellicle on the surface. Sometimes a dry folded growth.

Potato-At first growth is moderately elevated, greyish-yellow, shining, slimy ; eventually becomes much wrinkled and elevated.

Milk-At $20^{\circ} \mathrm{C}$. does not curdle milk but digests slowly and becomes alkaline; at $26^{\circ} \mathrm{C}$. curdles in 6 days and digests. Cream ripened with this organism produces butter with no aroma or flavour.

Aërobic-Facultative anaërobic. Non-pathogenic. 
MHCH BACILLUS OF MOHLLER.

Source and habitat-Pasteurised milk of Belzig.

Morphology - Bacillus. Morphologically resembling tubercle bacillus. Singly and in groups.

Staining reaction - Acid-fast and alcohol proof, especially in young cultures.

Motility-Non-motile.

Spore Formation-No spores demonstrated.

Biology: cultural characters (including biochemical features).

Bouillon-Grows at blood heat and room temperature. Liquid but little turbid and no deposit. Surface membrane of fatty aspect and amber colour, adherent to tube walls.

Gelatine plates and tubes-White wrinkled cūlture of creamy nature.

Glycerine-agar - At about 3 weeks the growth is white, uniform, of a creamy nature, at times slightly wrinkled, dry; in old cultures at times dry, at times glazed, of a yellowish colour which later turns to a reddish tint. The culture itself becoming of a wrinkled appearance (see Frontispiece).

Glycerine potato - At first a white creamy growth very little raised above the surface of the medium, as it grows older the culture becomes wrinkled and of a deep yellowish tint, almost red. An abundant precipitate in the liquid at the bottom of the tube, very coherent.

Milk-It grows quickly and luxuriantly, forming an ochreyellow ring round the surface edge of the medium.

Anaërobic or aërobic - Aërobe and facultative anaërobe.

Vitality - Vitality and resistance considerable.
Pathogenesis-Pathogenic, produces nodules in the organs of inoculated animals; virulence increased if inoculated with butter.

\section{BACILLUS MUSCI LACTIS}

(Conn).

Source and habitat-Milk.

Morphology $-1 \mu$ by 2 to $5 \mu$; forms long chains "which look like strings of sausages." Sometimes forms a tangled mass in the scum on gelatine.

Staining reaction - Ordinary aniline stains.

Biology: cultural characters (including biochemical features).

Bouillon-Masses of growth produced which float in the clear bouillon. Scum later.

Gelatine plates and tubes-Diffuse colonies growing under surface of gelatine; thicker in centre; characteristic networks of fibres. Freelike growth in needle track of stabcultures. Liquefaction takes place slowly, producing a liquid cone with central granular axis.

Agar plates-Widely spreading colonies with branches on surface like cotton threads.

Potato-Grows chiefly under the surface, which becomes rough and white, and somewhat broken.

Milk-Is curdled after 3 weeks and becomes slowly digested into a translucent mass full of flakes, and showing a skin on the surface of a ground-glass appearance.

Aërobic.

Non-pathogenic.

BACILLUS MYCOIDES LACTIS, Nos. 1, 2, 8 (Conn).

Source and habitat-Milk.

Morphology-These three organisms resemble somewhat the $B$. mycoides of Flügge, but as they show differ- 


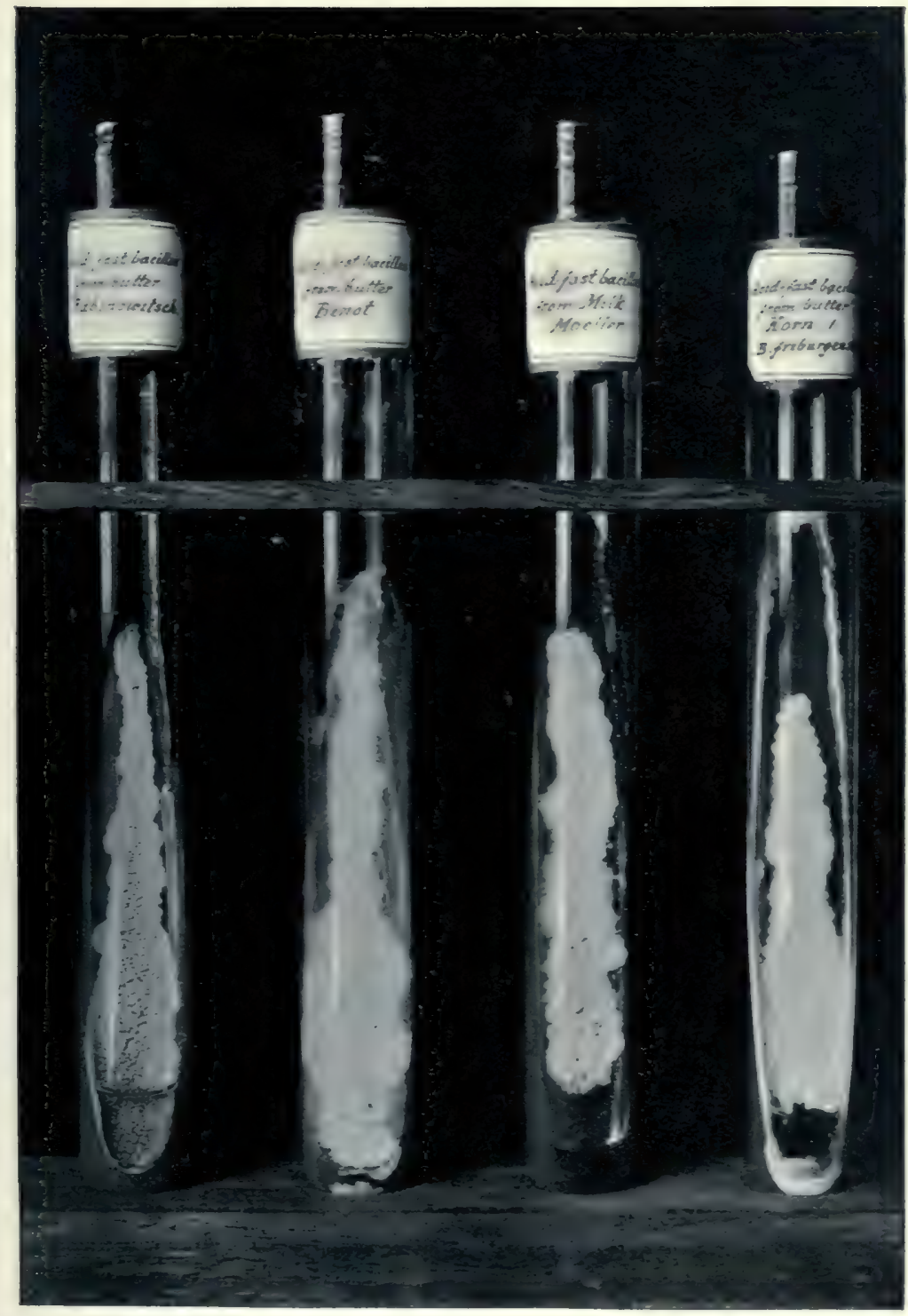

Acid-fast organisms from Milk and Butter.

Tylical growths upon glycerino-azar. 

ences Conn named them as varieties. Some of the individual elements show square ends; I $\mu$ by $2 \mu$; grows in long chains and threads.

Staining reaction-Ordinary aniline stains.

Capsule-A well-marked capsule.

Spore formation - Oval spores in centre of bacillus, becoming very prominent.

Biology : cultural characters (including biochemical features).

Gelatine plates and tubes-No. I produces a proteus-like colony. Liquefaction. In stab cultures a narrow funnel with dense deposit. No. 2 forms a tangled mass of threads like anthrax colonies. Liquefaction. No. 3, radiating growth in stab-culture, obliquely placed.

Agar plates and tubes-No. I, tough, dry growth easily broken into fragments ; later becomes very white from being covered with spores. No. 2, tough white growth breaking into lumps.

Potato-No. I, dry, rough, snowwhite growth. No. 2, thin, dry, putty-like growth. No. 3 , a velvety growth spreading over surface, later becomes very white and dry.

Milk-No. I, curdles in 2 days at $20^{\circ}$ or $36^{\circ} \mathrm{C}$. into soft alkaline curd. Digests slowly, producing a rancid odour. No effect on butter. No. 2, curdles rapidly at $36^{\circ}$, slowly at $20^{\circ} \mathrm{C}$., digests into a cloudy liquid which is colourless or amber coloured.

Non-pathogenic.

\section{BACILLUS PALLESCFNS (Henrici).}

Isolated from cheese. Non-motile short rods; I $\mu$ thick, singly or in pairs. Sometimes grows like $B$. arogenes, but less superficially.
Gas formation. Bouillon remains clear with sedimentation. $B$. pallens vesiculosum and castellum of the same author differ only from the above in that they make bouillon turbid with formation of surface membrane.

\section{BACILLUS PRODIGIOSUS.}

Source and habitat-On starchy substances, especially in autumn.

Morphology-Very short bacillus like a micrococcus (hence Micrococcus prodigiosus) pointed or rounded ends; about $1 \mu$ long. Sometimes in bouillon long forms and even threads occur.

Staining reaction-Ordinary stains; not by Gram's method.

Flagella; motility - Active motility; 6 to 8 long flagella; old cultures non-motile.

Biology: cultural characters (including biochemical features) - Best growth at $22^{\circ}$ to $25^{\circ} \mathrm{C}$.; no pigment at $37^{\circ} \mathrm{C}$.

Bovillon-Diffuse marked turbidity, with a delicate pellicle which may be red coloured. The bouillon eventually becomes oily. Red deposit.

Gelatine plates and tubes-Greyish-white round colonies; rapid liquefaction. Original colonies became red beginning in the centre. Some markings may occur on the colony. Atypical forms also occur. In stab-cultures liquefaction begins in 6 hours in saucer form. White or red flocculi occur. Eventually a deep red deposit appears at bottom of liquefied cylinder.

Agar plates and tubes-Small red colonies after 36 to 48 hours; irregularly round ; colonies become brown and then may lose their colour. Thread-like growth without nodules occurs in stab-cultures. Agar becomes tinted. 
Potato - Rosy-red moist flat growth with wavy smooth edge; becomes dark purple in colour, may develop atypically. Forms ammonia.

Milk-Is firmly coagulated after 24 hours. Later the coagulum is dissolved and a yellow colour is produced.

The red pigment-Is produced best on potato or agar and is insoluble in water. It is readily soluble in alcohol and ether, and is turned orange-yellow by alkalis. It contains no sulphur or phosphorus. The pigment is decolorised by hydrochloric acid and zinc. In light it fades rapidly.

Aërobe-And facultative anaërobe (but in anaërobic growth does not produce pigment).

Non-pathogenic.

\section{BACILLUS RUBFR LACTIS}

\section{(Conn).}

\section{Source and habitat-Milk}

Morphology-A straight rod; .9 $\mu$ broad, 2 to $4 \mu$ long. Sometimes occurs in long bent chains.

Staining reaction-Ordinary aniline stains.

Biology: cultural characters (including biochemical features).

Gelatine plates and tubes-White opaque granular colonies. Rapid liquefaction. In stab-culture a shallow funnel is formed with thin layer of liquefied gelatine. Dense sediment but no scum.

Agar plates and tubes-A thick, coarsely folded growth, at first yellowish in colour, subsequently pink.

Potato-Smooth, thick, glistening growth with pink or salmon tinge.

Milk-Curdled in 4 days at blood heat. Alkaline reaction, no curd- ling at $20^{\circ} \mathrm{C}$. Produces a good flavoured butter.

Aërobic.

Non-pathogenic.

BACILLUS RUDFNSIS (Connell).

Source and habitat-Dirty cheese vats, and in cheese.

Morphology-Bacillus short and sometimes curved, though usually straight. I $\mu$ to I.8 $\mu$ in length. Isolated, at times in pairs, but no long chain formation.

Staining reaction-Stains well with all ordinary stains, but best with carbol-fuchsine or aniline gentian violet.

Motility-Actively motile.

Spore formation-No spores demonstrated.

Biology: cultural characters (including biochemical features).

Bouillon-Slow growth with faint cloudiness of medium chiefly in lower half of tubes.

Gelatine plates and tubes-Faint white growth along needle track in 24 hours, showing as small discrete colonies under a lens in 48 hours. The growth has a faint yellowish tinge, and is as free in depth as towards surface. No liquefaction and no extension of growth over surface of medium. In gelatine slope cultures growth along needle track in form of discrete, small, rounded colonies, gradually assuming a reddishyellow tint.

Agar plates and tubes-Small white colonies with no pigment formation.

Potato-At $22^{\circ} \mathrm{C}$. in 24 hours, faint, yellowish colonies which soon acquire a red tint, and gradually become rust-coloured after 3 or 4 days. Colonies are small (seldom larger than a pin head), rounded, 
with raised centres and irregularly sloping edge.

Milk-Coagulation after 2 days at $22^{\circ} \mathrm{C}$., the coagulationg radually acquiring a faint yellow tint. At times pigmentation in form of faint reddish-yellow dots on surface of coagulum.

Anaërobic or aërobic-Aërobic and facultative aërobic, but grows more rapidly and with more pigment formation in presence of free oxygen.

Vitality-Is killed by heating to $68^{\circ} \mathrm{C}$., but readily withstands a temperature of $64^{\circ} \mathrm{C}$. for ten minutes (Connell).

Non-pathogenic.

\section{BACILLUS SCHAFFERI}

(Freudenreich).

Source and habitat - Isolated from blown cheese. Judged by Freudenreich to belong to the coli group, and much resembling $B$. coli communis.

Monphology-Rods ; motile ; 2 to $3 \mu$ long, by I $\mu$ broad. Found also in threads.

Staining reaction-Does not stain by Gram's method.

Gelatine-Deep colonies small, round, yellow, and granular. Superficial colonies much spread out, porcelain-white and with somewhat irregular edges. Cultures are highly viscous, and can be drawn out in long threads. In gelatine stab-culture, flat-headed, nail-like growth.

Agar-Grey, and later brownish.

Potato-A yellow growth.

Bouillon - Becomes turbid, and if peptone is present there are many gas bubbles.

Milk-Rarely coagulates.

Non-pathogenic.

\section{BACILLUS SUBTHIS.}

Source and habitat-Hay, straw, soil, dust, milk, etc.

Morphology-Short, thick rods with round ends, sometimes form threads. Sometimes also chains of long rods, short rods, and cocci. 0.8 to $\mathrm{I} \cdot 2 \mu$ broad, I. 3 to $3 \mu$ long. Often united in strings and threads.

Staining reaction-Stains by Gram's method.

Capsule; flagella; motility-Bacillus possesses a capsule and flagella which are long and numerous; short forms actively motile ; threads immotile.

Spore formation-Oval spores formed in presence of air, germinating at right angles to long diameter. Spores are set free in about 24 hours; size $\mathrm{I} \cdot 2$ by $.6 \mu$; widely distributed in nature, dust, air, excreta, etc.

Biology: cultural characters fincluding biochemical features).

Bovillon - Uniformly cloudy growth with marked pellicle, wrinkled and thick; copious spore formation.

Gelatineplatesandtubes-Saucerlike depressions; colonies have granular centres and folded margins. Surface growth in stabcultures is whitish-grey; colonies sink on liquefaction of medium ; liquefaction progresses in a cylindrical form, and a thick white scum is formed.

Agar plates and tubes-Small, irregular, greyish-white colonies; moist glistening growth along needle track in stab-cultures.

Potato-Dirty white to yellowish coloured growth; elevated, dull, spreading, with scolloped border; copious spore formation. 
Blood serum-A wrinkled growth develops.

Facultative anaèrobe.

Non-pathogenic.

\section{BACILLUS SYNOYANRUS}

(Ehrenberg).

$B$. cyanogenes of Flüge-Bacillus of blue milk. The typical organism of chromogenic milk.

Source and habitat-Milk, and probably soil, dust, water, etc.

Morphology; form and dimensionsRod varying in length from $2 \mu$ to $4 \mu$, and $0.5 \mu$ in breadth, with rounded ends ; no threads.

Manner of grouping-Form zooglea with a hyaline aureola; a sort of gelatinous capsule.

Spore formation-Ovoid spores a little larger than the rods, which swell at the point of production have been described (Hüppe). Probably they do not occur (Heim).

Polymorphism - In cultures on liquid media involution forms are produced.

Staining reaction-Easily stained by aniline dyes and Gram's method.

Flagella; motility-Special methods disclose from two to five flagella at ends of the rod; bacillus actively motile.

Biology_Cultures are easily obtained in the usual media; the microbe is exclusively aërobic. It grows well at the ordinary temperature, and less well towards $30^{\circ}$; at $40^{\circ}$ the cultures often die. The media soon give an alkaline reaction, even those which are acid. The production of pigment appears to depend, at least within certain limits, on the nutriment which the species has at its disposition, none being formed under special conditions. The shade of the coloration may vary; sometimes it is of a very decided blue, sometimes slightly violet. This microbe can also, under conditions still undetermined, lose the power of producing pigment and give a series of colourless cultures. This is a fact which has been observed with regard to many of the chromogenic bacteria. It is possible, by making this bacterium live under eminently favourable conditions, to make it recover its chromogenic power.

Gelatine plates-At the end of 2 days, small, whitish, moist colonies are seen, rounded and granular. The gelatine takes a blue-grey tint and is not liquefied. Grows better on acid gelatine than on that with a slight alkaline reaction $(0.2$ to 0.3 per cent. of lactic acid).

Gelatine tubes-In stab-cultures in gelatine tubes a thin whitish growth is seen in the needle track, and on the surface a small white disc ; the gelatine takes a greenishblue colour which turns brown with age. Culture grows least in depth of medium. In stroke-cultures, small white colonies develop along the line of inoculation, round which the gelatine takes a greenish tint which may turn into grey-blue. In thesegelatine cultures the organisms taken from the centre are much smaller than those on the edges; the first may measure only from I $\mu$ to I.4 $\mu$, whilst the others can be from $2.3 \mu$ to $3.5 \mu$ in length ; the breadth is about the same, from $0.4 \mu$ to $0.5 \mu$. On lactose gelatine a bluish-white growth is produced, with brown coloration of medium.

Agar - Shiny growth occurs; brown pigment may be markedly developed.

Potato-A band or series of yellowish-brown patches is produced ; the substance of the potato is deeply coloured grey-blue. Sometimes the colour of the growth 
on potato is blue-green or leadgrey instead of brown.

Bouillon-The liquid becomes turbid, and takes a greenish-blue tint; a thick dirty white deposit is formed. A pellicle is sometimes produced.

Milk-No coagulation or formation of acid, but with age a feeble alkaline reaction is noticeable. A bluish leaden coloration appears on the surface in patches, then extends over the whole superficial layer. When the milk has been sterilised, the blue tint is not imparted to the liquid, which is simply greyish at the top. In ordinary milk, on the contrary, the whole rapidly becomes a beautiful skyblue colour on account of lactic acid present. When grape sugar is added to sterile milk the bacillus produces a blue colour.

Mineral solutions-Hüppe recommends, as a culture medium, mineral solutions with tartrate of ammonia base. When one of these liquids is inoculated, a diffused flocculent turbidity is quickly produced throughout the whole. Then on the surface a thin white pellicle is formed, and the liquid takes a bluish tint. A green coloration then appears beneath the veil which invades the whole of the liquid in I4 days; the membrane itself becomes grey-green; it disintegrates from within, and its débris form a dirty white deposit at the bottom of the glass. The green tint of the liquid turns later to yellow, and the reaction is clearly alkaline. The green can be turned to blue by oxidation or by the addition of a little lactic acid. In simple solutions of sugar, peptone, glycerine or urea, no coloration is visible, but it appears when tartrate of ammonia is added. This property of secreting pigment does not appear to decrease by successive cultures as is the case with many chromogenic species. It remains as pronounced after a large number of generations and in very old cultures. The "optimum temperature for the formation of colouring matter is from $15^{\circ}$ to $18^{\circ}$, it is hindered at $25^{\circ}$, and no longer takes place at $37^{\circ}$.

Vitality-Does not resist much heat ; a temperature of $60^{\circ}$ will kill it in a few minutes. Therefore it is doubtful whether the formations described as spores should really be considered as such. Withstands desiccation for a long time.

The pigment has not been obtained in a pure state. It appears to be slightly soluble in acidulated water, insoluble in water, alcohol, and ether ; it also dissolves slightly in glycerine. It decomposes very quickly; solutions rapidly pale in the light, a little more slowly in the dark. Treated with organic acids or attenuated mineral acids the solution does not change colour; with ammonia it becomes violet, and with potash and soda rose-red; the blue colour is restored by acids. When treated with potash and left for some time, from 12 to 24 hours, from rose-red it becomes brickred, showing a very marked fluorescence; the colour is not restored to blue by acids, but turns yellow and decolorises little by little. Under the spectroscope the blue solution shows a thick band of absorption into the yellow, on the line D of Frauenhofer. As well as the characteristic blue pigment, this bacillus produces another colouring matter which gives to the medium a greenish fluorescence. Modifications of colour occur in varieties of the organism. 
This organism can develop in butter and cheese, which then show blue or greenish-blue patches in which the rods described are found in abundance.

The bacillus isolated from blue milk by Zangemeister and described by him under the name of $B$.cyanofluorescens, appears to be simply a variety of the $B$. syncyaneus. It only differs in some minute variations of form, the aspect of the colonies and the production of pigment.

Non-pathogenic - Even when inoculated in large quantities.

\section{BACILLUS SYNXANTHUS}

(B. of yellow milk).

Source and habitat-Milk.

Morphology-Short thin rods.

Staining reaction - Ordinary stains, and by Gram's method.

Motility - Actively motile.

Biology: cultural characters (includingbiochemical features)-Produces a yellow pigment, readily soluble in water, not in ether and alcohol ; decolorised by acids, but returns on treating with alkalis.

Bouillon-Uniform turbidity and a pellicle.

Gelatine plates and tubesLuxuriant yellow-grey growths; no liquefaction.

Milk-Is coloured abright yellow, the casein is dissolved, and the milk becomes alkaline.

Non-pathogenic.

\section{BACTLLUS TUBERCULOSIS OF KОCH.}

Source and habitat-Human sputum, and discharges from tubercular patients ; dust, milk, etc.

Morphology-Slender, slightly bent bacilli, with pointed ends; threads and true branched forms occur in sputum, and particularly in old cultures. They have also been obtained on acid potatoes. Clubshaped forms have also been described, I.5 to $4 \mu$ long by $0.4 \mu$ broad. Short in tissues, longer in milk ; solitary, or in twos or threes, often circumflex arrangement; in groups and bundles; discrete in human tissues and discharges; in colonies in animal tissues and discharges.

Staining reaction - Acid-fast; ZiehlNeelsen method; also by Gram's method.

Flagella; motility; spore formationUnstained vacuoles and granules often occur, but they are not true spores. There are no flagella in ordinary forms and no motility. The capsule stains.

Biology: cultural characters (including biochemical features)-Growth occurs from $22^{\circ}-42^{\circ} \mathrm{C}$. It is best at $37^{\circ} \mathrm{C}$.

Bouillon-Growth occurs in 7 or 8 days if glycerine added; from glycerinated cultures the bacillus may be removed and grown in ordinary bouillon. Klein obtained growth in bouillon to which white of egg had been added. Pellicle may be produced.

Glycerine-agar-Minute crumblike colonies, irregular in form, whitish-yellow in colour, elevated, sinuate. Later the entire growth becomes brownish in colour. The appearance is not unlike miniature mountain ranges. Sometimes the growth is dry, in other cases moist. Growth slow, commences 6 to 12 days; not unlike lichen.

Potato - Small, crumb-like, yellow, friable masses; dull and without moistness or lustre. Growth marked in about 2 to 3 weeks. Best if potato kept moist.

Blood serum - A slight growth in the form of light-coloured, dry, 


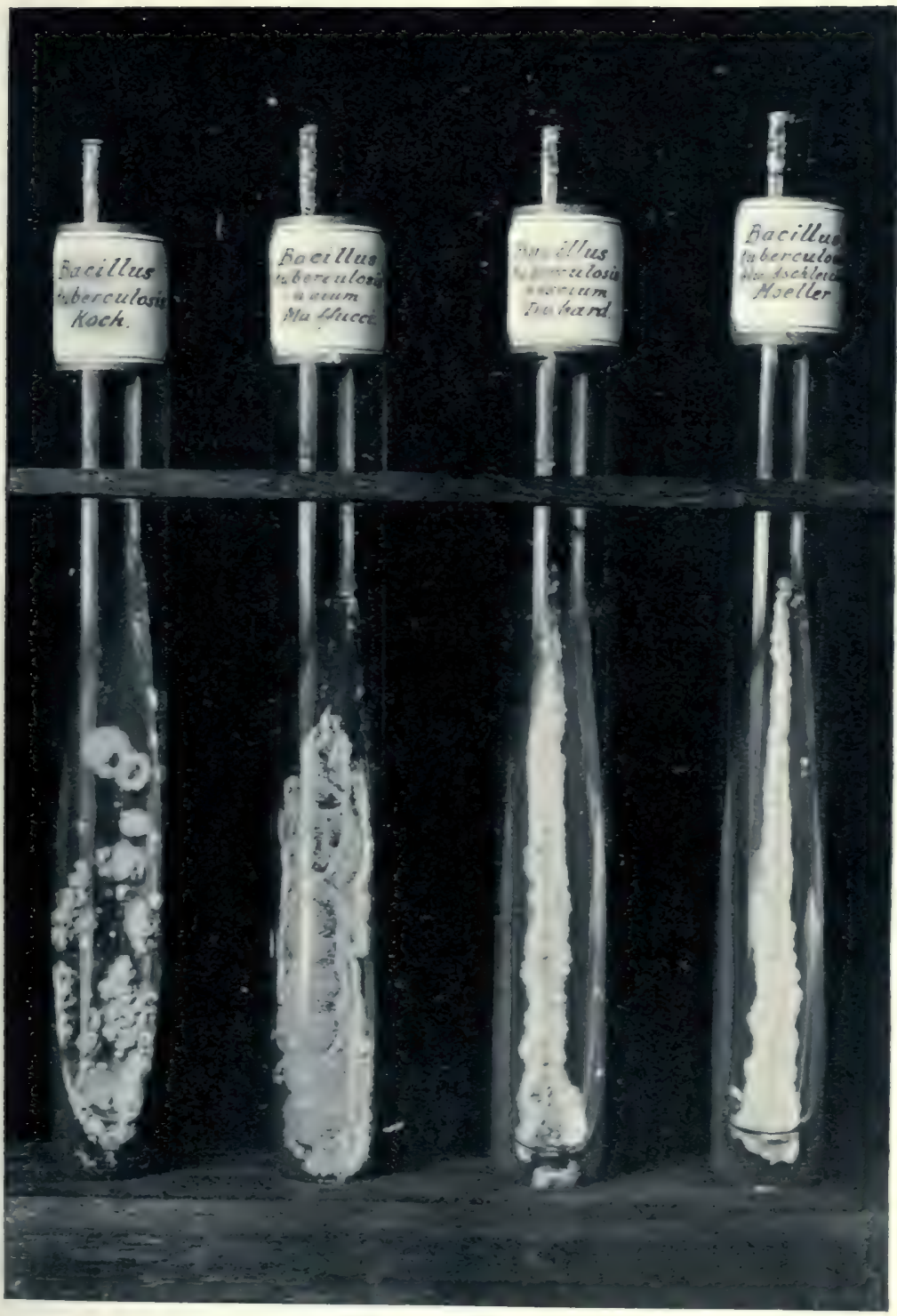

Varieties of B. Tuberculosis-cultures upon glycerine-agar.

a. Mammal, b. Birl. c. Fish. d. Reptilu. 

crumb-like scales which coalesce. Blood serum not liquefied. First growth appears 10 to 12 days.

\section{Aërobic.}

Vitality-Considerable, can withstand desiccation for months; moist heat at $70^{\circ} \mathrm{C}$. for 10 minutes kills.

Pathogenesis-Pathogenic; produces tuberculosis in man and the lower animals (bovine species of tubercle bacillus); very frequent in cows (perlsucht), less frequent in pigs, rare in sheep and horses; common in birds.

\section{BACILLUS PSEUDO-TUBER- CULOSIS (Pfeiffer).}

Source and habitat - Isolated from London milks ' 8 per cent.) by Klein.

Morphology - Resembles B. coli. Short, small bacilli; cylindrical; round ends. 0.4 to $0.5 \mu$ broad; $1 \cdot 2$ to $\mathrm{I} \cdot 8 \mu$ long.

Manner of grouping-Singly, or in couples or chains; sometimes filamentous forms, long chains in bouillon culture; most noticeable in unstained specimens.

Staining reaction - Alkaline Löffler's methyl-blue ; 15 minutes steaming ; also by Gram's method (Klein).

Capsule; flagella; motility - Nonmotile; occasionally a terminal flagellum.

Biology: cultural characters (including biochemical features).

Bovillon-In 24 hours a wellmarked granular cloudiness ; small flocculi float through the liquid; makes bouillon alkaline. Imperfect pellicle after several days' growth. No general turbidity.

Gelatine plates and tubesGrowth resembles $B$. coli, but the colonies are more circumscribed and granular; later, become tuberculated; growth slow, and colonies become more opaque, whiter, and less spread out than B. coli. No gas is formed in gelatine shake cultures. Slight growth in stab-culture. No liquefaction of gelatine.

Agar plates and tubes-Minute grey-white flat colonies; thick, less transparent centre. Stroke and stab-cultures similar to $B$. coli, but not so luxuriant.

Potato-Limited growth ; faintly brownish ; thin layer with crenated thicker margin of a whitish-yellow colour.

Milk-Grows well but leaves the milk unaltered; no curdling. Litmus milk retains its deep-blue colour.

Blood serum-Grows in colonies similar to the growth on agar; does not liquefy serum.

\section{Aërobic.}

Pathogenesis - Guinea - pigs inoculated subcutaneously with a small quantity of culture die in a few weeks. Their organs are found to be studded with yellow-white nodules containing the bacillus in pure culture. These nodules develop more rapidly than true tuberculosis, but do not contain any giant cells. If fed with food contaminated with this organism similar nodules develop in the walls of the intestine and mesenteric glands. Klein says: "The presence of the bacillus pseudo tuberculosis in milk may probably play a part in causing pseudotuberculous disease in the human subject." $\mathrm{He}$ found it present in 8 milks out of 100 milks examined in London (Rep. Loc. Gov. Bd, 1899-1900, pp. 355-384.) 


\section{BACILLUS TYPHOSUS}

(Eberth, Gaffky).

Source and habitat-Water, soil, etc., outside the body; spleen, lymphglands, blood, urine, excreta, etc., in the body of typhoid patients.

Morphology-Short plump rods when removed from organs; longer forms in culture, especially in potato having acid reaction. I to $3 \mu$ long by 0.6 to $0.8 \mu$ broad.

Staining reaction-Ordinary stains; not by Gram's method.

Capsule; flagella; motility-Actively motile ; many ( 8 to 14 ) long tortuous flagella attached all round capsule of bacillus; in threads; serpentine movement.

Spore formation-No spore formation. Vacuole formation is marked, apparent in unstained as well as stained preparations.

Biology: cultural characters (including biochemical features)-Grows best at $37^{\circ} \mathrm{C}$. on all ordinary media; grows less well on nonalbuminous media ; no pigment production; no indol. Does not produce gas in lactose media. The amount of acid produced in litmus-whey is very small compared with $B$. coli.

Bouillon-Turbid growth occurs with abundant sediment.

Gelatine plates and tubes-Small, yellowish-white punctiform colonies; round, shining; centre raised; periphery less opaque. Under magnification indistinct parallel curved lines appear on surface which give the appearance of wavy elevations and surface scratchings ; sometimes limpet-shell characters. In stab-cultures a thread-like, granular, greyish - white growth. On the surface, thin, white, iridescent colony, not elevated, and extending to the glass. In streak culture, the same appearance; non-liquefying.
Agarplates and tubes-Irregular, round, greyish-white colonies, shining and slightly raised; from the centre, dark, yellow, winding, jagged lines extend outward. In stab-culture a thread-like, granular, greyish growth with an irregular surface colony having an oily lustre, and eventually becomes yellowishgrey in colour. On streak culture a moderately spreading growth, wavy, with smooth edge and shining surface ; water of condensation clear with slight precipitate.

Milk-Is not coagulated; very little acid is formed. Unchanged appearance.

Potato-Growth spreads out from line of inoculation as a delicate, moist, "invisible" layer ; not always present; sometimes a greyish growth, and more rarely a brownish-yellow growth like $B$. coli. Condition depends upon reaction of potato.

For methods of differential diagnosis, see B. coli.

Anaërobic or aërobic-Grows best as aërobe, but also anaërobically and in $\mathrm{CO}_{2}$ 。

Vitality-Retain vitality dried for months in clothing or soil; withstand cold well; live in water for a few hours to many days. May be retained in the body for some weeks and even months.

Pathogenesis - Pathogenic for man, produces typhoid fever. Pathogenic for animals, but does not produce typhoid fever, the animal dying of intoxication, not infection.

BACILLUS VARIANS LACTIS, Nos. 1, 2, and 8 (Conn).

Source and habitat-Milk.

Morphology - A bacillus with blunt ends. $.8 \mu$ broad by $\mathrm{I} .5 \mu$ long. 
Staining reaction-Ordinary aniline stains.

Biology: cultural characters (including biochemical features).

Gelatine plates and tubes-Thin, spreading, transparent colonies, sinking into a pit in a dense, granular mass; sometimes a few lobe-like shoots branch out, and end in prominent knots. In stabcultures a shallow cone is produced. Rapid liquefaction. No. 2 produces a dry pit, and liquefaction is very slow. No. 3 does not liquefy.

Agar plates and tubes; potatoScanty, uncharacteristic growth.

Milk-Is curdled hard, and rendered amphoteric or sometimes acid. No digestion can be seen, but a watery whey subsequently deposits from a solid curd.

Aërobic.

Non-pathogenic.

BACILLUS VIOLACEUS.

Sonnce and habitat-Water, dust, air, milk.

Monphology-Bacillus 2 to $5 \mu$ long, 0.4 to $0.5 \mu$ broad; round ends; sometimes thread forms.

Staining reaction-Stains by Gram's method.

Motility - Non-motile. Sometimes feebly motile in fluid media.

Spore formation-Round or oval spores, same diameter as bacillus, in old cultures.

Biology: cultural characters (including biochemical features).

Gelatine plates-The organism attains its maximum growth in about three days. The colonies appear as small hyaline specks with sinuous borders and elliptic centres, opalescent and yellow. The central portion quickly becomes hollow, and liquefaction is rapid. Upon the surface of the liquefaction floats a thick, viscid, coherent membrane, which may be removed in its entirety. After a lapse of time this assumes a violet tinge, either as a whole or in part, and in concentric zones. At times, however, no coloration whatever appears.

In stab gelatine cultures liquefaction is very rapid. The liquefaction' becomes turbid, and a white pellicle is formed on the surface, adhering to the walls of the tube. At the end of some weeks this veil often shows a delicate, violet edging, and at the bottom of the tube a thick, whitish sediment is deposited. At other times the liquefaction is much slower, and especially if the proportion of gelatine in the medium is large ; and in this case the colony, which takes the form of a fairly thick growth of a pronounced violet colour, gradually sinks into the medium, when the liquefaction proceeds slowly and gradually. A thick, violet pellicle floats on the surface of the liquefied gelatine, and a white precipitate is formed, which at times has a slightly violet tinge. The liquid may at the same time be of a faintly roseviolet tint. The cultures often develop a strong odour of butyric acid.

Milk - Sometimes coagulated, usually fluid, and a violet cream layer forms.

Bouillon-Turbid; sometimes a pellicle, which may be violet in colour.

Agar-The cultures are more characteristic. On streak cultures there appears in two or three days, along the needle track, a thin, white line, which gradually grows larger, and forms a thick, folded 
film. This rapidly assumes a dark violet hue. The same odour of butyric acid may be perceived, but this is at times entirely wanting.

Potato-The growth is viscous, but not very thick. It gradually assumes a brown tinge, which at length covers all the surface of the medium. Along the original needle track, however, a distinct violet colour can be perceived.

Bouillon-Resembles that in liquefied gelatine.

Pigment-The most interesting peculiarity of the organism is its pigment production. The colouring matter is not produced in liquid media, or, if at all, in proportions altogether insignificant; but upon first cultures in agar it is very abundant. It is only formed, however, where the growth is in immediate contact with the air. If a thin layer of oil is poured upon the medium, growth is very slow and there is no coloration. The colouring matter is insoluble in water, but easily soluble in absolute alcohol, when it forms a liquid of a beautiful deep violet colour-resembling a solution of aniline violet when the proportion of the culture is sufficiently great.

Non-pathogenic.

BACILLUS VIscosus (Adametz).

Source and habitat-Water, milk, etc. Morphology $-2 \mu$ long by I $\mu$ broad. No chains are produced as a rule.

Staining reaction-Ordinary aniline dyes, marked polar staining.

Biology : cultural characters (including biochemical features).

Gelatine plates and tubes-Small, white colonies are produced, which rapidly liquefy. The medium is slightly tinged with green. In tubes a narrow tunnel is produced by the liquefaction, with a thick scum on the surface, which becomes wrinkled. The liquid becomes green, and later yellow and slimy.

Agar-A thin, rough, white, dry skin forms, which is very sticky. The agar becomes slightly green.

Milk-Is curdled at $20^{\circ} \mathrm{C}$. in 2 days into a soft, slimy clot, with no whey. The reaction is alkaline. There is an unpleasant odour. After 4 months in laboratory, this organism does not coagulate milk.

Non-pathogenic.

BACILLUS VISCOSUS, Nos, 1 and 2 (Van Laer).

Source and habitat-Beer wort; produces great viscosity in milk.

Morphology-Thin rods : $\mathrm{I} \cdot 6 \mu$ to $2.4 \mu$ in length by $.8 \mu$ in breadth. Isolated, and sometimes in pairs side by side; chain formation is rare.

Staining reaction-Ordinary aniline stains.

Biology: cultural characters (includ ing biochemical features).

Gelatine plates and tubes-On plates, round or ovoid colonies become visible in 48 hours at ordinary temperature ; surface colonies are white by transmitted, yellowish by reflected light. In tube cultures a whitish growth along the needle track, with sinuous borders. Gelatine is not liquefied.

Agar plates and tubes-Similar to that upon gelatine, but very rapid at about $33^{\circ} \mathrm{C}$.

Potato-A white surface growth; very viscous, and emitting an odour resembling that of stale fish.

Milk - Becomes very viscous with surface pellicle of a greenishyellow colour. The casein is precipitated and subsequently dis- 
solved, the serum shows a green fluorescence.

Aërobic.

Non-pathogenic.

No. 2 differs only from No. 1 in that the elements are thicker, and the viscosity produced in milk and other liquids is not so pronounced.

\section{CLOSTRIDIUM FCअTIDUM (Liborius).}

Source and habitat-Soil and milk.

Morphology; form-Bacillus. Rods I $\mu$ broad, singly and in filaments.

Staining reaction-Ordinary stains.

Motility-Active.

Spore formation-Present, resembling that of B. butyricus; oval, central, or terminal.

Biology: cultural characters (including biochemical features).

Gelatine plates and tubes-Gelatine is rapidly liquefied. Inoculated in the depth the liquefaction spreads from below upwards. There is much gas formation with unpleasant odour, and splitting up of the medium. Small irregular colonies.

Agar plates and tubes - The colonies form branching processes resembling those of $B$. adematis maligni; colonies small, yellow, and irregular in shape; gas formation.

Blood serum - Homogeneous cloudiness, and at end of puncture a few colonies.

Anaërobic.

Non-pathogenic.

\section{LEUCONOSTOC MESENTEROIDES}

(Crenkowsky and Van Tieghem).

Monphology; form-Cocci and rods singly and in chains; and in zoogloea surrounded by a thick gelatinous envelope. The spores,
I. 3 to $2 \mu$ in diameter are of round or ellipsoidal form, with thick membrane and glistening contents. In liquid media formation of small masses, very adherent. The zooglcea masses are of almost cartilaginous consistency. Particularly well cultivated in liquid media containing glucose. Spores have been demonstrated by Van Tieghem. Aërobic.

\section{MICROCOCCUS ACIDI LACTS}

(Krïger).

Source and habitat-Milk and butter.

Morphology-Oval coccus; 1 - $\mu$ to I.5 $\mu$ diameter; dippococci or tetrads.

Staining reaction-Ordinary aniline stains.

Biology : cultural ckaracters (including biochemical features).

Gelatineplates and tubes-Round, white colonies with ragged border; liquefying ; granular, white growth in stab-culture, liquefies in 2 to 3 days. A wrinkled pellicle floats on surface of liquefied gelatine.

Agar plates and tubes-Growth on surface becomes wrinkled, tenacious, and sticky; develops a yellow-salmon colour.

Potato - An abundant growth, folded, and of a salmon colour.

Milk-Forms lactic acid from milk sugar. Milk coagulated into a hard clot in 5 days $\left(15^{\circ}-35^{\circ} \mathrm{C}\right.$.). Eventually, albuminous bodies are peptonised, with production of sticky character and a pasty odour. Yellow lumps of fat appear on the surface. Butyric acid and alcohol are found to be present.

Anaërobic or Aerrobic-A facultative anzerobe.

Non-pathogenic. 
MICROCOCCUS ACIDI LACTIS,

Nos. 1, 2, and 8 (Marpmann and Conn).

Source and habitat-Milk.

Morphology-Coccus; .6 to $.8 \mu$ in diameter.

Grouping-Occurs in masses.

Staining reaction-Ordinary aniline dyes.

Biology: cultural characters (including biochemical features).

Gelatine plates and tubes-Finely granular round colonies with smooth edge. In stab-culture moderate "needle growth," rough and beaded. Surface growth, rough, irregular, and thick. No. 3, yellow at first, becoming white.

Agar plates and tubes-A white growth becoming yellow.

Potato-White, thick, spreading growth which becomes yellow. No. 3 grows very abundantly, thickly, and of a flesh colour.

Milk-Rendered acid at $20^{\circ} \mathrm{C}$. but not curdled except at $35^{\circ} \mathrm{C}$. Cream is thickened and rendered acid. Makes butter of good flavour.

Aërobic - A facultative anaërobe.

Non-pathogenic.

\section{MICROCOCCUS ARBORHSCENS LACTIS (Conn).}

Source and habitat-Milk.

Morphology - Coccus ; $7 \mu$ in diameter ; singly, not in chains.

Staining reaction-Ordinary aniline stains.

Biology: cultural characters (including biochemical features).

Bouillon-A very tough tenacious scum is formed.

Gelatine plates and tubes-Deep colonies are irregular, granular, with a broken edge. In stab-culture growth along needle track possesses radiating fibres. Surface growth white, not thick, but spreads over surface.

Agar plates and tubes; potatoNot characteristic,

Aërobic.

Non-pathogenic.

\section{MICROCOCCUS AUREUS LACTIS}

(Conn).

Source and habitat-Milk.

Morphology-Coccus ; $\cdot 8 \mu$ in diameter ; in pairs or clumps.

Staining reaction-Ordinary stains.

Biology: cultural characters (including biochemical features).

Gelatine plates and tubes-Round opaque colony with granular halo ; liquefaction. In stab-culture a shallow pit is produced which deepens into a horizontal layer with a yellow sediment.

Agar plates and tubes: potatoAn abundant, moist, glistening, yellow growth.

Milk-Curdled and alkaline after 3 weeks.

Aërobic.

Non-pathogenic.

\section{MICROCOCCUS CANDICANS}

(Flügge).

Source and habitat-Common in air, water, milk; also occurs in human hair.

Morphology-Round cocci, singly or in branches; $I .2 \mu$ in diameter. Sometimes showing a dividing line in centre.

Staining reaction-Ordinary aniline stains.

Biology: cultural characters (including biochemical features)-Grows at room and blood temperature on ordinary media.

Bouillon-Cloudy growth with some sediment; pellicle forms.

Gelatine plates and tubes-Round colonies, 2 to $3 \mu$ in diameter ; in 8 
days moist, shining, white, and elevated. Opaque at centre, transparent at edges. Thread-like granular white growth in stab-cultures. Wavy, shining, white growth in streak cultures, becoming dull and of consistence of butter. Does not liquefy gelatine.

Agar plates and tubes-As in gelatine but colonies more elevated and opaque ; white, spreading, oily growth in streak cultures.

Potato-Thick, white, porcelain growth, much elevated and with a wavy border.

Milk-Not coagulated in 14 days but becomes feebly acid.

Aërobic.

Non-pathogenic.

\section{MICROCOCCUS CINNABAREUS}

(Flügge).

Source and habitat-Air and water; thence to milk.

Monphology - Ovoid coccus $9 \mu$ or larger in diameter; isolated in pairs, or in tetrads.

Staining reaction-Ordinary aniline stains.

Biology : cultural characters (including biochemical features).

Bouillon-The medium becomes soon turbid, with a reddish deposit, very coherent and viscous, the medium itself acquiring also a certain viscosity.

Gelatine plates and tubes-Small, round colonies of a dull, red colour. In gelatine stab or stroke cultures, an abundant growth of a brick-red or rose colour. Slight liquefaction of the medium, but only after a considerable length of time.

Agar plates and tubes-Large, spatula-shaped colonies with sinuous borders, and of a yellowish brick-red colour, turning later to a rose tint. Soft, and with little coherence, separating itself easily from the surface of the medium.

Potato-Formation of a mucous layer of a lemon-yellow colour, sometimes red. Cultures have a distinctly unpleasant odour.

Aërobic.

Non-pathogenic.

\section{MIOROCOCCUS CITREUS LACTIS}

(Conn).

Source and habitat-Milk.

Monphology-Coccus ; $9 \mu$ in diameter. Staining reaction-Ordinary aniline stains.

Biology: cultural characters (including biochemical features).

Gelatineplatesandtubes-Smooth, opaque, surface colonies, thin, fiat, and decidedly yellow. Gelatine begins to dry before liquefaction occurs, In stab-cultures needle growth is abundant and there is a flat surface growth.

Agar plates and tubes-Growth sunken in middle. Abundant, moist, of a brilliant yellow.

Potato-Dry, abundant, yellow growth.

Milk-Rendered alkaline. No other change.

Aerobic.

Non-pathogenic.

\section{MICROCOCCUS COMMUNIS IAACTIS}

(Conn).

Source and habitat-Milk.

Morphology - Coccus, generally in form of streptococcus elements; .8 to $1 \mu$ in diameter.

Staiving reaction-Ordinary aniline stains.

Biology : cultural characters (including biochemical features).

Gelatine plates and tubes-Round brown colonies, irregular in shape; 
edge becomes light and surrounded by liquefaction. In stab-culture a narrow funnel is produced containing a dense cloudy liquid.

Agar plates and tubes-An almost snow-white growth is produced.

Potato-Thin and watery growth produced, spreading over surface.

Milk-Is curdled at $36^{\circ} \mathrm{C}$. in 3 days with amphoteric reaction. Curdles in 3 weeks at $20^{\circ} \mathrm{C}$. The curd is soft with no whey, and subsequent digestion is slight.

Non-pathogenic.

MICROCOCCUS FREUDENREICHII

(Guillebeau).

Source and habitat-Viscous milk.

Morphology-Large cocci ; more than $2 \mu$ in diameter; usually single, more rarely in chains, like a streptococcus.

Staining reaction-Ordinary stains.

Motility-Non-motile.

Biology : cultural characters (including biochemical features)-Growth occurs at any temperature from I $1^{\circ} \mathrm{C}$. to $35^{\circ} \mathrm{C}$.

Bouillon - At first becomes cloudy and then clear, with a flocculent deposit.

Milk gelatine - Round, white colonies, finely granular; after 2 days rapid liquefaction occurs.

Agar-A white growth.

Potato - A sulphur-yellow coloured growth, at times of brownish tint, often luxuriant.

Milk - Becomes tenacious and sticky with the formation of acid; coagulation occurs after a few days.

Aerrobic.

Non-pathogenic.

\section{MICROCOCCUS GIGANTEUS} LACTIS (Conn).

Source and habitat-Milk.

Morphology - Coccus; very large, I. $5 \mu$ in diameter.

Staining reaction - Ordinary aniline stains.

Biology: cultural characters (including biochemical features).

Bouillon-No visible growth.

Gelatine plates and tubes-An opaque white colony, not characteristic. In stab-culture an abundant "needle growth," but no surface growth.

Agar plates and tubes; potatoNo visible growth.

Milk - No effect upon milk, except that after 4 weeks very slight acid, reaction and curdling takes place.

Anaërobic.

Non-pathogenic.

\section{MICROCOCCUS LIQUEFACIENS} Nos. 1 and 2 (Conn).

Source and habitat-Milk.

Morphology- $\cdot 7$ to $\mathbf{I} \cdot \mathbf{I} \mu$ in diameter; grouped in pairs by dividing in two directions.

Staining reaction-Ordinary aniline stains.

Biology: cultural characters (including biochemical features).

Bouillon-Becomes cloudy.

Gelatine plates and tubes-No. I, granular colonies with folded edges; slowly liquefying, particularly in stab-cultures. No. 2, white opaque colonies ; slowly liquefying.

Potato-No. I, a dry snow-white opaque growth. No. 2, growth hardly visible.

Milk-No. I, curdles at $36^{\circ} \mathrm{C}$. in 5 days; hard curd; acid reaction, sour smell. No. 2, renders 
milk acid, but insufficiently to curdle it unless heated.

Aërobic.

Non-pathogenic.

\section{MICROCOCCUS ROSACUUS LACTIS (Conn).}

Source and habitat - Originally obtained from Uruguay, but also in Connecticut milk.

Morphology - Micrococcus ; $-8 \mu$ in diameter; grouped in fours.

Staining reaction-Ordinary stains.

Biology : cultural characters (including biochemical features).

Bovillon-After a few days a pink sediment appears. No scum.

Gelatine plates and tubes-Light pink colonies showing nucleus, pale zone around it ; centre raised, edges thin. In stab-cultures slight growth on surface, and along track of needle, pink coloration.

Agar plates and tubes; potatoMoist, thick, pink growth.

Milk-No curdling. Reaction slightly alkaline. Slight pink tinge appears after 2 weeks. Pink scum and sliminess of milk occur eventvally.

Aërobic.

Non-pathogenic.

\section{MICROCOCCUS RUBIDUS LACTIS} (Conn).

Source and habitat - Obtained only once in milk.

Morphology - Coccus; $\mathbf{1} \boldsymbol{\mu}$ in diameter. Staining reaction - Ordinary aniline stains.

Capsule; motility-Non-motile ; nonstaining capsule.

Biology: cultural characters (including biochemical features).

Gelatine plates and tubes Rapidly liquefying, with red pigment. In tubes a narrow funnel is produced with red deposit. Eventually whole gelatine liquefies and becomes red.

Agar plates and tubes; potatoBlood-red, thick, luxuriant growth. Pigment not produced at $35^{\circ} \mathrm{C}$, best at $23^{\circ} \mathrm{C}$.

Milk-Does not curdle milk or turn it red. No change in reaction.

Aërobic.

Non-pathogenic.

\section{MICROCOCCUS VARIANS I.ACTIS} (Conn).

Source and habitat-Milk and cream; found on plates exposed near cow.

Morphology-Coccus, variable; $\mathbf{I} \mu$ in diameter. Never forms chains.

Staining reaction-Stains readily with ordinary stains.

Motility - Non-motile.

Biology: cultural characters (including biochemical features).

Bouillon-In 2 days slight cloudiness, which in 6 days is very marked. Yellow sediment in 4 weeks.

Gelatine plates and tubes Whitish-yellow colonies pitting on liquefaction, which is rapid. In stab-cultures a broad shallow funnel of liquefaction occurs.

Agar plates and tubes-A dry, rough, yellow growth. Colour varies from white to orange.

Potato-A dry, granular, orangeyellow growth.

Milk - Curdles at $36^{\circ}$ in 3 days. Reaction amphoteric at $20^{\circ} \mathrm{C}$.; curdles in ro days.

Aerobe-A facultative anaërobe.

Non-pathogenic.

MHCROCOCCUS VISCOSUS LACTIS (Conn).

Source and habitat-Milk and cream. Morphology-Coccus; $\cdot 9 \mu$ in diameter; in pairs or chains. 
Staining reaction - Ordinary aniline dyes.

Biology: cultural characters (including biochemical features).

Bouillon-Abundant growth and pellicle on surface.

Gelatine plates and tubes Smooth, shining, white colonies. Slight needle growth in stabculture, with abundant white surface growth which becomes much raised.

Agar plates and tubes; potatoNot characteristic ; a white shining layer.

Milk-Becomes acid but does not curdle. It is soon rendered extremely slimy and can be drawn out into long threads. Sometimes coagulates, with production of butyric acid.

Aërobic.

Non-pathogenic.

MIST BACHLUS (Moeller).

Source and habitat-Manure of horses, cows, etc., thence to milk.

Morphology-Rods; I to $4 \mu$ long, 0.2 to $.4 \mu$ broad; often in masses, or at angles; variable forms, filaments ; on blood serum, short and grouped at angles, also "coccothrix" forms ; on gelatine, thicker ; in bouillon, filaments.

Staining reaction-Acid-fast; ZiehlNeelsen ; take Gram with difficulty ; Czaplewski's method.

Motility-Absent.

Biology: cultural characters (including biochemical features) - Best temperature $37^{\circ} \mathrm{C}$., on all usual media; slow growth at room temperature.

Bouillon - Abundant growth in 2 days, turbidity, yellow deposit, later a pellicle, adherent to sides of tube. Growth better in glycerine bouillon.

Gelatine plates and tubesGrowth best in depth; very little surface growth. No liquefaction.

Agar-Grows well in 48 hours, isolated colonies, slightly raised, eventually thick; at first greywhite, and later a chamois colour; better growth and more coloration in glycerine-agar.

Milk-Becomes acid in 4 to 5 days; sometimes coagulation occurs; red-yellow colour produced in pellicle.

Blood serum-Growth along the needle track ; yellow, not luxuriant.

Aërobic.

Pathogenesis-Similar to Timothy grass bacillus.

\section{OIDIUM LACTIS.}

Source and habitat-Sour milk.

Morphology; forms and dimensionsFruit-hyphæ simple, erect and colourless, bearing at their ends a series or chain of conidia. The conidia germinating into filaments of varying length, which by subdivision form septate mycelial hyphæ. These in their branching give rise to spores or conidia.

Staining reaction-Stains well with ordinary aniline stains.

Spore formation-Spores in form of short cylinders.

Biology : cultural characters.

Gelatine plates - On gelatine plates colonies appear first as white points, developing later into delicate stellate colonies, which eventually coalesce and form a fine mycelial network covering the surface of the medium.

Agar plates-Growth similar to that on gelatine.

Non-pathogene. 


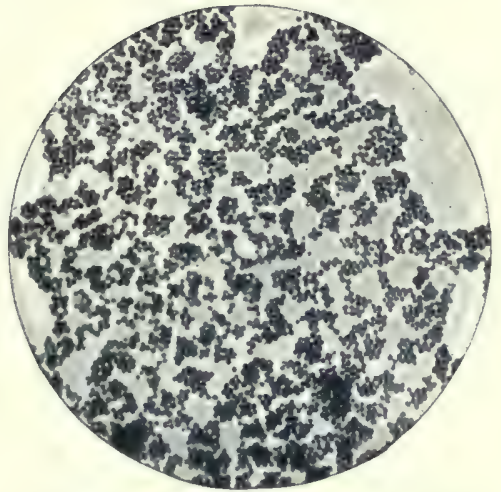

Staphylococens pyogenes aureus, from culture on algat at $37^{\circ}$ C. $\times 1000$.

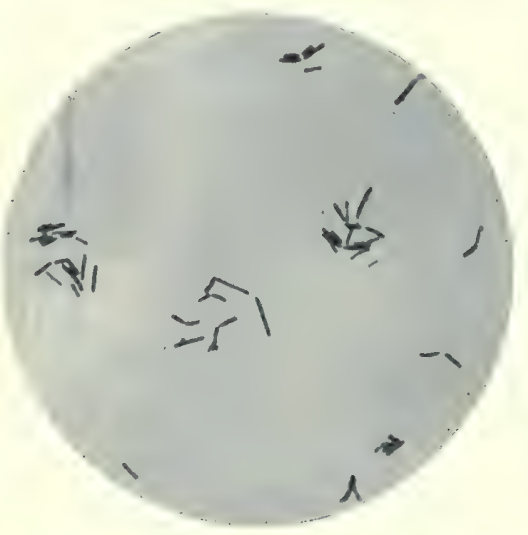

b. actimulucter (Dnclaux). Agar culture; 2 days' growth at $37^{\circ}$ C. Stained carbol-fuchsin dil. $\times 1000$.

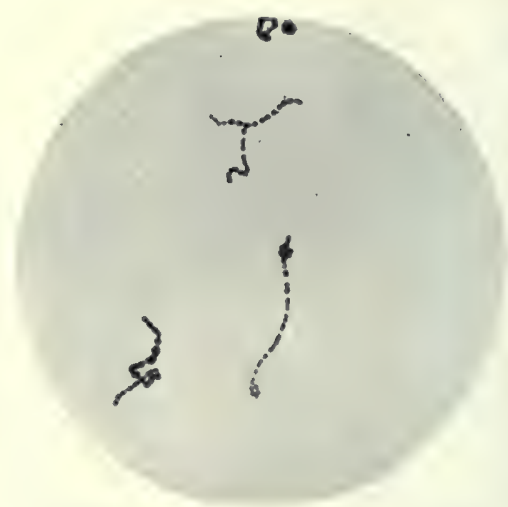

Streptococents pyogenes (pathogenic). $\times 1000$.

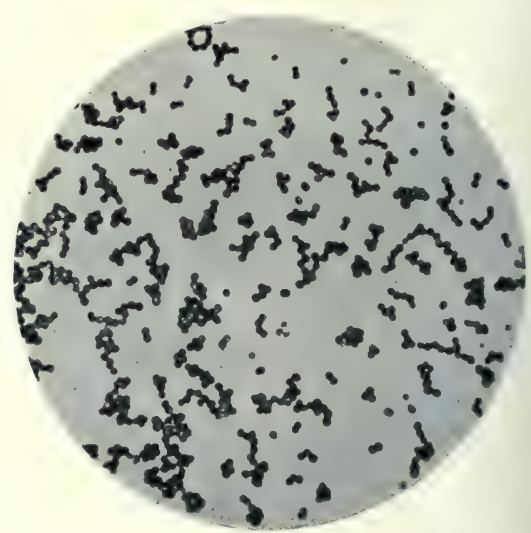

Mierococcus Freudenreichii (Guillibeau). Agar culture; 2 days' growth at $37^{\circ} \mathrm{C}$. Stained eartol. fuchsin dil. $\times 1000$. 


\section{PROTEUS VULGARIS.}

Source and habitat - Putrid meat, excreta, bad milk, etc.

Monphology-Slender thin rods or as threads; 1.6 to $4 \mu$ long, 0.4 to 0.5 $\mu$ broad.

Staining reaction-Ordinary stains, and by Gram's method.

Flagella; motility-Active motility; many long flagella.

Spore formation-None.

Biology: cultural characters (including biochemical features)-Rapid growth at all ordinary temperatures and in ice. Indol and $\mathrm{H}_{2} \mathrm{~S}$ are produced in cultivation.

Bouillon - Very cloudy and abundant precipitate.

Gelatine plates and tubes-Grey delicatecolonies; rapid liquefaction. Some small and punctiform, with sharp outlines and of a greyishyellow colour ; others larger, colourless, and with wavy lobulations.

Agar plates and tubes - Tube shaped liquefaction in stab-cultures and saucer liquefaction in plates; in both cases the liquefied fluid is turbid.

Potato - Scanty growth ; dull, raised, and of whitish-yellow colour.

Milk-Is coagulated in 2 to 3 days, and later is again liquefied, and eventually yellow in colour and acid in reaction. No gas.

Agar-Greyish-white colonies, non-characteristic; grey, shiny, moist surface growth, veil-like and of rapid growth. Water of condensation very cloudy, and whitishyellow in colour.

\section{Anaërobic and aërobic.}

Vitality - Considerable against chemical and thermal agencies.

Pathogenic for animals.

\section{PROTFUS ZENKFRI.}

Source and habitat - Decomposing meat, fluids, sewage, milk, etc.

Morphology - .5 to I $\mu$ thick, 2 to $4 \mu$ long; long threads in bouillon.

Staining reaction-Ordinary aniline stains.

Biology: cultural characters (including biochemical features).

Gelatine plates and tubes-Round colonies, with branching processes radiating widely. Non-liquefying. In stab-culture, along the needle track are formed lateral extensions or thin sheets (like an inverted fir tree). Surface growth thin and irregular.

Agar plates and tubes-Growth spreads rapidly from needle track, with radiating fibres of growth.

Potato - Dry, whitish-brown, rough growth.

Milk-Produces slightly alkalinity, and in time a sliminess.

Anaërobic or aërobic-Facultative anaërobe.

Pathogenesis-Hauser maintains that this bacilli is transformable into Proteus vulgaris, which possesses poisonous properties.

\section{STAPHYLOCOCCUS PYOGENES AUREYS.}

Source and habitat-Milk, water, air, on skin of healthy persons, in milk of healthy women; in many diseases, especially suppurative conditions.

Morphology-Micrococci in masses; round, small ; on an average about $0.8 \mu$ in diameter ; masses or pairs, or singly, usually in grape-like clusters.

Staining reaction-Ordinary stains.

Flagella: motility - No flagella; non-motile. 
Biology: cultural characters (including biochemical features)-Grows at room temperature, but better at $37^{\circ} \mathrm{C}$., on all media.

Bouillon - Marked uniform cloudiness ; delicate pellicle ; some sediment.

Gelatine plates and tubes-Small irregular, round colonies, yellowishwhite to yellow in colour; slow liquefaction; structure somewhat coarsely granular. In stab-culture, liquefaction along needle track in 2 to 3 days (conical, and later cylindrical); deposit pigmented yellow ; degree of liquefaction varies.

Agar plates and tubes-Round orange - yellow colonies, even margin ; granulation sometimes well marked. Feeble growth in stab-culture ; good surface growth ; smooth, shining, orange-yellow. In streak culture, similar growth; condensation fluid cloudy; precipitate, light orange in colour; odour like glue or stale paste ; most rapid method of isolation is by using agar.

Potato-At first white growth, becoming yellow-elevated, shining; old cultures widely spread, deep orange in colour, and dry. The best medium for chromogenic effects.

Milk-Firm coagulation in I to 8 days (Passet). Lactic acid produced.

Pigment produced only in presence of oxygen.

Anaërobic or aërobic_-Grows well aërobically, less well anaërobically.

Vitality - Considerable powers of vitality have been proved in the body ; in cultures, also tenacious of life. Alive after 56 to 100 days' desiccation; $70^{\circ} \mathrm{C}$. kills them if moist; alive after 66 days in ice ('Prudden).
Pathogenic-Much variation in virulence ; passage through animals and anaërobic cultivation increases virulence ; relative susceptibility to infection is shown in the following decreasing series-horse, dog, man, cattle, sheep, rabbits, guinea-pigs, and mice least of all. Chief pathological conditions set up are suppurative. Intravenous injection causes endocarditis.

$S$. pyogenes albus is almost identical with the $S$. pyogenes aureus, except that its colour production is white and not orangeyellow. S. pyogenes citreus is also similar, but that its colour in culture is lemon. It does not coagulate milk, and its liquefaction of gelatine is slow, with production of gas bubbles.

\section{STREPPOCOCCUS PYOGENFS}

(Rosenbach).

Source and habitat-In soil, water, outside the body-and in suppurative diseases inside the body. Milk, etc.

Morphology - Chains of cocci, long in fluid, short on solid medium.

Staining reaction - Ordinary stains and Gram's method.

Capsule-Definite mucoid capsule occasionally seen about the chain.

Biology: cultural characters (including biochemical features)-Growth rather slow, best at $37^{\circ} \mathrm{C}$.; above $47^{\circ} \mathrm{C}$. no growth. As a rule, more luxuriantly upon fully acid medium. Non-chromogenic. No gas formation.

Bouillon-Varies from diffuse cloudiness to compact sediment with clear medium. No indol.

Gelatine plates and tubes-Small white, round, flat colonies; slow growth, smooth margin. In stabcultures, at first thread-shaped, and later with small nodules. 
PLATE 32.

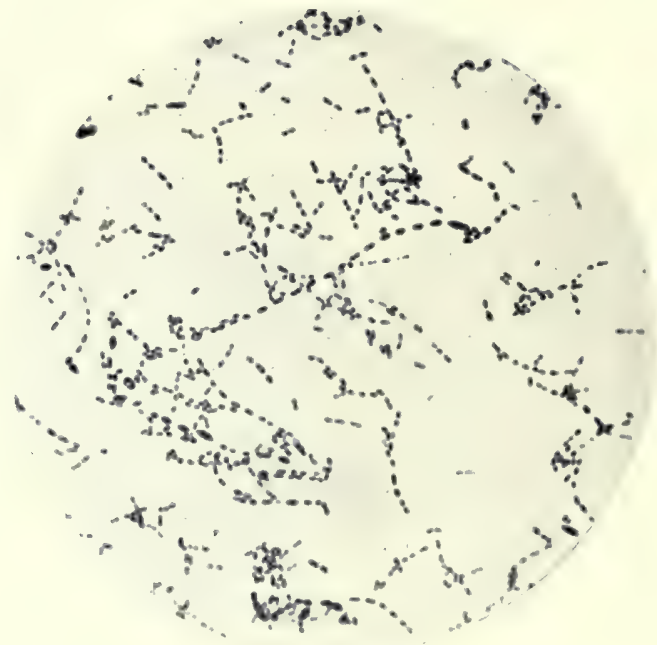

Streptococcus scarlatince (Gordon) from culture on agar, 48 hours' growth. Stained by Gram's method. $\times 1000$.

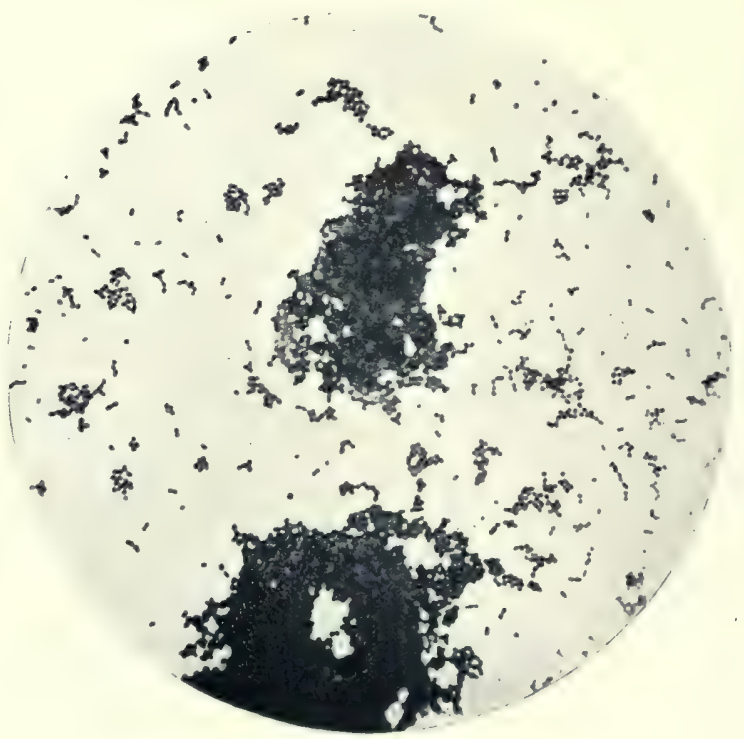

Streptococcus scarlatime (Gordun) from culture in bouillon, 96 hours' growth. Stained by Gram's method. $\times 100$. 
Agar plates and tubes-Small white colonies, granular, and sometimes showing lobulation. Threadlike, and later granular needle track. Growth in stab-cultures : a grey irregular surface growth.

Potato - "Invisible" growth, at times absent, rarely abundant.

Milk-Is usually firmly coagulated in from 4 to 24 hours.

Glycerine ascites agar-Luxuriant colonies; from periphery of colony coiling chains spread out; granulation in interior of colony more marked than upon agar.

Anaerrobic or aërobic-Facultative anaërobe.

Vitality-Considerable, retained several months, especially in dried pus. In cultures, usually only for a few weeks; but in bouillon to which hydrogen is admitted, the streptococcus will live for months.

Pathogenesis - Pathogenic for mice and rabbits, and less so for dogs and rats. In man streptococci have been successfully inoculated, producing erysipelas, suppuration, etc.

\section{STREPTOCOCCUS SCARLATIN A?}

(Klein and Gordon). STREPTOCOCCUS CONGLOMFRATUS (Kurth).

Source and habitat-Isolated from the blood, nasal and tonsillar discharge of persons suffering from scarlet fever in its earlier and later stages. Not from urine or skin. It has been isolated from blood of persons dying from scarlet fever. Assumed to be identical with streptococcus isolated from diseased udders of cows and from their milk. Found by Klein in ulcerations of teats and udders of certain cows.

Morphology-A streptococcus ; polymorphic ; showing tendency to oval and rod-shaped elements, especially in impression preparations. Pres- ence of wedge-shaped, spindlelike, rod-shaped elements in agar and gelatine, and the characteristic of coherent conglomeration differentiate this streptococcus from others of the same genus. Irregularity in size and shape of elements ; every transition between coccus and bacillus. Coccus shape prevails in bouillon, the bacillary being more common on agar (see Plate).

Staining reaction-Simple stains and Gram's method.

Biology: cultural characters (including biochemical features).

Bovillon-At $37^{\circ} \mathrm{C}$. after 24 hours, the medium remaining clear, a single, thick, white-grey mass, or several smaller masses, appear at the foot of the tube; coherent on shaking the tube, floats through the fluid medium as a flattened bunlike body. Kurth pointed out that when this mass was examined under the microscope, a conglomerate appearance was present. The mass is cohesive.

Gelatine plates and tubes-Slow growth forming small grey colonies, circular or oblong, with firm edge, and consisting of closely-set coherent mass of cocci. Older colonies become nodular. Non-liquefying. In gelatine at $37^{\circ} \mathrm{C}$. the same appearances occur as in bouillon, but often more marked. Chain formation from these cultures is more marked than in ordinary streptococcus. In gelatine at $37^{\circ} \mathrm{C}$. this organism grows similar to S. longus.

Agar plates and tubes - Three types of colonies occur after 24 hours : (a) grey, granular, irregularly-outlined tuberculated colonies; (b) colonies of similar kind, but having confluent appearance without tubercles; (c) younger and 
smaller colonies which have a fine frilling of chains around a more compact coherent centre. The most useful feature for differential purposes is the granular, glossy, coherent centre, combined with tuberculation. Grows more slowly than $S$. pyogenes, and on the whole its colonies on agar are smaller, more opaque and more irregular than those of the other streptococci present. Impression preparations are characteristic (see Plate).

Milk-Rapid coagulation; produces acid. Sometimes fails to clot milk.

Litmus milk-A firm, solid clot forms, as a rule, within the first 2 days at $37^{\circ} \mathrm{C}$. After 24 hours the acid-production is very strong, and commonily, when there is a clot as well, the lower half of the tube is yellow-white-the top layer being pink. This decolorisation of lower half of litmus milk is due to a reducing action of the streptococcus. Chain formation occurs more than in bouillon.

Blood serum-Grows well, producing colonies.

\section{Aërobic.}

Pathogenesis - Pathogenic for mice and rabbits. After passing through the mouse, the streptococcus takes on a bacillary form (Gordon), and other modifications, including the diminution of conglomeration, occur. Its virulence differentiates this streptococcus from streptococci present in non-scarlatinal throats, except $S$. pyogenes, which is more virulent to white mice than $S$. conglomeratus. Klein holds that this $S$. conglomeratus is causally related to scarlet fever in man, and is wholly distinct from S. pyogenes. Gordon has isolated the latter from the secretion on the surface of the tonsil in a case of clinically mild, uncomplicated, scarlatina. It has also been found like the $S$. conglomeratus in the nasal and aural discharge of scarlet fever patients. Gordon believes that both streptococci may play a part in the causation of scarlet fever, but that $S$. conglomeratus is the more important of the two, and that it occupies a position in the bacteriological kingdom between $S$. pyogenes and $B$. diphtheria. The streptococcus of Baginsky and Sommerfeld, and the streptococcus of Class may be different forms of the $S$. scarlatince. Gordon isolated this streptococcus from the throats of scarlet fever patients, and injected it into mice with fatal results, and producing symptoms in accord with those obtained by Klein when experimenting on mice with the organism obtained from the Hendon cow disease.

(For full record of Gordon's researches see Reports of Medical Officer to Loc. Gov. Bd., 1898-99, pp. 480-493; 1899-1900, pp. 385457 ; and 1900-1901, pp. 353-404.)

\section{TIMOTHY GRASS BAOTLLUS} (Moeller). B. phlei.

Source and habitat-Isolated from Phleum pratense, Timothy grass ; has also been met with in other plants, in grasses, in dust, etc., and thence into milk.

Morphology-Rod; I.4 $\mu$ long, 0.2 to $0.5 \mu$ broad; sometimes curved at angles, in filaments ; club-shaped. Longer in milk culture than on blood serum.

Staining reaction-Acid-fast; ZiehlNeelsen method; takes Gram.

Motility-Absent. 


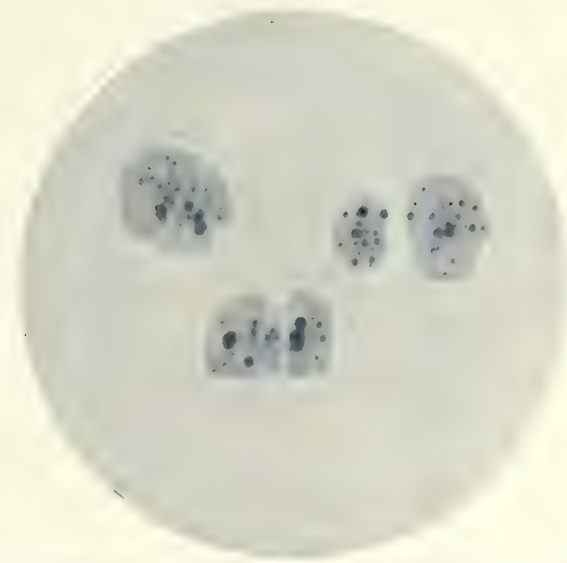

Stmptomens searlatime (Gordon). Colonies on utruent gelatine. Sixth week. Showing nodular conformation. $\times 3 \frac{1}{2}$.

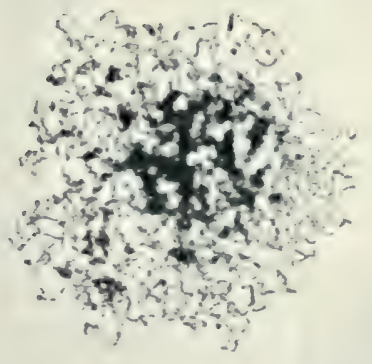

Streptoeocers scurlatino (Gordon). Culonies on uutrient agar. Impression preparation. $\times 250$. 

Biology : cultural characters (including biochemical features).

Bovillon-Turbidity and surface membrane after 3 days ; occasional chromogenicity. Abundant in glycerine bouillon, yellow membrane. No indol.

Gelatine plates and tubes-Slow, poor growth ; small colonies. No liquefaction. No gas production.

Agar plates and tubes-Rapid, abundant growth in 36 to 48 hours, yellow or white, wrinkled; resembles tubercle bacillus; yellow colour may turn to orange. Grows best in glycerine-agar ; translucent, irregular surface.

Potato - Thick, dry, yellow growth, very adherent to the medium.

Milk-Slow (5th day); small masses of growth, yellow; sometimes a yellow pellicle on the surface. No coagulation.

Blood serum-Numerous small colonies, remaining isolated and discrete; uncoloured.

Aerrobic - Slow, feeble growth anaërobically.

Pathogenesis - Moeller found this bacillus when injected intraperitoneally killed guinea-pigs in 2 or 3 days, and the bacillus, was isolated from the blood. In other cases, nodules were found on mesenteries, etc. (similar to Rabinowitsch's butter bacillus). Its effects most marked on guinea-pigs.

TORUI,A AMARA (Harrison).

Source and habitat-Isolated from milk-can washings, and maple leaves at Guelph, Canada. Produced a marked bitterness in milk.

Morphology-Oval cells, varying size, 7.5 to I0.0 $\mu$ long; after some days' growth vacuolation occurs in the cells. Budding occurs at the smaller end of the cell. Cells appear singly, or in small clumps or short chains. No spores.

Wort-At $25^{\circ} \mathrm{C}$, abundant growth. No pellicle; yeast rings occur in same medium at $37^{\circ} \mathrm{C}$.

Wort gelatine $-\left(20^{\circ} \mathrm{C}\right.$. $)$ pin - point colonies; in 4 days become round greyish-white, shining.

Gelatine-Like wort gelatine. In stab-cultures, beaded line of puncture, which in 20 to 30 days becomes dense, spiny growth, radiating from beads. Surface growth waxy, greyish - white, becoming brown at centre.

Wort agar-Rapid luxuriant growth at $37^{\circ} \mathrm{C}$.

Agar-Glistening, flat growth in 8 days at $25^{\circ} \mathrm{C}$.

Potato-Slightly raised growth in 3 days at $20^{\circ} \mathrm{C}$, at $37^{\circ} \mathrm{C}$. slightly yellower colour.

Milk-Bitter taste produced in 5 or 6 hours at $37^{\circ} \mathrm{C}$. In ro days milk is thickened, slightly acid, and very bitter. There is much gas formed. No butyric acid is present.

Non-pathogenic.

TYROTHRIX CATENULA (Duclaux).

Source and habitat-Cheese.

Morphology-Bacillus, $6 \mu$ broad by 3 to $5 \mu$ long. Grown in the absence of oxygen, the elements are short and thick; grown aërobically, they are thicker and of greater length. Isolated or in chains.

Motility-Actively motile when isolated; slow movement when in chains.

Spore formation-Mesial spores, voluminous, oval, and longer than the primitive elements. 


\section{Biology: cultural characters (includ- ing biochemical features).}

Milk - Milk becomes at first slightly acid; casein is slowly precipitated in flocculent masses; the precipitate is gradually redissolved. The liquid contains leucine, tyrosine, and butyric acid. The action of the organism is soon arrested, owing to the large production of acid. It can be caused to remain longer active by the addition of a little chalk, which partly neutralises the acid produced. The organism has no action upon casein already precipitated by acids or heat.

Anaërobic or aërobic-Facultative anaërobe, but grows more freely in the absence of oxygen.

Vitality-Is killed at a temperature of $90^{\circ} \mathrm{C}$.; spores perish at $105^{\circ} \mathrm{C}$.

Non-pathogenic.

\section{TYROTHRIX CLAVIFORMIS}

(Duclaux).

Source and habitat-Cheese.

Morphology-Bacillus, I $\mu$ broad, $2 \mu$ to $2.5 \mu$ long. Isolated or united in pairs ; never in chains.

Spore formation - Terminal spores; diameter about double that of mother-cells.

Biology: cultural characters (including biochemical features).

Milk-Rapid coagulation. Coagulum is dissolved after 24 hours, leaving a clear liquid with precipitate at the bottom of the tube. Casein and lactose are both attacked, with disengagement of gas. Liquid is slightly acid, and gives off a not unpleasant quincelike odour.

Anaërobic.

Non-pathogenic.

\section{TYROTHRIX DISTORTUS}

(Duclaux).

Source and habitat-Cheese.

Morphology -Bacillus, $\cdot 9 \mu$ broad, and with a length from 5 to ro times the breadth. Isolated or in chains. Motility-Actively motile when isolated; in chains of 4 to 5 elements the movement is slow, and it ceases altogether when the chains-become longer.

Biology: cultural characters (including biochemical features).

Gelatine plates and tubes-Gelatine is rapidly liquefied. There is formation of a thick surface pellicle and a dense precipitate at the bottom of the liquid. In old cultures the liquid is of a brownish tint.

Agar plates and tubes-Upon agar a greyish-white, shining growth, with transparent and finely cut edges,

Potato-Dry isolated colonies, at first yellowish-white, and later of a brownish tint.

Aërobic.

Vitality - The bacilli can support a temperature of $90^{\circ} \mathrm{C}$. to $95^{\circ} \mathrm{C}$, the spores to $100^{\circ} \mathrm{C}$.

\section{TYROTHRIX FIUIFORMIS}

(Duclaux).

Source and habitat-Cheese.

Monphology-Bacillus, $2 \mu$ in length by $.8 \mu$ in breadth. Isolated, united in pairs or in long chains.

Motility-Motile, with slow movement, without undulation.

Spore formation-Spores are formed generally at one extremity. The bacillus swells at the spot where the spore is produced, and takes the form of spindle or club.

Biology : cultural characters (including biochemical features).

Bouillon-The bouillon becomes 
turbid after some hours, with formation of a thick, white, velvety pellicle, which creeps up the walls of the tube. When the liquid has thickened, this veil, composed chiefly of spores, disintegrates and falls to the bottom of the tube.

Gelatine plates and tubes-Gelatine is rapidly liquefied in "stocking "form, greyish flocculi appear in the liquid, and there is surface formation of a thick, white pellicle.

Agar plates and tubes - Upon agar, a white mucous-like growth.

Potato-A thick, surface growth, at first white, turning yellow later.

Milk-In milk rapid formation of a wrinkled surface pellicle formed of filaments of fat globules and casein, at times flocons float in the liquid; there is very little if any coagulum. In 2 or 3 days the medium becomes suddenly transparent and almost clear.

Vitality - The bacilli can support a temperature of $100^{\circ} \mathrm{C}$. in fresh milk slightly alkaline, and in the same medium spores have given rise to fresh growth after having been submitted to a temperature of $120^{\circ} \mathrm{C}$.

The organism does not attack sugar, or milk, or glycerine.

Non-pathogenic.

\section{TYROTHRIX GENICULATUS}

(Duclaux).

Source and habitat-Cheese.

Morphology -Bacillus ; $\mathrm{I} \mu$ in breadth and of varying length, the filaments at times reaching to $\mu_{0}$. In filaments, often sharply bent, and entangled the one with the other.

Motility-Non-motile.

Spore formation-Forms spores.
Biology: cultural characters (including biochemical features).

Bovillon-In 6 hours at $25^{\circ} \mathrm{C}$. formation of flocons in the liquid. In 24 hours the liquid is limpid and full of floating filaments. The production of spores is more abundant than in milk, they form along the whole length of the filaments, and when liberated by dissolution of the membrane fall to the bottom of the tube in the form of a whitish precipitate. The liquid remains clear.

Gelatine plates and tubes-In gelatine stab the liquefaction is slow, and there develop along the line of the needle track numerous radiating ramified filaments, giving the growth a rootlet-like aspect.

Potato-A greyish warty-like growth.

Milk - Formation of flocons composed of matted filaments suspended in the liquid, and never uniting in the form of a surface soil.

\section{Aërobic.}

Vitality-The organism is killed in milk at a temperature of $80^{\circ} \mathrm{C}$. ; the spores can withstand a temperature of $105^{\circ} \mathrm{C}$.

Non-pathogenic.

TYROTHRIX SCABER (Duclaux).

Source and habitat-Cheese.

Morphology-Bacillus; $\mathbf{I} \cdot \mathbf{I} \mu$ to $\mathbf{I} \cdot 2 \mu$ broad by $2 \mu$ to $2 \cdot 2 \mu$ long. More often united in long chains.

Motility-Motile when the elements are young, but with slow and heavy movements. Non-motile or very slightly so when in long chains.

Spore formation-Forms spores.

Biology: cultural characters (including biochemical features).

Bouillon-Forms upon liquids a fragile surface pellicle, adherent 
to the tube walls, and veil composed chiefly of spores.

Gelatine - Gelatine is slowly liquefied.

Potato-A thick mucous growth of a dirty yellow colour.

Milk-No coagulation, but by degrees the liquid takes very slowly the colour and aspect of whey. The diastases are very little active, although manifestly produced. The liquid is alkaline and has a feeble odour; it contains leucine, tyrosine, and carbonate and valerianate of ammonia.

The organism in cheese does not develop until a late stage, when its predecessors have prepared for it nutritive matter easily assimilated.

Aërobic.

Vitality - The organism is killed by a temperature of $90^{\circ} \mathrm{C}$. to $95^{\circ} \mathrm{C}$ : ; the spores resist up to $105^{\circ} \mathrm{C}$. to $110^{\circ} \mathrm{C}$.

Non-pathogenic.

TTROTHRIX TENUIS (Duclaux).

Source and habitat-Milk, and cheese, in the maturation of which it plays an important part.

Morphology-Bacillus ; $3 \mu$ long by $\cdot 6$ $\mu$ broad. The elements at times take the form of very long filaments, straight or coiled, especially at low temperatures, or when there is scarcity of free oxygen.

Capsule; motility - No capsule, actively motile in rod form. A gentle undulatory movement when in filaments. No movement when the filaments are long or when the elements are formed in long chains.

Spore formation-Formation of ovoid spores.

Cultural characters - In bouillon, formation of small whitish flocons at the end of a few hours.
Milk-Formation of a wrinkled surface pellicle, little coherent; the milk is coagulated at first under the action of a minute quantity of rennet secreted by the organism, but the coagulum is softer than that produced by ordinary rennet. The casein precipitate is dissolved by degrees by the casease elaborated, and the liquid becomes opalescent. Division into elements takes place rapidly, and each of the elements produces an ovoid spore; the surface veil is soon almost entirely invaded with these spores. The organism is strictly aërobic.

Vitality-Its resistance to heat is remarkable. The spore forming elements, in a neutral liquid only perish at from $90^{\circ} \mathrm{C}$. to $95^{\circ} \mathrm{C}$.; in a medium feebly alkaline they can spore at $100^{\circ} \mathrm{C}$. The spores will bear a temperature of $115^{\circ} \mathrm{C}$. The optimum temperature for cultures is from $20^{\circ} \mathrm{C}$. to $25^{\circ} \mathrm{C}$.

Winckler has obtained from cultures of Duclaux several varieties of this species. One of which, liquefying gelatine, is a strong peptoniser of casein; another, which does not liquefy lactose gelatine, sets up a powerful lactic fermentation; another gives rise in liquid media to a greenish fluorescence and to formation of a reddish pigment upon potato.

\section{TYROTHRIX TURGIDUS} (Duclaux).

Source and habitat-Cheese.

Morphology - Bacillus with square ends sharply defined; $1 \mu$ broad 2 to $3 \mu$ long. Rarely isolated, more often united in chains of some length.

Capsule; motility-No capsule ; nonmotile.

Spore formation-Forms spores. 
Biology: cultural characters (including biochemical features).

Gelatine-Gelatine is slowly liquefied, with formation of a white surface pellicle.

Potato-Sparse growth of dull whitish appearance.

Milk-Formation of surface pellicle, very resistant, and composed of felted filaments. Light coagulation; the precipitate is dissolved, and the liquid becomes yellow and transparent. From the first days of fermentation, the liquid develops an odour similar to that observed in cheese-maturing cellars. The liquid is alkaline, containing carbonate and butyrate of ammonia. This last is gradually consumed during the fermentation process. Leucine and tyrosine are also found in the liquid.

Aërobic.

Non-pathogënic.

\section{TYROTHRIX UROCEPHALUM}

(Duclaux).

Source and habitat-Cheese. Widespread in nature, "one of the principal agents of putrefaction in animal matter."

Morphology-Bacillus ; I $\mu$ broad, $3 \mu$ in length or more. Isolated in pairs or united in long chains.

Motility-Actively motile.

Spore formation-Spherical spore formation at one extremity.

Biology: cultural characters (including biochemical features).

Gelatine-Gelatine stab-cultures; at first a white growth along the needle track followed by formation of gas bubbles right through the medium. Liquefaction commences late and proceeds slowly. Formation of gas bubbles on surface of liquid.

Potato-Yellowish white shining growth, turning to a brown colour with age.

Milk-Surface formation of small transparent gelatinous particles which may invade the whole mass, without, however, making it of a gelatinous consistency. After some time the milk liquid becomes muddy with a thick flocculent deposit formed chiefly of spores. Disengagement of gas when culture is made in $\mathrm{CO}_{2}$ the liquid having disagreeable garlic-like flavour when there is entire absence of oxygen.

Anaërobic or aërobic - Facultative anaërobic, but develops better in the absence of free oxygen.

Vitality - The organism is killed between $90^{\circ} \mathrm{C}$. and $95^{\circ} \mathrm{C}$. The spores perish at from $100^{\circ} \mathrm{C}$. to $105^{\circ} \mathrm{C}$. in a neutral liquid ; at from $95^{\circ} \mathrm{C}$. to $100^{\circ} \mathrm{C}$. in a liquid slightly acid.

\section{TYROTHRIX VIRGULA}

(Duclaux).

Source and habitat-Cheese.

Monphology-Bacillus; $5 \mu$ broad, about $2 \mu$ long. Isolated, or in chains composed of a small number of elements.

Motility - Non-motile.

Spore formation-Spores form at one extremity ; spherical and the same size as the mother cell.

Biology: cultural characters (including biochemical features).

Bouillon - Grows well in bouillon.

Gelatine-Grows with difficulty in gelatine.

It plays a rôle in the maturation of cheese, but only after other organisms have prepared a pabulum for it.

Aërobic.

Non-pathogenic. 


\section{CHAPTER XIII}

THE CONTROL OF THE MILK SUPPLY: (I) BY THE STATE

General Note. Milk Legislation in England and Wales. Model Milk Clauses. The Local Authority and the Milk Supply. Milk Legislation in Scotland: In Ireland: On the Continent of Europe : In the United States of America : In the British Colonies.

WE propose in this section to consider some of the main facts and principles having relation to the control of the milk supply. These facts arise very largely out of that which has gone before. The practical bearing of the present knowledge of the bacteriology of milk is a larger measure of control and regulation of the milk supply. More particularly is this necessary, when it is considered in relation to the growth of the towns and the resultant complexity of the management of the trade. When a family keeps its own cows and does its own milking and nothing more, the opportunities for pollution are small. But when, as now obtains, milk is transported long distances and is collected, not from one farm and for one family, but from hundreds of farms and for thousands of families, then the complexity of operations at once becomes obvious and the opportunities of pollution are manifold. It is idle to say that the control which met the necessities of our forefathers will also meet ours. The condition of things has during the last half century been revolutionised. The population has migrated from the country into the towns, and it has thus come about that what now constitutes the Milk Trade is comparatively a modern growth, and one which calls for totally new measures of control.

It is not, however, the growth of the milk trade alone which necessitates measures of control. There has also been during the last thirty years an enormous growth in our knowledge of milk and its relation to disease. Alongside the acquisition of the new facts about bacteria and milk, there has been an attempt to reduce its teaching to practical application, with the result that legislation and 152 
the more enlightened public opinion have not followed very far behind the new knowledge. What is now required in our view is a wider application of the methods and principles which have been found successful in a limited area.

In the first place, we consider that in a general way, before any new legislation is required, a vigorous and systematic enforcement of the legislation that already exists is of paramount importance. Certain legislation with regard to the management of the milk trade is now permissive or adoptive by local authorities. But many local authorities are suffering from supineness, or lack of interest in the trade, or perhaps too much interest in the trade, and nothing is done, though the statute book contains all that is immediately requisite. We should advocate in this case an enforcement of the existing law.

In the second place, we hold the view that what has been accomplished by private enterprise demonstrates beyond all possible doubt what is immediately practicable for public opinion to demand from the milk trade. The public demand a pure and ample water supply, because they have learned the evils resulting from an impure and inadequate supply. In the same way we believe that a knowledge of the facts of the case respecting the milk supply, what it is and what it might be, will have a similar effect. We are under no illusion as to the evils of impure milk. It has been our duty to point them out in the present volume, though we are aware that they may be readily exaggerated. The milk supply is not as important a matter as the water supply, and it is incorrect to suggest that it is. Nor can an impure milk supply have such wide-spread ill effect as a bad water supply. Nor do many of the bacteria in milk appear to have any bad effect on persons consuming it. The matter must be judged on the broad facts of the case; and these, we think, may be reduced to a very simple proposition. The milk supply, as a whole, is not at present under strict control, and much second-rate milk, and some actually diseased milk, is sold to the prejudice and injury of the purchaser. The public should know this, and should also know that with care and management and some systematising of control, this state of things can be at once corrected. The aim to be kept continually in view is a pure milk supply - a clean milk, of good quality; from healthy cows; properly strained and cooled, and protected from infection and contamination.

These chapters are divided substantially into three main portions. The first deals with what has already been done by the State in 
controlling and protecting the milk supply. The second chapter on this subject deals with the control by private enterprise. The third portion deals with what can be done by the trade in respect to-(a) milk herds ; (b) the housing of milk herds; $(c)$ milkers and milking; and $(d)$ the after treatment of the milk, and the suitable conditions of its sale.

\section{Legislation in England and Wales}

The legislation now in force in the United Kingdom in respect to the protection of milk had its origin, as far as Acts of Parliament are concerned, in the Contagious Diseases (Animals) Act of 1878, which authorised the Privy Council to make such general or special orders as seemed desirable for the purpose of registration and inspection of the trade of cowkeeping and dairying The main object was to insure sanitation and cleanliness, and for prescribing precautions to be taken for protecting milk against specific infection or contamination. ${ }^{1}$ This power conferred upon the Privy Council was subsequently transferred to the Local Government Board. In London the substance of these enactments was placed in the hands of the Metropolitan Board of Works (afterwards the London County Council) and the Corporation of the City of London. $^{2}$

Under the Contagious Diseases (Animals) Act of 1878 , the Privy Council therefore drew up an Order for these purposes. It was called the Dairies, Cowsheds, and Milk-shops Order, I $885,{ }^{3}$ and it is the only legislative measure wholly dealing with the protection of the milk supply. It revoked the first Order of 1879 , and applies to England, Wales, and Scotland. The chief matters dealt with are - $(a)$ the registration of all milk dealers; $(b)$ the construction and water supply of new dairies and cowsheds, with special reference to lighting, ventilation, etc. ; $(c)$ the health and good condition of the cattle therein, the cleanliness of milk utensils, and the protection of milk against infection derived from persons suffering from infectious diseases; $(d)$ the position of water-closets or privies in relation to the dairy or milk-shop; $(e)$ regulations for a variety of sanitary matters to be made and enforced by the local authority; and $(f)$ disease among cattle. Some question was raised after this Order came into force in 1886 , and after the power had been transferred from the Privy Council to the Local Government Board, and to the Local Authori-

1 See Appendix B, p. 550 ; Contagious Diseases (Animals) Act, 1878, Sec. 34.

2 See Appendix C, p. 551 ; Public Health (London) Act, 1891, Sec. 28.

3 See Appendix D, p. 555. 
ties under the Public Health Act, I875, as to whether or not there was any power to recover penalties for a breach of the Order. In consequence another Order was made by the Board in 1886, with the object of making it clear that such penalties could be recovered. ${ }^{1}$ In 1899 a further amendment became law $^{2}$ as the outcome of the findings of the Second Royal Commission on Tuberculosis. ${ }^{3}$ This Order of 1899 altered Article 15 of the 1885 Order so that, in addition to the diseases scheduled under the Contagious Diseases Acts, it should include, in the case of a cow, such disease of the udder as shall be certified by a veterinary surgeon to be tubercular. For the first time this brought tuberculosis under the control of the Order.

Further, just as we have the Dairies and Cowsheds Orders made under Section 34 of the Contagious Diseases (Animals) Acts, so we have regulations ${ }^{4}$ for the control of cattle and dairies made under Article 13 of the Dairies and Cowsheds Order, I885. These Regulations are adoptive by each local authority. They deal with the inspection of dairy cattle, and with the lighting, ventilation, and general sanitary requirements of Cowsheds (Parts i. and ii.), and of Dairies (Part iii.). They also secure the cleanliness of milk stores, milk-shops, and milk vessels, and prescribe precautions to be taken by purveyors of milk against infection or contamination. The penalty for offending any of the Regulations is $£ 5$, and in the case of a continuing offence, a further penalty of $£ 2$ for each day after written notice of the offence from the local authority.

As we have said, Regulations under the Dairies Order differ according to local circumstances, requirements, and standard. For the guidance of District Councils the Local Government Board caused some Model Regulations to be prepared. The Board's confirmation of such regulations adopted by District Councils is not required, but if at any time the Board are satisfied on inquiry with respect to any regulation that the same is of too

1 The Dairies, Cowsheds, and Milk-shops Amending Order, 1886. (See Appendix E, p. 559.)

2 The Dairies, Cowsheds, and Milk-shops Order, 1899. (See Appendix F, p. 561.)

${ }^{3}$ Report of the Royal Commission appointed to inquire into the administrative procedures for controlling danger to man through the use as food of the meat and milk of tuberculous animals, 1898 , part i., p. 22.

4 See Appendix G, p. 562 for Model Regulations (issued 1899). It will be understood that such regulations differ in minor particulars according to local requirements. 
restrictive a character or otherwise objectionable, they may direct its revocation. On account of this power possessed by the Local Government Board it has become the practice for local authorities to send up drafts of their prepared regulations to the Board before finally adopting them. Regulations under Article 13 are not very widely adopted, and where adopted are often not enforced. This is much to be regretted, as a study of them (see Appendix), will at once show their far-reaching value.

Now whilst the Dairies Order is the chief legislation dealing with the milk supply, there are others, and brief reference must now be made to them. In the first place, in addition to the Public Health Act, $1875,{ }^{1}$ there is the Infectious Diseases Prevention Act, I 890, Section 4 of which gives the medical officer powers of inspection of dairies, within or without his own district, and of animals therein, if there is evidence that any person is suffering from disease attributable to the milk therefrom. But there are three limiting conditions-namely, (a) that the medical officer must obtain an order from a justice having jurisdiction in the place where the premises are situated ; $(b)$ that he must be accompanied by a veterinary surgeon; and, (c) that he must report to his Authority, before any action whatever can be taken, and even then the action only results in putting the defendant upon his defence. ${ }^{2}$

In the second place, there is the Public Health (London) Act, I891, ${ }^{3}$ which authorises registration with the County Council (now transferred to the Metropolitan Borough Councils ${ }^{4}$ ) of all persons carrying on the trade of dairyman, and practically re-enacts the registration clauses of the Dairies Order for London. Under the London Act powers are also conferred on the Metropolitan Sanitary authorities for the inspection, examination, and seizure of " any article of food, whether solid or liquid, intended for the food of man, and sold or exposed for sale or deposited in any place for the purpose of sale or of preparation for sale." This of course includes milk. ${ }^{5}$ There is also a clause in this Act (Section 7 1 ) substantially the same as Section 4 of the Infectious Diseases (Prevention) Act; but the procedure contemplated involves considerable delay in the event of a sudden outbreak of milk-borne disease. Moreover, when milk

1 See Appendix I, p. 568 ; Public Health Act, 1875, Secs. 116, 117.

2 See Appendix H, p. 567 ; Infectious Diseases (Prevention) Act, 1890 , Sec. 4.

${ }^{3}$ See Appendix C, p. 551 ; Public Health (London) Act, 1891, Secs. 28, 47, 7 I.

${ }^{4}$ London Government Act, 1899 , Sec. 5.

${ }^{5}$ The same powers are conferred on districts outside London by the Public Health Act, 1875 (Secs. II6, I17, I18, I19, 167). See also Public Health Acts Amendment Act, 1890, Sec. 28. 
from a particular source has been found to be producing disease in one district, or even when it transpires that cases traceable to the same supply of milk are occurring in other districts, it would be necessary for the medical officer of each of these other districts separately to inspect the particular dairy from which the milk was supplied and comply with the other requirements of the section before the implicated milk could be excluded from his district. It seems obvious that when disease has been traced to a particular nilk supply the sale of such milk should be altogether stopped, and not merely that it should be excluded from the district in which the outbreak has occurred. The County Council has no power to exclude from London even a particular milk known to be the cause of disease. Moreover, when a sanitary authority in London excludes from its district the milk from a particular farm, notice is required to be given to the council of the county in which the farm is situated, but we know of no power of such county council to stop the sale of the milk. The section as it stands, is, in fact, practically worthless.

In the third place, there are various local acts which contain clauses dealing in particular with the milk supply.

The Sanitary Committee of the City of Manchester, inspired by the Report of the Royal Commission on Tuberculosis, determined, in September I898, to apply for similar powers to those enjoyed by Glasgow under its Police (Amendment) Act. As originally drafted, the Manchester Milk Clauses sought unlimited power of inspection of the cows on all farms supplying milk in Manchester, and provided for the exclusion, temporary or permanent, of milk from cows with udders affected with any disease, as well as of the milk from cows affected with advanced tuberculosis.

Some eight other corporations took the same course, but the opposition at first was concentrated on Manchester, and the clauses were materially curtailed before they reached Parliament. It was found impossible from the beginning to insert a clause providing for the slaughter of cows suffering from tuberculosis of the udder, or from advanced tuberculosis, though the necessity for this clause was clearly recognised. The corporations applying for powers were advised by the Local Government Board that they would be much more likely to get powers from Parliament if they could agree upon a common set of clauses.

The common clauses were considered by the Local Government Board, the Board of Agriculture, the Associated Chambers of Agriculture, and the Corporation concerned, and in their final form 
presented both additions to, and diminutions of, the powers asked for by the various corporations.

In general, it may be said that the clauses enacted in effect:-

I. That a cow known to be suffering from tuberculosis of the udder must be isolated (and by isolation was meant isolation on the same farm), and that the milk from such cow must not be used for human food.

2. Powers were given to inspect the cows, and to take samples of milk from particular teats, also to take samples of mixed milk produced, or sold, or intended for sale, within the city. These powers, however, can only be exercised outside the city on the production of an order of a justice having jurisdiction in the place in which the farm is situated.

3. If the Medical Officer of Health is of opinion that tuberculosis is caused, or is likely to be caused, to persons residing in his district from consumption of the milk supplied from any dairy, the dairyman may be summoned to appear before the Corporation to show cause why an order should not be made prohibiting him from supplying milk within the city.

4. A dairyman supplying milk within the city who has in his dairy any cow affected with, or suspected of, or exhibiting signs of, tuberculosis of the udder, shall forthwith give written notice of the fact to the Medical Officer of Health of the district which he supplies, stating his name and address and the situation of the premises where the cow is stabled. ${ }^{1}$

1 The following is a precis:-

r. Interpretation.

2. Penalty up to Ten Pounds on person selling milk from cow suffering from tuberculosis of the udder.

3. Penalty up to Five Pounds on dairyman continuing to keep diseased cow along with other dairy cattle.

4. Obligation on dairyman supplying milk within the City to notify to the City Medical Officer of Health any case of tuberculosis of the udder. Penalty up to $£ 20$.

5. (I) City Medical Officer of Health empowered to collect samples of milk for testing.

(2) Similar power of collecting samples outside City if provided with a Justice's Order.

6. (I) City Medical Officer of Health authorised, if accompanied by a Veterinary Surgeon, to enter dairies in City, inspect cows, and take samples direct for testing.

(2) Medical Officer of Health and Veterinary Surgeon, may report to Corporation, who may summon dairyman to appear before them to show cause why his milk supply should not be suspended. 
Dr Niven reports that the milk clauses in the Manchester Corpcration (General Powers) Act, I899, have been very serviceable, and by means of them a large number of tuberculous cows have been removed from Manchester cowsheds. ${ }^{1}$ The Corporation of Manchester has also carried out an admirable system of control of the milk supplied to the Corporation Fever Hospital. Such milk must contain 3.25 per cent. of fat, and 8.5 per cent. of solids not fat; it must be derived from cows free from tuberculosis, as tested by tuberculin; the cows must be properly housed and maintained in such a condition as required by the Medical Officer of Health of the City; and the milk is to be furnished cool and fresh. Frequent examinations of the milk and inspections of the cows and cowsheds are also made.

Other Local Authorities, for example that of Sunderland, have adopted a semi-official control of the milk supply by laying down certain conditions upon which they would encourage the sale of milk. Certificates are issued, stating that such and such milk is produced under special regulations drawn up by the Health Committee of the Corporation. Testing by tuberculin is one of

(3) Same powers as 6(1) and 6(2) in respect of dairies outside the City, provided a Justice's Order for inspection be obtained.

(4) Dairyman must assist Medical Officer of Health in his inspection, etc.

(5) Corporation may serve order on dairyman prohibiting him from supplying milk within the borough. If dairy is outside City, notice of this order is to be given to the Local Authority, County Council, and Local Government Board.

(6) Order to be withdrawn by Corporation when satisfied that the milk is no longer dangerous.

(7) Penalty up to $£ 5$ for contravening Order of Corporation.

(8) Dairyman not liable for breach of contract due to an order of Corporation.

7. As to public notice of provisions.

8. As to procedure for recovery of the penalties by the Corporation.

9. As to expenses incurred by the Corporation.

10. As to execution of the Act by a Committee of the Corporation.

Similar clauses to these were introduced into Local Acts between I899-I 90 I for the Cities and Towns of Manchester, Liverpool, Salford, Stockport, Sheffield, Halifax, Oldham, Bradford, Leeds, Southport, Scarborough, Blackpool, Derby, Harrogate, Ripon, Shipley, etc. Some of these places have, in addition to the "model" milk clauses, special clauses as to appeal (e.g. Liverpool, Bradford, Sheffield). As an example, the Liverpool milk clauses are printed in extenso in Appendix J, p. 569. In the Appendix will also be found a copy of a circular distributed among farmers sending milk into Manchester-Appendix K, p. 572.

1 See Reports on Health of City of Manchester, 1901 and 1902. 
the conditions, and others include notification of various infectious diseases, and a guarantee not to sell milk derived from diseased udders. The certificate has to be renewed every year; and is cancelled immediately any breach of the regulations takes place. At Sunderland, Dr Scurfield informs the writer that five farmers doing a large business are so certified, and have found the regulations to be most helpful to their business and beneficial to their herds. ${ }^{1}$

Nor must we fail to mention the excellent work which has been done by the Corporations of St Helens, Liverpool, Bradford, Battersea, and other places in the direction of supplying sterilised milk for infants and children. The idea seems to have originated in Dr Leon Dufour's establishment at Fécamp in Normandy, and has now been adopted in various places. In principle it consists in supplying "humanised" sterilised milk in stoppered bottles, each bottle containing sufficient food for one meal. Probably in England it is most largely carried out at Liverpool, where they have four branches with sterilising apparatus at two of them. The process is as follows :-When the milk is received, it is tested in a ListerGerber Tester, passed through a fine sieve, and the special modification made. It is then bottled by means of a special apparatus ${ }^{2}$ and the corks lightly fitted. The bottles are placed on trays, and the trays placed in the steriliser, ${ }^{3}$ which is then closed and the steam admitted. When the temperature reaches $200^{\circ} \mathrm{F}$, no further heat is applied, and this temperature is maintained for half an hour. The steriliser is then opened and the trays taken out. The stoppers are then fastened securely, and the bottles are placed in the wire baskets, seven or nine according to the table. Each basket is then labelled, and the milk is placed on sale. In addition to the Corporation Depôts, arrangements have been made with over 30 dairies, situated all over the town, to keep a stock of the milk, so that customers may obtain a supply with as little inconvenience as possible. The writer desires to make it clear that, however useful such sterilised milk is, the great requirement at the present time is a naturally pure milk supply. The promoters of these municipal supplies doubtless fully recognise that. (See footnote, p. 503.)

In passing, reference may be made to the ice cream regulations now in force in London, ${ }^{4}$ which require that ice cream must be made and stored in sanitary premises, and must not be made or

1 See Appendix L, p. 576, for Regulations in operation at Sunderland.

2 Made by the Palatine Engineering Co., Blackstock Street, Liverpool.

3 Made by R. A. Lister \& Co., Dursley.

4 London County Council (General Powers) Act, 1902, part viii., Secs. 42-45. 
stored in living-rooms; strict precautions must be taken as to protection from contamination; cases of infectious disease must be reported; and the name and address of the maker must appear on the street barrows. These regulations are new for London, but similar ones have been in existence in Glasgow since 1895, and in Liverpool since 1898 .

Finally, it should be noted that the adulteration of milk is dealt with in the Food and Drugs Acts. ${ }^{1}$ These Acts do not lay down restrictions respecting the food supply and its management, but are chiefly concerned with sampling, the detection of adulteration, and prosecution.

The Local Authority and the milk supply.-From what has been said above, it will be evident that the Local Authority is the chief body for the administration of milk legislation. It falls to its duty to enforce this legislation and thus exercise some control over the milk trade, and prevent, as far as practicable, outbreaks of disease due to an infected milk supply. But a little consideration will make evident a second and scarcely less important duty of the Local Authority. By means of legislation, and the powers of registration and inspection which it provides, the I.ocal Authority should obtain an accurate and intimate knowledge of the condition of the milk trade in the district under its supervision. The investigation of milk-borne infection can only be rapidly and thoroughly carried out, if the Local Authority is in possession of the facts in relation to (a) the sources of the milk supply in its district, and (b) the conditions under which the milk is stored and sold in its district. No State control of the milk supply can be efficient apart from this knowledge. The matter will therefore be briefly referred to in this place, and for convenience, the Metropolitan Borough of Finsbury (population 100,000), being a fairly representative district in the centre of London, will be taken as an example.2

\section{(a) The Sources of the Milk Supply in Finsbury.}

In a general way, it may be said, that vendors have three modes of obtaining milk. First, some 185 milk sellers in Finsbury obtain their supply through milk contractors, who deal with more than a thousand country farms. There are I4 such wholesale contractors trading in the district. Secondly, there are some 50 milk sellers

1 The Sale of Food and Drugs Act, 1875-1899.

2 See Report on the Milk Supply of Finsbury, 1903. 
who obtain their milk, through other milk sellers in the district, from country farms. Thirdly, there are, perhaps, a score of milk-shops which obtain part or all their milk from town cowsheds situated within the district. Speaking generally, therefore, it may be said that about 235 of the $26 \mathrm{I}$ milk sellers in Finsbury obtain their milk wholly or partly from country cowsheds, whereas about a score obtain their milk wholly or partly from town cowsheds.

The fourteen contractors deal with some II00 farms situated at varying distances from London. The following table and map sets out the main facts with regard to the distribution of the towns and villages where the farms are situated which send in milk:-

\begin{tabular}{|c|c|c|c|}
\hline Contractor. & $\begin{array}{l}\text { No. of } \\
\text { Farms. }\end{array}$ & Counties in which Farms are chiefly situated. & $\begin{array}{l}\text { No. of Milk } \\
\text { Sellers } \\
\text { supplied in } \\
\text { Finsbury. }\end{array}$ \\
\hline $\begin{array}{l}\text { C } \\
\text { D } \\
\text { E } \\
\\
\text { F } \\
\text { G } \\
\text { H } \\
I \\
J \\
\text { K } \\
\text { L } \\
M \\
N\end{array}$ & $\begin{array}{r}3 \\
400 \\
100 \\
30 \\
30 \\
3 \\
58 \\
70 \\
70 \\
1 \\
60 \\
60 \\
200\end{array}$ & 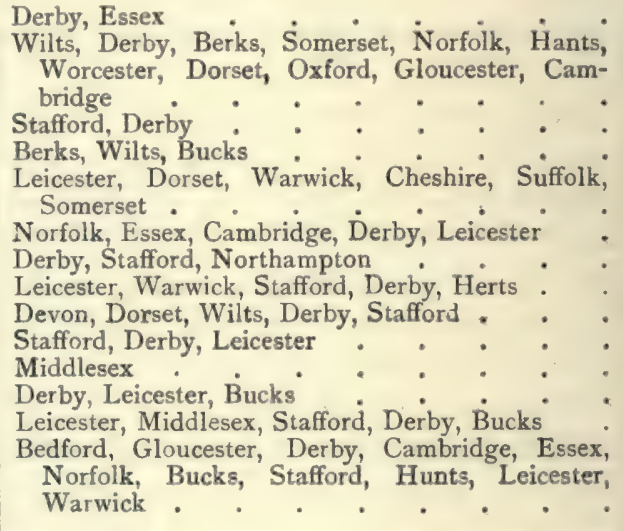 & $\begin{array}{r}6 \\
4 \\
18 \\
21 \\
31 \\
7 \\
16 \\
9 \\
24 \\
4 \\
2 \\
2 \\
\\
32\end{array}$ \\
\hline Totals & IIII & & 185 \\
\hline
\end{tabular}

In addition to the farms dealing with contractors, some 70 farms, situated in like manner in different parts of the country, deal direct with 2 I of the larger milk sellers, and in this way about 60 shops are supplied. Hence, about $\mathrm{I} 200$ farms send milk more or less regularly into this one district of London.

The country sources, as will be seen from the attached map, show a characteristic distribution. Much of the milk is derived from the great milk-producing counties of Derbyshire, Staffordshire, Leicestershire, Warwickshire, and Wiltshire, and very little appears to come from the home counties. To furnish some idea of distance, straight measurements have been marked on the map 


\section{CHART SHOWING SOURCES OF FINSBURY MILK-SUPPLY.}

This Spot Map indicates the geographical situation of 260 towns and villages where the farms are located, from which milk sold in Finsbury is derived. The measurements of distance from London are from point to point. Actually these distances would of course be slightly more than as stated on the Map.

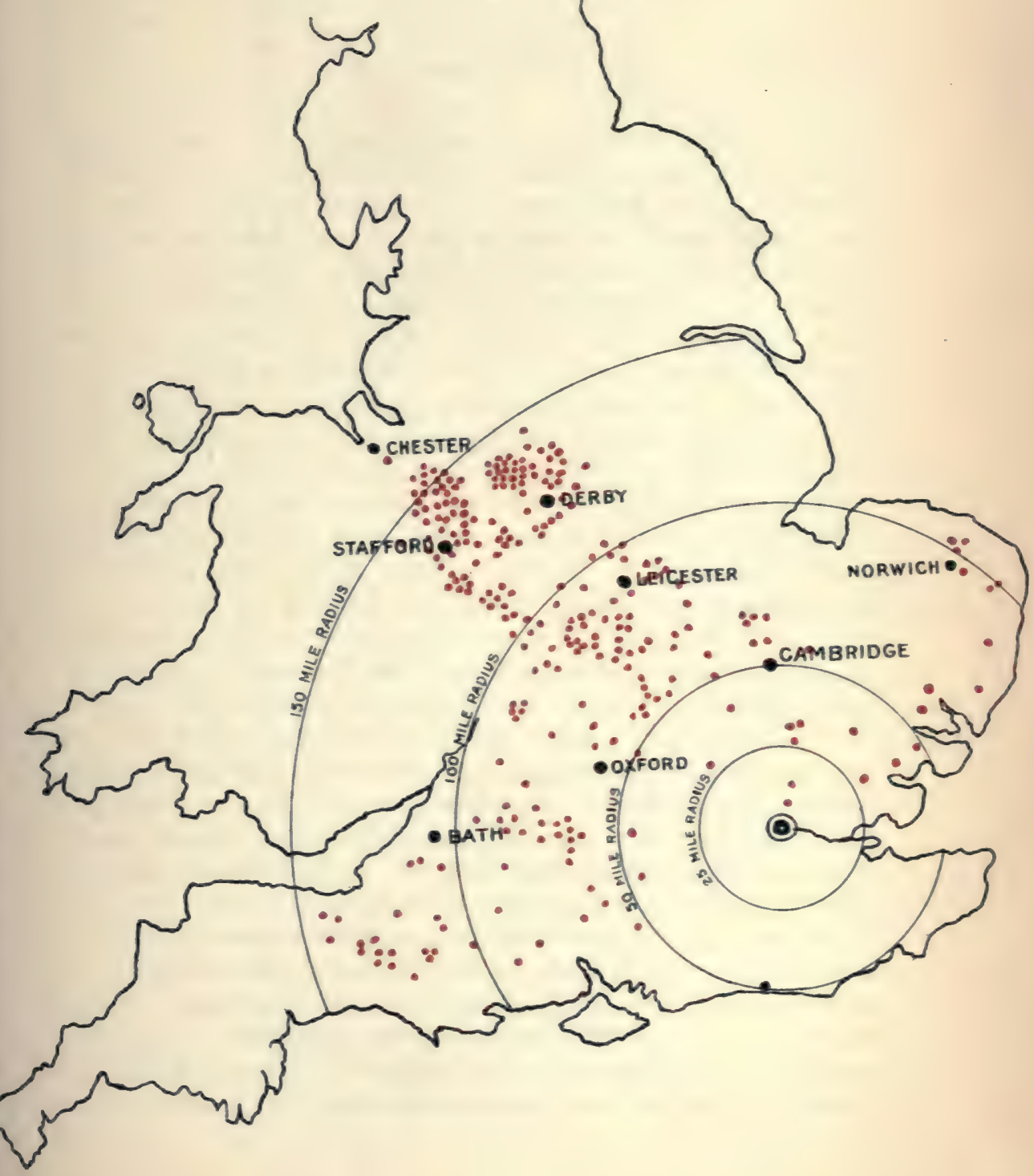



from London for $25,50,100$, and 150 miles. Of course, actually the distances by rail from London are much greater. But even this rough and ready method gives an approximate idea of the great distances which London milk travels before it reaches the milk-shop. On the map 280 towns and villages are marked. The distances of these places, in straight measurement, are as follows :-

\begin{tabular}{|c|c|c|c|c|c|}
\hline " & 50 & " & " & $13(4.6$ & $"$ \\
\hline " & 100 & ", & ” & I 1 I $(39.5$ & " \\
\hline$"$ & I 50 & , & " & $145(51 \cdot 9$ & ", \\
\hline , & 200 & , & ” & IO $(3.5$ & ", \\
\hline
\end{tabular}

It is evident that 95 per cent. of all the milk which comes into Finsbury from the country must of necessity spend several hours on the railway. From one cause or another this period of transit from farm to milk-shop averages ten to twelve hours. An example will illustrate what actually happens. A certain contractor in Finsbury obtains his milk from a number of farms in the Derbyshire and Staffordshire district. He possesses a more or less correct list of the farms with which he deals, and a correct list of the milk agents through whom he does his business. $\mathrm{He}$ receives between 1000 and 1500 gallons of milk daily, and it is delivered in ordinary milk churns at Euston or St Pancras Stations early in the morning. At R., in Staffordshire, he has an agent who obtains milk from some half-dozen farms within three or four miles of $\mathrm{R}$. railway station. Milking takes place between 4 and 6 P.M., and the milk is strained, and some of it cooled, and placed in churns and sent to $R$. railway station. The milk undergoes various vicissitudes on the railway (whose company does not provide refrigerator cars for its ordinary milk traffic), and eventually it arrives in London about three or four o'clock in the morning. The milk contractor (with his vans) meets the milk and distributes it, selecting so many churns for this van, and so many for that, and by six in the morning the milk is at the milk-shops in various parts of London. Exactly which milk reaches each milkshop is not known to the contractor or anybody else. What is known is that it is at least twelve hours old, and some of it eighteen hours old. A certain quantity, sometimes the whole supply, is taken from the railway stations to the depôt of the contractor, from which it is distributed to various districts. But in any case it has not yet reached its destination. For much of it is retailed (sometimes twice over) in small quantities to small shops which, whilst selling lamp 
oil, soap, pickles, candles, bacon, boot blacking, and toffee, also deal in a few quarts of milk. And so from hand to hand the milk passes, and under some circumstances does not reach the consumer until twenty-four hours after being drawn from the cow.

Lack of control by Local Authority over milk sources. - In a general way it may be said that country cowsheds and country cows are maintained at a lower standard of sanitation than town cows or cowsheds. The Dairy Order is administered in many rural districts with considerable laxity, and the personal interests of the members of the Local Authority lead to a certain degree of supineness. It is the exception rather than the rule for regulations under the Order to be adopted, or, if adopted, to be enforced. In towns, on the other hand, regulations are usually enforced with more or less strictness. But the difficulty is that towns at a distance from their milk-gathering ground have no direct control over the contributory farms. An illustration will make this evident.

(I) As to cozes.-In I90I, I 839 cows were inspected in Manchester cowsheds, and one only was found suffering from tuberculosis of the udder; in 1902, no cases of tuberculous udder were found in the city cowsheds.

But a very different result was obtained when country cows, the milk of which is sent into Manchester, were examined. The facts are set out in the following table:-

\begin{tabular}{|c|c|c|c|c|c|c|c|c|}
\hline & & & \multicolumn{2}{|c|}{$\begin{array}{c}\text { Farmers } \\
\text { sending Milk } \\
\text { to Manchester. }\end{array}$} & \multicolumn{2}{|c|}{$\begin{array}{l}\text { Farmers } \\
\text { sending,Tuber- } \\
\text { eulous Millk. }\end{array}$} & \multicolumn{2}{|c|}{$\begin{array}{l}\text { Percentage of } \\
\text { Tuberculous } \\
\text { Milk. }\end{array}$} \\
\hline & & & 1901. & 1902. & 1901. & 1902. & 1901. & 1902. \\
\hline $\begin{array}{l}\text { Cheshire } \\
\text { Derbyshire } \\
\text { Staffordshire }\end{array}$ & : & $: \quad:$ & $\begin{array}{r}\text { I } 72 \\
65 \\
25\end{array}$ & $\begin{array}{r}196 \\
104 \\
25\end{array}$ & $\begin{array}{r}18 \\
6 \\
2\end{array}$ & $\begin{array}{r}25 \\
9 \\
1\end{array}$ & $\begin{array}{r}10 \cdot 4 \\
9 \cdot 2 \\
8 \cdot 0\end{array}$ & $\begin{array}{r}12 \cdot 7 \\
8 \cdot 6 \\
4 \cdot 0\end{array}$ \\
\hline Totals & - & . $\quad$. & 262 & 325 & 26 & 35 & $9 \cdot 2$ & $8 \cdot 4$ \\
\hline
\end{tabular}

In this table only the three counties have been taken from which milk also comes into Finsbury. It is evident that a certain percentage of milk from these districts has been found to be tuberculous. But this is not all. Fortunately, the Manchester Corporation followed the matter up and endeavoured to remove the tuberculous cows. In 1902, they found that there were 3 I cows in these country farms suffering from tuberculosis of the udder. 
Sixteen of these were slaughtered on the instructions of the Corporation, but the other I 5 were disposed of on the open market. ${ }^{1}$ In one instance, when a search was being made to find the animal yielding the tuberculous milk, 75 farms were visited containing $18 \mathrm{I}$ cowsheds. The veterinary surgeon reports :-

"The great majority of the cows and cowsheds were in a very dirty condition, and a considerable number of cows examined, while not suffering from tuberculosis of the udder, were certainly otherwise tuberculous. The farms, in fact, with few exceptions, were totally unfit for dairy farms. Only a comparatively small proportion of the milk was sent to Manchester, the remainder, a very large quantity, being sent to London. . . . The dairyman stopped sending to Manchester for some time." "

In another case the farmer replied to the Manchester Corporation instruction to slaughter his tuberculous cow, "asking to be allowed to keep the cow, as he had discontinued sending milk to Manchester, and was sending to London. The Medical Officer of Health replied that the cow should be slaughtered." To this the Secretary of the local Milk Producers' Association replied, asking that the farmer should be allowed to sell the cow in the open market, as times were hard, etc. The Medical Officer again replied that the cow should be slaughtered. After much delay the cow was slaughtered, and the carcase was found to be "very extensively diseased and quite unfit for food." ${ }^{3}$ It had therefore been unfit for milking for a long period.

Other examples might be given revealing the ways in which tuberculous cows are passed from one farmer to another, or from one district to another, their milk, when its sale is discontinued in one district, being sent to another. It is surely a disgraceful traffic, and fortunately some of these dealers have been brought to justice. In London no control whatever is exercised over any of the farms or cows sending milk from the country.

(2) As to cowsheds. - The veterinary surgeon for Manchester reports as follows:-

In Cheshire: "Greater attempts are made at cleanliness, but

1 Report on Health of City of Manchester, IgO2, p. I4I. Lack of compulsory seizure and slaughter of dairy cows proved to be tuberculous, would appearj to be a grave flaw in our dairy legislation.
2 Ibid., p. 148.
I Ibid., p. 149.

4 The Public Health (London) Act, I89I, Sec. 7I, is a cumbersome arrangement for stopping a milk supply after it has been proved to cause disease. See Appendix C, p. 553. 
the cowsheds are, for the most part, dark, ill-ventilated, and either badly drained, or not drained at all." 1

In Derbyshire and Staffordshire: "The conditions show no improvement. The cowsheds are, on the whole, decidedly worse than those in Cheshire. In many cases the cubic space per cow is only about 200 cubic feet."

The above facts illustrate the need there is for placing in the hands of Local Authorities greater powers of control in respect to cows and cowsheds outside their boundary. They afford justification for the Milk Clauses of Manchester, Liverpool, and other places in self-defence, and they reflect little credit on country sanitary authorities.

\section{(b) Conditions under which Milk is sold.}

The milk trade is now a very complex one, largely owing, as we have pointed out, to the growth of the towns. Instead of a man owning his own cows or obtaining his necessary milk from neighbours in small quantities as required, it is now necessary to transport milk long distances in large quantities. Thus there has arisen the opportunity, or necessity, for the milk contractor. $\mathrm{He}$ is the middle man between the farmer and the milk vendor. The attached chart, which represents an actual state of affairs occurring in Finsbury, makes his position clear :-

The blue squares represent the 37 towns or villages in various counties in which the contributory farms are situated. The purple block in the middle represents the contractor through whose hands the milk passes on its way to the 32 milk-shops. These are represented by the round red spots, and are of two kinds. Some shops receive their milk supply direct from the contractor (e.g. Nos. I, I 5, 16, I7, 18, and 24). These are small retailers. Other similar retailers (e.g. Nos. 2, 4, 6, 8, 9, I I, I 2, etc.) receive their supply from other milk-shops in the district which receive milk from the same contractor $(e . g$. Nos. $3,5,7,10,13,19$, etc). These last-named shops do a wholesale and retail trade in the district. So it comes about that this one contractor supplies directly Io wholesale and retail milk vendors, also directly six retail milk vendors, and indirectly $\mathrm{I} 6$ retail milk vendors Thirty-two shops, in all, therefore, in this district get their milk from this contractor. But the ro wholesale dealers carry on a retail trade inside and outside the district. For example: No. 28

${ }^{1}$ Report on Health of City of Manchester, 1902, p. 143. See also Report on Health of Liverpool, 1902, p. 156. 


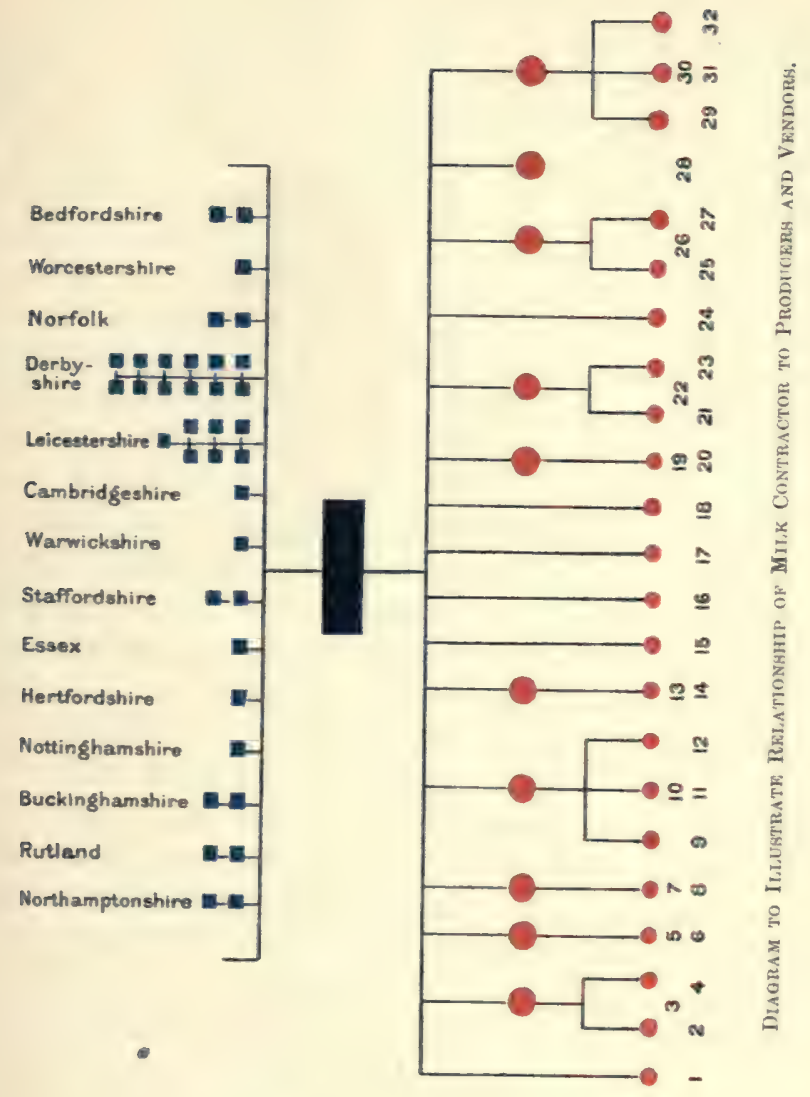



is such a milk-shop, doing no wholesale but only a retail trade in Finsbury. A brief consideration of this chart will reveal the complexity of the trade, and the extreme difficulty of tracing the exact source of the milk sold by wholesale or retail in any of these 32 shops. Nor is this a matter of merely theoretical interest. It involves the entire question of tracing infection and adulteration, and until a Local Authority possesses at least this minimum amount of information respecting the milk-shops under its supervision, it is unprepared for the emergency of a milk-borne outbreak of disease. It cannot be too clearly understood that a knowledge of the ramifications of the trade in every district is a requirement sine quâ non.

Information is also required by the Local Authority in respect to milk-shops in a district. Probably here also it will be the simplest course to pursue the matter in relation to Finsbury as an example.

There are registered in that district $26 \mathrm{I}$ milk vendors, of whom 40 are confectioners or maintain coffee-shops, leaving $22 \mathrm{I}$ milk vendors who sell milk for consumption off the premises. These 22 I milk-shops are divisible into two groups, namely (a) dairies and $(b)$ general shops selling milk. There are 39 so-called dairies, and 182 general shops. At the dairies only milk, butter, cheese, and mineral waters are sold as a rule. At the general shops every kind of provision and grocery is sold, and in many cases spices, soap, wood, paraffin oil, blacking, etc., in addition. This, therefore, is the great dividing line between these milk-shops, and, as would be expected, the dairies sell most milk (about 60 per cent. of the total), and are managed in a more satisfactory and cleanly manner than the general shops. It is, of course, evident that it is impossible to manage a general provision shop, selling all sorts of miscellaneous materials, in a cleanly way. Hence it comes about, that most of these general shops are open to criticism from the point of view of a pure milk supply.

Milk storage.-When the milk arrives from the farm or from the contractor, which it generally does, as we have seen, in the early morning, it is necessary to store it on the milk-shop premises. Such milk may be stored for two or three hours only, or for twentyfour hours or more. In 16 per cent. of the shops in Finsbury it is stored in the churns in which it arrives, or in special vessels kept for the purpose, and protected in a greater or lesser measure from pollution. In 84 per cent. the milk is at once placed in the counter pan (metal or earthenware) in the shop. As every one knows, these counter pans are exposed in the shop, and dipped 
into whenever a customer requires serving with milk. Commonly, such pans will contain from 2-4 gallons of milk, and this is the store which will last, more or less, throughout the day. As a rule, these pans and the other utensils used in milk-shops are fairly clean; but as the day goes by it is found that dirt collects in the pan and on the milk, owing to the fact that, as a rule, the pans are not covered. About 73 per cent. of the milk sellers do not take this most simple and elementary precaution to keep their milk from being polluted. It cannot be too strongly emphasised that milk becomes contaminated in milk-shops because it is not sufficiently protected. Such contamination arises from three sources:-(a) Constant dipping and manipulation; (b) Dust from the air; and $(c)$ Flies. If a pan of milk is exposed to the dusty air of a small general provision shop, for from two to twelve hours, it gains an almost incredible amount of dust and dirt. A frequent use of the dipper must inevitably convey some small quantity of dust to the milk. Dust also falls from the vendor's clothes, etc. Exposure to dusty atmosphere for a number of hours carries with the milk much more, and flies are responsible for a fourth dose of filth. Indeed, flies are responsible for a great deal of the pollution of milk. They pass from putrefying animal and vegetable matter in the street to the nearest milk-pan, and deposit in the milk the filth attaching to their bodies, mandibles, wings, and legs. When it is considered that a very large number of milk vessels and utensils are in daily use in the management of the milk trade, and that these vessels are handled by a large number of persons before the milk reaches the consumer, it will be evident that cleanliness is the great desideratum.

The Local Authority which would exercise its lawful control of the milk trade, and be prepared rapidly to investigate milk-borne disease should therefore $(a)$ obtain accurate information of the milk sources of its district, and (b) maintain a strict supervision over the storage and sale of milk in its district. Legislation for the control of the former is perhaps needed, although if every Local Authority in the country was doing its duty this would not be necessary. ${ }^{1}$ Legislation for the control of the latter practically exists in the Dairies Order, and the regulations under it.

1 Dr Barwise, the Medical Officer of Health for Derbyshire, considers that the cost of the supervision of dairy farms in the country which send milk to London and other cities should not fall on the local rates. "The cost of administrating these Orders," he writes, "should come from Imperial taxation."-Report on Health of Derbyshire, 1901, p. 19. 


\section{Legislation in Scotland}

In Scotland the most recent Public Health Act in the United Kingdom is now in force. ${ }^{1}$ It contains a clause with regard to inspection, examination, and seizure of food similar to Section 47 of the London Act, 1891. Another Section (58) is most valuable, and provides amongst other things that any person suffering from infectious disease or living in an infected house shall not milk any animal. Section 60 is constructed on the basis of Section 7I of the London Act, I89I, but with these two important differences, namely, that an order from a justice is unnecessary and that the medical officer may act, if he thinks the "milk from any such dairy is likely to cause disease and not only has caused disease." This is a great advance, and other modifications in these important clauses are of such a nature as greatly to expedite the preventive operations. Further, another clause (Section 6I) gives the local authority most useful powers in the event of an outbreak of milk-borne disease, in the direction of rapidly acquiring information from dairymen of their customers on the one hand, and their supplying farmers on the other. At the present time the milk clauses in this Act are the most advanced general legal enactments in the United Kingdom in respect to this matter, and they are, we understand, working well.

The following are the general Acts bearing on the subject :-

(I) Cattlesheds in Burghs (Scotland) Act, I 866.

(2) Contagious Diseases (Animals) Act, 1878, Section 34.

(3) Contagious Diseases (Animals) Act, r886, Section 9.

(4) Public Health (Scotland) Act, I 897.

(5) Sale of Food and Drug Acts, I 875 to I 899.

The before-mentioned sections of the Contagious Diseases (Animals) Acts were the only sections unrepealed. Following on these Acts there were issued:-

(a) The Dairies, Cowsheds, and Milk-shops Order of 1885 by the Privy Council;

(b) The Dairies, Cowsheds, and Milk-shops Amending Order of I 887 by the Board of Supervision; and

(c) The Dairies, Cowsheds, and Milkshops Order of 1899 by the Local Government Board for Scotland.

Under the Orders of 1885 and 1887 , the Privy Council and the Local Government Board respectively had merely to satisfy them-

1 See Appendix M, p. 578 ; Public Health (Scotland) Act, 1897, Secs. 60, 61. 
selves that dairy regulations were not of too restrictive a character, or otherwise objectionable, but under the Order of I 899 , dairy regulations were made subject to the same procedure as bye-laws under the Public Health (Scotland) Act, I 897, and duly confirmed by the Board. Since that date the Local Government Board have had occasion to consider dairy regulations submitted for confirmation, and the present regulations will be found to be considerably more stringent than most regulations previously in force; for example, such regulations as now exist for the Burghs of Dundee, Partick, sand Grangemouth, and for the Dunfermline District (Rural).

There are other enactments in Scotland for the protection of the milk supply, of which a typical example exists in Glasgow. This and similar legislation are of the nature of local Acts. Prior to the passing of the Private Legislation Procedure Act, I899, the Local Government Board were not consulted as to such Acts. During I900 and I9OI, however, clauses have appeared in the following local Acts :-

(I) Edinburgh Corporation Act, 1900 (Section 76 re Ice Cream: Application of Contagious Diseases (Animals) Act, etc.).

(2) Paisley Police and Public Health Act, I90I (Sections 8I-85).

(3) Greenock Corporation Order Confirmation Act, I90 I (Section 76, Regulation of Ice Cream shops).

The Glasgow Police (Amendment) Act, I890 above-mentioned contains milk provisions (Sections 24-27 ${ }^{1}$ ), and the Glasgow Corporation and Police Act, I895, Section 28, contains an ice cream provision.

The Dundee Extension and Improvement Act, I 892, Section 75, contains a provision imposing a penalty for selling milk of diseased cows. The Aberdeen Police and Water Works Amendment Act, 1867 (Sections 5 I and 52), deals with the cleansing of cowhouses and byres, and the licensing of premises in which cows are kept. By means of these Acts and the Cattlesheds in Burghs

1 This Act is one of the most typical local Acts in Scotland in regard to its milk clauses. Section 24 allows of inspection of byres and cattle; Section 25 insists on the owner rendering all necessary assistance; Section 26 demands intimation of any disease (including tuberculosis) in milch cows ; and Section 27 states the penalties for selling milk derived from diseased cows. This Act formed the starting point for the model milk clauses in various English Local Acts such as those existent in Manchester, Liverpool, etc. 
(Scotland) Act of 1866 , local authorities are enabled to prevent the sale of milk from diseased tuberculous cows, and to make stringent regulations for dairies and byres.

\section{Legislation in Ireland}

In Ireland there is not so much of what may be termed special legislation for the protection of the milk supply. The ordinary Acts (the Public Health Act, Contagious Diseases Act, Dairies, Cowsheds and Milk-shops Orders, etc.) are the only legislation available. Under the Public Health (Ireland) Act, 1896, the Irish Local Government Board is able to invest a rural sanitary authority with exceptional powers. The Board issues various orders and circulars, and in 1899 special orders were made in respect to tuberculosis.

\section{Needed Amendments in British Legislation.}

In a volume like the present criticisms of a passing or evanescent interest are perhaps out of place, and for this reason we have abstained in this chapter from entering, in detail, into anything other than matters of fact. It may be desirable, however, to remark that it appears that legislation in Great Britain in respect to milk is by no means as uniform or as consistent with modern knowledge and science as it ought to be. There seem to be a number of points demanding the attention of the legislature in respect to this article of food. But by far the most important requirement is an enforcement of the present law. For example, unless regulations under the Dairies Order are adopted, and their adoption is optional, it seems that a cow may be housed under very unsatisfactory conditions within the area of jurisdiction of the Local Authority failing to adopt regulations. The regulations are a sort of crystallisation of the principles of the Order, and in a vast number of districts the Order would be almost meaningless without regulations. Again, the I 899 Order only extends to tuberculosis in respect of mixing milk and selling it for human consumption. It still leaves the Local Authority without any power to make regulations for the inspection of tubercular cattle in dairies, and no power to prohibit the feeding of animals on tuberculous milk, although it is known that by such means tuberculosis may be spread. The Contagious Diseases (Animals) Act, 1886, Section 94, gives a Local Authority right of entry on premises, within its district, for purposes of the Dairies Order. But here again there is no application to tuberculosis, and hence the Local Authority cannot enter to examine for tubercular udders. The Act of 1886 applies, moreover, only to urban and rural 
sanitary authorities, and not to County Councils, which have no powers either in regard to dairies and cowsheds or in reference to tuberculosis. Again, the Local Government Board, the confirming authority under the Dairies Order, cannot require a Local Authority to make any particular regulation under Article I3. The Board can only disallow a bye-law which they consider to be unsatisfactory. Nor can they insist on a minimum cubic space, nor on a certain area of floor space, of a cowshed. Further, the Order only prohibits the sale of milk from a given tuberculous cow, it still leaves the animal in the possession of the cowkeeper. It is not impossible for him to remove it to another district. As a matter of fact this has been frequently done, and a cow considered in one district as tuberculous has been sold in the open market and conveyed elsewhere.

Another example is the Infectious Diseases (Prevention) Act, 1890, Section 4 of which gives the Medical Officer of Health powers of inspection of dairies in his district and of the animals therein, only if there is evidence that any person is suffering from disease attributable to the milk therefrom. Similar inspection of outside dairies may be made on a justice's order. This measure primarily protects tho consumer, and, secondarily, tends to improve the condition of cowsheds and cows. Unfortunately, however, the word "disease" is limited here to "notifiable infectious disease," which does not include tuberculosis.

Again, the elimination of tuberculosis from herds by means of tuberculin testing is a matter which has now reached a stage which calls for some legislation. The successful experience of Denmark led the Royal Commission to recommend that the Board of Agriculture be empowered to provide gratuitous testing of stock whose owners will undertake to keep them under satisfactory conditions and to isolate re-acting animals. If, in addition, the calves of tuberculous cows be fed on boiled milk, if healthy animals be re-tested once or twice a year, and if purchased animals be only such as have withstood this tuberculin test, Professor Bang believes that a markedly affected herd may be replaced by a healthy one. The writer ventures to think County Councils and County Borough Councils, who already have to provide a veterinary staff for the purposes of the Contagious Diseases (Animals) Act, might, with advantage in suitable cases, have the power of providing gratuitous tuberculin tests delegated to them by the Board of Agriculture.

Bacteriological standards of quality.-Many authorities consider that the time has come to judge the quality of milk by bacteriological standards. If a bacteriologist can show the milk to be highly contaminated with bacteria and capable, on inoculation, of killing experimental animals, it is held that such milk should be condemned. We are not satisfied that our knowledge of the bacteriology of milk is sufficiently established to justify penalties. It is to be hoped, however, that it may soon be so. In which case, fresh legislation will be required, as it would appear that such contamination could not be dealt with under the Public Health Act or the Sale of Food and Drugs Acts. Doubtless if legislation could be made for this purpose, and a bacteriological standard adopted, farmers, railway companies, and milk vendors would speedily see the necessity of reform in their management of the trade.

Powers of compensation are needed. When local authorities prevail on a milk vendor to cease the sale of suspected milk, compensation should be allowed if it is subsequently proved that the milk was not infective.

Information as to sources of milk and its destination.-Legislation is required in England upon this point. Scotland possesses powers to obtain such infor- 
mation, Public Health (Scotland) Act, 1897, Sec. 6r. See Appendix M, p. 578. Vendors selling to each other should also be required to keep records.

Protection of inspecting officers. - In any legislation for dealing with tuberculosis, it is most essential that a clause should be inserted similar to Section 265, Public Health Act, 1875, for the protection of all medical, veterinary, or other sanitary officers, from personal liability in respect of anything done bonâ fide for the purposes of carrying such legislation into effect.

\section{Legislation on the Continent of Europe}

In Paris cowhouses are classified, and authorisation is required for their establishment. There is special inspection, and the dairies are subject to regulations similar to those obtaining in England.

In Germany, Belgium, Denmark, Norway, and other European countries the milk supply is under more or less control. In Bavaria, for example, the sale of milk is prohibited under the following circumstances, namely, when it is obtained from cows suffering from disease (including tuberculosis); when the milk itself is tainted (blue milk, slimy milk, etc.); and when it is adulterated. Milk utensils and dairies have to be kept clean. ${ }^{1}$ Throughout Germany similar regulations are in force, and regular inspection takes place. Much the same legislation obtains in Belgium, with the addition that milk from cows which have been drugged or fed on poisonous plants may not be sold. It is necessary also for the milkman to print his name and address on all vehicles and vessels used for the conveyance of milk. ${ }^{2}$ In Denmark different towns and localities have their local bye-laws for protecting the milk supply, and a general law ${ }^{3}$ requires that no milk from cattle affected with tubercle of the udder may be sold or used in butter- or cheese-making, or as food for other animals, unless boiled. In Norway there is legislation against the sale of milk from tuberculous cows. In Stockholm, since I886, the milk trade has been organised. Milk-shops must be registered under the Bureau of Hygiene, and kept in good sanitary condition, well lighted, well ventilated, and clean. Immediately any infectious

1 Police Regulations, 1887. Imperial Law on Food Stuffs, 1879, etc.

2 Royal Decree, 1894.

${ }^{3}$ Law on the Contagious Diseases of Domestic Animals, I894, Sec. I I. 
disease breaks out notification must be sent to the Bureau, and the matter is at once inquired into.

\section{Milk legislation in the United States of America}

The development of the control of the dairy trade in the United States is instructive. Dairying was practised in America in colonial times, and butter and cheese are mentioned among the early exports from the settlement along the Atlantic coast, but this production was only a feature of general and pioneer farming. Dairying as a speciality did not appear in the United States to any extent until well on in the nineteenth century. During the early part of that century the care of milk and the making of butter and cheese were domestic matters, and wholly unorganised and seasonal. Winter dairying was practically unknown. These conditions continued without material change up to the middle of the century, and there was no order, no system, and no science in dairy operations. But the twenty-five years which followed I850 was a period of remarkable activity and progress in the dairy interests of the country. Agricultural exhibitions, the introduction of breeds noted for dairy qualities, co-operation, and the increasing demand were factors in the development. Williams, in $185 \mathrm{I}$, began the co-operative methods from which sprang the cheese factory system of the United States, and by I 869 such factories exceeded a thousand in number throughout the whole country. The effect of the establishment of cheese and butter factories was to transfer the making of these products from the farm to the factory, and this constitutes one of the landmarks in the progress of dairying. ${ }^{1}$ The same period witnessed the organisation of dairymen in voluntary associations for mutual benefit and the development of the trade. The American Dairymen's Association was established in I863, the North-Western Dairymen's Association in 1867, and the first of the State Dairy Associations in Vermont in 1870. Alongside of these various forms of co-operation there was the introduction of dairy cattle, and the efforts at herd improvement. The Shorthorn breed led in the

1 For details of the management of such factories see the Sixteenth Anmual Report of the Bureau of Animal Industry, 1899, Washington, pp. 247-254. 
introduction of improved cattle to the United States, and for a long time the representatives of this race imported from England included excellent dairy animals, and diffused new blood into American cattle. Consequent upon these changes the entire dairy trade rapidly rose to occupy an important place in the States, a position which was still further enhanced by two inventions, namely, the mechanical separation of cream from milk by the centrifuge, and the Babcock fat tester for milk.

These changes all tended in the direction of activity and development in the trade itself. They acted rather by way of incentive and stimulation than by way of control, and it soon became evident that the new enterprise must be legislated for. Such control naturally arose from the trade on the one hand, and the public, as represented by the State, on the other. Dairy schools, annual exhibitions strictly organised, weekly and monthly journals, and a knowledge of bacteriology may be said to have represented the sources of voluntary control. State laws and the regulations and supervision of the United States Department of Agriculture represent the strictly legislative control which arose out of public opinion and the competition of foreign markets. At the present time the dairy trade in America is not only one of the most enlightened and progressive in the world, but the measures of control which guide and guard its interests are far in advance of what may be found in many of the countries of Europe. In number of cows alone the growth of the trade in America has been remarkable, and it is now officially estimated that there are $17,500,000$ cows in the States. (Iowa, New York, Illinois, and Pennsylvania possess more than a million cows each.) The growth of legislation is still more instructive. Early in the sixties of the nineteenth century, States were constructing legislation controlling the trade. New Jersey was one of the first States to pass public laws protecting butter and cheese against adulteration. This was in I 864. Pennsylvania followed in 1869 with an act to authorise the councils in cities and boroughs in the Commonwealth to provide for inspection of milk. From that time to the end of the century innumerable State laws were made for the strict control of the trade, with the result that at the end of the century only three States out of 49 possessed no dairy laws. Eighteen States provide by law for officials known as Dairy Commissioners. These officers have a national association. At several large cities and centres of activity in the commerce of the dairy there are special Boards of Trade for supervisionary purposes. Nineteen States possess 
laws for the special inspection of dairies and the milk supply, nine States legislate in particular for the regular inspection of milch cows, and several of the more advanced States (for example, Pennsylvania and Wisconsin) have legislated against the addition of preservatives and antiseptics to milk or cream. The following selections representative of those three main lines of legislation may suffice to illustrate the State laws :-

"Milk inspection.-The dairy and food commissioner, or his agents, shall have full access and ingress to all premises, buildings, or dairies, where milk is stored, produced, or handled for the city milk trade, and is hereby empowered to enforce such measures as may be necessary to prevent the sale of milk from diseased cows, or from cows fed upon unwholesome food, and to require cleanliness in all barns, stables, milk-houses, or buildings where milk is produced or stored, for the city milk trade."-(Wisconsin, Laws of, I 897 , Sec. I, chap. 94.)

"Stabling and care of cozes.-When cows are kept by any person for dairy purposes, either for butter or cheese, or for the production of milk or cream for sale, and are confined in stables, such cows so confined shall each be allowed at least 800 cubic feet of air, and such cows so stabled shall not be confined facing each other when closer together than 6 feet, unless there shall be an airtight partition between such cows at least 4 feet in height; and all stables where such cows are kept, shall be well ventilated and kept in a good healthful condition; and if any suspected diseased cow or other animal belonging to, or kept in or about any dairy, the State dairy and food commissioners shall notify the State veterinarian; and if any dairy where cows are kept for the purposes above stated is found to be in a filthy and unhealthful condition, the commissioners may notify the proprietor, that said dairy must be put in a healthful condition within three days; and should said proprietor neglect or refuse to comply with such order, then the commissioner may employ other persons to perform such duty, and said proprietor shall pay all expenses of such labour."-(Oregon: General Laws, I893, p. 99, Sec. 4.)

"Preservatives and colouring matters.-That the sale or offering for sale of milk or cream for human consumption in this commonwealth to which has been added boracic acid salt, boracic acid, salicylic acid, salicylate of soda, or any other injurious compound, or substance for artificially colouring the same, shall be a misdemeanour, and punishable by a fine of not less than fifty nor more than one hundred dollars, or imprisonment not exceeding sixty days, or both, or either, at the discretion of the court."-(Pennsylvania: Adulteration Act, 1897, Sec. I.)

But American legislation having for its object the control of 
the milk supply and of dairy products is not confined to the State laws. Many of the larger municipalities, such as New York, Philadelphia, and Chicago, ${ }^{1}$ possess legislative powers of considerable magnitude. They are, in general, of a similar character in various cities, and it will be sufficient if we illustrate such municipal powers by the Sanitary Code of New York. The Board of Health of New York have powers to exercise control over milk by Sections 44, 5 I, 59, 63-66, 88, I 35 of the Sanitary Code. These sections provide against adulteration, ${ }^{2}$ fraudulent sale, and the inspection of dairies, byres, and cattle. No milk may be received, held, kept, or offered for sale, or delivered in the City of New York, without a permit in writing from the City Board of Health, and subject to the conditions laid down by the Board. The regulations in respect to tuberculosis in milch cattle in New York are also very strict, and include the tuberculin test. ${ }^{3}$ The Department of Health of New York also issue circulars of information to farmers respecting the collection and care of milk, and the relationship of bacteria to milk. Scrupulous cleanliness, rapid cooling of milk to $45^{\circ} \mathrm{F}$., and quick transport, are the three main principles upon which emphasis is laid. Information and advice is also sent to railroad officials. On the whole, the New York Health Department has probably done more in these matters than any other local authority in the world.

1 It is stated that since the Health Department of Chicago undertook the supervision of the milk supply, deaths of children under five years of age have fallen 5 per cent.

2 The term "adulterated" means in this code (Section 63)-(a) milk containing more than 88 per cent. of water or fluid; (b) milk containing less than 12 per cent. of milk solids; $(c)$ milk containing less than 3 per cent. of fat ; $(d)$ milk drawn from animals within fifteen days before or five days after parturition; (e) milk drawn from animals fed on distillery waste, or any substance in a state of fermentation, or putrefaction, or on any unhealthy food; $(f)$ milk drawn from cows kept in a crowded or unhealthy condition; $(g)$ milk from which any part of the cream has been removed; $(h)$ milk which has been adulterated with water or any other fluid, or to which has been added, or into which has been introduced any foreign substance whatever; (i) milk, the temperature of which is higher than $50^{\circ} \mathrm{F}$.

${ }^{3}$ All persons, corporations, or companies, bringing milch cows into the City of New York, shall furnish a certificate signed by a veterinarian who is a graduate of a recognised veterinary college, with the date of graduation, and the name of the college from which the degree was received, to the effect that the said cows are free from tuberculosis as far as may be determined by physical examination and the tuberculin test. Said certificate shall give a number which has been permanently attached to each cow, and a description sufficiently accurate for identification, stating the date (which must not be more 


\section{Milk legislation in the British Colonies}

The present position of the law in the British Colonies is perhaps more advanced than anywhere else in the world, though in all probability the law is not more thoroughly administered than in Great Britain. A study of colonial legislation for the protection of milk is of interest, and we may suitably pass from the United States of America to Canada. In Ontario power is given under various Acts ${ }^{1}$ for the enforcement of bye-laws for the regular inspection of byres, dairies, and milch cows, and also for periodical testing with tuberculin. Each animal found healthy is numbered, and any animal found diseased or giving a reaction to tuberculin, is at once removed and its milk disallowed for consumption. The province of Quebec obtained similar power in I89I, and by means of regulations under the Act lays down definite sanitary conditions for byres and milkshops. ${ }^{2}$ Winnipeg in Manitoba passed very stringent bye-laws in I 896 for dealing with the milk trade, bye-laws far in advance of anything in towns of the same size (population 50,000) in England. These bye-laws include-(a) registration of all milk vendors; $(b)$ veterinary inspection of all milch cows; $(c)$ systematic inspection of all premises used in the milk trade; $(d)$ a universal tuberculin test; $(e)$ strict and thorough sanitation; $(f)$ absolute veto as regards withholding license until bye-laws are strictly carried out. ${ }^{3}$ British Columbia and cities like Victoria and Vancouver have similar powers.

Nor is the matter of legislation for the protection of the milk trade any less advanced in the Australian colonies. Indeed, the

than sixty days prior to the time they are brought into the city), the place of examination, the temperature of the cow or cows, at intervals of three hours, for twelve hours before the subcutaneous injection of the tuberculin, the preparation of tuberculin used, the location of the injection, the quantity injected, the temperature at the tenth hour after the injection of the tuberculin, and every three hours after the aforesaid tenth hour for twelve hours, or till the reaction is complete. No cow with a certificate which stated that said cow gave a reaction of $2^{\circ} \mathrm{F}$. after the injection with 0.5 c.c. of the tuberculin prepared by the Department of Health of the City of New York (or its equivalent), diluted with ten times its volume of a 0.5 per cent. watery solution of carbolic acid, shall be brought into the City of New York.-Sanitary Code of New York, 1900, Sec. 135 (as amended 1899).

1 The Public Health Act, the Animals Contagious Diseases Act, and an Act for the Inspection of Meat and Milk Supplies in Cities and Towns, 1896.

2 Public Health Act, 1896, and Special Bye-laws, 1891.

3 Bye-law No. 1776 for the Licensing and Regulation of Dairies, etc., 1896.See Royal Com. Rep., 1898, Appendix, p. 419. 
vigour and thoroughness of inspection and control is said to be the chief cause in bringing about, for example, the decrease in the percentage of tuberculosis. As far back as I 883 Victoria appointed a Board of Experts to advise the Government as to the right steps for stamping out tuberculosis. In 1890 regulations were made for the inspection of dairy farms, milk stores, grazing grounds, cattle, and premises ; also for registration, cleansing, and disinfection, and for carrying out the requisite precautions for the protection of milk from infection or contamination. ${ }^{1}$ In South Australia, Victoria, and New Zealand, no person may keep or use any cow for the purpose of obtaining milk therefrom, either for use or sale, which is affected with tuberculosis. ${ }^{2}$ In Queensland the only enactment bearing on the subject is Part iii. of the Health Act Amendment Act, 1886. Registration and inspection are carried out, and at the year ending June I903, as many as 2440 dairies, 28 butter and cheese factories, and 24 creameries were registered, and 25,000 milch cows were inspected annually. A Dairy Act is badly needed. ${ }^{3}$ No preservatives whatever may be added to milk in these colonies. In Tasmania and New Zealand the law ${ }^{4}$ which has been in operation since 1894 is more advanced than any milk legislation we have in Great Britain.

A dairyman or milk vendor in New South Wales is required by the Act to report immediately any case of the following diseases which may occur on his dairy premises in any animal (that is in any animal whatever, and not in milch cows alone):-

Apthæ, cancer, pleuro-pneumonia, anthrax, tuberculosis, actinomycosis, and inflammation, eruptions, or warts on the udder.

1 Public Health Act (Victoria), 1889, Secs. 19, 22, etc.

2 Stock Diseases Act, 1893.

3 Report of Commissioner of Public Health, Queensland, 1903, p 10.

4 Bye-laws of Hobart, 1893, and the Dairy Industry Act (New Zealand), 1894. See Roy. Com. Rep. on Tuberculosis, 1898, Appendix, pp. 424-427. 


\section{CHAPTER XIV}

\section{THE CONTROL OF THE MILK SUPPLY : (2) BY PRIVATE ENTERPRISE}

The Aylesbury Dairy Company, London. The Dairy Supply Company of Copenhagen. The Danish Milk Company. The Milk Supply Pasteur, Copenhagen. The Philadelphia Milk Commission. The Rotch System.

WE propose under this heading to consider three main types of the control and protection of the milk supply by private enterprise as found in this country, in Denmark, and in America. The types we have selected are the Aylesbury Dairy Company in England, the Copenhagen Dairy Company in Denmark, and the Philadelphia Milk Commission in the United States. We are well aware that these three examples by no means exhaust the list of milk companies which have done much to raise the standard of the supply in these three countries. But it is unnecessary to burden the present volume with endless details, involving much repetition, of all the available "model" milk supplies. It may also be mentioned that we do not propose to discuss here the question of modified milk supplies for children and infants. The subject is a special one and not directly connected with the control of the milk supply. ${ }^{1}$

1 Recent information on this important subject of Infant Milk Supply will be found in the following references:-Theory and Practice of Infant Feeding, by H. D. Chapin, M.D., I902 ; Brit. Med. Jour., 1902, vol. ii., pp. 653-672, by T. M. Rotch, M.D.; Rev. d'Hyg. et Med. Infant, 1902, pp. 461-494, by H. de Rothschild, M.D.; The Strauss Milk Charity of New York City, 1896, by R. G. Freeman, M.D. ; Revue Scientifique, I902, p. II3 (Milk Dispensaries for Infants), by M. G. Variot, M.D. ; The Feeding of Infants, by E. Cautley, M.D., 1903 (second edition); Infant Feeding and the Milk supply, by T. D. Lister, M.D., 1903 ; also official reports by Dr Hope of Liverpool, Dr M'Cleary of Battersea, Dr Harris of St Helens, etc. See footnote, p. 503. On the whole question of the control of the milk supply, in addition to the supply of modified milk, see a series of articles in Brit. Med. Jour., 1903, p. 678 et seg. 


\section{The Aylesbury Dairy Company, London}

This Company supplies some I00,000 persons a day with milk Its trade is situated mostly in the West End of London but also includes many of the poorer classes. As much as $1,900,000$ gallons of milk are dealt with every year. The milk is received from about 70 farms in various parts of the provinces, the average distance of which is from London $70-80$ miles, though some of the contributory farms are 200 miles distant from London. The system carried out by this Company is, in the main, based upon a pure natural milk supply rather than an artificially purified milk supply. Thus the centre of gravity, so to speak, of the Company's work is on the farms and not in London. Reference may therefore be suitably made, in the first instance, to the farms, and then to the management of the milk in London.

The Farms.-Before taking on a new farm the Company requires the farmer to fill up a form entitled, "Proposal to supply milk." ${ }^{1}$ Although most of the particulars required are those of a commercial nature, five of them refer to the water supply, refrigeration, medical inspection, etc. Should this form be satisfactorily filled in, a second form ${ }^{2}$ is sent to the medical officer of health for the district, who is asked to make an inspection of the farm, to fill up the report, and to send samples of the water supply ${ }^{3}$ (which are examined by the Company's analyst). Many farms have to be rejected on account of faulty water supplies, though otherwise suitable. If the report is satisfactory and the water supply is good the Company enter into an agreement or contract with the farmer. ${ }^{4}$ Some of the chief conditions laid down in the contract are that all milk is to be of good quality, and nothing is to be added or subtracted. No milk is to be sent from any cow that is not in good health, or that is under physic, or that is newly calved. Attention is to be paid to the cleanliness of the udder and cleanliness in dairying generally. The milk is to be properly

1 Appendix N, p. 581 : Form A. These forms set forth so clearly the nature of the requirements insisted upon by the Company that it is unnecessary to burden the text with details.

Appendix O, p. 582 : Form B.

3 The chief items upon which the medical officer reports are the water supply, its source, gathering grounds, surroundings, possibility of contamination, sufficiency, conveyance ; sanitation of farm, cowsheds, dairy, and as to refrigeration, facilities for boiling water, etc. ; health of district and inmates of farm, particularly as regards infectious diseases; general character of farmer, etc.

4 Appendix P, p. 584: Form C. 
strained, and cooled immediately after milking over a refrigerator which shall be of a description satisfactory to the Company (commonly a Lawrence cooler is used). The churns are to be sealed. Attention may also be drawn to a clause in the contract which not only requires, under a penalty of $£ \mathrm{IOO}$, immediate notice of infectious disease occurring on the farm, but indemnifies the sender against loss from having given the information. We are informed that the Company has paid some hundreds of pounds under this clause for milk which has not been received. No milk is received again from a once-infected farm until it is certified as free from infection. ${ }^{1}$ The medical officer of health for the district also fills up a monthly report respecting the general and particular health of the inmates of the farm and district, especially as regards infectious disease, the sanitation and cleanliness of the farm, etc.

The veterinary surgeon to the Company for the district also makes an inspection monthly, or more frequently if he thinks desirable, and reports on the health of the cows, especially in regard to udder disease, cleanliness, and general byre sanitation. The district surgeons all work under the supervision of a consulting veterinary surgeon in London, who receives all their reports for his comments. There are upwards of 3000 cows yielding milk to the Company. About 20 or 30 of these are suspended annually as being unsatisfactory. Many of the herds are regularly tested with tuberculin. If the tubercle bacillus is found in any milk yielded by a cow having suspicious symptoms of tuberculosis, the cow is condemned. If the bacillus is not found the farmer may withdraw the cow or submit to the tuberculin test.

Should the medical officer of health or the veterinary surgeon report anything which would render the supply of milk from a farm a danger to health, the farmer receives telegraphic instructions not to send his milk.

Should any case of infectious disease occur amongst the customers of the Company, they are supplied by a "Special Service," of messengers, cans, etc., so as not to infect the general supply.

1 As proof of the fact that the medical and veterinary inspection of the farms, etc., and the laboratory work of the Company is of a serious and genuine character, it may be remarked that at the annual meeting of the Company, in February 1903, we notice that the accounts showed that a sum of $£ 2324$ had been expencled during I 902 on these items alone. 
For the supply of hospitals the Company slightly modify their system. Briefly, it differs from that in vogue for the ordinary milk supply, in that the various officers report direct to the hospital, and a daily analysis is also supplied.

Management in London.-The health of the employés at the chief depôt or branches is under the supervision of medical officers appointed by the Company. Notices are posted in conspicuous positions in the dairies informing employés that they must, under penalty of instant dismissal, report all cases of sickness in themselves or their families, and a form is sent to the medical officer for his report; the wages of men suspended from duty through infectious illness are paid in full.

The contract, as we have seen, requires that all milk must be cooled to as low a temperature as can be obtained by a refrigerator, dispatched by a certain train (which is the earliest available), and sent in sealed churns. On arriving at the London terminus the Company's vans await the milk and convey it to the chief or branch depôts. Here after the quantity of milk is noted and its temperature, specific gravity, and, if necessary, its acidity determined and approved, the milk is passed over a refrigerator, cooled with brine from the freezing plant (which reduces its temperature to $40^{\circ} \mathrm{F}$.) and collected in a tank, from which the churns, bottles, etc, in which it is sent out, are filled. Milk from certain selected farms is bottled and sealed for delivery as nursery milk. A certain proportion of the milk is pasteurised, and then cooled by running in succession over coolers supplied by water from the main, and a refrigerator cooled by brine. Another portion is used for the manufacture of cream, the milk is separated while hot, and the cream and skim milk cooled separately with coolers on the water and brine systems. Another portion is used for the manufacture of milk preparations, and this is cooled at the farm by a freezing plant, and sterilised on its arrival in London. The churns in which the milk arrives and the vessels in which it goes out are all washed in a soda bath, cleansed by special machinery, and finally sterilised by blowing high pressure steam into them, before being returned to the farmer, who is required to rinse them with boiling water before use.

The clerical department is so organised that the Company can, at any time, tell from reference to their books not only the quantity of milk that each customer is supplied with, but where it comes from and what its analysis is. In the laboratory, a sample of milk from each farm is daily examined, as well as a sample of every bulk of milk that leaves the premises; all milk having a specific 
gravity below IO3I or above 1034 is at once set aside for further examination. In addition samples are regularly taken from the carriers in the streets, and from the small quantities they return unsold. The Company uses neither preservatives nor colouring matters of any kind in the milk or cream that is sold, and find that there is but little public demand for bright yellow milk that will keep for two days or longer.

The contract, it may be remarked, requires the farmer to deliver his milk at the nearest railway station in the Company's churns, which must be sealed. Thus a check is placed upon fraudulent adulteration in transit. Milk churns not sealed are set on one side on arrival. Any milk returned unsold to the Company's depôt from the retailers is separated at once, and the cream churned after two or three days' ripening. The Company also prepares various kinds of "modified" and "humanised" milks for special purposes in accordance with commercial or professional demand. ${ }^{1}$

\section{The Dairy Supply Company of Copenhagen ${ }^{2}$}

Copenhagen is probably better supplied with milk than any other town in Europe, and has the largest number of model dairies. The town is served principally by two dairy companies. The oldest of these companies, the "Kjöbenhavns Mælkeforsyning" of Busck (Dairy Supply Company of Copenhagen) was the model not only for its rival the "Danske Mælke-Compagni" (Danish Dairy Company), but also for many similar enterprises formed in certain centres in other countries. These two companies, very similar in their organisation and working, differ in the fact that the first delivers fresh milk, preserved by means of ice, whilst the second sells pasteurised milk. They both have a very large custom, for, although they do not treat the milk in the same manner, they both provide their customers with a product of excellent quality.

Until I 878 milk was sold in Copenhagen by milkmen, grocers,

${ }^{1}$ It is perhaps desirable to add that this Company has merely been taken as an illustration of what is done in England. Other English companies follow similar lines.

${ }^{2}$ Since the description of this Company's operations which we had prepared was written, and which had been obtained from various sources, there has appeared the excellent report of Dr Henri de Rothschild, who was appointed by the French Government to inspect this Company's work, and report. We have, therefore, abstracted the following account from Dr Rothschild's statement.Rev. d Hyg. et Med. Infant, 1902, pp. 46 I-494. 
drink-sellers, etc., at varying prices, and under conditions of hygiene and purity which left much to be desired. It was in order to furnish the inhabitants of this town with pure milk, produced by healthy cows, "ren mealk af sunde koer," that the Company which bears the name of "Kjöbenhavns Mælkeforsyning" was formed.

The distinctive character of the "Kjöbenhavns Mælkeforsyning" lies in the fact that this Company only delivers fresh milk to the consumers, that is is to say, it is neither pasteurised nor sterilised. In order that milk used as a food or a remedy may not cause ill results from the presence in the liquid of pathogenic organisms, it should fulfil three essential conditions: (I) It should be produced by cows certified as healthy by veterinary examination and fed in a certain way; (2) it should be manipulated, from the time of milking until its sale in a rigorously aseptic manner; (3) it should be preserved during this time by some method which will render impossible the development of such bacteria as it may contain.

Source of the milk.-The Company has no farms of its own, nor does it keep cows, but only acts as intermediary between the producer and the consumer. All it sells is furnished by farms, large and small, situated within a radius of several kilometres round the town. It accepts milk from all farmers or proprietors who are willing to deliver it under certain conditions, stipulated in a contract which binds them to the Company. This contract cannot be modified to suit any individual, and every one is bound to adhere strictly to the clauses, under penalty of a fine or of cancellation of the contract. ${ }^{1}$

The conditions, set down in the regulations, may appear somewhat tyrannical, but, when it is considered that they are a sure means of the Company's obtaining milk of a superior quality absolutely guaranteed, they possess numerous advantages for the farmer who submits to them. They oblige him to renounce old-fashioned methods of feeding which are defective and unproductive, and to substitute those which experiment has proved to be better and more renumerative; they guard against his own ignorance of matters of hygiene by forcing him to watch the sanitary state of his cattle and of his staff, and thus avoid the injury and loss which the appearance of any epidemic disease on his farm would cause him. These conditions are, moreover, accepted to-day by all Danish farmers, whether affiliated to a co-operative society or not. On the strict observance of such prophylactic

1 See Appendix Q, p. 589, for Contracts and Regulations. 
regulations depends the prosperity of his business and the favour of his customers.

In order to ensure the strict application of the regulations, the Company has the farms regularly visited by veterinary surgeons paid by it, and whom it holds responsible. These, to the number of seven, have to inspect the farms of the district assigned to them every fortnight, and report to the Company on the entire management of the farms.

Production of milk.-It is under this permanent control of the general hygiene and feeding of the cows that the production of milk is carried on. As can be seen by glancing at the form to be filled in by the veterinary surgeon, the farmer can undertake to supply the Company with the milk of a certain number only of his cows, and reserve that of the others for himself or other customers. The cows certified as healthy are milked morning and evening by persons who, according to the regulations, wear a special costume and are provided with a towel so that they may wash and dry their hands whenever necessary. The milking is carried out with the greatest care and cleanliness, in daylight or with sufficient light to enable the milker to see clearly what he is doing.

The Company particularly insists on the necessity of keeping in special byres cows unfit to produce milk under the desired conditions, that is to say, the cows which have lately calved, and those which the veterinary has declared diseased, or which are suspected of disease, in order to avoid the two sorts of milk being mixed. The Company refuses to receive the milk of cows within a fortnight of calving, as well as that of cows which do not give more than 6 litres of milk, and they have to be informed of the use made by the farmer of the proceeds of the milking not delivered to them.

The milk, collected in buckets, is, immediately after milking, passed through a sieve covered with a clean woollen cloth into a refrigerating apparatus (Lawrence cooler), which lowers the temperature to $+4^{\circ} \mathrm{R}$. $\left(4 \mathrm{I}^{\circ} \mathrm{F}\right.$ ). The farmer can either buy this apparatus, or hire it from the Company; whatever the season, he has to keep the milk in the apparatus at the above temperature until the moment of dispatch, so that he must always have a reserve of at east $30 \mathrm{lbs}$. of ice to every 100 litres of milk.

The case of superintending the production of the milk in all its details is confided to women inspectors, who have to report to the Company at least twice a year on the state of cleanliness and hygiene of each farm. In their unexpected visits they have to see 
for themselves whether the milking and manipulation of the milk are carried out according to the regulations, whether there are repairs necessary, whether the provision of ice is sufficient, whether the refrigeration is carried out by means of apparatus working under the desired conditions, etc. The answers to these questions are entered on a form of which we give a model (No. 3).

On the farms the times of calving are regulated in such a way that the quantity of milk to be delivered in the later months of the year, notably in September and October, is not less than the average furnished in the four first months of the year. By this means the producers can supply the needs of the consumer with regularity.

Transport of the milk. - Having been cooled to $+4^{\circ}$ R. ( $4 \mathrm{I}^{\circ} \mathrm{F}$.) immediately after milking, the milk is poured into vessels provided by the Company for the transport of their merchandise. We have already said that the milk has to be kept until the moment of departure from the farm in vessels placed in tanks filled with ice. Once or twice a day, according to the needs of the Company, the milk is dispatched from the farm at fixed hours, so that the arrival at the station may not be long before the departure of the train. During their short stay at the station, the vessels must not be exposed to the sun, and during the heat of summer they are covered with an awning. The distance to their destination is generally short (the milk coming from the immediate neighbourhood of the town), and they are conveyed in special well-ventilated vans belonging to the Company. The empty vessels are cleaned and returned to the farms, where they are again rinsed with cold water in order to remove any dust or impurity they may have gathered on the way. Until wanted again they are placed in a fresh airy place, protected from all impurity, upside down, and in such a position that the air can freely penetrate to them. These vessels are kept exclusively for the transport of milk.

After treatment of the milk.-The works of the Company are situated in a suburb of Copenhagen, and cover a considerable area of ground. On entering, one finds oneself in a large square courtyard, surrounded at the end by the cart-sheds and stables, on the left by the offices, and on the right by the buildings containing the special premises used for the various manipulations of the milk:The reception and weighing; the preservation and filtration; the placing in pots and bottles; the cleaning and rinsing of the utensils; the butter-making; and the pasteurisation of the milk for infants. The floor of the works is macadamised, and is frequently flushed with 
water, not only in order to keep it thoroughly clean, but also in order to obtain, especially in summer, the lowest possible temperature. The different functions are carried out by 180 employés divided into two shifts, one commencing work at 3.30 in the morning, and leaving off at 3 in the afternoon, having in this time taken three hours rest, and the other working from 1.30 in the afternoon to 2 o'clock in the morning, with four hours rest (from 6 to 10 in the evening). The employés wear white duck clothing which is provided and washed for them; the men, coat, apron, and cap; the women, a sort of blouse, apron, and cap ; and everyone wears sabots. Smoking and spitting on the ground are absolutely forbidden. The steam necessary for the pasteurisation of the milk for infants is produced by machinery. There is also a machine for washing, sterilising, and drying the sand used for filtering the milk. In order to keep the milk at a low temperature during the different manipulations, from its arrival to its departure, no less than two million kilos of ice per annum are used, of which the greater part comes in summer from Norway. There is also a special railway station for the reception and dispatch of the milk.

Arrival and reception of the milk. - The milk from the morning and evening milkings does not arrive in Copenhagen in the same form; that of the evening milking alone is delivered as it is produced, that of the morning milking, after having been kept in ice all day, is creamed at the farm and dispatched with the evening milking as cream and creamed milk still containing about I per cent. of fatty matter. The two sorts of milk, creamed and not creamed, are, as will be seen later, resold under their true names at different prices. The transport plant, vans, and boxes, belong to the Company: they are all sealed when sent off, and should arrive with seals intact at the station of the Solbjerg works. Generally, all the day's milk (creamed and uncreamed) leaves the farms in the evening so as to arrive in Copenhagen between nine o'clock and midnight.

As soon as the first vans arrive the milk cans are unloaded without delay. The Company receives about 600 cans daily, each of which contains 50 litres. The total volume of milk received daily is, therefore, approximately about 30,000 litres. It is on this enormous quantity that the 180 employés are going to work in various ways from nine in the morning until evening.

The vans alongside the platform of the works are discharged into trucks, which transport the boxes into the hall where a group 
of employés await them. One of these unseals them one by one, raises the cover and takes the temperature of the milk, which at that moment should not be higher than $11^{\circ} \mathrm{R} .\left(57^{\circ} \mathrm{F}\right.$.). Another places the can on the weighing machine, weighs it and announces its weight with the number of the farm from which it comes, whilst a third takes a sample with a tube, which he gives in a large spoon to a woman specially employed for this purpose, to taste. All indications relative to the number, weight, temperature, and flavour of each can of milk are entered in the books by an inspector whose duty it is to direct and overlook these various operations, and who also takes from different cans samples destined to be analysed both chemically and bacteriologically.

Any milk which the inspectors suspect of not being perfectly good is at once set aside, and samples taken with a view to revealing the cause of the anomaly. As regards the analyses of the milk found to be good (and there is rarely an exception), they show the Company the variations in the composition of the milk, as well as the productive value of the different herds.

Filtration of the milk.-After the cans have been checked, the creamed and uncreamed milks are transported into a large hall where they are placed in tanks half filled with ice in which they remain until their turn comes to be passed through the filter.

The system of filtration employed is the invention of $\mathrm{M}$. Busck. It consists of two enamelled iron tubs on different levels and joined by a bent pipe, the longest part of which is adjusted vertically to the bottom of the higher tub, and the shorter to the bottom of the lower tub. This latter is properly speaking the filter. When the milk is poured into the higher tub it descends by the pipe and rises again from the bottom of the lower tub to the level of two issue pipes, by which it flows, all filtered, into a reservoir placed immediately below.

Formerly, filtration was carried out by means of sponges. This method was replaced about 1890 by a system less costly and more practical, which consists in using gravel and sand disposed in three layers, the lowest of which is composed of gravel as large as peas, the centre of less coarse sand, and the third of very fine sand. The three layers of sand are separated one from the other by sheets of tin pierced by a multitude of small holes; on the upper sheet are laid four layers of fine cloth. The whole is kept in place by the pressure exerted on the edges of the sheets by a special arrangement. 
Every day the gravel, sand, and cloths, as well as the filtration apparatus, are washed; boiling water to which carbonate of soda has been added is poured through until the water flows out clear; then they are sterilised by passing steam through them at a high temperature. This double process of cleaning and sterilisation radically cleans the filter from all impurities which the passage of the milk may leave, and destroys all microorganisms which it may have retained. The filtration of the cream, the creamed milk, the uncreamed milk, and the milk for infants is effected in four different forms of apparatus, in order to avoid any mixing of the different sorts. The filter reserved for cream is small, but the three others are of considerable dimensions, those used for the creamed and uncreamed milk filtering 4000 litres in three hours.

Under the lower tub of the filter is placed an enamelled iron reservoir into which the filtered milk flows. Adjusted to the centre of the bottom of this reservoir a horizontal pipe communicates by a right-angled bend with a pipe placed vertically in the middle of the recipient. This pipe, pierced throughout its length with a multitude of holes, collects the milk from the different layers and conducts it, so mixed as to show an equal average composition, into the horizontal pipe through which it flows into the vessels destined to transport it to the town.

It is from these reservoirs that the two kinds of milk, uncreamed and half-creamed, each filtered in a special apparatus, are drawn into the cans in which they are delivered in the town. The can is filled, then weighed and provided with a label indicating the quality of the milk it contains; the lid is kept shut by means of a wire whose two ends are passed into a leaden seal, on one side of which is impressed the date and on the other the Company's trade-mark. The cans thus sealed are then replaced in the tanks filled with ice until their departure for the town.

Bottling the cream.-On arrival at the works the cream is weighed and tasted, then declared, according to its quality, No. I Cream or No. 2 Cream. The two qualities are filtered separately, and then put into bottles containing 50 and 25 centilitres. Those containing No. I Cream are marked with a wide crosswise mark, those containing No. 2 Cream with two marks. All the bottles have at the bottom the registered trade-mark of the Company, and the initials K.M.F. The bottles are only filled to the beginning of the neck, where a mark indicates the exact quantity of the contents. The corks have a groove on 
the top through which passes a wire which goes round the neck, and whose ends are fixed in a leaden seal dated and marked. The Company also provides whipped cream of first quality in litre and half litre bottles, which in addition to the above indications bear after the horizontal mark the letter " $\mathrm{P}$," (pidske= whipped). The bottled cream is kept in ice until its departure for the town.

Milk for infants.-Special arrangements of protection and modification are adopted in respect of infants' milk, which comes from chosen cows which are the object of special care and feeding. This special milk is sent to the works, where it is treated in the same manner as the milk for adults, under the name of Bornemalk (Infants' milk). The farmer receives a higher price for this milk.

Transport of milk to the town.-Besides the ordinary closed vans which take the milk to the depôts, the Company also possesses for the retail sale in the streets of the town, carts and hand-carts. These vehicles all alike in construction, only differ in size; they are in the form of a chest mounted on two wheels for the hand-carts, and on four wheels for the carts. This chest is laden by means of three doors which open in the two sides and in the back. The cans are placed in twos and threes on each of three sides, so that the taps come into the opening below each door; above the taps is inscribed the quality of the milk for sale and the price. There is another smaller door on one side only, above the back wheel, which opens into a compartment reserved for milk and cream to be sold in bottles. Each cart has, besides the indication of quality and price of its merchandise, a number, the name of the Company, and its trade-mark. When the carts are laden the doors shutting in the cans of milk to be sold by the litre or half litre are sealed, and at fixed hours they go, accompanied by two men each, to stand in the street by the side of the footpath at certain points in the town, or to drive through the more distant parts and suburbs until they have sold all their provisions.

Sale of the milk.-The delivery of the milk is carried out by 200 employés, 50 vans, and 68 horses. The employés, drivers, sellers, all wear a cap bearing the trade-mark of the Company. The vans which deliver the milk to the depôts, pharmacies, and grocers, must arrive there with seals intact, and it is only on this condition that the consignment is accepted. The carts which sell retail must return to the works as they left them, that is to say, with the seals on the cans and doors intact. In this way adulteration by dilution and creaming on the part of the sellers is impossible; 
they can only sell the milk by drawing it from the taps which open and shut in the openings below the doors of the cart; it is also impossible for them to defraud by selling one quantity of milk for another, for above each tap is printed in full the indication whole milk or creamed milk. The seller is also bound to give his customer a ticket of a different colour for each quality, on which ticket is printed the price and quantity of the milk bought as well as a number and the trade-mark of the Company. This is the same for the bottles of cream and infants' milk sold with the seals intact. In this way the different qualities of milk reach the consumer as guaranteed by the Company. In summer the cans and bottles are surrounded by ice in the carts, which keeps the temperature as low as possible.

The sale of whole and creamed milk absorbs the greater part of the 30,000 litres which the Company receives daily; the quantity of milk sold under the name of milk for infants, either whole and fresh, or modified and pasteurised, is above 4000 litres per day.

Price of the milk. - At present, the Company buys milk at $10 \frac{1}{2}$ ores ( $14 \frac{1}{2}$ centimes) the litre. This is a higher average price than that which the French farmer gets for the milk he sells wholesale (I I to I 3 centimes), but for this price the Company expects absolutely irreproachable milk. To cut short any attempt at adulteration, any milk of apparently inferior quality is refused without compensation; on the other hand, in order to obtain milk free from pathogenic germs, they agree to pay for, without receiving, the milk of any cow that the farmer suddenly suspects of disease. The Company sells it at the following prices:-

Whole milk .

Half-creamed milk, containing about

I per cent. of fat . .

Whole milk for infants . .

Cream of Ist quality . .

Cream of 2nd quality . . . of $\cdot 225$ ( 16 ores) per litre.

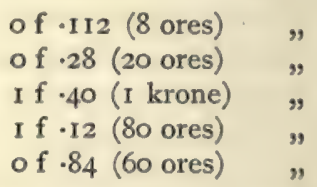

The Company, after having paid for the milk a higher price than is paid for it in France, after having treated it in the complicated manner described, resells it at less than half the price that it is sold by Parisian retailers.

Cleansing of the cans and bottles.-On their return to the works the vans bring back the empty cans and bottles from the depôts and customers' houses, as well as those which had contained the milk sold in the streets, still with seals intact. As soon as they 
arrive, they are taken on trucks into the cleansing-room; that reserved for the cans and large vessels being situated in the immediate neighbourhood of the railway station. It is a spacious hall, and at certain times of the day is the scene of the greatest activity. In the morning, there is the cleansing of the cans used for the transport of the milk from the farm to the works, in the evening comes the turn of the cans which have been used for the delivery in the town. The work is distributed in this manner; certain employés take the dirty cans and place them obliquely, with openings downwards, on the spokes of a large wooden wheel mounted on a tank filled with carbonated soda water in which it turns, successively submerging all the cans for several instants. After this first operation the cans are passed to another relay of employés who wash them with hot water. A third relay sterilises them with jets of steam under pressure, and a fourth rinses them with filtered water. After these several processes the cans are transported to a drying-room, where they remain upside down until their return to the farm or the town.

The milk and cream bottles are cleaned in another room in much the same manner. On leaving the carbonated soda water, they are washed with a bottle-brush turned by machinery, through the hollow stem of which, pierced by numerous holes, boiling or cold water can be spouted at will. They are then washed in filtered water and placed in a special drying-room.

The dairy.-The whole milk and the cream which come back unsold to the works, are taken to that part of the premises called the dairy, where they are at once made into butter, which is sold at a slightly lower price than first quality butter. The residue of this manufacture and the unsold half-creamed milk, after being pasteurised, are resold at very low prices to the farms for feeding young pigs. The staff is composed of office employés, drivers, sellers, carriers, workers for the heavier work such as moving the cans, filtering the milk, loading the vans, cleaning the premises and utensils, etc., and of women for the lighter work such as tasting the milk, bottling it, cleaning the glass utensils, making butter, etc., and numbers about 180 or 200 persons. Each group of men and women has to perform certain kinds of work at fixed hours, to the exclusion of all other. Thanks to the excellent distribution of the work, the various operations described above are accomplished with remarkable regularity and rapidity.

The Company provides the employés engaged in the interior of the works gratuitously with the white duck working clothes of 
which we have already spoken; the drivers, sellers, and carriers with a sort of livery composed of a cap and a blouse with distinctive facings. These last receive, in addition to a fixed salary, a percentage on the profits of the sale after deducting a small sum for the payment of the young boys of from I 2 to 16 who accompany the vans in the morning to help in the distribution of the milk. All the employés are allowed to drink as much milk as they like in the works.

A most rigorous discipline is exercised on the staff. Any infraction of the rules, however slight, is severely punished; on the other hand, bonuses are offered from time to time to those employés signalised by their strict observance of rules and their application to work.

The regulations relating to the staff include one of great interest from the point of view of the transmission of infectious disease by milk. It sets forth the line of conduct to be followed in the case of any illness capable of infecting the milk and thus being transmitted to the consumer, appearing in the family of an employé or in the house in which he lives. In the beginning of this article we pointed out with what care the Company choses the cows, with what constant attention it watches their health, and that of their proprietors, with what rigour they exclude from the herd any animal even suspected of illness. It extends the same prophylactic regulations to the staff. Its motto: Pure milk from healthy cows might be amplified and completed thus: Pure milk provided by healthy cows and treated by a healthy staff of workers. The employé who, according to the rules, goes of his own accord and informs the Company and its medical officers of a case of illness in his family or neighbourhood, is knocked off work until all danger is over, but the whole time of this enforced stoppage of work he receives the whole of his salary. It is therefore to his interest to act honestly, all the more that by omitting to make the notification he runs the risk of instant dismissal if the fact becomes known.

Thanks to this system of notification the "Kjöbenhavns Mælkeforsyning" can deliver the milk guaranteed as it received it, that is to say, absolutely pure and healthy. These are two essential qualities of milk destined for consumption, and it is towards procuring them that all the efforts of the enterprise are directed. ${ }^{1}$

1 A somewhat similar Company to the above is Det Danske Malke-Compagni which was founded about six years ago with the object of exploiting Casse's 
The Milk Supply, Pasteur, was a "bottled-milk" Company in Copenhagen. It employed 300 workers. About 40,000 bottles of milk were pasteurised daily. The farms supplying the Company were under control and inspection. There were fixed regulations as to the fodder of the cows, the treatment of the milk, and the notification of tuberculosis. On reaching the factory the milk was duly weighed and screened, and then pumped to a gravel-filter. After filtration the milk was cooled and pasteurised at $85^{\circ} \mathrm{C}$. for three minutes, after which it was again cooled to $3^{\circ} \mathrm{C}$. From the cooler the milk was conveyed to the bottle-feeding apparatus. After bottling the bottles were carefully sealed with glass stoppers. Throughout the process air was prevented from gaining access to the milk. Bacteriological and chemical examinations were made daily of the milk. The employés were all under medical supervision. Dr Schierbeck claimed that by this process : (a) all danger patent process, which consists in partially freezing the milk immediately after being milked, so as to keep it entirely fresh for several days.

The Company possesses in various places co-operative dairies whose members are bound to the same regulations as the farmers of the "Kjöbenhavns Mælkeforsyning " as regards the feeding of the cattle, the hygiene of the byres, and of the farm employés. The most important of the dairies is at Marslev near Odense, in Fionie, and sends about ro,000 kilos of milk daily.

The milk collected in the dairies is poured into enormous cans of 500 kilos capacity, and in these same cans it is submitted to a very low temperature which partially'freezes it. The cans are then placed in refrigerator vans for transport to Copenhagen, where they arrive with a third of the total mass of milk frozen into a block which floats in the mass of liquid. The vans are unladen, the cans weighed, and then taken to the large tanks into which they are emptied by being tilted mechanically, after a sample has been taken for analysis. From these tanks the milk passes into filters after it is melted. It is then pasteurised and cooled again in apparatus capable of treating 8000 kilos of milk per hour. The three processes, filtration, pasteurisation, and cooling, only take about a quarter of an hour for the same quantity of milk.

On leaving the pasteuriser the milk is immediately cooled, then taken to a room where it is bottled. The bottles are fusiform for retail sale, and cylindrical for wholesale ; there are two sizes of each, I litre and $\frac{1}{2}$ litre. When filled and corked they are placed in an ice house at a temperature of $4^{\circ} \mathrm{C}$. until their departure. The bottles reach the consumer without their contents having been, since placing in the pasteuriser, in contact with the outer air. They all bear a red label with the name and trade-mark of the Company, the date of pasteurisation, and a short notice regarding pasteurised milk.

The milk is sold wholesale either in cans or in baskets of 20 bottles, it is delivered retail in litres and half litres. Out of the 50,000 to 60,000 litres of milk received daily, the Company sells from 30,000 to 35,000 litres as whole pasteurised milk, about 3000 litres as cream, as half-creamed milk the milk from which the cream has been removed, and as pasteurised milk for infants several thousands of litres from special cows. 
from the possibility of infection of the milk, either at the farms or dairy transit is avoided; $(b)$ the distribution of the milk in sealed bottles is a safeguard against its infection or contamination in the milk-shops or in the streets; $(c)$ the milk awaiting consumption in the houses is kept free from contamination by means of flies, dust, or polluted air. We understand that this Company is now merged in the Copenhagen Dairy Company. ${ }^{1}$

\section{Philadelphia Milk Commission}

This Commission (established 1899) appoints experts to examine the dairy and its products in regard to the health of the cows, the cleanliness of the stables, milk-room, and apparatus, and the health of the employés; also in regard to the chemical composition and purity of the milk, and the number and kinds of bacteria present. The Commission grants or refuses certificates in accordance with the result of the experts' examination. These certificates are in such form that they can be handed to the consumer with each bottle of milk or cream. The dairyman agrees to abide by the decisions of the Commission and to pay for the examinations.

I. The procedures so far followed have been, first, that the agreements have been signed when the dairymen notified the Commission that they wished to have their dairy examined. The Commission, when so notified, instructs its experts to examine the dairy and its products. The experts then report to the Commission on blanks provided for that purpose, and finally, where the examination has shown the proper conditions to be present, certificates have been issued in accordance with the contract.

2. As the result of action thus far, others are being stimulated to improve their dairies so as to enable them to obtain the certificate of the Commission. It does not seem too much to hope that ultimately the whole milk supply of the city may be improved, owing to the demonstration of the practicability of such improvement, and to the demand for certified good milk that will surely follow the education of the public.

3. The Commission shall consist of four members besides the president of the society, who shall be a member ex-officio. The

1 The principles and practice of the Danish system have recently been introduced into England and are now being carried out by $\mathrm{Mr}$ C. W. Sorensen at the White Rose Dairy, West Huntington, York. This dairy farm may be taken as a type of the best practice now in vogue in England. It illustrates the sort of control of the milk supply which, in the opinion of the writer, should be adopted throughout the country, and which is the most important immediate requirement. See Appendix S, p. 594. 
members shall be appointed yearly by the president as soon as possible after his election. The Commission shall elect a chairman and a secretary from their number.

4. No statement for publication or information to any dairyman shall be given by or in the name of any individual member, but only after consideration by the Commission and in the name of "The Milk Commission of the Philadelphia Pediatric Society."

5. The Commission will hold itself in readiness to examine milk from dairies desiring this examination, and to certify to the good quality of milk which comes up to the standards fixed by it. It is understood that only the milk of dairies, and not that of milkmen who merely serve milk bought by them, will be examined by the Commission.

6. The method of examination and certification to which the dairyman or his agent shall agree to submit shall be as follows :-

7. The Commission shall select a bacteriologist, a chemist, and a veterinary inspector. The bacteriologist shall procure a specimen of milk from the dairy, or, preferably, from delivery waggons, at intervals to be arranged between the Commission and the dairy, but in no case at a longer interval than one month. The exact time of the procuring shall be without previous notice to the dairy. He shall test this milk for the number and nature of bacteria present in it, to the extent which the needs of safe milk demand. He shall also make a microscopic examination of the milk for pus cells. Milk free from pus and injurious germs and having not more than I0,000 germs of any kind to the cubic centimetre shall be considered to be up to the required standard of purity.

8. The chemist shall in a similar manner procure and examine the milk for the percentages of proteids, fat, sugar, mineral matter, and water present. $\mathrm{He}$ shall also test its chemical reaction and specific gravity, and shall examine it for the presence of foreign colouring or other matters or chemicals added as preservatives. Standard milk shall range from 1029 to 1034 specific gravity, be neutral or very faintly acid in reaction, contain not less than from 3.5 per cent. to 4.5 per cerit. proteid; from 4 to 5 per cent. sugar, and not less than 3.5 to 4.5 per cent. fat, and shall be free from all contaminating foreign matter, and from all addition of chemical substances or colouring matters. Richness of cream in fat shall be specified and shall vary not more than I per cent. above or below the figure named in selling. Neither milk nor cream shall have been subjected to heat before the examination has been made, nor at any time, unless so announced to the consumer. 
9. The veterinary inspector shall, at intervals equal to those of the bacteriologist and chemist, and without previous warning to the dairy, inspect the cleanliness of the dairy in general, the care and cleanliness observed in milking, the care of the various utensils employed, the nature and quality of the food used, and all other matters of a hygienic nature bearing upon the health of the cows and the cleanliness of the milk, including also so far as possible the inquiry into the health of the employés on their farms. He shall also see that the cows are free from tuberculosis or other disease.

Io. The charges made by the experts shall be: For the veterinarian $\$ 10$, and $\$ 5$ for each of the others, for each examination; this amount to be paid by the dairy at the time of the examination and without regard to whether the report is favourable or unfavourable. The experts shall make their examinations when, and only when, notified to do so by the commission. Any dairy the milk of which shall be found by the examiners to be up to the standard of the Commission shall receive a certificate from the Commission, which shall read as follows :-

\section{MILK COMMISSION OF THE PHILADELPHIA PEDIATRIC SOCIETY.}

\section{Date} $\mathrm{Mr}$

The Veterinary Inspector of the Commission has examined the dairy of clean, and the cows to be in a healthy condition.

The Bacteriologist reports that the milk does not contain germs beyond the limits of the standards of the Commission.

The Chemist reports that the milk is of standard richness, and that he has discovered in it no impurities, colouring matters, chemical preservatives, or harmful substances.

The Commission certifies to these statements of the examiners. It is understood and agreed to by the said $\mathrm{Mr}$ is good for not more than examination is to be made. that this certificate from date, when another

[Signed by the Commission.]

\section{Form for Veterinarian's Report.}

MILK COMMISSION, PHILADELPHIA PEDIATRIC SOCIETY. Report of Veterinarian.

Philadelphia, 190

\section{Secretary of the Milk Commission.}

DEAR SIR,-I have examined, as requested by the Commission, the dairy of

conditions :, at and find the following 
I. No. of milking cows; hospital cows ; dry cows ; cows added since last report; cows now in quarantine; cows recently calved; cows sick since last report.

Total number in the herd of which have

been tested with tuberculin.

II. Condition of milch cows at present, good.

III. Food employed.

IV. Condition of stables; ventilation; heat ; floors ; troughs ; cleanliness, etc ; dimensions of stables ; condition of other buildings, good.

V. Health of employés and their families, so far as ascertained, is good.

VI. Source and character of water in dairy and stables.

VII. The general precautions of cleanliness in milking, and the care of the milk are satisfactory.

I therefore recommend that milk from this dairy be submitted to the Bacteriologist and Chemist of the Commission for their examination.

Yours truly,

V.M.D., Veterinarian.

Form for Bacteriologist's Report.

MILK COMMISSION, PHILADELPHIA PEDIATRIC SOCIETY.

Report of Bacteriologist.

PHILAdelphia, 190

Secretary of the Commission.

DEAR SIR,-At the request of the Commission, received on. I90_ milk from the dairy of__ labelled obtained by me on _ $190 \_$at__ Street, at Street, at_o'clock -m., and examined at_ o'clock —m., with the following results :-

Number of bacteria per c.c. of milk,

I have been unable to detect any pathogenic organisms, or evidence of purulent inflammation of the udder.

I therefore recommend the milk as coming up to the bacteriological standards adopted by the Commission.

I find the milk bottles to be sealed in the manner prescribed by the Commission.

Yours truly,

Bacteriologist.

Form for Chemist's Report.

MILK COMMISSION, PHILADELPHIA PEDIATRIC SOCIETY.

Report of Chemist.

Philadelapta, 190

Secretary of the Commission.

DEAR SIR, - At the request of the Commission, received on $190 \ldots$, milk from the dairy of labelled was 
obtained by me on $190 \ldots$ at Street, at o'clock .m., and examined at o'clock $\longrightarrow$. m., with the following results :-

Total solids (by evaporation of weighed amount in platinum basin),

Fat (Leffmann-Beam method),__. Ash (by turning off solids obtained as above),___ Total proteids (Kjeldaht-Gunning method, factor 6.25), — Preservatives and added colour, none. Specific gravity,

I therefore recommend the milk as coming up to the chemical standards adopted by the Commission.

I find the milk bottles to be sealed in the manner prescribed by the Commission.

Yours truly,

, Chemist.

The objects of the Commission are to establish correct clinical standards of purity for cow's milk; to become responsible for a periodical inspection of the dairy under its patronage; provide for chemical and biological examinations of the product, and the frequent scrutiny of the stock by competent veterinarians.

Three general requirements or standards of quality for the milk have been formulated :-

First. An absence of large numbers of micro-organisms and the entire freedom of the milk from pathogenic varieties.

Second. Unvarying resistance to early fermentative change in the milk, so that it may be kept under ordinary conditions without extraordinary care.

Third. Having a constant nutritive value of known chemical composition and a uniform relation between the percentage of the fats, proteids, and carbohydrates. ${ }^{1}$

Similar companies or commissions exist in New York, ${ }^{2}$ New Jersey, ${ }^{3}$ Boston, and other cities and districts. ${ }^{4}$ The New York Commission lays down a standard of milk for certification as containing 3.5 per cent. of fat, 2 per cent. of acidity, and not more than

1 Seventeenth Annual Report of the Bureau of Animal Industry (U.S.A. Department of Agriculture), I900, p. 174.

${ }^{2}$ On very similar lines to the Philadelphia system, and largely due to the influence of Dr Chapin.

${ }^{3}$ One of the largest and most successful model dairies in America is located in New Jersey. Its success is due to the support which it has received from a Milk Commission organised for its exclusive benefit a few years ago. There are now over 400 cows supplying more than 4000 quarts of milk daily, sold at 9 cents a quart to the producer. The suggestion for the establishment of this Commission originated with Dr Henry L. Coit of Newark, New Jersey, some ten years ago.

4 The Australian Health Society arranged for the provision of certified milk under much the same conditions as the American Commissions in 1897. 
30,000 bacteria per c.c. Much has also been done in America in the direction of improving and modifying the milk supply for children, particularly by Dr Chapin of New York, ${ }^{1}$ and Dr Rotch of Harvard. ${ }^{2}$

1 Theory and Practice of Infant Feeding, by Henry Dwight Chapin, M.D., 1902.

2 This system was introduced by Dr Thomas Morgan Rotch of Harvard University, and is now in operation in some eighteen or twenty cities in the United States and Canada, and has also a centre in London. The system, which has for its object the betterment of infant feeding, consists in controlling the milk supply by controlling the farms and establishing a chain of protection from the time the milk leaves the cow until it arrives at the mouth of the infant. But in addition to this scheme of protection, there is also combined with it a scheme of modification of the milk to make it meet more exactly the requirements of infant feeding. The two-fold function of the Rotch System may be briefly referred to:-(a) Protection of the Milk.-With this object in view Rotch made a number of recommendations similar to those laid down by various Milk Commissions, which latter indeed took many of Rotch's proposals for their model. At the farms supplying milk under this system, the breed of the cow and its food are matters which receive primary attention. In America the Holstein has been found to be the best for its adaptability for infant feeding. The cow itself must be regularly and wisely fed on the basis to which reference has been made. There is regular grooming and good housing. The cowhouse has cemented walls, ceilings, and floors, and is properly drained and frequently cleansed. A most careful supervision of the cow's health is maintained, and if in any way abnormal the cow is isolated until in normal health. Careful tuberculin testing is made of each cow used, and the milk of each cow also undergoes micsoscopical examination for the purposes of detecting pus cells, colostrum cells, bacteria, etc. The milkers are under strict medical supervision and regulations are enforced in respect of "cleanliness." Cows are milked in their own stalls, but immediately after milking the milk is taken in closelycovered vessels to the milk-room where it is cooled and screened. The milkroom is a specially prepared chamber having smooth surfaces of polished cement, and specially constructed ventilators with cold water sprays to moisten the air and prevent dust gaining access, asepsis being the requirement. The milk is now ready for the laboratory. (b) Modification.-The object of the Walker-Gordon Laboratories is first to insure and distribute the naturally pure milk ; and secondly, to provide a place where different combinations of milk may be put up, according to the prescriptions of medical men, with accuracy, and under such conditions of cleanliness and asepsis as to insure the best possible food for infant feeding. The necessity for modification arises from two facts, namely, that milk varies in constituent percentages, and to obtain a regular and uniform constitution modification is necessary; and secondly, some children require for one reason or another a milk containing certain percentages of the various constituents. Thus the patient can receive on the physician's order a mixture of the percentages called for, made up of either separated cream or gravity cream, separated milk or whole milk. Twelve years' experience in Boston, U.S.A., seem to indicate the practicability of this system in preventing the summer diarrhœa of infants due to contaminated milk. The first laboratory 
I. The cows are properly selected, are free from disease, and are tested individually with tuberculin. A weekly inspection of the cows and their stables also takes place.

2. The employés are under the care of a physician, who examines them once a fortnight. Employés who are indisposed or suffering from infectious disease are not allowed to come to the laboratory or farm, and recive full pay during absence.

3. The care of the milk is strictly hygienic; no dirt is allowed to contaminate it, as the stables and cows are kept scrupulously clean. The milk is filtered as soon as drawn; it is put into sterile bottles and sealed in a clean milk-house 100 yards from the stables, and exposed to the air as little as possible to prevent its becoming bacteriologically contaminated. It is delivered to the consumer a few hours after milking.

4. The stables and dairy are sanitarily drained, and the cows have 1500 cubic feet of air space each.

5. The milk pails are provided with a layer of cotton-wool through which the milk passes before entering the pail. The milkers wear white overalls.

6. This milk is especially protected against infection. Every vessel used in the milk-house, the milking pails, and milkers' clothing are sterilised before being used, and sterilising apparatus is in use at all times for this purpose. This milk is, therefore, a perfectly safe milk to use in its natural state, and for safety against disease needs no sterilising or pasteurising. The laboratory produces all its own milk, and its cows are all tested with tuberculin. This milk is neither coloured or preserved in any way.

7. A resident bacteriologist and chemist makes periodical examinations and analyses in the laboratory or the farm. The laboratory, modifying rooms, milk cooling rooms, sterilising chambers, and washhouses constitute a building by themselves, separate from the cow stables, and are fitted up with the most improved sanitary arrangements and fittings. ${ }^{1}$

of this kind was established in Boston in I89I, under the name of the WalkerGordon Laboratory. Eighteen or twenty such laboratories now exist in America, and one in London. The London farm and laboratory is carried on in a similar manner to those in the United States. The farm is situated at Finchley, and the laboratory in West London.

1 A full account of the principles and methods of the Rotch system will be found in the Brit. Med. Jour., I902, vol. ii., pp. 653-672, by T. M. Rotch, M.D.

It may be desirable briefly to state here the writer's view of the modified milk movement which is represented in England by a branch of the Rotch system (Walker-Gordon Laboratory), and the Municipal Sterilised Milk Depôts 
at Liverpool, St Helens, Battersea, etc. There can be little doubt that this kind of milk supply may be of great service to the children of the poor, in the reduction of infantile mortality due to the use of contaminated or infected milk, and in special cases calling for special treatment. It is not, however, of the nature of control of the milk supply, but rather, of a specialised supply, to meet special needs. There is evidence to show that at Liverpool, Battersea, and other places, it has had beneficial results in this special direction, and has also done good service in protecting the milk from home contamination. The system has, however, several limitations unless properly managed. Its object being the,saving of life and prevention of infant diseases, it is necessary that the system should be individualised. Each mother must be separately advised, each infant inspected and weighed periodically, each home supervised, the condition of the milk regularly tested, and the source of the milk kept under control, the cows and cowsheds, from which the milk is derived, being supervised by a veterinary surgeon and the Medical Officer of Health. And here, in any event, the quality of the milk used must reach a high standard, chemically and bacteriologically. If these conditions are not fulfilled, it would appear that a municipal sterilised milk supply can only be a palliative measure of transient usefulness. The desideratum is a naturally pure milk supply, rather than an artificially purified and humanised supply. The latter question is one certainly requiring careful consideration, but of a different nature to the former. If undertaken by a Local Authority, it would appear desirable to do it very thoroughly, after the manner of Budin's work in Paris, each case being under strict medical supervision.

Further, at the present time it is not fully established that epidemic diarrhœa is avoided by sterilisation of the milk. In some places and cases it appears to be so, but the subject requires more investigation. On the whole, it may be said that general management of the child in the home, domestic cleanliness, clean milk bottles, and a pure milk supply are the chief desiderata in contra-distinction to sterilised milk. On the one hand, if the cow's milk is clean, pure, and unadulterated, sterilisation is not needed, though modification may be; on the other, a bad milk cannot be made good by sterilisation. Nor must it be forgotten that for a municipality to furnish a sterilised milk supply, raises special difficulties as to the function of a municipality, which surely should be to control the milk supply and insist upon its purity rather than itself to trade in a sterilised milk. If success attend such an enterprise, private dealers will take the matter up, and it will be carried on in an uncontrolled manner. For these reasons, it appears to the writer, $(a)$ that the education of the public, particularly of elder girls, in the practice of domestic cleanliness and infant feeding, is of more importance than a supply of sterilised milk by the municipality; (b) that municipalities should use all their powers, and as far as necessary, obtain extended powers for the supervision of the milk supply to large towns; and $(c)$ that milk laboratories, sterilised milk depôts, and the like, should be directed to special needs where existent, and then only under the control of the medical profession, each case treated being carefully observed. One of the most recent and lucid accounts of the milk depôt system will be found in the Jour. of State Medicine, Oct. I903, p. 599, written by Dr Mussen of Liverpool. 


\section{CHAPTER XV}

THE CONTROL OF THE MILK SUPPLY: (3) BY THE TRADE

General Note ; (I) Milk Herds:-Freedom from Disease, Inspection, Bacteriological Examination, Tuberculin; (2) The Housing of Milk Herds; (3) Milkers and Milking ; (4) Treatment of Milk after Milking :-Clean Milking, Straining, Cooling, Transport ; (5) The Final Treatment of Milk :-Filtration, Preservation, Pasteurisation, Sterilisation ; the Retail Sale. Summary.

Although much has been done by legislation, and doubtless much more would be done if the present law were wisely but firmly enforced, and although much has been done by several enterprising milk companies and commissions, the real control of the milk trade is largely in the hands of the public. The trade will supply what the customer demands, provided the dairy farmer and the milk purveyor know what to do and how to do it. Questions of price, outlay, or compensation, have a tendency to right themselves in the light of a well-informed public opinion and a rightly-cultivated public taste. A pure milk supply, that is, a clean milk of good quality; from healthy cows; properly strained and cooled, and protected from infection and contamination-which is the ideal we have already suggested-could be obtained throughout the United Kingdom in twelve months' time, if the public chose to insist that should be so.

The writer, therefore, proposes in this chapter to offer suggestions by which the trade and the public can obtain such a satisfactory milk supply. The term, "the trade" should be understood in the broadest sense.

\section{Milk Herds}

It is probable that the chief pollution of milk gains access in ways other than through the udder, and that few of the common diseases conveyed by milk have a bovine origin. Nevertheless the importance of healthy milch herds is beyond question. To obtain milk from unhealthy cows is similar to 
obtaining drinking water from contaminated sources. Therefore, at the outset of any consideration of the control of the milk supply it is necessary to deal with milch herds.

Present position.-It is a matter of common knowledge that clean dairy herds do not obtain, as a general rule. Of I44I cows inspected for the Manchester Corporation in I90I, as many as 379 were "very dirty," which is equal to a percentage of 26-09. The veterinary inspector writes: "Nearly always bad cowsheds and dirty cows are found together though occasionally this does not hold good; but, as a general rule, where you find bad insanitary cowsheds you find dirty cows. When I speak of dirty cows I mean not only those with dirty quarters and tails, but those in which the udders are also dirty. In fact at one farm a really abominable state of filth was seen. . . . So plastered up with dung and dirt do we find some udders that the dirt effectually masks any slight abnormality, such as commencing induration." 1 Nor is uncleanliness the only fault. In the majority of farms supplying milk, the question of breed, and really careful management of the milk herd, do not arise. The animals are considered and treated in a most casual way. On large farms, and by well-informed and experienced farmers, the matter is, of course, otherwise, but on hundreds of small dairy farms no real study of the milch herd takes place. If milk is yielded, and can be sold, that is deemed sufficient. But, in fact, that is not sufficient.

Recommendations.-Breed-It is evident that the present volume is not the place to discuss the question of breed. We will content ourselves with two brief references. "The breed of dairy cattle to be most profitable must have in addition to a large capacity to consume food and the quality to long sustain a large flow of rich milk, the ability to resist tuberculous and other diseases; it must be prolific in calf-bearing; longevity should be a strong characteristic; the animals must have kind dispositions; and all these must be blended as a strongly flowing stream in the heredity of the breed " (Hays). ${ }^{2}$ To improve milking stock should be one of the objects of the dairy farmer, and he should make it his business to study the question in practical fashion. Ernest Mathews has laid down five simple rules by which such improvement can be brought about, namely-(I) only keep the best cows and use bulls descended from the best milking dams; (2) select the calves with the best escutcheons and do not over-feed them;

1 Report on the Health of the City of Manchester, I90I, p. 247.

2 United States Year-Book, Department of Agriculture, 1901. 
(3) weigh all the milk and record the same against each cow, and where the butter is made, test the value of each cow for butter-making by the churn; (4) do not discard heifers with their first calf because they do not milk as well as some of the cows, as frequently those heifers that yield too well with their first calves turn out weak in constitution; (5) never exhibit any heifer intended to be kept for the dairy in the inspection classes at any agricultural show, as she will be over-fed and spoilt. ${ }^{1}$ Whatever means and methods are adopted, in breeding or in purchase, it is important to remember that some breeds are physiologically incapable of producing as good a quality and quantity of milk as certain other breeds. Hence the dairyman must first obtain a suitable breed if he desires to obtain compensatory results.

General hygiene, feeding, and cleanliness.-It is necessary here also only to add that the environment, food, cleanliness, and general management must be maintained in such a way as will ensure a high standard of good health and a yield of pure milk. All food given to the cows should be fresh, in good condition, and of such a nature as will not impart any unpleasant taste or taint or other undesirable property to the milk. Many suggestive hints in respect of food will have been gained by a perusal of the best methods as they are worked in practice in Europe and America, and the details of which are found in the present volume. We may add that Mathews states that too great a proportion of albuminoids tends to fatten only, whilst an excess of carbohydrates improves the quality of the milk. Milk is also reduced in quality in his opinion by giving foods which contain a large quantity of water. He adds that "the foods that produce the best flavoured milk are as follows: crushed oats, bran, maize and bean meal, linseed and cotton cakes, cabbages, kohlrabi, carrots, parsnips, and mangels later in the season, with of course good hay and straw." 2 There should always be a pure and sufficient water supply easy of access. Cleanliness is an absolute necessity in general management. Each cow should be regularly cleaned and groomed, and the udder should always be cleansed before milking. Hair in the region of the udder should be clipped. The hind quarters and flanks should also be cleansed before milking.

1 Economies in Dairy Farming, by Ernest Mathews, 1903, p. 31. This little book contains many useful hints on the management of dairy herds.

${ }^{2}$ Ibid., p. 45. 


\section{Freedom from Disease.}

The milk of no cow should be used unless the cow itself is free from disease, particularly disease of the udder. A cow that eats its food and gives a fair supply of milk is in the dairy farmer's view a healthy cow, and local diseases, eruptions on the udder, even ulcers, are not always looked upon as circumstances prohibiting the use of the milk. Yet they should be, for it is now well known that many serious diseases exhibit only transient or comparatively slight symptoms, and yet are infectious. Hence, any disease however insignificant or local, especially if affecting the udder, be it eruption, ulceration, or otherwise, and attended or not with a rise of temperature, should be considered to render the cow absolutely unfit to produce milk for human consumption so long as the illness exists. Foremost among these diseases is tuberculosis, but such conditions as contagious abortion, acute mastitis, mammary abscess, or any form of udder disease, persistent diarrhœea (scouring), actinomycosis, or febrile diseases generally, should be included as conditions prohibitive of milking for human food. Care should also be taken to protect other animals on the farm from contracting disease. The infection of healthy animals by tuberculosis, for example, takes place readily from cohabitation, the bacilli gaining entrance by the respiratory organs in most cases, but by the digestive tract in many, especially in calves when suckled by diseased mothers; and in calves, pigs, and other animals fed on milk, or on dairy bye-products containing living bacilli; and in pigs when allowed to eat tuberculous matter found in carcases and slaughter-house refuse given them as food; probably, too, in rare instances, from eating the flesh of tuberculous animals. It is to the buildings themselves, we must look sometimes for the chief source of infection, and to neglect of disinfection and sanitation must we attribute, in a great measure the continuance of disease and re-infections. There are on record repeated instances of herds cleared out and new purchases made with every care in purchasing, being followed by new cases of tuberculosis; so that too much trouble cannot be taken to eradicate disease, to make sure that the buildings are aseptic and that sufficient air space is provided, as well as a rational system of ventilation, whereby pure air is admitted and foul air driven out, and that properly trapped drains carry off the liquid excrements, and prevent the return of noxious vapours. 
For all these matters play a direct part in the propagation of disease among cattle. ${ }^{1}$

There are three chief methods for detection of disease, such as tuberculosis, in milch cows, namely-(a) clinical inspection, (b) bacteriological examination, and (c) diagnostic inoculations.

(a) Inspection.-The clinical diagnosis of tuberculosis, even by the most expert clinician, except in advanced cases, is always unreliable, as the disease may exist in such a stage as would be impossible of diagnosis by this means. Still much may be done towards obtaining a diagnosis even on clinical grounds. If the disease affects the respiratory organs there is a frequent cough, disturbance of the respiration, quickened breathing. Dull sounds may be elicited from the lungs. The superficial glands in the throat, between the jaws, under the ear or the udder, may be hard and swollen. If the disease is mostly abdominal the symptoms of defective nutrition are early apparent-emaciation, lessened secretion of milk, indigestion, breathlessness and general failure, more or less rapid.

Inspection to be of value must be regular, frequent, and thorough, as carried out in Denmark. The moment a sick animal is detected it must be eliminated from the milk herd and isolated by itself. Many of the great dairy companies, both in Europe and America, have their own expert veterinary inspectors for the purpose of regularly overhauling the milk herds and examining into the conditions of their stabling, etc. The value of inspection is well shown in the records appertaining to Manchester and the London County Council (see p. 212).

(b) Bacteriological examination of the milk yields, of course, a result primarily having relation to the milk alone. But indirectly such examinations may be used as an indication of the condition of the udder, its healthfulness or otherwise, and in this way is some test as to the herd. Nocard and others have recommended as the simplest and surest method of making a diagnosis the use of a trocar for examining any portion of the udder desired. ${ }^{2}$ By means of this trocar one can extract from

1 See also La propagation de la Tuberculose (Commission de la Tuberculose), 1900, pp. 264-277.

2 These trocars are 2-3 millimetres in diameter. The point is conical instead of three-sided, thus avoiding the action of the sharp angles of the ordinary trocar that sometimes give rise to slight hæmorrhage and tinge the milk with blood for two or three days; but the conically pointed trocar penetrates the skin with difficulty, so it is necessary to make a small incision with a lancet in the skin through which to pass it into the mammary gland. 
an indurated or apparently diseased udder a small fragment of tissue for examination. "The use of this trocar," says Nocard, "has helped me greatly, and has led me to determine in an hour when cows are affected by tuberculous mammitis, where I should otherwise have had to have kept them under surveillance for several weeks if I had had to wait for the result of the injection of milk into the peritoneum of guinea-pigs." Bacteriological examination may be applied in other ways in the testing of milch cows. For example, discharges from the nostrils, matter coughed up, or scraped or sponged off the pharynx, pus from suppurating tumours, discharge from ulcers on the udder or elsewhere, tissue of glands, etc.

The Manchester method. - The combined use of sampling, inspection, and bacteriological examination has been well shown in Manchester where the method now in practice is, according to Dr Niven, as follows :-

In connection with the procedure taken under the Milk Clauses, it may be well to state how cows having suspicious udders are discovered, and the nature of the disease definitely proved.

I. The first step in connection with milk supplied from the country is to take samples at the railway stations. In taking samples of milk, whether at the railway stations or from suspicious cows at farms, special care is required so that they be taken under strict antiseptic precautions, and everything possible is done to prevent outside contamination. All samples are taken in sterilised bottles, and accompanying these are rubber corks, tin ladles, and numbered metal cases to contain the whole. All of these are sterilised at the Pathological Laboratory, whence they are supplied to the Public Health Office by Professor Delépine. The samples are taken at the railway stations by the Food and Drugs Inspectors, and the samples, after the necessary notes have been taken, are labelled and taken to Professor Delépine, who tests them for tubercular infection.

2. The bacteriological examination occupies about a month, when a report is received at the Public Health Office giving the result of the test. When the sample has been found to cause tuberculosis, the farm from which the tuberculous sample has been sent to Manchester is visited by the deputy of the Medical Officer of Health and the veterinary surgeon, but not until an order from a Justice of the Peace, authorising such visit, has been first obtained. 
3. The veterinary surgeon examines the milking cows on the farm, and also takes samples from any having suspicious udders. These samples are taken under strict precautions as to cleanliness, and sterilised bottles, etc., are again used for the purpose. These samples are labelled in the same way as are the station samples, and are then taken for examination to Professor Delépine. The veterinary surgeon also makes a thorough examination of the condition of the cowsheds, draws plans, and suggests structural improvements.

4. In examining samples of milk from suspicious cows, Professor Delépine usually makes two reports: one giving the result of the microscopical examination of the sediment, obtained by centrifuging the sample of milk, for tubercle bacilli, and the other giving the result of testing the sample of milk by inoculation into animals. Samples of milk from cows found to have suspicious or diseased udder by clinical examination, without previous station samples, are examined in the same way.

In no case is any action taken against the farmer until it has been definitely proved that the milk taken by the veterinary surgeon from a cow having a diseased or suspicious udder has been shown to be tuberculous by one or other of these tests. Control examinations are also made with the object of insuring(a) that a second case of tuberculosis has not been overlooked, and (b) that the animal found has been removed from the herd. When a cow with tuberculosis of the udder is found, the farmer is urged by the Medical Officer of Health to slaughter the animal as soon as possible. During 1902, 31 such cows were found and 16 were slaughtered; the remaining 15 tuberculous cows were sold in the open market. ${ }^{1}$

(c) Tuberculin.-In recent years the method of testing herds for tuberculosis by means of tuberculin has come into vogue, and it will be necessary to refer briefly to this subject. The discovery by Koch, in I 890 , of the production of fever, indicated by a rise in temperature, in tuberculous animals into which he injected a sterilised glycerine extract of pure cultures of tubercle bacilli, while it produced no effect whatever when the animals were free from that disease, furnished us with a simple but very reliable diagnostic agent.

How tuberculin is made. - Tuberculin is a soluble product of cultures of tubercle bacilli, of which a glycerine extract is made,

1 For particulars respecting the Manchester method and its results, see Reports on the Health of the City of Manchester, I901 and 1902 (Dr Niven). 
which is sterilised by heat and filtered through porcelain, so that it contains no living germs, and therefore cannot produce tuberculosis in animals injected with it. It has, therefore, no effect on healthy animals; in some cases the disease is aggravated by it when it exists, but it cannot be produced by it. The lymph must not be exposed to sunlight ; it must not be frozen, and must be kept well corked to exclude air.

Koch's "old tuberculin" is made from glycerine veal broth cultures of $B$. tuberculosis by means of evaporation and precipitation with alcohol. The liquid cultures are thus concentrated to onetenth of their original bulk, and then passed through a Chamberland filter. The brown and viscid filtrate is the tuberculin. Buchner and Römer pointed out that the proteins of other bacteria have a similar effect upon tuberculosis, that is, cause a reaction with rise of temperature. In 1897 , Koch was able to improve his tuberculin, and under the name "Tuberculin T. R." recommended a new preparation. In point of fact the new preparation takes three forms, distinguished by the letters T. A. (alkaline tuberculin), T. O. (upper tuberculin, Germ. ober), and T. R. (residual tuberculin). T. A. is extracted from a young and virulent culture of $B$. tuberculosis by a means of a one-tenth normal solution of caustic soda, and the solution is filtered. The reaction on inoculation is intense and may be accompanied with abscesses. Accordingly its clinical use is open to objection. T. O. and T. R. are prepared by vigorously pounding in a mortar dried cultures of the tubercle bacilli and then adding distilled water. The emulsion is thoroughly centrifugalised. The clear opalescent fluid collecting at the upper part of the tube contains no tubercle bacilli, and constitutes in the first centrifugalisation T. O. The débris or residuum of tubercle bacilli remaining at the bottom of the tube, is used for the production of T. R. This residue is dried, triturated with distilled water, and centrifugalised repeatedly until hardly any residue remains. Twenty per cent. of glycerine is then added to both preparations for purposes of preservation. T. R. alone is used clinically. The dose is $\frac{1}{500}$ milligramme gradually increased up to 20 milligrammes.

How tuberculin is used.-Tuberculin injection has no bad effects on the secretion of milk, either in quantity or in quality. The consensus of opinion of those most experienced is that it does not lessen the secretion of milk in dairy cattle, consequently they may be tested even when in full milk without disturbing its secretion, unless it be during the few hours of its absorption.

It does not cause abortion in cows, or sterility in bulls. 
The consensus of unprejudiced opinion is that it is harmless to cattle, even when tubercle is present.

The dose varies with the size and age. As issued by the Department of Agriculture in Canada, it is ready for use, with doses marked on the bottle, viz. : twenty drops for calves, forty for small or medium-sized animals, sixty for larger, and eighty drops for very large ones.

When second tests are considered necessary, at least ninety days should elapse, and the doses be slightly increased. Second tests, however, are quite unreliable and often deceptive.

How to deal with a diseased herd.-When tuberculosis is discovered in a herd, immediately remove the diseased ones from the healthy to an isolated stable, or a part of the byre may be partitioned off by close boards as far as possible from the rest of the herd.

In the case of low-priced cattle the owner will best serve his own interests by slaughtering them at once. When they are especially valuable and in calf, the experiments of Professor Bang and others show that the calf may be saved by removing it as soon as born, and before the cow has licked it, or it has been suckled by its diseased mother. By placing it in an uninfected building and feeding it on milk from tested cows it will, in all probability, grow up free from tuberculosis. The herd should be tested every six months, and those which react likewise removed till all trace of it disappears. ${ }^{1}$

Disinfection of premises.-Most careful and complete disinfection of buildings and yards in which diseased cattle have been kept should be employed to rid them of disease germs. In doing this, before sweeping, sprinkle the floors and walls thoroughly with water to prevent dust rising, and remove drinking troughs, feedboxes, and stall divisions. The floors must be specially scrubbed, the walls, ceilings, and partitions should be carefully washed, and all freely sprayed with a disinfectant solution, such as carbolic acid (one pint of crude acid to four gallons of water) or lime-wash. It may be applied by a whitewash brush or a spraying pump, care being taken to see that every corner, crack, and joint is thoroughly penetrated by it. The cleansing and disinfection should extend to drinking troughs and fences of the farmyard to make disinfection complete.

1 For a full discussion of this question see paper by Professor Sheridan Delépine entitled, "How can the Tuberculin Test be utilised for the stamping out of Bovine Tuberculosis."-Trans. British Congress on Tuberculosis, 1901. vol ii., pp. $235-278$. 
Disposal of tuberculous carcases.-Should the disease be local, as is frequently the case, being confined to one or more glands, the flesh can be used as food with impunity. Infection from flesh is an extremely rare occurrence, and most unlikely to occur if it has been thoroughly cooked. This can be most relied on when it is cooked in the minced form ; deep-seated portions of large roasts may not be exposed to sufficient heat to kill the bacilli.

When on post-mortem examination the disease is found to be generalised, the carcase should be put into the rendering vat; the diseased viscera and tubercular masses should be burned or buried deeply in lime. No restriction is necessary on the sale and removal of hides, horns, hoofs, or hair.

Nocard summarises the matter by saying that experience proves the incomparable diagnostic value of tuberculin everywhere and in all countries. The latest and most limited lesions are revealed with the same precision, the same certainty, as those which have invaded the greater part of the lungs. For stamping out tuberculosis among cattle, Nocard advocates $(a)$ the complete separation of unhealthy from healthy animals; (b) the slaughtering without delay of those sick animals which show clinical signs of the disease, and especially of cows attacked with tuberculous mastitis; (c) the interdiction from selling tuberculous animals for a destination other than the slaughter-house; and (d) the pasteurisation of all the sub-products of butter and cheese manufactories.

Objections to tuberculin.-The test of tuberculin is recognised to be of great value, but it is not infallible. McFadyean states the defects which have been experienced as chiefly three: (a) That for a period after infection, a period that is sometimes considerable, an animal will not react. (b) That in some advanced cases of tuberculosis no distinct reaction is obtainable. (c) That in a considerable proportion of cases a second reaction is not obtainable for some days or weeks after the first. This immunity is rarely lasting. Nocard, though recognising that tuberculin has certain defects, holds that "when a cow reacts clearly to tuberculin, it may be considered tuberculous." The absence of action has not the same absolute value. A tuberculous cow may not react, first, because the disease is very advanced, in which case clinical examination will usually discover its presence; or secondly, because the animal has recently been subjected to the tuberculin test, and is therefore still tolerant to it. This latter pussibility must be taken into consideration in the case of a recently 
purchased animal, in which case the test may be delayed a month or so. ${ }^{1}$

\section{The Housing of Milk Herds}

Present conditions. - There can be little question that much of the polluted milk finding its way on to the market at the present time is due to the bad housing of milch cattle. The evil conditions existing in these places are well known. Lack of ventilation and light, uncleanliness, unsuitable storage of milk, and the proximity of the byre to other buildings, are conditions which abound. In some cases horses are stabled with milch cows, which is open to serious objection. Often there is overcrowding of the cows, and bad drainage and bad floors are in many districts the rule rather than the exception. Two or three illustrations will be ample, and they shall be selected from recent Government reports:-

"I found that no heed had been paid to providing anything approaching the 800 cubic feet per cow which the regulations prescribe. Most of these cow byres indeed seemed to contain as many cows as could be crowded within their four walls. Some have no ventilating openings whatever, others are provided with a few air shafts insufficient for the purpose. Often byres are badly paved and drained, and have no convenient supply of water. Almost all are imperfectly lighted. The superstition that a cow kept in foul air and in the dark is a 'better milker' is here still prevalent; the risk run by persons who consume milk from cows so kept is unheeded." 2

Again: "The greater number of the cowsheds [in the district] have a brick, or partly brick floor, the surface of which is irregular and often dilapidated, allowing the liquid manure to settle in small pools, and to soak between the bricks. The liquid manure which is not absorbed in the cowsheds finds its way by means of an open brick gutter passing at the rear of the beasts' stalls to the farm midden, where the dung and litter is deposited to remain until cleared away, as may be convenient. The usual arrangement is for the cowsheds to occupy one, two, or three sides of a square, the partially enclosed space forming the midden. Some [cowsheds] are built wholly of wood. Few of these buildings could be

1 For further particulars respecting the value, and methods of use, of tuberculin, books on general bacteriology and veterinary medicine may be consulted. Much interesting matter on this subject may be found in the Trans. British Congress on Tuberculosis, Igor, vols. i.-iv.; The Year-Book of the Department of Agriculture, U.S.A., Washington, I90I, pp. 58I-592 (D. E. Salmon); and official reports issued in Germany, England, and America.

2 Twenty-Eighth Report of Local Government Board, 1898-99 (Medical Officer's Supplement), p. IO4 (Sanitary Condition of Alnwick Urban District). 
considered clean. .. Most of the cows are milked in the stalls." 1

Again: "The condition of the cowsheds visited is bad. The cubic air space allotted to the cows is often insufficient, as also is the ventilation. Large quantities of manure were seen stored adjacent to cow byres. Surprise was expressed that these arrangements should be considered faulty." 2

The evidence obtainable from the country farms supplying Manchester is in the same direction. ${ }^{3}$ The country farms visited were, as a rule, found in a dirty condition. The water supply was often of a questionable quality. Altogether I I cowsheds were inspected, and the greater number were found to be insanitary. Some of the particulars are as follow :-

\begin{tabular}{|l|c|c|c|c|c|}
\hline & $\begin{array}{c}\text { Cowsheds } \\
\text { examined. }\end{array}$ & Dirty. & $\begin{array}{c}\text { Bad } \\
\text { Light. }\end{array}$ & $\begin{array}{c}\text { Deficiant } \\
\text { Ventilation. }\end{array}$ & $\begin{array}{c}\text { Bad } \\
\text { Drainage. }\end{array}$ \\
\cline { 2 - 5 } & III & $\begin{array}{l}48 \\
\text { Totals }\end{array}$ & $\begin{array}{l}73 \\
66 \cdot 2\end{array}$ & $\begin{array}{l}68 \\
61 \cdot 2\end{array}$ & $\begin{array}{l}64 \\
57 \cdot 6\end{array}$ \\
\hline
\end{tabular}

Such figures are in no way exceptional. In a large number of cowsheds the only means of lighting at all is the door, which is, however, generally closed. Ventilation and drainage are notoriously bad. The materials used in the construction of the floors in the majority of cowsheds are not conducive either to an impervious floor or to an efficient system of drainage. Large accumulations of manure are frequently allowed in the immediate vicinity of the cowshed and the water supply. Every kind of filth, and large pools of decomposing refuse water, are familiar adjuncts surrounding cowsheds throughout England. ${ }^{4}$

1 Twenty-Ninth Report of Local Government Board, 1899-1900 (Medical Officer's Supplement), p. 152 (Sanitary Condition of Aldershot).

${ }^{2}$ Loc. Cit. Thirtieth Report, 1900-1901, p. 136 (Sanitary Condition of Stroud and Nailsworth).

${ }^{3}$ Report on Health of City of Manchester, I901, p. 248 ; I902, p. 143.

4 It is unnecessary to quote further evidence in support of the deplorable conditions existing in cowsheds more or less throughout the United Kingdom. Reference may be made, if desired, to Report of Medical Officer of Northamptonshire County Council, I901, pp. 96-99; Report of Medical Officer County Palatine of Chester, I90I, p. II2; Report of Medical Officer of Staffordshire County Council, I900, pp. 98-IOI ; I90I, pp. 96, 97. Reports from Derbyshire, Wiltshire. West Riding of Yorkshire, Leicestershire, Bedfordshire, Herts, Sussex, Surrey, etc., all contain the same record of insanitary conditions in cowhouses. Take an illustration from Yorkshire. In IgOI, Dr Kaye inspected 24 cowsheds in 
Such examples could be multiplied almost ad libitum, in every part of the country, even in dairying districts. We have visited during the last four or five years a number of the dairying districts in England, and without exception we have found in these districts cowsheds in almost every particular open to grave criticism. The cows live under insanitary conditions, and are kept in uncleanliness. The milking itself is also carried by dirty people in dirty stalls. A pure milk supply is impossible under these circumstances.

Recommendations. - The Royal Commission on Tuberculosis, I898, recommended as follows :-

"Io. We recommend that the Local Government Board be empowered to require Local Authorities to adopt regulations as to dairies, cowsheds, etc., where that shall be found not to have been done already.

"I I. That in future no cowsheds, byre, or shippon, other than those already registered, shall be permitted or registered in urban districts within 100 feet of any dwelling-house; and that the discontinuance of any one already existing shall be ordered on the certificate, eitlicr of the Medical Officer of Health that it is injurious to the health of human beings residing near it, or of the Veterinary Inspector that it is not a place wherein cows. ought to be kept for the purpose of milk supply, and that it is incapable of being made so.

" 12 . That the conditions of the attached cowsheds that shall warrant the registering of a dairy in a populous place, whether technically urban or rural, in the future shall include the following :-

"I. An impervious floor.

" 2. A sufficient water supply for flushing.

"3. Proper drainage.

the Selby urban and rural districts. A study of his admirable return respecting these places shows that all, except one, and that a new shed, were open to criticism.-Report on Sanitary Condition of Selby Union, No. 8, 1901, pp. I 5, I6. In 1902, the same medical officer visited 40 cowsheds in the Pontefract Union of which 5 appear to have been passable.-Report on Sanitary Condition of Pontefract Union, 1902, pp. 12-16. One cowshed, Dr Kaye reports, as consisting of "an upturned railway carriage with wooden sides and roof of corrugated iron."

The footwalk was laid in bricks, badly and loosely set. Three cows were kept in this place, and the privy entrance was direct from the cowshed. Or take Staffordshire, one of the counties supplying milk to the south as Yorkshire does to the north. Dr Reid, the medical officer for Staffordshire, reporting in 1903, writes: "I very rarely came across a dairy farm which is satisfactory as regards the cowsheds; most are ill-lit, overcrowded, badly ventilated, and badly drained." 
"4. A depôt for the manure at a sufficient distance from the byres.

"5. A minimum cubic contents as regards such byres of from 600 to 800 cubic feet for each adult beast, varying according to the average weight of the animals.

"6. A minimum floor space of 50 feet to each adult beast.

"7. Sufficient light and ventilation.

"While we have prescribed a minimum cubic contents and floor space, without mentioning definite dimensions affecting ventilation and lighting, we are distinctly of opinion that these are by far the most important, and that requirements as to cubic and floor space are mainly of value as tending to facilitate adequate movement of air. Existing cowsheds should be obliged to conform to the prescribed regulations within a period of twelve months from the time of the regulations coming into force.

"I3. The same conditions as those recommended for populous places should apply to cowsheds in sparsely-populated places, except in so far as cubic contents per cow are concerned; as regards these cubic contents such space per cow should be provided as would, in view of the surrounding circumstances, secure reasonable ventilation without draught. But, the physical circumstances prevailing in different localities being so various, we do not find it practicable to prescribe uniform minimum requirements in this respect."

The regulations under the Milk-shops, Dairies, and Cowsheds Order prescribe and regulate the lighting, ventilation, cleansing, drainage, and water supply of cowsheds (see Appendix). But the difficulty is that the regulations (under Section 13) are adoptive, and not in force until adopted by each Local Authority. Accordingly their benefit and the control which they would exercise is limited by local opinion or prejudice.

This is not the place to enter into detail respecting the structure, arrangements, and management of cow byres. ${ }^{1}$ We desire simply to draw attention to two points; in the first place, to the evil conditions at present obtaining due in large measure to the supineness of many Local Authorities having charge of these matters; and, in the second place, we desire to draw public attention to the absolute and imperative necessity of reform in this

${ }^{1}$ Some practical suggestions will be found below. We may also refer to Jour. of State Medicine, 1897, p. 537 ; Public Health, 1899-1900, pp. 529 and 775-792 (Speir); 1902-03, pp. 441 and 457. 
particular, if the milk supply of England is to be a pure one. Generally we may state the requirements to be:-(a) that the conditions of life under which cows pass the winter shall be as natural as possible; $(b)$ that the cow byre shall be constructed with a view to thorough cleanliness, and smooth impervious floors and walls are as essential to this requirement as abundant light; and (c) that as regards purity of atmosphere the desideratum is equable and efficient ventilation rather than cubic capacity. For the convenience of those who may desire details in amplification of these principles (hygiene, cleanliness, light, air, etc.) we quote some of the particulars issued by the Sanitary Committee of the West Riding of Yorkshire in I90I (Dr J. R. Kaye). ${ }^{1}$ Such suggestions

\section{1. New Buildings, or premises proposed to be newly occupied as Cowsheds.}

Construction.-The walls should be covered internally to a height of 4 feet 6 inches with a smooth impervious material, so as to provide an easily washable surface. The roof should be externally covered with slate or tiles (not corrugated iron, because of the difficulty in regulating temperature, etc.). Gutters or spouts should be provided to carry the rain water to a fall pipe, which should not discharge into the midden but into the surface drainage system so as to avoid dampness of walls and foundations. In places where other water supply is not convenient the rain water might be conducted into a cistern, and used for cleansing the manure channel, floor, etc.

The distance of the cowshed from dwelling-houses, schools, workshops, or public buildings should not be less than 25 feet. The cowshed should have two sides at least free from obstruction, so as to admit of free lighting and ventilation. The building should be not less than 8 feet high internally at the eaves. The width or distance between the front and back main walls should be sufficient to allow for a feeding passage between the wall and manger, a trough manger and hay-rack, stall, a manure trench, and a cleansing passage. For example :-

3 feet 3 inches to 4 feet for the passage between the wall and manger.

2 feet for the trough manger and brick work.

5 feet 9 inches to 6 feet for the stall.

2 feet to 2 feet 6 inches for the manure trench, which should be 3 inches deep and carried from one end of the shed to the other.

5 feet to 5 feet 6 inches for the cleansing passage.

Where a double row of stalls is wanted, the section might be the duplicate of the above, with the exception that the raised passage in the centre for general use might remain at 5 feet 6 inches in width.

The width of each stall should be about 4 feet for a single cow, and from 6 feet 3 inches to 7 feet for a double stall.

Lighting.-Light should penetrate every nook and corner. To obtain this every cowshed should be provided with windows, or lights in the roof. Three square feet of window space per cow is suggested as a good working average.

Ventilation.-The windows should be so constructed that one-half will open inwards so as to admit air and deflect it upwards above the animals. It is well to provide a number of permanent openings in the walls, so designed that the 
act in some measure as a crystallisation of the theory of the regulations under the Dairies Orders, 1885-1899. We may also

external aperture is four or five times smaller than the internal orifice. These are placed at about 7 feet above the floor, and the air which enters by such coneshaped openings becomes readily diffused. Another simple method is to place 2 -inch tile pipes in the wall near the eaves with 2 inches of rise from the outside inwards. Outlets for the egress of foul air should be provided in the roof-or, if there is a loft or ceiling a chimney is desirable. Louvre ventilators may be employed, or the ridge may be elevated and provided with outlets at proper intervals. Roughly speaking, 30 to 40 square inches of inlet area per animal is advisable, while the outlets should be at least equal to the inlets or, preferably, a little more. The external door should be divided crosswise, so that the top half may be left open when desired.

Increased cubic space is of little value without proper ventilation.

Flooring.- The floor of a cowshed should not in any part be below the surface of the ground. It should be properly laid and graded so as to take away all liquids falling upon it. Concrete covered with cement properly furrowed is perhaps the best flooring, because no filth can accumulate in the joints as in ordinary paving. If flags or setts are used they should be laid on a bed of concrete and jointed with cement.

Drainage.-The floor of the standing should have a fall of 2 or 3 inches from the manger to the manure channel, whilst the floor of the channel itself should have a fall outwards of at least balf-an-inch in cross section, and a fall longitudinally of half-an-inch per single stall, so as to drain away the liquids; the floor itself should have a similar fall. The channel should discharge on to a trapped gully outside the cowshed leading to a liquid manure tank. No covered drain should exist inside the cowshed.

Midden.-A proper middenstead should be provided in accordance with the bye-laws as to prevention of nuisances. Its walls should be made of hard impervious material, and the roof should be supported by means of piers, thereby providing for adequate ventilation and the exclusion of rain. The flooring should also be impervious.

Yard.-The yard and approaches to the cowshed should be properly formed, graded and paved to take away the surface water, which should not discharge into the midden.

Manger.-A half-round salt-glazed trough, rising 12 or 15 inches above the floor, is much superior to a wooden tub. A hay-rack immediately over this trough obviates the placing of fodder on the floor. Neither the foddering passage nor any part of the cowshed should be used for the storage of foods. A proper food store should be provided.

Water.-Where the water supply is not derived from a public source, great attention should be given to its protection.

General.-No poultry or pigs should be kept in any part of the cowshed, and it is desirable also that horses and calves should be excluded.

A thermometer in the cowshed is useful.

\section{Existing Cowsheds.}

There are no doubt some buildings used as cowsheds which were not originally constructed for that purpose and which cannot, except by reconstruction, be 
refer to the suggestions in the seventeenth report of the Bureau of Animal Industry at Washington, as an illustration of the official view of this matter in the United States. ${ }^{1}$

made to satisfy even the most elementary hygienic requirements. On the other hand, there are a great number of existing cowsheds which, although not fulfilling all the conditions set forth above, may yet be rendered workable by providing them with abundance of light, efficient ventilation, impervious flooring, and an adequate supply of water. These essentials CAN be secured, and should be insisted upon by the Sanitary Authority.

\section{Register.}

A Register should be kept by every Sanitary Authority in which to record the results of periodical inspections. No cowshed should be used for housing a greater number of cows than is specified on the register. Amongst other information locally desirable the following items are suggested :-

I. Name and address of person registered.

2. Situation of premises.

3. Owner of premises.

4. Number of sheds-with size, length, width, height, and cubic space per cow.

5. Number of cows in each shed.

6. Flooring-material and condition of.

7. Walls, material-rough or smooth.

8. Water supply - source, position.

9. Lighting-number and size of windows or other lights.

10. Ventilation-number and size of permanent openings. Number. and size of openings capable of regulation.

11. Mirden-distance from cowshed, open or covered, disposal of liquid manure.

12. Yard-formation, paving, drainage.

13. Cleanliness - general condition of floors, walls, midden, yard.

I4. General drainage system.

15. Disposal of drainage.

The reader may also be recommended to study the excellent notes on this subject which appear in Public Health, vol. xii., 1900, pp. 775-792. ("The Best Means of Housing Cows," by John Speir.)

1 "The stable shall be arranged with a view to the comfort of the animals, and so as to facilitate the work of cleaning, milking, etc.

"The floor shall be smooth and incapable of absorbing liquids, and sloping sufficiently to cause good drainage.

"The gutters behind the cows shall be open and with sufficient incline to cause good drainage.

"The side walls and ceiling shall be so tight as to prevent dust sifting through, and they shall be so constructed as to prevent cobwebs and dust from collecting, and easily to be cleaned.

"There shall be windows in at least two sides of the stable, providing not less than 3 square feet of unobstructed window glass to each animal. 
In the United States of America and in Denmark, great advance has already been made in respect to the management of milk farms. In Denmark it is the rule rather than the exception to find systematic control of such farms. Such control exists in England of course, but it is the exception rather than the rule. Whereas in Denmark most of the Local Authorities have framed regulations under the law of 28 th March 1868, forbidding, for example, the accumulation of dung in the vicinity of farm wells. Local regulations again provide for the protection of private or public pumps against damage, and all drainage from the dung heap and stables must be prevented from reaching the water supply.

"Each animal shall be allowed at least as many cubic feet of air space, as the number of pounds of its live weight.

"The ventilation shall be so efficient that one will not notice a stale, disagreeable, or strong animal odour on entering the building.

"The stalls shall be comfortable, at least 3 feet wide, or $3 \frac{1}{2}$ feet for a large cow, and so long that the animal need not habitually stand with feet in the gutter.

"The stable-yard shall be well drained so as to be usualiy dry, and no pools allowed to form.

"A suitable place, at least 200 feet distant from the stable building, shall be provided for cows not approved by the veterinarian, and those separated from the herd for any cause except calving.

"A special room, conveniently located, shall be provided for the milkers to wash in before and during milking.

"The stable to be kept scrupulously clean.

"The interior walls shall be kept clean and light coloured. If whitewash is used, a fresh coat shall be applied at least three times a year, and oftener if necessary, to keep the walls clean and white. Mould spots shall not be permitted.

"The accumulation of dirt, cobwebs, rubbish, and materials not needed for stable work shall not be permitted.

"At least half an hour before milking time, stables shall be thoroughly cleaned and ventilated, and manure removed from the building.

"The stable floors shall be sprinkled when necessary, to keep down the dust.

"When cows are kept in the stable continuously (as in stormy weather), it shall be cleaned often enough to be kept as free as possible from manurial odours. If necessary, land plaster shall be used for absorbing liquids and odours.

"At least once every two months, the mangers shall be scrubbed with a brush and water and soap, lye, or washing powder.

"Animals of other species shall not be kept in the same room with milch cows.

"No strong smelling material shall be allowed in or near the stable. If manure is on the premises, it shall be at least 100 feet distant from the stable."

(R. A. Pearson, Assistant Chief of Dairy Division, Bureau of Animal Industry, Washington.-Seventeenth Report, 1900, p. 158 et seq.) 
The farm drains must remove all liquid filth, and pumps must be covered and protected against access of surface water. The immediate surroundings of all pumps must be raised above the level of the adjacent ground. These are but illustrations. They are sufficient to emphasise the fact that in actual practice milk farms in Denmark are better kept and managed than similar farms in England.

The conclusion in respect to this matter, which was arrived at by two English Local Government Board inspectors as the result of a visit to Denmark may be quoted. It should be added that this conclusion is supported by very substantial evidence which we need not quote, but to which we give the reference. They report :-

"We visited during our stay a variety of cowhouses, both great and small, and the general conclusion at which we arrived was that the Danes possess a somewhat higher estimate of the value of cleanliness in the cowhouse than, according to our experience, is possessed in this country. Still, we wish to state plainly that we saw nothing in this particular alone which is not frequently attained to in England, and which, by the exercise of more care and intelligence, could not be reached in many more instances-in a word, it was not the superiority of the Danish cowhouses which impressed itself upon us so much as the intelligent manner in which the Danes make use of the often humble facilities at their disposal." 1

\section{Milkers and Milking}

Present conditions. - Visits to cowsheds where and when milking is being carried on, will reveal conditions of filth which cannot do otherwise than lead to the contamination of the milk. This is an almost universal experience in Great Britain. But if we follow the milk into the dairy, we generally find a cleanliness rendered all the more impressive by contrast with the cowshed. Yet this dairy cleanliness, essential though it is, unfortunately comes too late; the mischief has been already done by the antecedent dirt in the byre. It is in the cowshed, therefore, and at the time of milking, that the chief precautions must be taken.

Recommendations.-(a) Time of milking:-We have already pointed out that the quality of milk varies, especially as regards fat, according to the difference in the interval of time which elapses between one milking and the next. Milk yielded by the

1 Report of Departmental Committee on Food Preservatives, 1900. Appendix containing report of visit of Dr Bulstrode and Mr Huddart to Denmark, and other places. Appendix No. 3, pp. 283 and 285. 
cow in the morning is almost invariably less rich in milk fat than that yielded in the evening. But if the day be divided into two equal periods and no unnecessary disturbance of the cows occurs during the day, the difference between the evening and morning milk is a matter of small amount. The aim should therefore be, to milk at equal periods. Such an arrangement is by no means always practicable, for owing to distance from market, the exigencies of the train service, the requirements imposed on the trade by the consumer, and the difficulty of obtaining a supply of competent milkers precisely at the required times, it is impossible in some localities to arrange for milking at equal periods of time. But it would be well to bear in mind that such a course is desirable and should be adopted wherever practicable. In any case milking should be commenced at the same hour every morning and evening.

(b) Preparation of the cow. - The cow should not be excited by hard driving, abuse, or unnecessary disturbance. The entire body of the cow should be cleansed and the coat well groomed daily. The tail of the cow should be cut in winter as in Denmark if its length interferes with cleanliness. Before milking the flanks and hind quarters of the cow should be cleansed, as there can be no doubt that it is from this source that dirt gains ready access to milk in the process of milking. Such dirt, of course, abounds in bacteria. Particular care should be taken that the udder of the cow is clean before milking begins. Some milkers consider it inadvisable to wash the udder, in which case it may be wiped with a clean, damp cloth before milking. Other milkers advocate thorough washing of the udder. If the hair is long the udder should be clipped from time to time. One of the chief British Milk Companies lays down the following instructions for milking :"The greatest possible care and cleanliness shall be observed in every detail connected with the work of milking. The hind quarters and udder of the cow shall be cleaned with a brush and damp cloth before milking takes place. Soap, water, and towel shall be provided for the milkers to wash and dry their hands with before beginning to milk, and as often as necessary during milking. The practice of milking in the field during summer is strongly recommended. In autumn before the cows are housed at night the long hair on the tail, udder, and hind quarters of the cows shall be clipped. The shippons shall be well lighted, particularly behind the cows, so that the milkers may see what they are doing and whether everything is clean and in order." Many of these conditions are derived from the practice in vogue in Denmark. 
(c) The milker.-The passing of the female milker is to be regretted, for now it happens that frequently odd men about the farm are employed as milkers amongst their other functions. They are often ill-trained, and unclean in person and manipulation. Yet the milker should be clean in all respects. He should, of course, be free from infectious disease of any kind, including tuberculosis. He should thoroughly cleanse his hands just before milking, and should, if possible, wear a clean smock, used only when milking, and kept in a clean place at other times. Milkers would also do well to rinse their hands in clean water between the milking of each cow. Milking should be done quietly and thoroughly, and the first few streams from each teat thrown away, for such milk is often somewhat watery, and always contains the air bacteria which have gained access to the teat during the interval since the last milking. Milking should be done with dry hands, which should not come into contact with the milk. The Hegelund system of milking manipulation has been extensively tested in Denmark and the State of Wisconsin with much success and a marked increase of the milk yield.

\section{Treatment of Milk after Milking}

The Danes recognise in a manner and to an extent at present unknown generally in Great Britain, that the destruction, inhibition, and control of bacterial activity in milk is the basis of all successful dairying. They recognise the multitudinous opportunities which milk has of becoming polluted from the time it leaves the udder of the cow to the time it reaches the consumer's mouth, and they further recognise that milk is a highly favourable medium, and that, therefore, the bacteria which gain access multiply at an enormous rate. In consequence of this knowledge the Danes adopt three simple principles:-

First, to prevent organisms gaining access, by cleanly methods of dairying.

Secondly, to strain thoroughly.

Thirdly, to inhibit by refrigeration the growth of such organisms as may have, by accident, gained access to the milk. Clean milking, careful straining, rapid cooling-are, so to speak, the three cardinal parts of the dairy creed in Denmark.

I. Clean milking.-This desideratum is obtained in considerable degree if the cows, the milkers, and the byres are clean. With these matters we have already dealt. There remain, however, 
several points to which reference must be made. Great attention should be paid to the cleansing of the utensils and churns used in dairy work. Churns of defective construction, imperfectly fastened, and admitting of the entrance of dirt should not be used. Above all, unclean churns should be avoided. In Denmark, and also now in other parts, the farmers' cans are cleansed in water heated by the injection of steam, each farmer having his own cans, which are returned to him immediately after cleansing. As a general rule the big farms sterilise their own churns, and the small farms have this done for them by the Company taking the milk. Such a Company treats the vessels used in its establishment in the same way. Bottles are cleansed by revolving brushes applied to the interior, and finally by steam injected into the inverted bottles. Churns are also cleansed by a jet of steam. It is of the greatest importance that all buckets, pails, churns, cans, utensils, etc., used on the farm or in the dairy in connection with milk should be scrupulously clean. ${ }^{1}$ Where possible they should be sterilised by steam.

After cleansing, the utensils should be kept inverted in pure air and sunlight, if possible, until actually wanted for use. Old cans, in which parts of the tin are worn off or where there are seams and cracks, it is impossible to keep really clean, and therefore such utensils should not be employed. A second matter requiring attention is the prompt removal of the pail of milk from the cowshed where the milking has taken place. The milk should not stand in the byre, nor should it be screened in the byre. Many devices have been suggested and practised for reducing the amount of external contamination during milking. One of the most obvious, and which is to be recommended, consists in using wholly or partly covered milk pails. One such pail is said to keep out 66 per cent. of the dirt.

2. Screening or straining.-As we have seen, milk contains even under good conditions of dairying a great deal of particulate matter, and the object of screening is its removal. Hairs, dust, and particulate matter generally are likely to be coated with bacteria, and therefore must be at once removed from the milk. The Danish system is, immediately after milking, to pass the milk

1 At the Copenhagen Milk Supply Company (Busck) the cans are cleaned by brushing with hot water and soda, and later washing out with hot water and lime, a ladleful of soda, about $\frac{1}{2} \mathrm{lb}$., being used for each pail of water, and $20 \mathrm{lbs}$. of lime to 900 litres of water. The outside is then washed and steam injected into the cans. Bottles are washed with soda and water, then placed over revolving brushes, and then steam sprayed. The corks are only used once. 
into the churn through a fine metal gauze screen, covered with a clean white cloth. A company in England lays down as one of its regulations controlling farms contributing milk that each "bucketful of milk shall, as soon as drawn from the cow, be strained through a fine wire strainer covered with a clean woollen cloth." The New York Milk Commission lays down a similar condition, the metal screen to have a flannel cloth or layer of cotton. The details are matters of convenience and custom, but the principle of prompt and careful straining is essential to success. ${ }^{1}$ A similar advantage is gained by centrifugalising the milk. At the Fabrick voor Gesteriliseerde Mælk, Amsterdam, it is the practice after cooling and screening to centrifugalise the milk. On receipt at the premises in Amsterdam the milk is at once screened, and then raised to a temperature of $30^{\circ} \mathrm{C} .\left(86^{\circ} \mathrm{F}\right.$ ) in order to facilitate the centrifugalising process which follows. The milk and cream thus separated are intimately remixed subsequently, by a series of revolving rollers. In the process of centrifugalisation there is left behind on the inside of the separator the usual residue, which consists of a viscous looking mass of slime containing much débris, hair, inorganic mud, and other particulate matter which has not been arrested by straining. This separation may rightly be regarded as a cleansing operation. We have frequently made examination of the slime from the separator, and have found it crowded with bacteria. B. tuberculosis has thus been separated, and owing to this fact it is usual in Denmark to burn the slime after centrifugalisation to prevent pigs from being fed upon it. ${ }^{2}$

1 One of the best strainers now on the market is the "Ulax." See Appendix S, p. 594 .

2 Though the straining of milk may be of some service in ridding it of any large particles of dirt or other matter which it may contain, it is of but little use in removing the dust, which, after all, constitutes the great bulk of filth which dirty milk contains. The effect of straining, so far as dust is concerned, was well shown in an experiment recently carried out at one of the American agricultural stations. In this experiment it was found that though the straining of milk left it cleaner, so far as the large particles were concerned, it proved of but very little advantage in excluding dust bacteria. It was found that about 60 per cent. of the dirt from manure and the air was soluble in water, and that no amount of straining would prove of any service in depriving the milk of this soluble material. With the object of testing as to what extent dust and bacteria found their way into the milk, trials were made with milking into covered pails with apertures only 6 inches in width at the top, and into others with from two or three times that extent of opening in the pail. With the 6-inch opening it was found that the number of germs present in a certain quantity of milk was 2300 , whereas in the milk drawn into the ordinary pail with the wide top the 
3. Cooling.-After screening the milk must be cooled. The Copenhagen Milk Supply Company lay down as a standard that immediately after being drawn from the udder and during all seasons of the year, the milk must be cooled down with ice water to $40^{\circ} \mathrm{F}$, and that every contractor must be provided with a Lawrence cooler obtainable from the Company. Thirty lbs. of ice, making due allowance for water, must be kept in stock for every $100 \mathrm{lbs}$. of milk produced, calculated from the fortnightly trial milkings. The Danske Mrlke-Compagni of Frederiksberg, near Copenhagen, also insists that the farms contributing milk must have ice for cooling milk, and the milk must not reach the establishment at a temperature above $10^{\circ} \mathrm{C} .\left(50^{\circ} \mathrm{F}\right.$.).

One of the English Companies supplying pure milk has closely followed the practice in Denmark, and laid down the following regulation to be complied with by all farms contributing milk: The milk shall be immediately (after milking) cooled down irrespective of weather or season of the year to $40^{\circ} \mathrm{F}$, by passing it over a cooling apparatus of approved pattern, kept cool with ice to be provided by the farmer, and the milk shall be kept at that temperature in the milk-cans provided by the Company until time for it to be sent to the station. Should the Company not require the farmer to use ice, the milk, after being strained through a fine wire strainer covered with a clean woollen cloth, must be immediately cooled down, irrespective of weather or season of the year, to within $4^{\circ}$ of the temperature of the coolest water on the farm, by passing it over a cooling apparatus of approved pattern, and the milk shall be kept at that temperature in the milk-cans provided by the Company until time for it to be sent to the station. Where natural ice is used, at least $30 \mathrm{lbs}$. of ice shall be available for $100 \mathrm{lbs}$. (Io gallons) milk produced. In years when with reasonable care on the part of the farmer, sufficient natural ice or snow cannot be got, the Company shall deliver at the nearest station such ice as the farmer may require, at the nominal price of $12 \mathrm{~s} .6 \mathrm{~d}$. per ton.

Ice is not necessary to cooling, as combined aerators and coolers suitable for use with well water or ice water can be

number of bacteria present worked out at over 43,000 . The advantage of using the narrow-mouthed pail with the object of excluding the bacteria, in this case, was well shown by the fact, that whereas the milk drawn into the narrow-mouthed vessel did not go sour until after being kept sixty-four hours, that milked into the open pail-and subsequently found to contain 43,000 odd bacteria-went sour in forty-seven hours. 
had at any dairy supply at small cost. There are many such mechanical coolers now in the market, allowing the milk to flow in a thin layer over corrugated copper cylinders or coils, through which is passing a current of cold water. ${ }^{1}$.

Low temperatures, it is true, do not easily destroy life, but they have a most beneficial effect upon the keeping quality of milk. It has been suggested that at the outset of the process of cooling, currents of air inimical to bacteria are started in the milk. If the temperature be lowered sufficiently, the contained bacteria become inactive and torpid, and eventually are unable to multiply or produce their characteristic fermentations. At about $50^{\circ} \mathrm{F}$. $\left(10^{\circ} \mathrm{C}\right.$.) the activity ceases, and at temperatures of $45^{\circ} \mathrm{F} .\left(7^{\circ} \mathrm{C}\right.$.) and $39^{\circ} \mathrm{F} .\left(4^{\circ} \mathrm{C}\right.$ ) ) organisms are deprived of their injurious powers. If it happens that the milk is to be conveyed long distances, then even a lower temperature is desirable.

The most important point with regard to the cooling of milk is that it should take place at once. It must not be forgotten that cooling processes are not sterilising processes. They do not necessarily kill bacteria; they only inhibit activity, and under favourable circumstances the torpid bacteria may again acquire their injurious faculties. Hence, during the cooling of milk, greater care must be taken to prevent aërial contamination than is necessary during the process of sterilising milk. The cooling apparatus should be kept clean, and the cooling carried out under clean and proper conditions. Climate makes little or no difference to the practical desirability of cooling milk, yet it is obvious that less cooling will be required in the cold season.

Transport.-When ready for transport to the town, the milk, screened and cooled, should be placed in sterilised churns which should be locked or sealed. The objects gained by careful sealing will be twofold, viz., there will be no opportunity for the admission of dirt and dust to the milk, and there will be no mixing of the milk at railway stations. Consignors and vendors have frequently alleged that railway companies will not transmit sealed churns. This is incorrect. ${ }^{2}$

${ }^{1}$ Appliances of this kind may be obtained from Lawrence \& Co., $22 \mathrm{St}$ Mary Axe, London, E.C., and 132 Latimer Road, London, W.; the Dairy Supply Company, Limited, 28 Museum Street, London, W.C.; R. A. Lister \& Co., Dursley, Gloucestershire ; or any other good firm.

${ }^{2}$ The evidence given before the Departmental Committee on Milk included a letter dated 29th May 1899, in which the Assistant Secretary of the Board of Agriculture inquired of the Railway Companies' Association, through the Board of Trade, if it was their practice to require milk churns to be unlocked, 
The question of the railway transport of milk is a large one, and of some complexity. But there can be little doubt that there is need in this country for much improved arrangements and a more rapid service. Special vans should always be employed for the conveyance of milk, and refrigerators wherever possible and where the quantity to be conveyed is considerable. There is also a great lack of milksheds at stations receiving large numbers of churns, which are at present frequently left standing on station platforms in sunlight for considerable periods. Quick transit and sealed churns are also a requirement.

\section{Final Treatment of Milk}

A consideration of the methods of control and protection of milk would not be complete without mentioning some of the ways in which milk is treated after milking. The chief of these are fltration, the use of preservatives, pasteurisation, and sterilisation. But at the outset, we desire to state, that if dairy work is properly carried on and the herds, cowsheds, and milking operations are of the standard suggested in the foregoing pages, these final processes are not required for milk which is produced and consumed in the same locality. Under the circumstances of modern civilisation, however, we admit that it may be necessary that some such processes should be adopted. Milk comes into the hands of large contractors or companies for purposes of distribution to all parts of the country and under such circumstances it is deemed necessary by many authorities to filter, pasteurise, or preserve it. Personally, we are strongly of opinion that what is required is a naturally pure milk supply in contradistinction to an artificially purified milk supply. As things are at present, however, we admit every kind of protection is needed.

(a) Filtration has been practised for some time by the Copen-

and if in consequence the consignor is unable to take any precautions to prevent persons tampering with their contents. In reply, he was informed by the Railway Companies' Association, in a communication dated 12th October, and signed by Sir Henry Oakley, that "senders have been for a long time allowed to send milk in sealed cans, the companies accept the declaration of the senders as to the quantity conveyed, no extra charge being made; the only condition the companies require to be fulfilled is that the tare weight of the cans shall be stamped on the outside of the can, so that in case of doubt the quantity of milk within the churn can be approximately ascertained by allowing 101 lbs. for each gallon of milk declared. It does not appear to the companies that there is any difficulty in the senders protecting themselves against alleged Joss of milk in transit by sealing, padlocking, or otherwise fastening the cans." 
hagen Dairy Company, the Manchester Pure Milk Supply Company, Bolle of Berlin, and others. The first-named company filter the milk they receive, on their own premises, through four layers of gravel of varying sized pebbles, and three layers of cloth. There is also a small filter with two layers of gravel and two of cloth to be used for smaller amounts of milk. The process of filtration in both cases is upward, first through the lowest layers of coarse gravel, then through the intervening finer layers of gravel, and eventually through the cloth of close texture. The milk rises upwards through the filter under gravitation pressure, and from the top of the filter is run off into coolers. The filter is cleaned by washing the gravel with hydrochloric acid, also with soda and water, and is sterilised at $120^{\circ} \mathrm{C}$. after each time of using. It is then dried in a high-pressure steam oven. Another milk company near Copenhagen filters all milk received through a filter of large sized pebbles by an upward flow, just prior to being bottled. The filtering material is thrown away after each time of using. A third company, the Milk Supply Pasteur, receive all their milk into a reservoir where it is duly weighed and strained, and from this it is pumped to a filter consisting of layers of gravel and felt, the whole apparatus being carefully sterilised daily. The Manchester Pure Milk Supply Company also filtered their milk. On receipt the milk was cooled to $34^{\circ} \mathrm{F}$. in ice tanks, and then filtered through three layers of sand 2 inches deep, from which filter the milk was passed to the steriliser, cooled, and bottled. The sand of the filter was regularly cleaned, first with cold water, soda water, and hot water; then it was steamed, sieved, and dried in an oven. It was said that by means of these gravel and sand filters the number of bacteria contained in the milk was reduced by one-third. Other forms of filtration have been adopted including filtration through felt, compressed absorbent cotton, and such materials. Dr Seibert has reported that milk passed through a half-inch layer of compressed cotton removes seven-eighths of the bacteria, and a second filtration will further reduce the number to one-twentieth. A considerable quantity of extremely fine particles of dust is also waylaid in the filter. But only one quart of milk can thus be filtered in fifteen minutes. Filtering is said to have the disadvantage of holding back some fat, and is at best somewhat cumbersome technique. Hence its adoption has not been very wide.

Preservatives. - In Great Britain the addition of preservatives to milk is unfortunately widespread. In London from io to 40 
per cent. of all milk sold contains preservatives of some kind and in some degree. ${ }^{1}$ It varies with the season, being greater, of course, in summer, and as a rule greater on Sundays than week days. Fifty per cent. of the London dairymen use preservatives. In Birmingham from 5 to 20 per cent. of the milk contains preservative, in South Wales the returns are about the same. ${ }^{2}$ In a large city in Yorkshire quite 50 per cent. of the milk sold contains preservative, and somewhat generally throughout the country such substances are added, the practice being common or otherwise, according to the strictness of the supervision and action of the Local Authority. ${ }^{3}$

Milk is, however, consigned to London by certain dealers under contract prohibiting the addition of preservatives. It is carefully strained and cooled, and transmitted 120 miles without difficulty, and without souring. In Denmark the use of all preservatives in milk is strictly prohibited, and the prohibition is stringently enforced. ${ }^{4}$

The chief preservatives used in milk are boracic acid, formalin, carbonate of soda, and salicylic acid. The commonest is that named first. It has been found in milk in Great Britain, in quantities varying from 5 to 200 grains per gallon, and on occasion as much as 80 grains to the pint. Probably these large amounts have occurred owing to cumulative dosage. For it has been shown that milk is constantly subjected to successive treatments of preservative before it reaches the consumer. The farmer sometimes applies it, the wholesale purveyor or contractor does the same; and so does the retail dealer. The domestic use of preservatives is also said to be increasing, and hence milk may receive four doses of preservative before being consumed. ${ }^{5}$

There are two chief objections to the addition of preservatives to milk. The first is the ill effect the preservative may have on the consumers of the milk. Particularly will this be the case of invalids, and young children who consume milk largely, and who are most susceptible. Substances capable of destroying bacteria in milk are not likely to be indifferent in their action upon the living tissues, especially the cells supplying the digestive

1 Report of Departmental Committee on Preservatives and Colouring Matters in Food, 19or, pp. xii.-xvii.
2 Ibid., p. xvii.
3 Tbid., p. xvii.
4 Tbid., p. xxvii.

5 Ibid., p. xxvii. See also Lancet, 1899, vol. ii., pp. 1427, I 577 (Foulerton), and p. 1282 (Annett); Brit. Med. Jour., 1900, vol. ii., p. 1 ; Public Health, 1899, Pp. 554,86 I. 
juices. In this way digestive ferments essential to the human economy may be prejudicially affected. Again, on the other hand, some preservatives lessen the digestibility of foods containing them. Formalin enters into combination with the proteid constituents of the food, the compound formed being less digestible than the original substance, thereby entailing a nutritive loss to the consumer (Halliburton). ${ }^{1}$ A French Commission has reported against the use of salicylates in milk on account of their injurious effects. The second objection to preservatives in milk is that they may be, and doubtless are, relied on to protect those engaged in the trade against the immediate results of neglect of scrupulous cleanliness. Under the influence of preservatives milk may be exposed without sensible injury to conditions which otherwise would render it unsatiable. It may remain sweet to taste and smell, and yet have incorporated in it disease germs of various kinds, the activity of which may only be suspended for a time by the action of the preservative, but may be resumed subsequently.

Beside these considerable disadvantages, the gains are small. These preservatives cannot be added in quantities sufficient to kill the contained bacteria. They merely stifle them for the time being. They prevent rapid multiplication of bacteria and increasing acidity. ${ }^{2}$ They disguise the true condition of the milk in which they exist. Their organised addition to milk places a premium on uncleanly and improper dairying. For these reasons and in the light of these facts, we feel strongly that the addition of preservatives to milk should be prohibited. We find ourselves in agreement with the conclusions of the Departmental Committee which was appointed by the Local Government Board in 1899 and reported in 1901 :-

I. That the use of formaldehyde, or formalin, or preparations thereof in foods or drinks be absolutely prohibited, and that salicylic acid be not used in greater proportion than one grain per pint in

1 Report of Departmental Committee on Preservatives and Colouring Matters in Food, 19or, pp. xxiv. and xxv.

2 As a result of experiments it has been shown that milk without preservative curdles on the third day; with formalin added, I to 100,000 parts, milk curdles on the third day, but with slightly less acidity; with formalin added, I to 50,000 parts, milk curdles on the fourth day, I to 20,000 on the sixth day, I to 10,000 on the eighth day, and I to 5000 on the ninth day. The usual quantity of formalin added to milk in the United States of America is I to ro,ooo parts. See Report State Board of Health, Massachusetts, 1898, No. 34, p. 559. Tests for the various milk preservatives are described in the same report. 
liquid food, and one grain per pound in solid food, its presence in all cases to be declared.

2. That the use of any preservative or colouring matter whatever in milk offered for sale in the United Kingdom, be constituted an offence under the Sale of Food and Drugs Acts.

3. That the only preservative which it shall be lawful to use in cream be boric acid or mixtures of boric acid and borax, and in amount not exceeding 0.25 per cent. expressed as boric acid. The amount of such preservative to be notified by a label upon the vessel.

4. That the only preservative permitted to be used in butter and margarine be boric acid or mixtures of boric acid and borax, to be used in proportions not exceeding 0.5 per cent. expressed as boric acid.

5. That in the case of all dietetic preparations intended for the use of invalids or infants, chemical preservatives of all kinds be prohibited.

6. That the use of copper salts in the so-called "greening" of preserved foods be prohibited.

7. That means be provided, either by the establishment of a separate court of reference or by the imposition of more direct obligation on the Local Government Board, to exercise supervision over the use of preservatives and colouring matters in foods, and to prepare schedules of such as may be considered inimical to the public health.

\section{Pasteurisation and Sterilisation}

The chief facts respecting the effect of heat on milk have already been stated, and reference has also been made to the bactericidal properties of heat in relation to pathogenic organisms, such as tubercle bacilli. Here it only remains to discuss briefly the application of heat to milk as a protective agency, in the methods of pasteurisation and sterilisation.

Pasteurisation was introduced, as the term denotes, by Pasteur, who recommended heating to $70^{\circ} \mathrm{C}$. for a short time, as sufficient to destroy all the chief forms of adult bacilli. The thermal deathpoint of almost all organisms, pathogenic or benign, is below $70^{\circ} \mathrm{C}$. The spores were left untouched by such a temperature, and on cooling of the milk would naturally commence development. Consequently "intermittent" and "continuous " pasteurisation was recommended, alternating heat at $70^{\circ} \mathrm{C}$. with a cool period during which the spores might develop and then be killed at the 
next heating before sporulation. Thus eventually the milk would be sterilised. But the method in practice is tedious and unreliable. The term pasteurisation, therefore, is now used to designate a process of heating at any temperature between $60^{\circ} \mathrm{C}$. and $90^{\circ} \mathrm{C}$. for a short period (not exceeding twenty minutes). In practice, a temperature of between $75^{\circ}$ and $85^{\circ}$ is generally adopted. There is no fixed standard for the temperature of pasteurisation, except that it must be above the thermal death-point of pathogenic bacteria, and yet below the boiling point. As a matter of fact $70^{\circ}$ to $80^{\circ} \mathrm{C}$. will kill lactic bacteria as well as most disease-producing bacteria found in milk. If therefore the milk has been kept at that temperature for ten to fifteen minutes, it is said to have been "pasteurised." It is now held that agitation of the milk is necessary to efficient pasteurisation. B. tuberculosis in milk has been destroyed by a temperature of $60^{\circ} \mathrm{C}$. in ten minutes, the milk being agitated (Russell), but as a rule it is considered that pasteurisation is not to be relied upon as invariably bactericidal to the tubercle bacillus, and probably never destroys spores.

The methods of pasteurisation are continually being modified and improved, especially in America, Denmark, and Germany, and it will be unnecessary to burden these pages with the large body of facts and experiments we have collected during several years' study of the matter.

It will be sufficient to refer to three common methods now in vogue, and the first to be considered may well be that practised in Denmark. The milk is here commonly pasteurised in small bottles ( $\mathrm{I} \cdot 7$ pint) for twenty minutes at $75^{\circ} \mathrm{C}$. ( $167^{\circ} \mathrm{F}$.), at which temperature it is left for half an hour, and then, in order to destroy any "cooked" flavour, it is rapidly cooled down to about $10^{\circ} \mathrm{C}$. In some places the milk is not pasteurised in bottles but in vats holding 500 litres, and the temperature is $85^{\circ}$ or $90^{\circ} \mathrm{C}$., instead of $75^{\circ} \mathrm{C}$, and it is kept at such temperature for three or five minutes. This method is in vogue in various countries. In England modifications of it are in common use where milk is pasteurised. For instance, it is adopted at the Hospital for Sick Children in Great Ormond Street, which is in advance of other London hospitals in this respect. Milk is received from a well-known metropolitan dairy company in quantities of 200 quarts daily, most being delivered in the morning, and a smaller quantity in the evening. The milk is derived from healthy cows, and sanitary cowsheds, the farms being placed under strict supervision. On receipt the milk is filtered through muslin, by downward and 
upward filtration, and passed directly into a bottle-filling machine. Clean stoppered bottles are kept in readiness. When filled, the bottles are placed in circles in the cage at the bottom of the pasteuriser. Into the centre of the apparatus is placed the thermometer. The lid is closed down and clamped, and the steam is admitted from below. The temperatures used are $160^{\circ} \mathrm{F}$. (or $71^{\circ} \mathrm{C}$.) for twenty minutes in winter, and $180^{\circ} \mathrm{F}$. (or $82^{\circ} \mathrm{C}$.) for twenty minutes in summer. After the elapse of this period, the lid is removed, the stoppers of the bottles are fixed down, and hot water is admitted into the floor of the apparatus. To this hot water is slowly added cold water, and in about forty minutes the pasteurised milk has been cooled down, and is ready for use in the wards. The apparatus is readily cleansed after use, and the various parts, including the bottles, stoppers, etc., are cleaned daily. ${ }^{1}$ A somewhat similar apparatus is in use by a Health Association at York, ${ }^{2}$ which has recently started (1903) the York Infants' Milk Depôt, after the manner of the Liverpool and Battersea system. The apparatus provided for this work is one of the latest construction. It consists of an ordinary oval cylinder disinfecting chamber, having doors at both ends. The apparatus is lagged, and with outside steel casing, provided with a steam distributor inside, steam gauge, safety valve, thermometers, etc., ready for steam supply from boiler. In connection with this apparatus there is also provided a trolley of convenient size upon three wheels, together with a steel frame holding three separate platforms, which can be taken apart to suit bottles or vessels of larger sizes. This frame is mounted also upon wheels running in grooves, and channels are fitted inside the steriliser to correspond. The steam rises around the bottles from the bottom of the cylinder. The trolley is fitted for both ends, and when duplicated a "charge" can be taken from one end of the apparatus, and a fresh one inserted at the other. This apparatus can be used as a steriliser or a pasteuriser.

A second kind of method is typified in what is known as Lawrence's pasteuriser. This consists of a vessel with fluted walls, over the outside of which flows a thin layer of milk, whilst

1 This apparatus was constructed by L. de Wyttenbach, Torrington Square, London, W.C.

2 The York Health and Housing Reform Association, established Igor. Secretary, Miss Hutchinson, 63 Gillygate, York. Apparatus by Wyttenbach : A central depôt in Gillygate, and branch depôts elsewhere in the city. For conditions controlling source of milk used by the York Society, see Appendix T, p. 596. 
the inside is filled with water kept at or near the boiling point. Steam is used in some forms of apparatus instead of water, and the surface may be flat or corrugated, or even a revolving cylinder. But the principle is the same. Care has to be taken that the stream of milk is not too rapid, otherwise it cannot reach the necessary temperature. A combined Lawrence pasteuriser and cooler is constructed on the same basis, which possesses certain advantages. It consists of two machines, the one a scalder and the other a refrigerator. The milk or cream is placed in a receiver whence it flows over the scalder, where it is heated to within a few degrees of the boiling point. The hot or scalded milk or cream is then allowed to run over the refrigerator, and is cooled to within $2^{\circ}$ or $3^{\circ} \mathrm{F}$. of the water rising within. The heat in the scalder is maintained by the water in constant circulation heated by a steam jet, so that the temperature is never raised to boiling point, and thus the objectionable flavour of boiled milk is avoided.

A third form of pasteuriser which was described by H. L. Russell of Wisconsin, consists of a scalder, a water cooler, and an ice cooler. The pasteuriser is heated by hot water in the outside casement. Agitation is obtained by movable rods suspended vertically in the milk, and thus the heat is rapidly diffused through the whole mass. The pasteurised milk is led at once into the coolers without exposure to air. The coolers are of two kinds, which may be used separately or conjointly. In one set of cylinders there is cold circulating water, in the other finely crushed ice. ${ }^{1}$

Summary.-Without entering into a long discussion upon the various methods adopted, we may summarise some of the chief essential conditions. It need scarcely be said that the operation must be efficiently conducted, and in such a way as to maintain absolute control over the time and temperature. The apparatus should be simple enough to be easily cleaned and sterilised, and economical in use. Arrangements must also be made to protect the milk from re-infection during and after the process. The entire preparation of the milk for market may be summed up in four items :-

I. Pasteurisation in heating apparatus.

2. Rapid cooling in water or ice coolers.

${ }^{1}$ Report from Wisconsin Agricultural Expt. Sta., 1896. Many modifications of this system are in vogue in England and Denmark as well as America, especially under the management of large dairy companies. The important points in either of the three methods referred to above, are (I) exact and clean management of details; and (2) control of the source and handling of the milk to be pasteurised. 
3. All cans, pails, bottles, and other utensils to be thoroughly sterilised in steam before use.

4. The prepared milk to be placed in sterilised bottles and sealed up.

The quality of the milk to be pasteurised is an important point. All milks are not equally suited for the purpose, and those containing a large quantity of contamination, especially of spores, are distinctly unsuitable. Such milks, to be purified, must be sterilised. Dr Russell has laid down a standard test for the degree of contamination which may be corrected by pasteurisation by estimating the degree of acidity; a low acidity (e.g. 0.2 per cent.) usually indicating a smaller number of spore-bearing germs than that which contains a high percentage of acid.

Lastly, while the heating process is, of course, the essential feature of efficient pasteurisation, it must not be forgotten that rapid and thorough cooling is almost equally important. As we have seen, pasteurisation differs from complete sterilisation in that it leaves behind a certain number of organisms or their spores. Cooling inhibits the germination and growth of this organismal residue. If, after the heating process, the milk is cooled and kept in a refrigerator, it will probably keep sweet from three to six days, and may do so for three weeks.

Domestic pasteurisation.-For domestic use there are various forms of apparatus. Many of them are constructed on the waterbath principle of one vessel containing milk, inside another containing water. Some are steam jacketed pans with revolving stirrers; others have automatic arrangements for the purpose of avoiding a boiling temperature, and adapted for use with fire, gas burners, or spirit lamps.

In the opinion of those best able to judge, the Aymard Milk Steriliser is one of the most satisfactory and efficient of household sterilisers. It is made of rolled copper heavily coated with tin, and constructed on the water-bath principle, the milk being placed in the inner vessel. Hot water to the depth of about $\frac{1}{2}$ inch is placed in the lower saucepan, covered with the lid and allowed to boil. Next the lid is removed, and the milk chamber (containing the requisite quantity of milk) placed in position, the large lid on the top. Steam will be given off freely from the lid; in the I pint size in about ten minutes. To avoid any formation of scum, the milk must be cooled by placing the upper portion of the steriliser in a basin of cold water, care being taken not to remove the inner lid until the milk is cooled. This will take, for the I pint size, 
about five minutes. The milk can now be poured off into a clean jug, and protected from the air by covering with a clean cloth.

For cleansing, soda is not required, but the chamber should be filled with warm water at once, and wiped out quite clean. The lids should be left off the steriliser until required for further use.

In the larger sizes it is necessary to stir occasionally during heating and cooling by drawing the handle of the stirrer up and down the spout. The milk is distinctly more palatable than when boiled. The method is simple and efficacious and avoids the difficulty of the separation of the fatty constituents, which occurs in the so-called "bottle sterilisers." The milk chamber acts as a condenser for the steam generated, and no steam is given off from the lids until the milk has reached the temperature of $195^{\circ} \mathrm{F}$. If left on the fire, the milk can only run up to $212^{\circ} \mathrm{F}$. The boiling point of milk is $213^{\circ} \mathrm{F}$.

In another form of domestic steriliser which is intended for use on an ordinary fire, a tube, which carries an alarm bell at the top, is inserted through the lid of the enamelled saucepan. When the desired temperature $\left(85^{\circ} \mathrm{C}\right.$. or $185^{\circ} \mathrm{F}$.) is reached, a trigger contained in the tube is automatically released, and the bell rings, thus warning the attendant to take the pan off the fire. In another form the action is entirely automatic. A saucepan containing the sensitive trigger is placed on a gas stove, and when the proper temperature is reached, the release of the trigger causes the supply of gas to be automatically cut off. A third is arranged for use with a spirit lamp. When the necessary temperature is reached the release of the trigger brings a cap over the lamp and extinguishes the flame.

In pasteurisation the taste and nutritive quality of the milk is but little altered, although it does not look so "rich" as unpasteurised milk. It is a method much practised in Amsterdam, and is said to have resulted in bringing about a reduction in infant mortality due to milk-borne disease. In Denmark pasteurisation is very general, though not universal. Some authorities maintain that pasteurisation, and, indeed, all forms of sterilisation of milk are harmful to children consuming such milk. It is said that infantile scurvy and malnutrition follow the consumption of milk which has been submitted to prolonged heating at a high temperature (Barlow, Ransom, Still, and others). It is for these reasons, so it is alleged, that some milk supply companies do not sterilise their milk. Physiologically, prolonged heating of milk is likely to alter the lact-albumen and render less digestible the proteids in 
milk, but any specific toxic action brought about by boiling milk is probably very slight (Halliburton). There is a further disadvantage which must not be lost sight of. Pasteurisation kills off the normal acid-producing organisms in milk, and, in addition, many ordinary saprophytic and fermentative bacteria, but it cannot be relied upon to destroy the more resistant, which are therefore left behind in the milk unchecked by their antagonists, and under favourable conditions for multiplication. Flügge and other workers have isolated such organisms, some of them anaërobic and sporulating, from pasteurised milk.

Results of pasteurisation.-Before leaving this subject we may glance for a moment at the bacterial results of pasteurisation and sterilisation. The two chief of these are the enhanced keeping quality and the removal of disease-producing germs. The former is due in part to the latter, and also to the removal of the lactic acid and other fermentative bacteria. As a general rule, these bacteria do not produce spores, and hence they are easily annihilated by pasteurisation. True, a number of indifferent bacteria are untouched, and also some of the peptonising species. The cooling itself contributes to the increased keeping power of the milk, especially in transit to the consumer.

Pasteurised milks have the following three economical and commercial advantages over sterilised milks, namely, $(a)$ they are more digestible; $(b)$ the flavour is not altered; $(c)$ and the fat and the lact-albumen are unchanged. Professor Hunter Stewart of Edinburgh, about three years ago, compiled from a number of experiments the following instructive and comprehensive table :-

\begin{tabular}{|c|c|c|c|c|c|c|}
\hline $\begin{array}{l}\text { No. of } \\
\text { Experi- } \\
\text { ments. }\end{array}$ & $\begin{array}{c}\text { Average No. } \\
\text { of } \\
\text { Microbes } \\
\text { per c.c. in } \\
\text { Millk before } \\
\text { Treatment. }\end{array}$ & $\begin{array}{c}\text { Temperature } \\
\text { and } \\
\text { Duration } \\
\text { of } \\
\text { Pasteurisation } \\
\text { in minutas. }\end{array}$ & $\begin{array}{l}\text { No. of Microbes } \\
\text { per c.e. in } \\
\text { Pasteurised Mink } \\
\text { after } 24 \text { hours. }\end{array}$ & $\begin{array}{c}\text { Solnble } \\
\text { Albumen } \\
\text { in } \\
\text { Fresh } \\
\text { Mullk } \\
\text { per cent. }\end{array}$ & $\begin{array}{c}\text { Boluble } \\
\text { Albumen } \\
\text { in } \\
\text { Pasteurised } \\
\text { Millk } \\
\text { per cont. }\end{array}$ & $\begin{array}{l}\text { Taste of } \\
\text { Pastenrised Milk. }\end{array}$ \\
\hline 5 & I 36,262 & Ió $60^{\circ} \mathrm{C}$. & I722 average & 0.423 & 0.418 & Unaffected. \\
\hline 4 & 53,656 & $30^{\circ} 60^{\circ} \mathrm{C} .\{$ & $\begin{array}{l}\text { I sterile } \\
3 \text { averaged } 955\end{array}$ & 0.435 & 0.427 & " \\
\hline 12 & 78,562 & I0' $65^{\circ}$ & $\begin{array}{l}6 \text { sterile } \\
6 \text { averaged } 686\end{array}$ & 0.395 & 0.362\{ & $\begin{array}{c}\text { Not appreciably } \\
\text { affected. }\end{array}$ \\
\hline 12 & I 32,833 & $30^{\prime} 65^{\circ} \mathrm{C} .\{$ & $\begin{array}{l}9 \text { sterile } \\
3 \text { averaged } 233\end{array}$ & 0.395 & 0.333 & (5) \\
\hline 13 & 49,867 & $10^{\circ} 70^{\circ} \mathrm{C}$. & sterile & 0.422 & 0.269 & Slightly boiled. \\
\hline 9 & $3^{8}, 320$ & $30^{\circ} 70^{\circ} \mathrm{C}$. & " & $0.42 I$ & 0.253 & \\
\hline 2 & 77,062 & $10^{\prime} 75^{\circ} \mathrm{C}$ & " & 0.38 & 0.07 & Boiled. \\
\hline 3 & 48,250 & $30^{\circ} 75^{\circ} \mathrm{C}$ & " & $0.3^{8}$ & 0.05 & $n$ \\
\hline I & $1,107,000$ & $10^{\prime} 80^{\circ} \mathrm{C}$. & $"$ & 0.375 & 0.00 & $"$ \\
\hline I & $I, 107,000$ & $30^{\circ} 80^{\circ} \mathrm{C}$. & $n$ & 0.375 & 0.00 & $"$ \\
\hline
\end{tabular}


It will be admitted that this table exhibits much in favour of pasteurisation; yet the crucial test must ever be the effect upon pathogenic bacteria. Flugge has conducted a series of experiments upon the destruction of bacteria in milk, and he states that a temperature of $158^{\circ} \mathrm{F}$. $\left(70^{\circ} \mathrm{C}\right.$.) maintained for thirty minutes will kill the specific organisms of tubercle, diphtheria, typhoid fever, and cholera. Macfadyen and Hewlett have demonstrated, ${ }^{1}$ by sudden alternate heating and cooling, that $70^{\circ} \mathrm{C}$. maintained for half a minute is generally sufficient to kill suppurative organisms and such virulent types of pathogenic bacteria as B. diphtheria, B. typhosus, and B. tuberculosis. ${ }^{2}$

Respecting the numerical diminution of bacteria brought about by pasteurisation and sterilisation respectively, we may take the following two sets of experiments. Dr H. L. Russell ${ }^{3}$ tabulates the immediate results of pasteurisation as follows :-

\begin{tabular}{|c|c|c|c|c|c|c|}
\hline \multicolumn{7}{|c|}{ Number of Micro-organisms per cubic centimetre. } \\
\hline & \multicolumn{3}{|c|}{ Unpasteurised. } & \multicolumn{3}{|c|}{ Pasteurised. } \\
\hline & Minimum. & Maximum. & Average. & Minimum. & Maximum. & Average. \\
\hline Full cream milk & 25,300 & $18,827,000$ & $3,674,000$ & $\circ$ & 37,500 & 6,140 \\
\hline Cream . . & 425,000 & $32,800,000$ & $8,700,0 \mathrm{co}$ & $\circ$ & 57,000 & 24,250 \\
\hline
\end{tabular}

As regards the later effect of the process, he states that in fifteen samples of pasteurised milk examined from November to December, nine of them revealed no organisms, or so few that they might almost be regarded as sterile; in those samples examined after January the lowest number was 100 germs per c.c., while the average was nearly 5000. With the pasteurised cream a similar condition was to be observed. Other workers hold that from 95 to 99 per cent. of all bacteria are removed by pasteurisation.

Sterilisation, as a term, means that milk has been boiled, with or without pressure, for a sufficient period of time to kill all. bacteria and their spores. Probably only a small amount of "sterilisation" reaches this standard. Nevertheless such is the standard and it is obtainable in one of two ways ; either by heating

1 Jenner Institute of Preventive Medicine, Transactions (First series).

2 See also Archives of Pediatrics, 1896 (August), R. G. Freeman.

${ }^{3}$ Centralblatt fïr Bakteriologie, etc., 1 I Abteilung. 
considerably above the boiling point and maintaining such a temperature for some minutes, or by intermittent or discontinuous heating. Both methods have disadvantages. The former requires special apparatus (autoclave), and has an unfavourable effect upon the milk, the latter is tedious and cumbersome. Practically speaking the ordinary sterilisation which is used in general is boiling (at $212^{\circ} \mathrm{F}$.), and in some quarters the demand for such milk is increasing. Undoubtedly, the only safe, simple, practicable method for domestic protection against infected milk is to boil it, and we have no hesitation at all in recommending the private consumer, either to treat his milk as described on pp. Io or 537 as domestic pasteurisation, or to boil it. If all dairy work were properly done we hold such boiling would not be necessary, but under the present circumstances this form of simple "sterilisation" is advisable.

The application of sterilisation to milk is now very widely adopted. We have already noted its adoption by Liverpool and other Corporations for special purposes, by many of the great dairy companies, and by domestic custom. Recently we have had the opportunity of studying an excellent system in vogue in Essex, ${ }^{1}$ and which we mention in particular because it seems to us to emphasise principles which might be practised all over England. Briefly it may be said that Mr Carson's system lays emphasis on five chief points :-

1. The cows used are carefully selected for milking purposes, are regularly inspected, and have been tested with the tuberculin test. The farm is well kept and maintained under strict sanitary control, the health and cleanliness of the cows being the principal thing aimed at.

2. The cows are milked in the byre adjoining the sterilising plant. The udders are cleansed before milking, and it is required that milkers also should be clean in person and management of milking.

3. Immediately after milking, the milk is removed into an adjoining room, strained through a metal screen, and at once separated by an ordinary Laval separator. This separation is adopted for purposes of purification only. The milk and cream are again mixed, and poured by means of a mechanical automatic bottle-filler into bottles.

4. The milk in bottles is then, within a few minutes of leaving the udder, placed in the steriliser and maintained at $212^{\circ} \mathrm{F}$. for

1 J. Carson, Crystalbrook Farm, Theydon Bois, Essex. 
twenty minutes. The bottles have been previously sterilised at $220^{\circ} \mathrm{F}$. for sixty minutes.

5. After sterilisation the milk is cooled to $53^{\circ} \mathrm{F}$. and kept at that temperature till required for delivery.

We have examined this milk chemically and bacteriologically, and have found it to be of excellent quality. It is unquestionably an advantage to have milk which is to be sterilised, brought under treatment at once after milking. This cannot always be done, and hence it is the custom of some dairy companies and institutions (to which reference has been made) to sterilise milk on its delivery. There are a large number of appliances and different forms of apparatus now on the market, having for their object the sterilisation of milk. Our object has not been the recommendation of any special apparatus or process, but an explanation of the principles underlying the efficient pasteurisation and sterilisation of milk.

The retail sale of milk, etc.-Much still remains to be done to improve the conditions under which milk is sold by retail in milk-shops, hawked in the streets on milk-barrows, or stored in the house. It is unnecessary to say that it is of great importance that milk should only be stored in sanitary premises, where no effluvia or contamination may gain access to it. Further, it should be stored in clean-covered vessels in a cool place, free from dust. If it is necessary to sell milk from counter-pans, such vessels should be kept scrupulously clean, and always covered with a muslin or other light cover. The counter-pan should be cleansed daily with boiling water or steam. The "dippers" and other cans and utensils used in manipulating the milk should be treated in the same way. The whole milk-shop should be maintained in a strictly clean manner. Dust and fies are the two chief agencies of pollution of milk in milk-shops and in the house, and measures should be directed towards reducing such agencies to a minimum. Cases of infectious disease, sore throat, epidemic diarrhœa, etc., occurring at the milk-shop should be at once reported to the Sanitary Authority.

The education of the public, and particularly of girls and young women, as to the importance of a pure milk supply, and the necessity of feeding infants on clean milk from clean milk bottles, should not be any longer neglected. Such education in elementary schools and in the homes of the people is an important measure of preventive medicine, and one likely to yield beneficial results. 


\section{Summary of Recommendations.}

For the sake of convenience we may broadly summarise our recommendations for the control of the milk supply very briefly as follows :-

I. Legislation having for its object the registration and regulation of milk-sellers and dairymen should be enforced by law and not left to be adopted or not by the Local Authorities. In the first place, registration should be granted only to suitable premises and management; in the second place, healthy milch herds, clean dairying, sufficient water supply, etc., should be looked upon not as luxuries, but as essential to a pure milk supply. Legislation now on the statute book requires systematising and enforcing uniformly throughout the country. Milk contractors should be included under the same restrictions as milk sellers. There are also a number of minor alterations in legislation required, particularly in respect to the expeditious stamping out of milkborne epidemics. The one great aim of all such legislation should be to obtain such a regular, uniform, and systematic control of the milk supply that outbreaks of disease are prevented from occurring, and a pure milk supply is ensured.

2. The sampling of milk under the Sale of Food and Drugs Act should be carried out not only at the milk-shop but when the milk is at the farm, whilst in transit, before it reaches the contractor, and between the contractor and vendor. Bacteriological examinations should also be made periodically, though the time has probably not yet come for fixing a minimum standard of the number of organisms permissible, or for prosecution if that standard be passed. The addition of preservatives should be restricted, if prohibition is found to be impracticable.

3. The railway companies should provide special vans and refrigerators, and also milk-sheds at stations having a large milk traffic. Quick transit and sealed churns are also desiderata, at all events on some lines and in some districts.

4. Education of public opinion as to the importance of a pure milk supply, and the practicability of obtaining it by private and trade enterprise on public demand, and the training of girls in domestic hygiene, should be looked upon as an essential requirement in any radical reform of the milk supply. Cleanliness in everything that concerns milk at the farm, in transit, and in the home, is the thing most needed. 

A P PENDICES 



\section{APPENDIX A}

\section{ELEMENTARY GLASS BLOWING WITH BUNSEN FLAME ${ }^{?}$}

The glass used should be that known as "soft," "soda," or Thuringian glass. It is supplied in tube or rod form by all dealers in laboratory apparatus, and in lengths of from 4 to 5 feet.

I. Pasteur pipette.-Method of preparation.-(I) Lengths of soft glass tubing of about $25 \mathrm{~cm}$. in length and 5-7 mm. internal diameter as shown at $a$ on Fig. 35, are plugged lightly at each end with cotton-wool, and then sterilised in the hot air steriliser at $150^{\circ} \mathrm{C}$. for one hour. A number of these should be prepared at the same time and held ready in a box or other receptacle. The ordinary lengths of glass tubing as supplied by laboratory furnishers will be found to cut up into four of these lengths, each of which will make two pipettes.

(2) Holding the tube near its extremities between the first two fingers and thumb of each hand, and at an angle of about $30^{\circ}$ from the vertical so as to

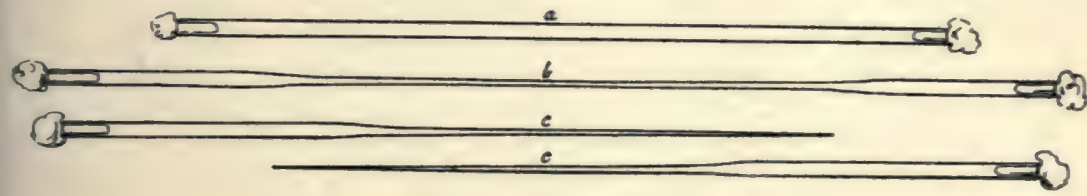

Fro. 85.-Method of making a Pasteur pipetto.

utilise as great a length of flame as possible, turn it steadily with a constant motion in the large flame of a Bunsen burner in such a manner that 3 to 4 inches in the centre of the tube will soften under the influence of the heat.

(3) As soon as the glass is sufficiently softened, withdraw the tube from the flame, and, holding it horizontally, draw the ends steadily apart until the heated portion assumes the form shown at $b$ on the figure. The interior dimensions of the capillary portions of the pipette will depend upon ( $I$ ) the length of tube which has been softened, and (2) the rapidity with which the above action is carried out. The smaller the portion of the tube heated, and the more rapid the drawing apart, the finer will be the capillary portion of the pipette.

(4) Divide the drawn-out portion of the tube at its centre and seal off the

1 The ordinary laboratory Bunsen burner will be found sufficient, but one giving as large a flame as possible is recommended. 
ends of each in the flame. Two sterile pipettes with sealed ends are thus formed, as shown at $c$ on the figure.

2. To make a bulb pipette.-Take a Pasteur pipette as described above, and holding it horizontally between the thumb and fingers of each hand, turn it constantly and with a regular motion in the flame at the point at which it is desired to make the bulb. As the glass at this point softens under the influence of the heat it must be "gathered" by gentle pressure inwards, without however interfering with the turning motion. When thoroughly softened, remove from the flame, and holding the pipette at an angle of about $45^{\circ}$ from the vertical, blow down the tube with a succession of short "puffs," still maintaining the turning motion with the fingers. As the glass cools the pipette portion below the bulb can be set to any desired angle with the fingers.

3. To make a constriction in a tube.- Hold the tube in a horizontal position and turn it constantly and with a regular motion in the point of the Bunsen flame at the spot at which it is desired to make the constriction. As soon as the glass has sufficiently softened, remove from the flame and draw the hands steadily apart until the desired constriction is made, maintaining the turning motion with the fingers until the glass has set. The constriction will be abrupt or elongated according to the size of the flame to which the tube is exposed.

4. To constrict, or close, the opening of a tube of small dimensions.-Holding the tube with its extremity in the edge of the hottest portion of the Bunsen flame, turn it constantly with the fingers until the orifice is seen to contract to the extent required. If it is desired to close the tube entirely, continue the motion until the fusion of the glass is complete and the extremity of the tube has taken a hemispherical form.

5. To bend a tube of small diameter.-This is preferably done in the flame of an ordinary batswing burner, as the Bunsen flame is apt to produce too sharp and weak a bend, and the soot which gathers on the bent portion of the tube is of advantage in preventing the too rapid cooling and consequent cracking of the tube through unequal contraction. The tube must be rotated slowly and with an even motion between the fingers whilst in the flame, and when it has become sufficiently pliable is removed and bent to the required angle. Should $a$ " $U$ " bend be required, it should be made by bringing the hands together in an upward direction, i.e. with the two ends of the tube in a vertical position and the bend downwards. If too much heat has been applied the sides of the bend will have a tendency to flatten. Should this tendency be observed it can in a great measure be obviated by closing up one end of the tube with the finger, and while the glass is still soft, blowing somewhat forcibly down the other. Should a bend of more than ordinary neatness be desired it can be obtained by filling the tube with fine dry sand and closing the ends with small corks, or wooden stoppers, before submitting it to the action of the flame.

6. Capillary tubes and glass inoculating needles.-These are made by heating glass tubing or rod in the flame as described above under the head of Pasteur pipettes. When the glass has thoroughly softened, withdraw from the flame, and holding the tube tightly between the fingers, separate the hands in a horizontal line with a steady motion until the desired fineness is obtained.

7. To cut glass tubing or rod.-In the case of tubes or rods of small diameter it will be sufficient to make a deep scratch or notch at the desired point with a small triangular file, or steel knife especially tempered for this purpose. The notch should be made by holding the tube in the left hand, the file or knife 
being supported by the third and fourth fingers of the right. The tube should be placed at right angles upon the cutting edge, and pressed upon it with the right thumb. It is then turned with the left hand in such a manner that about one-sixth of its circumference comes in contact with the cutting edge. If the tube is now grasped in both hands with the thumb placed at the opposite side of the notch (which should be outwards) and a gentle pressure be applied as though it were desired to bend the glass at the point of the notch, a clean and neat fracture will be the result. The sharp edges however should in every case be rounded off by turning the extreme ends in the edge of the flame.

If the tube is of larger dimensions a different procedure must be adopted. The simplest method is to bend a length of iron wire in the form of a semicircular hook of the same diameter as the tube it is desired to cut. The wire is heated to redness, and held firmly with the opening of the hook upwards. The tube is then placed in the hook and turned gently against the heated wire. If simple contact with the hot iron is insufficient, the tube should be removed after having been made to turn several times against it, and the heated portion of the tube touched with a cold iron. A clean circular fracture should result if the operations have been carefully performed.

The above manipulations are such as can be carried out with the aid of the Bunsen flame alone. For the welding of tubes and other more advanced work some form of blow pipe is essential, and a good text-book on the subject should be consulted.

\section{THERMOMETER}

Comparison of degrees Centigrade and degrees Fahrenheit.

\begin{tabular}{|c|c|c|c|c|c|c|c|c|c|c|c|c|c|c|c|}
\hline है & है & ڤ્ & 氞 & है & है & छُ & 苟 & ठُّ & है & 苞 & 苞 & s. & 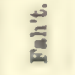 & है & है \\
\hline 0. & 32. & 13. & $55 \cdot 4$ & 26. & 78.8 & $39^{\circ}$ & $102 \cdot 2$ & 52. & 125.6 & $65^{\circ}$ & $149^{\circ}$ & 78. & 172.4 & 91. & 195.8 \\
\hline I. & 33.8 & $14^{\circ}$ & $57 \cdot 2$ & $27^{\circ}$ & 80.6 & $4^{\circ}$ & 104. & $53^{\circ}$ & $127 \cdot 4$ & 66. & 50.8 & $79^{\circ}$ & $174 \cdot 2$ & 92. & 197.6 \\
\hline 2. & $35 \cdot 6$ & I5. & $59^{\circ}$ & 28. & $82 \cdot 4$ & $4 I$. & 105.8 & $54^{\circ}$ & $129 \cdot 2$ & 67. & 152.6 & 80. & 176. & $93^{\circ}$ & 199.4 \\
\hline 3. & $37 \cdot 4$ & 16. & 60.8 & $29^{\circ}$ & $84 \cdot 2$ & 42. & 107.6 & $55^{\circ}$ & $13 \%$ & 68. & I 54.4 & $8 \mathrm{I}$. & 177.8 & $94^{\circ}$ & $20 \mathrm{I} \cdot 2$ \\
\hline 4 & $39 \cdot 2$ & $17^{\circ}$ & 62.6 & 30. & 86. & $43^{\circ}$ & $109 \cdot 4$ & $5^{6}$. & I 32.8 & 69. & 156.2 & 82. & 179.6 & 95. & $203^{\circ}$ \\
\hline 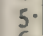 & 41. & 18. & $64 \cdot 4$ & $3 \mathrm{I}$. & 87.8 & $44^{\circ}$ & III $\cdot 2$ & $57^{\circ}$ & 134.6 & 70 & 158. & 83. & 181.4 & 96. & 204.8 \\
\hline 6 & 42.8 & I9. & $66 \cdot 2$ & 32. & $89 \cdot 6$ & $45^{\circ}$ & I1 $33^{\circ}$ & 58. & 136.4 & 71. & 159.8 & $84^{\circ}$ & 183.2 & $97^{\circ}$ & $206 \cdot 6$ \\
\hline 7. & $44 \cdot 6$ & 20. & 68. & $33^{\circ}$ & $9 I \cdot 4$ & 46. & $\mathrm{II}_{4} \cdot 8$ & $59^{\circ}$ & 138.2 & 72. & 161.6 & $85^{\circ}$ & 18 & 98. & 208.4 \\
\hline . & $4^{6} \cdot 4$ & 21. & 69.8 & $34^{\circ}$ & $92 \cdot 2$ & $47^{\circ}$ & $116-6$ & 60. & T 40. & 73. & 163.4 & 86. & 186.8 & 99. & 210.2 \\
\hline 9. & $4^{8 \cdot 2}$ & $22^{\circ}$ & $71 \cdot 6$ & $35^{\circ}$ & 95. & 48. & II 8.4 & $6 \mathrm{I}$. & 141.8 & $74^{\circ}$ & 165.2 & 87. & 188.6 & 100. & 212. \\
\hline I0. & 50. & $23^{\circ}$ & 73.4 & 36. & 96.8 & 49. & $120 \cdot 2$ & 62. & 143.6 & $75^{\circ}$ & $167^{\circ}$ & 88. & 190.4 & $\cdots$ & $\ldots$ \\
\hline II. & 51.8 & $24^{\circ}$ & $75 \cdot 2$ & $37^{\circ}$ & 98.6 & $50^{\circ}$ & I 22. & $63^{\circ}$ & $145 \cdot 4$ & 76. & 168.8 & 89. & 192.2 & . & .. \\
\hline 12 & $53 \cdot 6$ & $25^{\circ}$ & $77^{\circ}$ & 38. & 100.4 & $5 \mathrm{I}^{\circ}$ & 123.8 & $64^{\circ}$ & $147 \cdot 2$ & $77^{\circ}$ & 170.6 & 90. & $194^{\circ}$ & ... & $\ldots$ \\
\hline
\end{tabular}

To convert degrees Centigrade to Fahrenheit-multiply by 9 , divide by 5 , and add 32.

To convert degrees Fahrenheit to Centigrade-deduct 32, multiply by 5 , and divide by 9 . 


\section{APPENDIX B}

\section{THE CONTAGIOUS DISEASES (ANIMALS) ACT, 1878}

An Act for making better provision respecting Contagious and Infectious Diseases of Cattle and other Animals; and for other purposes.

34. The Privy Council may from time to time make such general or special orders as they think fit, subject and according to the provisions of the Act, for the following purposes, or any of them :-

(i) For the registration with the local authority of all persons carrying on the trade of cowkeepers, dairymen, or purveyors of milk.

(ii) For the inspection of cattle in dairies, and for prescribing and regulating the lighting, ventilation, cleansing, drainage, and water-supply of dairies and cowsheds in the occupation of persons following the trade of cowkeepers or dairymen.

(iii) For securing the cleanliness of milk-stores, milk-shops, and of milkvessels used for containing milk for sale by such persons.

(iv) For prescribing precautions to be taken for protecting milk against infection or contamination.

(v) For authorising a Local Authority to make Regulations for the purposes aforesaid, or any of them, subject to such conditions (if any) as the Privy Council prescribe. 


\section{APPENDIX C}

\section{PUBLIC HEALTH (LONDON) ACT, I891}

\section{Registration, Inspection, etc.}

Section 28.-(1) The Local Government Board may make such general or special orders as they think fit for the following purposes, or any of them, that is to say :-

(a) For the registration with the County Council of all persons carrying on the trade of dairymen.

(b) For the inspection of cattle in dairies, and for prescribing and regulating the lighting, ventilating, cleansing, drainage, and water-supply of dairies in the occupation of persons carrying on the trade of dairymen.

(c) For securing the cleanliness of milk-vessels used for containing milk for sale by such persons.

(d) For prescribing precautions to be taken for protecting milk against infection or contamination.

(e) For authorising the County Council to make byelaws for the purposes aforesaid, or any of them.

(2) The County Council for the purpose of enforcing the said orders and any byelaws made thereunder, shall have the same right to be admitted to any premises as a Sanitary Authority have under this Act for the purpose of examining as to the existence of a nuisance liable to be dealt with summarily, and the provisions of this Act shall apply accordingly as if they were herein re-enacted and in terms made applicable to this section, and in particular with the substitution of the County Council for the Sanitary Authority.

(3) The Local Government Board may, by any such order, impose the like fines for offences against orders made under this section as may be imposed for offences against the byelaws of a Sanitary Authority under this Act.

(4) In the application of this section to the City of London, the mayor, commonalty, and citizens of the City acting by the Council shall be substituted for the County Council, and their expenses in the execution of this section shall be paid out of the consolidated rate.

\section{Inspection and Seizure of Unsound Food}

Section 47.-(r) Any Medical Officer of Health or Sanitary Inspector may at all reasonable times enter any premises and inspect and examine

(a) any animal intended for the food of man which is exposed for sale, or deposited in any place for the purpose of sale, or of preparation for sale, and 551 
(b) any article, whether solid or liquid, intended for the food of man and sold or exposed for sale or deposited in any place for the purpose of sale or of preparation for sale,

the proof that the same was not exposed or deposited for any such purpose or was not intended for the food of man, resting with the person charged; and if any such animal or article appears to such Medical Officer or Inspector to be diseased, or unsound, or unwholesome, or unfit for the food of man he may seize and carry away the same himself, or by an assistant, in order to have the same dealt with by a justice.

(2) If it appears to a justice that any animal or article which has been seized or is liable to be seized under this section is diseased, or unsound, or unwholesome, or unfit for the food of man, he shall condemn the same, and order it to be destroyed or so disposed of as to prevent it from being exposed for sale or used for the food of man; and the person to whom the same belongs or did belong at the time of sale or exposure for sale, or deposit for the purpose of sale or preparation for sale, or in whose possession or on whose premises the same was found shall be liable on summary conviction to a fine not exceeding fifty pounds for every animal, or article, or if the article consists of fruit, vegetables, corn, bread or flour, for every parcel thereof so condemned, or, at the discretion of the court, without the infliction of a fine, to imprisonment for a time of not more than six months with or without hard labour.

(3) Where it is shown that any article liable to be seized under this section, and found in the possession of any person, was purchased by him from another person for the food of man, and when so purchased was in such a condition as to be liable to be seized and condemned under this section, the person who so sold the same shall be liable to the fine and imprisonment above mentioned, unless he proves that at the time he sold the said article he did not know, and had no reason to believe, that it was in such condition.

(4) Where a person convicted of an offence under this section has been within twelve months previously convicted of an offence under this section, the court may, if it thinks fit, and finds that he knowingly and wilfully committed both such offences, order that a notice of the facts be affixed, in such form and manner, and for such period not exceeding twenty-one days, as the court may order, to any premises occupied by that person, and that the person do pay the costs of such affixing; and if any person obstructs the affixing of such notice, or removes, defaces, or conceals the notice while affixed during the said period, he shall for each offence be liable to a fine not exceeding five pounds.

(5) If the occupier of a licensed slaughter-house is convicted of an offence under this section, the court convicting him may cancel the licence for such slaughter-house.

(6) If any person obstructs an officer in the performance of his duty under any warrant for entry into any premises granted by a justice in pursuance of this Act for the purposes of this section, he shall, if the court is satisfied that he obstructed with intent to prevent the discovery of an offence against this section, or has within twelve months previously been convicted of such obstruction, be liable to imprisonment for any term not exceeding one month in lieu of any fine authorised by this Act for such obstruction.

(7) A justice may act in adjudicating on an offender under this section, whether he has or has not acted in ordering the animal or article to be destroyed or disposed of. 
(8) Where a person has in his possession any article which is unsound or unwholesome or unfit for the food of man, he may, by written notice to the Sanitary Authority, specifying such article, and containing a sufficient identification of it, request its removal, and the Sanitary Authority shall cause it to be removed as if it were trade refuse.

\section{Control of Milk Supply on Outbreak of Disease}

Section 71.- (I) If the Medical Officer of Health of any district has evidence that any person in the district is suffering from a dangerous infectious disease, attributable to milk supplied within the district from any dairy, situate within or without the district, or that the consumption of milk from such dairy is likely to cause any such infectious disease to any person residing in the district, such Medical Officer shall, if authorised by an order of a justice having jurisdiction in the place where the dairy is situate, have power to inspect the dairy, and if accompanied by a veterinary inspector or some other properly qualified veterinary surgeon to inspect the animals therein, and if on such inspection the Medical Officer of Health is of opinion that any such infectious disease is caused from consumption of the milk supplied therefrom, he shall report thereon to the Sanitary Authority, and his report shall be accompanied by any report furnished to him by the said veterinary inspector or veterinary surgeon, and the Sanitary Authority may thereupon serve on the dairyman notice to appear before them within such time, not less than twenty-four hours, as may be specified in the notice, to show cause why an order should not be made requiring him not to supply any milk therefrom within the district until the order has been withdrawn by the Sanitary Authority.

(2) The Sanitary Authority, if, in their opinion, he fails to show such cause, may make the said order, and shall forthwith serve notice of the facts on the County Council of the county in which the dairy is situate, and on the Local Government Board, and, if the dairy is situate within the district of another Sanitary Authority, on such Authority.

(3) The said order shall be forthwith withdrawn on the Sanitary Authority, or their Medical Officer of Health on their behalf, being satisfied that the milk supply has been changed, or that the cause of the infection has been removed.

(4) If any person refuses to permit the Medical Officer of Health, on the production of a justice's order under this section, to inspect any dairy, or if so accompanied as aforesaid to inspect the animals kept there, or, after any such order has been made, supplies any milk within the district in contravention of the order, or sells it for consumption therein, he shall, on the information of the Sanitary Authority, be liable to a fine not exceeding five pounds, and, if the offence continues, to a fine not exceeding forty shillings for every day during which the offence continues.

(5) Provided that-

(a) proceedings in respect of the offence shall be taken before a court having jurisdiction in the place where the dairy is situate; and

(b) a dairyman shall not be liable to an action for breach of contract if the breach be due to an order under this section.

(6) Proceedings may be taken under this section in respect of a dairy situate in the district of a Local Authority under the Public Health Acts, and the notice of the facts shall be served on the Local Authority as if they were a Sanitary Authority within the meaning of this Act. 
(7) Nothing in or done under this section shall interfere with the operation or effect of the Contagious Diseases (Animals) Acts, 1878 to I886, or this Act, or of any order, licence, or Act of the Board of Agriculture or the Local Government Board thereunder, or of any order, byelaw, regulation, licence, or act of a Local Authority made, granted, or done under any such order of the Board of Agriculture or the Local Government Board, or exempt any dairy, building, or thing, or any person, from the provisions of any general Act relating to dairies, milk, or animals. 


\section{APPENDIX D}

\section{THE DAIRIES, COWSHEDS AND MILK-SHOPS ORDER OF 1885}

\section{At the Council Chamber, Whitehall,}

The 15 th day of June, 1885 ,

By Her Majesty's Most Honourable Privy Council.

\section{Present :-Lord President, Mr Trevelyan.}

The Lords and others of Her Majesty's Most Honourable Privy Council, by virtue and in exercise of the powers in them vested under the Contagious Diseases (Animals) Act, 1878, and of every other power enabling them in this behalf, do order, and it is hereby ordered, as follows :-

Short Title.-I. This Order may be cited as the Dairies, Cowsheds and Milk-shops Order of 1885 .

Extent.-2. This Order extends to England and Wales and Scotland only.

Commencement.-3. This Order shall commence and take effect from and immediately after the Thirtieth day of June, One thousand eight hundred and eighty-five.

\section{Interpretation.-4. In this Order-}

The Act of 1878 means the Contagious Diseases (Animals) Act, 1878.

Other terms have the same meaning as in the Act of 1878 .

Revocation of former Order.-5. The Dairies, Cowsheds and Milk-shops Order of July, 1879, is hereby revoked: Provided that nothing in this Order shall be deemed to revive any Order of Council thereby revoked or to invalidate or make unlawful anything done before the commencement of this Order, or interfere with the institution or prosecution of any proceeding in respect of any offence committed against, or any penalty incurred under, the said Order hereby revoked.

Registration of Dairymen and others.-6. (I) It shall not be lawful for any person to carry on in the District of any Local Authority the trade of cowkeeper, dairyman or purveyor of milk unless he is registered as such therein in accordance with this Article.

(2) Every Local Authority shall keep a Register of persons from time to time carrying on in their District the trade of cowkeepers, dairymen, or purveyors of milk, and shall from time to time revise and correct the Register. (3) The Local Authority shall register every such person, but the fact of 
such registration shall not be deemed to authorise such person to occupy as a dairy or cowshed any particular building or in any way preclude any proceedings being taken against such person for non-compliance with, or infringement of, any of the provisions of this Order or any Regulation made thereunder.

(4) The Local Authority shall from time to time give public notice by advertisement in a newspaper circulating in their District, and, if they think fit, by placards, hand-bills, or otherwise, of registration being required, and of the mode of registration.

(5) A person who carries on the trade of cowkeeper or dairyman for the purpose only of making and selling butter or cheese or both, and who does not carry on the trade of purveyor of milk, shall not, for the purposes of registration, be deemed to be a person carrying on the trade of cowkeeper or dairyman, and need not be registered.

(6) A person who sells milk of his own cows in small quantities to his workmen or neighbours, for their accommodation, shall not, for the purposes of registration, be deemed, by reason only of such selling, to be a person carrying on the trade of cowkeeper, dairyman, or purveyor of milk, and need not, by reason thereof, be registered.

Construction and Water Supply of New Dairies and Cowsheds.-7. ( $r$ ) It shall not be lawful for any person following the trade of cowkeeper or dairyman to begin to occupy as a dairy or cowshed any building not so occupied at the commencement of this Order, unless and until he first makes provision, to the reasonable satisfaction of the Local Authority, for the lighting, and the ventilation including air-space, and the cleansing, drainage, and water-supply of the same, while occupied as a dairy or cowshed.

(2) It shall not be lawful for any such person to begin so to occupy any such building without first giving one month's notice in writing to the Local Authority of his intention so to do.

Sanitary State of all Dairies and Cowsheds.-8. It shall not be lawful for any person following the trade of cowkeeper or dairyman to occupy as a dairy or cowshed any building, whether so occupied at the commencement of this Order or not, if and as long as the lighting, and the ventilation including airspace, and the cleansing, drainage, and water-supply thereof, are not such as are necessary or proper-

(a) for the health and good condition of the cattle therein ; and

(b) for the cleanliness of milk-vessels used therein for containing milk for sale ; and

(c) for the protection of the milk therein against infection or contamination.

Contamination of Milk.- -9. It shall not be lawful for any person following the trade of cowkeeper or dairyman or purveyor of milk, or being the occupier of a milk-store or milk-shop-

(a) to allow any person suffering from a dangerous infectious disorder, or having recently been in contact with a person so suffering, to milk cows or to handle vessels used for containing milk for sale, or in any way to take part or assist in the conduct of the trade or business of the cowkeeper or dairyman, purveyor of milk, or occupier of a milk-store 
or milk-shop, so far as regards the production, distribution, or storage of milk; or

(b) if himself so suffering, or having recently been in contact as aforesaid, to milk cows, or handle vessels used for containing milk for sale, or in any way to take part in the conduct of his trade or business, as far as regards the production, distribution, or storage of milk-

until in each case all danger therefrom of the communication of infection to the milk or of its contamination has ceased.

IO. It shall not be lawful for any person following the trade of cowkeeper or dairyman or purveyor of milk, or being the occupier of a milk-store or milkshop, after the receipt of notice of not less than one month from the Local Authority calling attention to the provisions of this Article, to permit any watercloset, earth-closet, privy, cesspool, or urinal to be within, communicate directly with, or ventilate into any dairy or any room used as a milk-store or milk-shop.

II. It shall not be lawful for any person following the trade of cowkeeper or dairyman or purveyor of milk, or being the occupier of a milk-store or milkshop to use a milk-store or milk-shop in his occupation, or permit the same to be used, as a sleeping apartment, or for any purpose incompatible with the proper preservation of the cleanliness of the milk-store or milk-shop, and of the milk-vessels and milk therein, or in any manner likely to cause contamination of the milk therein.

12. It shall not be lawful for any person following the trade of cowkeeper or dairyman or purveyor of milk to keep any swine in any cowshed or other building used by him for keeping cows, or in any milk-store or other place used by him for keeping milk for sale.

Regulations of Local Authority. - 13. A Local Authority may from time to time make Regulations for the following purposes, or any of them :-

(a) For the inspection of cattle in dairies.

(b) For prescribing and regulating the lighting, ventilation, cleansing, drainage, and water-supply of dairies and cowsheds in the occupation of persons following the trade of cowkeepers or dairymen.

(c) For securing the cleanliness of milk-stores, milk-shops, and of milkvessels, used for containing milk for sale by such persons.

(d) For prescribing precautions to be taken by purveyors of milk, ard persons selling milk by retail against infection or contamination.

Provisions as to Regulations of Local Authority.-14. The following provisions shall apply to Regulations made by a Local Authority under this Order :-

(I) Every Regulation shall be published by advertisement in a newspaper circulating in the District of the Local Authority.

(2) The Local Authority shall send to the Privy Council a copy of every Regulation made by them not less than one month before the date named in such Regulation for the same to come into force.

(3) If at any time the Privy Council are satisfied on inquiry, with respect to any Regulation, that the same is of too restrictive a character, or otherwise objectionable, and direct the revocation thereof, the same shall not come into operation, or shall thereupon cease to operate, as the case may be. 
$55^{8}$

APPENDICES

Existence of Disease among Cattle.-I5. If at any time disease exists among the cattle in a dairy or cowshed, or other building or place, the milk of a diseased cow therein-

(a) shall not be mixed with other milk; and

(b) shall not be sold or used for human food; and

(c) shall not be sold or used for food of swine, or other animals, unless and until it has been boiled.

Acts of Local Authorities.-I6. (I) All Orders and Regulations made by a Local Authority under the Dairies, Cowsheds and Milk-shops Order of July, 1879, on any Order revoked thereby, and in force at the making of this Order shall, as far as the same are not varied by or inconsistent with this Order, remain in force until altered or revoked by the Local Authority.

(2) Forms of Registers and other forms which have been before the making of this Order prepared for use by a Local Authority under the Dairies, Cowsheds and Milk-shops Order of July, 1879, or any Order revoked thereby may be used, as far as they are suitable for the purposes of this Order.

Scotland.-17. Nothing in this Order shall be deemed to interfere with the operation of the Cattle Sheds in Burghs (Scotland) Act, 1866.

C. L. PEEL.

(This Order was amended in 1886 and 1889 , see copy of amending Orders.) 


\section{APPENDIX E}

\section{THE DAIRIES, COWSHEDS AND MILK-SHOPS AMENDING ORDER, 1886}

To the Mayor and Commonalty and Citizens of the City of London, acting by the Mayor, Aldermen, and Commons of that City in Common Council assembled ;-

To the Metropolitan Board of Works ;-

To the several Urban and Rural Sanitary Authorities for the time being in England and Wales;-

And to all others whom it may concern.

Whereas by Section 34 of the Contagious Diseases (Animals) Act, 1878 (hereinafter referred to as "the principal Act"), it was enacted that Her Majesty's Most Honourable Privy Council (hereinafter referred to as "the Privy Council") might from time to time make such general or special Orders as they should think fit, subject and according to the provisions of the Act, for the purposes specified in that Section;

And whereas on the 15 th day of June, 1885 , the Privy Council, in pursuance of the powers vested in them by the principal Act, made a General Order, known as "The Dairies, Cowsheds and Milk-shops Order of 1885" (hereinafter referred to as "the Order of 1885 "); and such Order extends to the whole of England and Wales;

And whereas by Article 14 of the Order of 1885 , it is provided that a copy of every Regulation therein referred to shall be sent to the Privy Council, and that if at any time the Privy Council are satisfied on inquiry with respect to any Regulation that the same is of too restrictive a character, or otherwise objectionable, and direct the revocation thereof, the same shall not come into operation, or shall thereupon cease to operate, as the case may be ;

And whereas by Section 9 of the Contagious Diseases (Animals) Act, 1886 (hereinafter referred to as "the Act of 1886 "), it is enacted as follows :-

"(I) The powers vested in the Privy Council of making general or special Orders under Section 34 of the principal Act, for the purposes in that Section mentioned, are hereby transferred to and shall henceforth be excerciseable by the Local Government Board ; every such Order shall have effect as if enacted in this Section, and shall be published in such manner as the Local Government Board may direct, and the said Board may from time to time alter or revoke any such Order."

"(2) For the purposes of the said Section and this Section, and of any Order in force thereunder, the expression Local Authority, unless the context 
otherwise requires, in the Metropolis has the same meanings as in the principal Act, and elsewhere has the same meanings as in the Public Health Act, 1875."

"(5) The like penalties for offences against Orders or Regulations made for the purposes of Section 34 of the principal Act as amended by this Section may be imposed by the Local Government Board or Local Authority making the same, and such offences may be prosecuted and penalties recovered in a summary manner, and subject to the like provisions, as if such Orders or Regulations were byelaws of a Local Authority under the Public Health Act, 1875, and as if the Local Authority mentioned in that Act included a Local Authority in the Metropolis as defined in this Section."

"6 (a). The Dairies, Cowsheds and Milk-shops Order of 1885, and any Regulations thereunder, or having effect in pursuance thereof, made by any Local Authority under the principal Act, other than the Local Authority of a county, shall be deemed to have been made respectively by the Local Government Board and by a Local Authority under this Section."

And whereas it is expedient that the Order of 1885 should be altered as hereinafter mentioned, and that penalties should be imposed for offences against such Order :

Now therefore, We, the Local Government Board, in pursuance of the powers vested in Us by the Act of 1886 , hereby Order as follows :-

Article 1.-This Order may be cited as "the Dairies, Cowsheds and Milkshops Amending Order of $1886 . "$

Article 2.- Article 14 of the Order of 1885 shall be altered by the substitution therein of the words "Local Government Board" for the words "Privy Council" occurring therein.

Article 3.- If any person is guilty of an offence against the Order of 1885 , he shall for every such offence be liable to a penalty of Five Pounds, and in the case of a continuing offence to a further penalty of Forty Shillings for each day after written notice of the offence from the Local Authority.

Provided, nevertheless, that the justices or court before whom any complaint may be made, or any proceedings may be taken in respect of any such offence, may, if they think fit, adjudge the payment as a penalty of any sum less than the full amount of the penalty imposed by this Order.

Article 4.-In this Order the expression "Local Authority" means-

In the City of London and the Liberties thereof, the Mayor and Commonalty and Citizens of the City of London, acting by the Mayor, Aldermen, and Commons of that City in the Common Council assembled :

In the Metropolis, except the City of London and the Liberties thereof, the Metropolitan Board of Works :

Elsewhere than in the Metropolis, the Urban or Rural Sanitary Authority.

Given under the Seal of Office of the Local Government Board, this First day of November, in the year One thousand eight hundred and eighty-six.

HUGH OWEN,

Secretary.

Chas. T. Ritchie, President. 


\section{APPENDIX F}

\section{THE DAIRIES, COWSHEDS AND MILK-SHOPS ORDER OF 1899}

To the London County Council ;-

To the Mayor and Commonalty and Citizens of the City of London, acting by the Mayor, Aldermen, and Commons of that City in Common Council assembled ;-

To the Mayor, Aldermen, and Burgesses of the several County Boroughs for the time being in England and Wales;-

To the several Urban and Rural District Councils for the time being in England and Wales;-

And to all others whom it may concern.

Whereas on the 15 th day of June, 1885, Her Majesty's Most Honourable Privy Council (hereinafter referred to as "the Privy Council "), in pursuance of the statutory provisions in that behalf, made an Order (hereinafter referred to as "the Order") which is known as "The Dairies, Cowsheds and Milk-shops Order of $1885^{\prime \prime}$;

And whereas certain powers of the Privy Council, including the power of altering or revoking the Order, have been transferred to Us, the Local Government Board; and, in pursuance of such powers, the Order has been altered by an Order (hereinafter referred to as "the Amending Order"), which was made by Us on the Ist day of November, 1886, and is known as "The Dairies, Cowsheds, and Milk-shops Amending Order of 1886";

And whereas it is expedient that the Order is altered by the Amending Order should be further altered :

Now therefore, in pursuance of the powers vested in Us in that behalf, We hereby Order as follows :-

Article I.-This Order may be cited as "The Dairies, Cowsheds and Milkshops Order of $1899 . "$

Article 2.-Article 15 of the Order shall be altered so that, for the purposes of the provisions of paragraphs $(a)$ and $(b)$ thereof the expressions in the said Article which refer to disease shall include, in the case of a cow, such disease of the udder as shall be certified by a veterinary surgeon to be tubercular; and the Order and the Amending Order shall apply and be construed with the modifications necessary to give effect to this Article.

Given under the Seal of Office of the Local Government Board, this Seventh day of February, in the year One thousand eight hundred and ninety-nine.

S. B. PROVIS, 561
Henry Chaplin, President. 


\section{APPENDIX G}

\section{MODEL REGULATIONS: DAIRIES, COWSHEDS AND MILK-SHOPS}

Regulations made by the ${ }^{1}$

with respect to dairies, cowsheds and milk-shops in the ${ }^{2}$

Interpretation.-I. Throughout these Regulations the expression "The Council" means the 1

the expression "the district" means the ${ }^{2}$

the expression "cowshed" includes any dairy in which milking-cows may be kept, and the expression "cowkeeper means any person following the trade of a cowkeeper or dairyman who is, or is required to be, registered under the Dairies, Cowsheds and Milk-shops Order of 1885.

For the Inspection of Cattle in Dairies.-2. Every occupier of a dairy wherein any cattle may be kept, and which the Medical Officer of Health, or the Inspector of Nuisances, or any other officer of the Council specially authorised by them in that behalf, may visit for the purpose of inspecting cattle, and every person for the time being having the care or control of any such dairy, or of any cattle therein, shall afford such Medical Officer of Health, Inspector of Nuisances, or officer, all reasonable assistance that may, for the purpose of the inspection, be required by him.

\section{Sanitation of Cowsheds}

For prescribing and regulating the Lighting, Ventilation, Cleansing, Drainage, and Water-Supply of Cowsheds and Dairies in the occupation of persons following the Trade of Cowkeepers or Dairymen.

\section{PART I.}

The Regulations in this part shall apply to cowsheds, the cows from which are habitually grazed on grass land during the greater part of the year, and when not so grazed, are habitually turned out during a portion of each day.

Lighting.-3. Every cowkeeper shall provide that every cowshed in his occupation shall be sufficiently lighted with windows, whether in the sides or roof thereof.

Ventilation.-4. Every cowkeeper shall cause every cowshed in his occupa-

1 "Mayor, aldermen, and burgesses, of the borough of the Council" or "Urban (or Rural) District Council of case may be.

2 "Borough" or "Urban (or Rural) District of

, acting by ," as the may be. 
tion to be sufficiently ventilated, and for this purpose to be provided with a sufficient number of openings into the external air to keep the air in the cowshed in a wholesome condition.

Cleansing.-5. (I) Every cowkeeper shall cause every part of the interior of every cowshed in his occupation to be thoroughly cleansed from time to time as often as may be necessary to secure that such cowshed shall be at all times reasonably clean and sweet.

(2) Such person shall cause the ceiling or interior of the roof, and the walls of every cowshed in his occupation, to be properly limewashed trvice at least in every year, that is to say, once during the month of May, and once during the month of October, and at such other times as may be necessary.

Provided that this requirement shall not apply to any part of such ceiling, roof, or walls, that may be properly painted, or varnished, or constructed of or covered with any material such as to render the limewashing unsuitable or inexpedient, and that may be otherwise properly cleansed.

(3) He shall cause the floor of every such cowshed to be thoroughly swept, and all dung and other offensive matter to be removed from such cowshed as often as may be necessary, and not less than once in every day.

Drainage.-6. (I) Every cowkeeper shall cause the drainage of every cowshed in his occupation to be so arranged that all liquid matter which may fall or be cast upon the floor may be conveyed by a suitable open channel to a drain inlet situate in the open air at a proper distance from any door or window of such cowshed, or to some other suitable place of disposal which is so situate.

(2) He shall not cause or suffer any inlet to any drain of such cowshed to be within such cowshed.

Water-Supply.-7. (1) Every cowkeeper shall keep in, or in connection with, every cowshed in his occupation a supply of water suitable and sufficient for all such purposes as may from time to time be reasonably necessary.

(2) He shall cause any receptacle which may be provided for such water to be emptied and thoroughly cleansed from time to time as often as may be necessary to prevent the pollution of any water that may be stored therein, and where such receptacle is used for the storage only of water he shall cause it to be properly covered and ventilated, and so placed as to be at all times readily accessible.

\section{PART II.}

The Regulations in Part I., and also the following Regulation, shall apply to all cowsheds other than those the cows from which are habitually grazed on grass land during the greater part of the year, and when not so grazed, are habitually turned out during a portion of each day.

8. A cowkeeper shall not cause or allow any cowshed in his occupation to be occupied by a larger number of cows than will leave not less than eight hundred feet of air-space for each cow.

Provided as follows :-

(a) In calculating the air-space for the purposes of this Regulation, no space shall be reckoned which is more than sixteen feet above the floor; but if the roof or ceiling is inclined, then the mean height of the same above the floor may be taken as the height thereof for the purposes of this Regulation. 
(b) This Regulation shall not apply to any cowshed constructed and used before the date of these Regulations coming into effect, until two years after that date.

\section{Sanitation of Dairies}

\section{PART III.}

9. In this part, the expression "Dairy" means a dairy in which cattle are not kept.

Lighting.-10. Every cowkeeper shall provide that every dairy in his occupation shall be sufficiently lighted with windows, whether in the sides or roof thereof.

Ventilation.-Ir. Every cowkeeper shall cause every dairy in his occupation to be sufficiently ventilated, and for this purpose to be provided with a sufficient number of openings into the external air to keep the air in the dairy in a wholesome condition.

Cleansing.-12. (I) Every cowkeeper shall cause every part of the interior of every dairy in his occupation to be thoroughly cleansed from time to time as often as may be necessary to secure that such dairy shall be at all times reasonably clean and sweet.

(2) He shall cause the floor of every such dairy to be thoroughly cleansed with water at least once in every day.

Drainage.-13. (I) Every cowkeeper shall cause the drainage of every dairy in his occupation to be so arranged that all liquid matter which may fall or be cast upon the floor may be conveyed by a suitable open channel to the outside of such dairy, and may there be received in a suitable gulley communicating with a proper and sufficient drain.

(2) He shall not cause or suffer any inlet to any drain of such dairy to be within such dairy.

Water-Supply.-14. (I) Every cowkeeper shall cause every dairy in his occupation to be provided with an adequate supply of good and wholesome water for the cleansing of such dairy and of any vessels that may be used therein for containing milk, and for all other reasonable and necessary purposes in connection with the use thereof.

(2) He shall cause every cistern or other receptacle in which any such water may be stored to be properly covered and ventilated, and so placed as to be at all times readily accessible.

(3) He shall cause every such cistern or receptacle to be emptied and thoroughly cleansed from time to time as often as may be necessary to prevent the pollution of any water that may be stored therein.

\section{Sanitation of Milk-shops}

For securing the Cleanliness of Milk-Stores, Milk-Shops, and of Milk-Vessels used for containing Milk for Sale by persons following the trade of Cowkeepers or Dairymen.

Cleanliness of Milk-Stores and Milk-Shops.-15. Every cowkeeper who is the occupier of a milk-store or milk-shop shall cause every part of the interior of such milk-store or milk-shop to be thoroughly cleansed from time to time as often as may be necessary to maintain such milk-store or milk-shop in a thorough state of cleanliness. 
Cleanliness of Milk-Vessels.-16. (I) Every cowkeeper shall from time to time as often as may be necessary cause every milk-vessel that may be used by him for containing milk for sale to be thoroughly cleansed with steam or clean boiling water, and shall otherwise take all proper precautions for the maintenance of such milk-vessel in a constant state of cleanliness.

(2) He shall, on every occasion when any such vessel shall have been used to contain milk, or shall have been returned to him after having been out of his possession, cause such vessel to be forthwith so cleansed.

\section{For prescribing Precautions to be taken by Purveyors of Milk and Persons selling Milk by retail against Infection or Confamination.}

17. (I) Every purveyor of milk or person selling milk by retail shall take all reasonable and proper precautions, in and in connection with the storage and distribution of the milk, and otherwise, to prevent the exposure of the milk to any infection or contamination.

(2) He shall not deposit or keep any milk intended for sale-

(a) in any room or place where it would be liable to become infected or contaminated by impure air, or by any offensive, noxious, or deleterious gas or substance, or by any noxious or injurious emanation, exhalation, or effluvium ; or

(b) in any room used as a kitchen or as a living room ; or

(c) in any room or building, or part of a building communicating directly by door, window, or otherwise with any room used as a sleeping room, or in which there may be any person suffering from any infectious or contagious disease, or which may have been used by any person suffering from any such disease and may not have been properly disinfected; or

(d) in any room or building or part of a building in which there may be any direct inlet to any drain.

(3) He shall not keep milk for sale, or cause or suffer any such milk to be placed, in any vessel, receptacle, or utensil which is not thoroughly clean.

(4) He shall cause every vessel, receptacle, or utensil used by him for containing milk for sale to be thoroughly cleansed with steam or clean boiling water after it shall have been used, and to be maintained in a constant state of cleanliness.

\section{Cleanliness of Cows and Milkers}

(5) He shall not cause or suffer any cow belonging to him or under his care or control to be milked for the purpose of obtaining milk for sale-

(a) unless, at the time of milking, the udder and teats of such cow are thoroughly clean ; and

(b) unless the hands of the person milking such cow, also, are thoroughly clean and free from all infection and contamination.

\section{Penalties}

18. Every person who shall offend against any of the foregoing Regulations shall be liable for every such offence to a penalty of five pounds, and in the case of a continuing offence to a further penalty of forty shillings for each day after written notice of the offence from the Council.

Provided, nevertheless, that the justices, or court before whom any com- 
plaint may be made or any proceedings may be taken in respect of any such offence may, if they think fit, adjudge the payment as a penalty of any sum less than the full amount of the penalty imposed by this Regulation.

Commencement of the Regulations.-19. These Regulations shall come into force on and after the day of 18 .

Revocation of Regulations. ${ }^{1-20}$. From and after the date on which these Regulations shall come into force, all Regulations heretofore made under, or having effect in pursuance of the Dairies, Cowsheds and Milk-shops Order of 1885, shall, so far as the same are now in force in the district, be revoked.

\section{(Enclosure No. 5.)}

Extract from General Order, dated 23rd March, 1891, prescribing Regulations as to * * Inspectors of Nuisances.

Article 19 (7).-The Inspector of Nuisances "shall from time to time, and forthwith upon complaint, visit and inspect the shops and places kept or used for the preparation or sale of butchers' meat, poultry, fish, fruit, vegetables, corn, bread, flour, milk, or any other article to which the provisions of the Public Health Act, 1875 , in this behalf shall apply, and examine any animal, carcase, meat, poultry, game, flesh, fish, fruit, vegetables, corn, bread, flour, milk, or other article as aforesaid, which may be therein; and in case any such article appear to him to be intended for the food of man, and to be unfit for such food, he shall cause the same to be seized, and take such other proceedings as may be necessary in order to have the same dealt with by a justice : Provided that in any case of doubt arising under this clause, he shall report the matter to the Medical Officer of Health, with the view of obtaining his advice thereon."

1 If this clause is not intended in the series submitted to the Local Government Board for approval, it should be stated whether or not there are any Regulations in force upon the subject. 


\section{APPENDIX H}

\section{INFECTIOUS DISEASES (PREVENTION) ACT, 1890}

\section{Inspecting of Dairies in certain cases : Power to prokibit Supply of Milk.}

4. In case the Medical Officer of Health is in possession of evidence that any person in the district is suffering from infectious disease attributable to milk supplied within the district from any dairy situate within or without the district, or that the consumption of milk from such dairy is likely to cause infectious disease to any person residing in the district, such Medical Officer shall $(a)$ if authorised in that behalf by any order of a justice having jurisdiction in the place where such dairy is situate, have power to inspect such dairy, and (b) if accompanied by a veterinary inspector or some other properly qualified veterinary surgeon to inspect the animals therein, and if on such inspection the Medical Officer of Health shall be of opinion that infectious disease is caused from consumption of the milk supplied therefrom, he shall report thereon to the Local Authority, and his report shall be accompanied by any report furnished to him by the said veterinary inspector or veterinary surgeon, and the Local Authority may thereupon give notice to the dairyman to appear before them within such time, not less than twenty-four hours, as may be specified in the notice, to show cause why an order should not be made requiring him not to supply any milk therefrom within the district until such order has been withdrawn by the Local Authority, and if, in the opinion of the Local Authority, he fails to show such cause, then the Local Authority may make such order as aforesaid; and the Local Authority shall forthwith give notice of the facts to the Sanitary Authority and County Council (if any) of the district or county in which such dairy is situate, and also to the Local Government Board.

An order made by a Local Authority in pursuance of this section shall be forthwith withdrawn on the Local Authority or the Medical Officer of Health on its behalf being satisfied that the milk supply has been changed, or that the cause of the infection has been removed. Any person refusing to permit the Medical Officer of Health on the production of such order as aforesaid to inspect any dairy, or if so accompanied as aforesaid to inspect the animals kept there, or after any such order not to supply milk as aforesaid has been given, supplying any milk within the district in contravention of such order, or selling it for consumption therein, shall be deemed guilty of an offence against this Act. Provided always, that proceedings in respect of such offence shall be taken before the Justices of the Peace having jurisdiction in the place where the said dairy is situate. Provided also, that no dairyman shall be liable to an action for breach of contract if the breach be due to an order from the Local Authority under this Act. 


\section{APPENDIX I}

\section{PUBLIC HEALTH ACT, 1875}

Unsound Meat, Milk, etc.-Power of Medical Officer of Health to inspect.116. Any Medical Officer of Health or Inspector of Nuisances may at all reasonable times inspect and examine any animal carcase, meat, poultry, game, flesh, fish, fruit, vegetables, corn, bread, flour, or milk exposed for sale, or deposited in any place for the purpose of sale, or of preparation for sale, and intended for the food of man, the proof that the same was not exposed or deposited for any such purpose, or was not intended for the food of man, resting with the party charged; and if any such animal carcase, meat, poultry, game, flesh, fish, fruit, vegetables, corn, bread, flour, or milk appears to such medical officer or inspector to be diseased or unsound, or unwholesome, or unfit for the food of man, he may seize and carry away the same himself or by an assistant, in order to have the same dealt with by a justice.

Power of Justice to Order Destruction of Unsound Meat, etc.-II7. If it appears to the justice that any animal carcase, meat, poultry, game, flesh, fish, fruit, vegetables, corn, bread, flour, or milk so seized is diseased, or unsound, or unwholesome, or unfit for the food of man, he shall condemn the same, and order it to be destroyed or so disposed of so as to prevent it from being exposed for sale or used for the food of man ; and the person to whom the same belongs, or did belong at the time of exposure for sale, or in whose possession or on whose premises the same was found, shall be liable to a penalty not exceeding twenty pounds for every animal carcase, or fish, or piece of meat, flesh, or fish, or any poultry or game, or for the parcel of fruit, vegetables, corn, bread, or flour, or for the milk so condemned, or, at the discretion of the justice, without the infliction of a fine, to imprisonment for a term of not more than three months.

The justice who, under this section, is empowered to convict the offender may be either the justice who may have ordered the article to be disposed of or destroyed, or any other justice having jurisdiction in the place. 


\section{APPENDIX J}

\section{CITY OF LIVERPOOL-TUBERCULOSIS AND MILK}

NOTICE IS HEREBY GIVEN, that by the Liverpool Corporation Act, 1900, it is enacted as follows:-

\section{7.- In this Part of this Act-}

"Dairy," shall include any farm, farmhouse, cowshed, milk-store, milkshop, or other place from which milk is supplied, or in which milk is kept for purposes of sale.

"Dairyman," shall include any cowkeeper, purveyor of milk, or occupier of a dairy.

"Medical officer," means the medical officer of health for the City, and includes any person duly authorised to act temporarily as medical officer of health.

I8.-Every person who knowingly sells, or suffers to be sold, or used for human consumption within the City, the milk of any cow which is suffering from tuberculosis of the udder, shall be liable to a penalty not exceeding $£ 10$.

19.-Any person, the milk of the cows, in whose dairy is sold or suffered to be sold or used for human consumption within the City, who, after becoming aware that any cow in his dairy is suffering from tuberculosis of the udder, keeps or permits to be kept, such cow, in any field, shed, or other premises along with other cows in milk, shall be liable to a penalty not exceeding $£ 5$.

20.-Every dairyman who supplies milk within the City, and has in his dairy any cow affected with, or suspected of, or exhibiting signs of tuberculosis of the udder, shall forthwith give written notice of the fact to the medical officer, stating his name and address, and the situation of the dairy or premises where the cow is.

Any dairyman failing to give such notice shall be liable to a penalty not exceeding $\&_{2}^{2}$.

21.- (I) It shall be lawful for the medical officer or any person provided with, and if required, exhibiting the authority in writing of such mediral officer, to take within the City for examination, samples of milk produced or sold or intended for sale within the City.

(2) The like powers in all respects may be exercised outside the City by the medical officer or such authorised person, if he shall first have obtained from a justice, having jurisdiction in the place where the sample is to be taken, an order authorising the taking of samples of the milk, which order any such justice is hereby empowered to make. 
22.-(I) If milk from a dairy situate within the City is being sold or suffered to be sold or used within the City, the medical officer or any person provided with, and if required, exhibiting the authority in writing of the medical officer, may if accompanied by a properly qualified veterinary surgeon, at all reasonable hours, enter the dairy, and inspect the cows kept therein; and if the medical officer or such person has reason to suspect that any cow in the dairy is suffering from tuberculosis of the udder, he may require the cow to be milked in his presence, and may take samples of the milk, and the milk from any particular teat shall, if he so requires, be kept separate, and separate samples thereof be furnished.

(2) If the medical officer is of opinion that tuberculosis is caused, or is likely to be caused to persons residing in the City from consumption of the milk supplied from a dairy situate within the City, or from any cow kept therein, he shall report thereon to the Corporation, and his report shall be accompanied by any report furnished to him by the veterinary surgeon, and the Corporation may thereupon serve on the dairyman notice to appear before them within such time, not less than twenty-four hours as may be specified in the notice, to show cause why an order should not be made, requiring him not to supply any milk from such dairy within the City, until the order has been withdrawn by the Corporation.

(3) If the medical officer has reason to believe that milk from any dairy situate outside the City from which milk is being sold or suffered to be sold, or used within the City, is likely to cause tuberculosis in persons residing within the City, the powers conferred by this section, may, in all respects, be exercised in the case of such dairy, provided that the medical officer or other authorised person shall first have obtained from a justice, having jurisdiction in the place where the dairy is situate, an order authorising such entry and inspection, which order any such justice is hereby empowered to make.

(4) Every dairyman and the persons in his employment shall render such reasonable assistance to the medical officer or such authorised person or veterinary surgeon, as aforesaid, as may be required by such medical officer, person, or veterinary surgeon, for all or any of the purposes of this section, and any person refusing such assistance or obstructing such medical officer, person, or veterinary surgeon in carrying out the purposes of this section, shall be liable to, a penalty not exceeding $£ 5$.

(5) If in their opinion the dairyman fails to show cause why such an order may not be made as aforesaid, the Corporation may make the said order, and shall forthwith serve notice of the facts on the county council of any administrative county in which the dairy is situate, and on the Local Government Board, and if the dairy is situate outside the City, on the council of the borough or country district in which it is situate.

(6) The said order shall be forthwith withdrawn on the Corporation or their medical officer, being satisfied that the milk supply has been changed, or that it is not likely to cause tuberculosis to persons residing in the City.

(7) If any person, after any such order has been made, supplies any milk within the City in contravention of the order, or sells it for consumption therein, he shall be liable to a penalty not exceeding $£ 5$, and if the offence continues to a further penalty not exceeding $£ 2$ for every day during which the offence continues.

(8) A dairyman shall not be liable to an action for breach of contract, if the breach be due to an order under this section. 
(9) The dairyman may appeal against an order of the Corporation under this section, or the refusal of the Corporation to withdraw any such order, either to a petty sessional court having jurisdiction within the City, or at his option, if the dairy is situate outside the City, to the Board of Agriculture, who shall appoint an officer to hear such appeal. Such officer shall fix a time and place of hearing within the City, and give notice thereof to the dairyman and the town clerk, not less than forty-eight hours before the hearing. Such officer shall, for the purposes of the appeal, have all the powers of a petty sessional court.

(Io) The Board of Agriculture may at any stage require payment to them by the dairyman, of such sum as they deem right, to secure the payment of any costs incurred by the Board of Agriculture in the matter of the appeal.

(II) The Court or the Board of Agriculture (as the case may be) may confirm, vary, or withdraw the order which is the subject of the appeal, and may direct to, and by whom the costs of the appeal (including any sum paid or payable to the Board of Agriculture as aforesaid) are to be paid, but pending the decision of the appeal, the order shall remain in force unless previously withdrawn by the Corporation.

23. - The Corporation shall cause to be given public notice of the effect of the provisions of this Part of this Act by advertisement in local newspapers, and by handbills, and otherwise in such manner as they think sufficient, and this Part of this Act shall come into operation at such time, not being less than one month after the first publication of such an advertisement as aforesaid, as the Corporation may fix.

24.- - Offences under this Part of this Act may be prosecuted, and penalties may be recovered by the Corporation before a petty sessional court, having jurisdiction in the place where the dairy is situate, or the offence is committed, and not otherwise.

25. - All expenses incurred by the Corporation in carrying into execution the provisions of this Part of this Act shall be chargeable on the City fund and City rate, and the Corporation may also charge on the same rate any expenses incurred by them in the application by a veterinary surgeon of the tuberculin or other reasonable test for the purpose of discovering tuberculosis, to any cow whose milk is, or was recently being supplied within the City. Provided that no such test shall be applied except with the previous consent of the owner of such cow.

26. - This Part of this Act may be carried into execution by a Committee of the Council formed in accordance with, and subject to, the provisions of the Fourth Schedule to the Diseases of Animals Act, 1894, except that the Committee shall consist wholly of members of the Council.

AND NOTICE IS HEREBY FURTHER GIVEN, that the foregoing provisions will come into operation on the Ist December Igoo.

$$
\text { By order, }
$$

EDWARD R. PICKMERE, Town Clerk.

TOWN Clerk's OfFice, Liverpool, 25th October 1900 . 


\section{APPENDIX K}

The following circular was originally sent to all farms known to be sending milk into Manchester. It is now sent to those farms from which tuberculous milk has been found to be coming into Manchester, and to all new farms :-

\section{Public Health Office, Town Hali, Manchestek, 24th October 1898.}

DEAR SIR,-I am instructed by a resolution of the Sanitary Committee, passed at a meeting held on 3Ist August of this year, to send to all farmers supplying milk for consumption in the City of Manchester the accompanying memorandum on certain points to which they desire more particularly to draw the attention of farmers. Any matter of difficulty which may be experienced in regard to the memorandum, if referred to the Medical Officer of Health, will receive careful attention. It will be understood that the memorandum is intended not by way of compulsion, but for purposes of guidance.Yours faithfully,

JAMES NIVEN, Medical Officer of Health.

\section{Suggestions addressed to Farmers supplying Milk for consumption in the City of Manchester}

The Committee appointed by the Corporation of Manchester to consider questions relating to the milk supply of the citizens, having had under their consideration the milk which is sent into the City from outside districts, have been led, by the investigations which have been made for them, to the conclusion that the milk supply leaves much to be desired, both as regards cleanliness and as regards the transmission of disease. They are advised that where milk is not kept clean it is one of the chief agents in producing fatal diarrhœa amongst children. They are also advised that consumption of the bowels in children and consumption of the brain are largely produced by the use of tuberculous milk, and they are in possession of proof that such milk is being largely imported into the City from surrounding districts. They feel obliged, therefore, to call on all farmers and cowkeepers supplying milk to the inhabitants of their district to take such precautions as will ensure a supply of clean, pure, and wholesome milk to the City.

With this object in view, they would point out that no milk should be sent into the City during the warm season of the year which has been kept at the farmhouse for more than a few hours, either separately or mixed with other milk, and that previous to despatch such milk should be kept cool. It is 572 
indeed much to be desired that milk should be kept cool on its way to the consumer in the City, but individuals could scarcely be expected to incur the expense of this proceeding.

Special precautions should be taken in respect of the following particulars :-

1. Cleanliness of the cows. The flanks and udders of the cows should at all times be kept in a state of strict cleanliness. There is good reason for believing that the absence of care in this matter not only leads to great fatality among children, but is one of the chief reasons why tuberculosis spreads amongst the stock.

2. No milker should be allowed to milk cows unless the hands have been thoroughly cleaned immediately before milking, and conveniences for cleaning the hands with soap and water should be supplied to the milkers.

3. The cowshed should at all times be kept rigorously clean. It should be cleaned out by sweeping twice a day, at such periods that no dust will be floating in the atmosphere of the cowshed during the period of milking.

4. The dairy should be kept in a state of strict cleanliness, and all utensils used for receiving, storing, or conveying milk should be washed with scalding water before each time of using.

5. The manure from the cowshed, so soon as it is removed from the cowshed, should be taken to the midden-stead, which must not be in the immediate vicinity of the cowshed. The object aimed at will, however, be partly attained by the erection at some distance from the cowshed of a midden-stead provided with an impermeable floor and sides, and either covered over or drained into an impermeable cesspool, at a distance of at least 20 yards from the cowshed. If the farm is supplied with water from a well, the well should be at least 10 feet from any collection of manure or midden.

6. The cowshed should be thoroughly washed out from time to time in the warm season, when it can rapidly get dry, so as to remove all traces of tuberculous matter and other impurities.

Strict cleanliness of the animals and of the cowshed, sufficient to remove infectious dry material and to prevent its accumulation, is the most essential thing, both in preventing milk from causing diarrhoea and in the struggle with tuberculosis.

\section{Structural Conditions.}

Certain structural conditions are requisite in order that the milk may be clean and free from disease :-

A. The floor of each cowshed must be even, and should be composed of concrete slightly ribbed in that portion of the stall where the cows stand. Behind the cows should be a cement concrete channel leading to a trapped drain outside the cowshed. Between the channel and the wall, if any, behind should interpose a sufficient space so that the cow's dung does not splash upon the wall. Many floors are at present so uneven and otherwise imperfect that it is extremely difficult, and may be impossible, to cleanse them.

B. The cowshed should be well lighted throughout, otherwise cleanliness is not to be looked for. There is no reason why every cowshed, fit for the purpose, should not have abundant light, so that every part of it should be visible during ordinary daylight.

C. Every cowshed should be sufficiently and properly ventilated. That is to say, there should be introduced into it a sufficient supply of fresh air in such a 
manner as not to cause draughts, and provision should be made for extracting the fresh air supplied after it has become contaminated in the cowshed. The supply of fresh air should be spread out as much as possible, so as not to cause draughts, and extraction should be conducted on the same principle. Thus, air may be admitted under the eaves by spaces which should, however, be accessible for cleaning, and extracted continuously by the ridge of the roof. Or, if a hayloft is over the cowshed, a double set of openings should be made in the walls for the admission and extraction of air, the latter being near the ceiling level. In the latter case, also, the ceiling of the cowshed should be rendered impermeable to dust.

D. A cowshed should not have less than 50 superficial feet per cow, nor be less than 12 feet high. If the cowshed is large, the height should be correspondingly increased, but the floor space should not be diminished.

The Committee are aware that many existing cowsheds do not conform to these suggestions, but they consider it desirable to place milk producers in possession of their views on the subject.

\section{Miscellaneous.}

Farmers are also advised that-

I. Their stock should be guaranteed free from tuberculosis. In order to get rid of the disease it will be necessary to have their existing stock tested and tuberculous cows removed from the cowshed, and fresh cows purchased under a guarantee of freedom from tuberculosis. This will, at first, entail a loss, but the ultimate gain will more than compensate for the initial loss. In order to mitigate the initial loss, they may isolate the cows found to be tuberculous and fatten them for slaughter. This will require, however, some provision for isolation. On the other hand, from the public health point of view, it would be better to slaughter the affected animals at once and to replace them by fresh cows, where this could be done. It is largely a matter of expense. This operation will evidently be most easily conducted while the cows are outside in summer, but, with sufficient arrangements for isolation, may be effected at any season.

2. No milk should be sent into the City from a cow affected with knots, indurations, or other diseased conditions of the udder.

3. The water consumed by the cows should be pure and wholesome. Where this is supplied by ponds, each pond should be fenced round so as to prevent excreta being washed into it, and means should be devised to prevent the cows from fouling the water in the act of drinking.

4. The water supply of the homestead should be free from contamination, for the sake of the household. It will be sufficient as regards utensils if all water used in washing these is scalding hot, providing the utensils are thoroughly cleaned.

5. A supply of clean wholesome water should be carried into the cowshed, and stored in a clean covered cistern, both in order to supply drinking water to the cows and with a view to cleansing the cowshed. Where, however, wholesome water is supplied under pressure, it will suffice to have a tap in the cowshed, though it is desirable also to fix a tap to each trough connected with a common pipe running along the back of the troughs.

6. To prevent the propagation of tuberculosis, each cow should have a separate stall and a separate earthenware feeding trough, bedded in cement 
in such a manner as to leave no corners at the head of the stall in which infectious matter may lodge.

A feeding rack should also be provided.

The Committee are desirous of impressing, both on cowkeepers and on dairymen, the urgency, and, so far as their own jurisdiction extends, the necessity of strict cleanliness in respect of the cows, milkers, cowsheds, dairy, and milk utensils.

[Dr Niven also sends a circular letter to all farmers sending milk into Manchester explanatory of Clause 4 of the Manchester Milk Clauses, which requires notification of tuberculosis of the udder. This circular both contains advice as to diagnosis of udder disease, and offers expert opinion in cases of difficulty. Its general purpose is to draw marked attention to the Manchester Milk Clauses and the manner of their operation upon dairy farmers.] 


\section{APPENDIX L}

\section{BOROUGH OF SUNDERLAND}

Certificates will be granted by the Health Committee to Dairy Farmers, respecting the milk supplied from their farms, if, in addition to complying with the Regulations made by the Council, under the Dairies, Cowsheds and Milkshops Order of 1885 , they also carry out the following Regulations as to the Construction and Management of their Farms and Dairies.

Construction.-I. The byre must be well lighted, ventilated, paved, and drained.

(In a well-lighted byre, every part of the byre should be easily visible in the day time with the doors closed.)

(In a well-ventilated byre the air will not feel oppressively close, or smell disagreeably when the cows are all housed and the doors shut.)

2. The dairy must not communicate directly with the house, and must be well ventilated.

3. The place used for washing and boiling the milk utensils must not communicate directly with the house, and must have a proper water-supply.

4. An efficient refrigerator or cooler for the milk must be provided.

Management.-I. Only cows which pass a veterinary surgeon's examination, the examination to include the application of the tuberculin test, must be kept. The veterinary surgeon's certificate for each cow, together with the temperature chart after the application of the tuberculin test, must be sent to the Medical Officer of Health. Newly-bought cows must be kept apart from the others till they have been examined and tested.

2. The milk must be of first-rate quality.

(Samples of the milk will be taken from time to time to ascertain that the quality is really first-rate.)

3. The cows must be kept as clean as possible.

4. The byre must be kept as clean as possible. The ceiling should be cleared of dust and cobwebs at least every three months, and the walls and ceilings whitewashed every six months. The manure should be taken out twice a day, and the walks and gutters flushed with water.

5. The farmer must at once notify any case of infectious disease, including consumption, measles, and whooping-cough, occurring on the farm, or in the families of his employees, and take measures, satisfactory to the Medical Officer of Health, for preventing the possibility of the infection of the milk by such case. 
6. Hay or food must not be stored in the byre, but kept in an adjoining building.

7. The dairy must only be used as a dairy, and the place for washing the milk utensils for that purpose only.

Milking.-I. The air of the byre must be kept as free from dust as possible, and at milking-time especially so.

2. The udders and teats must be cleaned before milking.

(It is also recommended that the tail and hind-quarters of the cows should be clipped.)

3. The milker must wash his or her hands thoroughly before milking, and also rinse the hands in water after milking each cow.

4. The milk must not remain a moment longer in the byre than is absolutely necessary, and must be at once strained and cooled.

5. The milk of any cow showing signs of disease of the udder, or of other disease, must not be used for sale. 


\section{APPENDIX M}

\section{PUBLIC HEALTH (SCOTLAND) ACT, 1897}

\section{Powers to be Exercised in Event of Outbreak of Milk-Borne Disease.}

Section 60.-(I) If the Medical Officer of any district has evidence that any person in the district is suffering from an infectious disease attributable to milk supplied within the district from any dairy situate within the district, or that the milk from any such dairy is likely to cause any such disease to any person residing in the district, such Medical Officer shall visit such dairy, and the Medical Officer shall examine the dairy and every person engaged in the service thereof or resident upon the premises or who may be resident in any premises where any person employed in such dairy may reside, and if accompanied by a veterinary surgeon approved as aforesaid, shall examine the animals therein, and the Medical Officer shall forthwith report the results of his examination accompanied by the report of the veterinary surgeon, if any, to the Local Authority or any committee of the Local Authority appointed under section fourteen to deal with such matters.

(2) If the Medical Officer of any district has evidence that any person in the district is suffering from any infectious disease attributable to milk from any dairy without the district, or that the milk from any such dairy is likely to cause any such disease to any person residing in the district, such Medical Officer shall forthwith intimate the same to the Local Authority of the district in which such dairy is situate, and such other Local Authority shall be bound, forthwith, by its Medical Officer to examine the dairy and the persons aforesaid, and by a veterinary surgeon approved as aforesaid, to examine the animals therein, previous notice of the time of such examination having been given to the Local Authority of the first-mentioned district, in order that the Medical Officer or any veterinary surgeon approved as aforesaid may, if they so desire, be present at the examinations referred to, and the Medical Officer of the second-mentioned Local Authority shall forthwith report the results of his examination, accompanied by the report of the veterinary surgeon, if any, to that Local Authority or any committee of that Local Authority appointed under section fourteen of this Act to deal with such matters.

(3) The Local Authority of the district in which the dairy is situated, or any committee appointed for the purpose, shall meet forthwith and consider the reports together with any other evidence that may be submitted by parties concerned, and shall either make an order requiring the dairyman not to supply any milk from the dairy until the order has been withdrawn by the Local Authority, or resolve that no such order is necessary. 
(4) Where proceedings are taken or any order is made under this section by the Local Authority of a district other than a burgh, it shall not be competent to appeal against the said proceedings or against said order to the County Council.

(5) The Local Authority may, if the dairy is within the district, require the dairyman not to supply milk either within or without the district, and shall give notice of the fact to the Local Authority of any district within which they believe milk to be supplied from such dairy.

(6) Any such order shall be forthwith withdrawn on the Local Authority, or their Medical Officer on their behalf, being satisfied that the milk from the dairy is no longer likely to cause infectious disease.

(7) It shall be open to any Local Authority or dairyman aggrieved by any such resolution or order, or withdrawal of order, to appeal in a summary manner to a sheriff having jurisdiction in the district in which the dairy is situated, and the sheriff may either make an order requiring the dairyman to cease from supplying milk, or may vary or rescind any order which has been made by the Local Authority, and he may at any time withdraw any order made under this section. Pending the disposal of any such appeal, the order shall remain in force.

(8) If any person refuses to permit the Medical Officer or veterinary surgeon of either Local Authority to make examination as above provided, or, after any order has been made under this section, supplies milk in contravention of the order, he shall be liable to a penalty not exceeding ten pounds, and, if the offence continues, to a further penalty not exceeding five pounds for every day during which the offence continues.

(9) Provided that-

(a) proceedings in respect of the offence shall be taken before a sheriff having jurisdiction in the place where the dairy is situate; and

(b) a dairyman shall not be liable to an action for breach of contract if the breach be due to an order under this section.

(Io) Nothing in or done under this section shall interfere with the operation or effect of the Contagious Diseases (Animals) Acts, 1878 to 1886, or of any order, licence, or act of the Privy Council or the Board thereunder, or of any regulation, licence, or act of a Local Authority, made, granted, or done under any such order of the Privy Council or the Board, or exempt any dairy, building, or thing, or any person from the provisions of any general Act relating to dairies, milk, or animals.

\section{Sources and Destination of Milk.}

Section 61.-Whenever it shall be certified to the Local Authority, by the Medical Officer or other legally qualified medical practitioner, that the outbreak or spread of infectious disease within the district is, in the opinion of such Medical Officer or medical practitioner, attributable to milk supplied by any dairyman, whether wholesale or retail, or to milk supplied by one or other of several such dairymen, whether wholesale or retail,-

(I) The Local Authority may require such dairyman, whether within or without the district, to furnish to them within a time to be fixed by them, being not less than twenty-four hours, a full and complete list of the names and addresses of all his customers within the district so far as known to him, and such dairyman shall furnish such list accord- 
ingly, and the Local Authority shall pay to him for every such list at the rate of sixpence for every twenty-five names contained therein; and every person who shall wilfully or knowingly offend against this enactment shall for each such offence be liable to a penalty not exceeding five pounds, and to a daily penalty not exceeding forty shillings ;

(2) The Local Authority may require such dairyman to furnish to them within a time to be fixed by them, a full and complete list of the names and addresses of the farmers, dairymen, or other parties from whom during a period to be specified, the milk, or any part of the milk which they sell or distribute, was obtained, and, if required, to produce and exhibit to the Medical Officer, or to any person deputed by him, all invoices, pass-books, accounts, or contracts, connected with the consignment or purchase of milk during such period, and such dairymen or others shall furnish such lists and produce and exhibit such invoices, pass books, accounts, or contracts, accordingly ; and every person who shall wilfully or knowingly offend against this enactment shall for every such offence be liable to a penalty not exceeding five pounds, and to a daily penalty not exceeding forty shillings;

(3) In any case where the person liable to any penalty under this section is not resident within the district, such penalty may be sued for at the instance of the procurator fiscal before the sheriff of the county in which such person is either domiciled or carries on business. 


\title{
APPENDIX N
}

\author{
FORM A
}

\section{PROPOSAL TO SUPPLY MILK}

TO

\section{THE AYLESBURY DAIRY COMPANY, LIMITED}

Full Christian and Surname of Proposer. Full Postal Address. Shortest Telegraphic Address. Name of Telegraph Office and distance from same. Railway Station from which Milk will be sent, and distance from Station. If there is any other Railway Station within working distance, state name, and on what line of Railway. Time of arrival in London of Trains by which the Milk could be sent :-Week days-Morning, Evening ; Sundays-Morning, Evening. Average number of Cows kept. About lowest Winter quantity, Imperial Gallons. About highest Summer quantity, Imperial Gallons. Source of Water Supply for Cooling and Washing purposes. Is Water from any other source ever used in the Dairy? Is there a boiler properly fixed and provided with an ample supply of Water which is not used for household purposes? Is there a refrigerator; if not, is Proposer willing to fix one. Name and Address of the Medical Officer of Health for the District. Price asked, delivered in London (each month).

Any other particulars :-

Signed

Date

N.B.-The Aylesbury Dairy Company find Churns, and pay by cheque every Friday for all Milk supplied up to the previous Saturday. 


\title{
APPENDIX O
}

\author{
FORM B
}

\section{REPORT OF MEDICAL OFFICER OF HEALTH}

ON THE FARM IN THE OCCUPATION OF

$\mathbf{A T}$

I.-WATER SUPPLY,-

I. Source of water, whether from a public water supply, a well, a running stream, or other source?

(a) For drinking purposes.

(b) For cleansing cans.

(c) For refrigerator.

2. If a well, how is it supplied? What is the position of the source in regard to -

(a) Farmhouse or other dwellings?

(b) Cowsheds, stables, pigstyes, farmyard, etc. ?

(c) Arable land?

(d) Pasture land?

(Indicate, if possible, the course of the supply with regard to $a, b, c$, and $d$.)

3. What are the relative levels of the source, the various buildings, etc., in the vicinity?

(The level of a well should be taken as the point to which the water reaches.) Is there any possibility of contamination arising from these?

4. What is the depth of the well? and distance of water below the surface?

5. Of what material are the sides of the well? Are they perfectly sound?

6. Are the fields in the vicinity dressed with fertilisers? If so, what is used?

7. For all sources of supply: is any variation noticed according to the seasons, e.g. notable increase after heavy rains, or diminution after drought?

8. How is the water conveyed from source of supply? If in pipes state whether they are of iron or other material. 582 
9. From what geological formation is the supply derived?

10. State your impressions as to the cleanliness of water supply, and any other points to which you think it desirable to call our attention.

2.-Sanitation.-

(a) Are there any conditions in connection with either the farmhouse, the farm buildings, or the residences of any of the persons employed about the dairy, such as to lead you to suppose that they might be the means of engendering any contagious or infectious disease, or likely in any way to contaminate the Milk?

(b) Especial attention being given to the dairy premises, are you quite satisfied as to the sanitary arrangements?

(c) Where is the refrigerator fixed, and how is the waste water from it dealt with?

(d) Where is the copper situated, and is it used solely for dairy purposes?

(c) Is the drainage perfectly satisfactory?

3.-Health.-

(a) Is the general health of the inmates of the farmhouse good?*

(b) And that of the persons employed upon the farm, and their families?*

* Note. $-(a)$ The skin - for evidence of desquamation or skin disease.

(b) The throat-for evidence of Diphtheria.

(c) The digestive organs-for evidence of Typhoid.

(c) If not in good health, are any of them suffering from any disease of a contagious or infectious character, and, if so, what?

(d) Is the general health of the surrounding neighbourhood good?

(e) Are there any cases of a zymotic character in your district?

(f) How lately has there been a case, and, if so, how far from the farm?

\section{4.-General Remarks.-}

(a) Are there any special local circumstances in connection with this farm, to which you, as Medical Officer of Health, deem it necessary to draw our attention?

(b) Are any alterations necessary, and if so, what do you recommend?

(c) Is there any other point occurring to you upon which we should be informed?

(d) We enclose copy of our contract, and shall be glad to know whether you consider that the occupier understands the responsibility, and is likely to observe the regulations set forth therein.

Signed 


\section{APPENDIX P}

FORM C

I, the undersigned.

of

hereinafter called the sender, do hereby contract to supply cows' milk to The Aylesbury Dairy Company, Limited, hereinafter referred to as the company, on the following terms and conditions-that is to say:- I agree to supply all the milk produced on my farm

in the following quantities and at the following prices:-

\begin{tabular}{|c|c|c|c|c|c|c|c|c|}
\hline \multicolumn{3}{|c|}{ Frox } & \multicolumn{3}{|c|}{ To } & \multirow[t]{2}{*}{$\begin{array}{l}\text { Not LEss THAN } \\
\text { Imperial Gallons } \\
\text { dailly. }\end{array}$} & \multirow[t]{2}{*}{$\begin{array}{l}\text { Not MORE THAN } \\
\text { Imperial Gallons } \\
\text { daily. }\end{array}$} & \multirow[t]{2}{*}{$\begin{array}{l}\text { AT THE PRICE } \\
\text { PER IMPERIAL } \\
\text { GALLON OF }\end{array}$} \\
\hline April & . & Ist & April & 3oth & 190 & & & \\
\hline
\end{tabular}

[Each month is returned in the same way.]

The company to have power to deduct from the sender's account the sum of sixpence per imperial gallon on every gallon below the above minimum quantities (see back) except in case of the sender's cows being slaughtered by order of the Government or other legally constituted authority. The milk to be delivered free of cost to the company twice daily at by the trains arriving at_a.m. and days, and a.m. and p.m. on Sundays, p.m. on week

All milk sent to the company during each week, ending on Saturday, to be paid for by cheque on the following Friday. (As any irregularity in sending in the account causes great inconvenience and extra trouble, each time that an account for the milk supplied up to the previous Saturday does not reach the company by the following Wednesday, the sender will be fined five shillings.)

\section{Churns.}

Churns to be provided by the company, to be returned on the expiration of the contract, or at any earlier perind if demanded by the company.

Every churn of milk to be sealed, and for this purpose the company to provide the necessary seals and sealing pliers, the latter to be returned on expiration of the contract, or at any earlier period if demanded by the company. 
Milk received in a churn which has not been securely sealed, will be made into butter, and paid for according to market price of butter.

A ticket or label provided by the company to be sent with every consignment of milk, stating the quantity consigned. On receipt of the consignment the quantity will be carefully ascertained by the company, and in case of any excess or deficiency, the quantity found to have been actually received will be stated on one of the company's post-cards, in the form printed below, and sent to the sender, who shall be bound by, and shall be paid for, the quantity so stated.

\section{Quality of Milk.}

All milk to be of good quality, and to be delivered in a pure and good condition.

The whole of the produce of each cow to be sent at each meal, that is, the sender on no account to allow his cows to be partly milked and to send the first portion only to the company, and keep back the "strippings" for feeding calves or for any other purpose.

No cream to be removed or in any way abstracted from the milk.

No water or any other ingredient or material to be added to, or mixed with the milk.

Milk yielding less than 3.25 per cent. "fat" and 8.75 per cent. "solids not fat," to be regarded as not complying with the term "good quality," the reports of the company's analyst to be accepted without dispute.

No milk to be sent from any cow that is not in good health, or that is under physic.

No milk to be sent from a newly calved cow or from a newly purchased cow until she shall have been in the possession of the sender one clear day; or from any cow whose calf has been removed, for the space of one clear day after removal.

No mixed milk of two separate meals to be sent.

\section{Straining and Cooling.}

All milk to be sent away fresh, that is-the morning's milk to be sent the same morning; and the evening's milk the same evening -summer and winter.

All milk to be properly strained as soon after being taken from the cow as possible, and before it is put into the churns.

All milk to be thoroughly cooled, immediately after milking, over a refrigerator, which shall be of a description satisfactory to the company, and to which an ample supply of water from the source or sources only passed by the Medical Officer of Health for the district and by the company's analyst, and described at the end of this contract, "shall be provided, and the drainage from which shall not be connected with any covered drain. Milk to be considered as not having been thoroughly cooled, when the temperature, on arrival at the company's dairy is found to be higher than the average temperature of milk of the same meal received from other farms.

N.B. - The sender to daily examine his refrigerator and the in-flow and outflow pipes, etc., in order to see that everything is in thorough repair, and that there is not any leakage. 


\section{Utensils, etc.}

All pails, strainers, railway churns, refrigerators, fittings, and other vessels and implements brought into contact with the milk, to be thoroughly inspected before being used, to be properly cleansed, scalded, and dried immediately after being used, and exposed to the air in a clean place, without lids or covers, and turned bottom up so as to admit free circulation of air inside.

No other than boiling water or water that has been boiled in a boiler not used for household purposes, properly fixed in a suitable place, and provided with an ample supply of water from the source or sources only passed by the Medical Officer of Health for the district and by the company's analyst, and described at the end of this contract, to be brought into contact with any vessel or implement used for the reception, cooling, or storage of milk.

\section{Conditions as to Farms and Cleanliness.}

The sender to send no purchased milk, that is, no milk other than that produced on his own farm, unless by agreement with the company. In case it be agreed that a sender be allowed to purchase milk, it shall only be on condition that he is equally responsible for such purchased milk as he is for that from his own cows, under all heads of this contract.

Sender not to interfere in any way with the drains or make any structural alteration from the condition in which they were when seen by the company's inspecting engineer without first notifying the same to the company, fully describing the intended alteration or repairs-and on the completion of such alterations, the sender to inform the company of the same, in order that they may send to inspect them, if necessary.

All cows from which milk is sent, to be supplied with good water, and all improper substances to be excluded from their food.

Attention to be paid to the cleanliness of the cows' udders, and the hands of the milkers.

All buildings in which the cows are kept, to be sufficiently and efficiently ventilated.

The sender to furnish the company with the name of the Medical Officer of Health for the district in which his farm is situated, and should there be a change at any time, to at once notify the same to the company.

Should the water at the source or sources described at the end of this contract, become impure from any cause whatever, and be so certified by the Medical Officer of Health for the district, or by the company's analyst, the company shall be at liberty to at once cease taking the milk, and to determine this contract if they so desire.

\section{Infectious Disease.}

Should any disease of a contagious or infectious character, such as diphtheria, small-pox, typhoid, or scarlet fever occur in the house or family of the sender, or in that of any of his servants employed on the farm, or should any member of his family, or any member of the families of his servants, or any visitor or temporary resident in the house of the sender, or in the house of any person employed upon the farm, have suffered from any such disease within one month of the date of such person's arrival, the person so affected, or who may have been affected, and those persons living in the same house who have in any way 
personal communication with such person, to be prohibited and prevented from coming into or near the dairy or cowsheds, or from taking part in the dairy operations, and the sender to immediately give information to the company in order that the directors of the company may use their own judgment as regards the use of the milk, they guaranteeing that the sender shall not sustain any loss from having given such information ; but should any disease so exist, or have existed, and the sender shall neglect to give information as aforesaid, then he, the sender, to be liable for and pay to the company the sum of one hundred pounds, as and for liquidated damages. (See back.)

Should anything be found to exist, at any time, either on the farm, or in the family of the sender, or in that of any of his servants, that shall be deemed by the company to be, or likely to become, in any way dangerous to the public health, the company to have power to at once cease taking the milk, and, if they please, to determine this contract.

The company or its representative to be allowed to enter upon and inspect the farm and everything connected therewith at any time, and to have the liberty of taking samples of milk at any time, either at the farm, or whilst the milk is in course of delivery.

The company to have the right to reject any milk which does not comply with the above conditions, and if they deem fit, to determine this contract. In case of milk received and sent out by the company for distribution to its customers being found, generally, by the customers on the beat on which it has been distributed, to be out of condition, the senders to pay for such milk at the price the customers would have paid the company for it.

The sender to be liable for all damages sustained by the company that may arise from disregard or neglect of all or any of the above conditions.

The above contract is made subject to the sanitary condition of the farm being entirely satisfactory to the Company's Inspecting Engineer and the Local or other Medical Officer of Health, and its being so maintained.

Dated this. day of I90

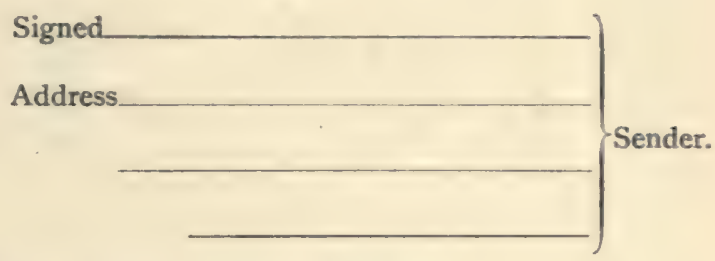

Accepted for The Aylesbury Dairy Company, Limited.

Director.

Secretary.

Senders are particularly requested to read the conditions of their contract with the greatest possible care, as under no circumstances can they be departed from, and in case of a breach of any of the clauses, the terms of the contract will be enforced. 
Precautions suggested to occupiers of farms for the purpose of ensuring a healthy supply of milk, and of complying with the terms of their contract with The Aylesbury Dairy Company as above.

I. Constant and periodical inquiries as to the health of all persons working on the farm, and of their families, more especially with regard to those whose duties bring them into the dairy and cowsheds, and in contact with the milk.

2. Immediate consultation with the Medical Officer of Health for the district in the event of any illness in sender's house or in the house of anyone in his employ.

N.B.-Sore throat occurring in young persons, say under I6 years of age, although apparently of ordinary character, should be brought to the notice of the Medical Officer of Health, without a moment's delay.

3. Immediate suspension from work of anyone who is either suffering, or supposed to be suffering, from any infectious disease, or who is brought in contact with anyone so suffering.

4. Immediate notice to The Aylesbury Dairy Company on the discovery of the existence of any disease of a contagious or infectious character, among men or cattle, on the farm in the neighbourhood, and, in the former case, immediate stoppage of the milk supply to the company.

5. Immediate stoppage of the milk supply to the company, should sender have any reason to suspect the pollution or contamination of the milk from any other source whatever, or of the water used for dairy purposes.

6. Absolute cleanliness in the dairy and in the sheds; the use of none other than boiling water, or water that has been boiled in the boiler set specially apart for dairy purposes, and from the authorised source or sources only; and the constant cleansing and flushing of all drains.

A sender of milk to The Aylesbury Dairy Company renders himself liable to a penalty of $£ \mathrm{I} 00$ if he fails to report, immediately, any case of contagious or infectious disease which may occur in his own household, or in the houses in which any of his workpeople reside, or from which any visitor or temporary resident therein may have suffered within one month previous to such person's arrival. As an inducement to him to report such cases without the slightest possible delay, and to act as he may think it advisable in the company's interests as well as his own, the company undertakes to pay for all milk stopped under any of the above contingencies, just as if it were sent, and for any loss or expense that may be otherwise incurred by senders.

The Aylesbury Dairy Company would further suggest that the cards of rules which they issue for the guidance of persons employed in the dairies and cowsheds, be placed in prominent positions therein. 


\section{APPENDIX $Q$}

\section{COPENHAGEN DAIRY COMPANY'S REGULATIONS}

Regulations regarding the supply of milk to the "Kjöbenhavns Malkeforsyming."

\section{A.-Feeding and Treatment of the Cows.}

1. The food for the cows should be quite fresh and in a good state of preservation, without anything liable to communicate abnormal flavour or colour to the milk.

2. In summer the cows should be put out in pasture, and have no other food than grass and clover; only in case of need is it permissible to give dry food and cut corn, and then only in the open air. It is forbidden to leave them in the byres at this period of the year.

3. The farmer should arrange with the company beforehand about the food to be given to the cows in the winter.

4. In any case he must conform to the following rules:-

(a) Roots. Carrots and beet should be given in the proportion of $1 \frac{1}{2}$ bushels for each cow, but on condition that they are mixed with at least 5 lbs. of corn, bran, and oil-cake. Cows which furnish milk for infants should only be given them in the proportion of $\frac{1}{2}$ bushel. Turnips, chonx-raves, rutabaga, turnip tops are to be excluded.

(b) Oil-cake. Colza and tournesol-cake should only be given in the proportion of at most I lb., mixed with at least $5 \mathrm{lbs}$. of corn and bran. Cows which furnish milk for infants should not have any.

(c) The use of any distillery refuse is forbidden.

5. Before bringing the cows in in the autumn, their tails, hind-quarters, and udders should be clipped.

6. The times of calving should be regulated so that the quantity of milk dispatched to the company during the months of September and October, is not less than the average quantity during the first four months of the year.

7. The farmer who wishes to deliver a larger quantity of milk than usual, must arrange with the company beforehand.

8. The milk of recently calved cows must not be used during the first fourteen days of lactation; the company will also refuse the milk of diseased cows or of cows giving more than a maximum of 6 litres a day. 


\section{B.-Treatment of the Milk.}

9. The milking should be carried out with the greatest care and cleanliness, under the following conditions :-

(a) The milker should wear a special costume, and be provided with a towel in case it should be necessary to wash his hands.

(b) During the milking the byre should be properly lighted, especially behind the cow, so that the milker may see to do his work with care and cleanliness.

(c) Immediately after the milking the milk should be passed through a metal sieve covered with a clean fine cloth.

(d) The milk should then, whatever the season, be passed through a refrigerator, to lower the temperature to $4^{\circ} \mathrm{R}$., at which it should be maintained until its despatch.

(e) The manure should be removed in the morning after the milking, and in the afternoon at least an hour before the evening milking.

( $f$ ) The milk of cows just entering on the period of lactation, that of cows declared diseased, and that of cows giving more than 6 litres a day, should be collected separately, so that it cannot get mixed or mistaken for the normal milk destined for the company.

Io. The farmer is bound to keep a provision of at least 30 lbs. of ice for each 100 litres of milk.

I1. A Lawrence apparatus, which can be hired from the company, should be used for cooling the milk.

\section{C.-Delivery of the Milk.}

12. The milk should be delivered once or twice a day, according to the needs of the company, at the nearest railway station, either as whole milk, or as "half-creamed" milk and cream. It should only leave the farm in time to arrive at the station at the proper time. In summer the vans which convey the milk-cans to the station should be covered with an awning as a shelter from the sun. milk.

13. The company provides the necessary cans for the conveyance of the

14. On their return to the farm the cans should be carefully rinsed with cold water, the outsides freed from all dirt or dust which they may have collected, then placed upside down in a well-ventilated place until required again.

I5. The use of the cans for any other purpose than the conveyance of milk is strictly prohibited.

\section{D.-Various Obligations.}

16. The farmers are bound on their word of honour to give the company every information regarding the milk.

17. They are bound to allow the veterinary of the company to visit their byres as often as he may think necessary. They must convey the veterinary to and from the nearest station, and strictly carry out his instructions.

18. Those cows declared tuberculous by the veterinary should immediately be separated from the rest of the herd, to be sold or slaughtered as soon as possible. 
19. All calves raised for the production of milk must, since 1896, be submitted to the tuberculin test, and the injections must be repeated at least once a year in the case of those which do not react to the test.

Since the 9 th January 1898 , the tuberculin test has become obligatory in the case of cows and young cattle separated on suspicion of disease.

20. If during the interval between the veterinary's visits, one of the cows should be suspected of disease, the farmer should immediately notify the company, who will suspend the delivery of the milk until the veterinary sball have made his report; the milk refused by the company will be paid for, in the meantime, as if it were delivered.

2I. The farmers are bound to pay every attention to the state of health of all persons residing or employed on the farm, as well as their families. They are bound, in case of any infectious illness, to immediately notify the company of the fact, who will then take the necessary measures.

In this case the company refuses to take the milk until all trace of illness has disappeared, but pays for it all the same.

22. If the milk should be found to be below the normal in quality and consequently unfit for sale, the company reserves the right to refuse it without giving the farmer compensation.

23. Either of the contracting parties may, after six months' notice, withdraw on the rst January.

24. If, by reason of an epidemic or any unforeseen cause, the sale of the milk should be suspended in Copenhagen, the farmer will have to keep his milk and will have no right to any idemnity from the company.

I accept the above conditions.

(Signature of the Farmer.) 


\section{APPENDIX R}

\section{TUBERCULOSIS IN LONDON COWSHEDS}

In addition to the returns appearing on p. 213 , Mr Shaw, F.R.C.V.S., Veterinary Inspector to the London County Council, has also made two separate investigations as to the degree of tuberculosis present in London cows. The first, in 1898 , was an examination, by tuberculin, of 73 cows selected haphazard in six different metropolitan cowsheds. Sixteen or 20.5 per cent. of these cows reacted to the tuberculin, and were therefore judged to be suffering from tuberculosis. In only 2 of these 16 cows was there any clinical sign of disease, the others appeared to be healthy. It should be added that in one shed 44 per cent. of the cows were tuberculous, in another 36 per cent. were tuberculous, and in two sheds ( 28 cows) there were no cases of tuberculosis.

The second investigation made by Mr Shaw was at Colney Hatch Asylum in July 1900 , when he tested 53 cows with tuberculin. Ten, or 18.8 per cent. of them were found to be tuberculous. If these returns are compared with results obtained in connection with the milk supply of Manchester or Liverpool, it will be found that London cows appear to suffer less from tuberculosis than cows in the country. The reasons for this are three : First, good class animals come to the London cowsheds, because the owners have to send them to the butcher when dry, and, therefore, inferior animals are a poor investment. Secondly, there is a very regular and thorough inspection by the veterinary inspector of the London County Council. Thirdly, there is no inbreeding. Consequently, general tuberculosis, which probably affects 30 per cent. of milch cows in the country (McFadyean, Lloyd, Delépine, etc.), only affects 20 per cent. in London, and whereas 2 per cent. of country cows have tuberculosis of the udder, only 0.02 per cent. of London cows suffer in the same way.

\section{TUBERCULOSIS IN MANCHESTER AND LIVERPOOL COWSHEDS}

Manchester.-In I902, 5649 inspections of cows were made in Manchester (which means that many cows were examined more than once), and no case of tuberculous udder was found. In two cases, however, there was clinical evidence of generalised tuberculosis, and the cows were slaughtered.

In 1902, 420 samples were taken at the various Manchester railway stations from 345 farmers sending milk in from the country. Of these 345 farmers-

196 resided in Cheshire, of whom 25 sent tuberculous milk $=12.72$ per cent.

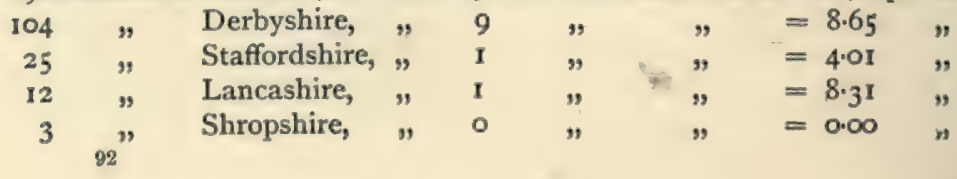


As a result of following up tuberculous samples from the railway stations a total of 3656 cows were inspected. Out of the 420 samples 36 were found by Prof. Delépine to be tuberculous $=10.43$ per cent. These samples came from $3^{1}$ cows, 15 of which were slaughtered, the remaining 16 being disposed of on the open market or otherwise (Report on Health of Manchester, 1902, pp. 137-14i).

Liverpool.-In addition to the returns appearing on pp. 215 and 216 , it may be stated that in 1902,442 cowsheds existed within the boundaries of the City of Liverpool containing 6068 cows. Out of this number 13 cows were, after inspection, suspected of suffering from tuberculosis, and 5 were found to be tuberculous.

In I902, 297 samples of milk were taken at Liverpool railway stations, and 29 or Io per cent. were found to be tuberculous (as compared with I per cent. in 213 town samples); 22 farms comprising 66 cowsheds were therefore visited and 760 cows inspected. Out of this total of 760,16 were obviously suffering from tuberculosis, and $\mathrm{I} 8$ presented suspicious features; 24 cows were isolated and ultimately disposed of (Report on Health of City of Liverpool, 1902, pp. 152-157).

\section{TUBERCULOUS MILK AT MELBOURNE}

Special investigations have been made in the City of Melbourne as to the condition of the milk supply. During the past three years 90 samples have been examined, of which 5 (or 5.5 per cent.) were found to be tuberculous by inoculation in guinea-pigs. There is some evidence to show that Australian guineapigs are more resistant to bovine tuberculosis than European. Fifty samples of milk were examined for ordinary bacteria. The highest number of bacteria present was $8,892,000$ per c.c., the lowest was 4800 per c.c. One pasteurised milk gave 3000 and one gave 30,000 bacteria per c.c. Five of the 50 samples showed over two million bacteria per c.c. (Annual Report on Health of Melbourne, 1902, J. Jamieson, M.D.). 


\section{APPENDIX S}

\section{THE DANISH METHOD OF MILK SUPPLY IN ENGLAND}

The principles and practice of the Copenhagen Milk Supply Company have been introduced into England, and are being carried out by $\mathrm{Mr}$ C. W. Sorensen at the White Rose Dairy, West Huntington, York. Mr Sorensen is a nephew of Mr Busck, of the Copenhagen Company, and has been trained in the Danish methods. His dairy farm at York is carried on in a similar manner to the Copenhagen Company's work, with this difference, that whilst the latter obtain their milk from contributory farms, Mr Sorensen works his own farm, and the control and management of the cows is under his direct and immediate supervision. The writer had an opportunity recently of visiting this dairy farm near York, and a brief description of the most important points may be added here.

I. The health of the cows is secured by a special monthly inspection by the York Corporation Veterinary Officer, Mr Wm. Fawdington, M.R.C.V.S., who has authority to order the disposal of any unhealthy or even suspected animal, and whose reputation and experience affords a guarantee of efficiency in this important point. There are about 50 cows in all, Io of which are Jerseys. The feeding of the cows is scientifically carried out. No brewers' grains, turnip-tops, or other unsuitable foods are used, and especial care is exercised in the selection and feeding of the cows supplying "Table or Nursery Milk" so as to maintain a high standard of richness and flavour. To ensure an abundant supply of pure water for the cows to drink, as well as for cleansing purposes, the farm has been connected with the York City Water Supply, which is provided in a continuous trough at the head of the stalls. The cleanliness and ventilation of the cow-houses receives special attention, and is in every way excellent.

2. While no money has been wasted on fancy fittings (which make the milk no better, but simply increase the cost), the proprietor's aim has been to keep everything sweet and clean from the cows themselves down to the smallest utensil. A high pressure boiler has been put in for sterilising all utensils, cans, etc., with steam.

The udders of the cows are cleansed before milking. The milkers are clothed in overalls, and wash before, and if necessary, during milking. The operation of milking is carried out under cleanly conditions and with clean utensils. After milking the milk is strained by a "Ulax" strainer. This apparatus consists of a fine metal sieve through which the milk is first passed. Then a conical funnel ending in a short cylinder, just above which is a ledge 594 
supporting two gauze metal discs which act as strainers. Between the strainers is placed the filtering medium which is a pure and innocuous prepared cotton, the edge of which projects equally all round the strainers, this projecting edge-in order that no milk may pass unfiltered between the edges of the strainers and the side of the funnel-being tucked under the bottom strainer; the edge of the bottom strainer should be first moistened with water, to permit of the cotton being turned more easily. These are covered by a cupshaped lid provided with apertures to allow the milk to pass through, the whole being kept in position by means of a turned cross piece which acts as a spring. The filtering process may be carried out during the operation of milking-the milk from each cow being poured into the filter, and so immediately freeing it from impurities. Immediately after each filtering operation, the medium is burned. The "Ulax" is probably the best strainer now on the market.

3. Immediately after the milk is strained, prompt and efficient cooling is obtained by allowing it to flow in a thin layer over a corrugated copper cylinder, inside which cold water and ice are passed, thus reducing the temperature in a few seconds to a point at which germ life cannot develop. Clean milk, so treated, needs no "preservation." If kept in a cool place it will remain perfectly sweet for several days, even in the hottest weather. Therefore no preservation or sterilisation is necessary.

4. The usual practice of slopping milk about from one can to another in the street - exposed to contamination from clouds of dust, the not always clean hands, or in wet weather, the dripping garments of the driver-is one so objectionable that only long usage and the absence of anything better has made it tolerated. The ideal system, without doubt, is delivery in glass bottles, filled and sealed at the dairy, and placed straight on the table without the intervention of jugs, basins, or what not. Next comes delivery in cans, likewise filled and sealed at the dairy. After that comes the system of drawing the milk by tap from a sealed can, which, however, is much preferable to the plan of dipping into an open can. The entire system at this dairy farm is so arranged as to supply a clean whole milk from healthy cows kept under hygienic conditions, and protected from dust and infection. This desirable object is attained by $(a)$ clean milking, $(b)$ straining, $(c)$ cooling, and (d) bottling promptly, efficiently, and at the dairy farm. On the whole, Mr Sorensen's methods appear to represent the high tide of dairy farm work in England. But nothing is done by him which could not be done by every dairy farmer in the country. It will be understood that this system is an illustration of how to obtain a naturally pure milk supply without modification or sterilisation. 


\section{APPENDIX T}

\section{INFANT MILK DEPÔTS}

It has been pointed out elsewhere that one of the essential requirements for the satisfactory working of a milk depôt is the control of the source of the milk. The following are the conditions of contract for the supply of milk and cream to the York Infants' Milk Depôt :-

I. The farm, water supply, drainage system, farm-buildings, dairy and cattle shall be open at any reasonable time to the inspection by the Association's Representatives.

2. The cows shall be subject, if required, to periodical veterinary inspection, and the contractor undertakes not to supply milk from, and as soon as possible to get rid of, any cow considered by the Association's Veterinary Surgeon to be diseased.

3. The cows shall during summer, be pastured, and during winter so fed that no taint is imparted to the milk. The contractor undertakes to use no brewers' grains (wet or dry), turnip-tops, or vetches, and to be strictly moderate in the use of swedes and turnips.

4. The udders of the cows shall be carefully cleaned before milking, and the utmost possible cleanliness observed at every point connected with the cows, cow-house, utensils, and attendants.

5. The milk shall be carefully strained and cooled to at least $60^{\circ} \mathrm{F}$., immediately after milking, over a cooler of approved design.

6. Cans for delivery will be supplied by the Association, and shall be used for no other purpose whatever. They will be washed and sterilised at the Depôt.

7. The milk shall be pure milk, free from preservatives or other chemicals, and shall contain not less than 3.2 per cent. of butter-fat. The cream (generally required in the proportion of half a pint to each gallon of milk) shall contain not less that 35 per cent. of fat, be sweet, smooth, and free from lumps, preservatives or other chemicals.

8. The contractor undertakes to supply no milk or cream except that produced on his own farm without the express consent in writing of the Association's Representative.

9. Delivery may be made in cool weather in the afternoon (afternoon's milk), in summer in the morning (morning's milk), not later than 8 o'clock.

N.B.-Double quantities will be required for Saturday's sterilising, and none for Sundays. 
Io. Payment shall be made by cheque on the of each month, for milk and cream supplied the previous month. Such payment to be at the rate of___ per imperial gallon for milk, and___ per imperial pint for cream, of quality as provided in par. 7 .

II. One month's notice, in writing, shall be required of either party to terminate this agreement.

I agree to the above.

(Signed) Contractor.

September 1903. 


\title{
USEFUL DATA AND FORMULÆ
}

\author{
WEIGHTS AND MEASURES
}

Comparison between Metric and British Systems.

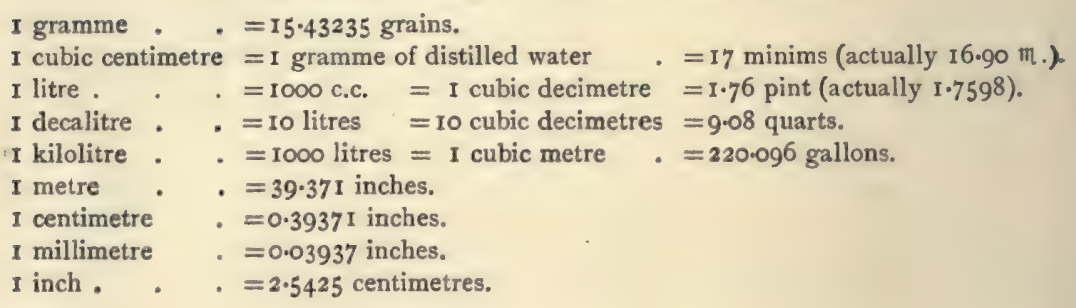

\section{CIRCULAR MEASURES}

Circumference of a circle $=$ diameter $\times 3.14$.

Area of a disc . . = square of diameter $\times .7854$.

Area of a sphere . . . = square of diameter $\times 3.14159$.

\section{USEFUL FACTORS}

To convert Multiply by

Grammes to ounces . . . . . . . . . . . . . . .

Ounces to grammes . . . . . 28.36

Litres to gallons . . . . . . .22

Gallons to litres . . . . . . 4.54

Centimetres to inches . . . . . 4

Inches to centimetres . . . . . 2.5

Kilogrammes to pounds . . . . . $\mathbf{2} 2$

Pounds to kilogrammes . . . . . 45 


\section{INDEX}

AcID-FAST organisms, 253 ; classification of, 253 ; of butter and milk, 254; of grass, manure, and hay, 256 ; differential diagnosis of, 257 ; pathogenic differences of, $25^{8}$

Acidity of milk, I33, 154

Aërobic organisms, examination of, 64-74

Agar media, 38-40

Air, bacteria of, 98

Air, examination of, 98-110; by Kóch's plate method, I00; by Miquel's method, 102 ; by Laveran's method, I05; by Strauss and Wurtz' method, 106; by Hesse's method, IO7; by Sedgwick and Tucker's method, ro9; by Petri's method, Iro; by Frankland's method, 110

Albuminoids of milk, 5

Alcoholic fermentation in milk, 162

Amendments in milk legislation, 471

America, milk legislation in, 474

Amylolytic ferments, I52

Anaërobic organisms, examination of, 78 ; in hydrogen, $80,88,89,93$; in Vignal tube, 83 ; in Roux tube, 85 ; in nitrogen, 86 ; in Buchner tube, 86 ; by Wright's method, 87 ; by Klein's method, 88 ; in vacuo, 88, 89, 93; in Esmarck roll cultures, 90 ; in Fraenkel's tube, 91 ; by yeast flask method, 92 ; by Veillon's method, 92 ; by oxygen absorption method, 93 ; in Pasteur's tubes, 95

Anaërobic technique, 78-97

Andrewes on air examination, 99

Antibiosis, 140

Antitoxin treatment of diphtheria, 39 $\mathrm{r}$

Aylesbury Dairy Co., 48I-484, 58 I-588

Aymard's pasteuriser, 537

BABCOCK on milk composition, 3

Bacilli, acid-fast, 253

Bacillas aciai lactici, 393 ; actinobacter, 180 , 599
393 ; airews (Adametz), 394 ; Nos, I417 (Adametz), 394, 395; albus (Löffler), I78; arborescens lactis, 395,396 ; arana, 396; aurantiacus, 396 ; aureus acidi, 397 ; aureus lactis, 397 ; anureus minutissimns, 397 ; bernowis, 397 ; of bitter milk, 177 ; butyricus, 178, 398-400; butter (PetriRabinowitsch), 254, 400; butter (Binot), 401 ; butter (Mark1), 402 ; butter (Grassberger), 403 ; butter (Tobler, I-5), 403406; cancasium, 406; circulans, 407; citrews acidi, 407; citress arborescens, 407 ; citrews lactis, 408 ; coli communis, $69,408-412$; communis Lactis, 412 ; cyanogenes, 172 ; diphtheriar, $68,34 \mathrm{I}, 4 \mathrm{I} 2-$ $4 \mathrm{I} 4$; dispora lactis, $4 \mathrm{I} 4$; enteritidis sporogenes, 69, 4I4; filiformis lactis, 415; fiworescens liquefaciens, 4I5; fiworescens non-liquefaciens, 416; finorescens Schuylo killiensis, 4I7; No. 4I, 4I7 ; Friburgensis (I and 2), 417-419; of grass (Moeller), 256, 419; of Guillebeau, 419-420 ; gummosus, 183, 420; Hessi, 182, 420; lactis niger, 184,420 ; lactii pilwitosi, 183, 420 ; lactis saponacti, 179,421; lactis viscosus, 181 , 421 ; lactericus, 421 ; lacticus, 422 ; lactis of Flügge (I-I I ), 422-424; lactis erogenes, $183,189,424$; lactis albus, 424 ; lactis erythrogenes, 174, 424; liguefaciens lactis amari, 178 ; megatherium, 425 ; mesentericus fuscus, 425 ; mesentericus vulgatus, 183; milk of Moeller, 426 ; musci lactis, 426 ; mycoides lactis, 426; pallescens, 427 ; prodigiosws, 427 ; ruber lactis, 428 ; rudensis, 428 ; Schafferi, 426; subtilis, 429 ; syncyanews, 430-432; synxanthous, I75, 432; tuberculosis (Koch), 70, 232253, 432 ; psendo-tuberculasis (Pfeiffer), 67,433 ; typhosus, 3II, 434; varians lactis, 434 ; violacens, 435 ; viscosus, 436 
Bacteria, aërobic, 64; anaërobic, 78 ; in air, 98 ; spore formation of, $140-144$; in separated milk, I44; in set milk, I45; in set cream, $145 ;$ in butter, 197; in cheese, I98-20I

Ballard on causes of epidemic diarrhœea, $36 r$

Batten on infantile tuberculosis, 245

Bergey on streptococcus in milk, 223

Binot on air bacteria, 98 ; butter bacillus, 40r

Bitter milk, I76 ; bacteria of, 177

Blood cells in milk, 29

Blue milk, I7I; bacteria of, I72

Booker on bacteriology of diarrhoea, 362

Bouillon, 35

Bovine tuberculosis, 234, 236, 247

Bowhill on examination for diphtheria bacillus, 34 I

Brown, Sir George, on Hendon disease, 286

Buchner's anaërobic method, 86

Burr on lactic fermentation, 158,190

Butter, examination of, 72 ; making, bacteria in, I9I, I94, 197; tubercle bacilli in, 220; acid-fast bacilli in, 254

Butter-milk, 8

Butyric fermentation, 159 ; bacteria of, $16 \mathrm{I}$

Capsule-staining, 64

Carbohydrates of milk, 4

Carbol-fuchsin, 57,60

Carr on primary lesion in human tuberculosis, 245

Carson on milk sterilisation, $54 \mathrm{I}$

Casease, I69

Casein, 5

Caseinogen, 5

Centrifugalisation of milk, 75,76

Cheese, bacteria, 199-20I ; making, bacteria in, I98 ; abnormalities, 205; inflated, 205 ; tainted, 207 ; bitter, 207 ; coloured 207 ; poisonous, 208

Chemical composition of milk, 2-17

Cholera, 373

Clearing films, 56

Clostridium fretiaum, 437

Coagulation of milk, 9, I53, I66, I70

Colostrum, 12, 27, 7 I

Colwell on preservatives in London milk, 123

Composition and properties of milk, 2-17 ; conditions affecting it, I3-16

Compound staining, 59-64
Conditions affecting bacteria in milk, I

Conn on bacteria in milk, 127 ; on lactic fermentation, I56 ; (and Esten) on cream ripening, 187, 188, 189, 193; artificial starters, 196; cheese ripening, 202

Contagious Diseases (Animals) Act, 454, 550

Control of milk supply, $45^{2}$; by the State, 452 ; by private enterprise, 480 ; by the trade, 504

Cooling of milk, 527

Copenhagen Dairy Co., 484-494, 589

Cover glass preparations, 53

Cowsheds, condition of, $5^{14}$; construction of, 516-522; disinfection of, 186,512

Cream, 6, 8, I45, I46

Cream ripening, 187,189

Culture media, I $7-19,30-48$

Curdling of milk, 8

DAIKY, disinfection of, $185, I 86,384$

Dairy Orders, 454, 555-566

Dairy Supply Co., Aylesbury, 48I ; Copenhagen, 484-494; (Danish), 494 ; Pasteur, 495

Davies, on Clifton epidemic of typhoid, 270, 271, 272, 379; on Clifton epidemic of scarlet fever, $38 \mathrm{I}$; Widal diagnosis in milk-borne epidemics, 387

Dean and Todd on bovine diphtheria, 339, 343

Delépine on farm contamination of milk, 24 ; on effect of temperature, I37-139; on cause of diarrhoea, 365 ; on tuberculosis, 228 ; on Widal diagnosis, 387

Dextrose, 5

Dextrose-free bouillon, 36

Diarrhoen, epidemic, 36I-373; bacteria of, 362 ; causes of, $36 \mathrm{I}$; conditions favourable to, 365 ; and milk, 368 ; preventive methods, 372

Diphtheria, bacillus of, 68,341 ; bovine, 338 ; channels of infection, 344 ; epidemics, milk-borne, 336-351; typical outbreaks, 346-35I

Diseases of cow communicable to man, 210

Diseases of milk, $17 \mathrm{r}$

Disinfection of cowsheds, 185, 186, 384, 512 ; of dairy, $185,186,384$

Drigalski and Conradi's medium, 37

Dropping staining bottles, 57

Duclaux on casease, 169 ; on ropy milk, 180 ; on cheese bacteria, 198 ; on Tyrothrix, 447-45I 
Dyes, aniline, 57

Dyscaseose, 9

EASTES on pus cells in milk, 29, 222

Eberth's bacillus of typhoid fever, 304, 3 II, $+34$

Economic bacteria in milk, 187

Effects of heat on milk, 9

Elsner's medium, 4I

Enteritis of cow, 2 I I

Enumeration, methods of, of colonies, 5 I

Enzymes, 152

Epidemic diseases, milk-borne, 259-39I ; characters of, 259; local incidence in, 263; incidence as to social position, 264 ; incidence on milk drinkers, 265 ; age and sex incidence, 269; incubation period, 272 ; sudden onset, 275 ; clinical characters, 276 ; mortality rate, 277 ; scarlet fever, 279-304; typhoid fever, 304-335; diphtheria, 336-35I ; throat illness, 352-36r ; epidemic diarrhoea, 36I-373; cholera, 373 ; investigation and prevention of, $372,375-391$

Epithelium in milk, 29

Ermengem's stain for flagella, 63

Esmarck's roll cultures, 90

Examination of milk, 75 ; for special organisms in milk, 67-69; of colostrum, 71; of butter, 72 ; of cheese, 72 ; of moulds, 72 ; for pus cells, 73 ; for gas production, 77 ; for indol, 77 ; of air, 98-110; of water, IIo-I I5; of cream, 145

External conditions affecting lactic fermentation, 157

Eyre on diphtheria bacilli in milk, 34I, 342

FAT of milk, 2, 3, 6

Fermentations of milk, 148, I52; methods of observing, 77 ; kinds of, 152 ; lactic acid, 153 ; butyric, 159; alcoholic, I62 ; coagulative, I66 ; diseases of milk, I7I

Film, preparation of, 54

Films, clearing of, 56

Filtration of milk, 529

Firth and Horrocks, on typhoid bacilli in soil, 310 ; do., clothes, 313

Fixation of film, methods of, 55

Flagella, staining of, $6 \mathrm{I}-64$

Flemming's solution, 73

Foot-and-mouth disease, 2 II

Fraenkel on staining, $6 \mathrm{I}$; on anaërobic culture, 9 I
Frankland on air examination, I10

Freudenreich on bacteria in milk, I26, 127

\section{GaLACtose, 5}

Garget, I80

Gas production, 77

Gelatine media, 37, 38

Glass blowing, 547

Glucose bouillon, 36 ; formate agar, 40

Glycerine agar, 39

Gordon on scarlet fever streptococcus, 445 ; on $B$. coli, 408

Gram's method of staining, 59

Great Ormond Street Hospital, milk pasteurisation at, 534

Guthrie on primary lesions in human tuberculosis, 245

Halliburton on caseinogen, 5 ; on preservatives, 532

Hamilton on bovine and human tuberculosis, 229

Heat, effect of, on milk, 9

Hendon outbreak, 282

Herds, milk, 504-510; housing of, 514

Hesse's tube, ro7

Hope on bovine tuberculosis at Liverpool, $215,218,592$

Horrocks, see Firth and Horrocks

Horton Smith on typhoid bacillus, 305, 307,308

Hüppe on lactic fermentation, 156 ; on blue milk, I7I-I72; on red milk, I74; on bitter milk, 176,178

Hutchison on koumiss, I64; on condensed milks, 37 I

Hydrogen methods, 80

INDOL, examination for, 77

Infectious Diseases (Prevention) Act, 456, 467

Influence of time on bacteria in milk, 127 ; of temperature, I 24-I 26

Inoculation of animals, 74

Inter-relationship of bacteria in milk, $\mathbf{J} 39$

Intra-peritoneal inoculation, 74

Inversive ferments, 153

Investigation and prevention of milk-borne epidemics, 375-391 ; notification, 375 ; milk-infected cases, 376 ; relationship of milk, 378 ; and prevention, 384 ; and bacteriological diagnosis, 385 ; and examination of throat, 385 ; and serum 
diagnosis, 386 ; and preventive inoculation, 390

Iodine solution (Gram), 59

KAYE on cowsheds, $515,516,518$

Kephir, 165

Klebs-Löfler bacillus of diphtheria, 68 , $34 \mathrm{I}, 4 \mathrm{I} 2$

Klein on $B$. enteritidis sporogenes, 69 ; on anaërobic culture, 88 ; on London milk, 217 ; on pseudo-tuberculosis, 236 ; on Hendon disease, 283 ; on cow disease and milk, 282 ; on bovine diphtheria, 338 , 342 ; on cause of diarrhœe, 364,369

Koch on bovine tuberculosis, 225-227; on plate method, 100 ; on standard of sterilisation, I25; on spore formation, I 4 I-I 43

Korn's acid-fast bacilli, 4I7, 418

Koumiss, 163

\section{LACTALBUMIN, 5}

Lactase, 4

Lactic fermentation, 153 ; bacteria in, 156 ; external conditions affecting, 157 ; function of, 159

Lacto-globulin, 5

Lactose, 4

Laveran's method of air examination, ros

Legislation, milk, in England and Wales, 454 ; by model milk clauses, 457 ; in America, 474; in British Colonies, 478 ; in Scotland, 469; in Ireland, 47I; amendments suggested, $47 \mathrm{I}$; in Europe, 473

Leucocytes in milk, $\mathbf{2 7}$

Leuconostoc mesenteroides, 437

Lister on sterile milk, I9; fermentation in milk, 155

Litmus gelatine, 38

Littlejohn on Edinburgh epidemic of scarlet fever, 27 I

Lloyd on fat globules, 2, 6, 7; on cheese making, 204, 206; on tainted cheese, 207

Lorffler's bacillus of diphtheria, 412 ; methylene-blue, $5^{8}$

London Milk Supply, 25, 122, 123, 215, $217,461-468$

Low temperatures, effect on tubercle bacillus, 252

MACConkry's liquid medium, 36 ; capsule-staining, 64
MacCrorie stain for flagella, 63

McFadyean on foot-and-mouth disease, 212 ; on tuberculosis, 215,225 ; on tuberculin, 513; Macfadyen and Rowland on low temperatures, 125; and Hewlett on milk sterilisation, 540

Martin on tuberculosis, 229-230; on virulence of tubercle bacillus, 243 ; on typhoid bacillus in soil, 310

Mastitis, 2 I I

Media for cultivation of milk bacteria, I7-19, 30-48; favourable to bacteria, 17-19; neutralisation of, 31-34; peptones used in, 34 ; preparation of, 35 ; bouillon, 35; glycerine bouillon, 35; glucose bouillon, 36 ; glucose formate bouillon, 36 ; phenol-bouillon, 36 ; dextrose free bouillon, 36 ; Parietti's bouillon, 36 ; MacConkey's medium, 36 ; Drigalski and Conradi's medium, 37 ; gelatine, 37 ; carbolised gelatine, 38 ; glucose formate gelatine, 38 ; glucose gelatine, 38 ; litmus gelatine, $3^{8}$; neutral litmus solution, 38 ; agar, 38; glycerine agar, 39; glucose agar, 39; agar gelatine, 39 ; glucose formate agar, 40 ; milk agar, 40 ; potato, 40 ; potato gelatine (Elsner), 4I ; milk, 42-44; litmus milk, 44 ; gelatinised milk, 44 ; whey, 45; gelatinised litmus whey, 45 ; Petruschky's whey, 46 ; litmus lactose solution, 46; lacto-serum, 46; extract of malt, 47 ; gelatinised beer wort, 47 ; peptone solution (Durham), 47; hay infusion, 47 ; choice of, 48 ; preservation of, 48

Melbourne, tuberculous milk at, 593

Method of obtaining sterile milk, 42-44; of milk dilution, 48-5I ; of slide preparation, 53-55; of cleaning slides, 53; of staining, 55-64; of examining milk, 30 et seq.; of heating milk, Io

Methylene-blue, $57,58,68$

Micrococcus acidi lactici, x88, 437-438; arborescens lactis, 438 ; aureus lactis, 438 ; of bitter milk, 177; candicans, 438 ; casei amari, 177 ; cinnabareus, 439 ; citreus lactis, 439; communis lactis, 439 ; Fretdenreichii, 182, 440; liquefaciens, 440 ; rosaceus lactis, 44I ; rubidus lactis, 441 ; varians lactis, 44I ; viscosus lastis, 44I

Milk as medium for bacteria, $x$ 7-20

Milk herds, 504-510

Milk legislation, $454-478$

Milk sugar, 4 
Milk pollution, 20-26

Milk, as culture medium, 42

Milkers and milking, 522-524

Milking, clean, 524

Miquel on air bacteria, 99 ; on air examination, 102,107

Mist bacillus, $44^{2}$

Model milk clauses, 457, 569

Moeller's acid-fast bacilli, 254-257; stain for spores, 64

Mohler's experiments in tuberculosis, 241

Montefusco on Naples milk, 119

Mucous threads in milk, 29

Municipal Milk Depôts, 460, 503, 535, 596

Mycoprotein, 17

NeELSEN's stain for tubercle-bacillus, 60

Neutral litmus solution, 38

Neutralisation of media, fractional method, 31 ; in bulk, 33

New York, milk of, 26, $117,120,128$

Newsholme, on throat illness at Brighton, 358 ; on epidemic diarrhoea, $366-368$; on infant milk consumption, 370

Niven, on tuberculous cows in Manchester, 214,218 ; on epidemic diarrhoea, 368 ; on Manchester milk clauses, 459 ; on examination of milk, 509 ; on farmers' circular, 572

Northrup on infantile tuberculosis, 245

Number of bacteria in milk, 116 at seq.; in butter, 197 ; in cheese, 199; in cream, 187

Nutrient media, 30

Oidium lactis, 203, 442

Organised ferments, 152

\section{- Pariettris bouillon, 36}

Park on bacteria in New York milk, 117 , I20, 128, 139

Parsons on Macclesfield epidemic, 267, 353

Pasteur pipettes, making of, 547

Pasteur tubes, 95 ; on lactic fermentation, I5I, I55; on butyric fermentation, I 59

Pasteurisation of milk, 533 ; methods of, 534 ; domestic, 237 ; results of, 539

Pathogenic bacteria in milk, 212; clinical evidence of, 212 ; bacteriological evidence of, 215

Pathology of milk, 27

Pattin on milk consumption, 315

Penicillium glaucwm, 203
Peptones, 34

Perlsucht, 236

Petri dishes, 52 ; acid-fast bacillus, 255 ; method of air examination, I Io

Petruschky's litmus whey, 46

Pfeiffer on pseudo-tuberculosis, 67

Phenol-phthalein as indicator, 3 I

Philadelphia Milk Commission, 496-500

Physiology of milk, 8

Pipettes, 48, 49, 547

Pitfield's flagella stain, 62

Pollution of milk, 2I-26

Potato as culture medium, 40

Power on York Town epidemic, 265, 266, 337 ; on Wimbledon epidemic, 267 ; on cow disease and milk, 280-285; on North London epidemic, 336 ; on Hendon disease, 337

Preparation of media, 30

Preservatives in milk, 530

Prevention of milk anomalies, $184 ;$ of milk-borne epidemics, 384

Properties of milk, 2-17

Proteids of milk, 5

Proteolytic ferments, 152

Protews vulgaris, 443 ; Zenkeri, 443

Pseudo-tuberculosis, 235

Public Health Act, $1875,456,568$

Public Health (London) Act, 456, 55 I

Public Health (Scotland) Act, 469, 578

Pus cells in milk, 28

QUALITATIVE examination of milk, 48,64

RABINoWITSCH, acid-fast bacillus of, 255 ; on tuberculous udders, $24 \mathrm{I}$

Railway transport of milk, 25, 26, 528, 529, 543

Ravenel, nutrient agar, 39 ; on tuberculosis, 228

Raw on bovine and human tuberculosis, 247

Reaction of milk, 3 ; media, $3 \mathbf{I}$

Recommendations, summary of, 543

Red milk, I74

Reed and Ward on streptococcus in milk, 223

Refrigeration of milk, 527

Regulations under the dairy orders, 455 , 462

Rennet, 167

Rennin, 9

Resistance of tubercle bacillus, $247-253$

Richards on infant milk consumption, 370 
Richmond on milk composition, 4,6 ; on seasonal variation in milk, 14,15

Ripening of cream, $187-189$

Ropy milk, 180

Rotch on variations in milk, 16 ; on modified milk supplies, $501-503$

Rotch system of milk supply, 501

Roux tubes, 85 ; on anaërobic culture, 96

Russell on artificial "starters," I95; on cheese ripening, 200, 204, 205 ; on effect of temperature on tubercle bacillus, $25 \mathrm{I}$; on pasteurisers, 536

ST PETERSBURg, milk of, II9

Salts of milk, 7

Scarlet fever, milk-borne, 279-304 ; Hendon disease, 282 ; channels of infection in, 298 ; typical outbreaks, 290-304

Schäfer on physiology of milk, 2 ; on colostrum, 12

Schwann on fermentation, 150

Scurfield on certified milk, 460,576

Secretion of milk, II

Sedgwick and Tucker on air examination, Iog

Separated milk, 6, I44

Separators, effect of, on milk bacteria, 147

Serum diagnosis of typhoid fever, 386

Set cream, I45, I46

Set milk, I45

Shaw on tuberculous London cows, 2I 4 , 592

Shennan on infantile tuberculosis, 245

Simpson on infectious diseases, 289 ; on cholera, 373

Skimmed milk, 6,8

Slides, preparations on, 53,54

Smith (Theobald) on tubercle bacillus, 234 ; on effect of temperature on tubercle bacillus, 249-25I

Soapy milk, 179

Sörensen's milk supply, 594

Sources of bacteria found in milk, 20 ; lactic acid bacteria, 190

Species of milk bacteria, 147, 392-45 I

Specificity of tuberculosis, $\mathbf{2 2 3}$

Spore formation, $840-144$

Staining methods, 55-64; simple stains, 57 ; for spores, 64 ; of capsule, 64 ; of flagella, 6I-64; of diphtheria bacillus, 68 ; by Gram's method, 59 ; by ZiehlNeelsen method, 60 ; of tubercle bacillus, 70
Standardisation of media, 31

Staphylococcus pyogenes aureus, 443

"Starters" in butter ripening, 194; in cheese making, 204

Steatolytic ferments, 153

Sterilisation of milk, 248-252, 533-542

Stewart on results of pasteurisation, 539

Still on infantile tuberculosis, 245,246

Stokes and Wegefarth on pus in milk, 28

Storch on artificial "starters," I93

Storch's mucoid, 5

Straining of milk, 525

Strauss and Wurtz on air examination, 107

Streptococci in milk, 22 I

Streptococcus Hollanaicus, 183, 203

Streptococcus pyogenes, 444

Streptococcus scarlatince, 445

Sunderland, certified milk at, 460,576

TAYLOR on milk-borne epidemics, 259-26I

Technique of milk examination, 30

Temperature, influence of, on milk, 124

Thermometer, scales of, compared, 549

Throat illnesses due to milk, 352 ; signs and symptoms of, 352 ; typical outbreaks, 354-36r

Timothy-grass bacillus, 446

Toettmaelk, 183

Torula amara, I78, 447

Tuberculosis, 210; in London cows, 213 , 592 ; in Manchester cows, 214, 592; in London milk, 217 ; bacillus of, in milk, 219; bacillus of in butter, 220; and the milk supply, 223; specificity of, 223 ; Koch's experiments on, .225; Martin's experiments on, 229; Woodhead's experiments on, 243 ; biology of bacillus, 232235 ; bovine and human, 234, 236, 247 ; entrance of bacillus into milk, 238 ; modes of infection in, 239; and milk, without udder disease, 240; of the udder, 237 ; virulence of bacillus of, 242-247 ; resistance of bacillus of, $247-253$; cultivation of bacillus of, 432; examination for bacillus of, 70

Tuberculin, 5 ro-513

Typhoid fever, milk-borne, 304-335 ; pathology of, 305 ; vehicle of infection of, 306 ; bacillus of, 3 II ; comparison of bacillus of, with $B$. coli, 4II-4I2; how milk infected by, 313 ; cause of outbreaks of, 316 ; typical outbreaks of, 316-335

Tyrothrix cateluna, 447 ; claviformis, 448 ; distortus, $44^{8}$; fliformis, $44^{8}$; genicula- 
thes, I78, 449; scaber, 449; tenuis, 450 ; turgidus, 450; wrocephalsm, 451 ; virgula, $45 I$

Tyrotoxicon, 208

ULAX strainer, 594

Unorganised ferments, 152

Unripened cream, 189

VAUGHAN on tyrotoxicon, 208

Veillon's anaërobic method, 92

Vignal's tubes, 83

Viscous milk, 180

WARRY on throat illness at Hackney, 356
Water, examination of, rro; bacteria in, II 2

Weights and measures, 598

Widal's serum diagnosis, 386

Woodhead, on virulence of tuberculous milk, 243 ; on effect of heat on tubercle bacillus, 249 ; on diphtheria bacillus in throat, 344

Wright's method of anaërobic culture, 87

YeLLow milk, 175

York, Milk Depôt at, 535, 596 ; pure milk supply at, 594

ZAMMTT on milk poisoning, 370

Ziehl-Neelsen, staining method, 60 






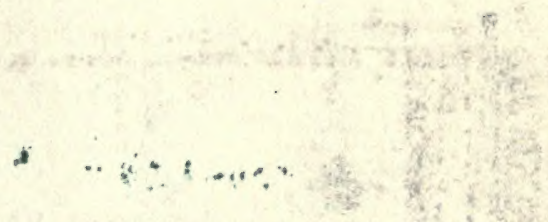

ty

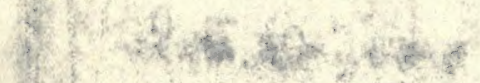

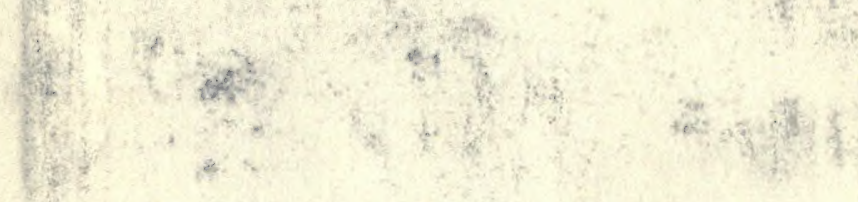




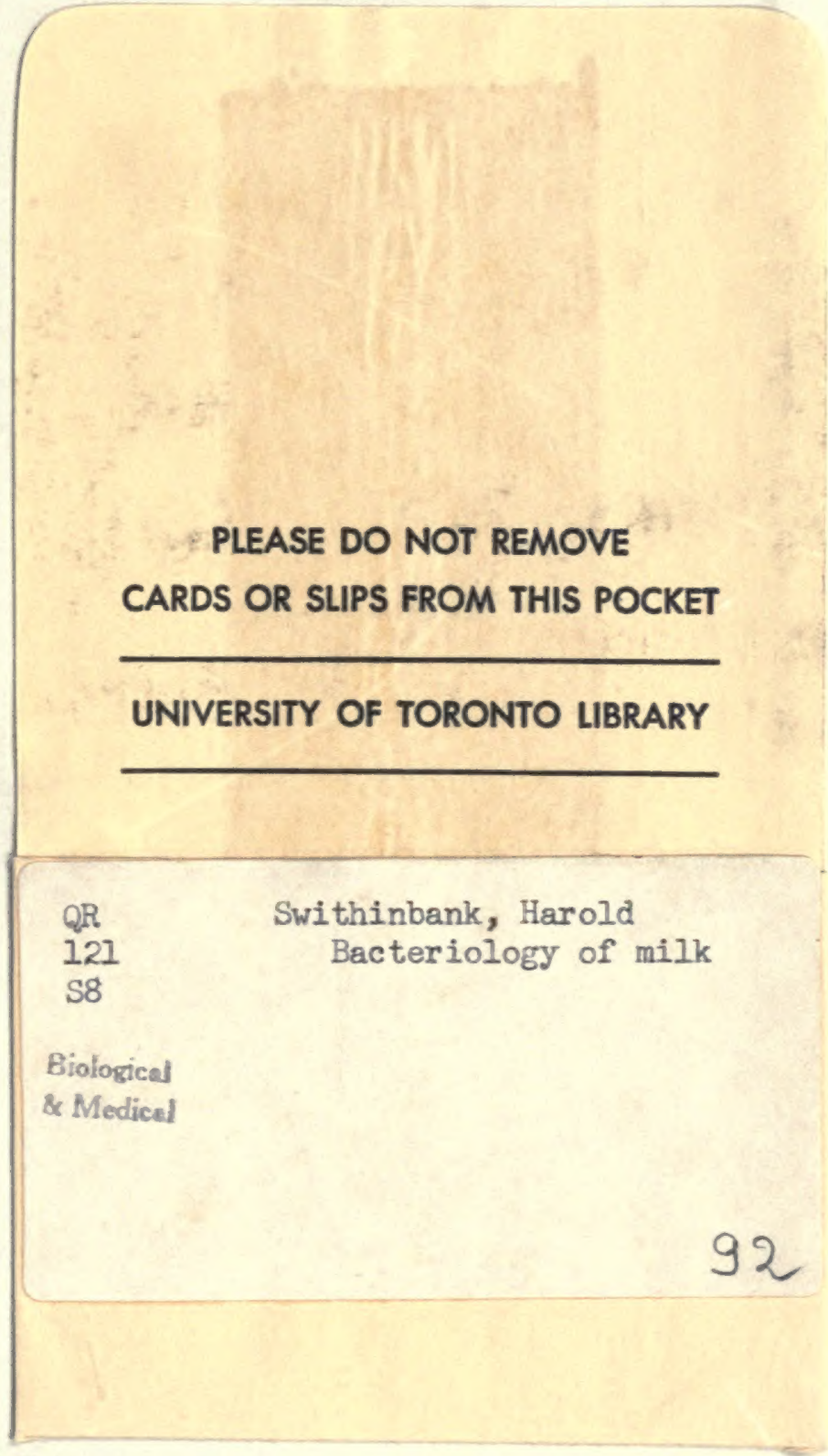


